\title{
Energy Levels and Classified Lines in the Second Spectrum of Thorium (Th II)
}

\author{
Romuald Zalubas and Charles H. Corliss
}

Institute for Basic Standards, National Bureau of Standards, Washington, D.C. 20234

(November 30, 1973)

\begin{abstract}
The analysis of Th II has been extended with improved observations of the spectrum between 2000 and $25000 \AA$. About 6500 lines are classified as transitions between 199 odd levels and 271 even levels. Of the 192 levels expected from the six odd configurations $5 f(6 d+7 s)^{2}$ and $(6 d+7 s)^{2} 7 p, 188$ are now known. In the even level system, all but one of the 37 levels of the three $(6 d+7 s)^{3}$ configurations are known. Of the 268 levels predicted for the configurations $5 f^{2} 7 s+5 f 7 s 7 p+5 f 6 d 7 p+5 f^{2} 6 d$, 235 are now known. The identifications are based on the theoretical calculations by N. Minsky. ${ }^{1}$ New Zeeman effect observations have increased the number of levels with known $g$-values to 406 out of 470 known levels. The classifications will be useful in establishing secondary standards of wavelength.
\end{abstract}

Key words: Energy levels of Th II; g-values of Th II; spectrum of Th II; Th II spectrum; thorium; wavelengths of Th II.

\section{Introduction}

The first work published on the analysis of the second spectrum of thorium (Th II) was done by McNally, Harrison, and Park [1942] ${ }^{2}$ at the M.I.T. Spectroscopy Laboratory. Their analysis was based on the new thorium wavelengths reported in the M.I.T. Wavelength Tables of Harrison [1939] and new Zeeman effect observations made with the Bitter magnet at fields of about 9 teslas. With resolved Zeeman patterns for more than 700 lines, they classified 1100 Th II lines as arising from 219 levels. Measured $g$ values were provided for each level. Many levels were identified from the Zeeman effect. The levels belonged to two unconnected groups of terms. In one group, the low even configurations were identified as $(6 d+7 s)^{3}$ and the higher odd configurations as $6 d^{2} 7 p, 6 d 7 s 7 p$, and $5 f 6 d 7 \mathrm{~s}$. In the other group the low odd levels were attributed to $5 f 7 s^{2}$ and $5 f 6 d 7 s$ and the higher evens to $5 f 7 s 7 p$ and $5 f 6 d 7 p$.

In a second paper, McNally [1945] investigated the infrared spectrum of Th II and established that the lowest odd level, $5 f 7 s^{2}{ }^{2} \mathrm{~F}_{21 / 2}^{\circ}$, lay $4490 \mathrm{~cm}^{-1}$ above the ground state. His paper gives 301 levels on a unified scale relative to the ground state. Of these levels, 21 are credited to the first paper of de Bruin, Schuurmans, and Klinkenberg [1943] (v.i.) and 17 others have been rejected since.

In an investigation which, because of the war, was entirely independent, de Bruin, Schuurmans, and

1 N. Minsky, Dissertation, Hebrew University, Jerusalem (1969).

${ }^{2}$ Literature references are at the end of this paper.
Klinkenberg [1943], [1944] at the Zeeman Laboratory in Amsterdam, classified about 1900 lines as arising from 232 levels. Measured g-values were published for every level but no list of classified lines could be printed. They found essentially the same two systems as those found at M.I.T. and in a note at the end of their second paper announced their discovery of the wavenumber difference between the two systems which later, and independently, was confirmed by McNally.

Their work was extended by Schuurmans [1946] with a discussion based on the theory of the configurations with $5 f$-electrons. He suggested tentative locations for the then undiscovered configuration $5 f^{2} 7 p$ and for $5 f^{3}$. He found 31 new levels, all but one of which are here confirmed.

In $1958 \mathrm{G}$. W. Charles prepared a compilation of available data on the first four spectra of thorium. For Th II Charles listed 165 odd and 191 even levels (including some not previously published) and 2850 classified lines, together with all known Zeeman patterns and isotope shifts. We have rejected 5 even and 14 odd levels in his list.

Recently Minsky [1969] has calculated all 13 of the important configurations of Th II as three groups in intermediate coupling with configuration interaction. They are the low even group $(6 d+7 s)^{3}$, the high even group $5 f^{2} 7 s+5 f 7 s 7 p+5 f 6 d 7 p+5 f^{2} 6 d$, and the six odd configurations $5 f 7 s^{2}+5 f 6 d 7 s+5 f 6 d^{2}+6 d 7 s 7 p$ $+7 s^{2} 7 p+6 d^{2} 7 p$. Thus Minsky's work provides a modern theoretical analysis for all the important configurations of Th II.

In addition to his extensive theoretical analysis, 
Minsky discovered 29 new levels, all of which are confirmed in this paper. Furthermore, his predictions of the locations of the undiscovered levels of the thirteen configurations have been most helpful in guiding our searches and, of course, in identifying newly found levels.

Recently Brewer [1971] has predicted the position of the lowest terms of $5 f^{2} 7 p$ at $48000: 2000 \mathrm{~cm}^{-1}, 5 f 7 p^{2}$ at $54000 \pm 4000 \mathrm{~cm}^{-1}$, and $5 f^{3}$ at $55000 \pm 5000 \mathrm{~cm}^{-1}$. In this paper we have confirmed his predictions for $f^{2} p$ and $f p^{2}$.

\section{Observations}

A summary of the observations of thorium spectra at NBS and elsewhere prior to 1960 has been given by Zalubas [1960]. His monograph presented new observations of wavelengths and intensities in electrodeless lamps and spark sources for 15000 lines of Th I and Th II in the spectral range 2000 to $11550 \AA$. It superseded all previous published descriptions of Th I and Th II in both accuracy and extent. The monograph was used by Minsky [1969] for his extension of the classification of Th II.

With the completion of the new description, work on the analysis of Th I was begun by Zalubas [1959]. As the work proceeded it became clear that the analysis could be carried on to a considerably more advanced state if the precision of the wavelengths could be improved still further. New observations were made at NBS over the period 1965-1970 with instruments of higher resolution and with improved standards. These observations will be described in detail in a forthcoming publication on thorium spectra by Zalubas.

In the region 9200 to $30000 \AA$ lines observed with the C.N.R.S. (Orsay) Fourier transform spectrometer of Connes et al. [1970] were generously furnished to us by Giacchetti. That list is discussed and published by Giacchetti, Blaise, Corliss, and Zalubas [1974]. Earlier work in the infrared region of the thorium spectra was done by Steers [1967] and by Bernage, Houdart, and Niay [1971].

The list of thorium lines now at hand has nearly 40000 lines, of which about 14,000 are regarded as belonging to Th II. This list will be published to replace the earlier list of Zalubas [1960].

In addition to the wavelength observations described above, we have Zeeman effect plates taken at the Argonne National Laboratory in 1964 in the range 2690 to $9100 \AA$ at a magnetic field of 2.4 teslas. These spectra, made with electrodeless thorium iodide lamps, provided Zeeman patterns mostly for Th I, but there were also many good resolved patterns for lines of Th II. These data were used to calculate $g$-values for Th II levels for which published values were not already available.

\section{Searches for Energy Levels}

Before we undertook searches for new energy levels in Th II, we had to improve the accuracy of the pub- lished level values, most of which were in error by more than $0.03 \mathrm{~cm}^{-1}$ and a few by more than $0.2 \mathrm{~cm}^{-1}$. With the aid of the proposed secondary-standard wavelengths of Giacchetti, Stanley, and Zalubas [1970] and Giacchetti's Fourier transform infrared list, we determined three decimal wavenumber values $\left(\mathrm{cm}^{-1}\right)$ for 82 even and 110 odd levels. With these values and our new line list we then determined two decimal values for the remaining known levels.

The searches for new energy levels were carried out on the NBS Univac 1108 using the programs called "Combo," written by Jack Tech. They were guided by the predictions of Minsky [1969]. At the conclusion of the searchers we had found 71 new even and 37 new odd levels.

This paper incorporates the results of seven investigations, each of which increased the number of known energy levels in Th II. The contribution of each to the total number of levels now regarded as real is listed below.

\begin{tabular}{|c|c|c|c|c|}
\hline Symbol & & Even & Odd & Total \\
\hline $\mathrm{H}$ & McNally et al. [1942] ......... & 112 & 102 & 214 \\
\hline B & de Bruin et al. [1943], [1944]. & 37 & 28 & 65 \\
\hline $\mathbf{R}$ & McNally [1945]................... & 8 & 5 & 13 \\
\hline $\mathrm{S}$ & Schuurmans [1946].. & 21 & 10 & 31 \\
\hline $\mathrm{C}$ & Charles [1958]........ & 7 & 3 & 10 \\
\hline M & 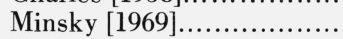 & 15 & 14 & 29 \\
\hline \multirow[t]{2}{*}{$\mathrm{Z}$} & Present paper............. & 71 & 37 & 108 \\
\hline & Total... & 271 & 199 & 470 \\
\hline
\end{tabular}

Although, as mentioned earlier, the results of McNally, Harrison and Park and of de Bruin, Schuurmans and Klinkenberg were for the most part the same, we have credited the levels in common to McNally et al. because of prior publication.

\section{Results}

\subsection{Energy' Levels}

The known energy levels are listed in numerical order in two tables: (1) the even parity levels and (2) the odd levels. The relative positions of the configurations are illustrated by the energy diagram in figure 1 .

The three low even configurations $(6 d+7 s)^{3}$ have 37 levels of which 31 were already known. We have found five of the remaining six levels, leaving only the highest level with $J=1 / 2$ undiscovered.

The four higher even configurations $4 f^{2} 6 s, 5 f 7 s 7 p$, $5 f 6 d 7 p$ and $5 f^{2} 6 d$ start between 24000 and 33000 $\mathrm{cm}^{-1}$ and consist of 268 levels, of which 235 are now known. Most of the unknown levels are above 50000 $\mathrm{cm}^{-1}$. In these configurations Minsky's correlation of observed energy levels with the calculated ones appears to be in error above $42000 \mathrm{~cm}^{-1}$ for $J=2 \frac{1}{2}$, above $41000 \mathrm{~cm}^{-1}$ for $J=3 \frac{1}{2}$, and above $43000 \mathrm{~cm}^{-1}$ for $J=4 \frac{1}{2}$. No designations are given here for these levels, only the configurations. New calculations will 
Th II electron configurations

$5 f, 6 d, 7 s$ and $7 p$ electrons

E

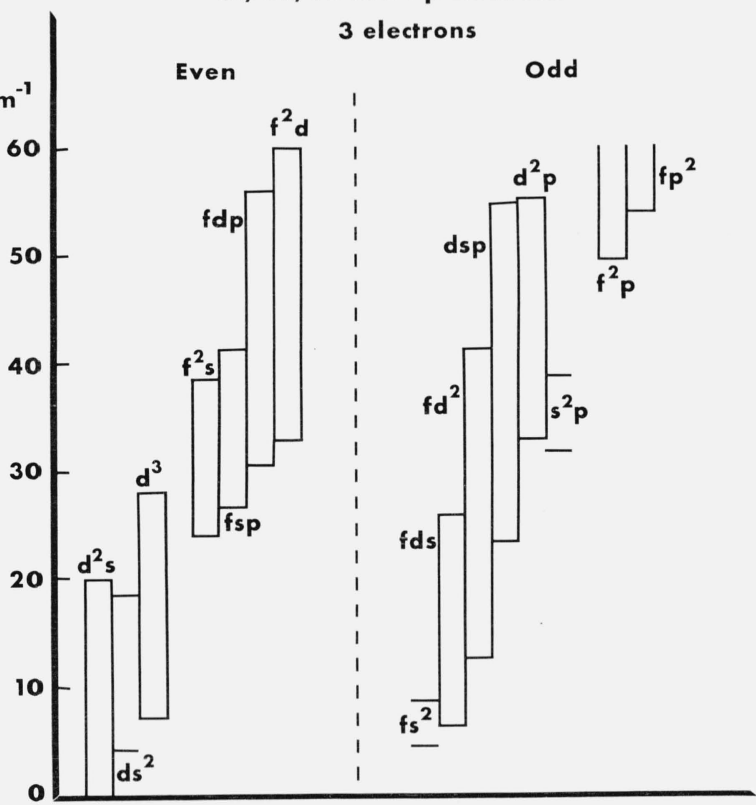

FIGURE 1. Relative positions of the 15 known electron configurations of $T h$ II.

be needed before proper correlation of energy levels with eigenvectors can be made in these cases.

The three low odd configurations $5 f(6 d+7 s)^{2}$, with 122 levels, are now all known. The lowest term, $5 f 7 s^{2}$ ${ }^{2} \mathrm{~F}^{\circ}$, was identified by McNally, Harrison, and Park [1942]. The three high odd configurations $(6 d+7 s)^{2} 7 p$ give 70 levels, of which only the two highest $J=1 / 2$ and $J=1 \frac{1}{2}$ levels have not been found.

The three higher odd configurations $5 f^{2} 7 p, 5 f 7 p^{2}$, and $5 f^{3}$ are predicted by Brewer [1971] to start near
$50000 \mathrm{~cm}^{-1}$. We have found eight likely $5 f^{2} 7 p$ levels. The lowest, at $49495.57 \mathrm{~cm}^{-1}$, may be ${ }^{4} \mathrm{I}_{41 / 2}^{\circ}$, which lies lowest, according to Hund's rule.

In tables 1 and 2 the configuration of the largest component in the eigenvector of the level is listed in the first column. The compositions are from Minsky [1969]. If this component is at least 50 percent, the term symbol is listed in the second column and serves as a designation for the level. If the largest component in the eigenvector is less than 50 percent, no name is given and the term symbol for the major component appears in column seven.

For example, the leading component of the ground state is 43 percent $6 d^{2} 7 s^{4} \mathrm{~F}_{11 / 2}$ with a second component of 27 percent $6 d 7 s^{2}{ }^{2} \mathrm{D}_{11 / 2}$. However, the level at $1859 \mathrm{~cm}^{-1}$ is 52 percent $6 d^{2} 7 s^{4} \mathrm{~F}_{11 / 2}$ and 36 percent $6 d 7 s^{2}{ }^{2} \mathrm{D}_{11 / 2}$. Therefore, it is impossible to assign a meaningful $L S$ designation to the ground level. In a spectrum with such strong intermediate coupling and configuration interactions, many levels have no meaningful names.

The $J$-value, observed level value, and observed $g$-value appear in the next three columns. The $g$-values are taken from Charles's [1958] compilation or from our own observations. The percentage contributed by the first component in the eigenvector of the level is given in the sixth column. In columns 8 and 9 appears the percentage and term for the second largest component. A very few of the levels of Th II have purity greater than 99 percent. In such cases the second component is omitted from the table.

The last columns show the approximate number of combinations observed for each level and a symbol (from the list in section 3) for the discoverer of the level.

The $6 d^{3}$ configuration has two ${ }^{2} \mathrm{D}$ terms, which are theoretically differentiated by the seniority number of Racah [1943]. We use the notations of Nielson and Koster [1963] for these terms; the ${ }^{2} \mathrm{D} 1$ term has seniority 1 and the ${ }^{2} \mathrm{D} 2$ term has seniority 3 .

TABLE 1. Even energy levels of Th II

\begin{tabular}{|c|c|c|c|c|c|c|c|c|c|}
\hline \multirow{3}{*}{$\begin{array}{l}\text { Configuration } \\
6 d^{2}\left({ }^{3} \mathrm{~F}\right) 7 s\end{array}$} & \multirow{2}{*}{ Term } & \multirow{2}{*}{$J$} & \multirow{2}{*}{$\begin{array}{l}\text { Level } \\
\left(\mathrm{cm}^{-1}\right)\end{array}$} & \multirow{2}{*}{$g$} & \multicolumn{3}{|c|}{ Leading percentages } & \multirow{2}{*}{\multicolumn{2}{|c|}{$n$, ref. }} \\
\hline & & & & & First & \multicolumn{2}{|r|}{ Second } & & \\
\hline & & $1 \frac{1 / 2}{2}$ & 0.000 & 0.639 & ${ }^{4} \mathrm{~F}$ & 27 & $6 d\left({ }^{2} \mathrm{D}\right) 7 s^{2}{ }^{2} \mathrm{D}$ & 74 & $\mathrm{H}$ \\
\hline $6 d^{2}\left({ }^{3} \mathrm{~F}\right) 7 s$ & ${ }^{4} \mathrm{~F}$ & $2^{1 / 2}$ & 1521.893 & 1.076 & 65 & 17 & $\left({ }^{1} \mathrm{D}\right){ }^{2} \mathrm{D}$ & 82 & $\mathrm{H}$ \\
\hline $6 d^{2}\left({ }^{3} \mathrm{~F}\right) 7 s$ & ${ }^{4} \mathrm{~F}$ & $1^{1 / 2}$ & 1859.936 & 0.586 & 52 & 36 & $6 d\left({ }^{2} \mathrm{D}\right) 7 s^{2}{ }^{2} \mathrm{D}$ & 64 & $\mathrm{H}$ \\
\hline $6 d\left({ }^{2} \mathrm{D}\right) 7 s^{2}$ & & $2^{1 / 2}$ & 4113.356 & 1.163 & 36 & 30 & $6 d^{2}\left({ }^{3} \mathrm{~F}\right) 7 s{ }^{4} \mathrm{~F}$ & 78 & $\mathrm{H}$ \\
\hline $6 d^{2}\left({ }^{3} \mathrm{~F}\right) 7 s$ & ${ }^{4} \mathrm{~F}$ & $3^{1 / 2}$ & 4146.575 & 1.232 & 96 & 2 & $\left({ }^{1} \mathrm{~F}\right){ }^{2} \mathrm{~F}$ & 68 & $\mathrm{H}$ \\
\hline $6 d^{2}\left({ }^{3} \mathrm{~F}\right) 7 s$ & ${ }^{4} \mathrm{~F}$ & $4^{1 / 2}$ & 6213.488 & 1.312 & 89 & 9 & $\left({ }^{1} \mathrm{G}\right){ }^{2} \mathrm{G}$ & 43 & $\mathrm{H}$ \\
\hline $6 d^{2}\left({ }^{3} \mathrm{P}\right) 7 s$ & ${ }^{4} \mathrm{P}$ & $1 / 2$ & 6244.294 & 2.112 & 70 & 13 & $\left({ }^{1} \mathrm{P}\right){ }^{2} \mathrm{P}$ & 31 & $\mathrm{H}$ \\
\hline $6 d^{3}$ & & $1 \frac{1 / 2}{2}$ & 7001.425 & 0.800 & 41 & 16 & $6 d\left({ }^{2} \mathrm{D}\right) 7 s^{2}{ }^{2} \mathrm{D}$ & 62 & $\mathrm{H}$ \\
\hline $6 d^{2}\left({ }^{1} \mathrm{P}\right) 7 s$ & & $1 / 2$ & 7828.562 & 1.254 & ${ }^{2} \mathrm{P}$ & 36 & $6 d^{3}{ }^{2} \mathrm{P}$ & 33 & $\mathrm{H}$ \\
\hline $6 d^{2}\left({ }^{3} \mathrm{P}\right) 7 s$ & ${ }^{4} \mathrm{P}$ & $11 / 2$ & 8018.191 & 1.608 & 89 & 3 & $6 d^{3}{ }^{4} \mathrm{~F}$ & 51 & $\mathrm{H}$ \\
\hline
\end{tabular}


TABLE 1. Even energy levels of Th II - Continued

\begin{tabular}{|c|c|c|c|c|c|c|c|c|c|c|}
\hline \multirow{3}{*}{$\begin{array}{l}\text { Configuration } \\
6 d^{3}\end{array}$} & \multirow{2}{*}{ Term } & \multirow{2}{*}{$J$} & \multirow{2}{*}{$\begin{array}{l}\text { Level } \\
\left(\mathrm{cm}^{-1}\right)\end{array}$} & \multirow{2}{*}{$g$} & \multicolumn{4}{|c|}{ Leading percentages } & \multirow{2}{*}{\multicolumn{2}{|c|}{$n$, ref. }} \\
\hline & & & & & \multicolumn{2}{|c|}{ First } & \multicolumn{2}{|r|}{ Second } & & \\
\hline & & $1^{1 / 2}$ & 8460.354 & 0.968 & 43 & ${ }^{4} \mathrm{~F}$ & 21 & $6 d\left({ }^{2} \mathrm{P}\right) 7 s^{2}{ }^{2} \mathrm{P}$ & 60 & $\mathrm{H}$ \\
\hline $6 d^{2}\left({ }^{1} \mathrm{~F}\right) 7 s$ & ${ }^{2} \mathrm{~F}$ & $2^{1 / 2}$ & 8605.841 & 0.986 & 62 & & 11 & $6 d\left({ }^{2} \mathrm{D}\right) 7 s^{2}{ }^{2} \mathrm{D}$ & 72 & $\mathrm{H}$ \\
\hline $6 d^{2}\left({ }^{3} \mathrm{P}\right) 7 s$ & ${ }^{4} \mathrm{P}$ & $2^{1 / 2}$ & 9061.101 & 1.419 & 71 & & 12 & $\left({ }^{1} \mathrm{~F}\right){ }^{2} \mathrm{~F}$ & 69 & $\mathrm{H}$ \\
\hline $6 d^{3}$ & ${ }^{4} \mathrm{~F}$ & $2^{1 / 2}$ & 9400.967 & 1.034 & 84 & & 6 & $6 d\left({ }^{2} \mathrm{D}\right) 7 s^{2}{ }^{2} \mathrm{D}$ & 69 & $\mathrm{H}$ \\
\hline $6 d^{2}\left({ }^{1} \mathrm{G}\right) 7 s$ & ${ }^{2} \mathrm{G}$ & $3^{1 / 2}$ & 9711.961 & 0.953 & 54 & & 27 & $6 d^{3}{ }^{2} \mathrm{G}$ & 64 & $\mathrm{H}$ \\
\hline $6 d^{2}\left({ }^{1} \mathrm{G}\right) 7 s$ & & $4^{1 / 2}$ & 10379.124 & 1.153 & 41 & ${ }^{2} \mathrm{G}$ & 30 & $6 d^{32}{ }^{2} \mathrm{G}$ & 45 & B \\
\hline $6 d^{3}$ & & $3^{1 / 2}$ & 10855.324 & 1.166 & 46 & ${ }^{4} \mathrm{~F}$ & 33 & $6 d^{2}\left({ }^{1} \mathrm{~F}\right) 7 s^{2} \mathrm{~F}$ & 57 & B \\
\hline $6 d^{2}\left({ }^{1} \mathrm{D}\right) 7 s$ & ${ }^{2} \mathrm{D}$ & $1^{1 / 2}$ & 12219.974 & 0.977 & 56 & & 11 & $6 d\left({ }^{2} \mathrm{D}\right) 7 s^{2}{ }^{2} \mathrm{D}$ & 55 & $\mathrm{H}$ \\
\hline $6 d^{3}$ & & $3^{1 / 2}$ & 12570.492 & 1.131 & 40 & ${ }^{4} \mathrm{~F}$ & 34 & $6 d^{2}\left({ }^{1} \mathrm{~F}\right) 7 s^{2} \mathrm{~F}$ & 56 & B \\
\hline $6 d^{3}$ & ${ }^{4} \mathrm{~F}$ & $4^{1 / 2}$ & 13248.709 & 1.242 & 60 & & 33 & $6 d^{2}\left({ }^{1} \mathrm{G}\right) 7 s^{2} \mathrm{G}$ & 36 & $\mathrm{H}$ \\
\hline $6 d^{2}\left({ }^{1} \mathrm{D}\right) 7 s$ & & $2^{1 / 2}$ & 13250.505 & 1.245 & 44 & ${ }^{2} \mathrm{D}$ & 20 & $6 d\left({ }^{2} \mathrm{D}\right) 7 s^{2}{ }^{2} \mathrm{D}$ & 48 & $\mathrm{H}$ \\
\hline $6 d^{3}$ & ${ }^{4} \mathrm{P}$ & $1 / 2$ & 14349.390 & 2.555 & 93 & & 5 & $6 d^{2}\left({ }^{1} \mathrm{P}\right) 7 s^{2} \mathrm{P}$ & 22 & $\mathrm{H}$ \\
\hline $6 d^{3}$ & ${ }^{4} \mathrm{P}$ & $1^{1 / 2}$ & 15236.639 & 1.592 & 71 & & 19 & $6 d^{2}\left({ }^{1} \mathrm{P}\right) 7 s^{2} \mathrm{P}$ & 36 & $\mathrm{H}$ \\
\hline $6 d^{3}$ & ${ }^{2} \mathrm{H}$ & $4^{1 / 2}$ & 15305.265 & 1.006 & 69 & & 16 & ${ }^{4} \mathrm{~F}$ & 35 & $\mathrm{H}$ \\
\hline $6 d^{3}$ & ${ }^{4} \mathrm{P}$ & $2^{1 / 2}$ & 15786.986 & 1.571 & 91 & & 4 & ${ }^{2} \mathrm{Dl}$ & 36 & B \\
\hline $6 d^{3}$ & ${ }^{2} \mathrm{G}$ & $3^{1 / 2}$ & 16818.067 & 0.916 & 58 & & 32 & $6 d^{2}\left({ }^{1} \mathrm{G}\right) 7 s^{2} \mathrm{G}$ & 45 & B \\
\hline $6 d^{3}$ & ${ }^{2} \mathrm{H}$ & $5^{1 / 2}$ & 17727.251 & 1.09 & 99 & & & & 20 & $\mathrm{Z}$ \\
\hline $6 d^{3}$ & & $1^{1 / 2}$ & 18118.705 & 0.93 & 44 & ${ }^{2} \mathrm{D} 2$ & 20 & ${ }^{2} \mathrm{D} 1$ & 35 & M \\
\hline $6 d^{2}\left({ }^{1} \mathrm{~S}\right) 7 s$ & ${ }^{2} \mathrm{~S}$ & $1 / 2$ & 19594.350 & & 80 & & 10 & $6 d^{32} \mathrm{P}$ & 12 & $\mathrm{Z}$ \\
\hline $6 d^{3}$ & ${ }^{2} \mathrm{G}$ & $4^{1 / 2}$ & 19880.083 & 1.08 & 59 & & 22 & ${ }^{2} \mathrm{H}$ & 23 & $\mathrm{Z}$ \\
\hline $6 d^{3}$ & ${ }^{2} \mathrm{D} 2$ & $2^{1 / 2}$ & 20158.744 & 1.19 & 67 & & 12 & $6 d^{2}\left({ }^{1} \mathrm{D}\right) 7 s^{2} \mathrm{D}$ & 38 & M \\
\hline $6 d^{3}$ & ${ }^{2} \mathrm{~F}$ & $2^{1 / 2}$ & 22106.437 & 0.92 & 71 & & 8 & $6 d^{2}\left({ }^{1} \mathrm{~F}\right) 7 s^{2} \mathrm{~F}$ & 36 & $\mathrm{Z}$ \\
\hline $6 d^{3}$ & ${ }^{2} \mathrm{~F}$ & $3^{1 / 2}$ & 22834.138 & 1.12 & 82 & & 15 & $6 d^{2}\left({ }^{1} \mathrm{~F}\right) 7 s^{2} \mathrm{~F}$ & 31 & M \\
\hline $5 f^{2}\left({ }^{3} \mathrm{H}\right) 7 s$ & ${ }^{4} \mathrm{H}$ & $3^{1 / 2}$ & 24381.802 & 0.70 & 74 & & 12 & $5 f 6 d\left({ }^{3} \mathrm{G}^{\circ}\right) 7 p{ }^{4} \mathrm{H}$ & 21 & M \\
\hline $5 f^{2}\left({ }^{3} \mathrm{H}\right) 7 s$ & & $4^{1 / 2}$ & 25246.298 & 0.96 & 45 & ${ }^{4} \mathrm{H}$ & 33 & $\left({ }^{3} \mathrm{H}\right){ }^{2} \mathrm{H}$ & 20 & $\mathrm{R}$ \\
\hline $6 d^{3}$ & & $1^{1 / 2}$ & 25381.927 & 1.25 & 46 & ${ }^{2} \mathrm{P}$ & 34 & $6 d^{2}\left({ }^{1} \mathrm{P}\right) 7 s^{2} \mathrm{P}$ & 9 & $\mathrm{Z}$ \\
\hline $5 f 7 s\left({ }^{3} \mathrm{~F}^{\circ}\right) 7 p$ & & $2^{1 / 2}$ & 26488.644 & 0.776 & 43 & ${ }^{4} \mathrm{G}$ & 16 & $\left({ }^{3} \mathrm{~F}^{\circ}\right){ }^{4} \mathrm{~F}$ & 18 & $\mathrm{H}$ \\
\hline $5 f^{2}\left({ }^{3} \mathrm{~F}\right) 7 s$ & & $1 \frac{1 / 2}{2}$ & 26762.272 & 0.47 & 42 & ${ }^{4} \mathrm{~F}$ & 17 & $5 f 6 d\left({ }^{3} \mathrm{~F}^{\circ}\right) 7 p{ }^{4} \mathrm{~F}$ & 18 & B \\
\hline $5 f 7 s\left({ }^{3} \mathrm{~F}^{\circ}\right) 7 p$ & & $3^{1 / 2}$ & 27257.144 & 1.032 & 32 & ${ }^{4} \mathrm{G}$ & 15 & $\left({ }^{1} \mathrm{~F}^{\circ}\right)^{2} \mathrm{G}$ & 23 & $\mathrm{H}$ \\
\hline $5 f^{2}\left({ }^{3} \mathrm{H}\right) 7 s$ & & $4^{1 / 2}$ & 27526.948 & 0.93 & 42 & ${ }^{2} \mathrm{H}$ & 37 & $\left({ }^{3} \mathrm{H}\right){ }^{4} \mathrm{H}$ & 20 & $\mathrm{~S}$ \\
\hline $5 f^{2}\left({ }^{3} \mathrm{~F}\right) 7 s$ & & $2^{1 / 2}$ & 27593.965 & 0.963 & 37 & ${ }^{4} \mathrm{~F}$ & 17 & $5 f 7 s\left({ }^{3} \mathrm{~F}^{\circ}\right) 7 p{ }^{4} \mathrm{G}$ & 23 & $\mathrm{~B}$ \\
\hline $5 f 7 s\left({ }^{3} \mathrm{~F}^{\circ}\right) 7 p$ & & $1 \frac{1}{2}$ & 27631.225 & 0.625 & 45 & ${ }^{4} \mathrm{~F}$ & 25 & $5 f^{2}\left({ }^{3} \mathrm{~F}\right) 7 s^{4} \mathrm{~F}$ & 14 & $\mathrm{H}$ \\
\hline $5 f^{2}\left({ }^{3} \mathrm{H}\right) 7 s$ & ${ }^{4} \mathrm{H}$ & $5 \frac{1}{2}$ & 27937.074 & 1.12 & 73 & & 14 & $\left({ }^{3} \mathrm{H}\right){ }^{2} \mathrm{H}$ & 13 & $\mathrm{Z}$ \\
\hline $6 d^{3}$ & ${ }^{2} \mathrm{Dl}$ & $1^{1 / 2}$ & 28011.159 & 0.717 & 52 & & 36 & ${ }^{2} \mathrm{D} 3$ & 11 & $\mathrm{H}$ \\
\hline $6 d^{3}$ & ${ }^{2} \mathrm{Dl}$ & $2^{1 / 2}$ & 28026.347 & 1.13 & 70 & & 14 & ${ }^{2} \mathrm{~F}$ & 15 & $\mathrm{~S}$ \\
\hline
\end{tabular}


TABLE 1. Even energy levels of Th II - Continued

\begin{tabular}{|c|c|c|c|c|c|c|c|c|c|c|}
\hline \multirow{3}{*}{$\begin{array}{l}\text { Configuration } \\
5 f 7 s\left({ }^{3} \mathrm{~F}^{\circ}\right) 7 p\end{array}$} & \multirow{3}{*}{ Term } & \multirow{3}{*}{\begin{tabular}{|c|}
$J$ \\
$21 / 2$
\end{tabular}} & \multirow{3}{*}{$\begin{array}{c}\begin{array}{c}\text { Level } \\
\left(\mathrm{cm}^{-1}\right)\end{array} \\
28823.649\end{array}$} & \multirow{3}{*}{$\begin{array}{c}g \\
0.987\end{array}$} & \multicolumn{4}{|c|}{ Leading percentages } & \multirow{2}{*}{\multicolumn{2}{|c|}{$n$, ref. }} \\
\hline & & & & & \multicolumn{2}{|c|}{ First } & \multicolumn{2}{|r|}{ Second } & & \\
\hline & & & & & 26 & ${ }^{4} \mathbf{F}$ & 15 & $\left({ }^{3} \mathrm{~F}^{\circ}\right){ }^{4} \mathrm{D}$ & 18 & $\mathrm{H}$ \\
\hline $5 f^{2}\left({ }^{3} \mathrm{~F}\right) 7 s$ & & $2^{1 / 2}$ & 29345.894 & 0.935 & 39 & ${ }^{2} \mathbf{F}$ & 29 & $\left({ }^{3} \mathrm{~F}\right){ }^{4} \mathrm{~F}$ & 24 & B \\
\hline $5 f^{2}\left({ }^{3} \mathrm{~F}\right) 7 s$ & ${ }^{4} \mathrm{~F}$ & $3^{1 / 2}$ & 29431.847 & 1.1 & 63 & & 11 & $5 f 6 d\left({ }^{3} \mathrm{~F}^{0}\right) 7 p{ }^{4} \mathrm{~F}$ & 23 & M \\
\hline $5 f^{2}\left({ }^{3} \mathrm{~F}\right) 7 s$ & & $4^{1 / 2}$ & 29515.132 & 1.030 & 32 & ${ }^{4} \mathbf{F}$ & 13 & $\left({ }^{1} \mathrm{G}\right){ }^{2} \mathrm{G}$ & 25 & $\mathrm{H}$ \\
\hline $5 f^{2}\left({ }^{3} \mathrm{~F}\right) 7 s$ & & $3^{1 / 2}$ & 29873.948 & 1.10 & 44 & ${ }^{2} \mathbf{F}$ & 18 & $\left({ }^{3} \mathrm{~F}\right){ }^{2} \mathrm{G}$ & 19 & B \\
\hline $5 f 6 d\left({ }^{3} \mathrm{H}^{\circ}\right) 7 p$ & & $4^{1 / 2}$ & 30452.723 & 1.035 & 41 & ${ }^{4} \mathbf{I}$ & 14 & $\left({ }^{3} \mathrm{H}^{\circ}\right){ }^{2} \mathrm{H}$ & 22 & $\mathrm{H}$ \\
\hline $5 f^{2}\left({ }^{3} \mathrm{H}\right) 7 s$ & ${ }^{2} \mathrm{H}$ & $5^{1 / 2}$ & 30484.713 & 1.08 & 72 & & 12 & $\left({ }^{3} \mathrm{H}\right){ }^{4} \mathrm{H}$ & 17 & M \\
\hline $5 f^{2}\left({ }^{3} \mathrm{H}\right) 7 s$ & ${ }^{4} \mathrm{H}$ & $6^{1 / 2}$ & 30548.67 & 1.23 & 87 & & 6 & $5 f 6 d\left({ }^{3} \mathrm{G}^{\circ}\right) 7 p{ }^{4} \mathrm{H}$ & 4 & $\mathrm{Z}$ \\
\hline $5 f 7 s\left({ }^{3} \mathrm{~F}^{\circ}\right) 7 p$ & & $3^{1 / 2}$ & 30879.418 & 1.213 & 35 & ${ }^{4} \mathbf{F}$ & 25 & $\left({ }^{3} \mathrm{~F}^{\circ}\right){ }^{4} \mathrm{D}$ & 22 & B \\
\hline $5 f 6 d\left({ }^{3} \mathrm{~F}^{\circ}\right) 7 p$ & & $2^{1 / 2}$ & 31259.295 & 0.781 & 27 & ${ }^{4} \mathrm{G}$ & 15 & $5 f^{2}\left({ }^{3} \mathrm{~F}\right) 7 s^{2} \mathrm{~F}$ & 22 & $\mathrm{H}$ \\
\hline $5 f 7 s\left({ }^{1} \mathbf{F}^{\circ}\right) 7 p$ & & $2^{1 / 2}$ & 31754.210 & 0.948 & 36 & ${ }^{2} \mathbf{F}$ & 23 & $\left({ }^{1} \mathrm{~F}^{\circ}\right){ }^{2} \mathrm{D}$ & 21 & $\mathrm{H}$ \\
\hline $5 f 7 s\left({ }^{3} \mathbf{F}^{\circ}\right) 7 p$ & & $4^{1 / 2}$ & 31773.077 & 1.222 & 35 & ${ }^{4} \mathrm{G}$ & 30 & $\left({ }^{3} \mathrm{~F}^{\circ}\right){ }^{4} \mathrm{~F}$ & 21 & $\mathrm{H}$ \\
\hline $5 f 7 s\left({ }^{1} \mathbf{F}^{\circ}\right) 7 p$ & & $3^{1 / 2}$ & 32576.745 & 1.024 & 22 & ${ }^{2} \mathrm{G}$ & 18 & $5 f^{2}\left({ }^{3} \mathbf{F}\right) 7 s^{2} \mathbf{F}$ & 27 & $\mathrm{H}$ \\
\hline $5 f^{2}\left({ }^{3} \mathrm{H}\right) 6 d$ & ${ }^{4} \mathrm{~K}$ & $5^{1 / 2}$ & 32620.859 & 0.826 & 72 & & 11 & $\left({ }^{3} \mathrm{H}\right){ }^{2} \mathrm{I}$ & 9 & $\mathrm{H}$ \\
\hline $5 f 6 d\left({ }^{3} \mathrm{H}^{\circ}\right) 7 p$ & & $3^{1 / 2}$ & 32736.186 & 0.904 & 24 & ${ }^{4} \mathrm{H}$ & 13 & $\left({ }^{3} \mathrm{H}^{\circ}\right){ }^{2} \mathrm{G}$ & 24 & $\mathrm{H}$ \\
\hline $5 f 6 d\left({ }^{1} \mathrm{D}^{\circ}\right) 7 p$ & & $1^{1 / 2}$ & 32959.48 & 0.874 & 23 & ${ }^{2} \mathrm{D}$ & 15 & $\left({ }^{1} \mathrm{D}^{\circ}\right){ }^{2} \mathrm{P}$ & 16 & $\mathrm{H}$ \\
\hline $5 f 7 s\left({ }^{3} \mathbf{F}^{\circ}\right) 7 p$ & & $3^{1 / 2}$ & 33209.40 & 1.051 & 15 & ${ }^{4} \mathrm{G}$ & 13 & $\left({ }^{1} \mathrm{~F}^{\circ}\right)^{2} \mathrm{~F}$ & 29 & $\mathrm{H}$ \\
\hline $5 f^{2}\left({ }^{3} \mathrm{~F}\right) 7 s$ & & $4^{1 / 2}$ & 33384.444 & 1.14 & 26 & ${ }^{4} \mathrm{~F}$ & 16 & $\left({ }^{1} \mathrm{G}\right){ }^{2} \mathrm{G}$ & 24 & B \\
\hline $5 f^{2}\left({ }^{3} \mathrm{~F}\right) 6 d$ & & $3^{1 / 2}$ & 33637.228 & 0.830 & 17 & ${ }^{4} \mathrm{H}$ & 11 & $\left({ }^{3} \mathrm{H}\right){ }^{4} \mathrm{H}$ & 22 & $\mathrm{H}$ \\
\hline $5 f^{2}\left({ }^{1} \mathrm{D}\right) 7 s$ & & $2^{1 / 2}$ & 33730.94 & 1.031 & 13 & ${ }^{2} \mathrm{D}$ & 8 & $5 f 6 d\left({ }^{1} \mathrm{D}^{\circ}\right) 7 p{ }^{2} \mathrm{D}$ & 23 & $\mathrm{H}$ \\
\hline $5 f^{2}\left({ }^{1} \mathrm{D}\right) 7 s$ & & $1^{1 / 2}$ & 34019.240 & 0.823 & 25 & ${ }^{2} D$ & 21 & $5 f 6 d\left({ }^{3} \mathrm{~F}^{\circ}\right) 7 p{ }^{2} \mathrm{D}$ & 18 & B \\
\hline $5 f^{2}\left({ }^{1} \mathrm{D}\right) 7 s$ & & $2^{1 / 2}$ & 34174.540 & 0.986 & 22 & ${ }^{2} \mathrm{D}$ & 12 & $5 f 6 d\left({ }^{3} \mathrm{~F}^{\circ}\right) 7 p{ }^{4} \mathrm{G}$ & 27 & $\mathrm{H}$ \\
\hline $5 f^{2}\left({ }^{3} \mathrm{H}\right) 6 d$ & & $4^{1 / 2}$ & 34270.25 & 0.938 & 42 & ${ }^{2} \mathrm{H}$ & 11 & $\left({ }^{3} \mathrm{~F}\right){ }^{2} \mathrm{H}$ & 22 & $\mathrm{H}$ \\
\hline $5 f^{2}\left({ }^{1} \mathrm{G}\right) 7 s$ & & $3^{1 / 2}$ & 34279.321 & 1.047 & 26 & ${ }^{2} \mathrm{G}$ & 14 & $5 f 7 s\left({ }^{1} \mathrm{~F}^{\circ}\right) 7 p{ }^{2} \mathrm{G}$ & 34 & $\mathrm{H}$ \\
\hline $5 f 7 s\left({ }^{3} \mathbf{F}^{\circ}\right) 7 p$ & & $2^{1 / 2}$ & 34543.551 & 1.003 & 28 & ${ }^{4} \mathrm{D}$ & 13 & $\left({ }^{3} \mathrm{~F}^{\circ}\right){ }^{4} \mathrm{~F}$ & 29 & $\mathrm{H}$ \\
\hline $5 f 7 s\left({ }^{3} \mathbf{F}^{\circ}\right) 7 p$ & & $4^{1 / 2}$ & 34553.950 & 1.069 & 19 & ${ }^{4} \mathrm{G}$ & 15 & $\left({ }^{3} \mathrm{~F}^{\circ}\right){ }^{4} \mathrm{~F}$ & 28 & $\mathrm{H}$ \\
\hline $5 f 6 d\left({ }^{3} \mathrm{H}^{\circ}\right) 7 p$ & ${ }^{4} \mathbf{I}$ & $5^{1 / 2}$ & 34661.718 & 0.998 & 50 & & 12 & $\left({ }^{3} \mathrm{H}^{\circ}\right){ }^{2} \mathrm{H}$ & 19 & $\mathrm{H}$ \\
\hline $5 f 6 d\left({ }^{3} \mathrm{~F}^{\circ}\right) 7 p$ & & $3^{1 / 2}$ & 34726.58 & 0.950 & 29 & ${ }^{4} \mathrm{G}$ & 18 & $5 f^{2}\left({ }^{3} \mathbf{F}\right) 6 d{ }^{4} G$ & 22 & $\mathrm{H}$ \\
\hline $5 f^{2}\left({ }^{3} \mathrm{P}\right) 7 s$ & ${ }^{4} \mathrm{P}$ & $1 / 2$ & 34782.74 & 1.67 & 57 & & 14 & $5 f 6 d\left({ }^{3} \mathrm{P}^{\circ}\right) 7 p{ }^{4} \mathrm{P}$ & 6 & $\mathrm{~S}$ \\
\hline $5 f 7 s\left({ }^{3} \mathbf{F}^{\circ}\right) 7 p$ & ${ }^{4} \mathrm{D}$ & $1^{1 / 2}$ & 35021.37 & 1.042 & 57 & & 15 & $\left({ }^{3} \mathrm{~F}^{\circ}\right){ }^{4} \mathrm{~F}$ & 18 & $\mathrm{~B}$ \\
\hline $5 f^{2}\left({ }^{3} \mathrm{H}\right) 6 d$ & ${ }^{4} \mathrm{~K}$ & $6^{1 / 2}$ & 35400.78 & 0.98 & 91 & & 3 & $\left({ }^{1} \mathrm{G}\right){ }^{2} \mathrm{I}$ & 6 & $\mathrm{Z}$ \\
\hline $5 f 7 s\left({ }^{3} \mathbf{F}^{\circ}\right) 7 p$ & ${ }^{4} \mathrm{D}$ & $1 / 2$ & 35425.627 & 0.265 & 88 & & 2 & $5 f^{2}\left({ }^{3} \mathbf{F}\right) 6 d{ }^{4} \mathrm{D}$ & 7 & $\mathrm{H}$ \\
\hline $5 f 6 d\left({ }^{1} \mathrm{G}^{\circ}\right) 7 p$ & & $4^{1 / 2}$ & 35456.181 & 1.054 & 23 & ${ }^{2} \mathrm{H}$ & 13 & $5 f^{2}\left({ }^{1} \mathrm{G}\right) 7 s^{2} \mathrm{G}$ & 25 & $\mathrm{H}$ \\
\hline $5 f^{2}\left({ }^{3} \mathrm{H}\right) 6 d$ & & $5^{1 / 2}$ & 35525.169 & 1.024 & 18 & ${ }^{2} \mathrm{H}$ & 17 & $\left({ }^{3} \mathrm{H}\right){ }^{4} \mathrm{~K}$ & 16 & B \\
\hline $5 f^{2}\left({ }^{3} \mathrm{H}\right) 6 d$ & ${ }^{4} \mathrm{I}$ & $4^{1 / 2}$ & 35545.60 & 0.826 & 71 & & 8 & $\left({ }^{1} \mathrm{G}\right){ }^{2} \mathrm{H}$ & 19 & $\mathrm{H}$ \\
\hline
\end{tabular}


TABLE 1. Even energy levels of Th II - Continued

\begin{tabular}{|c|c|c|c|c|c|c|c|c|c|c|}
\hline \multirow{2}{*}{ Configuration } & \multirow{2}{*}{ Term } & \multirow{2}{*}{$J$} & \multirow{2}{*}{$\begin{array}{l}\text { Level } \\
\left(\mathrm{cm}^{-1}\right)\end{array}$} & \multirow{2}{*}{$g$} & \multicolumn{4}{|c|}{ Leading percentages } & \multirow{2}{*}{\multicolumn{2}{|c|}{$n$, ref. }} \\
\hline & & & & & & First & & Second & & \\
\hline $5 f\left(6 d\left({ }^{3} \mathrm{~F}^{\circ}\right) 7 p\right.$ & & $2^{1 / 2}$ & 35741.295 & 0.954 & 19 & ${ }^{4} \mathrm{G}$ & 9 & $\left({ }^{3} \mathrm{~F}^{\circ}\right) \quad{ }^{2} \mathrm{D}$ & 29 & $\mathrm{H}$ \\
\hline $5 f 6 d\left({ }^{1} \mathrm{G}^{\circ}\right) 7 p$ & & $3^{1 / 2}$ & 35878.862 & 0.983 & 17 & ${ }^{2} \mathrm{~F}$ & 11 & $\left({ }^{3} \mathrm{H}^{\circ}\right){ }^{4} \mathrm{H}$ & 31 & $\mathrm{H}$ \\
\hline $5 f^{2}\left({ }^{3} \mathbf{F}\right) 6 d$ & & $1 / 2$ & 36040.85 & 1.210 & 29 & ${ }^{2} \mathrm{P}$ & 28 & $5 f^{2}\left({ }^{3} \mathrm{P}\right) 7 s{ }^{2} \mathrm{P}$ & 11 & $\mathrm{H}$ \\
\hline $5 f^{2}\left({ }^{3} \mathrm{~F}\right) 6 d$ & & $2^{1 / 2}$ & 36065.729 & 0.887 & 42 & ${ }^{4} \mathrm{G}$ & 10 & $\left({ }^{3} \mathrm{H}\right){ }^{4} \mathrm{G}$ & 26 & $\mathrm{H}$ \\
\hline $5 f^{2}\left({ }^{3} \mathrm{~F}\right) 6 d$ & & $4^{1 / 2}$ & 36125.335 & 1.035 & 22 & ${ }^{4} \mathrm{H}$ & 19 & $\left({ }^{3} \mathrm{H}\right){ }^{4} \mathrm{H}$ & 24 & $\mathrm{H}$ \\
\hline $5 f^{2}\left({ }^{3} \mathrm{P}\right) 7 s$ & ${ }^{4} \mathrm{P}$ & $1^{1 / 2}$ & 36328.61 & 1.615 & 64 & & 10 & $5 f 6 d\left({ }^{3} \mathrm{P}^{\circ}\right) 7 p{ }^{4} \mathrm{P}$ & 17 & $\mathrm{H}$ \\
\hline $5 f 6 d\left({ }^{1} G^{\circ}\right) 7 p$ & & $3^{1 / 2}$ & 36809.279 & 1.022 & 13 & ${ }^{2} \mathrm{~F}$ & 13 & $5 f^{2}\left({ }^{3} \mathrm{~F}\right) 6 d{ }^{4} \mathrm{H}$ & 33 & $\mathrm{H}$ \\
\hline $5 f^{2}\left({ }^{3} \mathrm{P}\right) 7 s$ & & $1 / 2$ & 36812.44 & 0.737 & 36 & ${ }^{2} \mathrm{P}$ & 17 & $5 f\left(6 d\left({ }^{3} \mathrm{D}^{\circ}\right) 7 p{ }^{2} \mathrm{P}\right.$ & 13 & $\mathrm{H}$ \\
\hline $5 f 7 s\left({ }^{3} \mathbf{F}^{\circ}\right) 7 p$ & & $5^{1 / 2}$ & 37053.44 & & 19 & ${ }^{4} \mathrm{G}$ & 14 & $5 f^{2}\left({ }^{3} \mathrm{H}\right) 6 d^{2} \mathrm{I}$ & 13 & $\mathrm{Z}$ \\
\hline $5 f 6 d\left({ }^{3} \mathrm{H}^{\circ}\right) 7 p$ & & $4^{1 / 2}$ & 37063.308 & 0.999 & 29 & ${ }^{4} \mathrm{H}$ & 13 & $5 f^{2}\left({ }^{3} \mathrm{~F}\right) 6 d{ }^{4} \mathrm{H}$ & 27 & $\mathrm{H}$ \\
\hline $5 f 6 d\left({ }^{3} \mathrm{~F}^{\circ}\right) 7 p$ & & $3^{1 / 2}$ & 37277.368 & 0.881 & 16 & ${ }^{4} \mathrm{G}$ & 13 & $5 f^{2}\left({ }^{3} \mathrm{H}\right) 6 d{ }^{4} \mathrm{G}$ & 26 & $\mathrm{H}$ \\
\hline $5 f 6 d\left({ }^{3} \mathbf{F}^{\circ}\right) 7 p$ & & $2^{1 / 2}$ & 37465.46 & 1.048 & 18 & ${ }^{4} \mathrm{D}$ & 15 & $\left({ }^{3} \mathrm{~F}^{\circ}\right)^{2} \mathrm{D}$ & 28 & $\mathrm{H}$ \\
\hline $5 f^{2}\left({ }^{3} \mathrm{P}\right) 7 s$ & & $1^{1 / 2}$ & 37542.17 & 1.003 & 21 & ${ }^{2} \mathrm{P}$ & 13 & $5 f 6 d\left({ }^{1} \mathrm{D}^{\circ}\right) 7 p{ }^{2} \mathrm{D}$ & 22 & $\mathrm{H}$ \\
\hline $5 f^{2}\left({ }^{3} \mathrm{H}\right) 6 d$ & ${ }^{4} \mathbf{I}$ & $5^{1 / 2}$ & 37562.27 & 1.008 & 61 & & 14 & $5 f 7 s\left({ }^{3} \mathbf{F}^{\circ}\right) 7 p{ }^{4} \mathrm{G}$ & 13 & $\mathrm{R}$ \\
\hline $5 f^{2}\left({ }^{1} \mathbf{I}\right) 7 s$ & ${ }^{2} \mathbf{I}$ & $6^{1 / 2}$ & 37575.24 & 1.088 & 77 & & 6 & $5 f 6 d\left({ }^{1} \mathrm{H}^{\circ}\right) 7 p{ }^{2} \mathbf{I}$ & 10 & $\mathrm{R}$ \\
\hline $5 f 7 s\left({ }^{3} \mathbf{F}^{\circ}\right) 7 p$ & & $5^{1 / 2}$ & 37679.71 & 1.13 & 31 & ${ }^{4} \mathrm{G}$ & 11 & $5 f 6 d\left({ }^{3} \mathrm{H}^{\circ}\right) 7 p{ }^{4} \mathrm{G}$ & 18 & $\mathrm{R}$ \\
\hline $5 f 6 d\left({ }^{3} \mathrm{G}^{\circ}\right) 7 p$ & & $3^{1 / 2}$ & 37787.89 & 1.062 & 26 & ${ }^{4} \mathrm{H}$ & 11 & $\left({ }^{3} G^{\circ}\right)^{4} G$ & 27 & B \\
\hline $5 f 7 s\left({ }^{3} \mathrm{~F}^{\circ}\right) 7 p$ & & $1^{1 / 2}$ & 37821.97 & 1.15 & 13 & ${ }^{2} \mathrm{D}$ & 7 & $5 f 6 d\left({ }^{3} \mathrm{~F}^{\circ}\right) 7 p \quad{ }^{4} \mathbf{F}$ & 17 & B \\
\hline $5 f^{2}\left({ }^{3} \mathbf{F}\right) 6 d$ & & $4^{1 / 2}$ & 37840.845 & 1.07 & 35 & ${ }^{4} \mathrm{G}$ & 15 & $\left({ }^{3} \mathrm{H}\right){ }^{4} \mathrm{G}$ & 21 & $\mathrm{~S}$ \\
\hline $5 f 7 s\left({ }^{3} \mathbf{F}^{\circ}\right) 7 p$ & & $2^{1 / 2}$ & 37945.104 & 0.893 & 32 & ${ }^{2} \mathrm{~F}$ & 16 & $5 f 6 d\left({ }^{1} \mathrm{G}^{\circ}\right) 7 p{ }^{2} \mathbf{F}$ & 28 & $\mathrm{H}$ \\
\hline $5 f^{2}\left({ }^{3} \mathrm{P}\right) 7 s$ & & $2^{1 / 2}$ & 38105.065 & 1.172 & 33 & ${ }^{4} \mathrm{P}$ & 9 & $5 f 6 d\left({ }^{3} \mathrm{D}^{\circ}\right) 7 p{ }^{4} \mathbf{P}$ & 25 & $\mathrm{H}$ \\
\hline $5 f^{2}\left({ }^{3} \mathrm{H}\right) 6 d$ & ${ }^{4} \mathrm{~K}$ & $7^{1 / 2}$ & 38107.11 & 1.08 & 99 & & & & 2 & $\mathrm{Z}$ \\
\hline $5 f 7 s\left({ }^{1} \mathbf{F}^{\circ}\right) 7 p$ & & $3^{1 / 2}$ & 38165.355 & 0.981 & 11 & ${ }^{2} \mathrm{G}$ & 7 & $5 f 6 d\left({ }^{3} \mathbf{G}^{\circ}\right) 7 p{ }^{2} \mathbf{F}$ & 30 & $\mathrm{H}$ \\
\hline $5 f 6 d\left({ }^{3} \mathrm{H}^{\circ}\right) 7 p$ & & $41 / 2$ & 38179.941 & 0.979 & 29 & ${ }^{2} \mathrm{H}$ & 14 & $\left({ }^{3} \mathrm{H}^{\circ}\right){ }^{4} \mathrm{I}$ & 25 & $\mathrm{H}$ \\
\hline $5 f 7 s\left({ }^{3} \mathbf{F}^{\circ}\right) 7 p$ & & $3^{1 / 2}$ & 38291.801 & 0.949 & 19 & ${ }^{2} \mathrm{G}$ & 12 & $5 f^{2}\left({ }^{3} \mathrm{~F}\right) 6 d{ }^{4} \mathrm{G}$ & 31 & $\mathrm{H}$ \\
\hline $5 f^{2}\left({ }^{3} \mathrm{~F}\right) 6 d$ & & $1^{1 / 2}$ & 38372.02 & 1.200 & 15 & ${ }^{4} \mathrm{P}$ & 15 & $5 f 6 d\left({ }^{1} \mathrm{D}^{\circ}\right) 7 p{ }^{2} \mathrm{P}$ & 13 & B \\
\hline $5 f 7 s\left({ }^{3} \mathrm{~F}^{\circ}\right) 7 p$ & & $3^{1 / 2}$ & 38389.368 & 1.088 & 16 & ${ }^{4} \mathrm{D}$ & 7 & $5 f 6 d\left({ }^{3} \mathrm{~F}^{\circ}\right) 7 p{ }^{2} \mathrm{G}$ & 27 & $\mathrm{H}$ \\
\hline $5 f^{2}\left({ }^{3} \mathrm{H}\right) 6 d$ & ${ }^{4} \mathbf{I}$ & $6^{1 / 2}$ & 38517.98 & 1.111 & 55 & & 33 & $5 f 6 d\left({ }^{3} \mathrm{H}^{\circ}\right) 7 p{ }^{4} \mathbf{I}$ & 8 & B \\
\hline $5 f 7 s\left({ }^{1} \mathrm{~F}^{\circ}\right) 7 p$ & & $4^{1 / 2}$ & 38617.68 & 1.16 & 39 & ${ }^{2} \mathrm{G}$ & 19 & $\left({ }^{3} \mathrm{~F}^{\circ}\right){ }^{4} \mathrm{~F}$ & 20 & M \\
\hline $5 f\left(6 d\left({ }^{1} \mathrm{D}^{\circ}\right) 7 p\right.$ & & $2^{1 / 2}$ & 38728.681 & 1.255 & 13 & ${ }^{2} \mathrm{~F}$ & 12 & $5 f^{2}\left({ }^{3} \mathrm{~F}\right) 6 d{ }^{4} \mathrm{P}$ & 31 & $\mathrm{H}$ \\
\hline $5 f^{2}\left({ }^{3} \mathrm{~F}\right) 6 d$ & & $5^{1 / 2}$ & 38740.45 & 1.188 & 27 & ${ }^{4} \mathrm{H}$ & 20 & $\left({ }^{3} \mathrm{H}\right){ }^{4} \mathbf{H}$ & 12 & $\mathrm{R}$ \\
\hline $5 f^{2}\left({ }^{1} \mathrm{D}\right) 7 s$ & & $1^{1 / 2}$ & 38757.190 & 0.935 & 14 & ${ }^{2} \mathrm{D}$ & 10 & $\left({ }^{1} \mathrm{P}\right)^{2} \mathrm{P}$ & 21 & $\mathrm{H}$ \\
\hline $5 f 6 d\left({ }^{3} \mathrm{D}^{\circ}\right) 7 p$ & & $1^{1 / 2}$ & 38836.20 & 1.013 & 21 & ${ }^{4} \mathrm{~F}$ & 9 & $5 f^{2}\left({ }^{3} \mathrm{P}\right) 7 s^{2} \mathrm{P}$ & 20 & $\mathrm{H}$ \\
\hline $5 f^{2}\left({ }^{1} \mathrm{I}\right) 7 s$ & & $5^{1 / 2}$ & 38862.817 & 1.083 & 47 & ${ }^{2} \mathrm{I}$ & 13 & $5 f 6 d\left({ }^{3} \mathrm{H}^{\circ}\right) 7 p{ }^{2} \mathrm{I}$ & 22 & $\mathrm{R}$ \\
\hline $5 f 6 d\left({ }^{3} \mathrm{H}^{\circ}\right) 7 p$ & & $2^{1 / 2}$ & 38863.85 & 0.967 & 15 & ${ }^{4} \mathrm{G}$ & 10 & $\left({ }^{3} G^{\circ}\right){ }^{4} G$ & 25 & $\mathrm{H}$ \\
\hline
\end{tabular}


TABlE 1. Even energy levels of Th II-Continued

\begin{tabular}{|c|c|c|c|c|c|c|c|c|c|c|}
\hline \multirow{2}{*}{ Configuration } & \multirow{2}{*}{ Term } & \multirow{2}{*}{$J$} & \multirow{2}{*}{$\begin{array}{l}\text { Level } \\
\left(\mathrm{cm}^{-1}\right)\end{array}$} & \multirow{2}{*}{$g$} & \multicolumn{4}{|c|}{ Leading percentages } & \multirow{2}{*}{\multicolumn{2}{|c|}{$n$, ref. }} \\
\hline & & & & & & irst & & Second & & \\
\hline $5 f 6 d\left({ }^{3} \mathrm{~F}^{\circ}\right) 7 p$ & & $1 / 2$ & 38867.170 & 0.947 & 34 & ${ }^{4} \mathrm{D}$ & 25 & $5 f^{2}\left({ }^{3} \mathrm{~F}\right) 6 d^{4} \mathrm{P}$ & 12 & $\mathrm{H}$ \\
\hline $5 f^{2}\left({ }^{3} \mathrm{~F}\right) 6 d$ & & $3^{1 / 2}$ & 39068.691 & 1.114 & 23 & ${ }^{2} \mathrm{~F}$ & 7 & $5 f 6 d\left({ }^{3} \mathrm{~F}^{\circ}\right) 7 p{ }^{4} \mathrm{D}$ & 30 & $\mathrm{H}$ \\
\hline $5 f^{2}\left({ }^{3} \mathrm{H}\right) 6 d$ & & $6^{1 / 2}$ & 39085.55 & 1.10 & 33 & ${ }^{2} \mathrm{I}$ & 25 & $\left({ }^{1} \mathrm{I}\right)^{2} \mathrm{I}$ & 8 & $\mathrm{~S}$ \\
\hline $5 f 6 d\left({ }^{3} \mathrm{~F}^{\circ}\right) 7 p$ & & $1^{1 / 2}$ & 39150.679 & 0.739 & 18 & ${ }^{4} \mathrm{D}$ & 12 & $\left({ }^{3} \mathrm{D}^{\circ}\right){ }^{4} \mathrm{D}$ & 21 & $\mathrm{H}$ \\
\hline $5 f 6 d\left({ }^{1} \mathrm{G}^{\circ}\right) 7 p$ & & $5^{1 / 2}$ & 39352.294 & 1.05 & 20 & ${ }^{2} \mathrm{H}$ & 10 & $5 f^{2}\left({ }^{3} \mathbf{F}\right) 6 d{ }^{4} \mathrm{H}$ & 15 & $\mathrm{~S}$ \\
\hline $5 f 7 s\left({ }^{1} \mathrm{~F}^{\circ}\right) 7 p$ & & $2^{1 / 2}$ & 39366.90 & 1.140 & 22 & ${ }^{2} \mathrm{D}$ & 13 & $\left({ }^{3} \mathrm{~F}^{\circ}\right) \quad{ }^{4} \mathrm{D}$ & 24 & $\mathrm{H}$ \\
\hline $5 f^{2}\left({ }^{3} \mathrm{~F}\right) 6 d$ & & $3^{1 / 2}$ & 39458.272 & 0.979 & 15 & ${ }^{4} \mathrm{G}$ & 9 & $5 f 6 d\left({ }^{3} \mathrm{G}^{\circ}\right) 7 p{ }^{4} \mathrm{G}$ & 24 & $\mathrm{H}$ \\
\hline $5 f 6 d\left({ }^{3} \mathrm{~F}^{\circ}\right) 7 p$ & & $4^{1 / 2}$ & 39552.244 & 1.100 & 13 & ${ }^{2} \mathrm{G}$ & 10 & $\left({ }^{1} \mathrm{G}^{\circ}\right){ }^{2} \mathrm{H}$ & 29 & $\mathrm{H}$ \\
\hline $5 f 6 d\left({ }^{3} \mathrm{H}^{\circ}\right) 7 p$ & & $2^{1 / 2}$ & 39700.583 & 1.090 & 16 & ${ }^{4} \mathrm{G}$ & 10 & $\left({ }^{3} \mathrm{~F}^{\circ}\right){ }^{4} \mathrm{~F}$ & 29 & $\mathrm{H}$ \\
\hline $5 f 6 d\left({ }^{3} \mathrm{G}^{\circ}\right) 7 p$ & & $4^{1 / 2}$ & 39895.42 & 1.024 & 13 & ${ }^{2} \mathrm{G}$ & 12 & $\left({ }^{3} \mathrm{~F}^{\circ}\right){ }^{4} \mathrm{G}$ & 29 & $\mathrm{H}$ \\
\hline $5 f^{2}\left({ }^{3} \mathrm{~F}\right) 6 d$ & & $5^{1 / 2}$ & 39939.115 & 1.12 & 25 & ${ }^{4} \mathrm{G}$ & 14 & $5 f^{2}\left({ }^{1} \mathrm{I}\right) 7 s^{2} \mathrm{I}$ & 17 & B \\
\hline $5 f^{2}\left({ }^{3} \mathrm{~F}\right) 6 d$ & & $1 / 2$ & 39948.40 & 1.542 & 29 & ${ }^{4} \mathrm{P}$ & 23 & $5 f 6 d\left({ }^{3} \mathrm{~F}^{\circ}\right) 7 p{ }^{4} \mathrm{D}$ & 9 & $\mathrm{H}$ \\
\hline $5 f 6 d\left({ }^{3} \mathrm{H}^{\circ}\right) 7 p$ & & $2^{1 / 2}$ & 40216.12 & 1.024 & 23 & ${ }^{4} \mathrm{G}$ & 10 & $5 f^{2}\left({ }^{1} \mathrm{D}\right) 7 s^{2} \mathrm{D}$ & 23 & $\mathrm{~S}$ \\
\hline $5 f 7 s\left({ }^{1} \mathrm{~F}^{\circ}\right) 7 p$ & & $1^{1 / 2}$ & 40222.903 & 0.738 & 25 & ${ }^{2} \mathrm{D}$ & 17 & $\left({ }^{3} \mathrm{~F}^{\circ}\right){ }^{2} \mathrm{D}$ & 18 & $\mathrm{H}$ \\
\hline $5 f 7 s\left({ }^{1} \mathrm{~F}^{\circ}\right) 7 p$ & & $1^{1 / 2}$ & 40278.13 & 0.705 & 14 & ${ }^{2} \mathrm{D}$ & 13 & $5 f^{2}\left({ }^{3} \mathrm{H}\right) 6 d{ }^{4} \mathrm{~F}$ & 22 & $\mathrm{H}$ \\
\hline $5 f 6 d\left({ }^{3} \mathrm{G}^{\circ}\right) 7 p$ & & $4^{1 / 2}$ & 40367.44 & 1.176 & 26 & ${ }^{4} \mathrm{G}$ & 8 & $\left({ }^{3} \mathrm{G}^{\circ}\right){ }^{4} \mathrm{~F}$ & 27 & B \\
\hline $5 f 6 d\left({ }^{1} \mathrm{D}^{\circ}\right) 7 p$ & & $3^{1 / 2}$ & 40411.48 & 1.106 & 18 & ${ }^{2} \mathrm{~F}$ & 11 & $\left({ }^{3} \mathrm{~F}^{\circ}\right){ }^{2} \mathrm{G}$ & 25 & $\mathrm{H}$ \\
\hline $5 f 6 d\left({ }^{3} \mathrm{P}^{\circ}\right) 7 p$ & & $1 / 2$ & 40523.74 & 0.50 & 41 & ${ }^{4} \mathrm{D}$ & 12 & $\left({ }^{3} \mathrm{D}^{\circ}\right){ }^{4} \mathrm{D}$ & 11 & $\mathrm{~S}$ \\
\hline $5 f 6 d\left({ }^{3} \mathrm{~F}^{\circ}\right) 7 p$ & & $3^{1 / 2}$ & 40570.63 & 1.1 & 19 & ${ }^{4} \mathrm{D}$ & 12 & $\left({ }^{3} \mathrm{G}^{\circ}\right){ }^{4} \mathrm{G}$ & 28 & M \\
\hline $5 f 6 d\left({ }^{3} \mathrm{H}^{\circ}\right) 7 p$ & & $5^{1 / 2}$ & 40574.76 & 1.140 & 14 & ${ }^{2} \mathrm{H}$ & 11 & $\left({ }^{3} \mathrm{H}^{\circ}\right){ }^{4} \mathrm{G}$ & 19 & B \\
\hline $5 f^{2}\left({ }^{3} \mathrm{~F}\right) 6 d$ & & $2^{1 / 2}$ & 40644.331 & 0.856 & 11 & ${ }^{2} \mathrm{~F}$ & 10 & $\left({ }^{3} \mathrm{~F}\right){ }^{4} \mathrm{P}$ & 35 & $\mathrm{H}$ \\
\hline $5 f^{2}\left({ }^{3} \mathrm{H}\right) 6 d$ & & $6^{1 / 2}$ & 40724.74 & 1.129 & 32 & ${ }^{4} \mathrm{I}$ & 26 & $5 f 6 d\left({ }^{3} \mathrm{H}^{\circ}\right) 7 p{ }^{4} \mathrm{I}$ & 11 & B \\
\hline $5 f 6 d\left({ }^{3} \mathrm{D}^{\circ}\right) 7 p$ & & $2^{1 / 2}$ & 40923.62 & 0.988 & 24 & ${ }^{4} \mathrm{~F}$ & 9 & $5 f^{2}\left({ }^{3} \mathrm{H}\right) 6 d^{4} \mathrm{~F}$ & 30 & $\mathrm{H}$ \\
\hline $5 f 6 d\left({ }^{3} \mathrm{~F}^{\circ}\right) 7 p$ & & $1^{1 / 2}$ & 40991.58 & 1.036 & 10 & ${ }^{4} \mathrm{D}$ & 9 & $5 f^{2}\left({ }^{3} \mathrm{H}\right) 6 d{ }^{4} \mathrm{~F}$ & 23 & $\mathrm{~S}$ \\
\hline $5 f 6 d\left({ }^{1} \mathrm{G}^{\circ}\right) 7 p$ & & $4^{1 / 2}$ & 41047.209 & 1.112 & 30 & ${ }^{2} \mathrm{G}$ & 20 & $\left({ }^{3} \mathrm{~F}^{\circ}\right){ }^{4} \mathrm{G}$ & 29 & B \\
\hline $5 f^{2}\left({ }^{3} \mathrm{H}\right) 6 d$ & ${ }^{4} \mathbf{I}$ & $71 / 2$ & 41099.07 & 1.17 & 83 & & 6 & $\left({ }^{3} \mathrm{H}\right){ }^{2} \mathrm{~K}$ & 5 & B \\
\hline $5 f 6 d\left({ }^{3} \mathrm{H}^{\circ}\right) 7 p$ & & $5^{1 / 2}$ & 41223.56 & 1.11 & 35 & ${ }^{4} \mathrm{H}$ & 26 & $\left({ }^{1} \mathrm{G}^{\circ}\right)^{2} \mathrm{H}$ & 19 & B \\
\hline $5 f 7 s\left({ }^{3} \mathbf{F}^{\circ}\right) 7 p$ & & $2^{1 / 2}$ & 41328.341 & 1.101 & 22 & ${ }^{2} \mathrm{D}$ & 7 & $5 f 6 d\left({ }^{3} \mathrm{~F}^{\circ}\right) 7 p{ }^{4} \mathrm{~F}$ & 32 & $\mathrm{H}$ \\
\hline $5 f^{2}\left({ }^{1} \mathrm{G}\right) 6 d$ & & $4^{1 / 2}$ & 41398.51 & 0.983 & 25 & ${ }^{2} \mathrm{H}$ & 10 & $5 f 6 d\left({ }^{3} \mathrm{G}^{\circ}\right) 7 p{ }^{4} \mathrm{H}$ & 28 & $\mathrm{~S}$ \\
\hline $5 f^{2} 6 d+5 f 6 d 7 p$ & & $3^{1 / 2}$ & 41488.15 & 1.010 & & & & & 31 & $\mathrm{H}$ \\
\hline $5 f^{2}\left({ }^{3} \mathrm{~F}\right) 6 d$ & & $1 \frac{1 / 2}{2}$ & 41676.85 & 1.220 & 21 & ${ }^{4} \mathrm{P}$ & 11 & $5 f 6 d\left({ }^{3} \mathrm{D}^{\circ}\right) 7 p{ }^{4} \mathrm{D}$ & 21 & $\mathrm{H}$ \\
\hline $5 f^{2} 6 d+5 f 6 d 7 p$ & & $31 / 2$ & 41688.44 & 0.940 & & & & & 29 & $\mathrm{H}$ \\
\hline $5 f^{2}\left({ }^{3} \mathrm{~F}\right) 6 d$ & & $6^{1 / 2}$ & 41882.39 & & 37 & ${ }^{4} \mathrm{H}$ & 23 & $\left({ }^{3} \mathrm{H}\right){ }^{4} \mathrm{H}$ & 8 & $\mathrm{Z}$ \\
\hline $5 f 6 d\left({ }^{3} \mathrm{~F}^{\circ}\right) 7 p$ & & $4^{1 / 2}$ & 41909.33 & 1.097 & 12 & ${ }^{2} \mathrm{G}$ & 10 & $\left({ }^{3} \mathrm{G}^{\circ}\right){ }^{4} \mathrm{~F}$ & 26 & B \\
\hline $5 f^{2}\left({ }^{3} \mathrm{~F}\right) 6 d$ & & $1^{1 / 2}$ & 41936.53 & 1.095 & 22 & ${ }^{4} \mathrm{P}$ & 16 & $\left({ }^{3} \mathrm{~F}\right){ }^{2} \mathrm{D}$ & 18 & C \\
\hline
\end{tabular}


TABLE 1. Even energy levels of Th II-Continued

\begin{tabular}{|c|c|c|c|c|c|c|c|c|c|c|}
\hline \multirow{2}{*}{ Configuration } & \multirow{2}{*}{ Term } & \multirow{2}{*}{$J$} & \multirow{2}{*}{$\begin{array}{c}\text { Level } \\
\left(\mathrm{cm}^{-1}\right)\end{array}$} & \multirow{2}{*}{$g$} & \multicolumn{4}{|c|}{ Leading percentages } & \multirow{2}{*}{\multicolumn{2}{|c|}{$n$, ref. }} \\
\hline & & & & & & First & & Second & & \\
\hline $5 f^{2}\left({ }^{3} \mathrm{~F}\right) 6 d$ & & $1 / 2$ & 42008.16 & 0.8 & 24 & ${ }^{2} \mathrm{P}$ & 17 & $5 f 6 d\left({ }^{3} \mathrm{D}^{\circ}\right) 7 p{ }^{4} \mathrm{D}$ & 7 & $\mathrm{Z}$ \\
\hline $5 f 6 d\left({ }^{3} \mathrm{G}^{\circ}\right) 7 p$ & & $5^{1 / 2}$ & 42146.54 & 1.158 & 31 & ${ }^{4} \mathrm{G}$ & 12 & $\left({ }^{3} \mathrm{G}^{\circ}\right){ }^{2} \mathrm{H}$ & 22 & B \\
\hline $5 f 6 d\left({ }^{3} \mathrm{~F}^{\circ}\right) 7 p$ & & $4^{1 / 2}$ & 42200.205 & 1.08 & 12 & ${ }^{4} \mathrm{G}$ & 11 & $\left({ }^{3} \mathrm{H}^{\circ}\right){ }^{2} \mathrm{G}$ & 32 & B \\
\hline $5 f^{2} 6 d+5 f 6 d 7 p$ & & $3^{1 / 2}$ & 42222.31 & 0.935 & & & & & 30 & $\mathrm{~S}$ \\
\hline $5 f 6 d\left({ }^{3} \mathrm{P}^{\circ}\right) 7 p$ & & $1 / 2$ & 42246.02 & 0.8 & 20 & ${ }^{4} \mathrm{P}$ & 13 & $5 f^{2}\left({ }^{3} \mathrm{~F}\right) 6 d{ }^{4} \mathrm{D}$ & 7 & $\mathrm{Z}$ \\
\hline $5 f^{2} 6 d+5 f 6 d 7 p$ & & $2^{1 / 2}$ & 42336.83 & 1.15 & & & & & 21 & $\mathrm{Z}$ \\
\hline $5 f^{2} 6 d+5 f 6 d 7 p$ & & $2^{1 / 2}$ & 42352.13 & 1.126 & & & & & 27 & $\mathrm{H}$ \\
\hline $5 f^{2} 6 d+5 f 6 d 7 p$ & & $3^{1 / 2}$ & 42518.907 & 1.080 & & & & & 31 & $\mathrm{H}$ \\
\hline $5 f 6 d\left({ }^{3} \mathrm{H}^{\circ}\right) 7 p$ & & $6^{1 / 2}$ & 42644.878 & 1.10 & 32 & ${ }^{4} \mathrm{I}$ & 28 & $\left({ }^{3} \mathrm{H}^{\circ}\right){ }^{4} \mathrm{H}$ & 10 & B \\
\hline $5 f^{2} 6 d+5 f 6 d 7 p$ & & $3^{1 / 2}$ & 42751.324 & 1.09 & & & & & 31 & $\mathrm{~S}$ \\
\hline $5 f 6 d\left({ }^{3} \mathrm{H}^{\circ}\right) 7 p$ & & $4^{1 / 2}$ & 42955.85 & 1.141 & 21 & ${ }^{4} \mathrm{G}$ & 15 & $\left({ }^{2} \mathrm{G}^{\circ}\right){ }^{4} \mathrm{~F}$ & 29 & $\mathrm{~B}$ \\
\hline $5 f^{2}\left({ }^{3} \mathrm{H}\right) 6 d$ & ${ }^{2} \mathrm{~K}$ & $6^{1 / 2}$ & 43014.143 & 0.976 & 57 & & 25 & (1I) ${ }^{2} \mathrm{~K}$ & 12 & $\mathrm{H}$ \\
\hline $5 f^{2} 6 d+5 f 6 d 7 p$ & & $2^{1 / 2}$ & 43096.54 & 0.982 & & & & & 21 & $\mathrm{H}$ \\
\hline $5 f 6 d\left({ }^{3} \mathrm{H}^{\circ}\right) 7 p$ & $2 I$ & $5^{1 / 2}$ & 43127.21 & 1.037 & 53 & & 9 & $\left({ }^{3} \mathrm{H}^{\circ}\right){ }^{4} \mathrm{H}$ & 22 & $\mathrm{~B}$ \\
\hline $5 f^{2} 6 d+5 f 6 d 7 p$ & & $2^{1 / 2}$ & 43227.86 & 1.153 & & & & & 22 & $\mathrm{H}$ \\
\hline $5 f^{2}\left({ }^{3} \mathrm{~F}\right) 6 d$ & & $1^{1 / 2}$ & 43244.86 & 1.08 & 28 & ${ }^{4} \mathrm{~F}$ & 13 & $\left({ }^{3} \mathrm{~F}\right){ }^{2} \mathrm{P}$ & 16 & $\mathrm{Z}$ \\
\hline $5 f^{2} 6 d+5 f 6 d 7 p$ & & $3^{1 / 2}$ & 43246.83 & 1.033 & & & & & 30 & $\mathrm{R}$ \\
\hline $5 f^{2} 6 d+5 f 6 d 7 p$ & & $2^{1 / 2}$ & 43772.47 & 1.04 & & & & & 28 & $\mathrm{Z}$ \\
\hline $5 f^{2} 6 d+5 f 6 d 7 p$ & & $3^{1 / 2}$ & 43803.975 & 1.026 & & & & & 32 & $\mathrm{H}$ \\
\hline $5 f 6 d\left({ }^{3} \mathrm{~F}^{\circ}\right) 7 p$ & & $1^{1 / 2}$ & 43807.55 & 1.211 & 23 & ${ }^{4} \mathrm{D}$ & 18 & $\left({ }^{3} \mathrm{D}^{\circ}\right){ }^{4} \mathrm{D}$ & 21 & $\mathrm{H}$ \\
\hline $5 f^{2} 6 d+5 f 6 d 7 p$ & & $41 / 2$ & 43809.26 & 1.102 & & & & & 27 & $\mathrm{~B}$ \\
\hline $5 f 6 d\left({ }^{3} \mathrm{P}^{\circ}\right) 7 p$ & & $1 / 2$ & 43965.62 & 1.555 & 20 & ${ }^{4} \mathrm{D}$ & 15 & $5 f^{2}\left({ }^{3} \mathrm{~F}\right) 6 d{ }^{4} \mathrm{D}$ & 12 & $\mathrm{~S}$ \\
\hline $5 f^{2} 6 d+5 f 6 d 7 p$ & & $4^{1 / 2}$ & 44096.26 & 1.14 & & & & & 27 & $\mathrm{Z}$ \\
\hline $5 f 6 d\left({ }^{3} \mathrm{G}^{\circ}\right) 7 p$ & & $5^{1 / 2}$ & 44177.26 & 1.12 & 18 & ${ }^{4} \mathrm{H}$ & 17 & $5 f^{2}\left({ }^{1} \mathrm{G}\right) 6 d{ }^{2} \mathrm{H}$ & 20 & $\mathrm{~B}$ \\
\hline $5 f^{2}\left({ }^{3} \mathrm{~F}\right) 6 d$ & & $1^{1 / 2}$ & 44300.548 & 1.342 & 19 & ${ }^{4} \mathrm{~F}$ & 9 & $5 f 6 d\left({ }^{3} \mathrm{~F}^{\circ}\right) 7 p{ }^{2} \mathrm{D}$ & 18 & $\mathrm{H}$ \\
\hline $5 f^{2} 6 d+5 f 6 d 7 p$ & & $2^{1 / 2}$ & 44388.77 & 1.158 & & & & & 29 & $\mathrm{H}$ \\
\hline $5 f^{2} 6 d+5 f 6 d 7 p$ & & $41 / 2$ & 44450.28 & 1.04 & & & & & 26 & M \\
\hline $5 f^{2} 6 d+5 f 6 d 7 p$ & & $3^{1 / 2}$ & 44503.80 & 1.058 & & & & & 26 & $\mathrm{H}$ \\
\hline $5 f^{2} 6 d+5 f 6 d 7 p$ & & $2^{1 / 2}$ & 44552.68 & 1.182 & & & & & 21 & $\mathrm{H}$ \\
\hline $5 f 6 d\left({ }^{3} \mathrm{~F}^{\circ}\right) 7 p$ & & $5^{1 / 2}$ & 44727.24 & 1.2 & 43 & ${ }^{4} \mathrm{G}$ & 10 & $\left({ }^{3} \mathrm{H}^{\circ}\right) \quad{ }^{4} \mathrm{G}$ & 17 & $\mathrm{M}$ \\
\hline $5 f 6 d\left({ }^{1} \mathrm{D}^{\circ}\right) 7 p$ & & $1 / 2$ & 44789.30 & 0.84 & 24 & ${ }^{2} \mathrm{P}$ & 18 & $\left({ }^{3} \mathrm{~F}^{\circ}\right){ }^{4} \mathrm{D}$ & 13 & $\mathrm{Z}$ \\
\hline $5 f^{2} 6 d+5 f 6 d 7 p$ & & $3^{1 / 2}$ & 44807.95 & 1.078 & & & & & 29 & $\mathrm{~B}$ \\
\hline $5 f^{2}\left({ }^{3} \mathrm{~F}\right) 6 d$ & & $1^{1 / 2}$ & 44889.81 & 1.346 & 36 & ${ }^{4} \mathrm{D}$ & 21 & $5 f 6 d\left({ }^{3} \mathrm{P}^{\circ}\right) 7 p{ }^{4} \mathrm{D}$ & 19 & C \\
\hline $5 f^{2} 6 d+5 f 6 d 7 p$ & & $3^{1 / 2}$ & 44898.77 & 1.181 & & & & & 29 & $\mathrm{H}$ \\
\hline $5 f 6 d\left({ }^{3} \mathrm{H}^{\circ}\right) 7 p$ & ${ }^{4} \mathrm{I}$ & $71 / 2$ & 45095.24 & & 58 & & 25 & $5 f^{2}\left({ }^{3} \mathrm{H}\right) 6 d^{2} \mathrm{~K}$ & 5 & $\mathrm{Z}$ \\
\hline
\end{tabular}


TABLE 1. Even energy levels of Th II-Continued

\begin{tabular}{|c|c|c|c|c|c|c|c|c|c|c|}
\hline \multirow{2}{*}{ Configuration } & \multirow{2}{*}{ Term } & \multirow{2}{*}{$J$} & \multirow{2}{*}{$\begin{array}{l}\text { Level } \\
\left(\mathrm{cm}^{-1}\right)\end{array}$} & \multirow{2}{*}{$g$} & \multicolumn{4}{|c|}{ Leading percentages } & \multirow{2}{*}{\multicolumn{2}{|c|}{$n$, ref. }} \\
\hline & & & & & & irst & & Second & & \\
\hline $5 f^{2} 6 d+5 f 6 d 7 p$ & & $4^{1 / 2}$ & 45126.24 & 1.21 & & & & & 24 & B \\
\hline $5 f^{2} 6 d+5 f 6 d 7 p$ & & $2^{1 / 2}$ & 45189.645 & 0.674 & & & & & 24 & $\mathrm{H}$ \\
\hline $5 f 6 d\left({ }^{3} \mathrm{P}^{\circ}\right) 7 p$ & & $11 / 2$ & 45306.16 & 0.6 & 27 & ${ }^{4} \mathrm{P}$ & 17 & $\left({ }^{3} \mathrm{D}^{\circ}\right){ }^{4} \mathrm{P}$ & 13 & $\mathrm{Z}$ \\
\hline $5 f^{2} 6 d+5 f 6 d 7 p$ & & $3^{1 / 2}$ & 45395.04 & 1.216 & & & & & 21 & $\mathrm{~S}$ \\
\hline $5 f^{2} 6 d+5 f 6 d 7 p$ & & $2^{1 / 2}$ & 45610.63 & 1.075 & & & & & 23 & $\mathrm{H}$ \\
\hline $5 j 6 d\left({ }^{3} \mathrm{H}^{\circ}\right) 7 p$ & & $7^{1 / 2}$ & 45646.25 & 1.13 & 35 & ${ }^{4} \mathrm{I}$ & 29 & $5 f^{2}\left({ }^{3} \mathrm{H}\right) 6 d{ }^{2} \mathrm{~K}$ & 3 & $\mathrm{Z}$ \\
\hline $5 f^{2}\left({ }^{3} \mathrm{H}\right) 6 d$ & & $5^{1 / 2}$ & 45735.10 & 1.141 & 34 & ${ }^{4} \mathrm{H}$ & 16 & $\left({ }^{3} \mathrm{~F}\right){ }^{4} \mathrm{H}$ & 20 & $\mathrm{~B}$ \\
\hline $5 f^{2} 6 d+5 f 6 d 7 p$ & & $2^{1 / 2}$ & 45800.27 & 1.3 & & & & & 26 & $\mathrm{Z}$ \\
\hline $5 f^{2} 6 d+5 f 6 d 7 p$ & & $4^{1 / 2}$ & 45904.40 & 1.02 & & & & & 17 & $\mathrm{~S}$ \\
\hline $5 f 6 d\left({ }^{3} \mathrm{~F}^{\circ}\right) 7 p$ & & $5^{1 / 2}$ & 46216.583 & 1.16 & 14 & ${ }^{4} \mathrm{G}$ & 14 & $5 f^{2}\left({ }^{3} \mathrm{~F}\right) 6 d^{4} \mathrm{G}$ & 21 & $\mathrm{~S}$ \\
\hline $5 f^{2} 6 d+5 f 6 d 7 p$ & & $4^{1 / 2}$ & 46253.33 & 1.01 & & & & & 23 & M \\
\hline $5 f 6 d\left({ }^{1} \mathrm{D}^{\circ}\right) 7 p$ & & $11 / 2$ & 46264.24 & 0.891 & 13 & ${ }^{2} \mathrm{P}$ & 10 & $\left({ }^{3} \mathrm{D}^{\circ}\right){ }^{4} \mathrm{D}$ & 17 & $\mathrm{H}$ \\
\hline $5 f^{2} 6 d+5 f 6 d 7 p$ & & $3^{1 / 2}$ & 46352.23 & 1.1 & & & & & 34 & $\mathrm{C}$ \\
\hline $5 f 6 d\left({ }^{3} \mathrm{H}^{\circ}\right) 7 p$ & & $6^{1 / 2}$ & 46378.86 & 1.13 & 29 & ${ }^{2} \mathrm{I}$ & 25 & $5 f^{2}\left({ }^{3} \mathrm{H}\right) 6 d{ }^{4} \mathrm{H}$ & 11 & B \\
\hline $5 f^{2} 6 d+5 f 6 d 7 p$ & & $3^{1 / 2}$ & 46385.42 & 1.07 & & & & & 31 & $\mathrm{Z}$ \\
\hline $5 f 6 d\left({ }^{3} \mathrm{G}^{\circ}\right) 7 p$ & & $1^{1 / 2}$ & 46395.98 & & 23 & ${ }^{4} \mathrm{~F}$ & 17 & $5 f^{2}\left({ }^{3} \mathrm{H}\right) 6 d{ }^{4} \mathrm{~F}$ & 15 & $\mathrm{Z}$ \\
\hline $5 f 6 d\left({ }^{3} \mathrm{G}^{\circ}\right) 7 p$ & & $5^{1 / 2}$ & 46555.61 & & 32 & ${ }^{4} \mathrm{H}$ & 20 & $\left({ }^{3} \mathrm{H}^{\circ}\right){ }^{4} \mathrm{G}$ & 15 & $\mathrm{Z}$ \\
\hline $5 f^{2} 6 d+5 f 6 d 7 p$ & & $2^{1 / 2}$ & 46581.30 & 1.018 & & & & & 28 & $\mathrm{C}$ \\
\hline $5 f^{2} 6 d+5 f 6 d 7 p$ & & $2^{1 / 2}$ & 46603.20 & 1.112 & & & & & 28 & $\mathrm{H}$ \\
\hline $5 f^{2} 6 d+5 f 6 d 7 p$ & & $3^{1 / 2}$ & 46706.25 & 1.081 & & & & & 19 & $\mathrm{H}$ \\
\hline $5 f 6 d\left({ }^{3} \mathrm{G}^{\circ}\right) 7 p$ & & $5^{1 / 2}$ & 46861.59 & 1.15 & 12 & ${ }^{4} \mathrm{H}$ & 12 & $5 f^{2}\left({ }^{1} \mathrm{I}\right) 6 d^{2} \mathrm{I}$ & 20 & $\mathrm{Z}$ \\
\hline $5 f^{2} 6 d+5 f 6 d 7 p$ & & $2^{1 / 2}$ & 46902.54 & 1.143 & & & & & 22 & $\mathrm{H}$ \\
\hline $5 f 6 d\left({ }^{3} \mathrm{H}^{\circ}\right) 7 p$ & & $6^{1 / 2}$ & 46910.78 & 1.18 & 26 & ${ }^{4} \mathrm{H}$ & 22 & $\left({ }^{3} \mathrm{H}^{\circ}\right)^{2} \mathrm{I}$ & 14 & $\mathrm{~S}$ \\
\hline $5 f^{2}\left({ }^{1} \mathrm{D}\right) 6 d$ & & $1^{1 / 2}$ & 46935.66 & 0.956 & 22 & ${ }^{2} \mathrm{P}$ & 19 & $\left({ }^{3} \mathrm{P}\right)^{2} \mathrm{P}$ & 23 & $\mathrm{H}$ \\
\hline $5 f 6 d\left({ }^{3} \mathrm{D}^{\circ}\right) 7 p$ & & $1 / 2$ & 47145.77 & & 19 & ${ }^{4} \mathrm{D}$ & 18 & $\left({ }^{3} \mathrm{D}^{\circ}\right){ }^{4} \mathrm{P}$ & 8 & C \\
\hline $5 f 6 d\left({ }^{3} \mathrm{P}^{\circ}\right) 7 p$ & & $1^{1 / 2}$ & 47148.60 & 1.09 & 11 & ${ }^{4} \mathrm{~S}$ & 9 & $5 f^{2}\left({ }^{3} \mathrm{~F}\right) 6 d{ }^{4} \mathrm{D}$ & 19 & $\mathrm{H}$ \\
\hline $5 f^{2} 6 d+5 f 6 d 7 p$ & & $4^{1 / 2}$ & 47171.38 & 1.15 & & & & & 23 & $\mathrm{Z}$ \\
\hline $5 f^{2} 6 d+5 f 6 d 7 p$ & & $2^{1 / 2}$ & 47324.39 & 1.189 & & & & & 35 & $\mathrm{H}$ \\
\hline $5 f 6 d\left({ }^{3} \mathrm{G}^{\circ}\right) 7 p$ & & $5^{1 / 2}$ & 47675.06 & 1.15 & 17 & ${ }^{2} \mathrm{H}$ & 14 & $5 f^{2}\left({ }^{3} \mathrm{H}\right) 6 d{ }^{4} \mathrm{G}$ & 6 & $\mathrm{Z}$ \\
\hline $5 f^{2} 6 d+5 f 6 d 7 p$ & & $4^{1 / 2}$ & 47731.50 & 1.1 & & & & & 24 & M \\
\hline $5 f^{2}\left({ }^{3} \mathrm{P}\right) 6 d$ & & $11 / 2$ & 47869.61 & & 18 & ${ }^{4} \mathrm{~F}$ & 14 & $\left({ }^{3} \mathrm{~F}\right){ }^{2} \mathrm{D}$ & 25 & $\mathrm{Z}$ \\
\hline $5 f^{2} 6 d+5 f 6 d 7 p$ & & $3^{1 / 2}$ & 47871.39 & 1.194 & & & & & 23 & $\mathrm{~S}$ \\
\hline $5 f^{2} 6 d+5 f 6 d 7 p$ & & $4^{1 / 2}$ & 48006.87 & 1.13 & & & & & 25 & $\mathrm{Z}$ \\
\hline $5 f^{2} 6 d+5 f 6 d 7 p$ & & $3^{1 / 2}$ & 48298.50 & 1.07 & & & & & 22 & $\mathrm{~S}$ \\
\hline $5 f^{2} 6 d+5 f 6 d 7 p$ & & $2^{1 / 2}$ & 48320.81 & & & & & & 20 & $\mathrm{Z}$ \\
\hline
\end{tabular}


TABLE 1. Even energy levels of Th II-Continued

\begin{tabular}{|c|c|c|c|c|c|c|c|c|c|c|}
\hline \multirow{2}{*}{ Configuration } & \multirow{2}{*}{ Term } & \multirow{2}{*}{$J$} & \multirow{2}{*}{$\begin{array}{l}\text { Level } \\
\left(\mathrm{cm}^{-1}\right)\end{array}$} & \multirow{2}{*}{$g$} & \multicolumn{4}{|c|}{ Leading percentages } & \multirow{2}{*}{\multicolumn{2}{|c|}{$n$, ref. }} \\
\hline & & & & & \multicolumn{2}{|c|}{ First } & \multicolumn{2}{|r|}{ Second } & & \\
\hline $5 f^{2} 6 d+5 f 6 d 7 p$ & & $3^{1 / 2}$ & 48453.12 & & & & & & 22 & $\mathrm{Z}$ \\
\hline $5 f^{2} 6 d+5 f 6 d 7 p$ & & $2^{1 / 2}$ & 48492.03 & & & & & & 20 & $\mathrm{Z}$ \\
\hline $5 f 6 d\left({ }^{3} \mathrm{G}^{\circ}\right) 7 p$ & & $6^{1 / 2}$ & 48612.23 & 1.08 & 49 & ${ }^{4} \mathrm{H}$ & 17 & $5 f^{2}\left({ }^{1} \mathrm{G}\right) 6 d^{2} \mathrm{I}$ & 9 & $\mathrm{Z}$ \\
\hline $5 f 6 d\left({ }^{3} \mathrm{D}^{\circ}\right) 7 p$ & & $1^{1 / 2}$ & 48689.95 & 0.922 & 19 & ${ }^{2} \mathrm{D}$ & 12 & $5 f^{2}\left({ }^{3} \mathrm{P}\right) 6 d{ }^{4} \mathrm{~F}$ & 24 & $\mathrm{H}$ \\
\hline $5 f^{2}\left({ }^{3} \mathrm{P}\right) 6 d$ & & $11 / 2$ & 48817.97 & 0.956 & 22 & ${ }^{4} \mathrm{~F}$ & 19 & $5 f 6 d\left({ }^{3} \mathrm{D}^{\circ}\right) 7 p^{2} \mathrm{D}$ & 17 & $\mathrm{H}$ \\
\hline $5 f^{2} 6 d+5 f 6 d 7 p$ & & $4^{1} / 2$ & 48844.96 & 1.1 & & & & & 25 & $\mathrm{~S}$ \\
\hline $5 f^{2} 6 d+5 f 6 d 7 p$ & & $2^{1 / 2}$ & 49068.82 & & & & & & 22 & $\mathrm{Z}$ \\
\hline $5 f^{2}\left({ }^{1} \mathrm{I}\right) 6 d$ & & $5^{1 / 2}$ & 49124.56 & & 18 & ${ }^{2} \mathrm{H}$ & 18 & $5 f 6 d\left({ }^{3} \mathrm{G}^{\circ}\right) 7 p^{4} \mathrm{G}$ & 18 & $\mathrm{Z}$ \\
\hline $5 f 6 d\left({ }^{3} \mathrm{G}^{\circ}\right) 7 p$ & & $5^{1 / 2}$ & 49357.99 & 1.15 & 30 & ${ }^{2} \mathrm{H}$ & 28 & $\left({ }^{1} \mathrm{H}^{\circ}\right)^{2} \mathrm{H}$ & 15 & $\mathrm{Z}$ \\
\hline $5 f^{2} 6 d+5 f 6 d 7 p$ & & $3^{1 / 2}$ & 49377.17 & 1.2 & & & & & 23 & M \\
\hline $5 f 6 d\left({ }^{3} \mathrm{P}^{\circ}\right) 7 p$ & & $11 / 2$ & 49414.65 & 1.003 & 18 & ${ }^{4} \mathrm{~S}$ & 14 & $5 f^{2}\left({ }^{3} \mathrm{P}\right) 6 d^{4} \mathrm{D}$ & 14 & $\mathrm{H}$ \\
\hline $5 f 6 d\left({ }^{3} D^{\circ}\right) 7 p$ & & $1 / 2$ & 49485.52 & & 17 & ${ }^{4} \mathrm{P}$ & 16 & $5 f^{2}\left({ }^{3} \mathrm{P}\right) 6 d^{4} \mathrm{D}$ & 10 & $\mathrm{Z}$ \\
\hline $5 f^{2}\left({ }^{1} \mathrm{I}\right) 6 d$ & & $6^{1 / 2}$ & 49527.320 & 1.1 & 26 & ${ }^{2} I$ & 25 & $5 f 6 d\left({ }^{3} \mathrm{G}^{\circ}\right) 7 p{ }^{4} \mathrm{H}$ & 7 & $\mathrm{Z}$ \\
\hline $5 f^{2} 6 d+5 f 6 d 7 p$ & & $4^{1} / 2$ & 49837.66 & & & & & & 19 & $\mathrm{Z}$ \\
\hline $5 f^{2} 6 d+5 f 6 d 7 p$ & & $2^{1 / 2}$ & 49873.098 & & & & & & 23 & $\mathrm{Z}$ \\
\hline $5 f^{2} 6 d+5 f 6 d 7 p$ & & $3^{1 / 2}$ & 49960.57 & 1.248 & & & & & 27 & $\mathrm{R}$ \\
\hline $5 f^{2} 6 d+5 f 6 d 7 p$ & & $3^{1 / 2}$ & 50407.27 & & & & & & 16 & M \\
\hline $5 f^{2} 6 d+5 f 6 d 7 p$ & & $4^{1 / 2}$ & 50470.04 & 1.18 & & & & & 30 & $\mathrm{~S}$ \\
\hline $5 f 6 d\left({ }^{1} \mathrm{H}^{\circ}\right) 7 p$ & & $5^{1 / 2}$ & 50631.12 & & 19 & ${ }^{2} I$ & 18 & $5 f^{2}\left({ }^{3} \mathrm{~F}\right) 6 d{ }^{2} \mathrm{H}$ & 14 & $\mathrm{Z}$ \\
\hline $5 f^{2} 6 d+5 f 6 d 7 p$ & & $2^{1 / 2}$ & 50663.62 & & & & & & 20 & $\mathrm{Z}$ \\
\hline $5 f 6 d\left({ }^{3} \mathrm{D}^{\circ}\right) 7 p$ & & $1^{1 / 2}$ & 50735.47 & 1.36 & 16 & ${ }^{2} \mathrm{P}$ & 9 & $\left({ }^{3} \mathrm{P}^{\circ}\right){ }^{4} \mathrm{~S}$ & 23 & C \\
\hline $5 f 6 d\left({ }^{3} \mathrm{D}^{\circ}\right) 7 p$ & & $11 / 2$ & 50907.79 & 1.3 & 26 & ${ }^{4} \mathrm{P}$ & 10 & $5 f^{2}\left({ }^{3} \mathrm{~F}\right) 6 d^{4} \mathrm{P}$ & 10 & $\mathrm{Z}$ \\
\hline $5 f^{2}\left({ }^{3} \mathrm{P}\right) 6 d$ & & $11 / 2$ & 51024.846 & 1.270 & 24 & ${ }^{4} \mathrm{D}$ & 15 & $5 f 6 d\left({ }^{3} \mathrm{P}^{\circ}\right) 7 p{ }^{4} \mathrm{P}$ & 16 & C \\
\hline $5 f^{2} 6 d+5 f 6 d 7 p$ & & $4^{1 / 2}$ & 51224.27 & & & & & & 19 & $\mathrm{Z}$ \\
\hline $5 f^{2} 6 d+5 f 6 d 7 p$ & & $3^{1 / 2}$ & 51268.12 & & & & & & 21 & $\mathrm{Z}$ \\
\hline $5 f^{2} 6 d+5 f 6 d 7 p$ & & $2^{1 / 2}$ & 51362.89 & & & & & & 21 & $\mathrm{Z}$ \\
\hline $5 f^{2}\left({ }^{3} \mathrm{P}\right) 6 d$ & & $1 / 2$ & 51653.96 & & 24 & ${ }^{2} \mathrm{P}$ & 17 & $\left({ }^{3} \mathrm{P}\right){ }^{4} \mathrm{D}$ & 10 & $\mathrm{Z}$ \\
\hline $5 f 6 d\left({ }^{1} \mathrm{P}^{\circ}\right) 7 p$ & & $11 / 2$ & 51676.08 & & 25 & ${ }^{2} \mathrm{D}$ & 11 & $5 f^{2}\left({ }^{3} \mathrm{P}\right) 6 d^{4} \mathrm{P}$ & 15 & Z \\
\hline $5 f^{2} 6 d+5 f 6 d 7 p$ & & $4^{1 / 2}$ & 51681.99 & & & & & & 17 & $\mathrm{Z}$ \\
\hline $5 f^{2} 6 d+5 f 6 d 7 p$ & & $3^{1 / 2}$ & 51830.53 & & & & & & 25 & $\mathrm{Z}$ \\
\hline $5 f^{2} 6 d+5 f 6 d 7 p$ & & $2^{1 / 2}$ & 51865.14 & & & & & & 25 & $\mathrm{Z}$ \\
\hline $5 f^{2} 6 d+5 f 6 d 7 p$ & & $2^{1 / 2}$ & 51935.68 & & & & & & 22 & $\mathrm{Z}$ \\
\hline $5 f^{2}\left({ }^{1} \mathrm{I}\right) 6 d$ & ${ }^{2} \mathrm{I}$ & $5^{1 / 2}$ & 52170.20 & 1.12 & 55 & & 16 & $5 f 6 d\left({ }^{1} \mathrm{H}^{\circ}\right) 7 p{ }^{2} \mathrm{H}$ & 16 & $\mathrm{Z}$ \\
\hline $5 f^{2} 6 d+5 f 6 d 7 p$ & & $3^{1 / 2}$ & 52272.33 & & & & & & 19 & $\mathrm{Z}$ \\
\hline $5 f 6 d\left({ }^{1} \mathrm{P}^{\circ}\right) 7 p$ & & $1 \frac{1}{2}$ & 52307.48 & & 22 & ${ }^{2} \mathrm{D}$ & 12 & $5 f^{2}\left({ }^{1} \mathrm{G}\right) 6 d^{2} \mathrm{D}$ & 15 & $\mathrm{Z}$ \\
\hline
\end{tabular}


TABLE 1. Even energy levels of Th II-Continued

\begin{tabular}{|c|c|c|c|c|c|c|c|c|c|c|}
\hline \multirow{2}{*}{ Configuration } & \multirow{2}{*}{ Term } & \multirow{2}{*}{$J$} & \multirow{2}{*}{$\begin{array}{l}\text { Level } \\
\left(\mathrm{cm}^{-1}\right)\end{array}$} & \multirow{2}{*}{$g$} & \multicolumn{4}{|c|}{ Leading percentages } & \multirow{2}{*}{\multicolumn{2}{|c|}{$n$, ref. }} \\
\hline & & & & & & irst & & Second & & \\
\hline $5 f^{2} 6 d+5 f 6 d 7 p$ & & $3^{1 / 2}$ & 52562.52 & & & & & & 19 & $\mathrm{Z}$ \\
\hline $5 f 6 d\left({ }^{3} \mathrm{P}^{\circ}\right) 7 p$ & & $1 \frac{1}{2}$ & 52735.74 & & 22 & ${ }^{2} \mathrm{D}$ & 16 & $\left({ }^{3} \mathrm{P}^{\circ}\right){ }^{2} \mathrm{P}$ & 17 & $\mathrm{Z}$ \\
\hline $5 f^{2}\left({ }^{1} \mathrm{I}\right) 6 d$ & & $6^{1 / 2}$ & 52918.92 & & 36 & ${ }^{2} \mathbf{I}$ & 18 & $\left({ }^{3} \mathrm{H}\right){ }^{2} \mathrm{I}$ & 10 & $\mathrm{Z}$ \\
\hline $5 f^{2} 6 d+5 f 6 d 7 p$ & & $4^{1 / 2}$ & 53520.890 & & & & & & 17 & $\mathrm{Z}$ \\
\hline $5 f^{2} 6 d+5 f 6 d 7 p$ & & $2^{1 / 2}$ & 53845.40 & & & & & & 20 & $\mathrm{Z}$ \\
\hline $5 f^{2} 6 d+5 f 6 d 7 p$ & & $3^{1 / 2}$ & 54010.17 & & & & & & 18 & $\mathrm{Z}$ \\
\hline $5 f^{2} 6 d+5 f 6 d 7 p$ & & $3^{1 / 2}$ & 54169.67 & & & & & & 14 & $\mathrm{Z}$ \\
\hline $5 f^{2} 6 d+5 f 6 d 7 p$ & & $2^{1 / 2}$ & 54493.99 & & & & & & 20 & M \\
\hline $5 f^{2} 6 d+5 f 6 d 7 p$ & & $4^{1 / 2}$ & 54845.32 & & & & & & 15 & $\mathrm{Z}$ \\
\hline $5 f 6 d\left({ }^{1} \mathrm{~F}^{\circ}\right) 7 p$ & & $1 \frac{1}{2}$ & 54922.10 & & 18 & ${ }^{2} \mathrm{D}$ & 17 & $5 f^{2}\left({ }^{3} \mathrm{P}\right) 6 d{ }^{4} \mathrm{P}$ & 15 & $\mathrm{Z}$ \\
\hline $5 f^{2} 6 d+5 f 6 d 7 p$ & & $4^{1 / 2}$ & 55496.83 & & & & & & 18 & $\mathrm{Z}$ \\
\hline $5 f 6 d\left({ }^{1} \mathrm{~F}^{\circ}\right) 7 p$ & & $1^{1 / 2}$ & 56235.23 & & 16 & ${ }^{2} \mathrm{D}$ & 15 & $5 f^{2}\left({ }^{3} \mathrm{P}\right) 6 d{ }^{4} \mathrm{P}$ & 14 & $\mathrm{Z}$ \\
\hline $5 f^{2} 6 d+5 f 6 d 7 p$ & & $2^{1 / 2}$ & 56391.01 & & & & & & 16 & $\mathrm{Z}$ \\
\hline $5 f^{2}\left({ }^{1} \mathrm{I}\right) 6 d$ & & $5^{1 / 2}$ & 56945.14 & 1.1 & 40 & ${ }^{2} \mathrm{H}$ & 26 & $5 f 6 d\left({ }^{1} \mathrm{H}^{\circ}\right) 7 p{ }^{2} \mathrm{H}$ & 11 & $\mathrm{Z}$ \\
\hline $5 f^{2} 6 d+5 f 6 d 7 p$ & & $4^{1 / 2}$ & 57078.52 & & & & & & 13 & $\mathrm{Z}$ \\
\hline $5 f^{2} 6 d+5 f 6 d 7 p$ & & $3^{1 / 2}$ & 59387.31 & & & & & & 14 & Z \\
\hline
\end{tabular}

TABLE 2. Odd energy levels of Th II

\begin{tabular}{|c|c|c|c|c|c|c|c|c|c|}
\hline \multirow{2}{*}{ Configuration } & \multirow{2}{*}{ Term } & \multirow{2}{*}{$J$} & \multirow{2}{*}{$\begin{array}{l}\text { Level } \\
\left(\mathrm{cm}^{-1}\right)\end{array}$} & \multirow{2}{*}{$g$} & \multicolumn{3}{|c|}{ Leading percentages } & \multirow{2}{*}{\multicolumn{2}{|c|}{$n$, ref. }} \\
\hline & & & & & First & & Second & & \\
\hline $5 f\left({ }^{2} \mathbf{F}^{\circ}\right) 7 s^{2}$ & ${ }^{2} \mathrm{~F}^{\circ}$ & $2^{1 / 2}$ & 4490.256 & 0.856 & 89 & 3 & $5 f\left({ }^{1} \mathrm{~S}^{\circ}\right) 6 d^{2}{ }^{2} \mathrm{~F}^{\circ}$ & 74 & $\mathrm{H}$ \\
\hline $5 f 6 d\left({ }^{3} \mathrm{H}^{\circ}\right) 7 s$ & ${ }^{4} \mathrm{H}^{\circ}$ & $3^{1 / 2}$ & 6168.351 & 0.718 & 78 & 17 & $\left({ }^{1} G^{\circ}\right){ }^{2} G^{\circ}$ & $7 \dot{6}$ & $\mathrm{H}$ \\
\hline $5 f 6 d\left({ }^{3} \mathrm{~F}^{\circ}\right) 7 s$ & ${ }^{4} \mathrm{~F}^{\circ}$ & $1 \frac{1 / 2}{2}$ & 6691.386 & 0.492 & 74 & 20 & $\left({ }^{1} D^{\circ}\right)^{2} D^{\circ}$ & 60 & $\mathrm{H}$ \\
\hline $5 f 6 d\left({ }^{3} \mathrm{H}^{\circ}\right) 7 s$ & & $4^{1 / 2}$ & 6700.183 & 1.018 & ${ }^{4} \mathrm{H}^{\circ}$ & 27 & $\left({ }^{1} G^{\circ}\right)^{2} G^{\circ}$ & 70 & $\mathrm{H}$ \\
\hline $5 f 6 d\left({ }^{3} \mathrm{~F}^{\circ}\right) 7 s$ & ${ }^{4} \mathrm{~F}^{\circ}$ & $2^{1 / 2}$ & 7331.485 & 1.061 & 54 & 25 & $\left({ }^{1} D^{\circ}\right){ }^{2} D^{\circ}$ & 77 & $\mathrm{H}$ \\
\hline $5 f\left({ }^{2} \mathrm{~F}^{\circ}\right) 7 s^{2}$ & ${ }^{2} \mathrm{~F}^{\circ}$ & $3^{1 / 2}$ & 8378.853 & 1.132 & 85 & 3 & $5 f\left({ }^{1} \mathrm{~S}^{\circ}\right) 6 d^{2}{ }^{2} \mathrm{~F}^{\circ}$ & 91 & $\mathrm{H}$ \\
\hline $5 f 6 d\left({ }^{1} \mathrm{G}^{\circ}\right) 7 s$ & ${ }^{2} \mathrm{G}^{\circ}$ & $3^{1 / 2}$ & 9202.264 & 0.911 & 50 & 17 & $\left({ }^{3} \mathrm{~F}^{\circ}\right){ }^{4} \mathrm{~F}^{\circ}$ & 86 & $\mathrm{H}$ \\
\hline $5 f 6 d\left({ }^{1} \mathrm{G}^{\circ}\right) 7 s$ & & $4^{1 / 2}$ & 9238.018 & 1.086 & ${ }^{2} \mathrm{G}^{\circ}$ & 31 & $\left({ }^{3} \mathrm{H}^{\circ}\right){ }^{4} \mathrm{H}^{\circ}$ & 76 & $\mathrm{H}$ \\
\hline $5 f 6 d\left({ }^{3} \mathrm{G}^{\circ}\right) 7 s$ & ${ }^{4} \mathrm{G}^{\circ}$ & $2^{1 / 2}$ & 9585.404 & 0.601 & 83 & 6 & $6 d^{2}\left({ }^{3} \mathrm{~F}\right) 7 p{ }^{4} \mathrm{G}^{\circ}$ & 87 & $\mathrm{H}$ \\
\hline $5 f 6 d\left({ }^{3} \mathrm{~F}^{\circ}\right) 7 s$ & ${ }^{4} \mathrm{~F}^{\circ}$ & $3^{1 / 2}$ & 9720.296 & 1.173 & 60 & 11 & $\left({ }^{3} \mathrm{G}^{\circ}\right){ }^{4} \mathrm{G}^{\circ}$ & 84 & $\mathrm{H}$ \\
\hline
\end{tabular}


TABLE 2. Odd energy levels of Th II - Continued

\begin{tabular}{|c|c|c|c|c|c|c|c|c|c|c|}
\hline \multirow{2}{*}{ Configuration } & \multirow{2}{*}{ Term } & \multirow{2}{*}{$J$} & \multirow{2}{*}{$\begin{array}{l}\text { Level } \\
\left(\mathrm{cm}^{-1}\right)\end{array}$} & \multirow{2}{*}{$g$} & \multicolumn{4}{|c|}{ Leading percentages } & \multirow{2}{*}{\multicolumn{2}{|c|}{$n$, ref. }} \\
\hline & & & & & & irst & & Second & & \\
\hline $5 f 6 d\left({ }^{3} \mathrm{H}^{\circ}\right) 7 s$ & ${ }^{4} \mathrm{H}^{\circ}$ & $5^{1 / 2}$ & 10189.063 & 1.128 & 81 & & & $\left({ }^{3} \mathrm{H}^{\circ}\right){ }^{2} \mathrm{H}^{\circ}$ & 62 & $\mathrm{H}$ \\
\hline $5 f 6 d\left({ }^{3} \mathrm{H}^{\circ}\right) 7 s$ & & $4^{1 / 2}$ & 10572.042 & 0.931 & 45 & ${ }^{2} \mathrm{H}^{\circ}$ & 27 & $\left({ }^{3} \mathrm{H}^{\circ}\right){ }^{4} \mathrm{H}^{\circ}$ & 79 & $\mathrm{H}$ \\
\hline $5 f 6 d\left({ }^{3} \mathrm{~F}^{\circ}\right) 7 s$ & & $2^{1 / 2}$ & 10673.137 & 1.088 & 40 & ${ }^{4} \mathbf{F}^{\circ}$ & 31 & $\left({ }^{1} \mathrm{D}^{\circ}\right)^{2} \mathrm{D}^{\circ}$ & 82 & $\mathrm{H}$ \\
\hline $5 f 6 d\left({ }^{3} \mathrm{G}^{\circ}\right) 7 s$ & ${ }^{4} \mathrm{G}^{\circ}$ & $3^{1 / 2}$ & 11116.584 & 0.983 & 54 & & 8 & $\left({ }^{1} G^{\circ}\right)^{2} G^{\circ}$ & 99 & $\mathrm{H}$ \\
\hline $5 f 6 d\left({ }^{1} \mathrm{D}^{\circ}\right) 7 s$ & ${ }^{2} \mathrm{D}^{\circ}$ & $1 / 2$ & 11576.402 & 0.832 & 51 & & 19 & $\left({ }^{3} \mathbf{F}^{\circ}\right){ }^{4} \mathbf{F}^{\circ}$ & 67 & $\mathrm{H}$ \\
\hline $5 f 6 d\left({ }^{3} \mathrm{D}^{\circ}\right) 7 s$ & ${ }^{4} \mathrm{D}^{\circ}$ & $1 / 2$ & 11725.431 & 0.255 & 53 & & 12 & $5 f\left({ }^{1} \mathrm{D}^{\circ}\right) 6 d^{2}{ }^{2} \mathrm{P}^{\circ}$ & 33 & $\mathrm{H}$ \\
\hline $5 f 6 d\left({ }^{3} \mathrm{~F}^{\circ}\right) 7 s$ & ${ }^{2} \mathrm{~F}^{\circ}$ & $2^{1 / 2}$ & 12472.178 & 0.982 & 55 & & 17 & $\left({ }^{1} \mathrm{D}^{\circ}\right){ }^{2} \mathrm{D}^{\circ}$ & 89 & $\mathrm{H}$ \\
\hline $5 f\left({ }^{3} \mathrm{~F}^{\circ}\right) 6 d^{2}$ & & $3^{1 / 2}$ & 12485.688 & 0.855 & 37 & ${ }^{4} \mathrm{H}^{\circ}$ & 20 & $\left({ }^{3} \mathrm{~F}^{\circ}\right)^{2} \mathrm{G}^{\circ}$ & 90 & $\mathrm{~B}$ \\
\hline $5 f 6 d\left({ }^{3} \mathrm{~F}^{\circ}\right) 7 s$ & ${ }^{4} \mathbf{F}^{\circ}$ & $4^{1 / 2}$ & 12488.288 & 1.245 & 53 & & 30 & $\left({ }^{3} G^{\circ}\right){ }^{4} G^{\circ}$ & 82 & B \\
\hline $5 f 6 d\left({ }^{3} \mathrm{D}^{\circ}\right) 7 s$ & & $1^{1 / 2}$ & 12902.378 & 1.167 & 48 & ${ }^{4} \mathrm{D}^{\circ}$ & 9 & $\left({ }^{1} \mathrm{D}^{\circ}\right){ }^{2} \mathrm{D}^{\circ}$ & 71 & $\mathrm{H}$ \\
\hline $5 f 6 d\left({ }^{3} \mathrm{H}^{\circ}\right) 7 s$ & ${ }^{4} \mathrm{H}^{\circ}$ & $6^{1 / 2}$ & 13406.439 & 1.231 & 96 & & 2 & $5 f\left({ }^{3} \mathrm{~F}^{\circ}\right) 6 d^{2}{ }^{4} \mathrm{H}^{\circ}$ & 28 & B \\
\hline $5 f 6 d\left({ }^{3} \mathrm{G}^{\circ}\right) 7 s$ & ${ }^{4} \mathrm{G}^{\circ}$ & $4^{1 / 2}$ & 13468.969 & 1.185 & 57 & & 20 & $\left({ }^{3} \mathrm{~F}^{\circ}\right){ }^{4} \mathbf{F}^{\circ}$ & 68 & B \\
\hline $5 f 6 d\left({ }^{3} \mathrm{~F}^{\circ}\right) 7 s$ & & $3^{1 / 2}$ & 13818.339 & 1.153 & 26 & ${ }^{2} \mathbf{F}^{\circ}$ & 17 & $\left({ }^{1} \mathbf{F}^{\circ}\right)^{2} \mathbf{F}^{\circ}$ & 94 & B \\
\hline $5 f 6 d\left({ }^{3} \mathrm{D}^{\circ}\right) 7 s$ & & $1 / 2$ & 14101.800 & 0.523 & 33 & ${ }^{4} \mathrm{D}^{\circ}$ & 29 & $5 f\left({ }^{1} \mathrm{D}^{\circ}\right) 6 d^{2}{ }^{2} \mathrm{P}^{\circ}$ & 38 & $\mathrm{~S}$ \\
\hline $5 f\left({ }^{3} \mathrm{~F}^{\circ}\right) 6 d^{2}$ & ${ }^{4} \mathrm{I}^{\circ}$ & $4^{1 / 2}$ & 14275.583 & 0.791 & 77 & & 10 & $5 f 6 d\left({ }^{3} \mathrm{H}^{\circ}\right) 7 s{ }^{2} \mathrm{H}^{\circ}$ & 75 & $\mathrm{H}$ \\
\hline $5 f 6 d\left({ }^{3} \mathrm{H}^{\circ}\right) 7 s$ & ${ }^{2} \mathrm{H}^{\circ}$ & $51 / 2$ & 14484.327 & 1.09 & 60 & & 15 & $5 f\left({ }^{1} \mathrm{D}^{\circ}\right) 6 d^{2}{ }^{2} \mathrm{H}^{\circ}$ & 59 & B \\
\hline $5 f 6 d\left({ }^{3} \mathrm{D}^{\circ}\right) 7 s$ & ${ }^{4} \mathrm{D}^{\circ}$ & $2^{1 / 2}$ & 14545.557 & 1.339 & 67 & & 6 & $\left({ }^{1} \mathrm{D}^{\circ}\right)^{2} \mathrm{D}^{\circ}$ & 80 & $\mathrm{~B}$ \\
\hline $5 f 6 d\left({ }^{3} \mathrm{G}^{\circ}\right) 7 s$ & & $3^{1 / 2}$ & 14790.996 & 0.862 & 40 & ${ }^{2} \mathrm{G}^{\circ}$ & 29 & $5 f\left({ }^{3} \mathrm{~F}^{\circ}\right) 6 d^{2}{ }^{4} \mathrm{H}^{\circ}$ & 91 & $\mathrm{H}$ \\
\hline $5 f 6 d\left({ }^{3} \mathrm{P}^{\circ}\right) 7 s$ & & $1^{1 / 2}$ & 15144.738 & 1.366 & 41 & ${ }^{4} \mathrm{P}^{\circ}$ & 14 & $\left({ }^{3} \mathrm{D}^{\circ}\right)^{4} \mathrm{D}^{\circ}$ & 65 & $\mathrm{H}$ \\
\hline $5 f\left({ }^{3} \mathrm{~F}^{\circ}\right) 6 d^{2}$ & ${ }^{4} \mathrm{H}^{\circ}$ & $4^{1 / 2}$ & 15242.905 & 1.00 & 59 & & 20 & $\left({ }^{3} F^{\circ}\right){ }^{2} G^{\circ}$ & 56 & B \\
\hline $5 f 6 d\left({ }^{3} \mathrm{P}^{\circ}\right) 7 s$ & ${ }^{4} \mathrm{P}^{\circ}$ & $1 / 2$ & 15324.239 & 2.565 & 87 & & 5 & $6 d 7 s\left({ }^{3} \mathrm{D}\right) 7 p{ }^{4} \mathrm{P}^{\circ}$ & 28 & $\mathrm{~S}$ \\
\hline $5 f 6 d\left({ }^{3} \mathrm{G}^{\circ}\right) 7 s$ & ${ }^{4} \mathrm{G}^{\circ}$ & $5^{1 / 2}$ & 15349.879 & 1.267 & 91 & & 4 & $6 d^{2}\left({ }^{3} \mathrm{~F}\right) 7 p^{4} \mathrm{G}^{\circ}$ & 50 & B \\
\hline $5 f 6 d\left({ }^{3} \mathrm{D}^{\circ}\right) 7 s$ & ${ }^{4} \mathrm{D}^{\circ}$ & $3^{1 / 2}$ & 15453.036 & 1.296 & 55 & & 17 & $\left({ }^{3} \mathbf{F}^{\circ}\right)^{2} \mathbf{F}^{\circ}$ & 92 & B \\
\hline $5 f 6 d\left({ }^{3} \mathrm{D}^{\circ}\right) 7 s$ & ${ }^{2} \mathrm{D}^{\circ}$ & $11 / 2$ & 15710.843 & 0.643 & 52 & & 21 & $\left({ }^{3} \mathrm{P}^{\circ}\right){ }^{4} \mathrm{P}^{\circ}$ & 67 & $\mathrm{R}$ \\
\hline $5 f 6 d\left({ }^{3} \mathrm{D}^{\circ}\right) 7 s$ & & $2^{1 / 2}$ & 16033.149 & 1.07 & 33 & ${ }^{2} \mathrm{D}^{\circ}$ & 32 & $\left({ }^{1} \mathbf{F}^{\circ}\right){ }^{2} \mathbf{F}^{\circ}$ & 77 & B \\
\hline $5 f\left({ }^{3} \mathrm{~F}^{\circ}\right) 6 d^{2}$ & ${ }^{4} \mathrm{I}^{\circ}$ & $5^{1 / 2}$ & 16564.633 & 0.98 & 86 & & 4 & $\left({ }^{1} \mathrm{G}^{\circ}\right)^{2} \mathrm{I}^{\circ}$ & 47 & B \\
\hline $5 f\left({ }^{3} \mathrm{~F}^{\circ}\right) 6 d^{2}$ & & $3^{1 / 2}$ & 16906.604 & 0.950 & 24 & ${ }^{2} \mathrm{G}^{\circ}$ & 17 & $\left({ }^{3} \mathrm{~F}^{\circ}\right){ }^{4} \mathbf{H}^{\circ}$ & 82 & $\mathrm{~S}$ \\
\hline $5 f\left({ }^{1} \mathrm{D}^{\circ}\right) 6 d^{2}$ & & $1^{1 / 2}$ & 17121.623 & 1.277 & 28 & ${ }^{2} \mathrm{P}^{\circ}$ & 28 & $5 f 6 d\left({ }^{3} \mathrm{P}^{\circ}\right) 7 s{ }^{2} \mathrm{P}^{\circ}$ & 60 & $\mathrm{H}$ \\
\hline $5 f 6 d\left({ }^{3} \mathrm{G}^{\circ}\right) 7 s$ & ${ }^{2} \mathrm{G}^{\circ}$ & $4^{1 / 2}$ & 17272.252 & 1.083 & 58 & & 12 & $5 f\left({ }^{1} \mathrm{D}^{\circ}\right) 6 d^{2}{ }^{2} \mathrm{G}^{\circ}$ & 79 & B \\
\hline $5 f 6 d\left({ }^{3} \mathrm{P}^{\circ}\right) 7 s$ & ${ }^{4} \mathrm{P}^{\circ}$ & $2^{1 / 2}$ & 17460.625 & 1.51 & 73 & & 8 & $\left({ }^{1} \mathrm{D}^{\circ}\right)^{2} \mathrm{D}^{\circ}$ & 83 & B \\
\hline $5 f\left({ }^{3} \mathrm{~F}^{\circ}\right) 6 d^{2}$ & & $4^{1 / 2}$ & 17722.990 & 1.08 & 45 & ${ }^{2} \mathrm{G}^{\circ}$ & 30 & $\left({ }^{3} \mathbf{F}^{\circ}\right){ }^{4} \mathbf{H}^{\circ}$ & 59 & M \\
\hline $5 f\left({ }^{3} \mathrm{~F}^{\circ}\right) 6 d^{2}$ & ${ }^{4} \mathrm{H}^{\circ}$ & $5^{1 / 2}$ & 17771.06 & 1.10 & 69 & & 13 & $\left({ }^{1} G^{\circ}\right){ }^{2} I^{\circ}$ & 45 & B \\
\hline $5 f 6 d\left({ }^{3} \mathrm{P}^{\circ}\right) 7 s$ & & $1 / 2$ & 17837.804 & 1.080 & 30 & ${ }^{2} \mathrm{P}^{\circ}$ & 25 & $5 f\left({ }^{3} \mathrm{~F}^{\circ}\right) 6 d^{2}{ }^{4} \mathrm{D}^{\circ}$ & 29 & M \\
\hline $5 f\left({ }^{3} \mathrm{~F}^{\circ}\right) 6 d^{2}$ & & $2^{1 / 2}$ & 17983.388 & 0.995 & 25 & ${ }^{2} \mathrm{~F}^{\circ}$ & 14 & $\left({ }^{3} \mathrm{~F}^{\circ}\right){ }^{4} \mathbf{D}^{\circ}$ & 72 & $\mathrm{~S}$ \\
\hline $5 f\left({ }^{3} \mathrm{~F}^{\circ}\right) 6 d^{2}$ & & $1^{1 / 2}$ & 18214.423 & 0.876 & 31 & ${ }^{4} \mathbf{F}^{\circ}$ & 14 & $\left({ }^{3} \mathbf{F}^{\circ}\right){ }^{4} \mathbf{D}^{\circ}$ & 48 & $\mathrm{H}$ \\
\hline
\end{tabular}


TABLE 2. Odd energy levels of Th II-Continued

\begin{tabular}{|c|c|c|c|c|c|c|c|c|c|c|}
\hline \multirow{3}{*}{$\begin{array}{l}\text { Configuration } \\
5 f\left({ }^{3} \mathrm{~F}^{\circ}\right) 6 d^{2}\end{array}$} & \multirow{2}{*}{ Term } & \multirow{2}{*}{$J$} & \multirow{2}{*}{$\begin{array}{l}\text { Level } \\
\left(\mathrm{cm}^{-1}\right)\end{array}$} & \multirow{2}{*}{$g$} & \multicolumn{4}{|c|}{ Leading percentages } & \multirow{2}{*}{\multicolumn{2}{|c|}{$n$, ref. }} \\
\hline & & & & & \multicolumn{2}{|c|}{ First } & \multicolumn{2}{|r|}{ Second } & & \\
\hline & & $1 / 2$ & 18568.27 & 1.007 & 44 & ${ }^{2} \mathrm{~S}^{\circ}$ & 25 & $\left({ }^{3} \mathrm{~F}^{\circ}\right){ }^{4} \mathrm{D}^{\circ}$ & 31 & $\mathrm{Z}$ \\
\hline $5 f\left({ }^{3} \mathrm{~F}^{\circ}\right) 6 d^{2}$ & ${ }^{4} \mathrm{I}^{\circ}$ & $6^{1 / 2}$ & 18816.87 & 1.10 & 72 & & 8 & $\left({ }^{1} \mathrm{G}^{\circ}\right)^{2} \mathbf{I}^{\circ}$ & 34 & B \\
\hline $5 f 6 d\left({ }^{1} \mathrm{~F}^{\circ}\right) 7 s$ & & $3^{1 / 2}$ & 18973.782 & 1.12 & 37 & ${ }^{2} \mathbf{F}^{\circ}$ & 14 & $5 f\left({ }^{3} \mathrm{~F}^{\circ}\right) 6 d^{2}{ }^{2} \mathrm{G}^{\circ}$ & 57 & $\mathrm{~S}$ \\
\hline $5 f\left({ }^{3} \mathrm{~F}^{\circ}\right) 6 d^{2}$ & & $1 \frac{1 / 2}{2}$ & 19050.832 & 0.888 & 33 & ${ }^{4} \mathbf{F}^{\circ}$ & 23 & $\left({ }^{3} \mathrm{~F}^{\circ}\right){ }^{4} \mathrm{D}^{\circ}$ & 45 & $\mathrm{H}$ \\
\hline $5 f 6 d\left({ }^{3} \mathrm{D}^{\circ}\right) 7 s$ & & $2^{1 / 2}$ & 19248.267 & 0.931 & 20 & ${ }^{2} \mathrm{D}^{\circ}$ & 16 & $\left({ }^{1} \mathbf{F}^{\circ}\right){ }^{2} \mathbf{F}^{\circ}$ & 63 & $\mathrm{H}$ \\
\hline $5 f\left({ }^{3} \mathrm{~F}^{\circ}\right) 6 d^{2}$ & ${ }^{4} \mathrm{H}^{\circ}$ & $6^{1 / 2}$ & 19912.34 & 1.16 & 56 & & 22 & $\left({ }^{3} \mathbf{F}^{\circ}\right){ }^{4} \mathbf{I}^{\circ}$ & 30 & B \\
\hline $5 f 6 d\left({ }^{1} \mathrm{~F}^{\circ}\right) 7 s$ & & $3^{1 / 2}$ & 20080.667 & 1.142 & 14 & ${ }^{2} \mathbf{F}^{\circ}$ & 12 & $5 f\left({ }^{3} \mathbf{F}^{\circ}\right) 6 d^{2}{ }^{2} \mathbf{F}^{\circ}$ & 60 & $\mathrm{H}$ \\
\hline $5 f\left({ }^{3} \mathrm{P}^{\circ}\right) 6 d^{2}$ & & $2^{1 / 2}$ & 20120.157 & 0.743 & 44 & ${ }^{4} \mathrm{G}^{\circ}$ & 15 & $\left({ }^{3} \mathbf{F}^{\circ}\right){ }^{4} \mathbf{F}^{\circ}$ & 64 & $\mathrm{R}$ \\
\hline $5 f\left({ }^{1} \mathrm{G}^{\circ}\right) 6 d^{2}$ & & $5^{1 / 2}$ & 20288.57 & 0.97 & 42 & ${ }^{2} \mathbf{I}^{\circ}$ & 26 & $\left({ }^{3} \boldsymbol{F}^{\circ}\right){ }^{4} \mathrm{H}^{\circ}$ & 37 & M \\
\hline $5 f\left({ }^{3} \mathrm{~F}^{\circ}\right) 6 d^{2}$ & & $2^{1 / 2}$ & 20310.944 & 1.161 & 17 & ${ }^{4} \mathrm{D}^{\circ}$ & 15 & $\left({ }^{3} \mathbf{F}^{\circ}\right){ }^{4} \mathrm{G}^{\circ}$ & 62 & M \\
\hline $5 f\left({ }^{3} \mathrm{~F}^{\circ}\right) 6 d^{2}$ & & $2^{1 / 2}$ & 20686.148 & 0.945 & 39 & ${ }^{4} \mathbf{F}^{\circ}$ & 8 & $\left({ }^{3} \mathrm{P}^{\circ}\right){ }^{4} \mathrm{G}^{\circ}$ & 59 & B \\
\hline $5 f\left({ }^{3} \mathrm{P}^{\circ}\right) 6 d^{2}$ & & $3^{1 / 2}$ & 20969.01 & 0.99 & 39 & ${ }^{4} \mathrm{G}^{\circ}$ & 14 & $\left({ }^{3} \mathbf{F}^{\circ}\right){ }^{4} G^{\circ}$ & 50 & M \\
\hline $5 f\left({ }^{1} \mathrm{D}^{\circ}\right) 6 d^{2}$ & & $4^{1 / 2}$ & 20989.81 & 0.953 & 27 & ${ }^{2} \mathrm{H}^{\circ}$ & 16 & $5 f 6 d\left({ }^{3} \mathrm{G}^{\circ}\right) 7 s^{2} \mathrm{G}^{\circ}$ & 63 & $\mathrm{~S}$ \\
\hline $5 f 6 d\left({ }^{1} \mathrm{P}^{\circ}\right) 7 s$ & & $1^{1 / 2}$ & 21131.81 & 1.24 & 24 & ${ }^{2} \mathrm{P}^{\circ}$ & 18 & $5 f\left({ }^{3} \mathrm{~F}^{\circ}\right) 6 d^{2}{ }^{2} \mathrm{P}^{\circ}$ & 47 & M \\
\hline $5 f\left({ }^{3} \mathbf{F}^{\circ}\right) 6 d^{2}$ & & $2^{1 / 2}$ & 21297.417 & 0.767 & 38 & ${ }^{4} \mathrm{G}^{\circ}$ & 21 & $\left({ }^{3} \mathrm{P}^{\circ}\right){ }^{4} \mathrm{G}^{\circ}$ & 62 & $\mathrm{H}$ \\
\hline $5 f\left({ }^{3} \mathrm{~F}^{\circ}\right) 6 d^{2}$ & & $3^{1 / 2}$ & 21682.747 & 1.248 & 41 & ${ }^{4} \mathrm{~F}^{\circ}$ & 17 & $\left({ }^{3} \mathbf{F}^{\circ}\right){ }^{4} \mathbf{D}^{\circ}$ & 51 & B \\
\hline $5 f 6 d\left({ }^{1} \mathrm{H}^{\circ}\right) 7 s$ & ${ }^{2} \mathrm{H}^{\circ}$ & $5^{1 / 2}$ & 22014.881 & 1.10 & 61 & & 18 & $5 f\left({ }^{1} \mathrm{D}^{\circ}\right) 6 d^{2}{ }^{2} \mathrm{H}^{\circ}$ & 47 & $\mathrm{~S}$ \\
\hline $5 f\left({ }^{3} \mathbf{F}^{\circ}\right) 6 d^{2}$ & ${ }^{4} \mathbf{I}^{\circ}$ & $7^{1 / 2}$ & 22028.00 & 1.18 & 97 & & 2 & $\left({ }^{1} \mathrm{G}^{\circ}\right)^{2} \mathrm{~K}^{\circ}$ & 11 & $\mathrm{~S}$ \\
\hline $5 f\left({ }^{1} \mathrm{G}^{\circ}\right) 6 d^{2}$ & & $4^{1 / 2}$ & 22139.66 & 1.090 & 23 & ${ }^{2} \mathrm{G}^{\circ}$ & 19 & $\left({ }^{1} \mathrm{G}^{\circ}\right){ }^{2} \mathrm{H}^{\circ}$ & 55 & $\mathrm{H}$ \\
\hline $5 f 6 d\left({ }^{1} \mathrm{P}^{\circ}\right) 7 s$ & & $1 / 2$ & 22355.208 & 0.77 & 35 & ${ }^{2} \mathrm{P}^{\circ}$ & 16 & $5 f\left({ }^{3} \mathrm{~F}^{\circ}\right) 6 d^{2}{ }^{2} \mathrm{P}^{\circ}$ & 23 & M \\
\hline $5 f\left({ }^{3} \mathrm{P}^{\circ}\right) 6 d^{2}$ & & $2^{1 / 2}$ & 22513.290 & 1.17 & 43 & ${ }^{2} \mathrm{D}^{\circ}$ & 21 & $\left({ }^{3} \boldsymbol{F}^{\circ}\right){ }^{4} \mathrm{D}^{\circ}$ & 40 & $\mathrm{C}$ \\
\hline $5 f 6 d\left({ }^{1} \mathrm{H}^{\circ}\right) 7 s$ & & $4^{1 / 2}$ & 22642.107 & 1.043 & 42 & ${ }^{2} \mathrm{H}^{\circ}$ & 9 & $5 f\left({ }^{1} \mathrm{D}^{\circ}\right) 6 d^{2}{ }^{2} \mathrm{G}^{\circ}$ & 50 & $\mathrm{H}$ \\
\hline $5 f\left({ }^{3} \mathbf{F}^{\circ}\right) 6 d^{2}$ & ${ }^{4} \mathrm{G}^{\circ}$ & $3^{1 / 2}$ & 22685.447 & 0.981 & 65 & & 12 & $\left({ }^{3} \mathrm{P}^{\circ}\right){ }^{4} \mathrm{G}^{\circ}$ & 45 & B \\
\hline $5 f\left({ }^{3} \mathrm{P}^{\circ}\right) 6 d^{2}$ & & $1 \frac{1 / 2}{2}$ & 23012.060 & 1.017 & 25 & ${ }^{4} \mathrm{~F}^{\circ}$ & 13 & $\left({ }^{3} \mathrm{P}^{\circ}\right){ }^{2} \mathrm{D}^{\circ}$ & 30 & $\mathrm{H}$ \\
\hline $5 f\left({ }^{1} \mathrm{G}^{\circ}\right) 6 d^{2}$ & & $6^{1 / 2}$ & 23187.02 & & 31 & ${ }^{2} \mathbf{I}^{\circ}$ & 31 & $\left({ }^{3} \mathbf{F}^{\circ}\right){ }^{4} \mathrm{H}^{\circ}$ & 23 & $\mathrm{Z}$ \\
\hline $6 d 7 s\left({ }^{3} \mathrm{D}\right) 7 p$ & & $11 / 2$ & 23372.582 & 1.067 & 29 & ${ }^{4} \mathrm{~F}^{\circ}$ & 9 & $5 f\left({ }^{3} \mathrm{P}^{\circ}\right) 6 d^{2}{ }^{4} \mathrm{~F}^{\circ}$ & 27 & $\mathrm{H}$ \\
\hline $5 f\left({ }^{3} \mathrm{~F}^{\circ}\right) 6 d^{2}$ & & $3^{1 / 2}$ & 23518.425 & 1.2 & 21 & ${ }^{4} \mathbf{F}^{\circ}$ & 18 & $\left({ }^{3} \mathrm{~F}^{\circ}\right){ }^{4} \mathrm{D}^{\circ}$ & 45 & M \\
\hline $5 f\left({ }^{3} \mathrm{P}^{\circ}\right) 6 d^{2}$ & & $3^{1 / 2}$ & 23697.66 & 1.0 & 26 & ${ }^{4} \mathrm{G}^{\circ}$ & 26 & $\left({ }^{1} \mathrm{G}^{\circ}\right){ }^{2} \mathrm{G}^{\circ}$ & 43 & $\mathrm{Z}$ \\
\hline $5 f\left({ }^{3} \mathbf{F}^{\circ}\right) 6 d^{2}$ & & $4^{1 / 2}$ & 23730.656 & 1.186 & 45 & ${ }^{4} \mathrm{~F}^{\circ}$ & 13 & $\left({ }^{1} \mathrm{G}^{\circ}\right){ }^{2} \mathrm{H}^{\circ}$ & 35 & $\mathrm{H}$ \\
\hline $5 f\left({ }^{3} \mathbf{F}^{\circ}\right) 6 d^{2}$ & ${ }^{4} \mathrm{P}^{\circ}$ & $11 / 2$ & 24132.037 & 1.429 & 60 & & 5 & $6 d 7 s\left({ }^{3} \mathrm{D}\right) 7 p{ }^{4} \mathrm{~F}^{\circ}$ & 30 & $\mathrm{H}$ \\
\hline $5 f\left({ }^{1} \mathrm{G}^{\circ}\right) 6 d^{2}$ & & $5^{1 / 2}$ & 24309.226 & 1.11 & 25 & ${ }^{2} \mathrm{H}^{\circ}$ & 25 & $\left({ }^{3} \boldsymbol{F}^{\circ}\right){ }^{2} \mathrm{H}^{\circ}$ & 31 & M \\
\hline $5 f\left({ }^{3} \mathbf{F}^{\circ}\right) 6 d^{2}$ & & $1^{1 / 2}$ & 24414.643 & 1.105 & 24 & ${ }^{4} \mathrm{~S}^{\circ}$ & 17 & $6 d 7 s\left({ }^{3} \mathrm{D}\right) 7 p{ }^{4} \mathrm{~F}^{\circ}$ & 29 & $\mathrm{H}$ \\
\hline $5 f\left({ }^{3} \mathrm{~F}^{\circ}\right) 6 d^{2}$ & ${ }^{4} \mathrm{P}^{\circ}$ & $2^{1 / 2}$ & 24463.792 & 1.396 & 64 & & 7 & $\left({ }^{3} \mathrm{~F}^{\circ}\right)^{2} \mathrm{D}^{\circ}$ & 43 & $\mathrm{H}$ \\
\hline $5 f\left({ }^{3} \mathbf{F}^{\circ}\right) 6 d^{2}$ & & $4^{1 / 2}$ & 24757.507 & 1.13 & 45 & ${ }^{4} \mathrm{G}^{\circ}$ & 40 & $\left({ }^{3} \mathrm{P}^{\circ}\right){ }^{4} \mathrm{G}^{\circ}$ & 37 & $\mathrm{~S}$ \\
\hline $6 d 7 s\left({ }^{3} \mathrm{D}\right) 7 p$ & & $2^{1 / 2}$ & 24873.981 & 1.081 & 26 & ${ }^{4} \mathbf{F}^{\circ}$ & 20 & $5 f\left({ }^{3} \mathbf{P}^{\circ}\right) 6 d^{2}{ }^{4} \mathbf{F}^{\circ}$ & 25 & $\mathrm{H}$ \\
\hline
\end{tabular}


TABLE 2. Odd energy levels of Th $\mathrm{II}-$ Continued

\begin{tabular}{|c|c|c|c|c|c|c|c|c|c|c|}
\hline \multirow{2}{*}{ Configuration } & \multirow{2}{*}{ Term } & \multirow{2}{*}{$J$} & \multirow{2}{*}{$\begin{array}{l}\text { Level } \\
\left(\mathrm{cm}^{-1}\right)\end{array}$} & \multirow{2}{*}{$g$} & \multicolumn{4}{|c|}{ Leading perc entages } & \multirow{2}{*}{\multicolumn{2}{|c|}{$n$, ref. }} \\
\hline & & & & & \multicolumn{2}{|c|}{ First } & \multicolumn{2}{|r|}{ Second } & & \\
\hline $5 f\left({ }^{1} \mathrm{G}^{\circ}\right) 6 d^{2}$ & & $3^{1 / 2}$ & 24982.451 & 0.990 & 30 & ${ }^{2} \mathrm{G}^{\circ}$ & 24 & $\left({ }^{1} \mathrm{D}^{\circ}\right){ }^{2} \mathrm{G}^{\circ}$ & 37 & $\mathrm{H}$ \\
\hline $5 f\left({ }^{3} \mathrm{P}^{\circ}\right) 6 d^{2}$ & & $1 / 2$ & 25027.04 & 0.6 & 28 & ${ }^{4} \mathrm{D}^{\circ}$ & 22 & $\left({ }^{3} \mathrm{~F}^{\circ}\right){ }^{4} \mathrm{P}^{\circ}$ & 6 & $\mathrm{Z}$ \\
\hline $5 f\left({ }^{3} \mathrm{P}^{\circ}\right) 6 d^{2}$ & & $1^{1 / 2}$ & 25188.124 & 0.937 & 19 & ${ }^{4} \mathrm{D}^{\circ}$ & 16 & $\left({ }^{3} \mathrm{P}^{\circ}\right){ }^{4} \mathrm{~F}^{\circ}$ & 23 & $\mathrm{H}$ \\
\hline $5 f\left({ }^{3} \mathrm{~F}^{\circ}\right) 6 d^{2}$ & & $1 / 2$ & 25266.498 & 0.725 & 32 & ${ }^{4} \mathrm{P}^{\circ}$ & 29 & $\left({ }^{3} \mathrm{P}^{\circ}\right){ }^{4} \mathrm{D}^{\circ}$ & 12 & $\mathrm{H}$ \\
\hline $5 f\left({ }^{3} \mathrm{~F}^{\circ}\right) 6 d^{2}$ & & $5^{1 / 2}$ & 25414.92 & 1.236 & 44 & ${ }^{4} \mathrm{G}^{\circ}$ & 28 & $\left({ }^{1} \mathrm{G}^{\circ}\right){ }^{2} \mathrm{H}^{\circ}$ & 33 & M \\
\hline $5 f\left({ }^{3} \mathrm{P}^{\circ}\right) 6 d^{2}$ & & $2^{1 / 2}$ & 25440.234 & 1.138 & 20 & ${ }^{4} \mathbf{F}^{\circ}$ & 13 & $\left({ }^{3} \mathrm{~F}^{\circ}\right){ }^{4} \mathrm{P}^{\circ}$ & 33 & $\mathrm{H}$ \\
\hline $5 f 6 d\left({ }^{1} \mathrm{P}^{\circ}\right) 7 s$ & & $1 / 2$ & 25594.88 & 1.27 & 17 & ${ }^{2} \mathrm{P}^{\circ}$ & 14 & $5 f\left({ }^{3} \mathrm{~F}^{\circ}\right) 6 d^{2}{ }^{2} \mathrm{~S}^{\circ}$ & 7 & $\mathrm{Z}$ \\
\hline $5 f\left({ }^{3} \mathrm{~F}^{\circ}\right) 6 d^{2}$ & & $4^{1 / 2}$ & 25607.12 & 1.09 & 23 & ${ }^{4} \mathrm{G}^{\circ}$ & 17 & $\left({ }^{3} \mathrm{P}^{\circ}\right){ }^{4} \mathrm{G}^{\circ}$ & 32 & M \\
\hline $5 f\left({ }^{3} \mathrm{P}^{\circ}\right) 6 d^{2}$ & & $2^{1 / 2}$ & 26424.48 & 1.169 & 29 & ${ }^{4} \mathbf{F}^{\circ}$ & 20 & $\left({ }^{3} \mathrm{P}^{\circ}\right){ }^{4} \mathrm{D}^{\circ}$ & 29 & $\mathrm{H}$ \\
\hline $5 f\left({ }^{3} \mathrm{P}^{\circ}\right) 6 d^{2}$ & & $1^{1 / 2}$ & 26586.27 & 0.76 & 28 & ${ }^{4} \mathbf{F}^{\circ}$ & 28 & $\left({ }^{3} \mathrm{P}^{\circ}\right)^{2} \mathrm{D}^{\circ}$ & 19 & $\mathrm{Z}$ \\
\hline $6 d 7 s\left({ }^{3} \mathrm{D}\right) 7 p$ & & $1 / 2$ & 26626.478 & 0.645 & 38 & ${ }^{4} \mathrm{D}^{\circ}$ & 19 & $\left({ }^{3} \mathrm{D}\right){ }^{2} \mathbf{P}^{\circ}$ & 8 & $\mathrm{H}$ \\
\hline $5 f\left({ }^{1} \mathrm{G}^{\circ}\right) 6 d^{2}$ & ${ }^{2} \mathrm{~K}^{\circ}$ & $6^{1 / 2}$ & 26647.80 & & 86 & & 7 & $\left({ }^{1} \mathrm{G}^{\circ}\right){ }^{2} \mathbf{I}^{\circ}$ & 11 & M \\
\hline $5 f\left({ }^{3} \mathrm{P}^{\circ}\right) 6 d^{2}$ & ${ }^{4} \mathrm{G}^{\circ}$ & $5^{1 / 2}$ & 26770.495 & 1.250 & 53 & & 33 & $\left({ }^{3} \mathrm{~F}^{\circ}\right){ }^{4} \mathrm{G}^{\circ}$ & 22 & $\mathrm{~S}$ \\
\hline $5 f\left({ }^{3} \mathrm{P}^{\circ}\right) 6 d^{2}$ & ${ }^{4} \mathrm{~F}^{\circ}$ & $3^{1 / 2}$ & 26963.92 & 1.20 & 73 & & 7 & $\left({ }^{3} \mathrm{~F}^{\circ}\right){ }^{4} \mathrm{~F}^{\circ}$ & 22 & $\mathrm{Z}$ \\
\hline $5 f\left({ }^{3} \mathrm{~F}^{\circ}\right) 6 d^{2}$ & & $1^{1 / 2}$ & 26965.202 & 1.384 & 35 & ${ }^{4} \mathrm{~S}^{\circ}$ & 17 & $\left({ }^{3} \mathrm{~F}^{\circ}\right)^{2} \mathbf{P}^{\circ}$ & 24 & $\mathrm{~B}$ \\
\hline $5 f\left({ }^{3} \mathrm{P}^{\circ}\right) 6 d^{2}$ & ${ }^{4} \mathrm{D}^{\circ}$ & $3^{1 / 2}$ & 27249.543 & 1.382 & 50 & & 18 & $\left({ }^{3} \mathrm{~F}^{\circ}\right){ }^{4} \mathrm{D}^{\circ}$ & 24 & $\mathrm{R}$ \\
\hline $5 f\left({ }^{1} \mathrm{G}^{\circ}\right) 6 d^{2}$ & & $4^{1 / 2}$ & 27357.36 & 1.02 & 25 & ${ }^{2} \mathrm{H}^{\circ}$ & 14 & $\left({ }^{3} \mathrm{P}^{\circ}\right){ }^{4} \mathbf{F}^{\circ}$ & 35 & M \\
\hline $5 f\left({ }^{1} \mathrm{D}^{\circ}\right) 6 d^{2}$ & & $1^{1 / 2}$ & 27403.18 & 1.002 & 20 & ${ }^{2} \mathrm{D}^{\circ}$ & 20 & $\left({ }^{3} \mathrm{P}^{\circ}\right){ }^{4} \mathrm{D}^{\circ}$ & 11 & $\mathrm{H}$ \\
\hline $5 f\left({ }^{1} \mathrm{G}^{\circ}\right) 6 d^{2}$ & & $4 / 2$ & 27787.84 & 1.16 & 31 & ${ }^{2} \mathrm{G}^{\circ}$ & 18 & $\left({ }^{1} \mathrm{D}^{\circ}\right)^{2} \mathrm{G}^{\circ}$ & 23 & M \\
\hline $6 d 7 s\left({ }^{3} \mathrm{D}\right) 7 p$ & & $2^{1 / 2}$ & 28243.812 & 0.922 & 25 & ${ }^{4} \mathbf{F}^{\circ}$ & 17 & $6 d^{2}\left({ }^{3} \mathrm{~F}\right) 7 p{ }^{4} \mathrm{G}^{\circ}$ & 22 & $\mathrm{H}$ \\
\hline $5 f\left({ }^{1} \mathrm{G}^{\circ}\right) 6 d^{2}$ & & $2^{1 / 2}$ & 28587.36 & 1.0 & 42 & ${ }^{2} \mathrm{~F}^{\circ}$ & 17 & $\left({ }^{3} \mathbf{F}^{\circ}\right)^{2} \mathbf{F}^{\circ}$ & 26 & $\mathrm{Z}$ \\
\hline $6 d 7 s\left({ }^{3} \mathrm{D}\right) 7 p$ & & $1^{1 / 2}$ & 28720.835 & 1.162 & 46 & ${ }^{4} \mathrm{D}^{\circ}$ & 9 & $\left({ }^{3} \mathrm{D}\right){ }^{2} \mathrm{P}^{\circ}$ & 16 & $\mathrm{H}$ \\
\hline $5 f\left({ }^{3} \mathrm{~F}^{\circ}\right) 6 d^{2}$ & & $2^{1 / 2}$ & 28923.20 & 1.07 & 22 & ${ }^{2} \mathrm{D}^{\circ}$ & 15 & $\left({ }^{1} \mathrm{D}^{\circ}\right)^{2} \mathrm{D}^{\circ}$ & 19 & $\mathrm{Z}$ \\
\hline $5 f\left({ }^{1} \mathrm{D}^{\circ}\right) 6 d^{2}$ & & $2^{1 / 2}$ & 29095.467 & 0.998 & 24 & ${ }^{2} \mathrm{~F}^{\circ}$ & 11 & $6 d^{2}\left({ }^{3} \mathrm{~F}\right) 7 p{ }^{4} \mathrm{G}^{\circ}$ & 26 & $\mathrm{H}$ \\
\hline $5 f\left({ }^{3} \mathrm{~F}^{\circ}\right) 6 d^{2}$ & & $1^{1 / 2}$ & 29720.32 & 0.990 & 21 & ${ }^{2} \mathrm{D}^{\circ}$ & 15 & $\left({ }^{1} \mathrm{D}^{\circ}\right)^{2} \mathrm{D}^{\circ}$ & 21 & $\mathrm{H}$ \\
\hline $5 f\left({ }^{3} \mathrm{P}^{\circ}\right) 6 d^{2}$ & ${ }^{4} \mathrm{~F}^{\circ}$ & $4^{1 / 2}$ & 29788.45 & & 67 & & 13 & $\left({ }^{1} \mathrm{D}^{\circ}\right){ }^{2} \mathrm{G}^{\circ}$ & 18 & $\mathrm{Z}$ \\
\hline $5 f\left({ }^{1} \mathrm{G}^{\circ}\right) 6 d^{2}$ & ${ }^{2} \mathrm{~F}^{\circ}$ & $3^{1 / 2}$ & 30101.42 & 1.13 & 68 & & 13 & $\left({ }^{3} \mathrm{~F}^{\circ}\right)^{2} \mathrm{~F}^{\circ}$ & 21 & $\mathrm{Z}$ \\
\hline $5 f\left({ }^{1} \mathrm{G}^{\circ}\right) 6 d^{2}$ & ${ }^{2} \mathrm{~K}^{\circ}$ & $71 / 2$ & 30223.14 & & 97 & & 2 & $\left({ }^{3} \mathrm{~F}^{\circ}\right){ }^{4} \mathrm{I}^{\circ}$ & 4 & $\mathrm{Z}$ \\
\hline $5 f\left({ }^{1} \mathrm{G}^{\circ}\right) 6 d^{2}$ & & $5^{1 / 2}$ & 30310.210 & 1.1 & 34 & ${ }^{2} \mathrm{H}^{\circ}$ & 23 & $\left({ }^{1} \mathrm{D}^{\circ}\right){ }^{2} \mathrm{H}^{\circ}$ & 19 & $\mathrm{Z}$ \\
\hline $5 f\left({ }^{3} \mathrm{~F}^{\circ}\right) 6 d^{2}$ & & $1 / 2$ & 30564.58 & 0.553 & 25 & ${ }^{2} \mathrm{P}^{\circ}$ & 20 & $\left({ }^{1} \mathrm{D}^{\circ}\right)^{2} \mathrm{P}^{\circ}$ & 7 & $\mathrm{H}$ \\
\hline $5 f\left({ }^{3} \mathrm{~F}^{\circ}\right) 6 d^{2}$ & ${ }^{2} \mathrm{H}^{\circ}$ & $4^{1 / 2}$ & 30956.568 & 0.94 & 50 & & 23 & $\left({ }^{1} \mathrm{D}^{\circ}\right)^{2} \mathrm{H}^{\circ}$ & 16 & Z \\
\hline $6 d^{2}\left({ }^{3} \mathrm{~F}\right) 7 p$ & & $2^{1 / 2}$ & 30972.166 & 1.217 & 16 & ${ }^{4} \mathrm{G}^{\circ}$ & 15 & $6 d 7 s\left({ }^{3} \mathrm{D}\right) 7 p^{2} \mathrm{D}^{\circ}$ & 16 & $\mathrm{H}$ \\
\hline $6 d 7 s\left({ }^{3} \mathrm{D}\right) 7 p$ & ${ }^{4} \mathrm{~F}^{\circ}$ & $3^{1 / 2}$ & 30994.263 & 1.199 & 53 & & 8 & $6 d^{2}\left({ }^{3} \mathrm{~F}\right) 7 p{ }^{4} \mathrm{G}^{\circ}$ & 19 & $\mathrm{H}$ \\
\hline $6 d 7 s\left({ }^{3} \mathrm{D}\right) 7 p$ & & $1 \frac{1}{2}$ & 31353.127 & 0.863 & 17 & ${ }^{4} \mathrm{~F}^{\circ}$ & 9 & $6 d^{2}\left({ }^{3} \mathrm{~F}\right) 7 p^{2} \mathrm{D}^{\circ}$ & 15 & $\mathrm{H}$ \\
\hline $7 s^{2}\left({ }^{1} \mathrm{~S}^{\circ}\right) 7 p$ & & $1 / 2$ & 31625.680 & 0.344 & 31 & ${ }^{2} \mathrm{P}^{\circ}$ & 26 & $6 d 7 s\left({ }^{3} \mathrm{D}\right) 7 p{ }^{4} \mathrm{D}^{\circ}$ & 10 & $\mathrm{H}$ \\
\hline
\end{tabular}


TABLE 2. Odd energy levels of Th II-Continued

\begin{tabular}{|c|c|c|c|c|c|c|c|c|c|c|}
\hline \multirow{3}{*}{$\begin{array}{l}\text { Configuration } \\
5 f\left({ }^{1} \mathrm{D}^{\circ}\right) 6 d^{2}\end{array}$} & \multirow{2}{*}{ Term } & \multirow{2}{*}{$J$} & \multirow{2}{*}{$\begin{array}{l}\text { Level } \\
\left(\mathrm{cm}^{-1}\right)\end{array}$} & \multirow{2}{*}{$g$} & \multicolumn{4}{|c|}{ Leading percentages } & \multirow{2}{*}{\multicolumn{2}{|c|}{$n$, ref. }} \\
\hline & & & & & \multicolumn{2}{|c|}{ First } & \multicolumn{2}{|r|}{ Second } & & \\
\hline & & $3^{1 / 2}$ & 31800.192 & 1.168 & & ${ }^{2} \mathrm{~F}^{\circ}$ & 11 & $\left({ }^{3} \mathrm{P}^{\circ}\right)^{2} \mathrm{~F}^{\circ}$ & 24 & $\mathrm{H}$ \\
\hline $6 d 7 s\left({ }^{3} \mathrm{D}\right) 7 p$ & & $2^{1 / 2}$ & 31810.548 & 1.118 & & ${ }^{4} \mathrm{D}^{\circ}$ & 12 & $6 d^{2}\left({ }^{3} \mathrm{~F}\right) 7 p^{2} \mathrm{D}^{\circ}$ & 19 & $\mathrm{H}$ \\
\hline $5 f\left({ }^{3} \mathrm{~F}^{\circ}\right) 6 d^{2}$ & & $5^{1 / 2}$ & 31924.599 & 0.99 & 26 & ${ }^{2} \mathrm{H}^{\circ}$ & 22 & $\left({ }^{3} \mathrm{~F}^{\circ}\right){ }^{2} \mathrm{I}^{\circ}$ & 16 & $\mathrm{Z}$ \\
\hline $5 f\left({ }^{3} \mathbf{F}^{\circ}\right) 6 d^{2}$ & & $1^{1 / 2}$ & 31928.714 & 0.891 & 25 & ${ }^{2} \mathrm{D}^{\circ}$ & 16 & $\left({ }^{1} \mathrm{G}^{\circ}\right)^{2} \mathrm{D}^{\circ}$ & 18 & $\mathrm{H}$ \\
\hline $5 f\left({ }^{1} \mathrm{D}^{\circ}\right) 6 d^{2}$ & & $2^{1 / 2}$ & 32850.07 & 1.093 & 18 & ${ }^{2} \mathrm{D}^{\circ}$ & 16 & $\left({ }^{3} \mathrm{~F}^{\circ}\right){ }^{2} \mathrm{D}^{\circ}$ & 16 & $\mathrm{H}$ \\
\hline $6 d^{2}\left({ }^{3} \mathrm{~F}\right) 7 p$ & ${ }^{4} \mathrm{G}^{\circ}$ & $3^{1 / 2}$ & 32957.432 & 1.072 & 58 & & 11 & $6 d 7 s\left({ }^{3} \mathrm{D}\right) 7 p{ }^{4} \mathrm{~F}^{\circ}$ & 17 & $\mathrm{H}$ \\
\hline $5 f\left({ }^{1} \mathrm{G}^{\circ}\right) 6 d^{2}$ & & $1^{1 / 2}$ & 33215.466 & 1.036 & 20 & ${ }^{2} \mathrm{D}^{\circ}$ & 17 & $6 d^{2}\left({ }^{3} \mathrm{~F}\right) 7 p^{4} \mathrm{~F}^{\circ}$ & 16 & $\mathrm{H}$ \\
\hline $5 f\left({ }^{1} \mathrm{~S}^{\circ}\right) 6 d^{2}$ & & $2^{1 / 2}$ & 33354.78 & 0.952 & 37 & ${ }^{2} \mathrm{~F}^{\circ}$ & 14 & $\left({ }^{3} \mathrm{P}^{\circ}\right){ }^{2} \mathrm{~F}^{\circ}$ & 18 & $\mathrm{H}$ \\
\hline $5 f\left({ }^{1} \mathrm{D}^{\circ}\right) 6 d^{2}$ & & $1^{1 / 2}$ & 33843.042 & 1.19 & 32 & ${ }^{2} \mathrm{P}^{\circ}$ & 13 & $5 f 6 d\left({ }^{3} \mathrm{P}^{\circ}\right) 7 s^{2} \mathrm{P}^{\circ}$ & 9 & $\mathrm{Z}$ \\
\hline $6 d^{2}\left({ }^{1} \mathrm{G}\right) 7 p$ & & $3^{1 / 2}$ & 33902.085 & 1.0 & 19 & ${ }^{2} \mathrm{G}^{\circ}$ & 15 & $\left({ }^{1} \mathrm{G}\right){ }^{2} \mathrm{~F}^{\circ}$ & 17 & $\mathrm{Z}$ \\
\hline $6 d 7 s\left({ }^{3} \mathrm{D}\right) 7 p$ & & $2^{1 / 2}$ & 34212.024 & 1.148 & 30 & ${ }^{4} \mathrm{D}^{\circ}$ & 10 & $\left({ }^{1} \mathrm{D}\right){ }^{2} \mathrm{~F}^{\circ}$ & 16 & $\mathrm{H}$ \\
\hline $6 d^{2}\left({ }^{3} \mathrm{P}\right) 7 p$ & & $1 / 2$ & 34330.500 & 1.503 & 29 & ${ }^{2} \mathrm{~S}^{\circ}$ & 24 & $\left({ }^{3} \mathrm{P}\right){ }^{4} \mathrm{P}^{\circ}$ & 9 & $\mathrm{H}$ \\
\hline $6 d 7 s\left({ }^{3} \mathrm{D}\right) 7 p$ & & $1^{1 / 2}$ & 34986.606 & 1.298 & 27 & ${ }^{4} \mathrm{D}^{\circ}$ & 22 & $\left({ }^{3} \mathrm{D}\right){ }^{4} \mathrm{P}^{\circ}$ & 13 & $\mathrm{R}$ \\
\hline $5 f\left({ }^{1} \mathrm{G}^{\circ}\right) 6 d^{2}$ & & $2^{1 / 2}$ & 35156.916 & 1.079 & 29 & ${ }^{2} \mathrm{D}^{\circ}$ & 13 & $\left({ }^{1} \mathrm{G}^{\circ}\right){ }^{2} \mathrm{~F}^{\circ}$ & 16 & $\mathrm{H}$ \\
\hline $5 f\left({ }^{3} \mathrm{~F}^{\circ}\right) 6 d^{2}$ & ${ }^{2} I^{\circ}$ & $6^{1 / 2}$ & 35165.36 & 1.08 & 58 & & 41 & $\left({ }^{1} \mathrm{G}^{\circ}\right)^{2} \mathrm{I}^{\circ}$ & 5 & $\mathrm{Z}$ \\
\hline $6 d^{2}\left({ }^{3} \mathrm{P}\right) 7 p$ & ${ }^{4} \mathrm{D}^{\circ}$ & $1 / 2$ & 35198.99 & 0.972 & 54 & & 16 & $6 d 7 s\left({ }^{3} \mathrm{D}\right) 7 p{ }^{4} \mathrm{P}^{\circ}$ & 9 & $\mathrm{H}$ \\
\hline $6 d^{2}\left({ }^{3} \mathrm{~F}\right) 7 p$ & & $4^{1} / 2$ & 35593.436 & & 38 & ${ }^{4} \mathrm{G}^{\circ}$ & 18 & $\left({ }^{3} \mathrm{~F}\right){ }^{4} \mathrm{~F}^{\circ}$ & 8 & $\mathrm{Z}$ \\
\hline $6 d 7 s\left({ }^{3} \mathrm{D}\right) 7 p$ & & $3^{1 / 2}$ & 35593.527 & 1.253 & 39 & ${ }^{4} \mathrm{D}^{\circ}$ & 10 & $5 f\left({ }^{3} \mathrm{P}^{\circ}\right) 6 d^{2}{ }^{2} \mathrm{G}^{\circ}$ & 17 & B \\
\hline $5 f\left({ }^{3} \mathrm{~F}^{\circ}\right) 6 d^{2}$ & & $5^{1 / 2}$ & 35602.42 & 1.02 & 38 & ${ }^{2} \mathrm{I}^{\circ}$ & 22 & $\left({ }^{1} G^{\circ}\right){ }^{2} I^{\circ}$ & 10 & $\mathrm{Z}$ \\
\hline $5 f\left({ }^{3} \mathrm{P}^{\circ}\right) 6 d^{2}$ & & $4^{1 / 2}$ & 36193.437 & 1.149 & 34 & ${ }^{2} \mathrm{G}^{\circ}$ & 18 & $6 d^{2}\left({ }^{1} \mathrm{G}\right) 7 p^{2} \mathrm{G}^{\circ}$ & 14 & $\mathrm{H}$ \\
\hline $6 d^{2}\left({ }^{3} \mathrm{~F}\right) 7 p$ & & $1 \frac{1 / 2}{2}$ & 36390.527 & 0.829 & 21 & ${ }^{4} \mathrm{~F}^{\circ}$ & 21 & $5 f\left({ }^{1} \mathrm{G}^{\circ}\right) 6 d^{2}{ }^{2} \mathrm{P}^{\circ}$ & 14 & $\mathrm{H}$ \\
\hline $6 d^{2}\left({ }^{3} \mathrm{P}\right) 7 p$ & & $1^{1 / 2}$ & 36444.815 & 1.182 & 47 & ${ }^{4} \mathrm{D}^{\circ}$ & 9 & $5 f\left({ }^{1} \mathrm{G}^{\circ}\right) 6 d^{2}{ }^{2} \mathrm{P}^{\circ}$ & 13 & $\mathrm{H}$ \\
\hline $6 d^{2}\left({ }^{3} \mathrm{~F}\right) 7 p$ & & $1^{1 / 2}$ & 36581.568 & 1.107 & 21 & ${ }^{4} \mathrm{~F}^{\circ}$ & 14 & $5 f\left({ }^{1} \mathrm{G}^{\circ}\right) 6 d^{2}{ }^{2} \mathrm{D}^{\circ}$ & 14 & $\mathrm{H}$ \\
\hline $6 d^{2}\left({ }^{1} \mathrm{D}\right) 7 p$ & & $3^{1 / 2}$ & 36583.82 & 1.140 & 21 & ${ }^{2} \mathrm{~F}^{\circ}$ & 19 & $6 d 7 s\left({ }^{1} \mathrm{D}\right) 7 p{ }^{2} \mathrm{~F}^{\circ}$ & 16 & $\mathrm{H}$ \\
\hline $6 d^{2}\left({ }^{3} \mathrm{~F}\right) 7 p$ & & $2^{1 / 2}$ & 36687.992 & 1.049 & 41 & ${ }^{4} \mathrm{~F}^{\circ}$ & 8 & $6 d 7 s\left({ }^{3} \mathrm{D}\right) 7 p{ }^{4} \mathrm{P}^{\circ}$ & 14 & $\mathrm{H}$ \\
\hline $5 f\left({ }^{1} \mathrm{G}^{\circ}\right) 6 d^{2}$ & ${ }^{2} \mathrm{P}^{\circ}$ & $1 / 2$ & 37130.340 & 0.521 & 70 & & 8 & $6 d^{2}\left({ }^{3} \mathrm{P}\right) 7 p{ }^{4} \mathrm{D}^{\circ}$ & 10 & $\mathrm{H}$ \\
\hline $6 d 7 s\left({ }^{3} \mathrm{D}\right) 7 p$ & ${ }^{4} \mathrm{~F}^{\circ}$ & $4^{1 / 2}$ & 37569.762 & 1.29 & 70 & & 21 & $6 d^{2}\left({ }^{3} \mathrm{~F}\right) 7 p{ }^{4} \mathrm{G}^{\circ}$ & 9 & B \\
\hline $6 d 7 s\left({ }^{3} \mathrm{D}\right) 7 p$ & & $1^{1 / 2}$ & 37601.699 & 1.267 & 24 & ${ }^{2} \mathrm{D}^{\circ}$ & 11 & $6 d^{2}\left({ }^{1} \mathrm{D}\right) 7 p^{2} \mathrm{P}^{\circ}$ & 15 & $\mathrm{H}$ \\
\hline $6 d 7 s\left({ }^{3} \mathrm{D}\right) 7 p$ & & $1 / 2$ & 37607.989 & 2.239 & 43 & ${ }^{4} \mathrm{P}^{\circ}$ & 33 & $6 d^{2}\left({ }^{3} \mathrm{P}\right) 7 p^{2} \mathrm{~S}^{\circ}$ & 7 & $\mathrm{H}$ \\
\hline $6 d^{2}\left({ }^{1} \mathrm{D}\right) 7 p$ & & $1 / 2$ & 37716.322 & 0.690 & 28 & ${ }^{2} \mathrm{P}^{\circ}$ & 18 & $\left({ }^{3} F\right){ }^{4} D^{\circ}$ & 5 & $\mathrm{H}$ \\
\hline $5 f\left({ }^{1} \mathrm{~S}^{\circ}\right) 6 d^{2}$ & & $3^{1 / 2}$ & 37756.790 & 1.118 & 27 & ${ }^{2} \mathrm{~F}^{\circ}$ & 20 & $\left({ }^{3} \mathrm{P}^{\circ}\right)^{2} \mathrm{~F}^{\circ}$ & 18 & $\mathrm{H}$ \\
\hline $6 d^{2}\left({ }^{3} \mathrm{P}\right) 7 p$ & & $2^{1 / 2}$ & 37846.174 & 1.165 & 17 & ${ }^{2} \mathrm{D}^{\circ}$ & 15 & $\left({ }^{3} \mathrm{P}\right){ }^{4} \mathrm{D}^{\circ}$ & 22 & $\mathrm{H}$ \\
\hline $6 d 7 s\left({ }^{3} \mathrm{D}\right) 7 p$ & & $1^{1 / 2}$ & 38436.169 & 1.419 & 33 & ${ }^{4} \mathrm{P}^{\circ}$ & 27 & $7 s^{2}\left({ }^{1} \mathrm{~S}\right) 7 p{ }^{2} \mathrm{P}^{\circ}$ & 12 & $\mathrm{H}$ \\
\hline $6 d^{2}\left({ }^{3} \mathrm{P}\right) 7 \mathrm{P}^{3}$ & & $2^{1 / 2}$ & 38493.769 & 1.217 & 18 & ${ }^{4} \mathrm{D}^{\circ}$ & 11 & $6 d 7 s\left({ }^{1} \mathrm{D}\right) 7 p^{2} \mathrm{D}^{\circ}$ & 21 & $\mathrm{H}$ \\
\hline $6 d^{2}\left({ }^{3} \mathrm{~F}\right) 7 p$ & & $2^{1 / 2}$ & 38581.627 & 1.105 & 20 & ${ }^{2} \mathrm{~F}^{\circ}$ & 16 & $6 d 7 s\left({ }^{3} \mathrm{D}\right) 7 p{ }^{2} \mathrm{~F}^{\circ}$ & 21 & $\mathrm{H}$ \\
\hline
\end{tabular}


TABLE 2. Odd energy levels of Th II-Continued

\begin{tabular}{|c|c|c|c|c|c|c|c|c|c|c|}
\hline \multirow{2}{*}{ Configuration } & \multirow{2}{*}{ Term } & \multirow{2}{*}{$J$} & \multirow{2}{*}{$\begin{array}{l}\text { Level } \\
\left(\mathrm{cm}^{-1}\right)\end{array}$} & \multirow{2}{*}{$g$} & \multicolumn{4}{|c|}{ Leading percentages } & \multirow{2}{*}{\multicolumn{2}{|c|}{$n$, ref. }} \\
\hline & & & & & \multicolumn{2}{|c|}{ First } & \multicolumn{2}{|r|}{ Second } & & \\
\hline $6 d^{2}\left({ }^{3} \mathrm{~F}\right) 7 p$ & & $3^{1 / 2}$ & 38764.652 & 1.098 & 19 & ${ }^{2} \mathrm{G}^{\circ}$ & 18 & $\left({ }^{3} \mathrm{~F}\right){ }^{2} \mathrm{~F}^{\circ}$ & 14 & B \\
\hline $6 d^{2}\left({ }^{1} \mathrm{G}\right) 7 p$ & & $3^{1 / 2}$ & 39396.55 & 1.131 & 24 & ${ }^{2} \mathrm{~F}^{\circ}$ & 17 & $5 f\left({ }^{1} \mathrm{~S}^{\circ}\right) 6 d^{2}{ }^{2} \mathrm{~F}^{\circ}$ & 19 & $\mathrm{H}$ \\
\hline $6 d^{2}\left({ }^{3} \mathrm{~F}\right) 7 p$ & & $4^{1 / 2}$ & 39442.996 & 1.145 & 30 & ${ }^{4} \mathrm{G}^{\circ}$ & 16 & $\left({ }^{3} \mathrm{~F}\right){ }^{2} \mathrm{G}^{\circ}$ & 11 & $\mathrm{H}$ \\
\hline $6 d 7 s\left({ }^{3} \mathrm{D}\right) 7 p$ & & $2^{1 / 2}$ & 39861.642 & 1.172 & 26 & ${ }^{4} \mathrm{P}^{\circ}$ & 11 & $6 d^{2}\left({ }^{1} \mathrm{G}\right) 7 p^{2} \mathrm{~F}^{\circ}$ & 19 & $\mathrm{H}$ \\
\hline $6 d^{2}\left({ }^{3} \mathrm{~F}\right) 7 p$ & & $3^{1 / 2}$ & 40184.111 & 1.230 & 20 & ${ }^{4} \mathrm{~F}^{\circ}$ & 16 & $\left({ }^{3} \mathrm{~F}\right){ }^{4} \mathrm{D}^{\circ}$ & 13 & B \\
\hline $6 d^{2}\left({ }^{3} \mathrm{~F}\right) 7 p$ & & $1^{1 / 2}$ & 40472.459 & 1.249 & 39 & ${ }^{4} \mathrm{D}^{\circ}$ & 14 & $6 d 7 s\left({ }^{1} \mathrm{D}\right) 7 p^{2} \mathrm{D}^{\circ}$ & 17 & $\mathrm{H}$ \\
\hline $5 f\left({ }^{3} \mathrm{P}^{\circ}\right) 6 d^{2}$ & & $2^{1 / 2}$ & 40654.460 & 0.943 & 21 & ${ }^{2} \mathrm{~F}^{\circ}$ & 14 & $\left({ }^{1} \mathrm{~S}^{\circ}\right){ }^{2} \mathrm{~F}^{\circ}$ & 17 & $\mathrm{H}$ \\
\hline $6 d^{2}\left({ }^{1} \mathrm{G}\right) 7 p$ & & $3^{1 / 2}$ & 40706.82 & 1.073 & 24 & ${ }^{2} \mathrm{G}^{\circ}$ & 15 & $5 f\left({ }^{1} \mathrm{~S}^{\circ}\right) 6 d^{2}{ }^{2} \mathrm{~F}^{\circ}$ & 15 & $\mathrm{H}$ \\
\hline $6 d^{2}\left({ }^{3} \mathrm{~F}\right) 7 p$ & ${ }^{4} \mathrm{D}^{\circ}$ & $1 / 2$ & 40825.607 & 0.261 & 51 & & 8 & $6 d 7 s\left({ }^{3} \mathrm{D}\right) 7 p{ }^{2} \mathrm{P}^{\circ}$ & 9 & $\mathrm{H}$ \\
\hline $6 d^{2}\left({ }^{3} \mathrm{~F}\right) 7 p$ & ${ }^{4} \mathrm{G}^{\circ}$ & $5^{1 / 2}$ & 41447.968 & 1.267 & 88 & & 4 & $5 f 6 d\left({ }^{3} \mathrm{G}^{\circ}\right) 7 s{ }^{4} \mathrm{G}^{\circ}$ & 6 & $\mathrm{R}$ \\
\hline $6 d^{2}\left({ }^{1} \mathrm{D}\right) 7 p$ & & $1^{1 / 2}$ & 42112.94 & 1.467 & 21 & ${ }^{2} \mathrm{P}^{\circ}$ & 16 & $\left({ }^{3} \mathrm{P}\right){ }^{4} \mathrm{~S}^{\circ}$ & 16 & $\mathrm{H}$ \\
\hline $6 d^{2}\left({ }^{3} \mathrm{~F}\right) 7 p$ & & $2^{1 / 2}$ & 42219.764 & 1.168 & 31 & ${ }^{4} \mathrm{D}^{\circ}$ & 15 & (1D) ${ }^{2} \mathrm{~F}^{\circ}$ & 21 & $\mathrm{H}$ \\
\hline $6 d^{2}\left({ }^{3} \mathrm{~F}\right) 7 p$ & & $4^{1} / 2$ & 42319.024 & 1.165 & 38 & ${ }^{4} \mathrm{~F}^{\circ}$ & 32 & $\left({ }^{1} \mathrm{G}\right){ }^{2} \mathrm{H}^{\circ}$. & 10 & $\mathrm{H}$ \\
\hline $6 d^{2}\left({ }^{3} \mathrm{~F}\right) 7 p$ & & $1^{1 / 2}$ & 42418.228 & 1.304 & 24 & ${ }^{4} \mathrm{D}^{\circ}$ & 14 & $\left({ }^{3} \mathrm{P}\right){ }^{2} \mathrm{D}^{\circ}$ & 16 & $\mathrm{H}$ \\
\hline $6 d^{2}\left({ }^{3} \mathbf{F}\right) 7 p$ & & $3^{1 / 2}$ & 42944.467 & 1.246 & 25 & ${ }^{4} \mathrm{D}^{\circ}$ & 23 & $6 d 7 s\left({ }^{3} \mathrm{D}\right) 7 p{ }^{2} \mathrm{~F}^{\circ}$ & 16 & $\mathrm{H}$ \\
\hline $6 d^{2}\left({ }^{3} \mathrm{P}\right) 7 p$ & & $1^{1 / 2}$ & 43287.642 & 1.564 & 40 & ${ }^{4} \mathrm{~S}^{\circ}$ & 32 & $\left({ }^{3} \mathrm{P}\right){ }^{4} \mathbf{P}^{\circ}$ & 14 & $\mathrm{H}$ \\
\hline $6 d^{2}\left({ }^{3} \mathrm{P}\right) 7 p$ & & $2^{1 / 2}$ & 43382.780 & 1.203 & 39 & ${ }^{4} \mathrm{D}^{\circ}$ & 18 & $\left({ }^{3} \mathrm{~F}\right){ }^{4} \mathrm{D}^{\circ}$ & 16 & $\mathrm{H}$ \\
\hline $6 d^{2}\left({ }^{3} \mathrm{P}\right) 7 p$ & ${ }^{4} \mathrm{P}^{\circ}$ & $1 / 2$ & 43744.11 & 2.219 & 54 & & 19 & $\left({ }^{3} \mathrm{P}\right){ }^{2} \mathrm{~S}^{\circ}$ & 9 & $\mathrm{H}$ \\
\hline $6 d^{2}\left({ }^{1} \mathrm{G}\right) 7 p$ & & $3^{1 / 2}$ & 43773.64 & 1.052 & 18 & ${ }^{2} \mathrm{G}^{\circ}$ & 16 & $\left({ }^{3} \mathrm{P}\right){ }^{4} \mathrm{D}^{\circ}$ & 16 & B \\
\hline $6 d^{2}\left({ }^{3} \mathrm{P}\right) 7 p$ & & $3^{1 / 2}$ & 44650.491 & 1.234 & 42 & ${ }^{4} \mathrm{D}^{\circ}$ & 11 & $\left({ }^{3} \mathrm{~F}\right){ }^{2} \mathrm{G}^{\circ}$ & 17 & $\mathrm{H}$ \\
\hline $6 d 7 s\left({ }^{3} \mathrm{D}\right) 7 p$ & & $2^{1 / 2}$ & 44821.897 & 0.999 & 30 & ${ }^{2} \mathrm{~F}^{\circ}$ & 10 & $5 f\left({ }^{3} \mathrm{P}^{\circ}\right) 6 d^{2}{ }^{2} \mathrm{~F}^{\circ}$ & 14 & $\mathrm{H}$ \\
\hline $6 d^{2}\left({ }^{3} \mathrm{P}\right) 7 p$ & & $2^{1 / 2}$ & 45221.54 & 1.347 & 47 & ${ }^{4} \mathrm{P}^{\circ}$ & 10 & $6 d 7 s\left({ }^{3} \mathrm{D}\right) 7 p{ }^{2} \mathrm{~F}^{\circ}$ & 15 & $\mathrm{H}$ \\
\hline $6 d^{2}\left({ }^{3} \mathrm{~F}\right) 7 p$ & ${ }^{2} \mathrm{G}^{\circ}$ & $4^{1 / 2}$ & 45489.129 & 1.089 & 61 & & 18 & $\left({ }^{3} \mathrm{~F}\right){ }^{4} \mathrm{~F}^{\circ}$ & 12 & $\mathrm{H}$ \\
\hline $6 d^{2}\left({ }^{3} \mathrm{~F}\right) 7 p$ & & $3^{1 / 2}$ & 45689.325 & 1.261 & 18 & ${ }^{2} \mathrm{~F}^{\circ}$ & 13 & $6 d 7 s\left({ }^{1} \mathrm{D}\right) 7 p^{2} \mathrm{~F}^{\circ}$ & 11 & $\mathrm{H}$ \\
\hline $6 d 7 s\left({ }^{1} \mathrm{D}\right) 7 p$ & & $1^{1 / 2}$ & 45697.78 & 1.18 & 30 & ${ }^{2} \mathrm{P}^{\circ}$ & 10 & $6 d^{2}\left({ }^{3} \mathrm{P}\right) 7 p^{2} \mathrm{D}^{\circ}$ & 10 & B \\
\hline $6 d^{2}\left({ }^{3} \mathrm{P}\right) 7 p$ & & $11 / 2$ & 45838.437 & & 31 & ${ }^{2} \mathrm{P}^{\circ}$ & 20 & $\left({ }^{1} \mathrm{D}\right){ }^{2} \mathrm{D}^{\circ}$ & 5 & $\mathrm{Z}$ \\
\hline $6 d^{2}\left({ }^{3} \mathrm{P}\right) 7 p$ & & $2^{1 / 2}$ & 46155.54 & 1.152 & 33 & ${ }^{2} \mathrm{D}^{\circ}$ & 12 & $6 d 7 s\left({ }^{3} \mathrm{D}\right) 7 p^{2} \mathrm{D}^{\circ}$ & 16 & $\mathrm{H}$ \\
\hline $6 d^{2}\left({ }^{1} \mathrm{G}\right) 7 p$ & ${ }^{2} \mathrm{G}^{\circ}$ & $4^{1 / 2}$ & 46368.21 & 1.088 & 51 & & 29 & $\left({ }^{1} \mathrm{G}\right){ }^{2} \mathrm{H}^{\circ}$ & 8 & $\mathrm{H}$ \\
\hline $6 d 7 s\left({ }^{3} \mathrm{D}\right) 7 p$ & & $11 / 2$ & 46423.76 & 1.118 & 20 & ${ }^{2} \mathrm{P}^{\circ}$ & 14 & $6 s^{2}\left({ }^{1} \mathrm{~S}\right) 7 p^{2} \mathrm{P}^{\circ}$ & 14 & $\mathrm{H}$ \\
\hline $6 d^{2}\left({ }^{1} \mathrm{~S}\right) 7 p$ & & $1 / 2$ & 46426.05 & 0.9 & 16 & ${ }^{2} \mathrm{P}^{\circ}$ & 14 & $6 d 7 s\left({ }^{3} \mathrm{D}\right) 7 p{ }^{2} \mathrm{P}^{\circ}$ & 6 & $\mathrm{Z}$ \\
\hline $6 d^{2}\left({ }^{1} \mathrm{G}\right) 7 p$ & ${ }^{2} \mathrm{H}^{\circ}$ & $5^{1 / 2}$ & 47466.262 & 1.13 & 83 & & 6 & $5 f 6 d\left({ }^{1} \mathrm{H}^{\circ}\right) 7 s{ }^{2} \mathrm{H}^{\circ}$ & 4 & $\mathrm{Z}$ \\
\hline $6 d 7 s\left({ }^{1} \mathrm{D}\right) 7 p$ & & $1 / 2$ & 48532.05 & & 30 & ${ }^{2} \mathrm{P}^{\circ}$ & 24 & $\left({ }^{3} \mathrm{D}\right){ }^{2} \mathrm{P}^{\circ}$ & 8 & $\mathrm{Z}$ \\
\hline $6 d^{2}\left({ }^{1} \mathrm{D}\right) 7 p$ & & $3^{1 / 2}$ & 49121.92 & 1.11 & 36 & ${ }^{2} \mathrm{~F}^{\circ}$ & 24 & $6 d 7 s\left({ }^{1} \mathrm{D}\right) 7 p{ }^{2} \mathrm{~F}^{\circ}$ & 9 & $\mathrm{Z}$ \\
\hline $6 d^{2}\left({ }^{1} \mathrm{D}\right) 7 p$ & & $2^{1 / 2}$ & 49289.524 & 1.208 & 47 & ${ }^{2} \mathrm{D}^{\circ}$ & 10 & $\left({ }^{3} \mathrm{P}\right){ }^{4} \mathrm{P}^{\circ}$ & 9 & $\mathrm{H}$ \\
\hline $5 f^{2} 7 p ?$ & & $4^{1 / 2}$ & 49495.57 & & & & & & 14 & $\mathrm{Z}$ \\
\hline $6 d^{2}\left({ }^{3} \mathrm{P}\right) 7 p$ & & $1^{1 / 2}$ & 51528.46 & 0.925 & 25 & $2 \mathrm{D}^{\circ}$ & 16 & $6 d 7 s\left({ }^{1} \mathrm{D}\right) 7 p^{2} \mathrm{D}^{\circ}$ & 11 & C \\
\hline $6 d^{2}\left({ }^{1} \mathrm{G}\right) 7 p$ & & $2^{1 / 2}$ & 51946.84 & 0.937 & 37 & ${ }^{2} \mathrm{~F}^{\circ}$ & 11 & $6 d 7 s\left({ }^{3} \mathrm{D}\right) 7 p^{2} \mathrm{~F}^{\circ}$ & 12 & B \\
\hline $5 f^{2} 7 p ?$ & & $2^{1 / 2}$ & 52678.41 & & & & & & 11 & $\mathrm{Z}$ \\
\hline
\end{tabular}


TABLE 2. Odd energy levels of Th II-Continued

\begin{tabular}{|c|c|c|c|c|c|c|c|c|c|}
\hline \multirow{2}{*}{ Configuration } & \multirow{2}{*}{ Term } & \multirow{2}{*}{$J$} & \multirow{2}{*}{$\begin{array}{l}\text { Level } \\
\left(\mathrm{cm}^{-1}\right)\end{array}$} & \multirow{2}{*}{$g$} & \multicolumn{3}{|c|}{ Leading percentages } & \multirow{2}{*}{\multicolumn{2}{|c|}{$n$, ref. }} \\
\hline & & & & & First & & Second & & \\
\hline $5 f 7 p^{2} ?$ & & $4^{1 / 2}$ & 54360.46 & & & & & 12 & Z \\
\hline $6 d 7 s\left({ }^{3} \mathrm{D}\right) 7 p$ & & $3^{1 / 2}$ & 54497.69 & 1.126 & $28 \quad{ }^{2} \mathrm{~F}^{\circ}$ & 27 & $6 d^{2}\left({ }^{1} \mathrm{G}\right) 7 p{ }^{2} \mathrm{~F}^{\circ}$ & 10 & C \\
\hline $6 d^{2}\left({ }^{3} \mathrm{~F}\right) 7 p$ & & $2^{1 / 2}$ & 54648.28 & & $21 \quad{ }^{2} \mathrm{D}^{\circ}$ & 15 & $\left({ }^{1} \mathrm{G}\right){ }^{2} \mathrm{~F}^{\circ}$ & 12 & Z \\
\hline $5 f^{2} 7 p ?$ & & $4^{1 / 2}$ & 55038.943 & & & & & 11 & Z \\
\hline $5 f^{2} 7 p ?$ & & $4^{1 / 2}$ & 56086.24 & & & & & 17 & $\mathrm{Z}$ \\
\hline $5 f^{2} 7 p ?$ & & $3^{1 / 2}$ & 56683.43 & & & & & 17 & $\mathrm{Z}$ \\
\hline $5 f^{2} 7 p ?$ & & $2^{1 / 2}$ & 56892.09 & & & & & 15 & $\mathrm{Z}$ \\
\hline $5 f^{2} 7 p ?$ & & $2^{1 / 2}$ & 59299.15 & & & & & 15 & Z \\
\hline & & $1 \frac{1 / 2}{2}$ & 59616.61 & 1.1 & & & & 13 & $\mathrm{Z}$ \\
\hline $5 f 7 p^{2} ?$ & & $1 \frac{1}{2}$ & 59987.43 & & & & & 11 & $\mathrm{Z}$ \\
\hline $5 f^{2} 7 p ?$ & & $4^{1 / 2}$ & 59993.66 & & & & & 11 & $\mathrm{Z}$ \\
\hline
\end{tabular}

\subsection{Classified Lines}

The classified lines of Th II (table 3) consist of 6500 lines in the wavelength range 2000 to $25000 \AA$. Following the measured wavelength in table 3 , the visually estimated intensities in electrodeless lamp and sliding spark spectra are given. Lines not observed as single sharp lines are described with the following symbols:

$b=$ blend (two wavelengths measured)

$d=$ double (one wavelength measured)

$h=$ hazy

$l=$ shaded to long wavelength

$r=$ reversed

$s=$ shaded to short wavelengths

$w=$ wide

In column 4 is the wavenumber in vacuum in units of $\mathrm{cm}^{-1}$. The classification is given by the values of the energy levels responsible for the transition, with their $J$-values as subscripts, and a superscript degree symbol to indicate odd parity.

The average absolute value of $\mathrm{O}-\mathrm{C}$ in $\mathrm{cm}^{-1}$ for 100 lines at each of seven wavelengths is shown below.

\begin{tabular}{cr}
\hline Wavelength & O-C \\
\hline 3000 & 0.007 \\
3500 & .009 \\
4000 & .010 \\
4500 & .012 \\
6000 & .012 \\
9000 & .018 \\
15000 & .002 \\
\hline
\end{tabular}

The general precision of the wave numbers of the lines was determined by measuring the precision of repeating differences between three pairs of odd levels. The average deviation of 180 differences from the means was $0.015 \mathrm{~cm}^{-1}$. Therefore the average deviation for a single wavenumber is about $0.011 \mathrm{~cm}^{-1}$.

The Th II lines that have not been classified will be published in an improved complete description of thorium spectra.

The authors express their appreciation for the help of Mrs. Ruth Peterson in handling the data during this work.

\section{References}

Bernage, P., Houdart, R., and Niay, P. [1971], Spectrochim. Acta 26B, 261.

Brewer. L. [1971], J. Opt. Soc. Am. 61, 1674.

Charles. G. W. [1958], Oak Ridge Nat. Lab., ORNL-2319.

Connes. J., Delouis. H., Connes. P., Guelachvili. G., Maillard. J., and Michel. G. [1970], Nouv. Rev. d'Optique Appl. 1,3.

de Bruin, T. L., Schuurmans, P., and Klinkenberg, P. F. A. [1943] Z. Phys. 121, 667.

de Bruin, T. L., Klinkenberg, P. F. A., and Schuurmans. P. [1944], Z. Phys. 122, 23.

Giacchetti. A., Blaise, J., Corliss, C. H., and Zalubas, R. [1974], J. Res. Nat. Bur. Stand. (U.S. 78A (Phys. and Chem.) No. 2, 247.

Giacchetti. A., Stanley, R. W., and Zalubas, R. [1970], J. Opt. Soc. Am. 60, 474 .

Harrison, G. R. [1939], MIT Wavelength Tables (John Wiley \& Sons. N.Y.).

MeNally, J. R., Harrison, G. R., and Park, H. B. [1942], J. Opt. Soc. Am. 32, 334.

McNally, J. R. [1945], J. Opt. Soc. Am. 35, 390

Minsky, N. [1969], Dissertation, Hebrew Univ., Jerusalem.

Nielson, C. W. and Koster. G. F., Spectroscopic Coefficients for the $p^{n}, d^{n}, f^{n}$ Configurations, p, 2 (MIT Press, Cambridge, Mass., 1963).

Racah. G. [1943], Phys. Rev. 63, 367.

Schuurmans. P. [1946], Dissertation, Univ. Amsterdam (N.V. NoordHollandsche Uitgevers Maatschappij, Amsterdam).

Steers. E. B. M. [1967], Spectrochim. Acta 23B, 135.

Zalubas. R. [1959], J. Res. Nat. Bur. Stand. (U.S.) 63A (Phys. and Chem.), No. 3. 275.

Zalubas. R. [1960], Nat. Bur. Stand. (U.S.), Monogr. 17, 103 pages. 
TABle 3. Classified lines of Th II

\begin{tabular}{|c|c|c|c|c|c|c|c|c|c|}
\hline \multirow{2}{*}{$\begin{array}{c}\text { Wavelength } \\
\AA\end{array}$} & \multicolumn{2}{|c|}{ Intensity } & \multirow{2}{*}{$\begin{array}{c}\text { Wavenumber } \\
\mathbf{c m}^{-1}\end{array}$} & \multirow{2}{*}{ Classification } & \multirow{2}{*}{$\begin{array}{c}\text { Wavelength } \\
\AA\end{array}$} & \multicolumn{2}{|c|}{ Intensity } & \multirow{2}{*}{$\begin{array}{c}\text { Wavenumber } \\
\mathbf{c m}^{-1}\end{array}$} & \multirow{2}{*}{ Classification } \\
\hline & Lamp & Spark & & & & Lamp & Spark & & \\
\hline 29068.481 & 1 & & 3439.214 & $10379_{9 / 2}-13818_{7 / 2}^{\circ}$ & 21121.474 & 1 & & 4733.226 & $10572_{9 / 2}^{\circ}-15305_{9 / 2}$ \\
\hline 25401.710 & 1 & & 3935.669 & $10855_{7 / 2}-14790_{7 / 2}^{\circ}$ & 21046.638 & 0 & & 4750.056 & $23187_{13 / 2}^{\circ}-27937_{11 / 2}^{\circ}$ \\
\hline 25181.424 & 1 & & 3970.098 & $4490_{5 / 2}^{\circ}-8460_{3 / 2}$ & 21014.864 & 2 & & 4757.238 & $9061_{5 / 2}-13818_{7 / 2}^{\circ}$ \\
\hline 24926.551 & 3 & & 4010.692 & $9238_{9 / 2}^{\circ}-13248_{9 / 2}^{\circ}$ & 20934.926 & 0 & & 4775.403 & $15305_{9 / 2}-20080_{7 / 2}^{\circ}$ \\
\hline 24887.997 & 2 & & 4016.905 & $14101_{1 / 2}^{\circ}-18118_{3 / 2}^{1 / 2}$ & 20692.063 & 6 & & 4831.452 & $1859_{3 / 2}-6691_{3 / 2}^{\circ}$ \\
\hline 24352.698 & 0 & & 4105.201 & $10379_{9 / 2}-14484_{11 / 2}^{\circ}$ & 20682.889 & 4 & & 4833.595 & $9711_{7 / 2}-14545_{5 / 2}^{\circ}$ \\
\hline 24345.717 & 0 & & 4106.378 & $9711_{7 / 2}-13818_{7 / 2}^{\circ}$ & 20649.114 & 1 & & 4841.501 & $22685_{7 / 2}^{\circ}-27526_{9 / 2}$ \\
\hline 24291.248 & 3 & & 4115.586 & $4490_{5 / 2}^{\circ}-8605_{5 / 2}^{\circ}$ & 20609.747 & 1 & & 4850.749 & $17983_{5 / 2}^{\circ}-22834_{7 / 2}$ \\
\hline 24060.000 & 1 & & 4155.142 & $6700_{9 / 2}^{\circ}-10855_{7 / 2}^{\circ}$ & 20575.702 & 0 & & 4858.775 & $22106_{5 / 2}-26965_{3 / 2}^{\circ}$ \\
\hline 23964.262 & 3 & & 4171.742 & $16818_{z / 2}-20989_{9 / 2}^{\circ}$ & 20554.521 & 3 & & 4863.782 & $10379_{9 / 2}-15242_{9 / 2}^{\circ}$ \\
\hline 23850.490 & 2 & & 4191.642 & $8378_{7 / 2}^{\circ}-12570_{7 / 2}$ & 20528.880 & 4 & & 4869.857 & $8378_{7 / 2}^{\circ}-13248_{9 / 2}$ \\
\hline 23745.817 & 1 & & 4210.119 & $13250_{5 / 2}-17460_{5 / 2}^{\circ}$ & 20521.311 & 2 & & 4871.653 & $8378_{7 / 2}^{\circ}-13250_{5 / 2}$ \\
\hline 23742.146 & 4 & & 4210.770 & $6168_{/ / 2}^{\circ}-10379_{9 / 2}$ & 20468.653 & 4 & & 4884.186 & $8018_{3 / 2}-12902_{3 / 2}^{\circ}$ \\
\hline 23696.506 & 1 & & 4218.880 & $14349_{1 / 2}-18568_{1 / 2}^{\circ}$ & 20450.627 & 1 & & 4888.491 & $7331_{5 / 2}^{\circ}-12219_{3 / 2}$ \\
\hline 23621.480 & 2 & & 4232.280 & $4146_{7 / 2}-8378_{7 / 2}^{\circ}$ & 20443.773 & 2 & & 4890.130 & $12570_{7 / 2}-17460_{5 / 2}^{\circ}$ \\
\hline 23597.934 & 1 & & 4236.503 & $18118_{3 / 2}-22355_{1 / 2}^{\circ}$ & 20430.283 & 0 & & 4893.359 & $18118_{3 / 2}-23012_{3 / 2}^{\circ}$ \\
\hline 23477.236 & 1 & & 4258.283 & $13468_{9 / 2}^{\circ}-17727_{11 / 2}^{\circ}$ & 20395.738 & 3 & & 4901.647 & $12219_{3 / 2}-17121_{3 / 2}^{\circ}$ \\
\hline 23458.743 & 0 & & 4261.640 & $20120_{5 / 2}^{\circ}-24381_{7 / 2}^{\circ}$ & 20389.710 & 4 & & 4903.096 & $6213_{9 / 2}-11116_{7 / 2}^{\circ}$ \\
\hline 23437.525 & 5 & & 4265.498 & $4113_{5 / 2 / 2}-8378_{7 / 2}^{\circ / 2}$ & 20358.092 & 0 & & 4910.711 & $4490_{5 / 2}^{\circ}-9400_{5 / 2}$ \\
\hline 23372.954 & 0 & & 4277.282 & $20969_{7 / 2}^{\circ}-25246_{9 / 2}^{\circ}$ & 20165.057 & 3 & & 4957.720 & $20288_{11 / 2}^{\circ}-25246_{9 / 2}^{\circ}$ \\
\hline 23316.550 & 1 & & 4287.629 & $17727_{11 / 2}-22014_{11 / 2}^{\circ}$ & 20139.866 & 1 & & 4963.921 & $13250_{5 / 2}-18214_{3 / 2}^{\circ}$ \\
\hline 23222.202 & 1 & & 4305.049 & $20158_{5 / 2}-24463_{5 / 2}^{\circ}$ & 20061.498 & 0 & & 4983.312 & $15305_{9 / 2 / 2}-20288_{1 / 2}^{\circ}$ \\
\hline 23075.703 & 2 & & 4332.380 & $12485_{7 / 2}^{\circ}-16818_{7 / 2}$ & 19998.121 & 1 & & 4999.105 & $23012_{3 / 2}^{\circ}-28011_{3 / 2}$ \\
\hline 23003.968 & 2 & & 4345.890 & $12472_{5 / 2}^{\circ}-16818_{7 / 2}$ & 19984.221 & 0 & & 5002.582 & $26770_{11 / 2}^{\circ}-31773_{9 / 2}^{\circ}$ \\
\hline 22937.124 & 2 & & 4358.555 & $6213_{9 / 2}-10572_{9 / 2}^{\circ}$ & 19844.520 & 0 & & 5037.799 & $25414_{1 / 1 / 2}^{\circ}-30452_{9 / 2}^{\circ}$ \\
\hline 22911.403 & 0 & & 4363.448 & $24982_{7 / 2 / 2}^{\circ}-29345_{5 / 2 / 2}^{\circ}$ & 19774.295 & 6 & & 5055.690 & $4146_{7 / 2}-9202_{7 / 2}^{\circ}$ \\
\hline 22785.383 & 1 & & 4387.581 & $10855_{7 / 2}-15242_{9 / 2}^{\circ}$ & 19719.303 & 1 & & 5069.789 & $25414_{11 / 2}^{\circ}-30484_{11 / 2}$ \\
\hline 22733.218 & 0 & & 4397.649 & $27526_{9 / 2}-31924_{11 / 2}^{0}$ & 19703.648 & 1 & & 5073.817 & $7828_{1 / 2}-12902_{3 / 2}^{\circ}$ \\
\hline 22631.728 & 0 & & 4417.370 & $9400_{5 / 2}-13818_{7 / 2}^{\circ}$ & 19703.283 & 1 & & 5073.911 & $10379_{9 / 2}-15453_{7 / 2}^{\circ}$ \\
\hline 22582.257 & 1 & & 4427.047 & $15453_{7 / 2}^{\circ}-19880_{9 / 2}$ & 19701.761 & 0 & & 5074.303 & $15236_{3 / 2}-20310_{5 / 2}^{\circ}$ \\
\hline 22571.571 & 1 & & 4429.143 & $19880_{9 / 2}-24309_{11 / 2}^{\circ}$ & 19683.409 & 5 & & 5079.034 & $9711_{7 / 2}-14790_{7 / 2}^{\circ}$ \\
\hline 22506.128 & 4 & & 4442.022 & $8460_{3 / 2}-12902_{3 / 2}^{\circ}$ & 19644.523 & 4 & & 5089.088 & $14790_{7 / 2 / 2}^{\circ}-19880_{9 / 2}^{\circ}$ \\
\hline 22467.732 & 1 & & 4449.613 & $15144_{3 / 2}^{\circ}-19594_{1 / 2}^{\circ}$ & 19593.390 & 0 & & 5102.369 & $19880_{9 / 2 / 2}-24982_{7 / 2}^{\circ}$ \\
\hline 22445.678 & 2 & & 4453.985 & $8018_{3 / 2}-12472_{5 / 2}^{\circ}$ & 19540.414 & 1 & & 5116.202 & $10189_{1 / 1 / 2}^{\circ}-15305_{9 / 2}^{\circ}$ \\
\hline 22343.861 & 3 & & 4474.281 & $13248_{9 / 2}-17722_{9 / 2}^{\circ}$ & 19535.499 & 0 & & 5117.489 & $22139_{9 / 2}^{\circ}-27257_{7 / 2}^{\circ}$ \\
\hline 22264.353 & 6 & & 4490.259 & $0_{3 / 2}-4490_{5 / 2}^{\circ}$ & 19503.105 & 1 & & 5125.989 & $23697_{/ / 2}^{\prime \prime}-28823_{5 / 2}$ \\
\hline 22098.511 & 0 & & 4523.957 & $15786_{5 / 2}-20310_{5 / 2}^{\circ}$ & 19474.463 & 2 & & 5133.528 & $19248_{5 / 2}^{\circ}-24381_{7 / 2}$ \\
\hline 21907.002 & 1 & & 4563.505 & $10673_{5 / 2}^{\circ}-15236_{3 / 2}$ & 19473.652 & 1 & & 5133.742 & $25414_{1 / 1 / 2}^{\circ}-30548_{13 / 2}^{\circ}$ \\
\hline 21906.445 & 1 & & 4563.621 & $9711_{z / 2}-14275_{9 / 2}^{\circ}$ & 19432.597 & 3 & & 5144.588 & $9400_{5 / 2}-14545_{5 / 2}^{\circ}$ \\
\hline 21871.813 & 1 & & 4570.847 & $4490_{5 / 2}^{\circ}-9061_{5 / 2}^{\circ}$ & 19402.768 & 2 & & 5152.497 & $12570_{7 / 2}-17722_{9 / 2}^{\circ}$ \\
\hline 21852.069 & 2 & & 4574.977 & $7001_{3 / 2}-11576_{3 / 2}^{\circ}$ & 19353.457 & 0 & & 5165.625 & $20080_{\gamma / 2}^{\circ}-25246_{9 / 2}$ \\
\hline 21743.999 & 5 & & 4597.715 & $10855_{\tau / 2}-15453_{7 / 2}^{\circ}$ & 19338.976 & 7 & & 5169.493 & $1521_{5 / 2}-6691_{3 / 2}^{\circ}$ \\
\hline 21662.376 & 0 & & 4615.039 & $22642_{9 / 2}^{\circ}-27257_{\tau / 2}$ & 19307.868 & 2 & & 5177.822 & $10855_{7 / 2}-16033_{5 / 2}^{\circ}$ \\
\hline 21558.955 & 2 & & 4637.178 & $15242_{9 / 2}^{\circ}-19880_{9 / 2}^{\circ}$ & 19179.419 & 3 & & 5212.499 & $8605_{5 / 2}-13818_{7 / 2}^{\circ}$ \\
\hline 21387.561 & 1 & & 4674.339 & $24757_{9 / 2}^{\circ}-29431_{7 / 2}$ & 19165.340 & 1 & & 5216.328 & $12902_{3 / 2}^{\circ}-18118_{3 / 2}$ \\
\hline 21329.914 & 1 & & 4686.972 & $6168_{/ / 2}^{\circ}-10855_{7 / 2}$ & 19145.601 & 7 & & 5221.706 & $4490_{/ / 2}^{\circ}-9711_{7 / 2}^{\circ}$ \\
\hline 21264.265 & 0 & & 4701.442 & $14349_{1 / 2}-19050_{3 / 2}^{\circ}$ & 19082.536 & 3 & & 5238.963 & $12488_{9 / 2}^{\circ}-17727_{11 / 2}$ \\
\hline 21262.841 & 1 & & 4701.757 & $12570_{\tau / 2 / 2}-17272_{9 / 2}^{\circ}$ & 19076.390 & 0 & & 5240.651 & $12219_{3 / 2}-17460_{5 / 2}^{\circ}$ \\
\hline 21162.697 & 5 & & 4724.006 & $7001_{3 / 2}-11725_{1 / 2}^{\circ}$ & 18961.873 & 1 & & 5272.301 & $25607_{9 / 2}^{\circ}-30879_{7 / 2}$ \\
\hline 21123.018 & 0 & & 4732.880 & $13250_{5 / 2}-17983_{5 / 2}^{\circ}$ & 18880.700 & 0 & & 5294.968 & $22642_{9 / 2}^{\circ}-27937_{11 / 2}$ \\
\hline
\end{tabular}


TABLE 3. Classified lines of Th II-Continued

\begin{tabular}{|c|c|c|c|c|c|c|c|c|c|}
\hline \multirow{2}{*}{$\begin{array}{c}\text { Wavelength } \\
\AA\end{array}$} & \multicolumn{2}{|c|}{ Intensity } & \multirow{2}{*}{$\begin{array}{c}\text { Wavenumber } \\
\mathrm{cm}^{-1}\end{array}$} & \multirow{2}{*}{ Classification } & \multirow{2}{*}{$\begin{array}{c}\text { Wavelength } \\
\AA\end{array}$} & \multicolumn{2}{|c|}{ Intensity } & \multirow{2}{*}{$\begin{array}{c}\text { Wavenumber } \\
\mathbf{c m}^{-1}\end{array}$} & \multirow{2}{*}{ Classification } \\
\hline & Lamp & Spark & & & & Lamp & Spark & & \\
\hline 18624.701 & 0 & & 5367.748 & $14790_{7 / 2}^{\circ}-20158_{5 / 2}$ & 16433.049 & 2 & & 6083.636 & $9061_{5 / 2}-15144_{3 / 2}^{\circ}$ \\
\hline 18557.117 & 1 & & 5387.297 & $22139_{9 / 2}^{\circ}-27526_{9 / 2}^{\circ}$ & 16428.820 & 2 & & 6085.202 & $8460_{3 / 2}-14545_{5 / 2}^{\circ}$ \\
\hline 18547.718 & 3 & & 5390.027 & $9400_{5 / 2}-14790_{7 / 2}^{\circ}$ & 16380.898 & 6 & & 6103.004 & $9202_{7 / 2}^{\circ}-15305_{9 / 2}$ \\
\hline 18469.362 & 1 & & 5412.894 & $12570_{7 / 2}-17983_{5 / 2}^{\circ}$ & 16269.133 & 3 & & 6144.930 & $10673_{5 / 2}^{\circ}-16818_{7 / 2}$ \\
\hline 18402.093 & 0 & & 5432.681 & $19594_{1 / 2}-25027_{1 / 2}^{\circ}$ & 16182.835 & 0 & & 6177.699 & $20310_{5 / 2}^{\circ}-26488_{5 / 2}$ \\
\hline 18381.291 & 3 & & 5438.829 & $4146_{7 / 2}-9585_{5 / 2}^{\circ}$ & 16163.327 & 3 & & 6185.155 & $8605_{5 / 2}-14790_{7 / 2}^{\circ}$ \\
\hline 18293.755 & 1 & & 5464.854 & $21297_{5 / 2}^{\circ}-26762_{3 / 2}$ & 15951.379 & 0 & & 6267.338 & $20989_{9 / 2}^{\circ}-27257_{7 / 2}$ \\
\hline 18271.367 & 5 & & 5471.550 & $1859_{3 / 2}-7331_{5 / 2}^{\circ}$ & 15939.011 & 1 & & 6272.201 & $6213_{9 / 2}-12485_{7 / 2}^{\circ}$ \\
\hline 18269.704 & 3 & & 5472.048 & $4113_{5 / 2}-9585_{5 / 2}^{\circ}$ & 15936.374 & 4 & & 6273.239 & $7828_{1 / 2}-14101_{1 / 2}^{\circ}$ \\
\hline 18239.409 & 5 & & 5481.137 & $6244_{1 / 2}-11725_{1 / 2}^{\circ}$ & 15932.409 & 6 & & 6274.800 & $6213_{9 / 2}-12488_{9 / 2}^{\circ}$ \\
\hline 18228.371 & 4 & & 5484.456 & $9061_{5 / 2}-14545_{5 / 2}^{\circ}$ & 15927.216 & 0 & & 6276.846 & $24982_{7 / 2}^{\circ}-31259_{5 / 2}$ \\
\hline 18112.220 & 1 & & 5519.627 & $20969_{7 / 2}^{\circ}-26488_{5 / 2}^{\circ}$ & 15898.637 & 1 & & 6288.129 & $20969_{7 / 2}^{\circ}-27257_{7 / 2}$ \\
\hline 18082.856 & 4 & & 5528.590 & $6691_{3 / 2}^{\circ}-12219_{3 / 2}$ & 15877.380 & 0 & & 6296.548 & $21297_{5 / 2}^{\circ}-27593_{5 / 2}$ \\
\hline 18075.163 & 1 & & 5530.943 & $9711_{7 / 2}-15242_{9 / 2}^{\circ}$ & 15843.848 & 0 & & 6309.874 & $9400_{5 / 2}-15710_{3 / 2}^{\circ}$ \\
\hline 17936.434 & 7 & & 5573.722 & $4146_{7 / 2}-9720_{7 / 2}^{\circ}$ & 15815.492 & 6 & & 6321.187 & $9711_{7 / 2}-16033_{5 / 2}^{\circ}$ \\
\hline 17900.307 & 4 & & 5584.971 & $9720_{7 / 2}^{\circ}-15305_{9 / 2}$ & 15767.555 & 3 & & 6340.405 & $13818_{7 / 2}^{\circ}-20158_{5 / 2}$ \\
\hline 17837.933 & 1 & & 5604.500 & $14275_{9 / 2}^{\circ}-19880_{9 / 2}^{\prime 2}$ & 15747.955 & 1 & & 6348.296 & $12219_{3 / 2}-18568_{1 / 2}^{\circ}$ \\
\hline 17830.167 & 5 & & 5606.941 & $4113_{5 / 2}-9720_{7 / 2}^{\circ}$ & 15706.454 & 2 & & 6365.070 & $4490_{5 / 2}^{\circ}-10855_{7 / 2}$ \\
\hline 17795.604 & 3 & & 5617.831 & $12219_{3 / 2}-17837_{1 / 2}^{\circ}$ & 15698.032 & 0 & & 6368.485 & $20120_{5 / 2}^{\circ}-26488_{5 / 2}$ \\
\hline 17721.115 & 4 & & 5641.445 & $8460_{3 / 2}-14101_{1 / 2}^{\circ}$ & 15640.438 & 5 & & 6391.936 & $9061_{5 / 2}-15453_{7 / 2}^{\circ}$ \\
\hline 17705.162 & 3 & & 5646.528 & $12472_{5 / 2}^{\circ}-18118_{3 / 2}$ & 15637.160 & 3 & & 6393.276 & $11725_{1 / 2}^{\circ}-18118_{3 / 2}$ \\
\hline 17699.808 & 0 & & 5648.236 & $23697_{7 / 2}^{\circ}-29345_{5 / 2}$ & 15631.483 & 1 & & 6395.598 & $15710_{3 / 2}^{\circ}-22106_{5 / 2}$ \\
\hline 17535.396 & 0 & & 5701.194 & $23730_{9 / 2}^{\circ}-29431_{7 / 2}^{\circ}$ & 15624.606 & 1 & & 6398.413 & $17983_{5 / 2}^{\circ}-24381_{7 / 2}$ \\
\hline 17534.504 & 5 & & 5701.484 & $11116_{7 / 2}^{\circ}-16818_{7 / 2}$ & 15615.505 & 1 & & 6402.142 & $6168_{7 / 2}^{\circ}-12570_{7 / 2}$ \\
\hline 17507.525 & 0 & & 5710.270 & $25246_{9 / 2}-30956_{9 / 2}^{\circ}$ & 15612.710 & 0 & & 6403.288 & $12570_{7 / 2}-18973_{7 / 2}^{\circ}$ \\
\hline 17462.260 & 3 & & 5725.072 & $13248_{9 / 2}-18973_{7 / 2}^{\circ}$ & 15593.649 & 1 & & 6411.115 & $13468_{9 / 2}^{\circ}-19880_{9 / 2}^{\circ}$ \\
\hline 17456.286 & 0 & & 5727.031 & $19880_{9 / 2}-25607_{9 / 2}^{\circ}$ & 15558.814 & 2 & & 6425.469 & $4146_{7 / 2}-10572_{9 / 2}^{\circ}$ \\
\hline 17455.753 & 0 & & 5727.206 & $24757_{9 / 2}^{\circ}-30484_{11 / 2}$ & 15425.673 & 2 & & 6480.928 & $22106_{5 / 2}-28587_{5 / 2}^{\circ}$ \\
\hline 17447.564 & 3 & & 5729.894 & $9061_{5 / 2}-14790_{7 / 2}^{\circ}$ & 15317.814 & 3 & & 6526.563 & $4146_{7 / 2}-10673_{5 / 2}^{\circ}$ \\
\hline 17413.587 & 2 & & 5741.074 & $9711_{7 / 2}-15453_{7 / 2}^{\circ}$ & 15315.929 & 5 & & 6527.366 & $8018_{3 / 2}-14545_{5 / 2}^{\circ}$ \\
\hline 17405.417 & 3 & & 5743.769 & $9400_{5 / 2}-15144_{3 / 2}^{\circ}$ & 15315.660 & 2 & & 6527.481 & $10379_{9 / 2}-16906_{7 / 2}^{\circ}$ \\
\hline 17346.089 & 1 & & 5763.414 & $12219_{3 / 2}-17983_{5 / 2}^{\circ}$ & 15293.025 & 0 & & 6537.142 & $20989_{9 / 2}^{\circ}-27526_{9 / 2}^{\circ}$ \\
\hline 17244.366 & 1 & & 5797.412 & $22139_{9 / 2}^{\circ}-27937_{11 / 2}$ & 15288.921 & 4 & & 6538.897 & $8605_{5 / 2}-15144_{3 / 2}^{\circ}$ \\
\hline 17235.699 & 1 & & 5800.327 & $13250_{5 / 2}-19050_{3 / 2}^{\circ}$ & 15280.959 & 2 & & 6542.304 & $11576_{3 / 2}^{\circ}-18118_{3 / 2}$ \\
\hline 17229.251 & 0 & & 5802.498 & $20686_{5 / 2}^{\circ}-26488_{5 / 2}$ & 15266.440 & 4 & & 6548.526 & $6700_{9 / 2}^{\circ}-13248_{9 / 2}$ \\
\hline 17208.215 & 8 & & 5809.591 & $1521_{5 / 2}-7331_{5 / 2}^{\circ}$ & 15241.775 & 1 & & 6559.123 & $6691_{3 / 2}^{\circ}-13250_{5 / 2}$ \\
\hline 17155.413 & 1 & & 5827.472 & $23518_{\tau / 2}^{\circ}-29345_{5 / 2}$ & 15240.242 & 7 & & 6559.783 & $4113_{5 / 2}-10673_{5 / 2}^{\circ}$ \\
\hline 17030.220 & 3 & & 5870.311 & $6700_{9 / 2}^{\circ}-12570_{7 / 2}$ & 15214.240 & 1 & & 6570.994 & $20686_{5 / 2}^{\circ}-27257_{7 / 2}$ \\
\hline 16958.427 & 1 & & 5895.163 & $15236_{3 / 2}-21131_{3 / 2}^{\circ}$ & 15188.855 & 5 & & 6581.976 & $17727_{11 / 2}-24309_{11 / 2}^{\circ}$ \\
\hline 16956.701 & 2 & & 5895.763 & $15786_{5 / 2}-21682_{7 / 2}^{\circ}$ & 15135.223 & 0 & & 6605.299 & $10855_{7 / 2}-17460_{5 / 2}^{\circ}$ \\
\hline 16941.790 & 0 & & 5900.952 & $7001_{3 / 2}-12902_{3 / 2}^{\circ}$ & 15034.068 & 3 & & 6649.742 & $9061_{5 / 2}-15710_{3 / 2}^{\circ}$ \\
\hline 16912.370 & 1 & & 5911.217 & $21682_{7 / 2}^{\circ}-27593_{5 / 2}^{\circ}$ & 15015.230 & 2 & & 6658.085 & $6244_{1 / 2}-12902_{3 / 2}^{\circ}$ \\
\hline 16906.064 & 0 & & 5913.422 & $23518_{\tau / 2}^{\circ}-29431_{7 / 2}^{\circ}$ & 15013.595 & 0 & & 6658.810 & $17722_{9 / 2}^{\circ}-24381_{7 / 2}^{\circ}$ \\
\hline 16890.066 & 5 & & 5919.023 & $7331_{5 / 2}^{\circ}-13250_{5 / 2}$ & 15009.909 & 2 & & 6660.445 & $22685_{7 / 2}^{\circ}-29345_{5 / 2}$ \\
\hline 16520.919 & 3 & & 6051.279 & $10855_{7 / 2}-16906_{7 / 2}^{\circ}$ & 14970.942 & 1 & & 6677.781 & $12570_{7 / 2}-19248_{5 / 2}^{\circ}$ \\
\hline 16518.765 & 3 & & 6052.068 & $9400_{5 / 2}-15453_{7 / 2}^{\circ}$ & 14969.772 & 1 & & 6678.303 & $25246_{9 / 2}-31924_{11 / 2}^{\circ}$ \\
\hline 16495.017 & 0 & & 6060.781 & $15236_{3 / 2}-21297_{5 / 2}^{\circ}$ & 14940.492 & 7 & & 6691.391 & $0_{3 / 2}-6691_{3 / 2}^{\circ}$ \\
\hline 16492.394 & 2 & & 6061.745 & $13818_{\tau / 2}^{\circ}-19880_{9 / 2}$ & 14939.197 & 1 & & 6691.971 & $12902_{3 / 2}^{\circ}-19594_{1 / 2}$ \\
\hline 16477.433 & 2 & & 6067.249 & $9238_{9 / 2}^{\circ}-15305_{9 / 2}$ & 14920.495 & 3 & & 6700.359 & $16818_{7 / 2}-23518_{7 / 2}^{\circ}$ \\
\hline 16453.368 & 0 & & 6076.123 & $20686_{5 / 2}^{\circ}-26762_{3 / 2}$ & 14899.905 & 2 & & 6709.618 & $15305_{9 / 2}-22014_{11 / 2}^{\circ}$ \\
\hline
\end{tabular}


TABLE 3. Classified lines of Th II-Continued

\begin{tabular}{|c|c|c|c|c|c|c|c|c|c|}
\hline \multirow{2}{*}{$\begin{array}{c}\text { Wavelength } \\
\AA\end{array}$} & \multicolumn{2}{|c|}{ Intensity } & \multirow{2}{*}{$\begin{array}{c}\text { Wavenumber } \\
\mathbf{c m}^{-1}\end{array}$} & \multirow{2}{*}{ Classification } & \multirow{2}{*}{$\begin{array}{c}\text { Wavelength } \\
\AA\end{array}$} & \multicolumn{2}{|c|}{ Intensity } & \multirow{2}{*}{$\begin{array}{c}\text { Wavenumber } \\
\mathbf{c m}^{-1}\end{array}$} & \multirow{2}{*}{ Classification } \\
\hline & Lamp & Spark & & & & Lamp & Spark & & \\
\hline 14.739 .988 & 0 & & 6782.412 & $14349_{1 / 2}-21131_{3 / 2}^{\circ}$ & 13395.691 & 4 & & 7463.046 & $4113_{5 / 2}-11576_{3 / 2}^{\circ}$ \\
\hline 14699.723 & 2 & & 6800.990 & $16033_{5 / 2}^{\circ}-22834_{7 / 2}$ & 13376.396 & 0 & & 7473.811 & $20120_{5 / 2}^{\circ}-27593_{5 / 2}$ \\
\hline 14690.666 & 0 & & 6805.183 & $20158_{5 / 2}-26963_{7 / 2}^{\circ}$ & 13373.859 & 3 & & 7475.229 & $17771_{11 / 2}^{\circ}-25246_{9 / 2}$ \\
\hline 14672.400 & 0 & & 6813.655 & $18568_{1 / 2}^{\circ}-25381_{3 / 2}$ & 13370.190 & 2 & & 7477.280 & $19880_{9 / 2}-27357_{9 / 2}^{\circ}$ \\
\hline 14633.086 & 3 & & 6831.961 & $13248_{9 / 2}-20080_{7 / 2}^{\circ}$ & 13319.676 & 1 & & 7505.637 & $9400_{5 / 2}-16906_{7 / 2}^{\circ}$ \\
\hline 14627.874 & 4 & & 6834.395 & $15305_{9 / 2}-22139_{9 / 2}^{\circ}$ & 13311.623 & 2 & & 7510.178 & $12570_{7 / 2}-20080_{7 / 2}^{\circ}$ \\
\hline 14579.735 & 6 & & 6856.961 & $1521_{5 / 2}-8378_{7 / 2}^{\circ}$ & 13310.052 & 1 & & 7511.064 & $20120_{5 / 2}^{\circ}-27631_{3 / 2}$ \\
\hline 14565.023 & 0 & & 6863.887 & $8460_{3 / 2}-15324_{1 / 2}^{\circ}$ & 13306.095 & 2 & & 7513.298 & $20080_{7 / 2}^{\circ}-27593_{5 / 2}$ \\
\hline 14557.004 & 4 & & 6867.668 & $10855_{7 / 2}-17722_{9 / 2}^{\circ}$ & 13304.846 & 2 & & 7514.003 & $19248_{5 / 2}^{\circ}-26762_{3 / 2}$ \\
\hline 14508.948 & 1 & & 6890.415 & $19880_{9 / 2}-26770_{11 / 2}^{\circ}$ & 13288.387 & 1 & & 7523.310 & $17722_{9 / 2}^{\circ}-25246_{9 / 2}$ \\
\hline 14503.235 & 6 & & 6893.129 & $10379_{9 / 2}-17272_{9 / 2}^{\circ}$ & 13283.226 & 1 & & 7526.233 & $21297_{5 / 2}^{\circ}-28823_{5 / 2}$ \\
\hline 14472.395 & 1 & & 6907.818 & $20686_{5 / 2}^{\circ}-27593_{5 / 2}$ & 13262.164 & 2 & & 7538.186 & $10189_{11 / 2}^{\circ}-17727_{11 / 2}$ \\
\hline 14462.402 & 0 & & 6912.591 & $16818_{\tau / 2}-23730_{9 / 2}^{\circ}$ & 13251.713 & 2 & & 7544.131 & $7001_{3 / 2}-14545_{5 / 2}^{\circ}$ \\
\hline 14433.541 & 1 & & 6926.413 & $8378_{7 / 2}^{\circ}-15305_{9 / 2}$ & 13251.713 & 2 & & 7544.131 & $17837_{1 / 2}^{\circ}-25381_{3 / 2}$ \\
\hline 14360.388 & 0 & & 6961.697 & $15144_{3 / 2}^{\circ}-22106_{5 / 2}$ & 13241.997 & 0 & & 7549.666 & $12570_{7 / 2}-20120_{5 / 2}^{\circ}$ \\
\hline 14343.260 & 6 & & 6970.010 & $4146_{7 / 2}-11116_{7 / 2}^{\circ}$ & 13223.385 & 3 & & 7560.292 & $9711_{7 / 2}-17272_{9 / 2}^{\circ}$ \\
\hline 14339.066 & 2 & & 6972.049 & $9061_{5 / 2}-16033_{5 / 2}^{\circ}$ & 13222.361 & 0 & & 7560.878 & $14545_{5 / 2}^{\circ}-22106_{5 / 2}$ \\
\hline 14275.223 & 0 & & 7003.230 & $4113_{5 / 2}-11116_{7 / 2}^{\circ}$ & 13188.919 & 2 & & 7580.049 & $9238_{9 / 2}^{\circ}-16818_{7 / 2}$ \\
\hline 14224.305 & 0 & & 7028.299 & $12219_{3 / 2}-19248_{5 / 2}^{\circ}$ & 13179.277 & 0 & & 7585.595 & $15786_{5 / 2}-23372_{3 / 2}^{\circ}$ \\
\hline 14159.556 & 0 & & 7060.438 & $13250_{5 / 2}-20310_{5 / 2}^{\circ}$ & 13145.904 & 7 & & 7604.852 & $6213_{9 / 2}-13818_{7 / 2}^{\circ}$ \\
\hline 14070.742 & 5 & & 7105.003 & $8605_{5 / 2}-15710_{3 / 2}^{\circ}$ & 13130.308 & 1 & & 7613.885 & $22106_{5 / 2}-29720_{3 / 2}^{\circ}$ \\
\hline 14043.929 & 1 & & 7118.568 & $15236_{3 / 2}-22355_{1 / 2}^{\circ}$ & 13126.998 & 1 & & 7615.805 & $9202_{7 / 2}^{\circ}-16818_{7 / 2}$ \\
\hline 14028.205 & 3 & & 7126.547 & $8018_{3 / 2}-15144_{3 / 2}^{\circ}$ & 13029.053 & 0 & & 7673.056 & $12485_{7 / 2}^{\circ}-20158_{5 / 2}$ \\
\hline 14025.216 & 2 & & 7128.066 & $10855_{7 / 2}-17983_{5 / 2}^{\circ}$ & 13016.640 & 5 & & 7680.373 & $1521_{5 / 2}-9202_{7 / 2}^{\circ}$ \\
\hline 14007.683 & 1 & & 7136.988 & $20120_{5 / 2}^{\circ}-27257_{7 / 2}$ & 13006.151 & 2 & & 7686.567 & $12472_{5 / 2}^{\circ}-20158_{5 / 2}$ \\
\hline 13972.014 & 1 & & 7155.208 & $10572_{9 / 2}^{\circ}-17727_{11 / 2}^{\circ}$ & 13004.273 & 4 & & 7687.677 & $17727_{11 / 2}-25414_{11 / 2}^{\circ}$ \\
\hline 13895.433 & 3 & & 7194.642 & $9711_{7 / 2}-16906_{7 / 2}^{\circ}$ & 12983.105 & 0 & & 7700.211 & $20310_{5 / 2}^{\circ}-28011_{3 / 2}$ \\
\hline 13836.909 & 0 & & 7225.072 & $15786_{5 / 2}-23012_{i / 2}^{\circ}$ & 12964.195 & 2 & & 7711.443 & $19050_{3 / 2}^{\circ}-26762_{3 / 2}$ \\
\hline 13822.377 & 0 & & 7232.668 & $9585_{5 / 2}^{\circ}-16818_{7 / 2}$ & 12940.654 & 7 & & 7725.471 & $1859_{3 / 2}-9585_{5 / 2}^{\circ}$ \\
\hline 13811.481 & 4 & & 7238.374 & $20288_{11 / 2}^{\circ}-27526_{9 / 2}$ & 12933.539 & 5 & & 7729.721 & $4490_{5 / 2}^{\circ}-12219_{3 / 2}$ \\
\hline 13788.405 & 1 & & 7250.488 & $8460_{3 / 2}-15710_{3 / 2}^{\circ}$ & 12930.664 & 1 & & 7731.440 & $15786_{5 / 2}-23518_{7 / 2}^{\circ}$ \\
\hline 13778.916 & 6 & & 7255.481 & $6213_{9 / 2}-13468_{9 / 2}^{\circ}$ & 12925.892 & 3 & & 7734.294 & $22139_{9 / 2}^{\circ}-29873_{7 / 2}^{\circ}$ \\
\hline 13777.235 & 1 & & 7256.366 & $12902_{3 / 2}^{\circ}-20158_{5 / 2}^{\circ}$ & 12901.919 & 2 & & 7748.665 & $9711_{7 / 2}-17460_{5 / 2}^{\circ}$ \\
\hline 13756.558 & 2 & & 7267.273 & $22834_{7 / 2}-30101_{7 / 2}^{\circ}$ & 12742.669 & 1 & & 7845.503 & $9061_{5 / 2}-16906_{7 / 2}^{\circ}$ \\
\hline 13709.552 & 1 & & 7292.190 & $22139_{9 / 2}^{\circ}-29431_{7 / 2}$ & 12684.798 & 3 & & 7881.296 & $13250_{5 / 2}-21131_{3 / 2}^{\circ}$ \\
\hline 13699.211 & 3 & & 7297.695 & $23187_{13 / 2}^{\circ}-30484_{11 / 2}$ & 12683.213 & 4 & & 7882.281 & $7828_{1 / 2}-15710_{3 / 2}^{\circ}$ \\
\hline 13665.979 & 0 & & 7315.441 & $14790_{\pi / 2}^{\circ}-22106_{5 / 2}$ & 12642.339 & 3 & & 7907.765 & $19880_{9 / 2}-27787_{9 / 2}^{\circ}$ \\
\hline 13664.604 & 3 & & 7316.177 & $7828_{1 / 2}-15144_{3 / 2}^{\circ}$ & 12591.895 & 1 & & 7939.444 & $16818_{7 / 2}-24757_{9 / 2}^{\circ}$ \\
\hline 13654.606 & 0 & & 7321.534 & $18118_{3 / 2}-25440_{5 / 2}^{\circ}$ & 12479.376 & 1 & & 8011.029 & $9711_{7 / 2}-17722_{9 / 2}^{\circ}$ \\
\hline 13636.063 & 5 & & 7331.490 & $0_{3 / 2}-7331_{5 / 2}^{\circ}$ & 12468.606 & 1 & & 8017.949 & $11576_{3 / 2}^{\circ}-19594_{1 / 2}$ \\
\hline 13626.116 & 4 & & 7336.842 & $15305_{9 / 2}-22642_{9 / 2}^{\circ}$ & 12458.068 & 1 & & 8024.731 & $19912_{13 / 2}^{\circ}-27937_{11 / 2}$ \\
\hline 13582.026 & 0 & & 7360.659 & $22513_{5 / 2}^{\circ}-29873_{7 / 2}$ & 12421.310 & 1 & & 8048.478 & $21297_{5 / 2}^{\circ}-29345_{5 / 2}$ \\
\hline 13544.406 & 1 & & 7381.103 & $15453_{7 / 2}^{\circ}-22834_{7 / 2}$ & 12402.749 & 1 & & 8060.523 & $9061_{5 / 2}-17121_{3 / 2}^{\circ}$ \\
\hline 13524.811 & 1 & & 7391.797 & $12488_{9 / 2}^{\circ}-19880_{9 / 2}$ & 12400.331 & 3 & & 8062.095 & $6213_{9 / 2}-14275_{9 / 2}^{\circ}$ \\
\hline 13520.057 & 1 & & 7394.396 & $12485_{7 / 2}^{\circ}-19880_{9 / 2}$ & 12398.153 & 2 & & 8063.511 & $1521_{5 / 2}-9585_{5 / 2}^{\circ}$ \\
\hline 13494.985 & 0 & & 7408.134 & $8378_{7 / 2}^{\circ}-15786_{5 / 2}$ & 12372.484 & 4 & & 8080.240 & $4490_{5 / 2}^{\circ}-12570_{7 / 2}^{\circ}$ \\
\hline 13460.143 & 3 & & 7427.310 & $8605_{5 / 2}-16033_{5 / 2}^{\circ}$ & 12356.076 & 2 & & 8090.970 & $12219_{3 / 2}-20310_{5 / 2}^{\circ}$ \\
\hline 13445.057 & 0 & & 7435.644 & $13250_{5 / 2}-20686_{5 / 2}^{\circ}$ & 12318.487 & 1 & & 8115.659 & $12570_{7 / 2}-20686_{5 / 2}^{\circ}$ \\
\hline 13427.136 & 3 & & 7445.568 & $10673_{5 / 2}^{\circ}-18118_{3 / 2}$ & 12285.424 & 1 & & 8137.500 & $20686_{5 / 2}^{\circ}-28823_{5 / 2}$ \\
\hline 13425.849 & 0 & & 7446.282 & $20080_{\tau / 2}^{\circ}-27526_{9 / 2}$ & 12276.652 & 1 & & 8143.315 & $7001_{3 / 2}-15144_{3 / 2}^{\circ}$ \\
\hline
\end{tabular}


TABle 3. Classified lines of Th II-Continued

\begin{tabular}{|c|c|c|c|c|c|c|c|c|c|}
\hline \multirow{2}{*}{$\begin{array}{c}\text { Wavelength } \\
\AA\end{array}$} & \multicolumn{2}{|c|}{ Intensity } & \multirow{2}{*}{$\begin{array}{l}\text { W avenumber } \\
\mathrm{cm}^{-1}\end{array}$} & \multirow{2}{*}{ Classification } & \multirow{2}{*}{$\begin{array}{c}\text { Wavelength } \\
\AA\end{array}$} & \multicolumn{2}{|c|}{ Intensity } & \multirow{2}{*}{$\begin{array}{l}\text { Wavenumber } \\
\mathrm{cm}^{-1}\end{array}$} & \multirow{2}{*}{ Classification } \\
\hline & Lamp & Spark & & & & Lamp & Spark & & \\
\hline 12251.360 & 0 & & 8160.126 & $22834_{7 / 2}-30994_{7 / 2}^{\circ}$ & 11071.877 & 1 & & 9029.419 & $6213_{9 / 2}-15242_{9 / 2}^{\circ}$ \\
\hline 12244.970 & 3 & & 8164.384 & $16818_{7 / 2}-24982_{7 / 2}^{\circ}$ & 11069.049 & 3 & & 9031.726 & $7001_{3 / 2}-16033_{5 / 2}^{\circ}$ \\
\hline 12200.702 & 1 & & 8194.007 & $18568_{1 / 2}^{\circ}-26762_{3 / 2}$ & 11013.293 & 1 & & 9077.450 & $12219_{3 / 2}-21297_{5 / 2}^{\circ}$ \\
\hline 12194.157 & 8 & & 8198.405 & $1521_{5 / 2}-9720_{7 / 2}^{\circ}$ & 11010.266 & 1 & & 9079.946 & $6244_{1 / 2}-15324_{1 / 2}^{\circ}$ \\
\hline 12170.869 & 0 & & 8214.092 & $21131_{3 / 2}^{\circ}-29345_{5 / 2}$ & 10981.861 & 1 & & 9103.431 & $8018_{3 / 2}-17121_{3 / 2}^{\circ}$ \\
\hline 12136.565 & 1 & & 8237.309 & $22642_{9 / 2}^{\circ}-30879_{7 / 2}$ & 10942.244 & 8 & & 9136.391 & $6213_{9 / 2}-15349_{1 / 2}^{\circ}$ \\
\hline 12102.779 & 1 & & 8260.304 & $17121_{3 / 2}^{\circ}-25381_{3 / 2}$ & 10941.617 & 4 & & 9136.914 & $6168_{7 / 2}^{\circ}-15305_{9 / 2}$ \\
\hline 12087.362 & 2 & & 8270.840 & $6213_{9 / 2}-14484_{11 / 2}^{\circ}$ & 10924.483 & 4 & & 9151.245 & $1521_{5 / 2}-10673_{5 / 2}^{\circ}$ \\
\hline 12086.503 & 3 & & 8271.428 & $9711_{7 / 2}-17983_{5 / 2}^{\circ}$ & 10892.630 & 0 & & 9178.005 & $15236_{3 / 2}-24414_{3 / 2}^{\circ}$ \\
\hline 12043.786 & 1 & & 8300.765 & $8605_{5 / 2}-16906_{7 / 2}^{\circ}$ & 10861.797 & 1 & & 9204.059 & $12902_{3 / 2}^{\circ}-22106_{5 / 2}$ \\
\hline 12011.883 & 0 & & 8322.811 & $7001_{3 / 2}-15324_{1 / 2}^{\circ}$ & 10835.309 & $2 w$ & & 9226.559 & $20288_{11 / 2}^{\circ}-29515_{9 / 2}$ \\
\hline 12007.854 & 5 & & 8325.604 & $4146_{7 / 2}-12472_{5 / 2}^{\circ}$ & 10834.611 & 2 & & 9227.153 & $15236_{3 / 2}-24463_{5 / 2}^{\circ}$ \\
\hline 11984.664 & 8 & & 8341.714 & $4146_{7 / 2}-12488_{9 / 2}^{\circ}$ & 10820.075 & 5 & & 9239.549 & $6213_{9 / 2}-15453_{7 / 2}^{\circ}$ \\
\hline 11979.873 & 2 & & 8345.050 & $22139_{9 / 2}^{\circ}-30484_{11 / 2}$ & 10794.056 & 3 & & 9261.821 & $9711_{7 / 2}-18973_{7 / 2}^{\circ}$ \\
\hline 11979.873 & 2 & & 8345.050 & $15786_{5 / 2}-24132_{3 / 2}^{\circ}$ & 10790.541 & 0 & & 9264.838 & $10855_{7 / 2}-20120_{5 / 2}^{\circ}$ \\
\hline 11974.695 & 1 & & 8348.658 & $16033_{5 / 2}^{\circ}-24381_{7 / 2}^{\circ}$ & 10757.771 & 0 & & 9293.060 & $7828_{1 / 2}-17121_{3 / 2}^{\circ}$ \\
\hline 11960.136 & 3 & & 8358.821 & $4113_{5 / 2}-12472_{5 / 2}^{\circ}$ & 10740.457 & 1 & & 9308.041 & $10572_{9 / 2}^{\circ}-19880_{9 / 2}$ \\
\hline 11940.834 & 2 & & 8372.333 & $4113_{5 / 2}-12485_{7 / 2}^{\circ}$ & 10723.921 & 7 & & 9322.394 & $4146_{7 / 2}-13468_{9 / 2}^{\circ}$ \\
\hline 11934.349 & 1 & & 8376.882 & $20969_{7 / 2}^{\circ}-29345_{5 / 2}$ & 10660.847 & 3 & & 9377.549 & $8605_{5 / 2}-17983_{5 / 2}^{\circ}$ \\
\hline 11911.501 & 4 & & 8392.950 & $10855_{7 / 2}-19248_{5 / 2}^{\circ}$ & 10658.585 & 1 & & 9379.539 & $18214_{3 / 2}^{\circ}-27593_{5 / 2}$ \\
\hline 11903.594 & 0 & & 8398.525 & $12570_{7 / 2}-20969_{7 / 2}^{\circ}$ & 10642.859 & 1 & & 9393.398 & $13248_{9 / 2}-22642_{9 / 2}^{\circ}$ \\
\hline 11874.199 & 3 & & 8419.316 & $12570_{7 / 2}-20989_{9 / 2}^{\circ}$ & 10605.455 & 4 & & 9426.527 & $25594_{1 / 2}^{\circ}-3502 l_{3 / 2}$ \\
\hline 11865.636 & 4 & & 8425.392 & $15305_{9 / 2}-23730_{9 / 2}^{\circ}$ & 10595.992 & 0 & & 9434.946 & $13250_{5 / 2}-22685_{7 / 2}^{\circ}$ \\
\hline 11846.202 & 2 & & 8439.214 & $8378_{7 / 2}^{\circ}-16818_{7 / 2}$ & 10587.588 & 1 & & 9442.435 & $8018_{3 / 2}-17460_{5 / 2}^{\circ}$ \\
\hline 11842.232 & 0 & & 8442.043 & $20989_{9 / 2}^{\circ}-29431_{7 / 2}$ & 10576.600 & 1 & & 9452.244 & $15305_{9 / 2}-24757_{9 / 2}^{\circ}$ \\
\hline 11808.476 & 0 & & 8466.176 & $12219_{3 / 2}-20686_{5 / 2}^{\circ}$ & 10572.824 & 1 & & 9455.620 & $10855_{7 / 2}-20310_{5 / 2}^{\circ}$ \\
\hline 11776.403 & 5 & & 8489.233 & $9238_{9 / 2}^{\circ}-17727_{11 / 2}$ & 10560.617 & 1 & & 9466.550 & $6244_{1 / 2}-15710_{3 / 2}^{\circ}$ \\
\hline 11739.687 & 2 & & 8515.783 & $8605_{5 / 2}-17121_{3 / 2}^{\circ}$ & 10539.400 & 1 & & 9485.607 & $10673_{5 / 2}^{\circ}-20158_{5 / 2}$ \\
\hline 11715.587 & 1 & & 8533.301 & $9585_{5 / 2}^{\circ}-18118_{3 / 2}$ & 10538.316 & 2 & & 9486.583 & $7331_{5 / 2}^{\circ}-16818_{7 / 2}$ \\
\hline 11695.652 & 0 & & 8547.846 & $18214_{3 / 2}^{\circ}-26762_{3 / 2}^{\circ}$ & 10483.360 & 4 & & 9536.313 & $9711_{7 / 2}-19248_{5 / 2}^{\circ}$ \\
\hline 11655.207 & 1 & & 8577.508 & $6213_{9 / 2}-14790_{7 / 2}^{\circ}$ & 10429.665 & 6 & & 9585.409 & $0_{3 / 2}-9585_{5 / 2}^{\circ}$ \\
\hline 11648.639 & 1 & & 8582.344 & $11576_{3 / 2}^{\circ}-20158_{5 / 2}$ & 10423.795 & 2 & & 9590.807 & $14790_{7 / 2}^{\circ}-24381_{7 / 2}$ \\
\hline 11631.948 & 3 & & 8594.659 & $10379_{9 / 2}-18973_{7 / 2}^{\circ}$ & 10419.574 & 7 & & 9594.692 & $1521_{5 / 2}-11116_{7 / 2}^{\circ}$ \\
\hline 11594.834 & 0 & & 8622.170 & $16818_{7 / 2}-25440_{5 / 2}^{\circ}$ & 10404.502 & 3 & & 9608.591 & $8605_{5 / 2}-18214_{3 / 2}^{\circ}$ \\
\hline 11542.494 & 3 & & 8661.268 & $8460_{3 / 2}-17121_{3 / 2}^{\circ}$ & 10402.349 & 1 & & 9610.580 & $17983_{5 / 2}^{\circ}-27593_{5 / 2}$ \\
\hline 11521.827 & 2 & & 8676.804 & $15786_{5 / 2}-24463_{5 / 2}^{\circ}$ & 10391.352 & 0 & & 9620.750 & $12485_{7 / 2}^{\circ}-22106_{5 / 2}$ \\
\hline 11515.375 & 0 & & 8681.665 & $16564_{11 / 2}^{\circ}-25246_{9 / 2}$ & 10373.462 & 0 & & 9637.342 & $15236_{3 / 2}-24873_{5 / 2}^{\circ}$ \\
\hline 11478.678 & 2 & & 8709.420 & $7001_{3 / 2}-15710_{3 / 2}^{\circ}$ & 10359.998 & 3 & & 9649.867 & $9400_{5 / 2}-19050_{3 / 2}^{\circ}$ \\
\hline 11455.648 & lu & & 8726.929 & $12570_{7 / 2}-21297_{5 / 2}^{\circ}$ & 10356.372 & 2 & & 9653.246 & $15786_{5 / 2}-25440_{5 / 2}^{\circ}$ \\
\hline 11412.071 & 2 & & 8760.253 & $4490_{5 / 2}^{\circ}-13250_{5 / 2}$ & 10316.006 & 1 & & 9691.018 & $10189_{11 / 2}^{\circ}-19880_{9 / 2}$ \\
\hline 11387.846 & 1 & & 8778.888 & $17983_{5 / 2}^{\circ}-26762_{3 / 2}$ & 10304.810 & 2 & & 9701.547 & $10379_{9 / 2}-20080_{7 / 2}^{\circ}$ \\
\hline 11374.713 & 6 & & 8789.024 & $4113_{5 / 2}-12902_{3 / 2}^{\circ}$ & 10301.161 & 6 & & 9704.984 & $4113_{5 / 2}-13818_{7 / 2}^{\circ}$ \\
\hline 11290.238 & 0 & & 8854.785 & $8605_{5 / 2}-17460_{5 / 2}^{\circ}$ & 10288.987 & 4 & & 9716.467 & $1859_{3 / 2}-11576_{3 / 2}^{\circ}$ \\
\hline 11252.925 & 1 & & 8884.146 & $20989_{9 / 2}^{\circ}-29873_{7 / 2}$ & 10249.314 & 1 & & 9754.077 & $8460_{3 / 2}-18214_{3 / 2}^{\circ}$ \\
\hline 11244.310 & $2 d$ & & 8890.952 & $13248_{9 / 2}-22139_{9 / 2}^{\circ}$ & 10247.419 & 1 & & 9755.881 & $17771_{11 / 2}^{\circ}-27526_{9 / 2}$ \\
\hline 11232.320 & 2 & & 8900.443 & $6244_{1 / 2}-15144_{3 / 2}^{\circ}$ & 10244.986 & 1 & & 9758.198 & $22014_{11 / 2}^{\circ}-31773_{9 / 2}$ \\
\hline 11204.818 & 2 & & 8922.289 & $9061_{5 / 2}-17983_{5 / 2}^{\circ}$ & 10241.463 & 2 & & 9761.555 & $13250_{5 / 2}-23012_{3 / 2}^{\circ}$ \\
\hline 11202.078 & 1 & & 8924.471 & $17837_{1 / 2}^{\circ}-26762_{3 / 2}$ & 10219.382 & 2 & & 9782.646 & $14349_{1 / 2}-24132_{3 / 2}^{\circ}$ \\
\hline 11186.716 & 0 & & 8936.727 & $20158_{5 / 2}-29095_{5 / 2}^{\circ}$ & 10208.285 & 0 & & 9793.281 & $20080_{7 / 2}^{\circ}-29873_{7 / 2}$ \\
\hline 11107.735 & 0 & & 9000.271 & $8460_{3 / 2}-17460_{5 / 2}^{\circ}$ & 10197.163 & 0 & & 9803.962 & $17722_{9 / 2}^{\circ}-27526_{9 / 2}$ \\
\hline
\end{tabular}


TABle 3. Classified lines of Th $\mathrm{II}-$ Continued

\begin{tabular}{|c|c|c|c|c|c|c|c|c|c|}
\hline \multirow{2}{*}{$\begin{array}{c}\text { Wavelength } \\
\AA\end{array}$} & \multicolumn{2}{|c|}{ Intensity } & \multirow{2}{*}{$\begin{array}{l}\text { Wavenumber } \\
\mathrm{cm}^{-1}\end{array}$} & \multirow{2}{*}{ Classification } & \multirow{2}{*}{$\begin{array}{c}\text { Wavelength } \\
\AA\end{array}$} & \multicolumn{2}{|c|}{ Intensity } & \multirow{2}{*}{$\begin{array}{c}\text { Wavenumber } \\
\mathbf{c m}^{-1}\end{array}$} & \multirow{2}{*}{ Classification } \\
\hline & Lamp & Spark & & & & Lamp & Spark & & \\
\hline 10180.909 & 0 & & 9819.614 & $8018_{3 / 2}-17837_{1 / 2}^{\circ}$ & 9362.640 & 1 & & 10677.819 & $9202_{7 / 2}^{\circ}-19880_{9 / 2}$ \\
\hline 10169.296 & 2 & & 9830.828 & $10855_{7 / 2}-20686_{5 / 2}^{\circ}$ & 9360.989 & 2 & & 10679.702 & $9400_{5 / 2}-20080_{7 / 2}^{\circ}$ \\
\hline 10133.559 & 7 & & 9865.497 & $1859_{3 / 2}-11725_{1 / 2}^{\circ}$ & 9349.246 & 1 & & 10693.116 & $6213_{9 / 2}-16906_{7 / 2}^{\circ}$ \\
\hline 10085.323 & 1 & & 9912.681 & $9061_{5 / 2}-18973_{7 / 2}^{\circ}$ & 9346.644 & 1 & 2 & 10696.092 & $22513_{5 / 2}^{\circ}-33209_{7 / 2}$ \\
\hline 10045.996 & 0 & & 9951.486 & $15236_{3 / 2}-25188_{3 / 2}^{\circ}$ & 9311.053 & 2 & 4 & 10736.978 & $30310_{11 / 2}^{\circ}-41047_{9 / 2}$ \\
\hline 10032.174 & 1 & & 9965.197 & $8018_{3 / 2}-17983_{5 / 2}^{\circ}$ & 9292.242 & 2 & 2 & 10758.713 & $35593_{7 / 2}^{\circ}-46352_{7 / 2}$ \\
\hline 9993.866 & 2 & & 10003.395 & $15242_{9 / 2}^{\circ}-25246_{9 / 2}$ & 9267.686 & 2 & & 10787.220 & $7331_{5 / 2}^{\circ}-18118_{3 / 2}$ \\
\hline 9988.024 & 2 & & 10009.246 & $7828_{1 / 2}-17837_{i / 2}^{0}$ & 9267.082 & 3 & & 10787.923 & $8460_{3 / 2}-19248_{5 / 2}^{\circ}$ \\
\hline 9943.061 & 6 & & 10054.508 & $1521_{5 / 2}-11576_{3 / 2}^{\circ}$ & 9253.268 & 1 & 3 & 10804.028 & $20969_{7 / 2}^{\circ}-31773_{9 / 2}$ \\
\hline 9926.172 & 0 & & 10071.616 & $12570_{7 / 2}-22642_{9 / 2}^{\circ}$ & 9252.860 & 0 & & 10804.504 & $18118_{3 / 2}-28923_{5 / 2}^{\circ}$ \\
\hline 9892.187 & 0 & & 10106.217 & $14275_{9 / 2}^{\circ}-24381_{7 / 2}$ & 9249.887 & 5 & 4 & 10807.977 & $27357_{9 / 2}^{\circ}-38165_{7 / 2}$ \\
\hline 9890.522 & 1 & & 10107.918 & $8460_{3 / 2}-18568_{1 / 2}^{\circ}$ & 9245.253 & 10 & 5 & 10813.394 & $20158_{5 / 2}-30972_{5 / 2}^{\circ}$ \\
\hline 9888.819 & 0 & & 10109.659 & $15305_{9 / 2}-25414_{11 / 2}^{\circ}$ & 9233.267 & 3 & 75 & 10827.431 & $10855_{7 / 2}-21682_{7 / 2}^{\circ}$ \\
\hline 9880.782 & 2 & & 10117.882 & $6700_{9 / 2}^{\circ}-16818_{7 / 2}$ & 9226.409 & 0 & 5 & 10835.479 & $20158_{5 / 2}-30994_{7 / 2}^{\circ}$ \\
\hline 9878.520 & 2 & & 10120.199 & $7001_{3 / 2}-17121_{3 / 2}^{\circ}$ & 9225.657 & 1 & 2 & 10836.363 & $14545_{5 / 2}^{\circ}-25381_{3 / 2}$ \\
\hline 9876.688 & 0 & & 10122.076 & $13250_{5 / 2}-23372_{3 / 2}^{\circ}$ & & & & & $7001_{3 / 2}-17837_{1 / 2}^{\circ}$ \\
\hline 9869.929 & 0 & & 10129.008 & $4146_{7 / 2}-14275_{9 / 2}^{\circ}$ & 9164.081 & 0 & 8 & 10909.175 & $26770_{11 / 2}^{\circ}-37679_{11 / 2}$ \\
\hline 9864.597 & 2 & 8 & 10134.483 & $10855_{7 / 2}-20989_{9 / 2}^{\circ}$ & 9157.414 & 2 & 20 & 10917.117 & $14349_{1 / 2}-25266_{1 / 2}^{\circ}$ \\
\hline 9863.866 & 1 & & 10135.234 & $12219_{3 / 2}-22355_{1 / 2}^{\circ}$ & 9133.422 & 0 & 5 & 10945.794 & $33354_{5 / 2}^{\circ}-44300_{3 / 2}$ \\
\hline 9834.008 & 3 & & 10166.006 & $17771_{11 / 2}^{\circ}-27937_{11 / 2}$ & & & & & $30101_{7 / 2}^{\circ}-41047_{9 / 2}$ \\
\hline 9817.034 & $3 \mathrm{~h}$ & 3 & 10183.583 & $19248_{5 / 2}^{\circ}-29431_{7 / 2}$ & 9129.691 & 3 & 5 & 10950.268 & $1521_{5 / 2}-12472_{5 / 2}^{\circ}$ \\
\hline 9780.808 & 1 & 1 & 10221.301 & $19880_{9 / 2}-30101_{7 / 2}^{\circ}$ & 9124.513 & 2 & 4 & 10956.482 & $9202_{7 / 2}^{\circ}-20158_{5 / 2}$ \\
\hline 9734.696 & 1 & & 10269.718 & $13248_{9 / 2}-23518_{7 / 2}^{\circ}$ & 9119.627 & 200 & 100 & 10962.352 & $16564_{11 / 2}^{\circ}-27526_{9 / 2}$ \\
\hline 9663.062 & 0 & & 10345.849 & $12488_{9 / 2}^{\circ}-22834_{7 / 2}$ & 9118.418 & 4 & 5 & 10963.805 & $1521_{5 / 2}-12485_{7 / 2}^{\circ}$ \\
\hline 9651.502 & 4 & 2 & 10358.241 & $15236_{3 / 2}-25594_{1 / 2}^{\circ}$ & 9113.459 & 2 & 10 & 10969.771 & $16818_{7 / 2}-27787_{9 / 2}^{\circ}$ \\
\hline 9642.471 & 4 & & 10367.942 & $8605_{5 / 2}-18973_{7 / 2}^{\circ}$ & 9112.672 & 2 & 15 & 10970.718 & $14275_{9 / 2}^{\circ}-25246_{9 / 2}$ \\
\hline 9613.689 & 5 & & 10398.982 & $4146_{7 / 2}-14545_{5 / 2}^{\circ}$ & 9109.777 & 2 & 10 & 10974.205 & $9711_{7 / 2}-20686_{5 / 2}^{\circ}$ \\
\hline 9583.076 & 3 & & 10432.201 & $4113_{5 / 2}-14545_{5 / 2}^{\circ}$ & 9107.667 & 2 & 15 & 10976.747 & $18118_{3 / 2}-29095_{5 / 2}^{\circ}$ \\
\hline 9578.636 & 0 & 5 & 10437.037 & $22139_{9 / 2}^{\circ}-32576_{7 / 2}$ & 9103.998 & 1 & 4 & 10981.171 & $26963_{7 / 2}^{\circ}-37945_{5 / 2}$ \\
\hline 9577.344 & 3 & & 10438.445 & $9720_{7 / 2}^{\circ}-20158_{5 / 2}$ & 9103.341 & 3 & 5 & 10981.964 & $7001_{3 / 2}-17983_{5 / 2}^{\circ}$ \\
\hline 9560.574 & 1 & 2 & 10456.755 & $21297_{5 / 2}^{\circ}-31754_{5 / 2}$ & 9096.811 & 1 & 3 & 10989.847 & $11116_{7 / 2}^{\circ}-22106_{5 / 2}$ \\
\hline 9558.370 & 1 & 2 & 10459.166 & $7001_{3 / 2}-17460_{5 / 2}^{\circ}$ & 9072.260 & 5 & 40 & 11019.587 & $9061_{5 / 2}-20080_{7 / 2}^{\circ}$ \\
\hline 9519.819 & 0 & 5 & 10501.521 & $26963_{\pi / 2}^{\circ}-37465_{5 / 2}$ & 9066.096 & 20 & 40 & 11027.079 & $6700_{9 / 2}^{\circ}-17727_{11 / 2}^{\circ}$ \\
\hline 9494.041 & 1 & & 10530.034 & $11576_{3 / 2}^{\circ}-22106_{5 / 2}$ & 9062.545 & 100 & 150 & 11031.400 & $4113_{5 / 2}-15144_{3 / 2}^{\circ}$ \\
\hline 9483.850 & 0 & & 10541.349 & $18973_{7 / 2}^{\circ}-29515_{9 / 2}^{\circ}$ & 9053.468 & 150 & 75 & 11042.460 & $1859_{3 / 2}-12902_{3 / 2}^{\circ}$ \\
\hline 9477.269 & 1 & 10 & 10548.669 & $23730_{9 / 2}^{\circ}-34279_{7 / 2}$ & 9046.321 & 0 & 5 & 11051.184 & $35165_{13 / 2}^{\circ}-46216_{11 / 2}$ \\
\hline 9456.023 & 0 & & 10572.370 & $19912_{13 / 2}^{\circ}-30484_{11 / 2}$ & 9040.106 & 50 & 75 & 11058.781 & $6213_{9 / 2}-17272_{9 / 2}^{\circ}$ \\
\hline 9455.297 & 0 & $15 w$ & 10573.182 & $20686_{5 / 2}^{\circ}-31259_{5 / 2}$ & 9038.675 & 4 & 15 & 11060.532 & $13248_{9 / 2}-24309_{11 / 2}^{\circ}$ \\
\hline 9439.854 & 0 & & 10590.479 & $8460_{3 / 2}-19050_{3 / 2}^{\circ}$ & 9032.634 & 0 & 8 & 11067.929 & $22834_{7 / 2}-33902_{7 / 2}^{\circ}$ \\
\hline 9432.280 & 3 & & 10598.983 & $9711_{7 / 2}-20310_{5 / 2}^{\circ}$ & 8974.923 & 1 & & 11139.099 & $20120_{5 / 2}^{\circ}-31259_{5 / 2}$ \\
\hline 9420.493 & $2 d$ & & 10612.244 & $1859_{3 / 2}-12472_{5 / 2}^{\circ}$ & 8974.326 & 0 & 5 & 11139.840 & $27249_{7 / 2}^{\circ}-38389_{7 / 2}$ \\
\hline 9398.155 & 2 & 4 & 10637.468 & $15786_{5 / 2}-26424_{5 / 2}^{\circ}$ & 8964.041 & 2 & 20 & 11152.621 & $12219_{3 / 2}-23372_{3 / 2}^{\circ}$ \\
\hline 9394.096 & 0 & & 10642.064 & $9238_{9 / 2}^{\circ}-19880_{9 / 2}$ & 8957.972 & 10 & 100 & 11160.177 & $12570_{7 / 2}-23730_{9 / 2}^{\circ}$ \\
\hline 9393.770 & 1 & & 10642.433 & $8605_{5 / 2}-19248_{5 / 2}^{\circ}$ & 8954.817 & 1 & 2 & 11164.109 & $13250_{5 / 2}-24414_{3 / 2}^{\circ}$ \\
\hline 9392.016 & 1 & & 10644.421 & $4146_{7 / 2}-14790_{7 / 2}^{\circ}$ & 8935.812 & 1 & 10 & 11187.853 & $15236_{3 / 2}-26424_{5 / 2}^{\circ}$ \\
\hline 9374.051 & 0 & & 10664.821 & $17272_{9 / 2}^{\circ}-27937_{11 / 2}$ & 8929.834 & 0 & 5 & 11195.343 & $35156_{5 / 2}^{\circ}-46352_{7 / 2}$ \\
\hline 9366.743 & $0 \mathrm{~d}$ & & 10673.141 & $0_{3 / 2}-10673_{5 / 2}^{\circ}$ & 8928.630 & 3 & 2 & 11196.852 & $32576_{7 / 2}-43773_{7 / 2}^{\circ}$ \\
\hline 9362.794 & 0 & & 10677.643 & $4113_{5 / 2}-14790_{7 / 2}^{\circ}$ & 8902.210 & 1 & 3 & 11230.082 & $8018_{3 / 2}-19248_{5 / 2}^{\circ}$ \\
\hline 9362.794 & 0 & & 10677.643 & $14349_{1 / 2}-25027_{1 / 2}^{\circ}$ & 8887.781 & 1 & 3 & 11248.314 & $22106_{5 / 2}-33354_{5 / 2}^{\circ}$ \\
\hline 9362.685 & 2 & 20 & 10677.767 & $34212_{5 / 2}^{\circ}-44889_{3 / 2}$ & 8880.875 & 1 & 2 & 11257.061 & $9711_{7 / 2}-20969_{7 / 2}^{\circ}$ \\
\hline
\end{tabular}


TABLE 3. Classified lines of Th II-Continued

\begin{tabular}{|c|c|c|c|c|c|c|c|c|c|}
\hline \multirow{2}{*}{$\begin{array}{c}\text { Wavelength } \\
\AA\end{array}$} & \multicolumn{2}{|c|}{ Intensity } & \multirow{2}{*}{$\begin{array}{c}\text { Wavenumber } \\
\mathrm{cm}^{-1}\end{array}$} & \multirow{2}{*}{ Classification } & \multirow{2}{*}{$\begin{array}{c}\text { Wavelength } \\
\AA\end{array}$} & \multicolumn{2}{|c|}{ Intensity } & \multirow{2}{*}{$\begin{array}{l}\text { Wavenumber } \\
\mathbf{c m}^{-1}\end{array}$} & \multirow{2}{*}{ Classification } \\
\hline & Lamp & Spark & & & & Lamp & Spark & & \\
\hline 8864.494 & 2 & 3 & 11277.863 & $9711_{7 / 2}-20989_{9 / 2}^{\circ}$ & 8403.787 & 100 & 300 & 11896.128 & $12485_{7 / 2}^{\circ}-24381_{7 / 2}$ \\
\hline 8842.067 & 15 & 2 & 11306.468 & $4146_{7 / 2}-15453_{7 / 2}^{\circ}$ & 8403.556 & 15 & 15 & 11896.455 & $9400_{5 / 2}-21297_{5 / 2}^{\circ}$ \\
\hline 8826.334 & 0 & 5 & 11326.622 & $24414_{3 / 2}^{\circ}-35741_{5 / 2}$ & 8399.688 & $5 s$ & 2 & 11901.933 & $26965_{3 / 2}^{\circ}-38867_{1 / 2}$ \\
\hline 8816.161 & 8 & 40 & 11339.692 & $4113_{5 / 2}-15453_{7 / 2}^{\circ}$ & 8397.074 & 2 & 5 & 11905.638 & $18973_{7 / 2}^{\circ}-30879_{7 / 2}$ \\
\hline 8798.457 & 1 & 5 & 11362.509 & $17983_{5 / 2}^{\circ}-29345_{5 / 2}$ & 8394.722 & 0 & 8 & 11908.974 & $44177_{11 / 2}-56086_{9 / 2}^{\circ}$ \\
\hline 8784.553 & 40 & 50 & 11380.493 & $1521_{5 / 2}-12902_{3 / 2}^{\circ}$ & 8392.547 & 2 & 50 & 11912.060 & $12219_{3 / 2}-24132_{3 / 2}^{\circ}$ \\
\hline 8777.333 & 1 & 3 & 11389.854 & $15236_{3 / 2}-26626_{1 / 2}^{\circ}$ & 8391.218 & 0 & 8 & 11913.947 & $24414_{3 / 2}^{\circ}-36328_{3 / 2}^{\circ}$ \\
\hline 8757.977 & 0 & 5 & 11415.027 & $24463_{5 / 2}^{\circ}-35878_{7 / 2}$ & 8387.089 & 150 & 200 & 11919.812 & $4113_{5 / 2}-16033_{5 / 2}^{\circ}$ \\
\hline 8749.761 & 1 & 1 & 11425.746 & $16818_{7 / 2}-28243_{5 / 2}^{\circ}$ & 8351.367 & 3 & 8 & 11970.798 & $9711_{7 / 2}-21682_{7 / 2}^{\circ}$ \\
\hline 8745.509 & 2 & 2 & 11431.301 & $32957_{7 / 2}^{\circ}-44388_{5 / 2}$ & 8351.052 & 2 & 8 & 11971.249 & $17460_{5 / 2}^{\circ}-29431_{7 / 2}$ \\
\hline 8736.376 & 1 & 3 & 11443.251 & $20310_{5 / 2}^{\circ}-31754_{5 / 2}$ & 8342.686 & 2 & 5 & 11983.254 & $28587_{5 / 2}^{\circ}-40570_{7 / 2}$ \\
\hline 8721.653 & 8 & 100 & 11462.568 & $15786_{5 / 2}-27249_{7 / 2}^{\circ}$ & 8321.156 & 0 & 10 & 12014.259 & $15242_{9 / 2}^{\circ}-27257_{7 / 2}$ \\
\hline 8719.623 & 5 & 50 & 11465.237 & $15305_{9 / 2}-26770_{11 / 2}^{\circ}$ & 8313.535 & 4 & 2 & 12025.272 & $25440_{5 / 2}^{\circ}-37465_{5 / 2}$ \\
\hline 8698.591 & 10 & 4 & 11492.958 & $33902_{7 / 2}^{\circ}-45395_{7 / 2}$ & 8310.076 & 1 & 10 & 12030.278 & $22513_{5 / 2}^{\circ}-34543_{5 / 2}$ \\
\hline 8695.106 & 2 & 1 & 11497.564 & $22139_{9 / 2}^{\circ}-33637_{7 / 2}$ & 8302.576 & 2 & 15 & 12041.145 & $22685_{7 / 2}^{\circ}-34726_{7 / 2}$ \\
\hline 8688.761 & $2 w$ & 1 & 11505.960 & $22513_{5 / 2}^{\circ}-34019_{3 / 2}$ & 8295.021 & 4. & 15 & 12052.112 & $15305_{9 / 2}-27357_{9 / 2}^{\circ}$ \\
\hline 8686.605 & 3 & 10 & 11508.816 & $13248_{9 / 2}-24757_{9 / 2}^{\circ}$ & 8282.232 & 5 & 15 & 12070.722 & $9061_{5 / 2}-21131_{3 / 2}^{\circ}$ \\
\hline 8686.074 & 10 & 25 & 11509.520 & $6213_{9 / 2}-17722_{9 / 2}^{\circ}$ & 8280.974 & 1 & 8 & 12072.556 & $25607_{9 / 2}^{\circ}-37679_{11 / 2}$ \\
\hline 8682.441 & 3 & 8 & 11514.336 & $8605_{5 / 2}-20120_{5 / 2}^{\circ}$ & 8263.029 & 0 & 8 & 12098.774 & $30101_{7 / 2}^{\circ}-42200_{9 / 2}$ \\
\hline 8629.132 & 8 & $40 w$ & 11585.469 & $9711_{7 / 2}-21297_{5 / 2}^{\circ}$ & 8258.994 & 4 & 3 & 12104.685 & $33384_{9 / 2}-45489_{9 / 2}^{\circ}$ \\
\hline 8628.062 & 1 & 4 & 11586.905 & $20989_{9 / 2}^{\circ}-32576_{7 / 2}$ & 8242.406 & 0 & 8 & 12129.045 & $35602_{11 / 2}^{\circ}-47731_{9 / 2}$ \\
\hline 8621.818 & 2 & 1 & 11595.297 & $32957_{7 / 2}^{\circ}-44552_{5 / 2}$ & 8241.355 & 2 & 15 & 12130.592 & $22139_{9 / 2}^{\circ}-34270_{9 / 2}$ \\
\hline 8620.179 & 10 & 15 & 11597.501 & $4113_{5 / 2}-15710_{3 / 2}^{\circ}$ & 8236.320 & 5 & 2 & 12138.008 & $35593_{7 / 2}^{\circ}-47731_{9 / 2}$ \\
\hline 8617.110 & 2 & 8 & 11601.632 & $18118_{3 / 2}-29720_{3 / 2}^{\circ}$ & 8235.216 & 0 & 5 & 12139.635 & $22139_{9 / 2}^{\circ}-34279_{7 / 2}$ \\
\hline 8600.910 & 3 & 10 & 11623.484 & $13250_{5 / 2}-24873_{5 / 2}^{\circ}$ & 8221.233 & 0 & 5 & 12160.282 & $25414_{11 / 2}^{\circ}-37575_{13 / 2}$ \\
\hline 8599.731 & 10 & 25 & 11625.077 & $9061_{5 / 2}-20686_{5 / 2}^{\circ}$ & 8220.764 & 3 & 10 & 12160.976 & $10673_{5 / 2}^{\circ}-22834_{7 / 2}$ \\
\hline 8597.434 & 5 & 10 & 11628.183 & $22642_{9 / 2}^{\circ}-34270_{9 / 2}$ & 8217.219 & 75 & 150 & 12166.223 & $13248_{9 / 2}-25414_{11 / 2}^{\circ}$ \\
\hline 8591.828 & 200 & 300 & 11635.770 & $10379_{9 / 2}-22014_{11 / 2}^{0}$ & 8216.352 & 0 & 15 & 12167.506 & $35156_{5 / 2}^{\circ}-47324_{5 / 2}$ \\
\hline 8590.751 & 2 & 40 & 11637.229 & $22642_{9 / 2}^{\circ}-34279_{7 / 2}$ & 8203.190 & 200 & 100 & 12187.029 & $12570_{7 / 2}-24757_{9 / 2}^{\circ}$ \\
\hline 8587.629 & 5 & 50 & 11641.459 & $20158_{5 / 2}-31800_{7 / 2}^{\circ}$ & 8198.067 & 1 & 1 & 12194.645 & $12219_{3 / 2}-24414_{3 / 2}^{\circ}$ \\
\hline 8582.539 & 2 & & 11648.364 & $25414_{11 / 2}^{\circ}-37063_{9 / 2}$ & 8184.393 & 1 & 10 & 12215.019 & $26647_{13 / 2}^{\circ}-38862_{11 / 2}$ \\
\hline 8580.560 & 2 & 1 & 11651.050 & $24414_{3 / 2}^{\circ}-36065_{5 / 2}$ & 8182.265 & 5 & 8 & 12218.196 & $37277_{7 / 2}-49495_{9 / 2}^{\circ}$ \\
\hline 8568.203 & 10 & 150 & 11667.853 & $18816_{13 / 2}^{\circ}-30484_{11 / 2}$ & 8181.307 & 2 & 10 & 12219.626 & $20989_{9 / 2}^{\circ}-33209_{7 / 2}$ \\
\hline 8531.872 & 3 & 5 & 11717.538 & $11116_{7 / 2}^{\circ}-22834_{7 / 2}$ & 8178.203 & 4 & 20 & 12224.264 & $17121_{3 / 2}^{\circ}-29345_{5 / 2}$ \\
\hline 8523.848 & 2 & 4 & 11728.568 & $15236_{3 / 2}-26965_{3 / 2}^{\circ}$ & 8177.169 & 5 & 15 & 12225.810 & $8460_{3 / 2}-20686_{5 / 2}^{\circ}$ \\
\hline 8522.191 & 2 & 5 & 11730.849 & $9400_{5 / 2}-21131_{3 / 2}^{\circ}$ & 8170.181 & 3 & 5 & 12236.267 & $9061_{5 / 2}-21297_{5 / 2}^{\circ}$ \\
\hline 8521.409 & 2 & 25 & 11731.925 & $13250_{5 / 2}-24982_{7 / 2}^{\circ}$ & 8166.441 & 150 & 100 & 12241.871 & $1859_{3 / 2}-14101_{1 / 2}^{\circ}$ \\
\hline 8512.591 & $5 \mathbf{h}$ & 50 & 11744.078 & $17771_{11 / 2}^{\circ}-29515_{9 / 2}$ & 8165.733 & 4 & 4 & 12242.932 & $17272_{9 / 2}^{\circ}-29515_{9 / 2}$ \\
\hline 8500.667 & 50 & 75 & 11760.551 & $10379_{9 / 2}-22139_{9 / 2}^{\circ}$ & 8165.153 & $8 \mathbf{h}$ & 8 & 12243.802 & $12219_{3 / 2}-24463_{5 / 2}^{\circ}$ \\
\hline 8498.514 & 2 & 5 & 11763.531 & $26965_{3 / 2}^{\circ}-38728_{5 / 2}$ & 8163.117 & 100 & 150 & 12246.855 & $7001_{3 / 2}-19248_{5 / 2}^{\circ}$ \\
\hline 8496.718 & 8 & 4 & 11766.017 & $22513_{5 / 2}^{\circ}-34279_{7 / 2}$ & 8152.391 & 8 & 40 & 12262.968 & $10379_{9 / 2}-22642_{9 / 2}^{\circ}$ \\
\hline 8481.756 & 2 & 4 & 11786.773 & $10855_{7 / 2}-22642_{9 / 2}^{\circ}$ & 8150.530 & 0 & 8 & 12265.768 & $20310_{5 / 2}^{\circ}-32576_{7 / 2}$ \\
\hline 8477.916 & 20 & 150 & 11792.111 & $43246_{7 / 2}-55038_{9 / 2}^{\circ}$ & 8145.474 & 2 & 5 & 12273.382 & $20686_{5 / 2}^{\circ}-32959_{3 / 2}$ \\
\hline 8453.046 & $2 \mathbf{h}$ & 1 & 11826.805 & $24982_{7 / 2}^{\circ}-36809_{7 / 2}$ & 8139.896 & 75 & 100 & 12281.792 & $9400_{5 / 2}-21682_{7 / 2}^{\circ}$ \\
\hline 8452.387 & 4 & 2 & 11827.727 & $39396_{7 / 2}^{\circ}-51224_{9 / 2}^{\circ}$ & 8130.213 & 15 & 8 & 12296.420 & $1521_{5 / 2}-13818_{7 / 2}^{\circ}$ \\
\hline 8450.675 & $75 b$ & 150 & 11830.123 & $10855_{7 / 2}-22685_{7 / 2}^{\circ}$ & 8127.630 & 2 & 8 & 12300.327 & $15710_{3 / 2}^{\circ}-28011_{3 / 2}$ \\
\hline 8436.118 & 0 & 5 & 11850.537 & $32957_{7 / 2}^{\circ}-44807_{7 / 2}$ & 8125.514 & 1 & 3 & 12303.531 & $12570_{7 / 2}-24873_{5 / 2}^{\circ}$ \\
\hline 8412.973 & 4 & 25 & 11883.139 & $15710_{3 / 2}^{\circ}-27593_{5 / 2}$ & 8123.655 & 2 & 5 & 12306.346 & $10379_{9 / 2}-22685_{\tau / 2}^{\circ}$ \\
\hline 8410.532 & 25 & 25 & 11886.588 & $4146_{7 / 2}-16033_{5 / 2}^{\circ}$ & 8112.024 & 1 & 1 & 12323.991 & $6244_{1 / 2}-18568_{1 / 2}^{\circ}$ \\
\hline 8405.770 & 3 & 8 & 11893.322 & $12570_{7 / 2}-24463_{5 / 2}^{\circ}$ & 8082.673 & 5 & 4 & 12368.743 & $23372_{3 / 2}^{\circ}-35741_{5 / 2}$ \\
\hline
\end{tabular}


TABLE 3. Classified lines of Th II-Continued

\begin{tabular}{|c|c|c|c|c|c|c|c|c|c|}
\hline \multirow{2}{*}{$\begin{array}{c}\text { Wavelength } \\
\AA\end{array}$} & \multicolumn{2}{|c|}{ Intensity } & \multirow{2}{*}{$\begin{array}{c}\text { Wavenumber } \\
\text { cm }^{-1}\end{array}$} & \multirow{2}{*}{ Classification } & \multirow{2}{*}{$\begin{array}{c}\text { Wavelength } \\
\AA\end{array}$} & \multicolumn{2}{|c|}{ Intensity } & \multirow{2}{*}{$\begin{array}{c}\text { Wavenumber } \\
\mathbf{c m}^{-1}\end{array}$} & \multirow{2}{*}{ Classification } \\
\hline & Lamp & Spark & & & & Lamp & Spark & & \\
\hline 8054.524 & 10 & 50 & 12411.970 & $12570_{7 / 2}-24982_{7 / 2}^{\circ}$ & 7743.934 & 5 & 25 & 12909.780 & $12472_{5 / 2}^{\circ}-25381_{3 / 2}$ \\
\hline 8053.584 & 1 & 8 & 12413.418 & $35593_{9 / 2}^{\circ}-48006_{9 / 2}^{\circ}$ & 7731.716 & 75 & 100 & 12930.181 & $9711_{7 / 2}-22642_{9 / 2}^{\circ}$ \\
\hline 8053.010 & 1 & 10 & 12414.303 & $22139_{9 / 2}^{\circ}-34553_{9 / 2}$ & 7729.542 & 2 & 4 & 12933.818 & $15786_{5 / 2}-28720_{3 / 2}^{\circ}$ \\
\hline 8036.828 & 20 & 10 & 12439.299 & $16906_{7 / 2}^{\circ}-29345_{5 / 2}$ & 7701.101 & 15 & 20 & 12981.583 & $14275_{9 / 2}^{\circ}-27257_{7 / 2}$ \\
\hline 8020.742 & 2 & 2 & 12464.246 & $25381_{3 / 2}-37846_{5 / 2}^{\circ}$ & 7676.212 & 100 & 15 & 13023.674 & $1521_{5 / 2}-14545_{5 / 2}^{\circ}$ \\
\hline 8015.626 & 25 & 300 & 12472.202 & $0_{3 / 2}-12472_{5 / 2}^{\circ}$ & 7637.767 & 1 & 3 & 13089.229 & $20120_{5 / 2}^{\circ}-33209_{7 / 2}$ \\
\hline 7984.363 & 8 & 10 & 12521.037 & $9585_{5 / 2}^{\circ}-22106_{5 / 2}$ & 7633.890 & 2 & 4 & 13095.876 & $20288_{11 / 2}^{\circ}-33384_{9 / 2}$ \\
\hline 7983.722 & 1 & 10 & 12522.042 & $22139_{9 / 2}^{\circ}-34661_{11 / 2}$ & 7624.311 & 5 & 8 & 13112.330 & $9400_{5 / 2}-22513_{5 / 2}^{\circ}$ \\
\hline 7981.670 & 5 & 50 & 12525.261 & $16906_{7 / 2}^{\circ}-29431_{7 / 2}$ & 7623.565 & 5 & 5 & 13113.613 & $8018_{3 / 2}-21131_{3 / 2}^{\circ}$ \\
\hline 7981.213 & 75 & 1 & 12525.978 & $8605_{5 / 2}-21131_{3 / 2}^{\circ}$ & 7623.440 & 1 & 1 & 13113.828 & $9720_{7 / 2}^{\circ}-22834_{7 / 2}$ \\
\hline 7973.517 & 5 & 4 & 12538.068 & $27357_{9 / 2}^{\circ}-39895_{9 / 2}$ & 7620.586 & 3 & 4 & 13118.739 & $7001_{3 / 2}-20120_{5 / 2}^{\circ}$ \\
\hline 7972.881 & $4 \mathrm{~h}$ & 50 & 12539.069 & $22014_{11 / 2}^{\circ}-34553_{9 / 2}$ & 7616.576 & 1 & 2 & 13125.646 & $4146_{7 / 2}-17272_{9 / 2}^{\circ}$ \\
\hline 7945.845 & 0 & 5 & 12581.733 & $27357_{9 / 2}^{\circ}-39939_{11 / 2}$ & 7614.810 & 3 & 8 & 13128.690 & $20080_{7 / 2}^{\circ}-33209_{7 / 2}^{\circ}$ \\
\hline 7945.057 & 5 & 50 & 12582.981 & $17727_{11 / 2}-30310_{11 / 2}^{\circ}$ & 7608.659 & 2 & 3 & 13139.303 & $10379_{9 / 2}-23518_{\tau / 2}^{\circ}$ \\
\hline 7936.475 & 2 & 15 & 12596.587 & $21682_{7 / 2}^{\circ}-34279_{7 / 2}$ & 7566.526 & 5 & 20 & 13212.467 & $17272_{9 / 2}^{\circ}-30484_{11 / 2}$ \\
\hline 7934.889 & 0 & 8 & 12599.105 & $21131_{3 / 2}^{\circ}-33730_{5 / 2}$ & 7556.892 & 1 & 4 & 13229.311 & $17727_{11 / 2}-30956_{9 / 2}^{\circ}$ \\
\hline 7933.275 & 2 & 4 & 12601.668 & $17272_{9 / 2}^{\circ}-29873_{7 / 2}$ & 7547.309 & 1 & 8 & 13246.108 & $21297_{5 / 2}^{\circ}-34543_{5 / 2}$ \\
\hline 7928.939 & 4 & 40 & 12608.559 & $16906_{7 / 2}^{\circ}-29515_{9 / 2}$ & 7536.407 & 50 & 10 & 13265.270 & $11116_{7 / 2}^{\circ}-24381_{7 / 2}$ \\
\hline 7914.333 & 0 & 5 & 12631.829 & $29720_{3 / 2}^{\circ}-42352_{5 / 2}$ & 7536.011 & 0 & 8 & 13265.967 & $24309_{11 / 2}^{\circ}-37575_{13 / 2}$ \\
\hline 7896.427 & $5 c$ & 40 & 12660.472 & $14101_{1 / 2}^{\circ}-26762_{3 / 2}$ & 7534.224 & 3 & 2 & 13269.113 & $1521_{5 / 2}-14790_{7 / 2}^{\circ}$ \\
\hline 7894.776 & 4 & 20 & 12663.120 & $10855_{7 / 2}-23518_{7 / 2}^{\circ}$ & 7525.507 & 200 & 150 & 13284.483 & $9400_{5 / 2}-22685_{7 / 2}^{\circ}$ \\
\hline 7892.892 & 3 & 5 & 12666.143 & $22355_{1 / 2}^{\circ}-35021_{3 / 2}$ & 7525.329 & 10 & 5 & 13284.797 & $1859_{3 / 2}-15144_{3 / 2}^{\circ}$ \\
\hline 7891.743 & 3 & 8 & 12667.987 & $8018_{3 / 2}-20686_{5 / 2}^{\circ}$ & 7522.654 & 2 & 10 & 13289.521 & $20989_{9 / 2}^{\circ}-34279_{7 / 2}$ \\
\hline 7883.986 & 1 & 8 & 12680.451 & $28026_{5 / 2}-40706_{7 / 2}^{\circ}$ & 7514.891 & 4 & 4 & 13303.250 & $7828_{1 / 2}-21131_{3 / 2}^{\circ}$ \\
\hline 7880.761 & 4 & 8 & 12685.640 & $1859_{3 / 2}-14545_{5 / 2}^{\circ}$ & 7514.604 & 1 & 4 & 13303.758 & $20080_{7 / 2}^{\circ}-33384_{9 / 2}$ \\
\hline 7877.057 & 15 & 50 & 12691.605 & $8605_{5 / 2}-21297_{5 / 2}^{\circ}$ & 7511.346 & 50 & 25 & 13309.528 & $7001_{3 / 2}-20310_{5 / 2}^{\circ}$ \\
\hline 7875.448 & 8 & 100 & 12694.198 & $15242_{9 / 2}^{\circ}-27937_{11 / 2}$ & 7509.517 & 1 & 1 & 13312.770 & $16033_{5 / 2}^{\circ}-29345_{5 / 2}$ \\
\hline 7871.284 & 4 & 1 & 12700.913 & $39861_{5 / 2}^{\circ}-52562_{7 / 2}$ & 7508.791 & 3 & & 13314.057 & $4146_{7 / 2}-17460_{5 / 2}^{\circ}$ \\
\hline 7853.482 & 0 & 15 & 12729.703 & $17722_{9 / 2}^{\circ}-30452_{9 / 2}$ & 7497.882 & 4 & 8 & 13333.428 & $24132_{3 / 2}^{\circ}-37465_{5 / 2}$ \\
\hline 7849.616 & 8 & 150 & 12735.972 & $14790_{7 / 2}^{\circ}-27526_{9 / 2}$ & 7490.104 & 8 & 5 & 13347.274 & $4113_{5 / 2}-17460_{5 / 2}^{\circ}$ \\
\hline 7836.703 & 0 & 5 & 12756.958 & $25188_{3 / 2}^{\circ}-37945_{5 / 2}$ & 7488.169 & 2 & 2 & 13350.723 & $15236_{3 / 2}-28587_{5 / 2}^{\circ}$ \\
\hline 7836.081 & 1 & 5 & 12757.971 & $12488_{9 / 2}^{\circ}-25246_{9 / 2}$ & 7487.713 & 5 & 5 & 13351.536 & $10379_{9 / 2}-23730_{9 / 2}^{\circ}$ \\
\hline 7835.266 & 2 & 40 & 12759.298 & $22834_{7 / 2}-35593_{9 / 2}^{\circ}$ & 7484.002 & 1 & 3 & 13358.156 & $24463_{5 / 2}^{\circ}-37821_{3 / 2}^{\circ}$ \\
\hline 7834.805 & 2 & $10 \mathrm{~h}$ & 12760.049 & $4146_{\tau / 2}-16906_{7 / 2}^{\circ}$ & 7483.374 & 2 & 8 & 13359.277 & $22834_{7 / 2}-36193_{9 / 2}^{\circ}$ \\
\hline 7834.446 & 100 & 200 & 12760.633 & $12485_{7 / 2}^{\circ}-25246_{9 / 2}$ & 7479.821 & 3 & 15 & 13365.623 & $23697_{7 / 2}^{\circ}-37063_{9 / 2}$ \\
\hline 7824.041 & 2 & 10 & 12777.603 & $17771_{11 / 2}^{\circ}-30548_{13 / 2}$ & 7454.096 & 2 & 4 & 13411.749 & $21131_{3 / 2}^{\circ}-34543_{5 / 2}^{\circ}$ \\
\hline 7818.331 & 4 & 40 & 12786.935 & $27787_{9 / 2}^{\circ}-40574_{11 / 2}$ & 7439.086 & 8 & 8 & 13438.810 & $13818_{7 / 2}^{\circ}-27257_{7 / 2}$ \\
\hline 7815.866 & 1 & 2 & 12790.968 & $30223_{15 / 2}^{\circ}-43014_{13 / 2}$ & 7438.494 & 2 & 8 & 13439.880 & $22685_{7 / 2}^{\circ}-36125_{9 / 2}^{\prime}$ \\
\hline 7811.159 & 2 & 40 & 12798.676 & $20158_{5 / 2}-32957_{7 / 2}^{\circ}$ & & & & & $23372_{3 / 2}^{\circ}-36812_{1 / 2}$ \\
\hline 7807.026 & 5 & 4 & 12805.451 & $24982_{7 / 2}^{\circ}-37787_{\tau / 2}$ & 7437.708 & 2 & 5 & 13441.300 & $22014_{11 / 2}^{\circ}-35456_{9 / 2}$ \\
\hline 7806.029 & 4 & 3 & 12807.087 & $12219_{3 / 2}-25027_{1 / 2}^{\circ}$ & 7431.680 & 4 & 2 & 13452.202 & $9061_{5 / 2}-22513_{5 / 2}^{\circ}$ \\
\hline 7787.793 & $75 b$ & 200 & 12837.076 & $8460_{3 / 2}-21297_{5 / 2}^{\circ}$ & 7431.378 & 5 & 3 & 13452.749 & $14484_{11 / 2}^{\circ}-27937_{11 / 2}^{\circ}$ \\
\hline 7786.444 & 2 & 5 & 12839.300 & $20120_{5 / 2}^{\circ}-32959_{3 / 2}$ & 7423.311 & 10 & 4 & 13467.368 & $6691_{3 / 2}^{\circ}-20158_{5 / 2}$ \\
\hline 7784.608 & 2 & 50 & 12842.328 & $10855_{7 / 2}-23697_{7 / 2}^{\circ}$ & 7414.052 & 5 & 4 & 13484.187 & $15236_{3 / 2}-28720_{3 / 2}^{\circ}$ \\
\hline 7777.302 & 0 & 5 & 12854.392 & $30101_{\tau / 2}^{\circ}-42955_{9 / 2}$ & 7412.008 & 4 & 5 & 13487.905 & $19248_{5 / 2}^{\circ}-32736_{7 / 2}^{\circ}$ \\
\hline 7773.429 & 2 & 1 & 12860.796 & $21682_{7 / 2}^{\circ}-34543_{5 / 2}$ & 7399.724 & 2 & 4 & 13510.296 & $22014_{11 / 2}^{\circ}-35525_{11 / 2}$ \\
\hline 7767.173 & 0 & 8 & 12871.155 & $21682_{7 / 2}^{\circ}-34553_{9 / 2}$ & 7396.009 & 8 & 8 & 13517.082 & $20120_{5 / 2}^{\circ}-33637_{7 / 2}$ \\
\hline 7764.645 & 3 & 10 & 128.75 .346 & $10855_{7 / 2}-23730_{9 / 2}^{\circ}$ & 7393.434 & 200 & 150 & 13521.790 & $13248_{9 / 2}-26770_{11 / 2}^{\circ}$ \\
\hline 7748.368 & 2 & 3 & 12902.393 & $0_{3 / 2}-12902_{3 / 2}^{\circ}$ & 7389.264 & 20 & 3 & 13529.421 & $14101_{1 / 2}^{\circ}-27631_{3 / 2}$ \\
\hline 7747.415 & 5 & 4 & 12903.980 & $36581_{3 / 2}^{\circ}-49485_{1 / 2}$ & 7380.156 & 8 & 8 & 13546.117 & $16906_{7 / 2}^{\circ}-30452_{9 / 2}$ \\
\hline
\end{tabular}


TABLE 3. Classified lines of Th II-Continued

\begin{tabular}{|c|c|c|c|c|c|c|c|c|c|}
\hline \multirow{2}{*}{$\begin{array}{c}\text { Wavelength } \\
\AA\end{array}$} & \multicolumn{2}{|c|}{ Intensity } & \multirow{2}{*}{$\begin{array}{c}\text { Wavenumber } \\
\mathbf{c m}^{-1}\end{array}$} & \multirow{2}{*}{ Classification } & \multirow{2}{*}{$\begin{array}{c}\text { Wavelength } \\
\AA\end{array}$} & \multicolumn{2}{|c|}{ Intensity } & \multirow{2}{*}{$\begin{array}{l}\text { Wavenumber } \\
\mathbf{c m}^{-1}\end{array}$} & \multirow{2}{*}{ Classification } \\
\hline & Lamp & Spark & & & & Lamp & Spark & & \\
\hline 7359.069 & 2 & 8 & 13584.933 & $20969_{7 / 2}^{\circ}-34553_{9 / 2}$ & 7098.675 & 1 & 3 & 14083.253 & $26963_{7 / 2}^{\circ}-41047_{9 / 2}$ \\
\hline 7358.347 & 50 & 20 & 13586.266 & $12902_{3 / 2}^{\circ}-26488_{5 / 2}$ & 7089.699 & 4 & & 14101.083 & $4113_{5 / 2}-18214_{3 / 2}^{\circ}$ \\
\hline 7354.610 & 2 & 3 & 13593.169 & $20686_{5 / 2}^{\circ}-34279_{7 / 2}$ & 7089.334 & 400 & 100 & 14101.809 & $0_{3 / 2}-14101_{1 / 2}^{\circ}$ \\
\hline 7353.034 & 8 & & 13596.083 & $9238_{9 / 2}^{\circ}-22834_{7 / 2}$ & 7087.595 & 0 & 5 & 14105.269 & $24757_{9 / 2}^{\circ}-38862_{11 / 2}$ \\
\hline 7353.007 & 15 & 8 & 13596.133 & $9238_{9 / 2}^{\circ}-22834_{7 / 2}$ & 7084.983 & 2 & 10 & 14110.469 & $22014_{11 / 2}^{\circ}-36125_{9 / 2}$ \\
\hline 7352.581 & 5 & & 13596.920 & $26770_{11 / 2}^{\circ}-40367_{9 / 2}$ & 7078.262 & 5 & 4 & 14123.867 & $22685_{7 / 2}^{\circ}-36809_{7 / 2}$ \\
\hline 7349.315 & 3 & 8 & 13602.963 & $18973_{7 / 2}^{\circ}-32576_{7 / 2}$ & 7075.331 & 200 & 150 & 14129.718 & $11116_{7 / 2}^{\circ}-25246_{9 / 2}$ \\
\hline 7347.048 & 8 & 15 & 13607.160 & $17272_{9 / 2}^{\circ}-30879_{7 / 2}$ & 7075.003 & 5 & 4 & 14130.373 & $7001_{3 / 2}-21131_{3 / 2}^{\circ}$ \\
\hline 7346.341 & 15 & 15 & 13608.469 & $10855_{7 / 2}-24463_{5 / 2}^{\circ}$ & 7053.614 & 300 & 75 & 14173.221 & $1859_{3 / 2}-16033_{5 / 2}^{\circ}$ \\
\hline 7338.591 & 5 & 3 & 13622.841 & $1521_{5 / 2}-15144_{3 / 2}^{\circ}$ & 7045.795 & 400 & 150 & 14188.950 & $\begin{aligned} 15242_{9 / 2}^{\circ} & -29431_{7 / 2}^{\circ} \\
1521_{5 / 2} & -15710_{3 / 2}^{\circ}\end{aligned}$ \\
\hline 7337.775 & 15 & 8 & 13624.356 & $9061_{5 / 2}-22685_{7 / 2}^{\circ}$ & & & & & \\
\hline 7332.015 & 3 & 3 & 13635.059 & $15710_{3 / 2}^{\circ}-29345_{5 / 2}$ & 7008.051 & 2 & 5 & 14265.368 & $20288_{11 / 2}^{\circ}-34553_{9 / 2}$ \\
\hline 7323.851 & 4 & 5 & 13650.258 & $20080_{7 / 2}^{\circ}-33730_{5 / 2}$ & 6995.930 & 2 & 5 & 14290.084 & $12472_{5 / 2}^{\circ}-26762_{3 / 2}$ \\
\hline 7312.255 & 2 & 4 & 13671.905 & $20989_{9 / 2}^{\circ}-34661_{11 / 2}$ & 6993.034 & 300 & 200 & 14296.002 & $22513_{5 / 2}^{\circ}-36809_{7 / 2}$ \\
\hline 7308.515 & 5 & 8 & 13678.901 & $15144_{3 / 2}^{\circ}-28823_{5 / 2}$ & & & & & $7001_{3 / 2}-21297_{5 / 2}^{\circ}$ \\
\hline 7305.397 & 100 & 75 & 13684.739 & $7001_{3 / 2}-20686_{5 / 2}^{\circ}$ & 6992.692 & 50 & 40 & 14296.701 & $9400_{5 / 2}-23697_{7 / 2}^{\circ}$ \\
\hline 7292.669 & 5 & 5 & 13708.623 & $13818_{7 / 2}^{\circ}-27526_{9 / 2}$ & 6985.467 & 20 & 40 & 14311.488 & $9061_{5 / 2}-23372_{3 / 2}^{\circ}$ \\
\hline 7289.438 & 3 & 8 & 13714.700 & $13250_{5 / 2}-26965_{3 / 2}^{\circ}$ & 6975.478 & 1 & 5 & 14331.982 & $25607_{9 / 2}^{\circ}-39939_{11 / 2}$ \\
\hline 7257.189 & 4 & 4 & 13775.644 & $13818_{7 / 2}^{\circ}-27593_{5 / 2}$ & 6973.043 & 0 & 5 & 14336.987 & $8018_{3 / 2}-22355_{1 / 2}^{\circ}$ \\
\hline 7250.587 & 15 & 20 & 13788.187 & $13468_{9 / 2}^{\circ}-27257_{7 / 2}$ & 6973.020 & 4 & 5 & 14337.034 & $8018_{3 / 2}-22355_{1 / 2}^{\circ}$ \\
\hline 7240.981 & 5 & 2 & 13806.479 & $9711_{7 / 2}-23518_{7 / 2}^{\circ}$ & 6965.415 & 2 & 5 & 14352.687 & $16906_{7 / 2}^{\circ}-31259_{5 / 2}$ \\
\hline 7225.125 & 20 & 5 & 13836.778 & $4146_{7 / 2}-17983_{5 / 2}^{\circ}$ & 6956.332 & 1 & 8 & 14371.428 & $14349_{1 / 2}-28720_{3 / 2}^{\circ}$ \\
\hline 7217.759 & 150 & 50 & 13850.898 & $1859_{3 / 2}-15710_{3 / 2}^{\circ}$ & 6955.504 & 2 & 20 & 14373.139 & $20288_{11 / 2}^{\circ}-34661_{11 / 2}$ \\
\hline 7216.158 & 4 & 4 & 13853.971 & $12570_{7 / 2}-26424_{5 / 2}^{\circ}$ & 6953.230 & 0 & 5 & 14377.839 & $22685_{7 / 2}^{\circ}-37063_{9 / 2}$ \\
\hline 7195.955 & 5 & 5 & 13892.867 & $15453_{7 / 2}^{\circ}-29345_{5 / 2}$ & 6952.961 & 50 & 50 & 14378.396 & $10379_{9 / 2}-24757_{9 / 2}^{\circ}$ \\
\hline 7194.925 & 3 & 5 & 13894.856 & $8460_{3 / 2}-22355_{1 / 2}^{\circ}$ & 6946.781 & 1 & 5 & 14391.187 & $18568_{i / 2}^{\circ}-32959_{3 / 2}$ \\
\hline 7191.128 & 500 & 300 & 13902.192 & $10855_{7 / 2}-24757_{9 / 2}^{\circ}$ & 6938.967 & 0 & 5 & 14407.393 & $23697_{7 / 2}^{\circ}-38105_{5 / 2}$ \\
\hline 7188.415 & 3 & 2 & 13907.439 & $8605_{5 / 2}-22513_{5 / 2}^{\circ}$ & 6937.080 & 0 & 25 & 14411.312 & $22642_{9 / 2}^{\circ}-37053_{11 / 2}$ \\
\hline 7187.438 & 2 & 4 & 13909.330 & $14101_{1 / 2}^{\circ}-28011_{3 / 2}$ & 6932.458 & 2 & 5 & 14420.920 & $15453_{7 / 2}^{\circ}-29873_{7 / 2}$ \\
\hline 7181.902 & 1 & 3 & 13920.051 & $16564_{11 / 2}^{\circ}-30484_{11 / 2}$ & 6929.689 & 2 & 3 & 14426.682 & $23518_{7 / 2}^{\circ}-37945_{5 / 2}$ \\
\hline 7179.048 & 1 & 8 & 13925.585 & $24463_{5 / 2}^{\circ}-38389_{7 / 2}$ & 6921.432 & 2 & 20 & 14443.893 & $21297_{5 / 2}^{\circ}-35741_{5 / 2}$ \\
\hline 7176.719 & 25 & 25 & 13930.104 & $10379_{9 / 2}-24309_{11 / 2}^{0}$ & 6917.036 & 3 & 3 & 14453.072 & $26770_{11 / 2}^{\circ}-41223_{11 / 2}$ \\
\hline 7176.184 & 20 & 10 & 13931.143 & $1521_{5 / 2}-15453_{7 / 2}^{\circ}$ & 6915.001 & $2 \mathrm{~b}$ & 8 & 14457.325 & $9061_{5 / 2}-23518_{7 / 2}^{\circ}$ \\
\hline 7172.987 & 1 & 2 & 13937.352 & $25414_{11 / 2}^{\circ}-39352_{11 / 2}$ & 6910.690 & 1 & 3 & 14466.344 & $20989_{9 / 2}^{\circ}-35456_{9 / 2}$ \\
\hline 7166.514 & 0 & 8 & 13949.940 & $35545_{9 / 2}-49495_{9 / 2}^{\circ}$ & 6909.845 & 100 & 200 & 14468.113 & $13468_{9 / 2}^{\circ}-27937_{11 / 2}$ \\
\hline 7165.988 & 20 & 10 & 13950.964 & $9061_{5 / 2}-23012_{3 / 2}^{\circ}$ & 6907.384 & 2 & 5 & 14473.268 & $20080_{7 / 2}^{\circ}-34553_{9 / 2}$ \\
\hline 7160.764 & 1 & 3 & 13961.142 & $19248_{5 / 2}^{\circ}-33209_{7 / 2}$ & 6903.086 & 2 & 40 & 14482.279 & $23697_{7 / 2}^{\circ}-38179_{9 / 2}$ \\
\hline 7157.067 & 0 & 5 & 13968.353 & $20310_{5 / 2}^{\circ}-34279_{7 / 2}$ & 6902.652 & 5 & 2 & 14483.190 & $15305_{9 / 2}-29788_{9 / 2}^{\circ}$ \\
\hline 7151.197 & 3 & 3 & 13979.819 & $31924_{11 / 2}^{\circ}-45904_{9 / 2}$ & 6898.146 & 0 & 8 & 14492.650 & $23187_{13 / 2}^{\circ}-37679_{11 / 2}$ \\
\hline 7148.191 & 5 & 5 & 13985.698 & $9711_{7 / 2}-23697_{7 / 2}^{\circ}$ & 6891.087 & 1 & 3 & 14507.496 & $28243_{5 / 2}^{\circ}-42751_{7 / 2}$ \\
\hline 7145.793 & 3 & 2 & 13990.391 & $6168_{7 / 2}^{\circ}-20158_{5 / 2}$ & 6889.297 & 300 & 200 & 14511.265 & $1521_{5 / 2}-16033_{5 / 2}^{\circ}$ \\
\hline 7140.454 & 10 & 15 & 14000.852 & $13248_{9 / 2}-27249_{7 / 2}^{\circ}$ & 6888.720 & 10 & 3 & 14512.481 & $25188_{3 / 2}^{\circ}-39700_{5 / 2}$ \\
\hline 7139.861 & 5 & 25 & 14002.015 & $17771_{11 / 2}^{\circ}-31773_{9 / 2}$ & 6877.881 & 1 & 10 & 14535.351 & $20989_{9 / 2}^{\circ}-35525_{11 / 2}$ \\
\hline 7132.509 & $4 \mathrm{~h}$ & & 14016.448 & $12472_{5 / 2}^{\circ}-26488_{5 / 2}$ & 6873.051 & 10 & 3 & 14545.566 & $0_{3 / 2}-14545_{5 / 2}^{\circ}$ \\
\hline 7124.286 & 0 & 5 & 14032.626 & $14790_{7 / 2}^{\circ}-28823_{5 / 2}$ & 6870.154 & 2 & 2 & 14551.699 & $8460_{3 / 2}-23012_{3 / 2}^{\circ}$ \\
\hline 7120.334 & 1 & 2 & 14040.414 & $20686_{5 / 2}^{\circ}-34726_{7 / 2}$ & 6869.274 & 2 & 5 & 14553.564 & $24309_{11 / 2}^{\circ}-38862_{11 / 2}$ \\
\hline 7119.977 & 0 & 5 & 14041.118 & $27357_{9 / 2}^{\circ}-41398_{9 / 2}$ & 6868.638 & 2 & 3 & 14554.911 & $14790_{7 / 2}^{\circ}-29345_{5 / 2}$ \\
\hline 7113.983 & 8 & 10 & 14052.949 & $8460_{3 / 2}-22513_{5 / 2}^{\circ}$ & 6854.496 & 10 & 15 & 14584.940 & $10855_{7 / 2}-25440_{5 / 2}^{\circ}$ \\
\hline 7109.371 & 0 & 5 & 14062.065 & $15453_{7 / 2}^{\circ}-29515_{9 / 2}$ & 6851.210 & 1 & 5 & 14591.935 & $22685_{7 / 2}^{\circ}-37277_{7 / 2}^{\circ}$ \\
\hline 7100.507 & 150 & 75 & 14079.619 & $8605_{5 / 2}-22685_{\tau / 2}^{\circ}$ & 6850.531 & 5 & 40 & 14593.382 & $17983_{5 / 2}^{\circ}-32576_{7 / 2}$ \\
\hline
\end{tabular}


TABLE 3. Classified lines of Th II-Continued

\begin{tabular}{|c|c|c|c|c|c|c|c|c|c|}
\hline \multirow{2}{*}{$\begin{array}{c}\text { Wavelength } \\
\AA\end{array}$} & \multicolumn{2}{|c|}{ Intensity } & \multirow{2}{*}{$\begin{array}{c}\text { W avenumber } \\
\mathrm{cm}^{-1}\end{array}$} & \multirow{2}{*}{ Classification } & \multirow{2}{*}{$\begin{array}{c}\text { Wavelength } \\
\AA\end{array}$} & \multicolumn{2}{|c|}{ Intensity } & \multirow{2}{*}{$\begin{array}{c}\text { Wavenumber } \\
\mathbf{c m}^{-1}\end{array}$} & \multirow{2}{*}{ Classification } \\
\hline & Lamp & Spark & & & & Lamp & Spark & & \\
\hline 6845.852 & 3 & 4 & 14603.356 & $10379_{9 / 2}-24982_{7 / 2}^{\circ}$ & 6628.169 & 1 & 3 & 15082.957 & $14790_{7 / 2}^{\circ}-29873_{7 / 2}$ \\
\hline 684.5 .132 & 1 & 2 & 14604.892 & $24463_{5 / 2}^{\circ}-39068_{7 / 2}$ & 6622.127 & 1 & 5 & 15096.719 & $20969_{7 / 2}^{\circ}-36065_{5 / 2}$ \\
\hline 6832.885 & 5 & 20 & 14631.069 & $15242_{9 / 2}^{\circ}-29873_{7 / 2}$ & 6619.940 & 200 & 200 & 15101.706 & $4146_{7 / 2}-19248_{5 / 2}^{\circ}$ \\
\hline 6832.196 & 2 & 3 & 14632.544 & $17121_{3 / 2}^{\circ}-31754_{5 / 2}$ & 6619.616 & 3 & 40 & 15102.445 & $22685_{7 / 2}^{\circ}-37787_{7 / 2}$ \\
\hline 6812.762 & 40 & 750 & 14674.285 & $10572_{9 / 2}^{\circ}-25246_{9 / 2}$ & 6617.054 & 25 & 50 & 15108.293 & $12485_{7 / 2}^{\circ}-27593_{5 / 2}$ \\
\hline 6810.539 & 40 & 100 & 14679.075 & $12570_{7 / 2}-27249_{7 / 2}^{\circ}$ & 6616.863 & 2 & 2 & 15108.729 & $12902_{3 / 2}^{\circ}-28011_{3 / 2}^{\circ}$ \\
\hline 6804.731 & 8 & 20 & 14691.603 & $12902_{3 / 2}^{\circ}-27593_{5 / 2}$ & 6615.482 & 1 & 10 & 15111.883 & $26770_{11 / 2}^{\circ}-41882_{13 / 2}$ \\
\hline 6796.776 & 10 & 10 & 14708.798 & $10673_{5 / 2}^{\circ}-25381_{3 / 2}$ & 6615.345 & 3 & 5 & 15112.196 & $20288_{11 / 2}^{\circ}-35400_{13 / 2}$ \\
\hline 6792.470 & 1 & 2 & 14718.123 & $24982_{7 / 2}^{\circ}-39700_{5 / 2}$ & 6611.194 & 3 & 15 & 15121.684 & $17837_{1 / 2}^{\circ}-32959_{3 / 2}$ \\
\hline 6789.687 & 1 & 3 & 14724.155 & $14790_{7 / 2}^{\circ}-29515_{9 / 2}$ & 6610.306 & 1 & 8 & 15123.716 & $19050_{3 / 2}^{\circ}-34174_{5 / 2}$ \\
\hline 6786.360 & 2 & 8 & 14731.374 & $18118_{3 / 2}-32850_{5 / 2}^{\circ}$ & 6605.406 & 200 & 150 & 15134.935 & $4113_{5 / 2}-19248_{5 / 2}^{\circ}$ \\
\hline 6784.629 & 1 . & 2 & 14735.132 & $24132_{3 / 2}^{\circ}-38867_{1 / 2}$ & 6604.106 & 2 & 20 & 15137.914 & $24757_{9 / 2}^{\circ}-39895_{9 / 2}$ \\
\hline 6784.159 & 1 & 2 & 14736.153 & $19594_{1 / 2}-34330_{1 / 2}^{\circ}$ & 6601.537 & 5 & 2 & 15143.805 & $9238_{9 / 2}^{\circ}-24381_{7 / 2}$ \\
\hline 6779.987 & 15 & 10 & 14745.221 & $12219_{3 / 2}-26965_{3 / 2}^{\circ}$ & 6601.124 & 5 & 3 & 15144.752 & $0_{3 / 2}-15144_{3 / 2}^{\circ}$ \\
\hline 6771.323 & 3 & 3 & 14764.088 & $22513_{5 / 2}^{\circ}-37277_{7 / 2}$ & 6594.891 & 2 & 4 & 15159.066 & $12472_{5 / 2}^{\circ}-27631_{3 / 2}$ \\
\hline 6770.101 & 150 & 100 & 14766.752 & $8605_{5 / 2}-23372_{3 / 2}^{\circ}$ & 6593.606 & 3 & 10 & 15162.020 & $9711_{7 / 2}-24873_{5 / 2}^{\circ}$ \\
\hline 6769.385 & 31 & 15 & 14768.314 & $21297_{5 / 2}^{\circ}-36065_{5 / 2}$ & 6592.555 & 0 & 8 & 15164.437 & $31259_{5 / 2}-46423_{3 / 2}^{\circ}$ \\
\hline 6769.126 & $4 \mathrm{~h}$ & 5 & 14768.879 & $12488_{9 / 2}^{\circ}-27257_{7 / 2}$ & 6591.790 & 0 & 8 & 15166.197 & $23697_{7 / 2}^{\circ}-38863_{5 / 2}$ \\
\hline 6768.164 & 1 & 3 & 14770.979 & $19248_{5 / 2}^{\circ}-34019_{3 / 2}$ & 6585.996 & 3 & 8 & 15179.539 & $9202_{7 / 2}^{\circ}-24381_{7 / 2}$ \\
\hline 6767.945 & 2 & 5 & 14771.456 & $12485_{\tau / 2}^{\circ}-27257_{\tau / 2}$ & 6584.276 & 8 & 3 & 15183.505 & $7828_{1 / 2}-23012_{3 / 2}^{\circ}$ \\
\hline 6766.341 & 8 & 10 & 14774.958 & $7331_{5 / 2}^{\circ}-22106_{5 / 2}$ & 6583.585 & 5 & 4 & 15185.098 & $28587_{5 / 2}^{\circ}-43772_{5 / 2}$ \\
\hline 6760.888 & 25 & 25 & 14786.875 & $12570_{7 / 2}-27357_{9 / 2}^{\circ}$ & 6583.310 & 8 & 100 & 15185.733 & $44807_{7 / 2}-59993_{9 / 2}^{\circ}$ \\
\hline 6757.310 & 0 & 5 & 14794.704 & $24757_{9 / 2}^{\circ}-39552_{9 / 2}$ & 6578.923 & 2 & 2 & 15195.859 & $25027_{1 / 2}^{\circ}-40222_{3 / 2}$ \\
\hline 6756.540 & $100 \mathrm{~b}$ & 75 & 14796.390 & $9585_{5 / 2}^{\circ}-24381_{7 / 2}$ & 6577.651 & 20 & 100 & 15198.797 & $15349_{11 / 2}^{\circ}-30548_{13 / 2}$ \\
\hline 6742.493 & 8 & 5 & 14827.216 & $4146_{7 / 2}-18973_{7 / 2}^{\circ}$ & 6576.800 & 0 & 5 & 15200.764 & $18973_{7 / 2}^{\circ}-34174_{5 / 2}$ \\
\hline 6737.643 & 50 & 8 & 14837.889 & $25440_{5 / 2}^{\circ}-40278_{3 / 2}$ & 6573.986 & 2 & 10 & 15207.271 & $15786_{5 / 2}-30994_{7 / 2}^{\circ}$ \\
\hline 6732.242 & 3 & 3 & 14849.793 & $17771_{11 / 2}^{\circ}-32620_{11 / 2}$ & 6573.482 & 2 & 10 & 15208.437 & $16564_{11 / 2}^{\circ}-31773_{9 / 2}$ \\
\hline 6725.376 & 3 & 1 & 14864.953 & $27357_{9 / 2}^{\circ}-42222_{7 / 2}$ & 6572.883 & 5 & 40 & 15209.823 & $15242_{9 / 2}^{\circ}-30452_{9 / 2}$ \\
\hline 6715.181 & 25 & 8 & 14887.521 & $6244_{1 / 2}-21131_{3 / 2}^{\circ}$ & 6570.097 & 1 & 2 & 15216.272 & $34279_{7 / 2}-49495_{9 / 2}^{\circ}$ \\
\hline 6704.048 & 50 & 25 & 14912.244 & $\begin{aligned} 8460_{3 / 2} & -23372_{3 / 2}^{\circ} \\
11576_{3 / 2}^{\circ} & -26488_{5 / 2}^{\circ}\end{aligned}$ & 6569.628 & 8 & 50 & 15217.358 & $12570_{7 / 2}-27787_{9 / 2}^{\circ}$ \\
\hline & & & & & 6569.057 & 5 & 2 & 15218.681 & $29431_{7 / 2}-44650_{7 / 2}^{\circ}$ \\
\hline 6703.883 & 10 & 8 & 14912.611 & $8605_{5 / 2}-23518_{\pi / 2}^{\circ}$ & 6566.192 & 0 & 8 & 15225.321 & $34270_{9 / 2}-49495_{9 / 2}^{\circ}$ \\
\hline 6703.348 & 5 & & 14913.801 & $22139_{9 / 2}^{\circ}-37053_{11 / 2}$ & 6565.909 & 3 & 8 & 15225.978 & $17983_{5 / 2}^{\circ}-33209_{7 / 2}$ \\
\hline 6700.487 & 2 & 5 & 14920.169 & $22642_{9 / 2}^{\circ}-37562_{11 / 2}$ & 6561.566 & 1 & 4 & 15236.055 & $18118_{3 / 2}-33354_{5 / 2}^{\circ}$ \\
\hline 6692.717 & 100 & 25 & 14937.491 & $4113_{5 / 2}-19050_{3 / 2}^{\circ}$ & 6561.462 & 0 & 5 & 15236.297 & $26963_{7 / 2}^{\circ}-42200_{9 / 2}$ \\
\hline 6664.956 & $4 h$ & 5 & 14999.708 & $15453_{7 / 2}^{\circ}-30452_{9 / 2}$ & 6561.244 & 0 & 8 & 15236.803 & $24463_{5 / 2}^{\circ}-39700_{5 / 2}$ \\
\hline 6662.624 & $10 \mathrm{~h}$ & 8 & 15004.958 & $15305_{9 / 2}-30310_{11 / 2}^{\circ}$ & 6560.055 & 15 & 200 & 15239.565 & $14275_{9 / 2}^{\circ}-29515_{9 / 2}$ \\
\hline 6659.496 & lh & 2 & 15012.006 & $22834_{7 / 2}-37846_{5 / 2}^{\circ}$ & 6559.088 & 2 & 50 & 15241.811 & $15242_{9 / 2}^{\circ}-30484_{11 / 2}$ \\
\hline 6651.164 & 2 & 3 & 15030.812 & $14484_{11 / 2}^{\circ}-29515_{9 / 2}$ & 6558.576 & 1 & 8 & 15243.001 & $24309_{11 / 2}^{\circ}-39552_{9 / 2}$ \\
\hline 6651.058 & 2 & 3 & 15031.051 & $19248_{5 / 2}^{\circ}-34279_{7 / 2}$ & 6550.542 & 8 & 5 & 15261.696 & $1859_{3 / 2}-17121_{3 / 2}^{\circ}$ \\
\hline 6651.000 & 1 & 2 & 15031.182 & $21297_{5 / 2}^{\circ}-36328_{3 / 2}$ & 6544.596 & 2 & 8 & 15275.562 & $17460_{5 / 2}^{\circ}-32736_{7 / 2}$ \\
\hline 6648.956 & 50 & 50 & 15035.803 & $10379_{9 / 2}-25414_{11 / 2}^{\circ}$ & 6540.141 & 4 & 1 & 15285.967 & $24414_{3 / 2}^{\circ}-39700_{5 / 2}$ \\
\hline 6648.496 & 50 & 50 & 15036.843 & $11725_{1 / 2}^{\circ}-26762_{3 / 2}$ & 6535.645 & 1 & 5 & 15296.483 & $18973_{7 / 2}^{\circ}-34270_{9 / 2}$ \\
\hline 6647.693 & 2 & 51 & 15038.660 & $12488_{9 / 2}^{\circ}-27526_{9 / 2}$ & 6532.223 & 5 & 20 & 15304.496 & $17272_{9 / 2}^{\circ}-32576_{7 / 2}$ \\
\hline 6646.542 & 40 & 50 & 15041.264 & $12485_{7 / 2}^{\circ}-27526_{9 / 2}$ & 6531.769 & 0 & 8 & 15305.560 & $18973_{7 / 2}^{\circ}-34279_{7 / 2}$ \\
\hline 6644.661 & 200 & 150 & 15045.522 & $9711_{7 / 2}-24757_{9 / 2}^{\circ}$ & 6529.947 & 0 & 40 & 15309.830 & $25414_{11 / 2}^{\circ}-40724_{13 / 2}$ \\
\hline 6643.374 & 1 & 8 & 15048.436 & $22014_{1 / 2 / 2}^{\circ}-37063_{9 / 2}$ & 6520.938 & 4 & 8 & 15330.981 & $23187_{13 / 2}^{\circ}-38517_{13 / 2}$ \\
\hline 6637.032 & 1 & 2 & 15062.816 & $9400_{5 / 2}-24463_{5 / 2}^{\circ}$ & 6518.963 & 1 & 5 & 15335.626 & $25188_{3 / 2}^{\circ}-40523_{1 / 2}$ \\
\hline 6633.453 & 10 & 20 & 15070.943 & $9061_{5 / 2}-24132_{3 / 2}^{\circ}$ & 6518.439 & 3 & 5 & 15336.859 & $13250_{5 / 2}-28587_{5 / 2}^{\circ}$ \\
\hline 6631.806 & 1 & 3 & 15074.686 & $36193_{9 / 2}^{\circ}-51268_{7 / 2}$ & 6511.356 & 5 & 15 & 15353.542 & $9061_{5 / 2}-24414_{3 / 2}^{\circ}$ \\
\hline
\end{tabular}


TABLE 3. Classified lines of Th II-Continued

\begin{tabular}{|c|c|c|c|c|c|c|c|c|c|}
\hline \multirow{2}{*}{$\begin{array}{c}\text { Wavelength } \\
\AA\end{array}$} & \multicolumn{2}{|c|}{ Intensity } & \multirow{2}{*}{$\begin{array}{c}\text { Wavenumber } \\
\mathbf{c m}^{-1}\end{array}$} & \multirow{2}{*}{ Classification } & \multirow{2}{*}{$\begin{array}{c}\text { Wavelength } \\
\AA\end{array}$} & \multicolumn{2}{|c|}{ Intensity } & \multirow{2}{*}{$\begin{array}{l}\text { Wavenumber } \\
\mathbf{c m}^{-1}\end{array}$} & \multirow{2}{*}{ Classification } \\
\hline & Lamp & Spark & & & & Lamp & Spark & & \\
\hline 6511.256 & 3 & 10 & 15353.778 & $7001_{3 / 2}-22355_{\mathrm{i} / 2}^{\circ}$ & 6381.966 & 1 & 101 & 15664.822 & $22014_{11 / 2}^{\circ}-37679_{11 / 2}$ \\
\hline 6510.997 & 5 & 15 & 15354.388 & $8018_{3 / 2}-23372_{3 / 2}^{\circ}$ & 6379.795 & 2 & 10 & 15670.153 & $16906_{7 / 2}^{\circ}-32576_{7 / 2}$ \\
\hline 6503.997 & 4 & 2 & 15370.914 & $14349_{1 / 2}-29720_{3 / 2}^{\circ}$ & 6379.170 & 5 & 10 & 15671.688 & $8460_{3 / 2}-24132_{3 / 2}^{\circ}$ \\
\hline 6503.898 & 5 & 2 & 15371.148 & $31800_{7 / 2}^{\circ}-47171_{9 / 2}$ & 6378.756 & 4 & 10 & 15672.705 & $13250_{5 / 2}-28923_{5 / 2}^{\circ}$ \\
\hline 6503.512 & 50 & 75 & 15372.060 & $11116_{7 / 2}^{\circ}-26488_{5 / 2}$ & 6378.494 & 1 & 3 & 15673.349 & $12570_{7 / 2}-28243_{5 / 2}^{\circ}$ \\
\hline 6501.819 & 0 & 8 & 15376.063 & $26770_{1 / 2}^{\circ}-42146_{11 / 2}$ & 6377.495 & 2 & 50 & 15675.804 & $23187_{13 / 2}^{\circ}-38862_{11 / 2}$ \\
\hline \multirow[t]{2}{*}{6493.531} & 3 & 3 & 15395.688 & $30956_{9 / 2}^{\circ}-46352_{7 / 2}$ & 6368.957 & 8 & 8 & 15696.818 & $13818_{7 / 2}^{\circ}-29515_{9 / 2}$ \\
\hline & & & & $31928_{3 / 2}^{\circ}-47324_{5 / 2}$ & 6363.271 & 8 & 5 & 15710.844 & $0_{3 / 2}-15710_{3 / 2}^{\circ}$ \\
\hline 6491.269 & 0 & 8 & 15401.053 & $37277_{7 / 2}-52678_{5 / 2}^{\circ}$ & 6359.132 & 15 & 100 & 15721.070 & $16033_{5 / 2}^{\circ}-31754_{5 / 2}$ \\
\hline 6490.583 & 5 & 10 & 15402.680 & $9061_{5 / 2}-24463_{5 / 2}^{\circ}$ & 6358.623 & 8 & 100 & 15722.328 & $19880_{9 / 2}-35602_{11 / 2}^{\circ}$ \\
\hline 6485.370 & 4 & 5 & 15415.061 & $6691_{3 / 2}^{\circ}-22106_{5 / 2}$ & 6356.218 & 2 & 8 & 15728.277 & $9711_{7 / 2}-25440_{5 / 2}^{\circ}$ \\
\hline 6481.226 & 0 & 5 & 15424.917 & $30310_{11 / 2}^{\circ}-45735_{11 / 2}$ & 6353.285 & 1 & 5 & 15735.538 & $15236_{3 / 2}-30972_{5 / 2}^{\circ}$ \\
\hline 6480.607 & 3 & 10 & 15426.390 & $15453_{7 / 2}^{\circ}-30879_{7 / 2}$ & 6348.559 & 2 & 50 & 15747.252 & $22642_{9 / 2}^{\circ}-38389_{7 / 2}$ \\
\hline 6479.558 & 0 & 5 & 15428.888 & $30956_{9 / 2}^{\circ}-46385_{7 / 2}$ & 6348.435 & 1 & 5 & 15747.559 & $17983_{5 / 2}^{\circ}-33730_{5 / 2}$ \\
\hline 6478.940 & 2 & 10 & 15430.360 & $20310_{5 / 2}^{\circ}-35741_{5 / 2}$ & 6347.103 & 8 & 40 & 15750.864 & $35156_{5 / 2}^{\circ}-50907_{3 / 2}$ \\
\hline 6471.345 & 0 & 8 & 15448.469 & $32850_{5 / 2}^{\circ}-48298_{7 / 2}$ & 6343.157 & 0 & 5 & 15760.663 & $23697_{7 / 2}^{\circ}-39458_{7 / 2}$ \\
\hline 6471.205 & 20 & 50 & 15448.803 & $12488_{9 / 2}^{\circ}-27937_{11 / 2}$ & 6339.442 & 0 & 40 & 15769.898 & $27357_{9 / 2}^{\circ}-43127_{11 / 2}$ \\
\hline 6470.289 & 1 & 4 & 15450.990 & $18568_{1 / 2}^{\circ}-34019_{3 / 2}$ & 6332.510 & 5 & 20 & 15787.161 & $35741_{5 / 2}-51528_{3 / 2}^{\circ}$ \\
\hline 6468.090 & 0 & 5 & 15456.243 & $25188_{3 / 2}^{\circ}-40644_{5 / 2}$ & & & & & $9400_{5 / 2}-25188_{3 / 2}^{\circ}$ \\
\hline 6467.558 & 2 & 1 & 15457.515 & $26762_{3 / 2}-42219_{5 / 2}^{\circ}$ & 6330.804 & 0 & 5 & 15791.415 & $25607_{9 / 2}^{\circ}-41398_{9 / 2}$ \\
\hline 6462.622 & $200 \mathrm{~b}$ & $200 \mathrm{~b}$ & 15469.321 & $29720_{3 / 2}^{\circ}-45189_{5 / 2}$ & 6328.754 & 4 & 3 & 15796.530 & $9585_{5 / 2}^{\circ}-25381_{3 / 2}$ \\
\hline 6455.482 & 1 & 5 & 15486.430 & $17722_{9 / 2}^{\circ}-33209_{7 / 2}$ & 6325.432 & 1 & 10 & 15804.826 & $18214_{3 / 2}^{\circ}-34019_{3 / 2}$ \\
\hline 6450.369 & 15 & 10 & 15498.706 & $26647_{13 / 2}^{\circ}-42146_{11 / 2}$ & 6324.598 & 0 & 8 & 15806.910 & $31924_{11 / 2}^{\circ}-47731_{9 / 2}$ \\
\hline 6448.726 & 3 & 8 & 15502.654 & $7331_{5 / 2}^{\circ}-22834_{7 / 2}$ & 6323.837 & 5 & 8 & 15808.813 & $8605_{5 / 2}-24414_{3 / 2}^{\circ}$ \\
\hline 6438.372 & 2 & 8 & 15527.585 & $13818_{7 / 2}^{\circ}-29345_{5 / 2}$ & 6322.204 & 2 & 4 & 15812.896 & $9061_{5 / 2}-24873_{5 / 2}^{\circ}$ \\
\hline 6432.950 & 1 & 4 & 15540.672 & $12485_{7 / 2}^{\circ}-28026_{5 / 2}$ & 6321.159 & 3 & 10 & 15815.510 & $10673_{5 / 2}^{\circ}-26488_{5 / 2}$ \\
\hline 6430.229 & 2 & 10 & 15547.248 & $22028_{15 / 2}^{\circ}-37575_{13 / 2}$ & 6315.533 & 0 & 8 & 15829.599 & $16906_{7 / 2}^{\circ}-32736_{7 / 2}$ \\
\hline 6428.524 & 1 & 2 & 15551.372 & $25440_{5 / 2}^{\circ}-40991_{3 / 2}$ & 6312.668 & 0 & 5 & 15836.783 & $20288_{11 / 2}^{\circ}-36125_{9 / 2}$ \\
\hline 6427.011 & 0 & 8 & 15555.033 & $26963_{7 / 2}^{\circ}-42518_{7 / 2}$ & 6311.278 & 1 & 5 & 15840.271 & $20969_{7 / 2}^{\circ}-36809_{7 / 2}$ \\
\hline 6424.809 & 5 & 200 & 15560.364 & $22014_{11 / 2}^{\circ}-37575_{13 / 2}$ & 6309.451 & 1 & 4 & 15844.858 & $18816_{13 / 2}^{\circ}-34661_{11 / 2}$ \\
\hline 6423.402 & 1 & 2 & 15563.772 & $28243_{5 / 2}^{\circ}-43807_{3 / 2}$ & 6309.414 & 2 & 4 & 15844.951 & $13250_{5 / 2}-29095_{5 / 2}^{\circ}$ \\
\hline 6421.673 & 1 & 3 & 15567.963 & $20310_{5 / 2}^{\circ}-35878_{7 / 2}$ & 6304.239 & 20 & 40 & 15857.957 & $8605_{5 / 2}-24463_{5 / 2}^{\circ}$ \\
\hline 6421.417 & 0 & 8 & 15568.583 & $24132_{3 / 2}^{\circ}-39700_{5 / 2}$ & 6304.087 & 0 & 5 & 15858.340 & $33637_{7 / 2}-49495_{9 / 2}^{\circ}$ \\
\hline 6416.097 & 25 & 75 & 15581.492 & $9400_{5 / 2}-24982_{7 / 2}^{\circ}$ & 6299.795 & 1 & 1 & 15869.144 & $36809_{7 / 2}-52678_{5 / 2}^{\circ}$ \\
\hline 6408.869 & 0 & 8 & 15599.065 & $30956_{9 / 2}^{\circ}-46555_{11 / 2}$ & 6289.483 & 5 & 20 & 15895.162 & $9711_{7 / 2}-25607_{9 / 2}^{\circ}$ \\
\hline 6408.193 & 2 & 4 & 15600.711 & $1859_{3 / 2}-17460_{5 / 2}^{\circ}$ & 6285.277 & 40 & 100 & 15905.799 & $11725_{1 / 2}^{\circ}-27631_{3 / 2}$ \\
\hline 6406.278 & 4 & 3 & 15605.374 & $37130_{1 / 2}^{\circ}-52735_{3 / 2}$ & 6279.164 & 100 & 200 & 15921.283 & $12902_{3 / 2}^{\circ}-28823_{5 / 2}$ \\
\hline 6405.870 & 0 & 8 & 15606.368 & $22685_{7 / 2}^{\circ}-38291_{7 / 2}$ & 6277.232 & 25 & 50 & 15926.183 & $6213_{9 / 2}-22139_{9 / 2}^{\circ}$ \\
\hline 6404.390 & 0 & 8 & 15609.974 & $24757_{9 / 2}^{\circ}-40367_{9 / 2}$ & 6274.115 & 300 & 200 & 15934.096 & $4146_{7 / 2}-20080_{7 / 2}^{\circ}$ \\
\hline 6402.932 & 3 & 8 & 15613.529 & $13818_{7 / 2}^{\circ}-29431_{7 / 2}$ & 6269.591 & 2 & 8 & 15945.593 & $20120_{5 / 2}^{\circ}-36065_{5 / 2}$ \\
\hline 6401.729 & 0 & 40 & 15616.463 & $25607_{9 / 2}^{\circ}-41223_{11 / 2}$ & 6269.172 & 0 & 5 & 15946.659 & $29788_{9 / 2}^{\circ}-45735_{11 / 2}$ \\
\hline 6399.797 & 0 & 10 & 15621.177 & $20120_{5 / 2}^{\circ}-35741_{5 / 2}$ & 6268.765 & 0 & 8 & 15947.694 & $24463_{5 / 2}^{\circ}-40411_{7 / 2}$ \\
\hline 6388.731 & 0 & 8 & 15648.235 & $22139_{9 / 2}^{\circ}-37787_{7 / 2}$ & 6266.172 & 75 & 50 & 15954.294 & $8460_{3 / 2}-24414_{3 / 2}^{\circ}$ \\
\hline 6388.136 & 2 & 8 & 15649.692 & $22642_{9 / 2}^{\circ}-38291_{7 / 2}$ & 6261.060 & 200 & 200 & 15967.320 & $4113_{5 / 2}-20080_{7 / 2}^{\circ}$ \\
\hline 6387.865 & 2 & 15 & 15650.356 & $22106_{5 / 2}-37756_{7 / 2}^{\circ}$ & 6258.600 & 20 & 50 & 15973.596 & $4146_{7 / 2}-20120_{5 / 2}^{\circ}$ \\
\hline 6387.473 & 0 & 8 & 15651.317 & $15305_{9 / 2}-30956_{9 / 2}^{\circ}$ & 6256.923 & 2 & 4 & 15977.877 & $1859_{3 / 2}-17837_{1 / 2}^{\circ}$ \\
\hline 6386.386 & 0 & 40 & 15653.981 & $24757_{9 / 2}^{\circ}-40411_{7 / 2}$ & 6256.112 & 3 & 2 & 15979.948 & $21297_{5 / 2}^{\circ}-37277_{7 / 2}$ \\
\hline 6384.094 & 5 & 5 & 15659.601 & $22834_{7 / 2}-38493_{5 / 2}^{\circ}$ & 6248.115 & 2 & 10 & 16000.401 & $14484_{11 / 2}^{\circ}-30484_{11 / 2}$ \\
\hline 6383.342 & 5 & 40 & 15661.445 & $17722_{9 / 2}^{\circ}-33384_{9 / 2}$ & 6247.117 & 3 & 5 & 16002.957 & $8378_{7 / 2}^{\circ}-24381_{7 / 2}$ \\
\hline 6383.222 & 5 & 40 & 15661.740 & $14790_{7 / 2}^{\circ}-30452_{9 / 2}^{\circ}$ & 6246.924 & 4 & 5 & 16003.451 & $8460_{3 / 2}-24463_{5 / 2}^{\circ}$ \\
\hline
\end{tabular}


TABLE 3. Classified lines of Th II-Continued

\begin{tabular}{|c|c|c|c|c|c|c|c|c|c|}
\hline \multirow{2}{*}{$\begin{array}{c}\text { Wavelength } \\
\AA\end{array}$} & \multicolumn{2}{|c|}{ Intensity } & \multirow{2}{*}{$\begin{array}{c}\text { Wavenumber } \\
\mathrm{cm}^{-1}\end{array}$} & \multirow{2}{*}{ Classification } & \multirow{2}{*}{$\begin{array}{c}\text { Wavelength } \\
\AA \\
\end{array}$} & \multicolumn{2}{|c|}{ Intensity } & \multirow{2}{*}{$\begin{array}{c}\text { Wavenumber } \\
\mathbf{c m}^{-1}\end{array}$} & \multirow{2}{*}{ Classification } \\
\hline & Lamp & Spark & & & & Lamp & Spark & & \\
\hline 6245.601 & 50 & 15 & 16006.841 & $4113_{5 / 2}-20120_{5 / 2}^{\circ}$ & 6163.249 & 1 & 10 & 16220.719 & $22642_{9 / 2}^{\circ}-38862_{11 / 2}$ \\
\hline 6245.034 & 5 & 15 & 16008.295 & $9238_{9 / 2}^{\circ}-25246_{9 / 2}$ & 6159.840 & 0 & 10 & 16229.696 & $24414_{3 / 2}^{\circ}-40644_{5 / 2}$ \\
\hline 6244.117 & 1 & 1 & 16010.646 & $7001_{3 / 2}-23012_{3 / 2}^{\circ}$ & 6154.119 & 0 & $8 b$ & 16244.784 & $21297_{5 / 2}^{\circ}-37542_{3 / 2}$ \\
\hline 6243.103 & 0 & 5 & 16013.246 & $15786_{5 / 2}-31800_{7 / 2}^{\circ}$ & 6147.454 & 3 & 2 & 16262.396 & $21682_{7 / 2}^{\circ}-37945_{5 / 2}$ \\
\hline 6241.694 & 2 & 4 & 16016.861 & $12570_{7 / 2}-28587_{5 / 2}^{\circ}$ & 6146.272 & 1 & 3 & 16265.523 & $24309_{11 / 2}^{\circ}-40574_{11 / 2}$ \\
\hline 6241.408 & 2 & 8 & 16017.595 & $11576_{3 / 2}^{\circ}-27593_{5 / 2}$ & 6145.287 & 20 & 50 & 16268.131 & $8605_{5 / 2}-24873_{5 / 2}^{\circ}$ \\
\hline 6239.543 & 2 & 2 & 16022.382 & $33354_{5 / 2}^{\circ}-49377_{7 / 2}$ & 6138.650 & 3 & 40 & 16285.719 & $11725_{1 / 2}^{\circ}-28011_{3 / 2}$ \\
\hline 6239.083 & 3 & 15 & 16023.564 & $15786_{5 / 2}-31810_{5 / 2}^{\circ}$ & 6138.526 & 0 & 8 & 16286.048 & $20158_{5 / 2}-36444_{3 / 2}^{\circ}$ \\
\hline 6238.970 & 1 & 5 & 16023.854 & $12219_{3 / 2}-28243_{5 / 2}^{\circ}$ & 6134.794 & 2 & 5 & 16295.955 & $17983_{5 / 2}^{\circ}-34279_{7 / 2}$ \\
\hline 6238.244 & 0 & 5 & 16025.719 & $22139_{9 / 2}^{\circ}-38165_{7 / 2}$ & 6132.228 & 2 & 5 & 16302.774 & $16906_{7 / 2}^{\circ}-33209_{7 / 2}$ \\
\hline 6235.793 & 1 & 8 & 16032.018 & $16818_{\tau / 2}-32850_{5 / 2}^{\circ}$ & 6131.967 & 3 & 1 & 16303.468 & $7828_{1 / 2}-24132_{3 / 2}^{\circ}$ \\
\hline 6235.346 & 2 & 2 & 16033.167 & $0_{3 / 2}-16033_{5 / 2}^{\circ}$ & 6125.749 & 25 & 150 & 16320.017 & $15453_{7 / 2}^{\circ}-31773_{9 / 2}$ \\
\hline 6234.295 & 1 & 10 & 16035.870 & $17983_{5 / 2}^{\circ}-34019_{3 / 2}$ & 6124.675 & 1 & 3 & 16322.879 & $22513_{5 / 2}^{\circ}-38836_{3 / 2}$ \\
\hline 6232.970 & 25 & 50 & 16039.279 & $9400_{5 / 2}-25440_{5 / 2}^{\circ}$ & 6122.337 & 0 & 15 & 16329.112 & $18214_{3 / 2}^{\circ}-34543_{5 / 2}$ \\
\hline 6231.380 & 4 & 40 & 16043.371 & $15710_{3 / 2}^{\circ}-31754_{5 / 2}$ & 6120.563 & 250 & 300 & 16333.845 & $14545_{5 / 2}^{\circ}-30879_{7 / 2}$ \\
\hline 6231.116 & 4 & 10 & 16044.051 & $9202_{7 / 2}^{\circ}-25246_{9 / 2}$ & 6113.969 & 3 & 10 & 16351.461 & $12472_{5 / 2}^{\circ}-28823_{5 / 2}$ \\
\hline 6229.597 & 5 & 3 & 16047.963 & $25440_{5 / 2}^{\circ}-41488_{7 / 2}$ & 6112.838 & 150 & 200 & 16354.486 & $1859_{3 / 2}-18214_{3 / 2}^{\circ}$ \\
\hline 6226.927 & 2 & 4 & 16054.844 & $11576_{3 / 2}^{\circ}-27631_{3 / 2}$ & 6108.922 & 3 & 2 & 16364.970 & $17272_{9 / 2}^{\circ}-33637_{7 / 2}$ \\
\hline 6226.619 & 0 & 5 & 16055.638 & $13818_{7 / 2}^{\circ}-29873_{7 / 2}$ & 6108.411 & 10 & 20 & 16366.339 & $26647_{13 / 2}^{\circ}-43014_{13 / 2}$ \\
\hline 6223.516 & 2 & 25 & 16063.643 & $20989_{9 / 2}^{\circ}-37053_{11 / 2}$ & 6106.613 & 5 & 4 & 16371.158 & $7001_{3 / 2}-23372_{3 / 2}^{\circ}$ \\
\hline 6221.575 & 2 & 50 & 16068.655 & $30310_{11 / 2}^{\circ}-46378_{13 / 2}$ & 6104.572 & 300 & 200 & 16376.631 & $8605_{5 / 2}-24982_{7 / 2}^{\circ}$ \\
\hline 6216.441 & 4 & 1 & 16081.925 & $25594_{i / 2}^{\circ}-41676_{3 / 2}$ & 6103.640 & 15 & 50 & 16379.132 & $9061_{5 / 2}-25440_{5 / 2}^{\circ}$ \\
\hline 6213.650 & 2 & 5 & 16089.149 & $10673_{5 / 2}^{\circ}-26762_{3 / 2}$ & 6100.578 & 1 & 1 & 16387.353 & $22106_{5 / 2}-38493_{5 / 2}^{\circ}$ \\
\hline 6211.663 & 3 & 75 & 16094.295 & $20969_{7 / 2}^{\circ}-37063_{9 / 2}$ & 6099.804 & 3 & 5 & 16389.432 & $27787_{9 / 2}^{\circ}-44177_{11 / 2}$ \\
\hline 6211.607 & 0 & 5 & 16094.441 & $26424_{5 / 2}^{\circ}-42518_{7 / 2}$ & 6099.083 & 100 & 200 & 16391.370 & $10379_{9 / 2}-26770_{11 / 2}^{\circ}$ \\
\hline 6207.477 & 2 & 2 & 16105.149 & $21682_{7 / 2}^{\circ}-37787_{7 / 2}$ & 6097.194 & 10 & 10 & 16396.448 & $8018_{3 / 2}-24414_{3 / 2}^{\circ}$ \\
\hline 6206.820 & 0 & 5 & 16106.853 & $24463_{5 / 2}^{\circ}-40570_{7 / 2}$ & 6094.023 & 4 & 4 & 16404.980 & $34330_{1 / 2}^{\circ}-50735_{3 / 2}$ \\
\hline 6205.254 & 3 & 5 & 16110.918 & $6244_{1 / 2}-22355_{1 / 2}^{\circ}$ & & & & & $13468_{9 / 2}^{\circ}-29873_{7 / 2}$ \\
\hline 6204.767 & 1 & 8 & 16112.183 & $17272_{9 / 2}^{\circ}-33384_{9 / 2}$ & 6092.033 & 8 & 8 & 16410.338 & $21131_{3 / 2}^{\circ}-37542_{3 / 2}^{\circ}$ \\
\hline 6204.123 & 4 & 8 & 16113.855 & $8018_{3 / 2}-24132_{3 / 2}^{\circ}$ & & & & & $\begin{array}{l}25266_{1 / 2}^{\circ}-41676_{3 / 2} \\
11116_{7 / 2}^{\circ}-27526_{9 / 2}^{\circ}\end{array}$ \\
\hline 6200.551 & 0 & 10 & 16123.138 & $20686_{5 / 2}^{\circ}-36809_{\tau / 2}$ & & & & & \\
\hline 6200.427 & 40 & 40 & 16123.460 & $1859_{3 / 2}-17983_{5 / 2}^{\circ}$ & 6090.816 & 8 & 8 & 16413.617 & $8460_{3 / 2}-24873_{5 / 2}^{\circ}$ \\
\hline 6199.054 & 2 & 5 & 16127.031 & $9061_{5 / 2}-25188_{3 / 2}^{\circ}$ & 6090.109 & 15 & 25 & 16415.523 & $24309_{11 / 2}^{\circ}-40724_{13 / 2}$ \\
\hline 6196.396 & 5 & 10 & 16133.949 & $6700_{9 / 2}^{\circ}-22834_{7 / 2}$ & 6087.576 & 0 & 8 & 16422.353 & $21682_{7 / 2}^{\circ}-38105_{5 / 2}$ \\
\hline 6194.315 & 0 & 5 & 16139.369 & $16818_{7 / 2}-32957_{\tau / 2}^{\circ}$ & 6087.387 & 3 & 3 & 16422.863 & $20158_{5 / 2}-36581_{3 / 2}^{\circ}$ \\
\hline 6193.978 & 0 & 5 & 16140.247 & $25188_{3 / 2}^{\circ}-41328_{5 / 2}$ & 6087.262 & 75 & 300 & 16423.200 & $15349_{11 / 2}^{\circ}-31773_{9 / 2}$ \\
\hline 6193.858 & 50 & 200 & 16140.560 & $11116_{7 / 2}^{\circ}-27257_{7 / 2}$ & 6085.998 & 4 & 5 & 16426.611 & $22642_{9 / 2}^{\circ}-39068_{7 / 2}$ \\
\hline 6189.417 & 0 & 50 & 16152.141 & $22139_{9 / 2}^{\circ}-38291_{\tau / 2}$ & 6085.430 & 2 & 40 & 16428.144 & $29788_{9 / 2}^{\circ}-46216_{11 / 2}$ \\
\hline 6188.051 & $20 \mathrm{~b}$ & 150 & 16155.707 & $30223_{i 5 / 2}^{\circ}-46378_{13 / 2}$ & 6085.257 & 40 & 75 & 16428.611 & $6213_{9 / 2}-22642_{9 / 2}^{\circ}$ \\
\hline 6184.737 & $10 \mathrm{~b}$ & 8 & 16164.363 & $4146_{7 / 2}-20310_{5 / 2}^{\circ}$ & 6078.968 & 40 & 8 & 16445.607 & $8018_{3 / 2}-24463_{5 / 2}^{\circ}$ \\
\hline 6184.469 & 2 & 15 & 16165.064 & $22014_{11 / 2}^{\circ}-38179_{9 / 2}$ & 6078.588 & 5 & 8 & 16446.636 & $27357_{9 / 2}^{\circ}-43803_{7 / 2}$ \\
\hline 6184.390 & 8 & 10 & 16165.270 & $23187_{13 / 2}^{\circ}-39352_{11 / 2}$ & 6077.358 & 15 & 10 & 16449.964 & $11576_{3 / 2}^{\circ}-28026_{5 / 2}$ \\
\hline 6179.847 & 8 & 50 & 16177.154 & $14275_{9 / 2}^{\circ}-30452_{9 / 2}$ & 6076.639 & 3 & 3 & 16451.911 & $27357_{\mathbf{9} / 2}^{\circ}-43809_{9 / 2}$ \\
\hline 6178.885 & 3 & 2 & 16179.672 & $27593_{5 / 2}-43773_{\tau / 2}^{\circ}$ & 6076.195 & 8 & 5 & 16453.113 & $18568_{1 / 2}^{\circ}-35021_{3 / 2}$ \\
\hline 6178.549 & 0 & 8 & 16180.552 & $24463_{5 / 2}^{\circ}-40644_{5 / 2}$ & 6073.710 & 3 & 3 & 16459.844 & $24463_{5 / 2}^{\circ}-40923_{5 / 2}$ \\
\hline 6172.044 & 1 & 2 & 16197.606 & $4113_{5 / 2}-20310_{5 / 2}^{\circ}$ & 6073.100 & 100 & 150 & 16461.498 & $1521_{5 / 2}-17983_{5 / 2}^{\circ}$ \\
\hline 6168.580 & 2 & 2 & 16206.701 & $31800_{\pi / 2}^{\circ}-48006_{9 / 2}$ & 6071.415 & 2 & 2 & 16466.066 & $24757_{9 / 2}^{\circ}-41223_{11 / 2}$ \\
\hline \multirow[t]{2}{*}{6167.907} & 0 & 5 & 16208.470 & $20120_{5 / 2}^{\circ}-36328_{3 / 2}$ & 6070.892 & 3 & 4 & 16467.485 & $25414_{11 / 2}^{\circ}-41882_{13 / 2}$ \\
\hline & & & & $23730_{9 / 2}^{\circ}-39939_{11 / 2}$ & 6070.034 & 4 & 10 & 16469.812 & $13250_{5 / 2}-29720_{3 / 2}^{\circ}$ \\
\hline 6167.059 & 0 & 5 & 16210.698 & $29095_{5 / 2}^{\circ}-45306_{3 / 2}$ & 6068.043 & 2 & 2 & 16475.216 & $22106_{5 / 2}-38581_{5 / 2}^{\circ}$ \\
\hline
\end{tabular}


TABLE 3. Classified lines of Th II-Continued

\begin{tabular}{|c|c|c|c|c|c|c|c|c|c|}
\hline \multirow{2}{*}{$\begin{array}{c}\text { Wavelength } \\
\AA\end{array}$} & \multicolumn{2}{|c|}{ Intensity } & \multirow{2}{*}{$\begin{array}{c}\text { Wavenumber } \\
\mathbf{c m}^{-1}\end{array}$} & \multirow{2}{*}{ Classification } & \multirow{2}{*}{$\begin{array}{c}\text { Wavelength } \\
\AA\end{array}$} & \multicolumn{2}{|c|}{ Intensity } & \multirow{2}{*}{$\begin{array}{c}\text { Wavenumber } \\
\mathbf{c m}^{-1}\end{array}$} & \multirow{2}{*}{ Classification } \\
\hline & Lamp & Spark & & & & Lamp & Spark & & \\
\hline 6067.077 & 20 & 100 & 16477.839 & $16906_{7 / 2}^{\circ}-33384_{9 / 2}$ & 6015.424 & 50 & 200 & 16619.329 & $15305_{9 / 2}-31924_{11 / 2}^{\circ}$ \\
\hline 6066.496 & 40 & 200 & 16479.417 & $26647_{13 / 2}^{\circ}-43127_{11 / 2}$ & 6011.349 & 25 & 20 & 16630.595 & $19248_{5 / 2}^{\circ}-35878_{7 / 2}$ \\
\hline 6066.330 & $4 \mathbf{h}$ & 3 & 16479.868 & $30101_{7 / 2}^{\circ}-46581_{5 / 2}$ & 6009.113 & 5 & 8 & 16636.783 & $23730_{9 / 2}^{\circ}-40367_{9 / 2}$ \\
\hline 6063.355 & 2 & 2 & 16487.954 & $31810_{5 / 2}^{\circ}-48298_{7 / 2}$ & 6007.591 & 4 & 8 & 16640.998 & $24757_{9 / 2}^{\circ}-41398_{9 / 2}$ \\
\hline 6063.068 & 8 & 15 & 16488.735 & $25188_{3 / 2}^{\circ}-41676_{3 / 2}$ & 5998.655 & 3 & 8 & 16665.787 & $6168_{7 / 2}^{\circ}-22834_{7 / 2}$ \\
\hline 6062.614 & 15 & 40 & 16489.969 & $22028_{15 / 2}^{\circ}-38517_{13 / 2}$ & 5993.268 & 3 & 8 & 16680.767 & $37679_{11 / 2}-54360_{9 / 2}^{\circ}$ \\
\hline 6062.431 & 3 & 2 & 16490.467 & $21297_{5 / 2}^{\circ}-37787_{7 / 2}$ & 5991.711 & 2 & 2 & 16685.102 & $10572_{9 / 2}^{\circ}-27257_{7 / 2}$ \\
\hline 6061.486 & 3 & 25 & 16493.038 & $19248_{5 / 2}^{\circ}-35741_{5 / 2}$ & 5990.792 & 0 & 20 & 16687.661 & $30223_{15 / 2}^{\circ}-46910_{13 / 2}$ \\
\hline 6060.230 & 3 & 2 & 16496.456 & $20969_{7 / 2}^{\circ}-37465_{5 / 2}$ & & & & & $31924_{11 / 2}^{\circ}-48612_{13 / 2}$ \\
\hline 6059.975 & 4 & & 16497.150 & $21682_{7 / 2}^{\circ}-38179_{9 / 2}$ & 5990.267 & 3 & 40 & 16689.124 & $20120_{5 / 2}^{\circ}-36809_{7 / 2}$ \\
\hline 6059.535 & 2 & 3 & 16498.348 & $20310_{5 / 2}^{\circ}-36809_{7 / 2}$ & 5989.987 & 1 & 4 & 16689.904 & $20989_{9 / 2}^{\circ}-37679_{11 / 2}$ \\
\hline 6058.612 & 15 & 20 & 16500.862 & $12219_{3 / 2}-28720_{3 / 2}^{\circ}$ & 5989.893 & 1 & 8 & 16690.166 & $21131_{3 / 2}^{\circ}-37821_{3 / 2}$ \\
\hline 6058.273 & 3 & 2 & 16501.785 & $30101_{7 / 2}^{\circ}-46603_{5 / 2}$ & 5989.200 & 0 & 5 & 16692.097 & $15236_{3 / 2}-31928_{3 / 2}^{\circ}$ \\
\hline 6058.180 & 15 & 20 & 16502.038 & $10855_{7 / 2}-27357_{9 / 2}^{\circ}$ & 5989.045 & $400 \mathrm{~s}$ & 500 & 16692.529 & $1521_{5 / 2}-18214_{3 / 2}^{\circ}$ \\
\hline \multirow[t]{2}{*}{6055.160} & 3 & 3 & 16510.269 & $31810_{5 / 2}^{\circ}-48320_{5 / 2}$ & 5985.204 & 2 & 8 & 16703.242 & $12219_{3 / 2}-28923_{5 / 2}^{\circ}$ \\
\hline & & & & $26586_{3 / 2}^{\circ}-43096_{5 / 2}$ & 5983.991 & 4 & 75 & 16706.627 & $21682_{7 / 2}^{\circ}-38389_{7 / 2}$ \\
\hline 6054.535 & 2 & 4 & 16511.973 & $22355_{1 / 2}^{\circ}-38867_{1 / 2}$ & 5983.378 & 5 & 15 & 16708.339 & $1859_{3 / 2}-18568_{1 / 2}^{\circ}$ \\
\hline 6052.173 & 2 & 1 & 16518.417 & $23697_{7 / 2}^{\circ}-40216_{5 / 2}$ & 5981.880 & 15 & 20 & 16712.523 & $9711_{7 / 2}-26424_{5 / 2}^{\circ}$ \\
\hline 6049.925 & 8 & 10 & 16524.555 & $21297_{5 / 2}^{\circ}-37821_{3 / 2}$ & 5981.385 & 1 & 5 & 16713.906 & $17460_{5 / 2}^{\circ}-34174_{5 / 2}$ \\
\hline 6049.771 & 15 & 25 & 16524.975 & $12570_{7 / 2}-29095_{5 / 2}^{\circ}$ & 5978.069 & 5 & 15 & 16723.177 & $22139_{9 / 2}^{\circ}-38862_{11 / 2}^{\circ}$ \\
\hline 6048.174 & 3 & 1 & 16529.339 & $39861_{5 / 2}^{\circ}-56391_{5 / 2}$ & 5976.437 & 3 & 3 & 16727.744 & $8460_{3 / 2}-25188_{3 / 2}^{\circ}$ \\
\hline 6045.467 & 2 & 2 & 16536.740 & $16818_{7 / 2}-33354_{5 / 2}^{\circ}$ & 5976.118 & 1 & 4 & 16728.637 & $20080_{7 / 2}^{\circ}-36809_{7 / 2}$ \\
\hline 6044.430 & 100 & 200 & 16539.577 & $4146_{7 / 2}-20686_{5 / 2}^{\circ}$ & 5975.404 & 5 & 50 & 16730.636 & $16906_{7 / 2}^{\circ}-33637_{7 / 2}$ \\
\hline 6042.962 & 25 & 50 & 16543.595 & $16033_{5 / 2}^{\circ}-32576_{7 / 2}$ & & & & & $24757_{9 / 2}^{\circ}-41488_{7 / 2}$ \\
\hline 6041.628 & $5 \mathrm{~h}$ & 3 & 16547.248 & $17722_{9 / 2}^{\circ}-34270_{9 / 2}$ & 5972.789 & 41 & 75 & 16737.960 & $24309_{11 / 2}^{\circ}-41047_{9 / 2}$ \\
\hline 6040.120 & 3 & 10 & 16551.379 & $30310_{11 / 2}^{\circ}-46861_{11 / 2}$ & 5972.436 & 15 & 15 & 16738.950 & $27357_{9 / 2}^{\circ}-44096_{9 / 2}^{\circ}$ \\
\hline 6039.665 & 2 & 1 & 16552.626 & $37945_{5 / 2}-54497_{7 / 2}^{\circ}$ & 5961.970 & 25 & 50 & 16768.334 & $9720_{7 / 2}^{\circ}-26488_{5 / 2}$ \\
\hline 6038.308 & 3 & 2 & 16556.346 & $17722_{9 / 2}^{\circ}-34279_{7 / 2}$ & 5960.372 & $4 d$ & 40 & 16772.830 & $22685_{7 / 2}^{\circ}-39458_{7 / 2}$ \\
\hline \multirow[t]{2}{*}{6036.088} & 2 & 3 & 16562.435 & $27403_{3 / 2}^{\circ}-43965_{1 / 2}$ & 5958.072 & 3 & 20 & 16779.304 & $20686_{5 / 2}^{\circ}-37465_{5 / 2}$ \\
\hline & & & & $22834_{7 / 2}-39396_{7 / 2}^{\circ}$ & 5957.736 & 5 & 2 & 16780.251 & $42518_{7 / 2}-59299_{5 / 2}^{\circ}$ \\
\hline 6034.545 & 10 & 10 & 16566.670 & $8460_{3 / 2}-25027_{\mathrm{i} / 2}^{\circ}$ & 5957.091 & 2 & 15 & 16782.067 & $25440_{5 / 2}^{\circ}-42222_{7 / 2}$ \\
\hline 6034.139 & 1 & 4 & 16567.785 & $35602_{11 / 2}^{\circ}-52170_{11 / 2}$ & 5956.813 & 2 & 5 & 16782.851 & $17771_{11 / 2}^{\circ}-34553_{9 / 2}$ \\
\hline 6032.665 & 0 & 8 & 16571.833 & $18973_{7 / 2}^{\circ}-35545_{9 / 2}$ & 5955.952 & 2 & 10 & 16785.277 & $25414_{11 / 2}^{\circ}-42200_{9 / 2}$ \\
\hline 6032.432 & 0 & 5 & 16572.473 & $20989_{9 / 2}^{\circ}-37562_{11 / 2}$ & 5952.350 & 5 & 8 & 16795.434 & $22355_{1 / 2}^{o}-39150_{3 / 2}$ \\
\hline 6032.320 & $25 b$ & 15 & 16572.780 & $4113_{5 / 2}-20686_{5 / 2}^{\circ}$ & 5950.888 & 2 & 2 & 16799.560 & $35878_{7 / 2}-52678_{5 / 2}^{\circ}$ \\
\hline 6031.911 & 10 & 25 & 16573.904 & $15236_{3 / 2}-31810_{5 / 2}^{\circ}$ & 5948.557 & 3 & 3 & 16806.143 & $8460_{3 / 2}-25266_{1 / 2}^{\circ}$ \\
\hline 6030.808 & 1 & 2 & 16576.935 & $24414_{3 / 2}^{\circ}-40991_{3 / 2}$ & 5946.922 & 1 & 1 & 16810.764 & $28011_{3 / 2}-44821_{5 / 2}^{\circ}$ \\
\hline 6028.863 & 10 & 5 & 16582.283 & $8605_{5 / 2}-25188_{3 / 2}^{\circ}$ & 5945.616 & 1 & 5 & 16814.456 & $24873_{5 / 2}^{\circ}-41688_{7 / 2}$ \\
\hline 6028.273 & 10 & 3 & 16583.906 & $18816_{13 / 2}^{\circ}-35400_{13 / 2}$ & 5945.012 & 1 & 2 & 16816.165 & $22642_{9 / 2}^{\circ}-39458_{7 / 2}^{\circ}$ \\
\hline 6028.240 & 2 & 10 & 16583.997 & $10673_{5 / 2}^{\circ}-27257_{7 / 2}$ & 5944.112 & 1 & 5 & 16818.711 & $17460_{5 / 2}^{\circ}-34279_{7 / 2}$ \\
\hline 6027.952 & 2 & 2 & 16584.789 & $10379_{9 / 2}-26963_{7 / 2}^{\circ}$ & 5944.054 & 2 & 8 & 16818.875 & $20969_{7 / 2}^{\circ}-37787_{7 / 2}$ \\
\hline 6027.481 & $5 s$ & 2 & 16586.085 & $7828_{1 / 2}-24414_{3 / 2}^{\circ}$ & 5942.792 & 40 & 50 & 16822.447 & $4146_{7 / 2}-20969_{7 / 2}^{\circ}$ \\
\hline 6025.616 & 4 & 5 & 16591.219 & $20686_{5 / 2}^{\circ}-37277_{7 / 2}$ & 5942.120 & 3 & 40 & 16824.349 & $16906_{7 / 2}^{\circ}-33730_{5 / 2}$ \\
\hline 6024.934 & 8 & 10 & 16593.097 & $25607_{9 / 2}^{\circ}-42200_{9 / 2}$ & 5938.571 & 20 & 40 & 16834.403 & $8605_{5 / 2}-25440_{5 / 2}^{\circ}$ \\
\hline 6022.223 & 8 & 10 & 16600.566 & $30310_{11 / 2}^{\circ}-46910_{13 / 2}$ & 5936.602 & 1 & 15 & 16839.987 & $23730_{9 / 2}^{\circ}-40570_{7 / 2}$ \\
\hline 6022.149 & 4 & 4 & 16600.771 & $22139_{9 / 2}^{\circ}-38740_{11 / 2}$ & 5934.714 & 1 & 25 & 16845.344 & $26963_{7 / 2}^{\circ}-43809_{9 / 2}$ \\
\hline 6021.409 & 40 & 200 & 16602.811 & $22014_{11 / 2}^{\circ}-38617_{9 / 2}$ & 5932.701 & 0 & 5 & 16851.060 & $20989_{9 / 2}^{\circ}-37840_{9 / 2}$ \\
\hline 6019.215 & 1 & 2 & 16608.862 & $22834_{7 / 2}-39443_{9 / 2}^{\circ}$ & 5932.128 & 4 & 10 & 16852.687 & $13248_{9 / 2}-30101_{7 / 2}^{\circ}$ \\
\hline 6018.998 & 20 & 100 & 16609.461 & $15144_{3 / 2}^{\circ}-31754_{5 / 2}$ & 5930.957 & 2 & 10 & 16856.015 & $20686_{5 / 2}^{\circ}-37542_{3 / 2}^{\circ}$ \\
\hline 6015.769 & 1 & 1 & 16618.376 & $26626_{i / 2}^{\circ}-43244_{3 / 2}$ & 5929.479 & 8 & 40 & 16860.216 & $12485_{7 / 2}^{\circ}-29345_{5 / 2}$ \\
\hline
\end{tabular}


TABLE 3. Classified lines of Th II-Continued

\begin{tabular}{|c|c|c|c|c|c|c|c|c|c|}
\hline \multirow{2}{*}{$\begin{array}{c}\text { Wavelength } \\
\AA\end{array}$} & \multicolumn{2}{|c|}{ Intensity } & \multirow{2}{*}{$\begin{array}{c}\text { W avenumber } \\
\mathrm{cm}^{-1}\end{array}$} & \multirow{2}{*}{ Classification } & \multirow{2}{*}{$\begin{array}{c}\text { Wavelength } \\
\AA\end{array}$} & \multicolumn{2}{|c|}{ Intensity } & \multirow{2}{*}{$\begin{array}{c}\text { Wavenumber } \\
\mathrm{cm}^{-1}\end{array}$} & \multirow{2}{*}{ Classification } \\
\hline & Lamp & Spark & & & & Lamp & Spark & & \\
\hline 5929.141 & 1 & 8 & 16861.178 & $30310_{11 / 2}^{\circ}-47171_{9 / 2}$ & 5849.999 & 1 & 4 & 17089.283 & $24309_{11 / 2}^{\circ}-41398_{9 / 2}$ \\
\hline 5927.949 & 1 & 15 & 16864.568 & $24463_{5 / 2}^{\circ}-41328_{5 / 2}$ & 5849.077 & 2 & 10 & 17091.976 & $21297_{5 / 2}^{\circ}-38389_{7 / 2}$ \\
\hline 5926.933 & 3 & 8 & 16867.459 & $8378_{7 / 2}^{\circ}-25246_{9 / 2}$ & 5838.945 & 75 & 20 & 17121.635 & $0_{3 / 2}-17121_{3 / 2}^{\circ}$ \\
\hline 5926.780 & 3 & 10 & 16867.894 & $18118_{3 / 2}-34986_{3 / 2}^{\circ}$ & 5837.485 & 0 & 5 & 17125.917 & $23518_{7 / 2}^{\circ}-40644_{5 / 2}^{\circ}$ \\
\hline 5924.389 & 3 & 150 & 16874.702 & $32620_{11 / 2}-49495_{9 / 2}^{\circ}$ & 5836.674 & 2 & 2 & 17128.297 & $6244_{1 / 2}-23372_{3 / 2}^{\circ}$ \\
\hline 5918.808 & 15 & 5 & 16890.613 & $17771_{11 / 2}^{\circ}-34661_{11 / 2}$ & 5835.881 & 5 & 5 & 17130.624 & $7001_{3 / 2}-24132_{3 / 2}^{\circ}$ \\
\hline 5916.347 & 1 & 4 & 16897.639 & $17121_{3 / 2}^{\circ}-34019_{3 / 2}$ & 5832.941 & 1 & 1 & 17139.259 & $27249_{7 / 2}^{\circ}-44388_{5 / 2}^{\circ}$ \\
\hline 5914.387 & 200 & 200 & 16903.239 & $9585_{5 / 2}^{\circ}-26488_{5 / 2}$ & 5830.492 & 10 & 20 & 17146.457 & $27357_{9 / 2}^{\circ}-44503_{7 / 2}$ \\
\hline 5912.104 & 4 & 5 & 16909.766 & $11116_{7 / 2}^{\circ}-28026_{5 / 2}$ & 5822.522 & 10 & 5 & 17169.928 & $8018_{3 / 2}-25188_{3 / 2}^{\circ}$ \\
\hline 5911.360 & 5 & 5 & 16911.894 & $25440_{5 / 2}^{\circ}-42352_{5 / 2}$ & 5820.381 & 15 & 25 & 17176.244 & $16033_{5 / 2}^{\circ}-33209_{7 / 2}^{\circ}$ \\
\hline 5910.507 & 5 & 40 & 16914.335 & $24309_{11 / 2}^{\circ}-41223_{11 / 2}$ & 5820.168 & 5 & 10 & 17176.872 & $9585_{5 / 2}^{\circ}-26762_{3 / 2}$ \\
\hline 5910.041 & 5 & 2 & 16915.669 & $32957_{7 / 2}^{\circ}-49873_{5 / 2}$ & 5817.733 & 15 & 5 & 17184.061 & $4113_{5 / 2}-21297_{5 / 2}^{\circ}$ \\
\hline 5908.403 & 251 & 50 & 16920.358 & $31924_{11 / 2}^{\circ}-48844_{9 / 2}$ & 5817.326 & 10 & 5 & 17185.264 & $9400_{5 / 2}-26586_{3 / 2}^{\circ}$ \\
\hline 5908.244 & 5 & 8 & 16920.814 & $10673_{5 / 2}^{\circ}-27593_{5 / 2}$ & 5816.994 & 8 & 10 & 17186.244 & $26586_{3 / 2}^{\circ}-43772_{5 / 2}$ \\
\hline 5906.316 & 5 & 40 & 16926.337 & $16033_{5 / 2}^{\circ}-32959_{3 / 2}$ & 5815.422 & 100 & 150 & 17190.890 & $1859_{3 / 2}-19050_{3 / 2}^{\circ}$ \\
\hline \multirow[t]{2}{*}{5902.560} & 0 & $8 b$ & 16937.108 & $36583_{7 / 2}^{\circ}-53520_{9 / 2}$ & 5812.107 & 1 & 1 & 17200.695 & $27249_{7 / 2}^{\circ}-44450_{9 / 2}$ \\
\hline & & & & $35741_{5 / 2}-52678_{5 / 2}^{\circ}$ & 5810.973 & 8 & 10 & 17204.052 & $23012_{3 / 2}^{\circ}-40216_{5 / 2}$ \\
\hline 5901.995 & 3 & 20 & 16938.729 & $17722_{9 / 2}^{\circ}-34661_{11 / 2}^{\circ}$ & 5809.430 & 8 & 5 & 17208.621 & $14545_{5 / 2}^{\circ}-31754_{5 / 2}^{\circ}$ \\
\hline 5901.754 & 0 & 5 & 16939.421 & $27787_{9 / 2}^{\circ}-44727_{11 / 2}$ & 5808.978 & 4 & 5 & 17209.960 & $22685_{7 / 2}^{\circ}-39895_{9 / 2}^{\circ}$ \\
\hline 5899.407 & 5 & 50 & 16946.160 & $12485_{7 / 2}^{\circ}-29431_{7 / 2}$ & 5808.079 & 20 & 50 & 17212.624 & $22139_{9 / 2}^{\circ}-39352_{11 / 2}^{\circ}$ \\
\hline 5896.361 & 15 & 40 & 16954.914 & $10572_{9 / 2}^{\circ}-27526_{9 / 2}$ & 5806.279 & 40 & 40 & 17217.960 & $12570_{7 / 2}-29788_{9 / 2}^{\circ}$ \\
\hline 5893.476 & 10 & 50 & 16963.214 & $14790_{7 / 2}^{\circ}-31754_{5 / 2}$ & 5804.589 & 1 & 2 & 17222.973 & $30101_{7 / 2}^{\circ}-47324_{5 / 2}$ \\
\hline 5888.260 & 15 & 15 & 16978.240 & $10379_{9 / 2}-27357_{9 / 2}^{\circ}$ & 5802.239 & 25 & 50 & 17229.948 & $25414_{11 / 2}^{i}-42644_{13 / 2}$ \\
\hline 5887.815 & 1 & 5 & 16979.523 & $25266_{i / 2}^{\circ}-42246_{1 / 2}$ & 5801.813 & 10 & 15 & 17231.214 & $20310_{5 / 2}^{\circ}-37542_{3 / 2}$ \\
\hline 5886.741 & 8 & 50 & 16982.621 & $20080_{7 / 2}^{\circ}-37063_{9 / 2}$ & 5798.910 & 2 & 3 & 17239.840 & $24982_{7 / 2}^{\circ}-42222_{7 / 2}$ \\
\hline 5886.346 & 5 & 10 & 16983.761 & $13468_{9 / 2}^{\circ}-30452_{9 / 2}^{\circ}$ & 5796.417 & 100 & 100 & 17247.254 & $11576_{3 / 2}^{\circ}-28823_{5 / 2}^{\circ}$ \\
\hline 5884.178 & 2 & 8 & 16990.018 & $19050_{3 / 2}^{\circ}-36040_{1 / 2}$ & 5795.963 & 15 & 20 & 17248.605 & $15710_{3 / 2}^{\circ}-32959_{3 / 2}^{\circ}$ \\
\hline 5881.414 & 2 & 5 & 16998.003 & $17272_{9 / 2}^{\circ}-34270_{9 / 2}$ & 5794.377 & 20 & 25 & 17253.326 & $22642_{9 / 2}^{\circ}-39895_{9 / 2}$ \\
\hline 5879.479 & $5 \mathrm{~h}$ & 15 & 17003.597 & $17722_{9 / 2}^{\circ}-34726_{7 / 2}$ & 5793.209 & 5 & 15 & 17256.805 & $29095_{5 / 2}^{\circ}-46352_{7 / 2}$ \\
\hline 5879.049 & 0 & 15 & 17004.841 & $35165_{13 / 2}^{\circ}-52170_{11 / 2}$ & 5790.136 & 3 & 4 & 17265.963 & $17460_{5 / 2}^{\circ}-34726_{7 / 2}^{\circ}$ \\
\hline 5878.279 & 8 & & 17007.068 & $17272_{9 / 2}^{\circ}-34279_{7 / 2}$ & 5788.193 & 10 & 15 & 17271.759 & $23372_{3 / 2}^{\circ}-40644_{5 / 2}$ \\
\hline 5875.501 & 2 & & 17015.109 & $22685_{7 / 2}^{\circ}-39700_{5 / 2}$ & 5787.541 & 10 & 10 & 17273.705 & $20288_{11 / 2}^{\circ}-37562_{11 / 2}^{\circ}$ \\
\hline 5875.279 & 1 & 4 & 17015.752 & $13468_{9 / 2}^{\circ}-30484_{11 / 2}$ & 5786.678 & 15 & 10 & 17276.281 & $14349_{1 / 2}-31625_{1 / 2}^{\circ}$ \\
\hline 5874.348 & 20 & 10 & 17018.449 & $4113_{5 / 2}-21131_{3 / 2}^{\circ}$ & 5785.498 & 3 & 8 & 17279.805 & $42336_{5 / 2}-59616_{3 / 2}^{\circ}$ \\
\hline 5872.604 & 15 & 40 & 17023.503 & $9400_{5 / 2}-26424_{5 / 2}^{\circ}$ & 5784.866 & $75 b$ & 50 & 17281.692 & $17272_{9 / 2}^{\circ}-34553_{9 / 2}$ \\
\hline 5872.312 & 0 & 5 & 17024.349 & $24463_{5 / 2}^{\circ}-41488_{7 / 2}$ & 5784.373 & 4 & 5 & 17283.165 & $15453_{7 / 2}^{\circ}-32736_{7 / 2}$ \\
\hline 5871.447 & 2 & 4 & 17026.857 & $12488_{9 / 2}^{\circ}-29515_{9 / 2}$ & 5783.757 & 1 & 1 & 17285.006 & $26488_{5 / 2}-43773_{7 / 2}^{\circ}$ \\
\hline 5870.554 & 25 & 150 & 17029.447 & $12485_{\frac{1}{7 / 2}}^{\circ}-29515_{9 / 2}$ & 5783.205 & 151 & 200 & 17286.656 & $20288_{11 / 2}^{\circ}-37575_{13 / 2}^{\circ}$ \\
\hline 5867.621 & 2 & 5 & 17037.960 & $17983_{5 / 2}^{\circ}-35021_{3 / 2}$ & 5782.499 & 1 & 1 & 17288.766 & $14484_{11 / 2}^{\circ}-31773_{9 / 2}$ \\
\hline 5867.348 & 0 & 5 & 17038.752 & $26770_{11 / 2}^{\circ}-43809_{9 / 2}$ & 5782.047 & 5 & 10 & 17290.118 & $22106_{5 / 2}-39396_{7 / 2}^{\circ}$ \\
\hline 5864.882 & 1 & 4 & 17045.916 & $21682_{7 / 2}^{\circ}-38728_{5 / 2}$ & 5778.082 & $15 \mathrm{~h}$ & 15 & 17301.983 & $20989_{9 / 2}^{\circ}-38291_{7 / 2}$ \\
\hline 5860.879 & $1 \mathrm{~h}$ & 1 & 17057.559 & $22028_{15 / 2}^{\circ}-39085_{13 / 2}$ & 5777.094 & 10 & 4 & 17304.942 & $6213_{9 / 2}-23518_{7 / 2}^{\circ}$ \\
\hline 5859.666 & 50 & 150 & 17061.090 & $13818_{7 / 2}^{\circ}-30879_{7 / 2}$ & 5775.795 & 1 & 2 & 17308.834 & $33354_{5 / 2}^{\circ}-50663_{5 / 2}$ \\
\hline 5859.524 & 15 & 40 & 17061.503 & $13248_{9 / 2}-30310_{11 / 2}^{\circ}$ & 5775.035 & 2 & 5 & 17311.111 & $25440_{5 / 2}^{\circ}-42751_{7 / 2}$ \\
\hline 5856.379 & 2 & 5 & 17070.665 & $22014_{1 / 2}^{\circ}-39085_{13 / 2}$ & 5773.204 & 3 & 4 & 17316.602 & $23730_{9 / 2}^{\circ}-41047_{9 / 2}^{\circ}$ \\
\hline 5855.032 & 2 & 8 & 17074.593 & $21297_{5 / 2}^{\circ}-38372_{3 / 2}$ & 5772.529 & 4 & 4 & 17318.626 & $22139_{9 / 2}^{\circ}-39458_{7 / 2}$ \\
\hline 5853.762 & 3 & 4 & 17078.297 & $13406_{13 / 2}^{\circ}-30484_{11 / 2}$ & 5771.144 & 20 & 25 & 17322.783 & $20969_{7 / 2}^{\circ}-38291_{7 / 2}$ \\
\hline 5853.059 & 5 & 20 & 17080.348 & $19248_{5 / 2}^{\circ}-36328_{3 / 2}$ & 5770.145 & $15 b$ & 50 & 17325.782 & $26770_{11 / 2}^{\circ}-44096_{9 / 2}$ \\
\hline 5852.188 & 8 & 15 & 17082.890 & $17460_{5 / 2}^{\circ}-34543_{5 / 2}$ & 5767.459 & 4 & 2 & 17333.851 & $15242_{9 / 2}^{\circ}-32576_{7 / 2}^{\circ}$ \\
\hline 5850.258 & 1 & 8 & 17088.526 & $37756_{7 / 2}^{\circ}-54845_{9 / 2}$ & 5766.470 & 3 & 8 & 17336.824 & $31353_{3 / 2}^{\circ}-48689_{3 / 2}^{\circ}$ \\
\hline
\end{tabular}


TABLE 3. Classified lines of Th II-Continued

\begin{tabular}{|c|c|c|c|c|}
\hline \multirow{2}{*}{$\begin{array}{c}\text { Wavelength } \\
\AA \\
\end{array}$} & \multicolumn{2}{|c|}{ Intensity } & \multirow{2}{*}{$\begin{array}{c}\text { Wavenumber } \\
\mathrm{cm}^{-1}\end{array}$} & \multirow{2}{*}{ Classification } \\
\hline & Lamp & Spark & & \\
\hline 5766.101 & 20 & 10 & 17337.933 & $10189_{11 / 2}^{\circ}-27526_{9 / 2}$ \\
\hline 5765.947 & 10 & 15 & 17338.396 & $27787_{9 / 2}^{\circ}-45126_{9 / 2}$ \\
\hline 5765.697 & 1 & 2 & 17339.148 & $26626_{1 / 2}^{0}-43965_{1 / 2}$ \\
\hline 5764.766 & 1 & 2 & 17341.948 & $30956_{9 / 2}^{\circ}-48298_{7 / 2}$ \\
\hline 5763.645 & 10 & 15 & 17345.321 & $20120_{5 / 2}^{\circ}-37465_{5 / 2}$ \\
\hline 5762.237 & 3 & 5 & 17349.559 & $23697_{7 / 2}^{\circ}-41047_{9 / 2}$ \\
\hline 5762.096 & $3 b$ & 5 & 17349.984 & $22834_{7 / 2}-40184_{7 / 2}^{\circ}$ \\
\hline 5761.018 & 4 & 3 & 17353.230 & $10673_{5 / 2}^{\circ}-28026_{5 / 2}$ \\
\hline 5758.920 & 10 & 5 & 17359.552 & $7828_{1 / 2}-25188_{3 / 2}^{\circ}$ \\
\hline 5757.648 & 5 & 4 & 17363.387 & $9061_{5 / 2}-26424_{5 / 2}^{\circ}$ \\
\hline 5757.551 & 5 & 5 & 17363.680 & $16906_{7 / 2}^{\circ}-34270_{9 / 2}$ \\
\hline 5757.099 & 15 & 10 & 17365.043 & $10572_{9 / 2}^{\circ}-27937_{11 / 2}$ \\
\hline 5754.551 & 50 & 40 & 17372.732 & $16906_{7 / 2}^{\circ}-34279_{7 / 2}$ \\
\hline 5752.303 & 5 & 8 & 17379.521 & $26424_{5 / 2}^{\circ}-43803_{7 / 2}$ \\
\hline 5751.173 & 2 & 4 & 17382.936 & $29788_{9 / 2}^{\circ}-47171_{9 / 2}$ \\
\hline 5750.556 & 25 & 20 & 17384.801 & $20080_{7 / 2}^{\circ}-37465_{5 / 2}$ \\
\hline 5750.268 & 15 & 10 & 17385.671 & $12488_{9 / 2}^{\circ}-29873_{7 / 2}$ \\
\hline 5750.117 & 2 & 3 & 17386.128 & $27403_{3 / 2}^{\circ}-44789_{1 / 2}$ \\
\hline 5749.582 & 40 & 50 & 17387.746 & $23187_{13 / 2}^{\circ}-40574_{11 / 2}$ \\
\hline 5749.383 & 200 & $200 \mathrm{~d}$ & 17388.348 & $1859_{3 / 2}-19248_{5 / 2}^{\circ}$ \\
\hline 5749.157 & 3 & 8 & 17389.031 & $24757_{9 / 2}^{\circ}-42146_{11 / 2}$ \\
\hline 5749.012 & 15 & 15 & 17389.470 & $17272_{9 / 2}^{\circ}-34661_{11 / 2}$ \\
\hline 5747.521 & 15 & 15 & 17393.981 & $16818_{7 / 2}-34212_{5 / 2}^{\circ}$ \\
\hline 5745.676 & 75 & 100 & 17399.566 & $20989_{9 / 2}^{\circ}-38389_{7 / 2}$ \\
\hline 5743.810 & 2 & 2 & 17405.219 & $23518_{7 / 2}^{\circ}-40923_{5 / 2}$ \\
\hline 5742.651 & 10 & 5 & 17408.731 & $10379_{9 / 2}-27787_{9 / 2}^{\circ}$ \\
\hline 5742.084 & 150 & 150 & 17410.450 & $13468_{9 / 2}^{\circ}-30879_{7 / 2}$ \\
\hline 5741.379 & 15 & 25 & 17412.588 & $22139_{9 / 2}^{\circ}-39552_{9 / 2}$ \\
\hline 5741.170 & 150 & 100 & 17413.222 & $7001_{3 / 2}-24414_{3 / 2}^{\circ}$ \\
\hline 5738.819 & 3 & 4 & 17420.356 & $20969_{7 / 2}^{\circ}-38389_{7 / 2}$ \\
\hline 5738.515 & 1 & 2 & 17421.278 & $30310_{11 / 2}^{\circ}-47731_{9 / 2}$ \\
\hline 5738.306 & $25 d$ & $100 \mathrm{~b}$ & 17421.913 & $17121_{3 / 2}^{\circ}-34543_{5 / 2}$ \\
\hline 5738.271 & 1 & $10 \mathrm{~b}$ & 17422.019 & $\begin{aligned} 20120_{5 / 2}^{\circ} & -37542_{3 / 2} \\
8018_{3 / 2} & -25440_{5 / 2}^{\circ}\end{aligned}$ \\
\hline 5736.142 & 4 & 3 & 17428.485 & $15786_{5 / 2}-33215_{3 / 2}^{\circ}$ \\
\hline 5734.526 & 1 & 2 & 17433.397 & $31924_{11 / 2}^{\circ}-49357_{11 / 2}$ \\
\hline 5732.977 & 200 & 200 & 17438.107 & $17727_{11 / 2}-35165_{13 / 2}^{\circ}$ \\
\hline 5732.043 & 8 & 8 & 17440.948 & $13818_{7 / 2}^{\circ}-31259_{5 / 2}$ \\
\hline 5731.466 & 10 & 15 & 17442.704 & $24757_{9 / 2}^{\circ}-42200_{9 / 2}$ \\
\hline 5726.151 & 21 & 1 & 17458.894 & $30994_{7 / 2}^{\circ}-48453_{7 / 2}$ \\
\hline 5725.579 & 50 & 75 & 17460.639 & $0_{3 / 2}-17460_{5 / 2}^{\circ}$ \\
\hline 5725.011 & 40 & 40 & 17462.371 & $7001_{3 / 2}-24463_{5 / 2}^{\circ}$ \\
\hline 5721.655 & 1 & 1 & 17472.613 & $18568_{1 / 2}^{\circ}-36040_{1 / 2}^{\circ}$ \\
\hline 5721.618 & 2 & 1 & 17472.726 & $24463_{5 / 2}^{\circ}-41936_{3 / 2}$ \\
\hline 5719.847 & 3 & 5 & 17478.136 & $24873_{5 / 2}^{\circ}-42352_{5 / 2}$ \\
\hline 5717.328 & 5 & 10 & 17485.837 & $29095_{5 / 2}^{\circ}-46581_{5 / 2}$ \\
\hline 5714.894 & 8 & 10 & 17493.284 & $15242_{9 / 2}^{\circ}-32736_{7 / 2}$ \\
\hline 5713.828 & 1 & 2 & 17496.548 & $30956_{9 / 2}^{\circ}-48453_{7 / 2}$ \\
\hline 5713.519 & 3 & 2 & 17497.494 & $14275_{9 / 2}^{\circ}-31773_{9 / 2}$ \\
\hline 5712.588 & 40 & 20 & 17500.345 & $12219_{3 / 2}-29720_{3 / 2}^{\circ}$ \\
\hline
\end{tabular}

\begin{tabular}{c|c|c|c|c}
\multirow{2}{*}{$\begin{array}{c}\text { Wavelength } \\
\AA\end{array}$} & \multicolumn{2}{|c|}{ Intensity } & \multirow{2}{*}{$\begin{array}{c}\text { Wavenumber } \\
\mathbf{c m}^{-1}\end{array}$} & \multirow{2}{*}{ Classification } \\
\cline { 2 - 3 } & Lamp & Spark & \\
\hline & & & & \\
\hline
\end{tabular}

\begin{tabular}{r|r|r|r|l}
5709.102 & 3 & 3 & 17511.031 & $20310_{5 / 2}^{\circ}-37821_{3 / 2}$
\end{tabular}

\begin{tabular}{l|l|l|l|rr}
5707.100 & 300 & 200 & 17517.174 & $6213_{9 / 2}-23730_{9 / 2}^{\circ}$
\end{tabular}

\begin{tabular}{l|c|c|c|c|}
5706.145 & 5 & 8 & 17520.105 & $25607_{9 / 2}^{\circ}-43127_{11 / 2}$
\end{tabular}

5703.942

5703.268

5700.914

5760.698

5700.521

5700.453

5700.060

50

40

17520.105

$18214_{3 / 2}^{\circ}-35741_{5 / 2}$

$1521_{5 / 2}-19050_{3 / 2}^{\circ}$

$4146_{7 / 2}-21682_{7 / 2}^{\circ}$

$9720_{7 / 2}^{\circ}-27257_{7 / 2}$

$22014_{11 / 2}^{\circ}-39552_{9 / 2}$

$9711_{7 / 2}-27249_{7 / 2}^{\circ}$

$21297_{5 / 2}^{\circ}-38836_{3 / 2}$

5699.370

5699.220

5696.084

5692.936

5692.853

5691.804

5691.628

5691.092

5688.931

5687.682

5684.171

5682.436

5681.249

5680.489

5680.211

5678.920

5676.763

5675.019

5674.119

5670.666

5669.842

5669.232

5668.890

5668.792

5668.340

5665.772

5665.625

5664.999

5663.441

5661.551

5660.015

5657.295

5657.183

5654.607

5654.022

5653.915

5653.209

5652.904

5652.664
100

$0 \quad 10$

$100 \quad 100$

3

17537.599

17538.809

1754.0 .932

1754.1.394

17551.051

17560.756

17561.012

17564.248

17564.792

17566.446

17573.119

17576.978

17587.834

17593.204

17596.880

17599.234

17600.096

17604.097

17610.786

17616.198

17618.992

17629.720

17632.283

17634.180

17635.243

17635.548

17636.955

17644.949

17645.406

17647.356

17652.211

17658.104

17662.896

17671.388

17671.738

17679.788

17681.617

17681.952

17684.160

17685.114

17685.865
$25414_{1 / 2}^{\circ}-42955_{9 / 2}$ $27357_{9 / 2}^{\circ}-44898_{7 / 2}$ $23372_{3 / 2}^{\circ}-40923_{5 / 2}$ $17460_{5 / 2}^{\circ}-35021_{3 / 2}$ $19248_{5 / 2}^{\circ}-36809_{7 / 2}$ $9400_{5 / 2}-26965_{3 / 2}^{\circ}$ $27257_{7 / 2}-44821_{5 / 2}^{\circ}$ $21297_{5 / 2}^{\circ}-38863_{5 / 2}$ $24309_{11 / 2}^{\circ}-41882_{13 / 2}$ $31800_{7 / 2}^{\circ}-49377_{7 / 2}$

$17837_{1 / 2}^{\circ}-35425_{1 / 2}$ $22355_{1 / 2}^{\circ}-39948_{1 / 2}$ $21131_{3 / 2}^{\circ}-38728_{5 / 2}$ $25414_{11 / 2}^{\circ}-43014_{13 / 2}$ $24309_{11 / 2}^{\circ}-41909_{9 / 2}$ $31810_{5 / 2}^{\circ}-49414_{3 / 2}$ $16033_{5 / 2}^{\circ}-33637_{7 / 2}$ $29095_{5 / 2}^{\circ}-46706_{7 / 2}$ $4490_{5 / 2}^{\circ}-22106_{5 / 2}$ $23372_{3 / 2}^{\circ}-40991_{3 / 2}$

$17771_{1 / / 2}^{\circ}-35400_{13 / 2}$ $23012_{3 / 2}^{\circ}-40644_{5 / 2}$ $20310_{5 / 2}^{\circ}-37945_{5 / 2}$ $15324_{1 / 2}^{\circ}-32959_{3 / 2}$ $39443_{9 / 2}^{\circ}-57078_{9 / 2}$ $16906_{7 / 2}^{\circ}-34543_{5 / 2}$ $24873_{5 / 2}^{\circ}-42518_{7 / 2}$ $9711_{7 / 2}-27357_{9 / 2}^{\circ}$ $16906_{7 / 2}^{\circ}-34553_{9 / 2}$ $15305_{9 / 2}-32957_{7 / 2}^{\circ}$

$28923_{5 / 2}^{\circ}-46581_{5 / 2}$ $19912_{13 / 2}^{\circ}-37575_{13 / 2}$ $28026_{5 / 2}-45697_{3 / 2}^{\circ}$ $9585_{5 / 2}^{\circ}-27257_{7 / 2}$ $26770_{11 / 2}^{\circ}-44450_{9 / 2}$ $6700_{9 / 2}^{\circ}-24381_{7 / 2}$ $39396_{7 / 2}^{\circ}-57078_{9 / 2}$ $21682_{7 / 2}^{\circ}-39366_{5 / 2}$ $17771_{11 / 2}^{\circ}-35456_{9 / 2}$ $20686_{5 / 2}^{\circ}-38372_{3 / 2}$ 
TABLE 3. Classified lines of Th II-Continued

\begin{tabular}{|c|c|c|c|c|c|c|c|c|c|}
\hline \multirow{2}{*}{$\begin{array}{c}\text { Wavelength } \\
\AA\end{array}$} & \multicolumn{2}{|c|}{ Intensity } & \multirow{2}{*}{$\begin{array}{c}\text { Wavenumber } \\
\mathrm{cm}^{-1}\end{array}$} & \multirow{2}{*}{ Classification } & \multirow{2}{*}{$\begin{array}{c}\text { Wavelength } \\
\AA\end{array}$} & \multicolumn{2}{|c|}{ Intensity } & \multirow{2}{*}{$\begin{array}{c}\text { Wavenumber } \\
\mathrm{cm}^{-1}\end{array}$} & \multirow{2}{*}{ Classification } \\
\hline & Lamp & Spark & & & & Lamp & Spark & & \\
\hline 5652.433 & 2 & 3 & 17686.588 & $28011_{3 / 2}-45697_{3 / 2}^{\circ}$ & 5611.775 & 3 & 2 & 17814.728 & $15144_{3 / 2}^{\circ}-32959_{3 / 2}$ \\
\hline 5652.164 & 5 & 4 & 17687.430 & $20158_{5 / 2}-37846_{5 / 2}^{\circ}$ & 5611.144 & 1 & 2 & 17816.731 & $36193_{9 / 2}^{\circ}-54010_{7 / 2}$ \\
\hline 5651.446 & 2 & 2 & 17689.677 & $19880_{9 / 2}-37569_{9 / 2}^{\circ}$ & 5609.300 & 20 & 15 & 17822.588 & $17722_{9 / 2}^{\circ}-35545_{9 / 2}$ \\
\hline 5649.218 & 2 & 8 & 17696.653 & $30310_{11 / 2}^{\circ}-48006_{9 / 2}$ & 5608.100 & 8 & 10 & 17826.402 & $18214_{3 / 2}^{\circ}-36040_{1 / 2}$ \\
\hline 5648.862 & 20 & 20 & 17697.769 & $16033_{5 / 2}^{\circ}-33730_{5 / 2}$ & 5606.539 & 8 & 10 & 17831.365 & $24414_{3 / 2}^{\circ}-42246_{1 / 2}$ \\
\hline 5647.878 & 1 & 1 & 17700.852 & $23697_{7 / 2}^{\circ}-41398_{9 / 2}$ & 5605.247 & 15 & 10 & 17835.475 & $18973_{7 / 2}^{\circ}-36809_{7 / 2}$ \\
\hline 5647.108 & 2 & 1 & 17703.266 & $20686_{5 / 2}^{\circ}-38389_{7 / 2}$ & 5604.666 & 15 & 20 & 17837.324 & $24309_{11 / 2}^{\circ}-42146_{11 / 2}$ \\
\hline 5646.359 & 8 & 5 & 17705.614 & $16564_{11 / 2}^{\circ}-34270_{9 / 2}$ & 5604.514 & 100 & 40 & 17837.808 & $0_{3 / 2}-17837_{1 / 2}^{\circ}$ \\
\hline 5645.894 & $100 \mathrm{~b}$ & $200 \mathrm{~d}$ & 17707.072 & $11116_{7 / 2}^{\circ}-28823_{5 / 2}$ & 5601.136 & 3 & 1 & 17848.566 & $9400_{5 / 2}-27249_{7 / 2}^{\circ}$ \\
\hline 5645.856 & $20 \mathrm{~b}$ & $25 b$ & 17707.191 & $20080_{7 / 2}^{\circ}-37787_{7 / 2}$ & 5599.300 & $15 b$ & 15 & 17854.418 & $20310_{5 / 2}^{\circ}-38165_{7 / 2}$ \\
\hline 5645.084 & 2 & 2 & 17709.613 & $22513_{5 / 2}^{\circ}-40222_{3 / 2}$ & 5596.152 & 10 & 15 & 17864.462 & $20080_{7 / 2}^{\circ}-37945_{5 / 2}$ \\
\hline 5644.231 & 1 & 1 & 17712.289 & $25414_{11 / 2}^{\circ}-43127_{11 / 2}$ & 5595.610 & 50 & 75 & 17866.192 & $17727_{11 / 2}-35593_{9 / 2}^{\circ}$ \\
\hline 5643.596 & 1 & 1 & 17714.282 & $26586_{3 / 2}^{\circ}-44300_{3 / 2}$ & 5594.583 & 20 & 25 & 17869.472 & $21682_{\tau / 2}^{\circ}-39552_{9 / 2}$ \\
\hline 5640.081 & 50 & 75 & 17725.322 & $22642_{9 / 2}^{\circ}-40367_{9 / 2}$ & 5593.612 & 100 & 50 & 17872.574 & $7001_{3 / 2}-24873_{5 / 2}^{\circ}$ \\
\hline 5639.849 & 4 & 8 & 17726.051 & $22685_{7 / 2}^{\circ}-40411_{7 / 2}$ & 5593.480 & 15 & 25 & 17872.995 & $20989_{9 / 2}^{\circ}-38862_{11 / 2}$ \\
\hline 5639.746 & 300 & 300 & 17726.375 & $1521_{5 / 2}-19248_{5 / 2}^{\circ}$ & 5593.266 & 25 & 15 & 17873.679 & $9720_{7 / 2}^{\circ}-27593_{5 / 2}$ \\
\hline \multirow[t]{2}{*}{5637.947} & 3 & 2 & 17732.031 & $21131_{3 / 2}^{\circ}-38863_{5 / 2}$ & 5592.796 & 5 & 5 & 17875.181 & $17727_{11 / 2}-35602_{11 / 2}^{\circ}$ \\
\hline & & & & $10855_{7 / 2}-28587_{5 / 2}^{\circ}$ & 5592.510 & 8 & 10 & 17876.095 & $26424_{5 / 2}^{\circ}-44300_{3 / 2}$ \\
\hline 5635.409 & 2 & 8 & 17740.017 & $37756_{7 / 2}^{\circ}-55496_{9 / 2}$ & 5592.321 & 15 & 15 & 17876.700 & $27249_{7 / 2}^{\circ}-45126_{9 / 2}$ \\
\hline 5634.221 & 15 & 15 & 17743.757 & $13250_{5 / 2}-30994_{7 / 2}^{\circ}$ & & & & & $19880_{9 / 2}-37756_{7 / 2}^{\circ}$ \\
\hline 5633.649 & $20 \mathrm{~b}$ & 25 & 17745.559 & $13248_{9 / 2}-30994_{7 / 2}^{\circ}$ & 5592.125 & 3 & 5 & 17877.326 & $24873_{5 / 2}^{\circ}-42751_{7 / 2}$ \\
\hline 5632.872 & 251 & 20 & 17748.007 & $10189_{11 / 2}^{\circ}-27937_{11 / 2}$ & 5591.270 & 3 & 4 & 17880.060 & $23518_{7 / 2}^{\circ}-41398_{9 / 2}^{\circ}$ \\
\hline 5632.036 & 15 & 25 & 17750.641 & $20989_{9 / 2}^{\circ}-38740_{11 / 2}$ & 5591.124 & 1 & 2 & 17880.527 & $22014_{11 / 2}^{\circ}-39895_{9 / 2}$ \\
\hline 5631.111 & 3 & 8 & 17753.557 & $35165_{13 / 2}^{\circ}-52918_{13 / 2}$ & 5589.671 & 15 & 15 & 17885.174 & $22685_{7 / 2}^{\circ}-40570_{7 / 2}$ \\
\hline 5630.922 & 8 & 4 & 17754.153 & $17771_{11 / 2}^{\circ}-35525_{11 / 2}$ & 5588.867 & 40 & 20 & 17887.747 & $6244_{1 / 2}-24132_{3 / 2}^{\circ}$ \\
\hline 5630.589 & 3 & 3 & 17755.203 & $22106_{5 / 2}-39861_{5 / 2}^{\circ}$ & 5588.662 & 4 & 81 & 17888.404 & $30956_{9 / 2}^{\circ}-48844_{9 / 2}$ \\
\hline 5630.411 & 10 & 40 & 17755.764 & $22139_{9 / 2}^{\circ}-39895_{9 / 2}$ & 5587.857 & 25 & 25 & 17890.981 & $24309_{11 / 2}^{\circ}-42200_{9 / 2}$ \\
\hline 5630.231 & 5 & 4 & 17756.332 & $15453_{7 / 2}^{\circ}-33209_{7 / 2}$ & 5587.734 & 75 & 10 & 17891.374 & $20288_{11 / 2}^{\circ}-38179_{9 / 2}$ \\
\hline 5629.856 & 2 & 4 & 17757.514 & $23730_{9 / 2}^{\circ}-41488_{7 / 2}$ & 5586.658 & 8 & 10 & 17894.820 & $20969_{7 / 2}^{\circ}-38863_{5 / 2}^{\circ}$ \\
\hline 5629.731 & 2 & 1 & 17757.909 & $17983_{5 / 2}^{\circ}-35741_{5 / 2}$ & 5586.453 & 40 & 20 & 17895.477 & $17983_{5 / 2}^{\circ}-35878_{7 / 2}$ \\
\hline 5629.174 & 3 & 4 & 17759.666 & $20969_{7 / 2}^{\circ}-38728_{5 / 2}$ & 5585.610 & 20 & 4 & 17898.178 & $22513_{5 / 2}^{\circ}-40411_{7 / 2}$ \\
\hline 5628.963 & 1 & 2 & 17760.331 & $18568_{1 / 2}^{\circ}-36328_{3 / 2}$ & 5585.121 & 4 & 1 & 17899.745 & $17121_{3 / 2}^{\circ}-35021_{3 / 2}$ \\
\hline 5628.624 & 3 & 5 & 17761.401 & $24757_{9 / 2}^{\circ}-42518_{7 / 2}$ & 5584.161 & 15 & 75 & 17902.822 & $9061_{5 / 2}-26963_{7 / 2}^{\circ}$ \\
\hline 5628.568 & 1 & 2 & 17761.578 & $37277_{\tau / 2}-55038_{9 / 2}^{\circ}$ & 5583.758 & 100 & 15 & 17904.114 & $9061_{5 / 2}-26965_{3 / 2}^{\circ}$ \\
\hline 5626.734 & 40 & 50 & 17767.367 & $19912_{13 / 2}^{\circ}-37679_{11 / 2}$ & 5583.073 & 2 & 15 & 17906.311 & $38179_{9 / 2}-56086_{9 / 2}^{\circ}$ \\
\hline \multirow[t]{2}{*}{5626.256} & 5 & 15 & 17768.877 & $27357_{9 / 2}^{\circ}-45126_{9 / 2}$ & 5581.283 & 20 & 20 & 17912.053 & $23187_{13 / 2}^{\circ}-41099_{15 / 2}^{\circ}$ \\
\hline & & & & $24982_{7 / 2}^{\circ}-42751_{7 / 2}$ & 5577.905 & 25 & 40 & 17922.901 & $22355_{i / 2}^{\circ}-40278_{3 / 2}$ \\
\hline 5626.085 & $3 \mathrm{~b}$ & $4 \mathrm{~b}$ & 17769.416 & $22642_{9 / 2}^{\circ}-40411_{7 / 2}$ & 5577.489 & 20 & 40 & 17924.238 & $22014_{11 / 2}^{\circ}-39939_{11 / 2}^{\circ}$ \\
\hline 5626.057 & $10 \mathrm{~b}$ & $25 b$ & 17769.505 & $11576_{3 / 2}^{\circ}-29345_{5 / 2}$ & 5576.510 & 2 & 4 & 17927.384 & $35593_{7 / 2}^{\circ}-53520_{9 / 2}$ \\
\hline 5625.495 & 1 & 1 & 17771.280 & $21297_{5 / 2}^{\circ}-39068_{7 / 2}$ & 5575.264 & 2 & 4 & 17931.391 & $15453_{7 / 2}^{\circ}-33384_{9 / 2}$ \\
\hline 5624.146 & 10 & 10 & 17775.543 & $21682_{7 / 2}^{\circ}-39458_{7 / 2}$ & 5574.876 & $5 b$ & 50 & 17932.639 & $22642_{9 / 2}^{\circ}-40574_{11 / 2}$ \\
\hline 5620.918 & 15 & 10 & 17785.751 & $14790_{7 / 2}^{\circ}-32576_{7 / 2}$ & 5573.873 & 40 & 25 & 17935.866 & $13818_{7 / 2}^{\circ}-31754_{5 / 2}$ \\
\hline 5619.422 & 2 & 2 & 17790.486 & $23697_{7 / 2}^{\circ}-41488_{7 / 2}$ & 5568.000 & $300 \mathrm{~b}$ & 150 & 17954.784 & $13818_{7 / 2}^{\circ}-31773_{9 / 2}$ \\
\hline 5615.728 & 75 & 100 & 17802.188 & $17722_{9 / 2}^{\circ}-35525_{11 / 2}$ & 5567.395 & 20 & 40 & 17956.735 & $26770_{11 / 2}^{\circ}-44727_{11 / 2}$ \\
\hline 5615.635 & 15 & 20 & 17802.483 & $26586_{3 / 2}^{\circ}-44388_{5 / 2}$ & 5566.730 & 3 & 3 & 17958.880 & $22685_{7 / 2}^{\circ}-40644_{5 / 2}$ \\
\hline 5615.008 & 10 & 8 & 17804.471 & $24132_{3 / 2}^{\circ}-41936_{3 / 2}$ & 5565.105 & 5 & 4 & 17964.124 & $8460_{3 / 2}-26424_{5 / 2}^{\circ}$ \\
\hline 5614.515 & 5 & 1 & 17806.034 & $36687_{5 / 2}^{\circ}-54493_{5 / 2}$ & 5565.005 & 50 & 75 & 17964.447 & $12488_{9 / 2}^{\circ}-30452_{9 / 2}$ \\
\hline 5614.337 & 3 & 5 & 17806.599 & $25440_{5 / 2}^{\circ}-43246_{7 / 2}$ & 5564.371 & 50 & 50 & 17966.494 & $15242_{9 / 2}^{\circ}-33209_{\tau / 2}$ \\
\hline 5614.187 & 3 & 4 & 17807.074 & $29095_{5 / 2}^{\circ}-46902_{5 / 2}$ & 5564.201 & 200 & 200 & 17967.043 & $12485_{7 / 2}^{\circ}-30452_{9 / 2}$ \\
\hline 5613.471 & 1 & 4 & 17809.346 & $33215_{3 / 2}^{\circ}-51024_{3 / 2}$ & 5563.375 & 5 & 4 & 17969.710 & $23518_{7 / 2}^{\circ}-41488_{7 / 2}$ \\
\hline
\end{tabular}


TABLE 3. Classified lines of Th II-Continued

\begin{tabular}{|c|c|c|c|c|c|c|c|c|c|}
\hline \multirow{2}{*}{$\begin{array}{c}\text { Wavelength } \\
\AA\end{array}$} & \multicolumn{2}{|c|}{ Intensity } & \multirow{2}{*}{$\begin{array}{c}\text { Wavenumber } \\
\mathbf{c m}^{-1}\end{array}$} & \multirow{2}{*}{ Classification } & \multirow{2}{*}{$\begin{array}{c}\text { Wavelength } \\
\AA\end{array}$} & \multicolumn{2}{|c|}{ Intensity } & \multirow{2}{*}{$\begin{array}{c}\text { Wavenumber } \\
\mathbf{c m}^{-1}\end{array}$} & \multirow{2}{*}{ Classification } \\
\hline & Lamp & Spark & & & & Lamp & Spark & & \\
\hline 5560.556 & 6 & 5 & 17978.820 & $15236_{3 / 2}-33215_{3 / 2}^{\circ}$ & 5495.806 & 75 & 75 & 18190.639 & $14545_{5 / 2}^{\circ}-32736_{7 / 2}$ \\
\hline 5560.339 & 5 & 8 & 17979.522 & $23012_{3 / 2}^{\circ}-40991_{3 / 2}$ & 5494.556 & 50 & 50 & 18194.777 & $19912_{13 / 2}^{\circ}-38107_{15 / 2}$ \\
\hline 5560.054 & 15 & 10 & 17980.443 & $8605_{5 / 2}-26586_{3 / 2}^{\circ}$ & 5493.931 & 2 & 4 & 18196.847 & $25607_{9 / 2}^{\circ}-43803_{7 / 2}^{\circ}$ \\
\hline 5559.141 & 8 & 2 & 17983.396 & $0_{3 / 2}-17983_{5 / 2}^{\circ}$ & 5493.478 & 3 & 4 & 18198.348 & $24757_{9 / 2}^{\circ}-42955_{9 / 2}$ \\
\hline 5557.311 & 75 & 75 & 17989.318 & $16564_{11 / 2}^{\circ}-34553_{9 / 2}$ & 5492.332 & 40 & 20 & 18202.145 & $25607_{9 / 2}^{\circ}-43809_{9 / 2}$ \\
\hline 5556.146 & 40 & 10 & 17993.090 & $4146_{7 / 2}-22139_{9 / 2}^{\circ}$ & 5492.061 & 20 & 25 & 18203.043 & $26586_{3 / 2}^{\circ}-44789_{1 / 2}$ \\
\hline 5555.115 & 2 & 1 & 17996.429 & $12488_{9 / 2}^{\circ}-30484_{11 / 2}$ & & & & & $17837_{1 / 2}^{\circ}-36040_{1 / 2}$ \\
\hline 5551.515 & 3 & 4 & 18008.099 & $33354_{5 / 2}^{\circ}-51362_{5 / 2}$ & 5491.528 & 20 & 25 & 18204.810 & $24132_{3 / 2}^{\circ}-42336_{5 / 2}$ \\
\hline 5551.370 & 100 & 75 & 18008.570 & $9585_{5 / 2}^{\circ}-27593_{5 / 2}$ & 5489.623 & 10 & 10 & 18211.127 & $20080_{7 / 2}^{\circ}-38291_{7 / 2}$ \\
\hline 5548.516 & 20 & 25 & 18017.833 & $21682_{7 / 2}^{\circ}-39700_{5 / 2}^{\circ}$ & 5489.452 & 3 & 3 & 18211.694 & $23697_{7 / 2}^{\circ}-41909_{9 / 2}$ \\
\hline 5547.824 & 3 & 2 & 18020.080 & $15710_{3 / 2}^{\circ}-33730_{5 / 2}$ & 5488.922 & 50 & 40 & 18213.453 & $6168_{7 / 2}^{\circ}-24381_{7 / 2}$ \\
\hline 5546.490 & 20 & 20 & 18024.414 & $20080_{7 / 2}^{\circ}-38105_{5 / 2}$ & 5488.622 & 75 & 15 & 18214.448 & $0_{3 / 2}-18214_{3 / 2}^{\circ}$ \\
\hline 5545.323 & 4 & 5 & 18028.207 & $38863_{5 / 2}-56892_{5 / 2}^{\circ}$ & 5488.518 & 2 & 3 & 18214.793 & $28720_{3 / 2}^{\circ}-46935_{3 / 2}$ \\
\hline 5545.051 & 5 & 3 & 18029.092 & $19248_{5 / 2}^{\circ}-37277_{7 / 2}$ & 5487.426 & 1 & 3 & 18218.418 & $29788_{9 / 2}^{\circ}-48006_{9 / 2}$ \\
\hline 5544.405 & 4 & 3 & 18031.192 & $14545_{5 / 2}^{\circ}-32576_{7 / 2}$ & 5487.133 & 2 & 4 & 18219.391 & $30101_{7 / 2}^{\circ}-48320_{5 / 2}$ \\
\hline 5543.672 & 2 & 2 & 18033.576 & $33902_{7 / 2}^{\circ}-51935_{5 / 2}$ & 5486.917 & 20 & 40 & 18220.108 & $24132_{3 / 2}^{\circ}-42352_{5 / 2}$ \\
\hline 5543.375 & 5 & 5 & 18034.543 & $15349_{11 / 2}^{\circ}-33384_{9 / 2}$ & 5486.180 & 2 & 2 & 18222.556 & $24873_{5 / 2}^{\circ}-43096_{5 / 2}$ \\
\hline 5542.766 & 50 & 75 & 18036.524 & $23187_{13 / 2}^{\circ}-41223_{11 / 2}$ & 5484.615 & $3 \mathrm{~b}$ & 2 & 18227.756 & $22139_{9 / 2}^{\circ}-40367_{9 / 2}$ \\
\hline 5542.472 & 5 & 5 & 18037.481 & $31800_{7 / 2}^{\circ}-49837_{9 / 2}$ & 5484.268 & $15 \mathrm{~h}$ & 15 & 18228.909 & $29095_{5 / 2}^{\circ}-47324_{5 / 2}$ \\
\hline 5540.102 & 8 & 4 & 18045.197 & $20120_{5 / 2}^{\circ}-38165_{7 / 2}$ & 5484.138 & 150 & 200 & 18229.341 & $11116_{7 / 2}^{\circ}-29345_{5 / 2}$ \\
\hline 5539.909 & 300 & 3000 & 18045.826 & $9585_{5 / 2}^{\circ}-27631_{3 / 2}$ & 5482.406 & 20 & 75 & 18235.100 & $21131_{3 / 2}^{\circ}-39366_{5 / 2}$ \\
\hline 5538.500 & 50 & 20 & 18050.416 & $7331_{5 / 2}^{\circ}-25381_{3 / 2}$ & 5481.961 & 20 & 20 & 18236.580 & $18816_{13 / 2}^{\circ}-37053_{11 / 2}$ \\
\hline 5537.663 & 1 & 1 & 18053.145 & $29095_{5 / 2}^{\circ}-47148_{3 / 2}$ & 5481.477 & 75 & 100 & 18238.190 & $22685_{7 / 2}^{\circ}-40923_{5 / 2}$ \\
\hline 5537.131 & 75 & 50 & 18054.879 & $9202_{7 / 2}^{\circ}-27257_{7 / 2}$ & 5480.886 & 50 & 25 & 18240.157 & $10855_{7 / 2}-29095_{5 / 2}^{\circ}$ \\
\hline 5533.146 & 20 & 10 & 18067.882 & $10855_{7 / 2}-28923_{5 / 2}^{\circ}$ & 5479.680 & 15 & 15 & 18244.171 & $18568_{1 / 2}^{\circ}-36812_{1 / 2}$ \\
\hline 5532.179 & $15 \mathrm{~h}$ & 15 & 18071.040 & $20686_{5 / 2}^{\circ}-38757_{3 / 2}$ & 5479.310 & 1 & 2 & 18245.403 & $24982_{7 / 2}^{\circ}-43227_{5 / 2}$ \\
\hline 5530.693 & 50 & 150 & 18075.896 & $9711_{7 / 2}-27787_{9 / 2}^{\circ}$ & 5477.380 & 2 & 1 & 18251.832 & $20120_{5 / 2}^{\circ}-38372_{3 / 2}$ \\
\hline 5529.782 & 10 & 8 & 18078.874 & $20989_{9 / 2}^{\circ}-39068_{7 / 2}$ & 5477.046 & 50 & 50 & 18252.945 & $17272_{9 / 2}^{\circ}-35525_{11 / 2}$ \\
\hline 5529.651 & 5 & 8 & 18079.302 & $26424_{5 / 2}^{\circ}-44503_{7 / 2}$ & 5474.856 & 50 & 20 & 18260.246 & $1859_{3 / 2}-20120_{5 / 2}^{\circ}$ \\
\hline 5528.001 & 50 & 50 & 18084.698 & $20080_{7 / 2}^{\circ}-38165_{7 / 2}$ & 5473.616 & 3 & 4 & 18264.383 & $24982_{7 / 2}^{\circ}-43246_{7 / 2}$ \\
\hline 5524.219 & 100 & 100 & 18097.079 & $16564_{11 / 2}^{\circ}-34661_{11 / 2}$ & 5473.404 & 25 & 40 & 18265.090 & $7001_{3 / 2}-25266_{1 / 2}^{\circ}$ \\
\hline 5523.431 & 20 & 15 & 18099.661 & $20969_{7 / 2}^{\circ}-39068_{7 / 2}$ & 5471.387 & 50 & 75 & 18271.824 & $22139_{9 / 2}^{\circ}-404.11_{7 / 2}$ \\
\hline 5520.753 & 10 & 5 & 18108.441 & $28243_{5 / 2}^{\circ}-46352_{7 / 2}$ & & & & & $18118_{3 / 2}-36390_{3 / 2}^{\circ}$ \\
\hline 5520.341 & 15 & 8 & 18109.792 & $8378_{7 / 2}^{\circ}-26488_{5 / 2}$ & 5470.937 & 15 & 20 & 18273.327 & $17272_{9 / 2}^{\circ}-35545_{9 / 2}$ \\
\hline 5518.722 & 15 & 15 & 18115.105 & $15786_{5 / 2}-33902_{7 / 2}^{\circ}$ & 5469.708 & 2 & 2 & 18277.432 & $20158_{5 / 2}-38436_{3 / 2}^{\circ}$ \\
\hline 5517.791 & 10 & 8 & 18118.161 & $15236_{3 / 2}-33354_{5 / 2}^{\circ}$ & 5468.742 & 20 & 15 & 18280.661 & $17460_{5 / 2}^{\circ}-35741_{5 / 2}$ \\
\hline 5514.743 & 10 & 15 & 18128.175 & $26424_{5 / 2}^{\circ}-44552_{5 / 2}$ & 5466.685 & 2 & 2 & 18287.539 & $24463_{5 / 2}^{\circ}-42751_{7 / 2}$ \\
\hline 5514.438 & 4 & 8 & 18129.178 & $28026_{5 / 2}-46155_{5 / 2}^{\circ}$ & 5466.266 & 4 & 2 & 18288.941 & $9238_{9 / 2}^{\circ}-27526_{9 / 2}$ \\
\hline 5513.871 & 8 & 15 & 18131.042 & $22513_{5 / 2}^{\circ}-40644_{5 / 2}$ & 5464.784 & 5 & 5 & 18293.901 & $19248_{5 / 2}^{\circ}-37542_{3 / 2}$ \\
\hline 5512.699 & 20 & 15 & 18134.897 & $38757_{3 / 2}-56892_{5 / 2}^{\circ}$ & 5462.611 & 150 & 150 & 18301.178 & $14275_{9 / 2}^{\circ}-32576_{7 / 2}$ \\
\hline 5512.191 & $8 \mathrm{~h}$ & 5 & 18136.568 & $14484_{11 / 2}^{\circ}-32620_{11 / 2}$ & 5461.890 & 20 & 25 & 18303.594 & $18973_{7 / 2}^{\circ}-37277_{7 / 2}$ \\
\hline 5510.680 & 50 & 40 & 18141.541 & $15242_{9 / 2}^{\circ}-33384_{9 / 2}$ & 5461.735 & 100 & 100 & 18304.113 & $13468_{9 / 2}^{\circ}-31773_{9 / 2}$ \\
\hline 5507.948 & $50 \mathrm{~s}$ & 25 & 18150.539 & $10673_{5 / 2}^{\circ}-28823_{5 / 2}$ & 5461.151 & 10 & 8 & 18306.071 & $9720_{7 / 2}^{\circ}-28026_{5 / 2}$ \\
\hline 5506.334 & 3 & 2 & 18155.859 & $17722_{9 / 2}^{\circ}-35878_{7 / 2}$ & 5458.403 & 4 & 2 & 18315.287 & $11116_{7 / 2}^{\circ}-29431_{7 / 2}$ \\
\hline 5504.964 & 3 & 8 & 18160.378 & $31800_{7 / 2}^{\circ}-49960_{7 / 2}$ & 5458.105 & 20 & 40 & 18316.287 & $23012_{3 / 2}^{\circ}-41328_{5 / 2}$ \\
\hline 5502.034 & 2 & 4 & 18170.049 & $23518_{7 / 2}^{\circ}-41688_{7 / 2}$ & 5453.355 & 2 & 3 & 18332.240 & $25440_{5 / 2}^{\circ}-43772_{5 / 2}$ \\
\hline 5501.944 & 50 & 50 & 18170.346 & $6244_{1 / 2}-24414_{3 / 2}^{\circ}$ & 5452.518 & 5 & 4. & 18335.055 & $20158_{5 / 2}-38493_{5 / 2}^{\circ}$ \\
\hline 5501.543 & 25 & 25 & 18171.670 & $20120_{5 / 2}^{\circ}-38291_{7 / 2}$ & 5452.334 & 15 & 20 & 18335.673 & $24309_{11 / 2}^{\circ}-42644_{13 / 2}$ \\
\hline 5497.752 & 15 & 15 & 18184.200 & $15453_{7 / 2}^{\circ}-33637_{7 / 2}$ & 5449.895 & 20 & 10 & 18343.879 & $4490_{5 / 2}^{\circ}-22834_{7 / 2}$ \\
\hline 5496.464 & 2 & 1 & 18188.461 & $9061_{5 / 2}-27249_{7 / 2}^{\circ}$ & 5449.479 & 200 & 200 & 18345.279 & $14275_{9 / 2}^{\circ}-32620_{11 / 2}$ \\
\hline
\end{tabular}


TABLE 3. Classified lines of Th II-Continued

\begin{tabular}{|c|c|c|c|c|c|c|c|c|c|}
\hline \multirow{2}{*}{$\begin{array}{c}\text { Wavelength } \\
\AA\end{array}$} & \multicolumn{2}{|c|}{ Intensity } & \multirow{2}{*}{$\begin{array}{c}\text { Wavenumber } \\
\mathrm{cm}^{-1}\end{array}$} & \multirow{2}{*}{ Classification } & \multirow{2}{*}{$\begin{array}{c}\text { Wavelength } \\
\AA\end{array}$} & \multicolumn{2}{|c|}{ Intensity } & \multirow{2}{*}{$\begin{array}{l}\text { Wavenumber } \\
\qquad \mathrm{cm}^{-1}\end{array}$} & \multirow{2}{*}{ Classification } \\
\hline & Lamp & Spark & & & & Lamp & Spark & & \\
\hline 5448.568 & $4 h$ & 5 & 18348.347 & $28587_{5 / 2}^{\circ}-46935_{3 / 2}$ & 5396.538 & 8 & 20 & 18525.248 & $20310_{5 / 2}^{\circ}-38836_{3 / 2}$ \\
\hline 5447.313 & 50 & 20 & 18352.574 & $22014_{11 / 2}^{\circ}-40367_{9 / 2}$ & 5393.113 & $15 b$ & 75 & 18537.013 & $20080_{7 / 2}^{\circ}-38617_{9 / 2}$ \\
\hline 5446.021 & 25 & 8 & 18356.928 & $12902_{3 / 2}^{\circ}-31259_{5 / 2}^{\circ}$ & 5392.569 & 200 & 75 & 18538.883 & $4146_{7 / 2}-22685_{7 / 2}^{\circ}$ \\
\hline \multirow[t]{2}{*}{5445.295} & 4 & 2 & 18359.375 & $28243_{5 / 2}^{\circ}-46603_{5 / 2}$ & 5392.356 & 20 & 40 & 18539.615 & $19248_{5 / 2}^{\circ}-37787_{7 / 2}$ \\
\hline & & & & $8605_{5 / 2}-26965_{3 / 2}^{\circ}$ & 5391.942 & 2 & 3 & 18541.038 & $25266_{1 / 2}^{\circ}-43807_{3 / 2}$ \\
\hline 5444.792 & 5 & 4 & 18361.071 & $27249_{7 / 2}^{\circ}-45610_{5 / 2}$ & 5391.071 & 20 & 10 & 18544.034 & $6213_{9 / 2}-24757_{9 / 2}^{\circ}$ \\
\hline 5444.589 & 2 & 4 & 18361.756 & $22685_{7 / 2}^{\circ}-41047_{9 / 2}$ & 5390.658 & 1 & 4 & 18545.454 & $31924_{11 / 2}^{\circ}-50470_{9 / 2}$ \\
\hline 5444.371 & 3 & 4 & 18362.491 & $20989_{9 / 2}^{\circ}-39352_{11 / 2}$ & 5390.458 & $150 \mathrm{~b}$ & 75 & 18546.142 & $6700_{9 / 2}^{\circ}-25246_{9 / 2}^{\circ}$ \\
\hline 5443.999 & 3 & 5 & 18363.746 & $25440_{5 / 2}^{\circ}-43803_{7 / 2}$ & 5390.197 & 10 & 25 & 18547.040 & $27357_{9 / 2}^{\circ}-45904_{9 / 2}$ \\
\hline 5443.114 & 100 & 50 & 18366.731 & $4146_{7 / 2}-22513_{5 / 2}^{\circ}$ & 5389.913 & 2 & 2 & 18548.018 & $22106_{5 / 2}-40654_{5 / 2}^{\circ}$ \\
\hline 5442.943 & 1 & 3 & 18367.308 & $25440_{5 / 2}^{\circ}-43807_{3 / 2}$ & 5389.453 & $50 \mathrm{~h}$ & 50 & 18549.601 & $16906_{7 / 2}^{\circ}-35456_{9 / 2}$ \\
\hline 5441.298 & 5 & 15 & 18372.861 & $24873_{5 / 2}^{\circ}-43246_{7 / 2}$ & 5389.126 & 15 & 50 & 18550.726 & $27249_{7 / 2}^{\circ}-45800_{5 / 2}$ \\
\hline 5437.385 & 50 & 50 & 18386.083 & $12570_{7 / 2}-30956_{9 / 2}^{\circ}$ & 5388.903 & 20 & 15 & 18551.494 & $13248_{9 / 2}-31800_{7 / 2}^{\circ}$ \\
\hline 5436.499 & 20 & 75 & 18389.079 & $30223_{15 / 2}^{\circ}-48612_{13 / 2}$ & 5386.783 & 15 & 10 & 18558.795 & $1521_{5 / 2}-20080_{7 / 2}^{\circ}$ \\
\hline 5435.893 & 150 & 200 & 18391.129 & $12488_{9 / 2}^{\circ}-30879_{7 / 2}$ & 5386.476 & $8 b$ & $10 \mathrm{~b}$ & 18559.853 & $22014_{11 / 2}^{\circ}-40574_{11 / 2}$ \\
\hline 5435.722 & 20 & 25 & 18391.708 & $9202_{7 / 2}^{\circ}-27593_{5 / 2}$ & 5386.425 & $8 g$ & $10 \mathrm{~b}$ & 18560.029 & $13250_{5 / 2}-31810_{5 / 2}^{\circ}$ \\
\hline 5435.126 & 100 & 100 & 18393.725 & $12485_{\tau / 2}^{\circ}-30879_{\tau / 2}$ & 5385.731 & 8 & 10 & 18562.420 & $20989_{9 / 2}^{\circ}-39552_{9 / 2}^{\circ}$ \\
\hline \multirow[t]{2}{*}{5434.947} & 25 & 50 & 18394.330 & $25414_{11 / 2}^{\circ}-43809_{9 / 2}$ & 5384.031 & 50 & 15 & 18568.281 & $0_{3 / 2}-18568_{1 / 2}^{\circ}$ \\
\hline & & & & $15242_{9 / 2}^{\circ}-33637_{7 / 2}$ & 5383.899 & 2 & 1 & 18568.736 & $21131_{3 / 2}^{\circ}-39700_{5 / 2}$ \\
\hline 5433.699 & 75 & 75 & 18398.555 & $11116_{7 / 2}^{\circ}-29515_{9 / 2}$ & 5383.746 & 2 & 2 & 18569.264 & $18118_{3 / 2}-36687_{5 / 2}^{\circ}$ \\
\hline 5433.287 & 20 & 8 & 18399.950 & $4113_{5 / 2}-22513_{5 / 2}^{\circ}$ & 5382.923 & 100 & 50 & 18572.103 & $4113_{5 / 2}-22685_{7 / 2}^{\circ}$ \\
\hline 5432.580 & 10 & 25 & 18402.345 & $17722_{9 / 2}^{\circ}-36125_{9 / 2}^{\circ}$ & 5380.211 & 8 & 20 & 18581.465 & $22642_{9 / 2}^{\circ}-41223_{11 / 2}^{\circ}$ \\
\hline 5431.774 & 10 & 25 & 18405.076 & $22642_{9 / 2}^{\circ}-41047_{9 / 2}$ & 5379.700 & 8 & 15 & 18583.229 & $20969_{7 / 2}^{\circ}-39552_{9 / 2}$ \\
\hline 5431.418 & 8 & 4 & 18406.282 & $8018_{3 / 2}-26424_{5 / 2}^{\circ}$ & 5379.374 & 2 & 10 & 18584.356 & $25188_{3 / 2}^{\circ}-43772_{5 / 2}$ \\
\hline 5428.961 & 10 & 20 & 18414.612 & $19050_{3 / 2}^{\circ}-37465_{5 / 2}$ & 5375.767 & 100 & 100 & 18596.825 & $15305_{9 / 2}-33902_{7 / 2}^{\circ}$ \\
\hline 5428.048 & 8 & 10 & 18417.709 & $20310_{5 / 2}^{\circ}-38728_{5 / 2}$ & 5375.349 & 200 & 100 & 18598.271 & $1521_{5 / 2}-20120_{5 / 2}^{\circ}$ \\
\hline 5426.526 & 1 & 1 & 18422.875 & $20158_{5 / 2}-38581_{5 / 2}^{\circ}$ & 5373.223 & 8 & 15 & 18605.630 & $19912_{13 / 2}^{\circ}-38517_{13 / 2}$ \\
\hline 5426.261 & 15 & 15 & 18423.775 & $12570_{7 / 2}-30994_{7 / 2}^{\circ}$ & 5373.148 & 4 & 8 & 18605.890 & $20158_{5 / 2}-38764_{7 / 2}^{\circ}$ \\
\hline 5425.678 & 300 & 300 & 18425.754 & $9585_{5 / 2}^{\circ}-28011_{3 / 2}$ & 5372.938 & 20 & 501 & 18606.617 & $17272_{9 / 2}^{\circ}-35878_{7 / 2}$ \\
\hline 5424.793 & 3 & 2 & 18428.760 & $27787_{9 / 2}^{\circ}-46216_{11 / 2}$ & 5372.456 & 10 & 8 & 18608.286 & $8018_{3 / 2}-26626_{1 / 2}^{\circ}$ \\
\hline 5424.135 & 50 & 75 & 18430.996 & $22139_{9 / 2}^{\circ}-40570_{\tau / 2}$ & 5372.397 & 2 & 3 & 18608.491 & $20120_{5 / 2}^{\circ}-38728_{5 / 2}$ \\
\hline 5421.837 & 75 & 75 & 18438.808 & $7001_{3 / 2}-25440_{5 / 2}^{\circ}$ & 5369.170 & $40 \mathrm{~h}$ & 50 & 18619.675 & $17121_{3 / 2}^{\circ}-35741_{5 / 2}$ \\
\hline 5421.205 & 10 & 10 & 18440.957 & $9585_{5 / 2}^{\circ}-28026_{5 / 2}$ & 5364.363 & 5 & 10 & 18636.359 & $22355_{1 / 2}^{\circ}-40991_{3 / 2}$ \\
\hline 5419.658 & 3 & 4 & 18446.221 & $20310_{5 / 2}^{\circ}-38757_{3 / 2}$ & 5364.175 & 20 & 50 & 18637.013 & $20120_{5 / 2}^{\circ}-38757_{3 / 2}$ \\
\hline \multirow[t]{2}{*}{5415.434} & $200 \mathrm{~b}$ & 200 & 18460.609 & $33215_{3 / 2}^{\circ}-51676_{3 / 2}$ & 5363.611 & 25 & 40 & 18638.972 & $16906_{7 / 2}^{\circ}-35545_{9 / 2}$ \\
\hline & & & & $14275_{9 / 2}^{\circ}-32736_{7 / 2}$ & 5362.257 & 20 & 20 & 18643.679 & $8605_{5 / 2}-27249_{7 / 2}^{\circ}$ \\
\hline 5414.530 & $40 \mathrm{~h}$ & 40 & 18463.691 & $15710_{3 / 2}^{\circ}-34174_{5 / 2}$ & 5361.766 & 2 & 4 & 18645.386 & $26965_{3 / 2}^{\circ}-45610_{5 / 2}$ \\
\hline 5413.805 & 20 & 25 & 18466.163 & $17727_{11 / 2}-36193_{9 / 2}^{\circ}$ & 5361.409 & 75 & 100 & 18646.627 & $24309_{11 / 2}^{\circ}-42955_{9 / 2}$ \\
\hline 5413.128 & 3 & 4 & 18468.473 & $20989_{9 / 2}^{\circ}-39458_{7 / 2}$ & 5360.998 & 15 & 15 & 18648.057 & $20080_{7 / 2}^{\circ}-38728_{5 / 2}^{\circ}$ \\
\hline 5412.808 & 15 & 50 & 18469.565 & $23730_{9 / 2}^{\circ}-42200_{9 / 2}$ & 5357.832 & 5 & 2 & 18659.076 & $41328_{5 / 2}-59987_{3 / 2}^{\circ}$ \\
\hline 5411.005 & 2 & 10 & 184.75 .719 & $33354_{5 / 2}^{\circ}-51830_{7 / 2}$ & 5357.446 & 1 & 3 & 18660.421 & $33902_{7 / 2}^{\circ}-52562_{7 / 2}$ \\
\hline 5410.258 & $10 \mathrm{~b}$ & 15 & 18478.270 & $22513_{5 / 2}^{\circ}-40991_{3 / 2}^{\circ}$ & 5356.471 & $10 \mathrm{~h}$ & 10 & 18663.817 & $14545_{5 / 2}^{\circ}-33209_{7 / 2}$ \\
\hline 5407.078 & $2 \mathbf{b}$ & 2 & 18489.137 & $25607_{9 / 2}^{\circ}-44096_{9 / 2}$ & 5356.199 & $3 b$ & 10 & 18664.765 & $23012_{3 / 2}^{\circ}-41676_{3 / 2}$ \\
\hline \multirow[t]{2}{*}{5406.336} & 20 & 50 & 18491.674 & $23730_{9 / 2}^{\circ}-42222_{7 / 2}$ & 5354.747 & 2 & 5 & 18669.826 & $31800_{7 / 2}^{\circ}-50470_{9 / 2}$ \\
\hline & & & & $18973_{7 / 2}^{\circ}-37465_{5 / 2}$ & 5352.334 & $3 b$ & 3 & 18678.243 & $13250_{5 / 2}-31928_{3 / 2}^{\circ}$ \\
\hline 5405.207 & 10 & 3 & 18495.537 & $4146_{7 / 2}-22642_{9 / 2}^{\circ}$ & 5351.614 & 4 & 10 & 18680.756 & $20686_{5 / 2}^{\circ}-39366_{5 / 2}$ \\
\hline 5402.780 & 1 & 8 & 18503.845 & $34174_{5 / 2}-52678_{5 / 2}^{\circ}$ & 5351.452 & 8 & 40 & 18681.321 & $25414_{11 / 2}^{\circ}-44096_{9 / 2}$ \\
\hline \multirow[t]{2}{*}{5400.872} & 4 & 4 & 18510.382 & $33354_{5 / 2}^{\circ}-51865_{5 / 2}$ & 5351.325 & 10 & 20 & 18681.765 & $23518_{7 / 2}^{\circ}-42200_{9 / 2}$ \\
\hline & & & & $16033_{5 / 2}^{\circ}-34543_{5 / 2}$ & 5350.495 & 2 & 10 & 18684.663 & $21682_{7 / 2}^{\circ}-40367_{9 / 2}$ \\
\hline 5396.705 & 3 & 3 & 18524.674 & $23697_{7 / 2}^{\circ}-42222_{7 / 2}$ & 5349.401 & 1 & 10 & 18688.484 & $35156_{5 / 2}^{\circ}-53845_{5 / 2}^{\circ}$ \\
\hline
\end{tabular}


TABlE 3. Classified lines of Th II-Continued

\begin{tabular}{|c|c|c|c|c|c|c|c|c|c|}
\hline \multirow{2}{*}{$\begin{array}{c}\text { Wavelength } \\
\AA\end{array}$} & \multicolumn{2}{|c|}{ Intensity } & \multirow{2}{*}{$\begin{array}{c}\text { Wavenumber } \\
\mathbf{c m}^{-1}\end{array}$} & \multirow{2}{*}{ Classification } & \multirow{2}{*}{$\begin{array}{c}\text { Wavelength } \\
\AA\end{array}$} & \multicolumn{2}{|c|}{ Intensity } & \multirow{2}{*}{$\begin{array}{c}\text { Wavenumber } \\
\mathbf{c m}^{-1}\end{array}$} & \multirow{2}{*}{ Classification } \\
\hline & Lamp & Spark & & & & Lamp & Spark & & \\
\hline 5347.432 & 10 & 15 & 18695.365 & $23187_{13 / 2}^{\circ}-41882_{13 / 2}$ & 5292.919 & 15 & 20 & 18887.911 & $21682_{7 / 2}^{\circ}-40570_{7 / 2}$ \\
\hline 5347.036 & $100 w$ & 150 & 18696.750 & $22028_{15 / 2}^{\circ}-40724_{13 / 2}$ & 5291.895 & 10 & 8 & 18891.565 & $16564_{11 / 2}^{\circ}-35456_{9 / 2}$ \\
\hline 5346.375 & 50 & 40 & 18699.061 & $9238_{9 / 2}^{\circ}-27937_{11 / 2}$ & 5291.133 & 3 & 3 & 18894.286 & $19050_{3 / 2}^{\circ}-37945_{5 / 2}$ \\
\hline 5345.000 & 15 & 25 & 18703.871 & $23518_{7 / 2}^{\circ}-42222_{7 / 2}$ & 5290.665 & 4 & 5 & 18895.958 & $27357_{9 / 2}^{\circ}-46253_{9 / 2}^{\circ}$ \\
\hline 5344.244 & 4 & 20 & 18706.517 & $31924_{11 / 2}^{\circ}-50631_{11 / 2}$ & 5289.895 & 50 & 25 & 18898.708 & $4113_{5 / 2}-23012_{3 / 2}^{\circ}$ \\
\hline 5343.297 & 15 & 50 & 18709.833 & $22014_{11 / 2}^{\circ}-40724_{13 / 2}$ & 5289.503 & $5 h$ & 5 & 18900.109 & $14484_{11 / 2}^{\circ}-33384_{9 / 2}$ \\
\hline 5342.373 & 2 & 3 & 18713.069 & $22685_{7 / 2}^{\circ}-41398_{9 / 2}$ & 5287.963 & 3 & 3 & 18905.613 & $20989_{9 / 2}^{\circ}-39895_{9 / 2}$ \\
\hline 5339.960 & $10 \mathrm{~b}$ & 10 & 18721.524 & $15453_{7 / 2}^{\circ}-34174_{5 / 2}$ & 5284.758 & 8 & 15 & 18917.078 & $19248_{5 / 2}^{\circ}-38165_{7 / 2}$ \\
\hline 5333.163 & 15 & 10 & 18745.384 & $18816_{13 / 2}^{\circ}-37562_{11 / 2}$ & 5284.542 & 50 & 50 & 18917.851 & $13818_{7 / 2}^{\circ}-32736_{7 / 2}$ \\
\hline 5331.226 & 2 & 1 & 18752.195 & $12219_{3 / 2}-30972_{5 / 2}^{\circ}$ & 5284.305 & 4 & 5 & 18918.700 & $21297_{5 / 2}^{\circ}-40216_{5 / 2}$ \\
\hline 5329.758 & 25 & 25 & 18757.360 & $1116_{7 / 2}^{\circ}-29873_{7 / 2}$ & 5280.286 & 5 & 5 & 18933.099 & $10855_{7 / 2}-29788_{9 / 2}^{\circ}$ \\
\hline 5329.651 & 5 & 8 & 18757.737 & $20310_{5 / 2}^{\circ}-39068_{7 / 2}$ & 5278.375 & 3 & 5 & 18939.954 & $14790_{7 / 2}^{\circ}-33730_{5 / 2}$ \\
\hline 5329.476 & 40 & 50 & 18758.352 & $18816_{13 / 2}^{\circ}-37575_{13 / 2}$ & 5278.228 & $2 \mathrm{~b}$ & 10 & 18940.481 & $26963_{7 / 2}^{\circ}-45904_{9 / 2}$ \\
\hline 5329.373 & 50 & 50 & 18758.715 & $10673_{5 / 2}^{\circ}-29431_{7 / 2}^{\circ}$ & 5277.500 & 300 & 200 & 18943.094 & $10572_{9 / 2}^{\circ}-29515_{9 / 2}^{\circ}$ \\
\hline 5328.339 & 5 & 15 & 18762.355 & $254.14_{11 / 2}^{\circ}-44177_{11 / 2}^{\circ}$ & 5277.219 & 10 & 50 & 18944.103 & $17121_{3 / 2}^{\circ}-36065_{5 / 2}^{\circ}$ \\
\hline 5325.559 & 3 & 4 & 18772.149 & $20686_{5 / 2}^{\circ}-39458_{7 / 2}$ & 5276.407 & 40 & 15 & 18947.018 & $8018_{3 / 2}-26965_{3 / 2}^{\circ}$ \\
\hline 5325.145 & 200 & 200 & 18773.609 & $12485_{7 / 2}^{\circ}-31259_{5 / 2}$ & 5276.293 & 1 & 10 & 18947.427 & $33730_{5 / 2}-52678_{5 / 2}^{\circ}$ \\
\hline 5324.650 & $20 \mathrm{~b}$ & 20 & 18775.354 & $16818_{7 / 2}-35593_{9 / 2}^{\circ}$ & 5275.775 & 3 & 4 & 18949.287 & $20989_{9 / 2}^{\circ}-39939_{11 / 2}$ \\
\hline 5324.043 & 3 & 20 & 18777.494 & $25188_{3 / 2}^{\circ}-43965_{1 / 2}$ & 5275.447 & 3 & 5 & 18950.466 & $19912_{13 / 2}^{\circ}-38862_{11 / 2}$ \\
\hline 5322.464 & $3 b$ & $10 \mathrm{~b}$ & 18783.065 & $24463_{5 / 2}^{\circ}-43246_{7 / 2}^{\circ}$ & 5272.643 & 75 & 75 & 18960.543 & $16564_{11 / 2}^{\circ}-35525_{11 / 2}$ \\
\hline 5322.432 & 4 & $20 \mathrm{~b}$ & 18783.178 & $20080_{7 / 2}^{\circ}-38863_{5 / 2}$ & 5271.614 & 8 & 15 & 18964.244 & $23372_{3 / 2}^{\circ}-42336_{5 / 2}$ \\
\hline 5321.315 & 15 & 75 & 18787.121 & $12472_{5 / 2}^{\circ}-31259_{5 / 2}$ & 5270.744 & 5 & 50 & 18967.375 & $30101_{7 / 2}^{\circ}-49068_{5 / 2}$ \\
\hline 5320.991 & 8 & 15 & 18788.265 & $23730_{9 / 2}^{\circ}-42518_{7 / 2}^{\circ}$ & 5270.124 & 2 & 8 & 18969.606 & $29720_{3 / 2}^{\circ}-48689_{3 / 2}$ \\
\hline 5320.494 & 2 & 4 & 18790.020 & $24982_{7 / 2}^{\circ}-43772_{5 / 2}$ & 5269.650 & 15 & 20 & 18971.312 & $18973_{7 / 2}^{\circ}-37945_{5 / 2}$ \\
\hline 5318.423 & 50 & 20 & 18797.337 & $8605_{5 / 2}-27403_{3 / 2}^{\circ}$ & 5269.385 & 40 & 50 & 18972.266 & $16906_{7 / 2}^{\circ}-35878_{7 / 2}$ \\
\hline 5315.755 & 1 & 3 & 18806.771 & $31928_{3 / 2}^{\circ}-50735_{3 / 2}^{\circ}$ & 5268.932 & 15 & 20 & 18973.898 & $18568_{1 / 2}^{\circ}-37542_{3 / 2}$ \\
\hline 5313.671 & 10 & $15 b$ & 18814.147 & $18973_{7 / 2}^{\circ}-37787_{7 / 2}$ & 5268.737 & 15 & 25 & 18974.600 & $17837_{1 / 2}^{\circ}-36812_{1 / 2}$ \\
\hline 5313.612 & 8 & 20 & 18814.356 & $30310_{11 / 2}^{\circ}-49124_{11 / 2}$ & 5268.716 & $2 \mathrm{c}$ & 8 & 18974.675 & $17837_{1 / 2}^{\circ}-36812_{1 / 2}$ \\
\hline 5310.864 & 15 & 15 & 18824.091 & $9202_{7 / 2}^{\circ}-28026_{5 / 2}$ & 5267.365 & 10 & 40 & 18979.542 & $23372_{3 / 2}^{\circ}-42352_{5 / 2}$ \\
\hline 5310.260 & 200 & 100 & 18826.232 & $1859_{3 / 2}-20686_{5 / 2}^{\circ}$ & 5267.030 & $4 b$ & 5 & 18980.749 & $21297_{5 / 2}^{\circ}-40278_{3 / 2}$ \\
\hline 5310.103 & 4 & 15 & 18826.788 & $24982_{7 / 2}^{\circ}-43809_{9 / 2}$ & 5264.956 & 50 & 50 & 18988.226 & $16033_{5 / 2}^{\circ}-35021_{3 / 2}$ \\
\hline 5309.728 & 15 & 20 & 18828.118 & $19912_{13 / 2}^{\circ}-38740_{11 / 2}^{\circ / 2}$ & 5263.118 & 8 & 25 & 18994.857 & $27357_{9 / 2}^{\circ}-46352_{7 / 2}^{\circ}$ \\
\hline 5308.430 & 20 & 50 & 18832.722 & $15710_{3 / 2}^{\circ}-34543_{5 / 2}$ & 5260.867 & 20 & 20 & 19002.985 & $22685_{7 / 2}^{\circ}-41688_{7 / 2}$ \\
\hline 5308.154 & 15 & 40 & 18833.701 & $23518_{7 / 2}^{\circ}-42352_{5 / 2}^{\circ}$ & 5258.471 & 8 & 4 & 19011.643 & $18118_{3 / 2}-37130_{1 / 2}^{\circ}$ \\
\hline 5307.710 & 2 & 200 & 18835.276 & $35525_{11 / 2}-54360_{9 / 2}^{\circ}$ & 5255.972 & 50 & 50 & 19020.682 & $23730_{9 / 2}^{\circ}-42751_{7 / 2}$ \\
\hline 5307.466 & 100 & 150 & 18836.142 & $16564_{11 / 2}^{\circ}-35400_{13 / 2}$ & 5253.939 & 2 & 4 & 19028.042 & $27357_{9 / 2}^{\circ}-46385_{7 / 2}$ \\
\hline 5306.033 & 1 & 5 & 18841.229 & $27526_{9 / 2}-46368_{9 / 2}^{\circ}$ & 5253.443 & 100 & 75 & 19029.839 & $15144_{3 / 2}^{\circ}-34174_{5 / 2}$ \\
\hline 5305.577 & 75 & 50 & 18842.849 & $9400_{5 / 2}-28243_{5 / 2}^{\circ}$ & 5253.249 & 5 & 75 & 19030.541 & $20120_{5 / 2}^{\circ}-39150_{3 / 2}$ \\
\hline 5305.495 & 4 & 5 & 18843.140 & $25607_{9 / 2}^{\circ}-44450_{9 / 2}$ & 5252.766 & 50 & 75 & 19032.291 & $22014_{11 / 2}^{i}-41047_{9 / 2}$ \\
\hline 5304.675 & $15 b$ & $20 \mathrm{~b}$ & 18846.053 & $22642_{9 / 2}^{\circ}-41488_{7 / 2}^{\circ}$ & 5252.280 & 3 & 10 & 19034.052 & $25266_{1 / 2}^{o}-44300_{3 / 2}$ \\
\hline 5304.620 & 25 & 25 & 18846.248 & $14790_{7 / 2}^{\circ}-33637_{7 / 2}$ & 5251.930 & 4 & 10 & 19035.321 & $25414_{11 / 2}^{\circ}-44450_{9 / 2}$ \\
\hline 5303.048 & 50 & 40 & 18851.835 & $12902_{3 / 2}^{\circ}-31754_{5 / 2}$ & 5249.853 & 2 & 8 & 19042.852 & $30452_{9 / 2}-49495_{9 / 2}^{\circ}$ \\
\hline 5302.698 & 3 & 4 & 18853.079 & $31810_{5 / 2}^{\circ}-50663_{5 / 2}$ & 5249.657 & 3 & 2 & 19043.563 & $19248_{5 / 2}^{\circ}-38291_{7 / 2}$ \\
\hline & & & & $17272_{9 / 2}^{\circ}-36125_{9 / 2}^{\circ}$ & 5248.855 & 3 & & 19046.472 & $24757_{9 / 2}^{\circ}-43803_{7 / 2}$ \\
\hline 5301.649 & 3 & 3 & 18856.809 & $19248_{5 / 2}^{\circ}-38105_{5 / 2}$ & 5247.654 & 500 & 100 & 19050.831 & $0_{3 / 2}-19050_{3 / 2}^{\circ}$ \\
\hline 5301.403 & 100 & 100 & 18857.684 & $14101_{i / 2}^{\circ}-32959_{3 / 2}$ & 5242.091 & 3 & 200 & 19071.048 & $22028_{15 / 2}^{\circ}-41099_{15 / 2}$ \\
\hline 5300.969 & 2 & 5 & 18859.228 & $27357_{9 / 2}^{\circ}-46216_{11 / 2}$ & 5240.200 & 8 & 75 & 19077.930 & $6168_{7 / 2}^{\circ}-25246_{9 / 2}$ \\
\hline 5296.676 & 10 & 40 & 18874.513 & $15144_{3 / 2}^{\circ}-34019_{3 / 2}$ & 5237.907 & 5 & 500 & 19086.282 & $17722_{9 / 2}^{\circ}-36809_{7 / 2}^{\circ}$ \\
\hline 5295.617 & $20 \mathrm{~h}$ & 15 & 18878.288 & $8378_{7 / 2}^{\circ}-27257_{7 / 2}^{\circ}$ & 5237.626 & 2 & 300 & 19087.306 & $26647_{13 / 2}^{\circ}-45735_{11 / 2}$ \\
\hline 5294.826 & 2 & 3 & 18881.108 & $30956_{9 / 2}^{\circ}-49837_{9 / 2}$ & 5236.425 & 4 & 10 & 19091.684 & $14545_{5 / 2}^{\circ}-33637_{7 / 2}$ \\
\hline
\end{tabular}


TABLE 3. Classified lines of Th II-Continued

\begin{tabular}{|c|c|c|c|c|c|c|c|c|c|}
\hline \multirow{2}{*}{$\begin{array}{c}\text { Wavelength } \\
\AA\end{array}$} & \multicolumn{2}{|c|}{ Intensity } & \multirow{2}{*}{$\begin{array}{c}\text { Wavenumber } \\
\mathbf{c m}^{-1}\end{array}$} & \multirow{2}{*}{ Classification } & \multirow{2}{*}{$\begin{array}{c}\text { Wavelength } \\
\AA\end{array}$} & \multicolumn{2}{|c|}{ Intensity } & \multirow{2}{*}{$\begin{array}{l}\text { Wavenumber } \\
\text { cm }^{-1}\end{array}$} & \multirow{2}{*}{ Classification } \\
\hline & Lamp & Spark & & & & Lamp & Spark & & \\
\hline 5233.225 & 150 & 175 & 19103.358 & $9720_{7 / 2}^{\circ}-28823_{5 / 2}$ & 5148.212 & 200 & 1500 & 19418.810 & $15242_{9 / 2}^{\circ}-34661_{11 / 2}$ \\
\hline 5232.985 & 0 & 25 & 19104.234 & $37787_{7 / 2}-56892_{5 / 2}^{\circ}$ & 5145.035 & $150 \mathrm{~s}$ & 200 & 19430.800 & $26965_{3 / 2}^{\circ}-46395_{3 / 2}^{\circ}$ \\
\hline 5231.715 & 3 & 4 & 19108.871 & $14275_{9 / 2}^{\circ}-33384_{9 / 2}$ & & & & & $7331_{5 / 2}^{\circ}-26762_{3 / 2}^{\circ / 2}$ \\
\hline 5227.642 & 2 & 10 & 19123.759 & $19248_{5 / 2}^{\circ}-38372_{3 / 2}$ & 5143.268 & 3001 & 500 & 19437.476 & $1859_{3 / 2}-21297_{5 / 2}^{\circ}$ \\
\hline 5225.579 & 3 & 75 & 19131.309 & $18973_{7 / 2}^{\circ}-38105_{5 / 2}$ & 5137.708 & 20 & 25 & 19458.511 & $25440_{5 / 2}^{\circ}-44898_{7 / 2}^{\circ}$ \\
\hline 5225.074 & 3 & 2 & 19133.158 & $12219_{3 / 2}-31353_{3 / 2}^{\circ}$ & 5135.232 & 10 & 10 & 19467.893 & $31800_{7 / 2}^{\circ}-51268_{7 / 2}$ \\
\hline 5224.116 & 5 & 2 & 19136.666 & $7828_{1 / 2}-26965_{3 / 2}^{\circ}$ & 5132.236 & 8 & 8 & 19479.257 & $14790_{7 / 2}^{\circ}-34270_{9 / 2}$ \\
\hline 5219.960 & 4 & 8 & 19151.902 & $13468_{9 / 2}^{\circ}-32620_{11 / 2}^{\circ}$ & 5131.490 & 4 & 5 & 19482.089 & $17983_{5 / 2}^{\circ}-37465_{5 / 2}^{\circ}$ \\
\hline 5218.527 & 75 & 100 & 19157.162 & $7331_{5 / 2}^{\circ}-26488_{5 / 2}$ & 5131.072 & 25 & 250 & 19483.676 & $15242_{9 / 2}^{\circ}-34726_{7 / 2}^{\circ}$ \\
\hline 5216.593 & 200 & 1 & 19164.264 & $1521_{5 / 2}-20686_{5 / 2}^{\circ}$ & 5129.844 & $5 \mathrm{~h}$ & 20 & 19488.340 & $14790_{7 / 2}^{\circ}-34279_{7 / 2}^{\circ}$ \\
\hline 5214.156 & 5 & 100 & 19173.221 & $19912_{13 / 2}^{\circ}-39085_{13 / 2}$ & 5124.430 & $3 \mathbf{h}$ & 10 & 19508.929 & $19248_{5 / 2}^{\circ}-38757_{3 / 2}$ \\
\hline 5211.574 & 5 & 8 & 19182.720 & $9061_{5 / 2}-28243_{5 / 2}^{\circ}$ & 5123.477 & 2 & 10 & 19512.558 & $21131_{3 / 2}^{\circ}-40644_{5 / 2}^{\circ}$ \\
\hline 5210.572 & 3 & 1 & 19186.408 & $9400_{5 / 2}-28587_{5 / 2}^{\circ}$ & 5122.896 & 3 & 3 & 19514.771 & $24873_{5 / 2}^{\circ}-44388_{5 / 2}^{\circ}$ \\
\hline 5206.659 & 15 & 50 & 19200.828 & $25607_{9 / 2}^{\circ}-44807_{7 / 2}$ & & & & & $22685_{7 / 2}^{\circ}-42200_{9 / 2}$ \\
\hline & & & & $10673_{5 / 2}^{\circ}-29873_{7 / 2}$ & 5119.564 & 0 & 15 & 19527.472 & $30310_{11 / 2}^{\circ}-49837_{9 / 2}^{\circ}$ \\
\hline 5206.489 & 25 & 20 & 19201.454 & $6213_{9 / 2}-25414_{11 / 2}^{\circ}$ & 5113.379 & 15 & 25 & 19551.091 & $4146_{7 / 2}-23697_{7 / 2}^{\circ}$ \\
\hline 5205.776 & 20 & 400 & 19204.084 & $15349_{11 / 2}^{\circ}-34.553_{9 / 2}$ & 5110.863 & 15 & 300 & 19560.716 & $16564_{11 / 2}^{\circ}-36125_{9 / 2}$ \\
\hline 5204.998 & 5 & 20 & 19206.955 & $17121_{3 / 2}^{\circ}-36328_{3 / 2}$ & 5108.743 & 0 & 25 & 19568.833 & $28923_{5 / 2}^{\circ}-48492_{5 / 2}^{\circ}$ \\
\hline 5196.042 & 15 & 15 & 19240.060 & $12570_{7 / 2}-31810_{5 / 2}^{\circ}$ & 5107.235 & 3 & 2 & 19574.611 & $7828_{1 / 2}-27403_{3 / 2}^{\circ}$ \\
\hline 5194.250 & 0 & 10 & 19246.698 & $20120_{5 / 2}^{\circ}-39366_{5 / 2}^{\circ}$ & 5104.778 & 2 & 1 & 19584.032 & $4146_{7 / 2}-23730_{9 / 2}^{\circ}$ \\
\hline 5193.822 & 75 & 100 & 19248.284 & $0_{3 / 2}-19248_{5 / 2}^{\circ}$ & 5104.563 & $3 \mathrm{~b}$ & 2 & 19584.857 & $7001_{3 / 2}-26586_{3 / 2}^{\circ}$ \\
\hline 5190.867 & 100 & 100 & 19259.241 & $4113_{5 / 2}-23372_{3 / 2}^{\circ}$ & 5103.765 & 5 & 100 & 19587.919 & $19248_{5 / 2}^{\circ}-38836_{3 / 2}^{\circ}$ \\
\hline 5189.674 & 10 & 300 & 19263.668 & $20288_{11 / 2}^{o}-39552_{9 / 2}$ & 5098.657 & $4 h$ & 2 & 19607.543 & $18214_{3 / 2}^{\circ}-37821_{3 / 2}^{\circ}$ \\
\hline \multirow[t]{2}{*}{5188.721} & 2 & 25 & 19267.206 & $22642_{9 / 2}^{\circ}-41909_{9 / 2}^{\circ}$ & 5098.039 & 100 & 75 & 19609.920 & $1521_{5 / 2}-21131_{3 / 2}^{\circ}$ \\
\hline & & & & $13468_{9 / 2}^{\circ}-32736_{7 / 2}^{\circ}$ & 5093.086 & 3 & 20 & 19628.990 & $14545_{5 / 2}^{\circ}-34174_{5 / 2}$ \\
\hline 5188.364 & 20 & 150 & 19268.532 & $12485_{7 / 2}^{\circ}-31754_{5 / 2}$ & 5090.752 & 300 & 400 & 19637.989 & $26965_{3 / 2}^{\circ}-46603_{5 / 2}^{\circ}$ \\
\hline 5184.730 & 10 & 75 & 19282.037 & $12472_{5 / 2}^{\circ}-31754_{5 / 2}^{\circ}$ & & & & & $8605_{5 / 2}-28243_{5 / 2}^{\circ}$ \\
\hline 5184.660 & 0 & 10 & 19282.298 & $28587_{5 / 2}^{\circ}-47869_{3 / 2}$ & 5089.224 & 3 & 1 & 19643.886 & $18973_{7 / 2}^{\circ}-38617_{9 / 2}^{\circ}$ \\
\hline 5183.987 & 100 & 250 & 19284.801 & $12488_{9 / 2}^{\circ}-31773_{9 / 2}^{\circ}$ & 5087.503 & 2 & 50 & 19650.531 & $20288_{11 / 2}^{\circ}-39939_{11 / 2}$ \\
\hline 5183.601 & 3 & 2 & 19286.237 & $20080_{7 / 2}^{\circ}-39366_{5 / 2}^{\circ}$ & 5085.117 & 2 & 10 & 19659.751 & $9061_{5 / 2}-28720_{3 / 2}^{\circ}$ \\
\hline 5182.527 & 20 & 30 & 19290.234 & $18816_{13 / 2}^{\circ}-38107_{15 / 2}$ & 5079.138 & 8 & 15 & 19682.893 & $11576_{3 / 2}^{\circ}-31259_{5 / 2}$ \\
\hline 5178.988 & 2 & 1 & 19303.415 & $36193_{9 / 2}^{\circ}-55496_{9 / 2}^{\circ}$ & 5076.600 & 5 & 50 & 19692.734 & $24757_{9 / 2}^{\circ}-44450_{9 / 2}^{\circ}$ \\
\hline 5177.097 & 0 & 25 & 19310.466 & $31353_{3 / 2}^{\circ}-50663_{5 / 2}$ & 5076.196 & 0 & 5 & 19694.301 & $29720_{3 / 2}^{\circ}-49414_{3 / 2}^{\circ}$ \\
\hline 5176.735 & 4 & 8 & 19311.816 & $15349_{11 / 2}^{\circ}-34661_{11 / 2}$ & 5075.470 & 15 & 150 & 19697.118 & $15324_{1 / 2}^{\circ}-35021_{3 / 2}^{o}$ \\
\hline 5175.073 & 2 & 5 & 19318.018 & $18973_{7 / 2}^{\circ}-38291_{7 / 2}$ & 5073.981 & 4 & 5 & 19702.898 & $20158_{5 / 2}-39861_{5 / 2}^{\circ}$ \\
\hline 5172.912 & 10 & 25 & 19326.088 & $10189_{11 / 2}^{\circ}-29515_{9 / 2}$ & 5073.080 & $5 s$ & 25 & 19706.397 & $19050_{3 / 2}^{\circ}-38757_{3 / 2}$ \\
\hline 5170.220 & 10 & 100 & 19336.151 & $11116_{7 / 2}^{\circ}-30452_{9 / 2}$ & 5072.930 & 0 & 1 & 19706.980 & $13250_{5 / 2}-32957_{7 / 2}^{\circ}$ \\
\hline 5169.684 & $2 \mathbf{h}$ & 1 & 19338.155 & $20120_{5 / 2}^{\circ}-39458_{7 / 2}$ & 5072.627 & 10 & 150 & 19708.157 & $16033_{5 / 2}^{\circ}-35741_{5 / 2}$ \\
\hline 5169.160 & 1 & 25 & 19340.116 & $23012_{3 / 2}^{\circ}-42352_{5 / 2}^{\circ}$ & 5072.480 & 3 & 125 & 19708.728 & $12219_{3 / 2}-31928_{3 / 2}^{\circ}$ \\
\hline \multirow[t]{2}{*}{5166.923} & $2 b$ & 25 & 19348.489 & $\begin{array}{l}29720_{3 / 2}^{\circ}-49068_{5 / 2} \\
22139_{9 / 2}^{\circ}-41488_{7 / 2}\end{array}$ & & & & & $13248_{9 / 2}-32957_{7 / 2}^{\circ}$ \\
\hline & & & & & 5072.331 & 0 & 25 & 19709.307 & $40278_{3 / 2}-59987_{3 / 2}^{\circ}$ \\
\hline 5166.875 & 3 & 20 & 19348.669 & $17460_{5 / 2}^{\circ}-36809_{7 / 2}$ & 5068.239 & 0 & 15 & 19725.220 & $31928_{3 / 2}^{\circ}-51653_{1 / 2}^{\circ}$ \\
\hline 5160.678 & 50 & 25 & 19371.903 & $4146_{7 / 2}-23518_{7 / 2}^{\circ}$ & 5064.330 & $20 \mathrm{~b}$ & 50 & 19740.445 & $13468_{9 / 2}^{\circ}-33209_{7 / 2}^{\circ}$ \\
\hline 5159.781 & 0 & 25 & 19375.270 & $28923_{5 / 2}^{\circ}-48298_{7 / 2}$ & 5061.219 & 25 & 200 & 19752.579 & $14790_{7 / 2}^{\circ}-34543_{5 / 2}^{\circ}$ \\
\hline \multirow[t]{2}{*}{5159.153} & 3 & & 19377.629 & $20989_{9 / 2}^{\circ}-40367_{9 / 2}$ & 5058.589 & lb & 2 & 19762.848 & $11116_{7 / 2}^{\circ}-30879_{7 / 2}^{\circ}$ \\
\hline & & & & $20080_{7 / 2}^{\circ}-39458_{7 / 2}$ & 5058.563 & $40 \mathrm{~h}$ & 200 & 19762.950 & $14790_{7 / 2}^{\circ}-34553_{9 / 2}$ \\
\hline 5154.893 & 8 & 8 & 19393.642 & $6213_{9 / 2}-25607_{9 / 2}^{\circ}$ & 5057.848 & 2 & & 19765.743 & $16818_{7 / 2}-36583_{7 / 2}^{\circ}$ \\
\hline 5154.867 & 0 & 5 & 19393.740 & $40222_{3 / 2}-59616_{3 / 2}^{\circ}$ & 5056.331 & 0 & 15 & 19771.673 & $30101_{7 / 2}^{\circ}-49873_{5 / 2}^{\circ}$ \\
\hline 5153.516 & 4 & 25 & 19398.824 & $15144_{3 / 2}^{\circ}-34543_{5 / 2}^{\circ}$ & 5055.338 & 300 & 50 & 19775.557 & $1521_{5 / 2}-21297_{5 / 2}^{\circ}$ \\
\hline 5151.855 & 50 & 20 & 19405.078 & $4113_{5 / 2}-23518_{7 / 2}^{\circ}$ & 5053.309 & 4 & 2 & 19783.497 & $8460_{3 / 2}-28243_{5 / 2}^{\circ}$ \\
\hline
\end{tabular}


TABLE 3. Classified lines of Th II-Continued

\begin{tabular}{|c|c|c|c|c|c|c|c|c|c|}
\hline \multirow{2}{*}{$\begin{array}{c}\text { Wavelength } \\
\AA\end{array}$} & \multicolumn{2}{|c|}{ Intensity } & \multirow{2}{*}{$\begin{array}{c}\text { Wavenumber } \\
\mathbf{c m}^{-1}\end{array}$} & \multirow{2}{*}{ Classification } & \multirow{2}{*}{$\begin{array}{c}\text { Wavelength } \\
\AA\end{array}$} & \multicolumn{2}{|c|}{ Intensity } & \multirow{2}{*}{$\begin{array}{l}\text { Wavenumber } \\
\mathbf{c m}^{-1}\end{array}$} & \multirow{2}{*}{ Classification } \\
\hline & Lamp & Spark & & & & Lamp & Spark & & \\
\hline 5051.331 & 5 & 75 & 19791.244 & $17771_{11 / 2}^{\circ}-37562_{11 / 2}$ & 4979.135 & 5 & 15 & 20078.207 & $20969_{7 / 2}^{\circ}-41047_{9 / 2}$ \\
\hline 5049.799 & 500 & 300 & 19797.248 & $6691_{3 / 2}^{\circ}-26488_{5 / 2}$ & 4978.308 & 2 & 8 & 20081.543 & $17460_{5 / 2}^{\circ}-37542_{3 / 2}$ \\
\hline 5047.431 & 3 & 100 & 19806.536 & $15786_{5 / 2}-35593_{7 / 2}^{\circ}$ & 4978.034 & 10 & 50 & 20082.648 & $22139_{9 / 2}^{\circ}-42222_{7 / 2}$ \\
\hline 5044.935 & 2 & 10 & 19816.335 & $19050_{3 / 2}^{\circ}-38867_{1 / 2}$ & 4976.591 & 50 & 75 & 20088.471 & $12488_{9 / 2}^{\circ}-32576_{7 / 2}^{\circ}$ \\
\hline 5040.555 & 8 & 5 & 19833.554 & $24132_{3 / 2}^{\circ}-43965_{1 / 2}^{\circ}$ & 4975.947 & 100 & 100 & 20091.071 & $12485_{7 / 2}^{\circ}-32576_{7 / 2}^{\circ}$ \\
\hline 5037.729 & 2 & 3 & 19844.680 & $36390_{3 / 2}^{\circ}-56235_{3 / 2}$ & 4975.572 & 3 & 4 & 20092.585 & $15453_{7 / 2}^{\circ}-35545_{9 / 2}$ \\
\hline 5037.285 & 1 & 4 & 19846.429 & $9585_{5 / 2}^{\circ}-29431_{7 / 2}$ & 4974.998 & 4 & 5 & 20094.903 & $18973_{7 / 2}^{\circ}-39068_{7 / 2}$ \\
\hline 5033.902 & 1 & 10 & 19859.767 & $21131_{3 / 2}^{\circ}-40991_{3 / 2}$ & 4974.737 & 1 & 5 & 20095.958 & $20120_{5 / 2}^{\circ}-40216_{5 / 2}$ \\
\hline 5029.632 & 5 & 75 & 19876.627 & $15144_{3 / 2}^{\circ}-35021_{3 / 2}$ & 4974.441 & 1 & 3 & 20097.154 & $28720_{3 / 2}^{\circ}-48817_{3 / 2}$ \\
\hline 5028.613 & $150 \mathrm{~b}$ & $200 \mathrm{~b}$ & 19880.655 & $10572_{9 / 2}^{\circ}-30452_{9 / 2}$ & 4973.769 & 5 & 10 & 20099.869 & $19050_{3 / 2}^{\circ}-39150_{3 / 2}$ \\
\hline 5023.049 & 3 & 50 & 19902.676 & $16906_{7 / 2}^{\circ}-36809_{7 / 2}$ & 4973.596 & 5 & 10 & 20100.568 & $20310_{5 / 2}^{\circ}-40411_{7 / 2}$ \\
\hline 5021.758 & 4 & 25 & 19907.793 & $26647_{13 / 2}^{\circ}-46555_{11 / 2}$ & 4973.384 & 1001 & 150 & 20101.425 & $15324_{1 / 2}^{\circ}-35425_{1 / 2}$ \\
\hline 5020.541 & 50 & 40 & 19912.618 & $13818_{7 / 2}^{\circ}-33730_{5 / 2}^{\circ}$ & 4973.092 & $5 b$ & $20 \mathbf{b}$ & 20102.605 & $28587_{5 / 2}^{\circ}-48689_{3 / 2}$ \\
\hline 5019.614 & 2 & 10 & 19916.296 & $24982_{7 / 2}^{\circ}-44898_{7 / 2}$ & 4973.050 & 8 & 50 & 20102.775 & $20120_{5 / 2}^{\circ}-40222_{3 / 2}$ \\
\hline 5019.324 & 150 & 150 & 19917.446 & $14101_{1 / 2}^{\circ}-34019_{3 / 2}$ & 4972.668 & 5 & 10 & 20104.319 & $13250_{5 / 2}-33354_{5 / 2}^{\circ}$ \\
\hline 5018.220 & 2 & 10 & 19921.828 & $27249_{7 / 2}^{\circ}-47171_{9 / 2}$ & 4972.174 & 50 & 150 & 20106.316 & $23697_{7 / 2}^{\circ}-43803_{7 / 2}$ \\
\hline 5017.255 & 300 & 400 & 19925.660 & $7331_{5 / 2}^{\circ}-27257_{7 / 2}$ & & & & & $15349_{11 / 2}^{\circ}-35456_{9 / 2}$ \\
\hline 5015.888 & 100 & 100 & 19931.090 & $10379_{9 / 2}-30310_{11 / 2}^{\circ}$ & 4971.186 & 2 & 3 & 20110.312 & $22834_{7 / 2}-42944_{7 / 2}^{\circ}$ \\
\hline 5014.751 & 50 & 50 & 19935.609 & $30972_{5 / 2}^{\circ}-50907_{3 / 2}$ & 4970.029 & $15 b$ & 75 & 20114.994 & $8605_{5 / 2}-28720_{3 / 2}^{\circ}$ \\
\hline 5013.994 & 1 & 3 & 19938.619 & $26963_{7 / 2}^{\circ}-46902_{5 / 2}$ & 4968.377 & 21 & 3 & 20121.682 & $17983_{5 / 2}^{\circ}-38105_{5 / 2}$ \\
\hline 5013.600 & 201 & 50 & 19940.186 & $23187_{13 / 2}^{\circ}-43127_{11 / 2}$ & 4967.058 & 5 & 3 & 20127.025 & $8460_{3 / 2}-28587_{5 / 2}^{\circ}$ \\
\hline 5012.729 & 2 & 5 & 19943.650 & $27787_{9 / 2}^{\circ}-47731_{9 / 2}$ & 4964.982 & 15 & $20 \mathrm{~b}$ & 20135.441 & $20080_{7 / 2}^{\circ}-40216_{5 / 2}$ \\
\hline 5009.976 & $50 \mathrm{~b}$ & 100 & 19954.609 & $20969_{7 / 2}^{\circ}-40923_{5 / 2}$ & 4964.116 & 100 & 150 & 20138.953 & $10855_{7 / 2}-30994_{7 / 2}^{\circ}$ \\
\hline 5009.442 & 10 & 20 & 19956.736 & $17722_{9 / 2}^{\circ}-37679_{11 / 2}$ & 4963.791 & 0 & 5 & 20140.272 & $26770_{11 / 2}^{\circ}-46910_{13 / 2}$ \\
\hline 5009.078 & 2 & 3 & 19958.187 & $20686_{5 / 2}^{\circ}-40644_{5 / 2}$ & 4963.550 & 3 & 25 & 20141.250 & $24757_{9 / 2}^{\circ}-44898_{7 / 2}$ \\
\hline 5008.377 & 1 & 3 & 19960.980 & $26424_{5 / 2}^{\circ}-46385_{7 / 2}$ & 4963.184 & 75 & 100 & 20142.735 & $11116_{7 / 2}^{\circ}-31259_{5 / 2}$ \\
\hline 5008.190 & 100 & 150 & 19961.725 & $17983_{5 / 2}^{\circ}-37945_{5 / 2}$ & 4962.959 & $25 b$ & $100 \mathrm{~b}$ & 20143.648 & $9202_{7 / 2}^{\circ}-29345_{5 / 2}$ \\
\hline 5007.377 & 8 & 8 & 19964.966 & $13250_{5 / 2}-33215_{3 / 2}^{\circ}$ & 4962.925 & $40 \mathrm{~b}$ & $75 b$ & 20143.786 & $24982_{7 / 2}^{\circ}-45126_{9 / 2}$ \\
\hline 5006.821 & 3 & 5 & 19967.183 & $20310_{5 / 2}^{\circ}-40278_{3 / 2}$ & 4960.492 & $25 b$ & 20 & 20153.666 & $9720_{7 / 2}^{\circ}-29873_{7 / 2}$ \\
\hline 5005.738 & 2 & 2 & 19971.503 & $26424_{5 / 2}^{\circ}-46395_{3 / 2}$ & 4959.738 & 1 & 1 & 20156.730 & $16906_{7 / 2}^{\circ}-37063_{9 / 2}$ \\
\hline 5003.503 & 2 & 10 & 19980.424 & $29515_{9 / 2}-49495_{9 / 2}^{\circ}$ & 4959.438 & 3 & 10 & 20157.949 & $20120_{5 / 2}^{\circ}-40278_{3 / 2}$ \\
\hline 5002.567 & 15 & 20 & 19984.162 & $17837_{1 / 2}^{\circ}-37821_{3 / 2}$ & 4958.974 & 1 & 8 & 20159.835 & $30310_{11 / 2}^{\circ}-50470_{9 / 2}$ \\
\hline 4999.937 & 100 & 100 & 19994.674 & $14275_{9 / 2}^{\circ}-34270_{9 / 2}$ & 4958.717 & 10 & 5 & 20160.880 & $1521_{5 / 2}-21682_{7 / 2}^{\circ}$ \\
\hline 4999.105 & 20 & 20 & 19998.002 & $14545_{5 / 2}^{\circ}-34543_{5 / 2}$ & 4956.836 & $5 b$ & 75 & 20168.531 & $24132_{3 / 2}^{\circ}-44300_{3 / 2}$ \\
\hline 4997.669 & 40 & 50 & 20003.748 & $14275_{9 / 2}^{\circ}-34279_{7 / 2}$ & 4956.370 & 1 & 2 & 20170.427 & $25440_{5 / 2}^{\circ}-45610_{5 / 2}$ \\
\hline 4997.326 & 40 & 50 & 20005.121 & $17272_{9 / 2}^{\circ}-37277_{7 / 2}$ & 4955.960 & 2 & 10 & 20172.096 & $29788_{9 / 2}^{\circ}-49960_{7 / 2}$ \\
\hline 4996.982 & 3 & 4 & 20006.498 & $22106_{5 / 2}-42112_{3 / 2}^{\circ}$ & 4954.657 & $150 \mathrm{~b}$ & 200 & 20177.401 & $14484_{11 / 2}^{\circ}-34661_{11 / 2}$ \\
\hline 4996.873 & 5 & 10 & 20006.934 & $31928_{3 / 2}^{\circ}-51935_{5 / 2}$ & 4954.555 & 50 & 150 & 20177.816 & $11576_{3 / 2}^{\circ}-31754_{5 / 2}$ \\
\hline 4992.275 & 2 & 4 & 20025.361 & $20158_{5 / 2}-40184_{7 / 2}^{\circ}$ & 4954.336 & 3 & 10 & 20178.708 & $26424_{5 / 2}^{\circ}-46603_{5 / 2}$ \\
\hline 4991.925 & 3 & 5 & 20026.765 & $19912_{13 / 2}^{\circ}-39939_{11 / 2}^{\circ}$ & 4951.826 & 8 & 15 & 20188.936 & $18568_{1 / 2}^{\circ}-38757_{3 / 2}^{\circ}$ \\
\hline 4991.007 & $10 \mathrm{~b}$ & 20 & 20030.448 & $15710_{3 / 2}^{\circ}-35741_{5 / 2}$ & 4951.385 & 8 & 15 & 20190.734 & $21297_{5 / 2}^{\circ}-41488_{7 / 2}$ \\
\hline 4990.472 & 5 & 4 & 20032.596 & $16033_{5 / 2}^{\circ}-36065_{5 / 2}^{\circ}$ & 4950.621 & 150 & 100 & 20193.850 & $9238_{9 / 2}^{\circ}-29431_{7 / 2}^{\circ}$ \\
\hline 4990.030 & 20 & 15 & 20034.370 & $9061_{5 / 2}-29095_{5 / 2}^{\circ}$ & 4950.167 & 10 & 20 & 20195.702 & $15349_{11 / 2}^{\circ}-35545_{9 / 2}$ \\
\hline 4987.149 & 200 & 300 & 20045.944 & $18816_{13 / 2}^{\circ}-38862_{11 / 2}^{\circ}$ & 4949.961 & 8 & 40 & 20196.542 & $21131_{3 / 2}^{\circ}-41328_{5 / 2}^{\circ}$ \\
\hline 4986.029 & 4 & 25 & 20050.446 & $24757_{9 / 2}^{\circ}-44807_{7 / 2}$ & 4947.315 & 4 & 10 & 20207.344 & $35878_{7 / 2}-56086_{9 / 2}^{\circ}$ \\
\hline 4984.301 & 1 & 1 & 20057.397 & $20989_{9 / 2}^{\circ}-41047_{9 / 2}$ & 4946.661 & 100 & 100 & 20210.016 & $19248_{5 / 2}^{\circ}-39458_{7 / 2}$ \\
\hline 4981.228 & $3 b$ & 4 & 20069.771 & $17771_{11 / 2}^{o}-37840_{9 / 2}$ & 4945.863 & 15 & 20 & 20213.277 & $15242_{9 / 2}^{\circ}-35456_{9 / 2}^{\circ}$ \\
\hline 4980.949 & 50 & 40 & 20070.895 & $6691_{3 / 2}^{\circ}-26762_{3 / 2}$ & 4945.249 & 2 & $3 b$ & 20215.786 & $23012_{3 / 2}^{\circ}-43227_{5 / 2}$ \\
\hline 4979.976 & 3 & 10 & 20074.817 & $23697_{7 / 2}^{\circ}-43772_{5 / 2}$ & 4942.844 & 15 & 15 & 20225.622 & $8018_{3 / 2}-28243_{5 / 2}^{\circ}$ \\
\hline 4979.438 & 1 & 4 & 20076.986 & $28243_{5 / 2}^{\circ}-48320_{5 / 2}$ & 4942.616 & 3 & 15 & 20226.555 & $21682_{7 / 2}^{\circ}-41909_{9 / 2}$ \\
\hline
\end{tabular}


TABLE 3. Classified lines of Th II-Continued

\begin{tabular}{|c|c|c|c|c|c|c|c|c|c|}
\hline \multirow{2}{*}{$\begin{array}{c}\text { Wavelength } \\
\AA\end{array}$} & \multicolumn{2}{|c|}{ Intensity } & \multirow{2}{*}{$\begin{array}{c}\text { Wavenumber } \\
\mathbf{c m}^{-1}\end{array}$} & \multirow{2}{*}{ Classification } & \multirow{2}{*}{$\begin{array}{c}\text { Wavelength } \\
\AA\end{array}$} & \multicolumn{2}{|c|}{ Intensity } & \multirow{2}{*}{$\begin{array}{c}\text { Wavenumber } \\
\mathbf{c m}^{-1}\end{array}$} & \multirow{2}{*}{ Classification } \\
\hline & Lamp & Spark & & & & Lamp & Spark & & \\
\hline 4941.621 & 1 & $2 \mathrm{~h}$ & 20230.628 & $28587_{5 / 2}^{\circ}-48817_{3 / 2}$ & 4885.985 & $2 \mathbf{b}$ & 3 & 20460.988 & $13818_{7 / 2}^{\circ}-34279_{7 / 2}$ \\
\hline 4939.948 & 8 & 15 & 20237.479 & $20686_{5 / 2}^{\circ}-40923_{5 / 2}$ & 4885.529 & 1 & 3 & 20462.898 & $18118_{3 / 2}-38581_{5 / 2}^{\circ}$ \\
\hline 4939.808 & 5 & 15 & 20238.053 & $22513_{5 / 2}^{\circ}-42751_{7 / 2}$ & 4884.684 & 2 & 3 & 20466.437 & $27403_{3 / 2}^{\circ}-47869_{3 / 2}$ \\
\hline 4937.962 & 2 & 5 & 20245.618 & $31924_{11 / 2}^{\circ}-52170_{11 / 2}$ & 4882.446 & 4 & 10 & 20475.819 & $14545_{5 / 2}^{\circ}-35021_{3 / 2}$ \\
\hline 4937.405 & 2 & $2 \mathrm{~b}$ & 20247.902 & $12488_{9 / 2}^{\circ}-32736_{7 / 2}^{\circ}$ & 4881.106 & 0 & 1 & 20481.440 & $28587_{5 / 2}^{\circ}-49068_{5 / 2}$ \\
\hline 4934.534 & 3 & 4 & 20259.683 & $20310_{5 / 2}^{\circ}-40570_{7 / 2}$ & 4880.230 & $3 b$ & 4 & 20485.116 & $22642_{9 / 2}^{\circ}-43127_{11 / 2}$ \\
\hline 4933.850 & $15 \mathrm{~b}$ & 75 & 20262.491 & $7331_{5 / 2}^{\circ}-27593_{5 / 2}$ & 4879.349 & 5 & 20 & 20488.815 & $16564_{11 / 2}^{\circ}-37053_{11 / 2}$ \\
\hline 4933.563 & $4 b$ & $5 b$ & 20263.670 & $10189_{11 / 2}^{\circ}-30452_{9 / 2}$ & 4879.186 & 0 & 1 & 20489.499 & $25414_{11 / 2}^{\circ}-45904_{9 / 2}$ \\
\hline 4933.480 & $3 \mathbf{b}$ & $5 b$ & 20264.011 & $12472_{5 / 2}^{\circ}-32736_{7 / 2}$ & 4879.154 & 5 & 4 & 20489.634 & $8605_{5 / 2}-29095_{5 / 2}^{\circ}$ \\
\hline 4932.587 & 0 & 3 & 20267.680 & $30956_{9 / 2}^{\circ}-51224_{9 / 2}$ & 4877.809 & 15 & 5 & 20495.283 & $1859_{3 / 2}-22355_{1 / 2}^{\circ}$ \\
\hline 4932.532 & $8 \mathrm{~h}$ & $5 \mathrm{~h}$ & 20267.906 & $18568_{1 / 2}^{\circ}-38836_{3 / 2}$ & 4877.704 & 1 & 2 & 20495.725 & $20158_{5 / 2}-40654_{5 / 2}^{\circ}$ \\
\hline \multirow[t]{2}{*}{4932.345} & 1 & 2 & 20268.674 & $18816_{13 / 2}^{\circ}-39085_{13 / 2}$ & 4877.002 & 25 & 150 & 20498.675 & $16564_{11 / 2}^{\circ}-37063_{9 / 2}$ \\
\hline & & & & $24381_{7 / 2}-44650_{7 / 2}^{\circ}$ & 4874.152 & 2 & 3 & 20510.661 & $27787_{9 / 2}^{\circ}-48298_{7 / 2}^{\circ}$ \\
\hline 4929.985 & 10 & 100 & 20278.377 & $14275_{9 / 2}^{\circ}-34553_{9 / 2}$ & 4874.030 & 1 & 2 & 20511.174 & $26424_{5 / 2}^{\circ}-46935_{3 / 2}$ \\
\hline 4929.378 & 2 & 15 & 20280.874 & $15144_{3 / 2}^{\circ}-35425_{1 / 2}$ & 4873.351 & 2 & 3 & 20514.032 & $27357_{9 / 2}^{\circ}-47871_{7 / 2}^{\circ}$ \\
\hline \multirow[t]{2}{*}{4927.587} & 4 & 15 & 20288.245 & $15453_{7 / 2}^{\circ}-35741_{5 / 2}$ & 4872.971 & 0 & 8 & 20515.631 & $17272_{9 / 2}^{\circ}-37787_{7 / 2}^{\circ}$ \\
\hline & & & & $15305_{9 / 2}-35593_{7 / 2}^{\circ}$ & 4872.537 & 1 & 1 & 20517.459 & $21682_{7 / 2}^{\circ}-42200_{9 / 2}^{\circ}$ \\
\hline 4926.969 & 1 & 1 & 20290.790 & $23518_{7 / 2}^{\circ}-43809_{9 / 2}$ & 4872.138 & 3 & 5 & 20519.139 & $20969_{7 / 2}^{\circ}-41488_{7 / 2}^{\circ}$ \\
\hline 4925.795 & $2 \mathrm{~b}$ & 3 & 20295.626 & $10189_{11 / 2}^{\circ}-30484_{11 / 2}$ & 4868.274 & 5 & 25 & 20535.425 & $18816_{13 / 2}^{\circ}-39352_{11 / 2}$ \\
\hline 4925.418 & 5 & 50 & 20297.179 & $15305_{9 / 2}-35602_{11 / 2}^{\circ}$ & 4863.163 & $300 \mathrm{~d}$ & 1000 & 20557.006 & $6213_{9 / 2}-26770_{11 / 2}^{\circ}$ \\
\hline 4925.002 & 3 & 75 & 20298.893 & $18568_{1 / 2}^{\circ}-38867_{1 / 2}$ & 4862.203 & 0 & 2 & 20561.065 & $35525_{11 / 2}-56086_{9 / 2}^{\circ}$ \\
\hline 4924.798 & 2 & 8 & 20299.734 & $7331_{5 / 2}^{\circ}-27631_{3 / 2}$ & 4862.128 & 1 & 3 & 20561.382 & $22685_{7 / 2}^{\circ}-43246_{7 / 2}$ \\
\hline 4924.420 & 15 & 50 & 20301.293 & $4113_{5 / 2}-24414_{3 / 2}^{\circ}$ & 4861.932 & 1 & 5 & 20562.211 & $30101_{7 / 2}^{\circ}-50663_{5 / 2}$ \\
\hline 4923.421 & 1 & 2 & 20305.412 & $20686_{5 / 2}^{\circ}-40991_{3 / 2}$ & 4860.421 & 3 & 200 & 20568.603 & $17272_{9 / 2}^{\circ}-37840_{9 / 2}^{\circ}$ \\
\hline 4922.946 & 15 & 100 & 20307.371 & $10572_{9 / 2}^{\circ}-30879_{7 / 2}$ & 4858.332 & 40 & 500 & 20577.448 & $10379_{9 / 2}-30956_{9 / 2}^{\circ}$ \\
\hline 4921.876 & 0 & 2 & 20311.786 & $22106_{5 / 2}-42418_{3 / 2}^{\circ}$ & 4858.241 & 0 & 5 & 20577.833 & $23518_{7 / 2}^{\circ}-44096_{9 / 2}^{\circ}$ \\
\hline 4921.612 & $50 \mathrm{~b}$ & 150 & 20312.875 & $9202_{7 / 2}^{\circ}-29515_{9 / 2}$ & 4858.096 & 5 & 300 & 20578.447 & $18973_{7 / 2}^{\circ}-39552_{9 / 2}^{\circ}$ \\
\hline 4920.554 & $2 b$ & 3 & 20317.243 & $4146_{7 / 2}-24463_{5 / 2}^{\circ}$ & 4857.159 & 2 & 75 & 20582.417 & $18568_{1 / 2}^{\circ}-39150_{3 / 2}$ \\
\hline 4920.526 & $10 \mathrm{~b}$ & 100 & 20317.358 & $8605_{5 / 2}-28923_{5 / 2}^{\circ}$ & 4856.957 & 1 & 2 & 20583.273 & $22513_{5 / 2}^{\circ}-43096_{5 / 2}^{\circ}$ \\
\hline 4919.816 & 500 & 500 & 20320.290 & $6168_{7 / 2}^{\circ}-26488_{5 / 2}$ & 4856.273 & 3 & 40 & 20586.172 & $10673_{5 / 2}^{\circ}-31259_{5 / 2}$ \\
\hline 4917.468 & 2 & $2 b$ & 20329.993 & $15710_{3 / 2}^{\circ}-36040_{1 / 2}$ & 4854.647 & 1 & 1 & 20593.067 & $23372_{3 / 2}^{\circ}-43965_{1 / 2}$ \\
\hline 4914.119 & 5 & 100 & 20343.847 & $17121_{3 / 2}^{\circ}-37465_{5 / 2}$ & 4851.898 & 2 & 40 & 20604.734 & $22642_{9 / 2}^{\circ}-43246_{7 / 2}^{\circ}$ \\
\hline 4912.526 & 50 & 100 & 20350.444 & $4113_{5 / 2}-24463_{5 / 2}^{\circ}$ & 4850.437 & 100 & 300 & 20610.941 & $4146_{7 / 2}-24757_{9 / 2}^{\circ}$ \\
\hline 4911.447 & 2 & 4 & 20354.915 & $15710_{3 / 2}^{\circ}-36065_{5 / 2}$ & 4850.263 & 0 & 2 & 20611.680 & $22139_{9 / 2}^{\circ}-42751_{7 / 2}^{\circ}$ \\
\hline 4911.147 & 8 & 75 & 20356.159 & $13818_{7 / 2}^{\circ}-34174_{5 / 2}$ & 4850.024 & 3 & 100 & 20612.696 & $20310_{5 / 2}^{\circ}-40923_{5 / 2}$ \\
\hline 4910.418 & 0 & 1 & 20359.180 & $26965_{3 / 2}^{\circ}-47324_{5 / 2}$ & & & & & $15453_{7 / 2}^{\circ}-36065_{5 / 2}^{\circ}$ \\
\hline 4910.312 & 1 & 1 & 20359.620 & $10189_{11 / 2}^{\circ}-30548_{13 / 2}$ & 4849.439 & 3 & 15 & 20615.182 & $10379_{9 / 2}-30994_{7 / 2}^{\circ}$ \\
\hline 4906.680 & 3 & 1 & 20374.690 & $24414_{3 / 2}^{\circ}-44.789_{1 / 2}$ & 4849.043 & 20 & 500 & 20616.866 & $22028_{15 / 2}^{\circ}-42644_{13 / 2}$ \\
\hline 4903.122 & 1 & 1 & 20389.475 & $9711_{7 / 2}-30101_{7 / 2}^{\circ}$ & 4848.826 & 1 & 2 & 20617.789 & $15710_{3 / 2}^{\circ}-36328_{3 / 2}$ \\
\hline 4899.158 & 2 & 8 & 20405.972 & $17983_{5 / 2}^{\circ}-38389_{7 / 2}$ & 4847.887 & 2 & 3 & 20621.782 & $18214_{3 / 2}^{\circ}-38836_{3 / 2}$ \\
\hline 4898.802 & 51 & 75 & 20407.455 & $17272_{9 / 2}^{\circ}-37679_{11 / 2}$ & 4845.936 & 3 & 100 & 20630.084 & $12219_{3 / 2}-32850_{5 / 2}^{\circ}$ \\
\hline 4898.457 & 8 & 100 & 20408.893 & $17771_{11 / 2}^{\circ}-38179_{9 / 2}^{\circ}$ & 4844.755 & 4 & 100 & 20635.113 & $8460_{3 / 2}-29095_{5 / 2}^{\circ}$ \\
\hline 4897.928 & 2 & 10 & 20411.097 & $22685_{7 / 2}^{\circ}-43096_{5 / 2}$ & 4844.559 & 8 & 200 & 20635.948 & $15242_{9 / 2}^{\circ}-35878_{7 / 2}^{\circ}$ \\
\hline 4895.660 & 2 & 5 & 20420.553 & $17121_{3 / 2}^{\circ}-37542_{3 / 2}$ & & & & & $9238_{9 / 2}^{\circ}-29873_{7 / 2}^{\circ}$ \\
\hline 4894.397 & 1 & 1 & 20425.822 & $15453_{7 / 2}^{\circ}-35878_{7 / 2}$ & 4844.257 & 3 & $100 \mathrm{~b}$ & 20637.234 & $14349_{1 / 2}-34986_{3 / 2}^{\circ}$ \\
\hline 4894.351 & 0 & 1 & 20426.014 & $24463_{5 / 2}^{\circ}-44889_{3 / 2}$ & 4844.164 & 50 & $300 \mathrm{~b}$ & 20637.631 & $11116_{7 / 2}^{\circ}-31754_{5 / 2}$ \\
\hline 4893.517 & 1 & 2 & 20429.495 & $20969_{\tau / 2}^{\circ}-41398_{9 / 2}^{\circ}$ & 4843.815 & 0 & 1 & 20639.118 & $21297_{5 / 2}^{\circ}-41936_{3 / 2}^{\circ}$ \\
\hline 4889.853 & 8 & 5 & 20444.803 & $8378_{7 / 2}^{\circ}-28823_{5 / 2}$ & 4842.562 & 1 & 1 & 20644.458 & $17460_{5 / 2}^{\circ}-38105_{5 / 2}^{\circ}$ \\
\hline 4888.498 & 2 & 2 & 20450.470 & $20120_{5 / 2}^{\circ}-40570_{7 / 2}$ & 4841.385 & 1 & 3 & 20649.477 & $27357_{9 / 2}^{\circ}-48006_{9 / 2}^{\circ}$ \\
\hline 4888.365 & 2 & 2 & 20451.026 & $14275_{9 / 2}^{\circ}-34726_{7 / 2}$ & 4841.320 & 2 & 20 & 20649.754 & $19050_{3 / 2}^{\circ}-39700_{5 / 2}^{\circ}$ \\
\hline
\end{tabular}


TABLE 3. Classified lines of Th II-Continued

\begin{tabular}{|c|c|c|c|c|c|c|c|c|c|}
\hline \multirow{2}{*}{$\begin{array}{c}\text { Wavelength } \\
\AA\end{array}$} & \multicolumn{2}{|c|}{ Intensity } & \multirow{2}{*}{$\begin{array}{c}\text { Wavenumber } \\
\mathrm{cm}^{-1}\end{array}$} & \multirow{2}{*}{ Classification } & \multirow{2}{*}{$\begin{array}{c}\text { Wavelength } \\
\AA\end{array}$} & \multicolumn{2}{|c|}{ Intensity } & \multirow{2}{*}{$\begin{array}{c}\text { Wavenumber } \\
\mathbf{c m}^{-1}\end{array}$} & \multirow{2}{*}{ Classification } \\
\hline & Lamp & Spark & & & & Lamp & Spark & & \\
\hline 4841.037 & 0 & 3 & 20650.961 & $33843_{3 / 2}^{\circ}-54493_{5 / 2}$ & 4800.921 & 0 & 1 & 20823.516 & $17121_{3 / 2}^{\circ}-37945_{5 / 2}$ \\
\hline 4840.471 & 40 & 300 & 20653.376 & $13248_{9 / 2}-33902_{7 / 2}^{\circ}$ & 4800.168 & 20 & 200 & 20826.782 & $6700_{9 / 2}^{\circ}-27526_{9 / 2}^{\circ}$ \\
\hline & & & & $1859_{3 / 2}-22513_{5 / 2}^{\circ}$ & 4800.050 & 0 & 2 & 20827.295 & $28587_{5 / 2}^{\circ}-4.9414_{3 / 2}$ \\
\hline 4839.738 & 3 & 75 & 20656.504 & $11116_{7 / 2}^{\circ}-31773_{9 / 2}$ & 4799.756 & 3 & 40 & 20828.570 & $12902_{3 / 2}^{\circ}-33730_{5 / 2}^{\circ}$ \\
\hline 4839.563 & 0 & 1 & 20657.251 & $24132_{3 / 2}^{\circ}-44.789_{1 / 2}^{\circ}$ & 4799.158 & 0 & 1 & 20831.165 & $17460_{5 / 2}^{\circ}-38291_{7 / 2}^{\circ}$ \\
\hline 4838.353 & 8 & 300 & 20662.417 & $19912_{13 / 2}^{\circ}-40574_{11 / 2}$ & 4797.569 & 2 & 10 & 20838.065 & $22106_{5 / 2}-42944_{7 / 2}^{\circ}$ \\
\hline 4837.701 & 3 & 100 & 20665.202 & $14790_{7 / 2}^{\circ}-35456_{9 / 2}$ & 4796.511 & 0 & 2 & 20842.661 & $29788_{9 / 2}^{\circ}-50631_{11 / 2}^{\circ}$ \\
\hline 4836.725 & 0 & 2 & 20669.372 & $25594_{i / 2}^{\circ}-46264_{3 / 2}$ & 4796.448 & 0 & 2 & 20842.935 & $20080_{7 / 2}^{\circ}-40923_{5 / 2}$ \\
\hline & & & & $21682_{7 / 2}^{\circ}-42352_{5 / 2}$ & 4794.918 & 2 & 4 & 20849.585 & $14349_{1 / 2}-35198_{1 / 2}^{\circ}$ \\
\hline 4836.179 & 2 & 15 & 20671.705 & $9202_{7 / 2}^{\circ}-29873_{7 / 2}$ & 4794.175 & 1 & 2 & 20852.817 & $17983_{5 / 2}^{\circ}-38836_{3 / 2}$ \\
\hline 4833.872 & 0 & 3 & 20681.571 & $29788_{9 / 2}^{\circ}-50470_{9 / 2}$ & 4793.669 & 2 & 40 & 20855.018 & $23697_{7 / 2}^{\circ}-44552_{5 / 2}$ \\
\hline 4833.826 & 25 & $40 \mathrm{~b}$ & 20681.767 & $31625_{1 / 2}^{\circ}-52307_{3 / 2}$ & 4792.910 & 4 & 2 & 20858.321 & $30972_{5 / 2}^{\circ}-51830_{7 / 2}$ \\
\hline 4832.799 & 75 & 300 & 20686.162 & $0_{3 / 2}-20686_{5 / 2}^{\circ}$ & 4790.434 & 5 & 40 & 20869.101 & $4113_{5 / 2}-24982_{7 / 2}^{\circ}$ \\
\hline 4831.645 & 0 & 3 & 20691.103 & $23697_{7 / 2}^{\circ}-44388_{5 / 2}$ & 4790.148 & 0 & 2 & 20870.347 & $23518_{7 / 2}^{\circ}-44388_{5 / 2}$ \\
\hline 4829.884 & 1 & 25 & 20698.647 & $20989_{9 / 2}^{\circ}-41688_{7 / 2}$ & 4789.903 & 2 & 40 & 20871.415 & $20120_{5 / 2}^{\circ}-40991_{3 / 2}$ \\
\hline 4828.952 & 2 & 8 & 20702.642 & $8018_{3 / 2}-28720_{3 / 2}^{\circ}$ & 4788.375 & 2 & 2 & 20878.075 & $19594_{1 / 2}-40472_{3 / 2}^{\circ}$ \\
\hline 4828.463 & 4 & 200 & 20704.738 & $17460_{5 / 2}^{\circ}-38165_{7 / 2}$ & 4787.827 & 2 & 50 & 20880.464 & $17983_{5 / 2}^{\circ}-38863_{5 / 2}$ \\
\hline 4824.647 & 5 & 100 & 20721.114 & $12488_{9 / 2}^{\circ}-33209_{7 / 2}$ & 4787.372 & 4 & 150 & 20882.449 & $15242_{9 / 2}^{\circ}-36125_{9 / 2}^{\circ}$ \\
\hline 4824.044 & 1 & 2 & 20723.704 & $12485_{7 / 2}^{\circ}-33209_{7 / 2}$ & 4785.721 & 1 & 2 & 20889.653 & $22355_{1 / 2}^{\circ}-43244_{3 / 2}$ \\
\hline 4823.692 & 2 & 5 & 20725.217 & $13818_{7 / 2}^{\circ}-34543_{5 / 2}$ & 4785.295 & 0 & $4 b$ & 20891.513 & $24414_{3 / 2}^{\circ}-45306_{3 / 2}$ \\
\hline 4823.319 & 3 & 75 & 20726.820 & $18973_{7 / 2}^{\circ}-39700_{5 / 2}$ & 4784.929 & 5 & 200 & 20893.110 & $17272_{9 / 2}^{\circ}-38165_{7 / 2}$ \\
\hline 4823.179 & $8 b$ & 200 & 20727.421 & $4146_{7 / 2}-24873_{5 / 2}^{\circ}$ & 4784.565 & 2 & 8 & 20894.700 & $17722_{9 / 2}^{\circ}-38617_{9 / 2}^{\circ}$ \\
\hline 4821.272 & 10 & 500 & 20735.620 & $13818_{7 / 2}^{\circ}-34553_{9 / 2}^{\circ}$ & 4784.237 & 1 & 3 & 20896.132 & $15144_{3 / 2}^{\circ}-36040_{1 / 2}$ \\
\hline 4821.035 & 0 & 2 & 20736.639 & $24873_{5 / 2}^{\circ}-45610_{5 / 2}$ & & & & & $12488_{9 / 2}^{\circ}-33384_{9 / 2}^{\circ}$ \\
\hline 4820.693 & 0 & 3 & 20738.110 & $26586_{3 / 2}^{\circ}-47324_{5 / 2}$ & 4783.904 & 8 & 15 & 20897.587 & $19050_{3 / 2}^{\circ}-39948_{1 / 2}$ \\
\hline 4819.017 & 3 & 40 & 20745.322 & $17983_{5 / 2}^{\circ}-38728_{5 / 2}$ & 4783.636 & 3 & 200 & 20898.758 & $12485_{7 / 2}^{\circ}-33384_{9 / 2}$ \\
\hline 4818.644 & 50 & 500 & 20746.928 & $17771_{11 / 2}^{\circ}-38517_{13 / 2}$ & 4783.167 & 0 & 2 & 20900.807 & $27631_{3 / 2}-48532_{1 / 2}^{\circ}$ \\
\hline 4817.826 & 2 & 8 & 20750.451 & $6213_{9 / 2}-26963_{7 / 2}^{\circ}$ & 4782.759 & 20 & 200 & 20902.590 & $6691_{3 / 2}^{\circ}-27593_{5 / 2}$ \\
\hline 4817.320 & 2 & 40 & 20752.630 & $23697_{7 / 2}^{\circ}-44450_{9 / 2}$ & 4782.204 & 2 & 2 & 20905.016 & $8018_{3 / 2}-28923_{5 / 2}^{\circ}$ \\
\hline 4816.859 & $3 \mathbf{b}$ & 100 & 20754.616 & $14790_{7 / 2}^{\circ}-35545_{9 / 2}$ & 4781.584 & 3 & 5 & 20907.726 & $17272_{9 / 2}^{\circ}-38179_{9 / 2}^{\circ}$ \\
\hline 4816.129 & 1 & 10 & 20757.762 & $24132_{3 / 2}^{\circ}-44889_{3 / 2}$ & 4779.587 & 5 & 2 & 20916.462 & $14484_{11 / 2}^{\circ}-35400_{13 / 2}$ \\
\hline 4815.927 & 0 & 2 & 20758.633 & $20288_{11 / 2}^{\circ}-41047_{9 / 2}$ & 4778.921 & 1 & 4 & 20919.377 & $17837_{1 / 2}^{o}-38757_{3 / 2}$ \\
\hline 4815.460 & 8 & 3 & 20760.646 & $4113_{5 / 2}-24873_{5 / 2}^{\circ}$ & 4778.883 & 4 & 200 & 20919.543 & $20989_{9 / 2}^{\circ}-41909_{9 / 2}^{\circ}$ \\
\hline 4813.591 & 0 & 1 & 20768.707 & $34270_{9 / 2}-55038_{9 / 2}^{\circ}$ & 4778.547 & 1 & 3 & 20921.014 & $15144_{3 / 2}^{\circ}-36065_{5 / 2}$ \\
\hline 4812.562 & 1 & 3 & 20773.147 & $23730_{9 / 2}^{\circ}-44503_{7 / 2}$ & 4777.649 & 3 & 75 & 20924.946 & $39068_{\tau / 2}-59993_{9 / 2}^{\circ}$ \\
\hline 4812.449 & 1 & 5 & 20773.635 & $25381_{3 / 2}-46155_{5 / 2}^{\circ}$ & 4777.350 & 0 & 2 & 20926.256 & $24873_{5 / 2}^{\circ}-45800_{5 / 2}$ \\
\hline 4812.020 & 3 & 50 & 20775.487 & $15349_{11 / 2}^{\circ}-36125_{9 / 2}$ & 4776.779 & 50 & 150 & 20928.757 & $17460_{5 / 2}^{\circ}-38389_{7 / 2}$ \\
\hline 4811.867 & 4 & 75 & 20776.147 & $16033_{5 / 2}^{\circ}-36809_{7 / 2}$ & 4776.076 & 0 & 2 & 20931.838 & $23518_{7 / 2}^{\circ}-44450_{9 / 2}$ \\
\hline 4811.369 & 2 & 25 & 20778.298 & $25607_{9 / 2}^{\circ}-46385_{7 / 2}$ & 4775.357 & $3 \mathbf{b}$ & 40 & 20934.989 & $20288_{11 / 2}^{\circ}-41223_{11 / 2}$ \\
\hline 4810.874 & 2 & 1 & 20780.436 & $38836_{3 / 2}-59616_{3 / 2}^{\circ}$ & 4775.067 & 2 & 50 & 20936.261 & $18214_{3 / 2}^{\circ}-39150_{3 / 2}$ \\
\hline 4807.079 & 3 & 50 & 20796.841 & $15786_{5 / 2}-36583_{7 / 2}^{\circ}$ & 4774.499 & 2 & 50 & 20938.751 & $16818_{7 / 2}-37756_{7 / 2}^{\circ}$ \\
\hline 4806.050 & 3 & 75 & 20801.294 & $13468_{9 / 2}^{\circ}-34270_{9 / 2}^{\circ}$ & 4774.331 & 2 & 5 & 20939.488 & $22834_{7 / 2}-43773_{7 / 2}^{\circ}$ \\
\hline 4805.963 & 4 & 200 & 20801.670 & $25414_{11 / 2}^{\circ}-46216_{11 / 2}$ & 4774.254 & $50 \mathrm{~b}$ & 200 & 20939.826 & $6691_{3 / 2}^{\circ}-27631_{3 / 2}$ \\
\hline 4805.880 & 2 & $15 b$ & 20802.029 & $20686_{5 / 2}^{\circ}-41488_{7 / 2}$ & 4773.991 & 1 & 3 & 20940.979 & $22014_{11 / 2}^{\circ}-42955_{9 / 2}$ \\
\hline 4805.547 & 3 & 50 & 20803.471 & $20120_{5 / 2}^{\circ}-40923_{5 / 2}^{\circ}$ & 4773.955 & 1 & 3 & 20941.137 & $27357_{9 / 2}^{\circ}-48298_{7 / 2}$ \\
\hline 4805.290 & 0 & 2 & 20804.583 & $35878_{7 / 2}-56683_{7 / 2}^{\circ}$ & 4773.482 & 0 & 1 & 20943.212 & $33902_{7 / 2}^{\circ}-54845_{9 / 2}$ \\
\hline 4803.954 & 8 & 200 & 20810.369 & $13468_{9 / 2}^{\circ}-34279_{\tau / 2}$ & 4773.104 & 3 & 50 & 20944.871 & $10855_{7 / 2}-31800_{7 / 2}^{\circ}$ \\
\hline 4803.483 & 50 & 300 & 20812.410 & $19912_{13 / 2}^{\circ}-40724_{13 / 2}$ & 4771.952 & 1 & 4 & 20949.927 & $28923_{5 / 2}^{\circ}-49873_{5 / 2}$ \\
\hline 4802.612 & 0 & 3 & 20816.184 & $22139_{9 / 2}^{\circ}-42955_{9 / 2}$ & 4771.862 & 2 & 10 & 20950.322 & $14790_{7 / 2}^{\circ}-35741_{5 / 2}$ \\
\hline 4802.422 & 1 & 3 & 20817.008 & $24309_{11 / 2}^{\circ}-45126_{9 / 2}$ & 4771.123 & lh & 2 & 20953.567 & $23012_{3 / 2}^{\circ}-43965_{1 / 2}^{\circ}$ \\
\hline
\end{tabular}


TABLE 3. Classified lines of Th II-Continued

\begin{tabular}{|c|c|c|c|c|c|c|c|c|c|}
\hline \multirow{2}{*}{$\begin{array}{c}\text { Wavelength } \\
\AA\end{array}$} & \multicolumn{2}{|c|}{ Intensity } & \multirow{2}{*}{$\begin{array}{c}\text { Wavenumber } \\
\mathrm{cm}^{-1}\end{array}$} & \multirow{2}{*}{ Classification } & \multirow{2}{*}{$\begin{array}{c}\text { Wavelength } \\
\AA\end{array}$} & \multicolumn{2}{|c|}{ Intensity } & \multirow{2}{*}{$\begin{array}{c}\text { Wavenumber } \\
\mathbf{c m}^{-1}\end{array}$} & \multirow{2}{*}{ Classification } \\
\hline & Lamp & Spark & & & & Lamp & Spark & & \\
\hline 4770.945 & 0 & 2 & 20954.349 & $31353_{3 / 2}^{\circ}-52307_{3 / 2}$ & 4726.254 & 2 & 4 & 21152.488 & $18214_{3 / 2}^{\circ}-39366_{5 / 2}$ \\
\hline 4770.744 & $2 b$ & 100 & 20955.232 & $10855_{7 / 2}-31810_{5 / 2}^{\circ}$ & 4725.943 & 5 & 100 & 21153.880 & $15236_{3 / 2}-36390_{3 / 2}^{\circ}$ \\
\hline 4769.429 & 1 & 3 & 20961.009 & $26770_{11 / 2}^{\circ}-47731_{9 / 2}$ & 4725.305 & 3 & 15 & 21156.736 & $20989_{9 / 2}^{\circ}-42146_{11 / 2}^{\circ}$ \\
\hline 4768.761 & 3 & 100 & 20963.945 & $25414_{11 / 2}^{\circ}-46378_{13 / 2}$ & 4724.822 & $5 b$ & $40 \mathrm{~b}$ & 21158.899 & $6244_{1 / 2}-27403_{3 / 2}^{\circ}$ \\
\hline 4768.170 & 3 & 75 & 20966.544 & $20080_{\mathrm{o} / 2 / 2}^{\circ}-41047_{9 / 2}$ & 4724.771 & $40 \mathrm{~b}$ & 300 & 21159.127 & $9720_{7 / 2}^{\circ}-30879_{7 / 2}^{\circ}$ \\
\hline 4768.054 & 5 & 100 & 20967.054 & $8378_{7 / 2}^{\circ}-29345_{5 / 2}$ & 4724.553 & 2 & 8 & 21160.104 & $32850_{5 / 2}^{\circ}-54010_{7 / 2}$ \\
\hline 4767.871 & 1 & 10 & 20967.859 & $19248_{5 / 2}^{\circ}-40216_{5 / 2}$ & 4724.155 & 2 & 50 & 21161.886 & $22642_{9 / 2}^{\circ}-43803_{7 / 2}$ \\
\hline 4766.961 & 5 & 150 & 20971.861 & $14484_{11 / 2}^{\circ}-35456_{9 / 2}$ & 4723.783 & 300 & 500 & 21163.553 & $1521_{5 / 2}-22685_{7 / 2}^{\circ}$ \\
\hline 4766.016 & $0 \mathrm{~b}$ & 25 & 20976.020 & $33384_{9 / 2}-54360_{9 / 2}^{\circ}$ & 4722.974 & $2 \mathrm{~b}$ & 25 & 21167.178 & $22642_{9 / 2}^{\circ}-43809_{9 / 2}$ \\
\hline 4.765 .655 & $5 b$ & 25 & 20977.608 & $24757_{9 / 2}^{\circ}-45735_{11 / 2}^{\circ}$ & 4722.948 & $\mathrm{lb}$ & $10 \mathrm{~b}$ & 21167.294 & $17983_{5 / 2}^{\circ}-39150_{3 / 2}^{\circ}$ \\
\hline 4763.890 & 1 & 5 & 20985.380 & $23518_{7 / 2}^{\circ}-44503_{7 / 2}$ & 4721.882 & 3 & 100 & 21172.073 & $19050_{3 / 2}^{\circ}-40222_{3 / 2}$ \\
\hline 4763.718 & 2 & 20 & 20986.138 & $22028_{15 / 2}^{\circ}-43014_{13 / 2}^{\circ}$ & 4719.979 & 201 & 400 & 21180.609 & $14275_{9 / 2}^{\circ}-35456_{9 / 2}^{\circ}$ \\
\hline 4763.398 & 0 & 2 & 20987.548 & $22139_{9 / 2}^{\circ}-43127_{11 / 2}$ & 4719.251 & 3 & 50 & 21183.877 & $15144_{3 / 2}^{\circ}-36328_{3 / 2}$ \\
\hline 4762.519 & 5 & 20 & 20991.422 & $1521_{5 / 2}-22513_{5 / 2}^{\circ}$ & 4718.616 & 25 & 300 & 21186.727 & $19912_{13 / 2}^{\circ}-41099_{15 / 2}^{\circ / 2}$ \\
\hline 4761.854 & 4 & 100 & 20994.353 & $31924_{11 / 2}^{\circ}-52918_{13 / 2}$ & 4717.277 & 1 & 5 & 21192.741 & $13468_{9 / 2}^{\circ}-34661_{11 / 2}$ \\
\hline 4761.631 & 0 & 1 & 20995.336 & $32850_{5 / 2}^{\circ}-53845_{5 / 2}^{\circ}$ & 4716.606 & 2 & 8 & 21195.756 & $14545_{5 / 2}^{\circ}-35741_{5 / 2}^{\circ}$ \\
\hline 4.761 .353 & 1 & 8 & 20996.562 & $23730_{9 / 2}^{\circ}-44727_{11 / 2}^{\circ}$ & 4715.998 & $3 \mathrm{~b}$ & 75 & 21198.488 & $16906_{7 / 2}^{\circ}-38105_{5 / 2}^{\circ}$ \\
\hline 4761.106 & 75 & 500 & 20997.651 & $16564_{11 / 2}^{\circ}-37562_{11 / 2}^{\circ}$ & 4715.429 & 20 & 200 & 21201.046 & $10572_{9 / 2}^{\circ}-31773_{9 / 2}$ \\
\hline 4760.937 & 3 & 75 & 20998.396 & $17837_{1 / 2}^{\circ}-38836_{3 / 2}$ & 4714.867 & 3 & 50 & 21203.573 & $27249_{7 / 2}^{\circ}-48453_{7 / 2}$ \\
\hline 4760.052 & 2 & 20 & 21002.301 & $20686_{5 / 2}^{\circ}-41688_{7 / 2}^{\circ}$ & 4713.839 & 2 & 8 & 21208.198 & $\begin{array}{l}20120_{5 / 2}^{\circ}-41328_{5 / 2}^{\circ} \\
15236_{3 / 2}-36444_{3 / 2}^{\circ}\end{array}$ \\
\hline 4759.582 & 3 & 75 & 21004.375 & $15324_{1 / 2}^{\circ}-36328_{3 / 2}$ & & & & & \\
\hline 4756.619 & 3 & 50 & & $17722_{9 / 2}^{\circ}-38740_{11 / 2}$ & 4712.393 & $50 \mathrm{~b}$ & 150 & 21214.705 & $9238_{9 / 2}^{\circ}-30452_{9 / 2}$ \\
\hline 4754.403 & 3 & 2 & 21027.254 & $26647_{13 / 2}^{\circ}-47675_{1 / 1 / 2}^{\circ}$ & 4711.141 & 2 & 10 & & $21131_{3 / 2}^{\circ}-42352_{5 / 2}^{\circ}$ \\
\hline 4754.208 & 2 & 40 & 21028.117 & $16818_{7 / 2}-37846_{5 / 2}^{\circ}$ & 4709.598 & 2 & 8 & 21227.295 & $19050_{3 / 2}^{\circ}-40278_{3 / 2}$ \\
\hline 4753.810 & 2 & 25 & $21029 . \varepsilon$ & $19248_{5 / 2}^{\circ}-40278_{3 / 2}$ & 4708.102 & 50 & 200 & 21234 & $11725_{1 / 2}^{\circ}-32959_{3 / 2}$ \\
\hline 4752.414 & 200 & 500 & 21036.055 & $6213_{9 / 2}-27249_{7 / 2}^{\circ}$ & 4707.581 & 2 & 25 & 21236.390 & $26770_{11 / 2}^{\circ}-48006_{9 / 2}^{\circ}$ \\
\hline 4751.328 & 2 & 20 & 21040.863 & $14484_{11 / 2}^{\circ}-35525_{11 / 2}$ & 4706.250 & 50 & $200 \mathrm{~b}$ & 21242.396 & $7001_{3 / 2}-28243_{5 / 2}^{\circ}$ \\
\hline 4751.117 & 2 & 1 & 21041.797 & $25381_{3 / 2}-46423_{3 / 2}^{\circ}$ & 4705.758 & 200 & 500 & 21244.617 & $9711_{7 / 2}-30956_{9 / 2}^{\circ}$ \\
\hline 4748.585 & $3 b$ & 75 & & $8378_{7 / 2}^{\circ}-29431_{7 / 2}$ & 4705.615 & 50 & 200 & 21245.262 & $12485_{/ / 2}^{\circ}-33730_{5 / 2}$ \\
\hline 4747.550 & 2 & 2 & 21057.606 & $24132_{3 / 2}^{\circ}-45189_{5 / 2}^{\circ}$ & 4705.294 & 2 & 10 & 21246.712 & $9238_{9 / 2}^{\circ}-30484_{11 / 2}^{\circ}$ \\
\hline 4743.693 & $150 \mathrm{w}$ & 300 & 21074.728 & $4113_{5 / 2}-25188_{3 / 2}^{\circ}$ & 4705.084 & 1 & 4 & 21247.660 & $20080_{\sigma / 2}^{\circ}-41328_{5 / 2}$ \\
\hline 4743.112 & 2 & 10 & 21077.309 & $23730_{9 / 2}^{\circ}-44807_{7 / 2}$ & 4704.657 & 2 & 25 & 21249.589 & $14275_{9 / 2}^{\circ}-35525_{11 / 2}$ \\
\hline 4742.259 & $10 \mathrm{~s}$ & 100 & 1.100 & $10673_{5 / 2}^{\circ}-31754_{5 / 2}$ & 4703.844 & 2 & 5 & 21253.261 & $20969_{7 / 2}^{\circ}-42222_{7 / 2}$ \\
\hline 4741.384 & 5 & 200 & 21084.9 & $13468_{9 / 2}^{\circ}-3455$ & 4703.577 & 1 & 3 & 21254 . & $25607_{9 / 2}^{\circ}-46861_{11 / 12}$ \\
\hline 4740.529 & 500 & 500 & 21088.793 & $6168_{7 / 2}^{\circ}-27257_{7 / 2}$ & 4703.404 & 3 & 4 & 21255.249 & $13406_{13 / 2}^{\circ}-34661_{11 / 2}^{\circ}$ \\
\hline 4739.860 & 5 & 100 & 21091.770 & $17771_{11 / 2}^{\circ}-38862_{11 / 2}$ & 4702.624 & 3 & 25 & 21258.775 & $16906_{7 / 2}^{\circ}-38165_{7 / 2}$ \\
\hline 4737.656 & 4 & 100 & & $15710_{3 / 2}^{\circ}-36812_{1 / 2}$ & & & & & $12472_{5 / 2}^{\circ}-33730_{5 / 2}$ \\
\hline 4736.399 & 4 & 200 & 2110 & $22139_{9 / 2}^{\circ}-43246_{7 / 2}$ & 4702.359 & 3 & $25 b$ & 21259.973 & $8460_{3 / 2}-29720_{3 / 2}^{\circ}$ \\
\hline 4735.780 & 2 & 5 & 21109.941 & $20288_{11 / 2}^{\circ}-41398_{9 / 2}^{\circ}$ & 4702.309 & 8 & 200 & 21260.199 & $9711_{7 / 2}-30972_{5 / 2}^{\circ}$ \\
\hline 4735.702 & 2 & 8 & 21110.288 & $23697_{7 / 2}^{\circ}-44807_{7 / 2}$ & 4700.574 & 1 & 4 & 21268.046 & $17460_{5 / 2}^{\circ}-38728_{5 / 2}$ \\
\hline 4735.245 & 2 & 15 & 21112.326 & $22014_{11 / 2}^{\circ}-43127_{11 / 2}$ & 4700.137 & 75 & 200 & 21270.023 & $14275_{9 / 2}^{\circ}-35545_{9 / 2}$ \\
\hline 4734.757 & 2 & 10 & 21114.502 & $8605_{5 / 2}-29720_{3 / 2}^{\circ}$ & 4699.460 & 1 & 10 & 21273.087 & $21682_{7 / 2}^{\circ}-42955_{9 / 2}$ \\
\hline 4734.626 & 2 & 10 & 21115.086 & $16564_{11 / 2}^{\circ}-37679_{11 / 2}^{\circ}$ & 4699.091 & 2 & 20 & 21274.758 & $14790_{7 / 2}^{\circ}-36065_{5 / 2}^{\circ}$ \\
\hline 4733.023 & 3 & $50 \mathrm{~h}$ & 21122.237 & $18816_{13 / 2}^{\circ}-39939_{1 / 1 / 2}^{\circ}$ & 4698.767 & 4 & 100 & 21276.225 & $16564_{1 / 2}^{\circ}-37840_{9 / 2}^{\circ}$ \\
\hline 4732.668 & 8 & 200 & 21123.821 & $22685_{7 / 2}^{\circ}-43809_{9 / 2}$ & 4698.270 & 2 & 50 & 21278.475 & $33215_{3 / 2}^{\circ}-54493_{5 / 2}$ \\
\hline 4730.877 & 4 & 10 & 21131.818 & $0_{3 / 2}-21131_{3 / 2}^{\circ}$ & 4695.835 & 1 & 5 & 21289.509 & $23518_{7 / 2}^{\circ}-44807_{7 / 2}^{\circ}$ \\
\hline 4729.875 & 50 & 200 & 21136.295 & $8378_{7 / 2}^{\circ}-29515_{9 / 2}$ & 4694.921 & 10 & 200 & 21293.654 & $4146_{7 / 2}-25440_{5 / 2}^{\circ}$ \\
\hline 4729.084 & $50 \mathrm{~b}$ & 40 & 21139.830 & $17722_{9 / 2}^{\circ}-38862_{11 / 2}^{\circ}$ & 4694.844 & 1 & 3 & 21294.003 & $9585_{5 / 2}^{\circ}-30879_{7 / 2}^{\circ / 2}$ \\
\hline 4726.462 & 4 & 200 & 21151.558 & $12485_{/ 2 / 2}^{\circ}-33637_{7 / 2}$ & 4694.783 & 51 & 401 & 21294.280 & $22513_{5 / 2}^{\circ}-43807_{3 / 2}$ \\
\hline 4726.333 & 5 & 40 & 21152.135 & $1859_{3 / 2}-23012_{3 / 2}^{\circ}$ & 4694.282 & 1 & 25 & 21296.552 & $17460_{5 / 2}^{\circ}-38757_{3 / 2}^{\circ}$ \\
\hline
\end{tabular}


TABle 3. Classified lines of Th II-Continued

\begin{tabular}{|c|c|c|c|c|c|c|c|c|c|}
\hline \multirow{2}{*}{$\begin{array}{c}\text { Wavelength } \\
\AA\end{array}$} & \multicolumn{2}{|c|}{ Intensity } & \multirow{2}{*}{$\begin{array}{c}\text { Wavenumber } \\
\mathrm{cm}^{-1}\end{array}$} & \multirow{2}{*}{ Classification } & \multirow{2}{*}{$\begin{array}{c}\text { Wavelength } \\
\AA\end{array}$} & \multicolumn{2}{|c|}{ Intensity } & \multirow{2}{*}{$\begin{array}{l}\text { Wavenumber } \\
\mathbf{c m}^{-1}\end{array}$} & \multirow{2}{*}{ Classification } \\
\hline & Lamp & Spark & & & & Lamp & Spark & & \\
\hline 4694.090 & 400 & 300 & 21297.423 & $0_{3 / 2}-21297_{5 / 2}^{\circ}$ & 4647.913 & 2 & 4 & 21509.010 & $16033_{5 / 2}^{\circ}-37542_{3 / 2}$ \\
\hline 4691.050 & 10 & 400 & 21311.225 & $19912_{13 / 2}^{\circ}-41223_{11 / 2}$ & 4646.140 & 1 & 3 & 21517.218 & $23372_{3 / 2}^{\circ}-44889_{3 / 2}$ \\
\hline 4690.680 & 10 & 200 & 21312.906 & $17837_{1 / 2}^{\circ}-39150_{3 / 2}$ & 4645.495 & 2 & 20 & 21520.205 & $14545_{5 / 2}^{\circ}-36065_{5 / 2}$ \\
\hline 4690.331 & 3 & 100 & 21314.492 & $17771_{11 / 2}^{\circ}-39085_{13 / 2}$ & 4642.889 & 1 & 5 & 21532.284 & $34553_{9 / 2}-56086_{9 / 2}^{\circ}$ \\
\hline 4689.596 & 1 & 5 & 21317.832 & $20080_{7 / 2}^{\circ}-41398_{9 / 2}$ & 4642.272 & 1 & 5 & 21535.146 & $22642_{9 / 2}^{\circ}-44177_{11 / 2}$ \\
\hline 4689.170 & 100 & $300 \mathrm{~b}$ & 21319.769 & $6691_{3 / 2}^{\circ}-28011_{3 / 2}$ & 4642.055 & 3 & 75 & 21536.153 & $20686_{5 / 2}^{\circ}-42222_{7 / 2}$ \\
\hline 4688.276 & 3 & 100 & 21323.834 & $14101_{1 / 2}^{\circ}-35425_{1 / 2}$ & 4641.435 & 2 & 3 & 21539.029 & $9720_{7 / 2}^{\circ}-31259_{5 / 2}$ \\
\hline 4686.568 & 4 & 75 & 21331.605 & $12570_{7 / 2}-33902_{7 / 2}^{\circ}$ & 4641.181 & 50 & 200 & 21540.208 & $23187_{13 / 2}^{\circ}-44727_{11 / 2}$ \\
\hline 4685.963 & 3 & 50 & 21334.359 & $14790_{7 / 2}^{\circ}-36125_{9 / 2}$ & 4640.045 & 50 & $200 \mathrm{~b}$ & 21545.482 & $10379_{9 / 2}-31924_{11 / 2}^{\circ}$ \\
\hline 4685.827 & 3 & 8 & 21334.979 & $6691_{3 / 2}^{\circ}-28026_{5 / 2}$ & 4639.704 & 75 & 300 & 21547.065 & $12472_{5 / 2}^{\circ}-34019_{3 / 2}$ \\
\hline 4685.494 & 2 & 200 & 21336.495 & $24463_{5 / 2}^{\circ}-45800_{5 / 2}$ & 4639.094 & 3 & 100 & 21549.898 & $20969_{7 / 2}^{\circ}-42518_{7 / 2}$ \\
\hline 4685.448 & 1 & 5 & 21336.704 & $27787_{9 / 2}^{\circ}-49124_{11 / 2}$ & 4633.830 & $40 \mathrm{~b}$ & 40 & 21574.378 & $6213_{9 / 2}-27787_{9 / 2}^{\circ}$ \\
\hline 4684.127 & 2 & 25 & 21342.722 & $35602_{11 / 2}^{\circ}-56945_{11 / 2}$ & 4631.760 & 150 & 400 & 21584.020 & $10189_{11 / 2}^{\circ}-31773_{9 / 2}$ \\
\hline 4683.533 & 1 & 2 & 21345.428 & $17272_{9 / 2}^{\circ}-38617_{9 / 2}$ & 4631.348 & 2 & 4 & 21585.940 & $7001_{3 / 2}-28587_{5 / 2}^{\circ}$ \\
\hline 4683.484 & 2 & 3 & 21345.652 & $17722_{9 / 2}^{\circ}-39068_{7 / 2}$ & 4630.353 & 2 & 50 & 21590.579 & $17272_{9 / 2}^{\circ}-38862_{11 / 2}$ \\
\hline 4681.649 & 2 & 2 & 21354.018 & $37945_{5 / 2}-59299_{5 / 2}^{\circ}$ & 4629.769 & 10 & 75 & 21593.302 & $9400_{5 / 2}-30994_{7 / 2}^{\circ}$ \\
\hline 4681.163 & 2 & 20 & 21356.235 & $15453_{7 / 2}^{\circ}-36809_{7 / 2}$ & 4629.375 & 25 & 200 & 21595.140 & $24309_{11 / 2}^{\circ}-45904_{9 / 2}$ \\
\hline 4680.640 & $40 \mathrm{~b}$ & $150 \mathrm{~b}$ & 21358.621 & $6168_{7 / 2}^{\circ}-27526_{9 / 2}$ & 4629.005 & 2 & 75 & 21596.866 & $18973_{7 / 2}^{\circ}-40570_{7 / 2}$ \\
\hline 4679.045 & 1 & 20 & 21365.902 & $20310_{5 / 2}^{\circ}-41676_{3 / 2}$ & 4.627 .628 & 3 & 75 & 21603.292 & $14275_{9 / 2}^{\circ}-35878_{7 / 2}$ \\
\hline 4678.594 & 1 & 2 & 21367.962 & $20120_{5 / 2}^{\circ}-41488_{7 / 2}$ & 4626.818 & 1 & 2 & 21607.074 & $17121_{3 / 2}^{\circ}-38728_{5 / 2}$ \\
\hline 4678.200 & 4 & 10 & 21369.761 & $24982_{7 / 2}^{\circ}-46352_{7 / 2}$ & 4626.666 & 2 & 50 & 21607.784 & $20080_{7 / 2}^{\circ}-41688_{7 / 2}$ \\
\hline 4676.936 & 3 & 5 & 21375.537 & $17460_{5 / 2}^{\circ}-38836_{3 / 2}$ & 4626.604 & 2 & 10 & 21608.074 & $17460_{5 / 2}^{\circ}-39068_{7 / 2}$ \\
\hline 4676.680 & 1 & 5 & 21376.707 & $23012_{3 / 2}^{\circ}-44388_{5 / 2}$ & 4626.133 & 2 & 4 & 21610.274 & $15453_{7 / 2}^{\circ}-37063_{9 / 2}$ \\
\hline 4676.502 & 2 & 75 & 21377.520 & $20310_{5 / 2}^{\circ}-41688_{7 / 2}$ & 4625.054 & 10 & 300 & 21615.315 & $16564_{11 / 2}^{\circ}-38179_{9 / 2}$ \\
\hline 4675.934 & 2 & 75 & 21380.117 & $18568_{1 / 2}^{\circ}-39948_{1 / 2}$ & 4624.136 & 10 & 200 & 21619.606 & $11116_{7 / 2}^{\circ}-32736_{7 / 2}$ \\
\hline 4675.290 & 2 & 15 & 21383.062 & $11576_{3 / 2}^{\circ}-32959_{3 / 2}$ & 4623.892 & 5 & 400 & 21620.747 & $24982_{7 / 2}^{\circ}-46603_{5 / 2}$ \\
\hline 4674.816 & 1 & 3 & 21385.230 & $16906_{7 / 2}^{\circ}-38291_{7 / 2}$ & & & & & $20288_{11 / 2}^{\circ}-41909_{9 / 2}$ \\
\hline 4674.730 & 1 & 3 & 21385.624 & $24414_{3 / 2}^{\circ}-45800_{5 / 2}$ & 4623.397 & 1 & 5 & 21623.062 & $12219_{3 / 2}-33843_{3 / 2}^{\circ}$ \\
\hline 4670.889 & 1 & 2 & 21403.209 & $17460_{5 / 2}^{\circ}-38863_{5 / 2}$ & 4622.065 & 2 & 5 & 21629.293 & $28243_{5 / 2}^{\circ}-49873_{5 / 2}$ \\
\hline 4666.991 & 4 & 20 & 21421.085 & $10379_{9 / 2}-31800_{7 / 2}^{\circ}$ & & & & & $17722_{9 / 2}^{\circ}-39352_{11 / 2}$ \\
\hline 4666.002 & 40 & 200 & 21425.626 & $6168_{7 / 2}^{\circ}-27593_{5 / 2}$ & 4620.725 & 3 & 20 & 21635.565 & $17121_{3 / 2}^{\circ}-38757_{3 / 2}$ \\
\hline 4665.955 & 2 & 25 & 21425.842 & $24309_{1 / 2}^{\circ}-45735_{11 / 2}$ & 4619.561 & 50 & 300 & 21641.017 & $14484_{11 / 2}^{\circ}-36125_{9 / 2}$ \\
\hline 4664.542 & 1 & 3 & 21432.332 & $16033_{5 / 2}^{\circ}-37465_{5 / 2}$ & 4619.480 & $100 \mathrm{~b}$ & 400 & 21641.396 & $9238_{9 / 2}^{\circ}-30879_{7 / 2}$ \\
\hline 4663.372 & 1 & 5 & 21437.709 & $18973_{7 / 2}^{\circ}-40411_{7 / 2}$ & 4613.978 & 2 & 100 & 21667.202 & $22106_{5 / 2}-43773_{7 / 2}^{\circ}$ \\
\hline 4661.423 & 2 & 100 & 21446.672 & $25414_{11 / 2}^{\circ}-46861_{11 / 2}$ & 4613.875 & 2 & 25 & 21667.686 & $15144_{3 / 2}^{\circ}-36812_{1 / 2}$ \\
\hline 4659.846 & 1 & 3 & 21453.930 & $21297_{5 / 2}^{\circ}-42751_{7 / 2}$ & 4613.762 & 2 & 50 & 21668.217 & $24.132_{3 / 2}^{\circ}-45800_{5 / 2}$ \\
\hline 4659.799 & 5 & 3 & 21454.147 & $22642_{9 / 2}^{\circ}-44096_{9 / 2}$ & 4613.468 & 3 & 100 & 21669.598 & $22139_{9 / 2}^{\circ}-43809_{9 / 2}$ \\
\hline 4658.727 & 2 & 15 & 21459.083 & $24757_{9 / 2}^{\circ}-46216_{11 / 2}$ & 4613.263 & 2 & 8 & 21670.561 & $18973_{7 / 2}^{\circ}-4.0644_{5 / 2}$ \\
\hline 4658.492 & $10 \mathrm{~b}$ & $10 \mathrm{~b}$ & 21460.166 & $11116_{7 / 2}^{\circ}-32576_{7 / 2}$ & 4612.544 & 200 & 150 & 21673.938 & $9585_{5 / 2}^{\circ}-31259_{5 / 2}$ \\
\hline 4656.748 & 2 & 8 & 21468.203 & $17272_{9 / 2}^{\circ}-38740_{11 / 2}$ & 4612.168 & 2 & 20 & 21675.705 & $16818_{7 / 2}-38493_{5 / 2}^{\circ}$ \\
\hline 4655.727 & 1 & 3 & 21472.911 & $19050_{3 / 2}^{\circ}-40523_{1 / 2}$ & 4611.859 & 50 & 300 & 21677.158 & $9202_{7 / 2}^{\circ}-30879_{7 / 2}$ \\
\hline 4654.272 & 2 & 2 & 21479.623 & $29788_{9 / 2}^{\circ}-51268_{7 / 2}$ & 4609.370 & 50 & 400 & 21688.863 & $12485_{7 / 2}^{\circ}-34174_{5 / 2}$ \\
\hline 4653.084 & 2 & 15 & 21485.107 & $35593_{9 / 2}^{\circ}-57078_{9 / 2}$ & 4606.501 & 25 & 200 & 21702.371 & $12472_{5 / 2}^{\circ}-34174_{5 / 2}$ \\
\hline 4652.851 & 2 & 15 & 21486.183 & $18214_{3 / 2}^{\circ}-39700_{5 / 2}$ & 4606.299 & 2 & 50 & 21703.322 & $22685_{7 / 2}^{\circ}-44388_{5 / 2}$ \\
\hline 4652.415 & 3 & 8 & 21488.197 & $15324_{1 / 2}^{\circ}-36812_{1 / 2}$ & 4605.232 & 2 & 75 & 21708.351 & $25440_{5 / 2}^{\circ}-47148_{3 / 2}$ \\
\hline 4.651 .986 & 75 & 200 & 21490.178 & $1521_{5 / 2}-23012_{3 / 2}^{\circ}$ & 4604.651 & 2 & 25 & 21711.090 & $16906_{7 / 2}^{\circ}-38617_{9 / 2}$ \\
\hline 4.651 .554 & $150 \mathrm{~b}$ & 500 & 21492.174 & $7331_{5 / 2}^{\circ}-28823_{5 / 2}$ & 4604.160 & 2 & 8 & 21713.405 & $15349_{11 / 2}^{\circ}-37063_{9 / 2}$ \\
\hline 4650.918 & 8 & 75 & 21495.113 & $8378_{7 / 2}^{\circ}-29873_{7 / 2}$ & 4603.907 & 2 & 100 & 21714.599 & $17121_{3 / 2}^{\circ}-38836_{3 / 2}$ \\
\hline 4650.815 & 50 & 75 & 21495.589 & $8605_{5 / 2}-30101_{7 / 2}^{\circ}$ & 4602.885 & 75 & 300 & 21719.420 & $7001_{3 / 2}-28720_{3 / 2}^{\circ}$ \\
\hline 4650.757 & 2 & 75 & 21495.857 & $25414_{11 / 2}^{\circ}-46910_{13 / 2}$ & 4601.952 & 2 & 50 & 21723.823 & $24982_{7 / 2}^{\circ}-46706_{7 / 2}$ \\
\hline
\end{tabular}


TABLE 3. Classified lines of Th II-Continued

\begin{tabular}{|c|c|c|c|c|c|c|c|c|c|}
\hline \multirow{2}{*}{$\begin{array}{c}\text { Wavelength } \\
\AA \\
\end{array}$} & \multicolumn{2}{|c|}{ Intensity } & \multirow{2}{*}{$\begin{array}{c}\text { Wavenumber } \\
\mathbf{c m}^{-1}\end{array}$} & \multirow{2}{*}{ Classification } & \multirow{2}{*}{$\begin{array}{c}\text { Wavelength } \\
\AA\end{array}$} & \multicolumn{2}{|c|}{ Intensity } & \multirow{2}{*}{$\begin{array}{c}\text { Wavenumber } \\
\mathbf{c m}^{-1}\end{array}$} & \multirow{2}{*}{ Classification } \\
\hline & Lamp & Spark & & & & Lamp & Spark & & \\
\hline 4.599 .522 & 2 & 5 & 21735.300 & $17722_{9 / 2}^{\circ}-39458_{7 / 2}$ & 4556.806 & 2 & 10 & 21939.046 & $14101_{i / 2}^{\circ}-36040_{1 / 2}$ \\
\hline 4599.353 & 8 & 10 & 21736.099 & $13250_{5 / 2}-34986_{3 / 2}^{\circ}$ & 4555.063 & 2 & 50 & 21947.441 & $21297_{5 / 2}^{\circ}-43244_{3 / 2}$ \\
\hline 4598.055 & 1 & 5 & 21742.235 & $17121_{3 / 2}^{\circ}-38863_{5 / 2}$ & 4554.792 & 3 & 75 & 21948.747 & $24757_{9 / 2}^{\circ}-46706_{7 / 2}^{\circ}$ \\
\hline 4597.915 & 1 & 50 & 21742.897 & $30564_{1 / 2}^{o}-52307_{3 / 2}$ & 4554.657 & 2 & 50 & 21949.397 & $21297_{5 / 2}^{\circ}-43246_{7 / 2}$ \\
\hline 4597.357 & 1 & 3 & 21745.536 & $17121_{3 / 2}^{\circ}-38867_{1 / 2}$ & 4554.565 & 3 & 25 & 21949.840 & $18973_{7 / 2}^{\circ}-40923_{5 / 2}$ \\
\hline 4596.937 & 1 & 5 & 21747.522 & $25188_{3 / 2}^{\circ}-46935_{3 / 2}$ & 4554.082 & 3 & 10 & 21952.169 & $9400_{5 / 2}-31353_{3 / 2}^{\circ}$ \\
\hline 4594.749 & 1 & 3 & 21757.878 & $18816_{13 / 2}^{\circ}-40574_{11 / 2}$ & 4553.837 & 4 & 100 & 21953.350 & $16564_{11 / 2}^{\circ}-38517_{13 / 2}^{\circ}$ \\
\hline 4593.979 & 3 & 75 & 21761.525 & $20989_{9 / 2}^{\circ}-42751_{7 / 2}$ & 4553.162 & 2 & 15 & 21956.604 & $22139_{9 / 2}^{\circ}-44096_{9 / 2}$ \\
\hline 4593.553 & 5 & 75 & 21763.543 & $16818_{7 / 2}-38581_{5 / 2}^{\circ}$ & 4553.118 & 2 & 40 & 21956.816 & $34726_{7 / 2}-56683_{7 / 2}^{\circ}$ \\
\hline 4593.283 & 3 & 200 & 21764.822 & $22685_{7 / 2}^{\circ}-44450_{9 / 2}$ & 4553.027 & $3 b$ & 200 & 21957.255 & $16906_{7 / 2}^{\circ}-38863_{5 / 2}$ \\
\hline 4592.781 & 8 & 25 & 21767.201 & $27357_{9 / 2}^{\circ}-49124_{11 / 2}$ & 4552.359 & 2 & 20 & 21960.477 & $25188_{3 / 2}^{\circ}-47148_{3 / 2}$ \\
\hline 4589.835 & 2 & 100 & 21781.172 & $17771_{1 / 2}^{\circ}-39552_{9 / 2}$ & 4551.541 & 3 & 20 & 21964.423 & $26647_{13 / 2}^{\circ}-48612_{13 / 2}$ \\
\hline 4589.667 & $50 \mathrm{~b}$ & 150 & 21781.970 & $12488_{9 / 2}^{\circ}-34270_{9 / 2}$ & 4551.205 & 1 & 8 & 21966.045 & $20989_{9 / 2}^{\circ}-42955_{9 / 2}^{\circ}$ \\
\hline 4589.445 & 1 & 3 & 21783.023 & $14545_{5 / 2}^{\circ}-36328_{3 / 2}$ & 4550.429 & 2 & 4 & 21969.791 & $15786_{5 / 2}-37756_{7 / 2}^{\circ}$ \\
\hline 4589.121 & 20 & 200 & 21784.561 & $12485_{7 / 2}^{\circ}-34270_{9 / 2}$ & 4550.377 & 1 & 4 & 21970.042 & $19912_{13 / 2}^{\circ}-41882_{13 / 2}^{\circ}$ \\
\hline 4.588 .226 & 10 & 300 & 21788.810 & $16033_{5 / 2}^{\circ}-37821_{3 / 2}$ & 4546.832 & 25 & 15 & 21987.171 & $13468_{9 / 2}^{\circ}-35456_{9 / 2}$ \\
\hline 4587.756 & 20 & 75 & 21791.043 & $12488_{9 / 2}^{\circ}-34279_{7 / 2}^{\circ}$ & 4546.132 & 10 & 40 & 21990.556 & $22513_{5 / 2}^{\circ}-44503_{7 / 2}$ \\
\hline 4587.209 & 3 & 50 & 21793.641 & $12485_{7 / 2}^{\circ}-34279_{7 / 2}$ & 4545.824 & 2 & 25 & 21992.046 & $12219_{3 / 2}-34212_{5 / 2}^{\circ}$ \\
\hline 4586.620 & 3 & 75 & 21796.440 & $17272_{9 / 2}^{\circ}-39068_{7 / 2}$ & 4545.348 & $3 b$ & 3 & 21994.349 & $13406_{13 / 2}^{\circ}-35400_{13 / 2}^{\circ}$ \\
\hline 4586.267 & 4 & 200 & 21798.117 & $24757_{9 / 2}^{\circ}-46555_{11 / 2}$ & 4545.270 & 2 & 4 & 21994.727 & $10855_{7 / 2}-32850_{5 / 2}^{\circ}$ \\
\hline 4586.061 & $50 \mathrm{~b}$ & 50 & 21799.097 & $21297_{5 / 2}^{\circ}-43096_{\tilde{s} / 2}$ & 4544.895 & 8 & 15 & 21996.541 & $1521_{5 / 2}-23518_{7 / 2}^{\circ}$ \\
\hline 4584.368 & 8 & 200 & 21807.147 & $12472_{5 / 2}^{\circ}-34279_{7 / 2}$ & 4544.512 & 150 & 200 & 21998.395 & $4490_{5 / 2}^{\circ}-26488_{5 / 2}$ \\
\hline 4584.159 & 1 & 5 & 21808.141 & $22642_{9 / 2}^{\circ}-44450_{9 / 2}^{\circ}$ & 4543.208 & 4 & 20 & 22004.709 & $10572_{9 / 2}^{\circ}-32576_{7 / 2}^{\circ}$ \\
\hline 4583.654 & 2 & 40 & 21810.544 & $15242_{9 / 2}^{\circ}-37053_{11 / 2}^{\circ}$ & 454.2 .427 & 2 & 15 & 22008.493 & $18214_{3 / 2}^{\circ}-40222_{3 / 2}$ \\
\hline 4582.778 & 2 & 50 & 21814.712 & $15786_{5 / 2}-37601_{3 / 2}^{\circ}$ & 4541.615 & 3 & 40 & 22012.427 & $15453_{7 / 2}^{\circ}-37465_{5 / 2}$ \\
\hline 4582.436 & 3 & 50 & 21816.341 & $22834_{7 / 2}-44650_{7 / 2}^{\circ}$ & 4541.204 & 4 & 10 & 22014.420 & $7331_{5 / 2}^{\circ}-29345_{5 / 2}$ \\
\hline 4581.815 & 1 & 1 & 21819.297 & $27249_{7 / 2}^{\circ}-49068_{5 / 2}$ & 4540.403 & 40 & 200 & 22018.303 & $14790_{7 / 2}^{\circ}-36809_{7 / 2}$ \\
\hline 4581.581 & 20 & 200 & 21820.412 & $15242_{9 / 2}^{\circ}-37063_{9 / 2}$ & 4540.087 & 4 & 15 & 22019.836 & $27357_{9 / 2}^{\circ}-49377_{7 / 2}^{\circ}$ \\
\hline 4581.229 & $25 b$ & 150 & 21822.088 & $16906_{7 / 2}^{\circ}-38728_{5 / 2}^{\circ}$ & 4538.844 & 4 & 25 & 22025.866 & $20310_{5 / 2}^{\circ}-42336_{5 / 2}$ \\
\hline 4580.758 & 2 & 15 & 21824.332 & $15453_{7 / 2}^{\circ}-37277_{7 / 2}$ & 4538.182 & 1 & 2 & 22029.079 & $17121_{3 / 2}^{\circ}-39150_{3 / 2}$ \\
\hline 4579.292 & 3 & 75 & 21831.319 & $15710_{3 / 2}^{\circ}-37542_{3 / 2}$ & 4537.072 & 20 & 200 & 22034.468 & $15242_{9 / 2}^{\circ}-37277_{7 / 2}$ \\
\hline 4578.995 & 3 & 25 & 21832.735 & $20686_{5 / 2}^{\circ}-42518_{7 / 2}$ & 4536.427 & 1 & 4 & 22037.601 & $22139_{9 / 2}^{\circ}-44177_{11 / 2}^{\circ}$ \\
\hline 4.575 .425 & 10 & 200 & 21849.770 & $14275_{9 / 2}^{\circ}-36125_{9 / 2}$ & 4535.708 & $2 \mathrm{~b}$ & 5 & 22041.094 & $14349_{1 / 2}-36390_{3 / 2}^{\circ}$ \\
\hline 4573.703 & 25 & 150 & 21857.996 & $6168_{7 / 2}^{\circ}-28026_{5 / 2}$ & 4534.117 & 75 & 200 & 22048.828 & $10572_{9 / 2}^{\circ}-32620_{11 / 2}^{\circ}$ \\
\hline 4572.935 & 1 & 8 & 21861.667 & $22642_{9 / 2}^{\circ}-44503_{7 / 2}$ & 4533.302 & 50 & 300 & 22052.792 & $9720_{7 / 2}^{\circ}-31773_{9 / 2}$ \\
\hline 4570.614 & 2 & 75 & 21872.768 & $19050_{3 / 2}^{\circ}-40923_{5 / 2}$ & 4533.240 & 50 & $40 \mathrm{~b}$ & 22053.094 & $16564_{11 / 2}^{\circ}-38617_{9 / 2}$ \\
\hline 4569.812 & 2 & 5 & 21876.607 & $23518_{7 / 2}^{\circ}-45395_{7 / 2}$ & 4532.430 & 3 & 50 & 22057.035 & $9202_{7 / 2}^{\circ}-31259_{5 / 2}$ \\
\hline 4568.236 & 2 & 10 & 21884.154 & $25440_{5 / 2}^{\circ}-47324_{5 / 2}$ & 4532.256 & 50 & 300 & 22057.882 & $12485_{7 / 2}^{\circ}-34543_{5 / 2}$ \\
\hline 4566.650 & 20 & 200 & 21891.754 & $7828_{1 / 2}-29720_{3 / 2}^{\circ}$ & 4531.713 & 8 & 300 & 22060.525 & $13818_{7 / 2}^{\circ}-35878_{7 / 2}^{\circ}$ \\
\hline 4566.282 & 1 & 50 & 21893.519 & $29788_{9 / 2}^{\circ}-51681_{9 / 2}$ & 4531.611 & 2 & 10 & 22061.021 & $20158_{5 / 2}-42219_{5 / 2}^{\circ}$ \\
\hline 4566.247 & 2 & & 21893.686 & $15236_{3 / 2}-37130_{1 / 2}^{\circ}$ & 4531.196 & $3 b$ & 50 & 22063.042 & $10673_{5 / 2}^{\circ}-32736_{7 / 2}$ \\
\hline 4564.178 & 15 & 200 & 21903.611 & $10673_{5 / 2}^{\circ}-32576_{7 / 2}$ & 4530.656 & 1 & 1 & 22065.671 & $12488_{9 / 2}^{\circ}-34553_{9 / 2}^{\circ}$ \\
\hline 4563.727 & 3 & 8 & 21905.775 & $26586_{3 / 2}^{\circ}-48492_{5 / 2}$ & 4529.484 & 4 & 200 & 22071.381 & $12472_{5 / 2}^{\circ}-34543_{5 / 2}$ \\
\hline 4563.291 & 50 & $500 \mathrm{~b}$ & 21907.868 & $18816_{13 / 2}^{\circ}-40724_{13 / 2}^{\circ}$ & 4529.376 & 2 & 3 & 22071.907 & $16033_{5 / 2}^{\circ}-38105_{5 / 2}^{\circ}$ \\
\hline 4563.219 & 10 & 300 & 21908.214 & $23187_{13 / 2}^{\circ}-45095_{15 / 2}$ & 4528.973 & 3 & 50 & 22073.871 & $8378_{7 / 2}^{\circ}-30452_{9 / 2}$ \\
\hline 4562.632 & 2 & 5 & 21911.032 & $9061_{5 / 2}-30972_{5 / 2}^{\circ}$ & 4528.409 & 2 & 50 & 22076.620 & $13468_{9 / 2}^{\circ}-35545_{9 / 2}^{\circ}$ \\
\hline 4562.565 & 2 & 15 & 21911.354 & $20310_{5 / 2}^{\circ}-42222_{7 / 2}$ & 4527.710 & $2 \mathrm{~b}$ & 150 & 22080.028 & $17272_{9 / 2}^{\circ}-39352_{11 / 2}^{\circ}$ \\
\hline 4562.439 & 3 & 25 & 21911.959 & $16033_{5 / 2}^{\circ}-37945_{5 / 2}$ & 4526.028 & 4 & 50 & 22088.234 & $9711_{7 / 2}-31800_{7 / 2}^{\circ}$ \\
\hline 4560.394 & 3 & 10 & 21921.785 & $7001_{3 / 2}-28923_{5 / 2}^{\circ}$ & 4525.090 & 10 & 200 & 22092.812 & $11116_{7 / 2}^{\circ}-33209_{7 / 2}$ \\
\hline 4558.029 & 2 & 10 & 21933.159 & $9061_{5 / 2}-30994_{i / 2}^{\circ}$ & 4524.838 & 25 & 150 & 22094.043 & $7001_{3 / 2}-29095_{5 / 2}^{\circ}$ \\
\hline
\end{tabular}


TABLE 3. Classified lines of Th II-Continued

\begin{tabular}{|c|c|c|c|c|c|c|c|c|c|}
\hline \multirow{2}{*}{$\begin{array}{c}\text { Wavelength } \\
\AA\end{array}$} & \multicolumn{2}{|c|}{ Intensity } & \multirow{2}{*}{$\begin{array}{c}\text { Wavenumber } \\
\mathbf{c m}^{-1}\end{array}$} & \multirow{2}{*}{ Classification } & \multirow{2}{*}{$\begin{array}{c}\text { Wavelength } \\
\AA\end{array}$} & \multicolumn{2}{|c|}{ Intensity } & \multirow{2}{*}{$\begin{array}{c}\text { Wavenumber } \\
\mathbf{c m}^{-1}\end{array}$} & \multirow{2}{*}{ Classification } \\
\hline & Lamp & Spark & & & & Lamp & Spark & & \\
\hline 4524.560 & 1 & 2 & 22095.400 & $14349_{1 / 2}-36444_{3 / 2}^{\circ}$ & 4471.978 & 8 & 20 & 22355.196 & $0_{3 / 2}-22355_{1 / 2}^{\circ}$ \\
\hline 4524.432 & 1 & 2 & 22096.025 & $21131_{3 / 2}^{\circ}-43227_{5 / 2}$ & 4471.764 & 2 & 200 & 22356.266 & $16033_{5 / 2}^{\circ}-38389_{7 / 2}$ \\
\hline 4522.783 & 2 & 150 & 22104.081 & $24757_{9 / 2}^{\circ}-46861_{11 / 2}$ & & & & & $20288_{11 / 2}^{\circ}-42644_{13 / 2}$ \\
\hline \multirow[t]{2}{*}{4521.458} & 1 & 5 & 22110.559 & $12219_{3 / 2}-34330_{1 / 2}^{\circ}$ & 4470.005 & 2 & 75 & 22365.063 & $15236_{3 / 2}-37601_{3 / 2}^{\circ}$ \\
\hline & & & & $17837_{1 / 2}^{\circ}-39948_{1 / 2}$ & 4469.754 & 2 & 3 & 22366.319 & $8605_{5 / 2}-30972_{5 / 2}^{\circ}$ \\
\hline 4520.949 & 2 & 75 & 22113.048 & $21131_{3 / 2}^{\circ}-43244_{3 / 2}$ & 4465.931 & 2 & 2 & 22385.465 & $22513_{5 / 2}^{\circ}-44898_{7 / 2}$ \\
\hline 4520.034 & 3 & 15 & 22117.524 & $24463_{5 / 2}^{\circ}-46581_{5 / 2}$ & 4465.341 & 200 & 200 & 22388.423 & $8605_{5 / 2}-30994_{7 / 2}^{\circ}$ \\
\hline 4519.621 & 1 & 2 & 22119.546 & $20080_{7 / 2}^{\circ}-42200_{9 / 2}$ & 4463.190 & 2 & 20 & 22399.213 & $9400_{5 / 2}-31800_{7 / 2}^{\circ}$ \\
\hline \multirow[t]{2}{*}{4518.636} & $3 b$ & 100 & 22124.367 & $25607_{9 / 2}^{\circ}-47731_{9 / 2}$ & 4463.080 & 1 & 25 & 22399.765 & $25607_{9 / 2}^{\circ}-48006_{9 / 2}^{\circ}$ \\
\hline & & & & $17771_{1 / 12}^{\circ}-39895_{9 / 2}$ & 4461.705 & 4 & 200 & 22406.668 & $18816_{13 / 2}^{\circ}-41223_{11 / 2}$ \\
\hline \multirow[t]{2}{*}{4517.036} & $4 b$ & 200 & 22132.204 & $24132_{3 / 2}^{\circ}-46264_{3 / 2}$ & 4461.125 & 4 & 150 & 22409.581 & $9400_{5 / 2}-31810_{5 / 2}^{\circ}$ \\
\hline & & & & $16033_{5 / 2}^{\circ}-38165_{7 / 2}$ & 4461.065 & 4 & 200 & 22409.882 & $13468_{9 / 2}^{\circ}-35878_{7 / 2}$ \\
\hline 4512.483 & 8 & 200 & 22154.535 & $11576_{3 / 2}^{\circ}-33730_{5 / 2}$ & 4457.082 & 2 & 4 & 22429.908 & $18214_{3 / 2}^{\circ}-40644_{5 / 2}$ \\
\hline 4510.946 & 2 & 3 & 22162.083 & $16906_{7 / 2}^{\circ}-39068_{7 / 2}$ & 4456.708 & 4 & 150 & 22431.790 & $10189_{11 / 2}^{\circ}-32620_{11 / 2}$ \\
\hline 4510.526 & 200 & 600 & 22164.147 & $10572_{9 / 2}^{\circ}-32736_{7 / 2}$ & 4455.715 & 2 & 100 & 22436.789 & $15242_{9 / 2}^{\circ}-37679_{11 / 2}$ \\
\hline 4509.581 & 2 & & 22168.791 & $9585_{5 / 2}^{\circ}-31754_{5 / 2}$ & 4455.576 & 3 & 2 & 22437.489 & $28587_{5 / 2}^{\circ}-51024_{3 / 2}$ \\
\hline 4508.841 & 1 & 2 & 22172.429 & $17722_{9 / 2}^{\circ}-39895_{9 / 2}$ & 4455.424 & 2 & 2 & 22438.255 & $20080_{7 / 2}^{\circ}-42518_{7 / 2}$ \\
\hline 4508.639 & 1 & 2 & 22173.423 & $12488_{9 / 2}^{\circ}-34661_{11 / 2}^{\circ}$ & 4455.294 & 5 & 20 & 22438.909 & $19880_{9 / 2}-42319_{9 / 2}^{\circ}$ \\
\hline 4508.163 & 5 & 40 & 22175.764 & $1521_{5 / 2}-23697_{7 / 2}^{\circ}$ & 4455.045 & 3 & 200 & 22440.164 & $19248_{5 / 2}^{\circ}-41688_{7 / 2}$ \\
\hline 4506.071 & 2 & 2 & 22186.059 & $17272_{9 / 2}^{\circ}-39458_{7 / 2}$ & 4455.005 & 2 & 10 & 22440.365 & $20310_{5 / 2}^{\circ}-42751_{7 / 2}$ \\
\hline 4498.108 & 1 & 1 & 22225.335 & $15349_{11 / 2}^{\circ}-37575_{13 / 2}$ & 4454.513 & 8 & 300 & 22442.844 & $11576_{3 / 2}^{\circ}-34019_{3 / 2}$ \\
\hline 4496.315 & 8 & 200 & 22234.197 & $19912_{13 / 2}^{\circ}-42146_{11 / 2}$ & 4452.791 & 1 & 8 & 22451.522 & $15305_{9 / 2}-37756_{7 / 2}^{\circ}$ \\
\hline 4495.489 & 3 & 100 & 22238.282 & $12488_{9 / 2}^{\circ}-34726_{7 / 2}$ & 4451.268 & 5 & 4 & 22459.204 & $23187_{13 / 2}^{\circ}-45646_{15 / 2}$ \\
\hline 4495.241 & 3 & 150 & 22239.509 & $17983_{5 / 2}^{\circ}-40222_{3 / 2}$ & 4451.051 & 2 & 100 & 22460.299 & $16906_{7 / 2}^{\circ}-39366_{5 / 2}$ \\
\hline 4494.964 & 4 & 200 & 22240.880 & $12485_{7 / 2}^{\circ}-34726_{7 / 2}$ & 4448.555 & 5 & 100 & 22472.901 & $4113_{5 / 2}-26586_{3 / 2}^{\circ}$ \\
\hline 4494.076 & 4 & 40 & 22245.274 & $17121_{3 / 2}^{\circ}-39366_{5 / 2}$ & 4448.133 & 1 & 2 & 22475.033 & $21297_{5 / 2}^{\circ}-43772_{5 / 2}$ \\
\hline 4493.651 & 1 & 2 & 22247.378 & $13818_{7 / 2}^{\circ}-36065_{5 / 2}$ & 4447.834 & 100 & 400 & 22476.544 & $6244_{1 / 2}-28720_{3 / 2}^{\circ}$ \\
\hline 4492.236 & 4 & 200 & 22254.386 & $12472_{5 / 2}^{\circ}-34726_{7 / 2}$ & 4447.787 & 8 & 20 & 22476.781 & $26647_{13 / 2}^{\circ}-49124_{11 / 2}$ \\
\hline 4491.884 & 2 & 100 & 22256.130 & $20080_{7 / 2}^{\circ}-42336_{5 / 2}$ & 4444.987 & 4 & 150 & 22490.940 & $15349_{1 / 2}^{\circ}-37840_{9 / 2}$ \\
\hline 4491.708 & 2 & 100 & 22257.002 & $20989_{9 / 2}^{\circ}-43246_{7 / 2}$ & 4443.086 & 20 & 300 & 22500.562 & $8378_{7 / 2}^{\circ}-30879_{7 / 2}$ \\
\hline 4491.378 & 2 & 100 & 22258.637 & $16033_{5 / 2}^{\circ}-38291_{7 / 2}$ & 4442.370 & 1 & 15 & 22504.189 & $22685_{7 / 2}^{\circ}-45189_{5 / 2}$ \\
\hline 4490.354 & 2 & 50 & 22263.713 & $14545_{5 / 2}^{\circ}-36809_{7 / 2}$ & 4441.905 & 2 & 25 & 22506.54 .5 & $21297_{5 / 2}^{\circ}-43803_{7 / 2}$ \\
\hline 4490.198 & 2 & 150 & 22264.486 & $15305_{9 / 2}-37569_{9 / 2}^{\circ}$ & 4440.866 & 200 & 200 & 22511.810 & $8460_{3 / 2}-30972_{5 / 2}^{\circ}$ \\
\hline 4489.611 & 2 & 2 & 22267.397 & $29095_{5 / 2}^{\circ}-51362_{5 / 2}$ & 4440.574 & 150 & 200 & 22513.291 & $0_{3 / 2}-22513_{5 / 2}^{\circ}$ \\
\hline 4489.527 & 2 & 40 & 22267.814 & $1116_{7 / 2}^{\circ}-33384_{9 / 2}$ & 4439.124 & 150 & 400 & 22520.644 & $11116_{7 / 2}^{\circ}-33637_{7 / 2}$ \\
\hline 4488.678 & 100 & 500 & 22272.026 & $4490_{5 / 2}^{\circ}-26762_{3 / 2}$ & 4437.722 & 20 & 20 & 22527.759 & $9400_{5 / 2}-31928_{3 / 2}^{\circ}$ \\
\hline 4488.628 & 3 & 20 & 22272.274 & $14790_{7 / 2}^{\circ}-37063_{9 / 2}$ & 4436.553 & 75 & 8 & 22533.695 & $14275_{9 / 2}^{\circ}-36809_{7 / 2}$ \\
\hline 4487.495 & 100 & 300 & 22277.897 & $4146_{7 / 2}-26424_{5 / 2}^{\circ}$ & 4436.375 & 1 & 8 & 22534.599 & $22355_{1 / 2}^{\circ}-44889_{3 / 2}$ \\
\hline 4486.631 & 15 & 300 & 22282.187 & $18816_{13 / 2}^{\circ}-41099_{15 / 2}$ & 4436.286 & 50 & 200 & 22535.051 & $9238_{9 / 2}^{\circ}-31773_{9 / 2}^{\circ}$ \\
\hline 4485.795 & 8 & 200 & 22286.340 & $10673_{5 / 2}^{\circ}-32959_{3 / 2}$ & 4436.145 & 1 & 15 & 22535.767 & $18118_{3 / 2}-40654_{5 / 2}^{\circ}$ \\
\hline 4484.291 & 4 & 75 & 22293.814 & $11725_{1 / 2}^{\circ}-34019_{3 / 2}$ & 4436.049 & 20 & 200 & 22536.255 & $10673_{5 / 2}^{\circ}-33209_{7 / 2}$ \\
\hline 4484.235 & 2 & 2 & 22294.092 & $23012_{3 / 2}^{\circ}-45306_{3 / 2}$ & 4434.829 & 4 & 50 & 22542.455 & $7331_{5 / 2}^{\circ}-29873_{7 / 2}$ \\
\hline 4484.106 & 2 & 25 & 22294.734 & $17983_{5 / 2}^{\circ}-40278_{3 / 2}$ & 4434.516 & 1 & 5 & 22544.046 & $22106_{5 / 2}-44650_{7 / 2}^{\circ}$ \\
\hline 4481.640 & 3 & 150 & 22307.001 & $13818_{7 / 2}^{\circ}-36125_{9 / 2}$ & 4434.332 & 4 & 75 & 22544.981 & $15242_{9 / 2}^{\circ}-37787_{7 / 2}$ \\
\hline 4481.178 & 2 & 150 & 22309.301 & $18214_{3 / 2}^{\circ}-40523_{1 / 2}$ & 4433.723 & 2 & 75 & 22548.078 & $23187_{13 / 2}^{\circ}-45735_{11 / 2}$ \\
\hline 4480.909 & 2 & 2 & 22310.640 & $22139_{9 / 2}^{\circ}-44450_{9 / 2}$ & 4432.962 & 500 & 800 & 22551.948 & $9202_{7 / 2}^{\circ}-31754_{5 / 2}$ \\
\hline 4479.158 & 3 & 150 & 22319.362 & $15242_{9 / 2}^{\circ}-37562_{11 / 2}$ & 4432.420 & 2 & 4 & 22554.706 & $1859_{3 / 2}-24414_{3 / 2}^{\circ}$ \\
\hline 4474.073 & 20 & 200 & 22344.728 & $13248_{9 / 2}-35593_{9 / 2}^{\circ}$ & 4431.628 & 1 & 5 & 22558.737 & $20686_{5 / 2}^{\circ}-43244_{3 / 2}$ \\
\hline 4472.275 & 5 & 200 & 22353.711 & $13248_{9 / 2}-35602_{11 / 2}^{\circ}$ & 4429.594 & 3 & 75 & 22569.095 & $14484_{11 / 2}^{\circ}-37053_{11 / 2}$ \\
\hline 44.72 .105 & 1 & 1 & 22354.561 & $18973_{7 / 2}^{\circ}-41328_{5 / 2}$ & 4429.258 & 50 & 100 & 22570.807 & $9202_{7 / 2}^{\circ}-31773_{9 / 2}$ \\
\hline
\end{tabular}


TABLE 3. Classified lines of Th II-Continued

\begin{tabular}{|c|c|c|c|c|c|c|c|c|c|}
\hline \multirow{2}{*}{$\begin{array}{c}\text { Wavelength } \\
\AA \\
\end{array}$} & \multicolumn{2}{|c|}{ Intensity } & \multirow{2}{*}{$\begin{array}{c}\text { Wavenumber } \\
\mathbf{c m}^{-1}\end{array}$} & \multirow{2}{*}{ Classification } & \multirow{2}{*}{$\begin{array}{c}\text { Wavelength } \\
\AA\end{array}$} & \multicolumn{2}{|c|}{ Intensity } & \multirow{2}{*}{$\begin{array}{c}\text { Wavenumber } \\
\mathrm{cm}^{-1}\end{array}$} & \multirow{2}{*}{ Classification } \\
\hline & Lamp & Spark & & & & Lamp & Spark & & \\
\hline 4427.786 & 4 & 50 & 22578.311 & $10379_{9 / 2}-32957_{7 / 2}^{\circ}$ & 4396.477 & 75 & 300 & 22739.097 & $9061_{5 / 2}-31800_{7 / 2}^{\circ}$ \\
\hline \multirow[t]{2}{*}{4427.655} & 50 & 300 & 22578.979 & $17121_{3 / 2}^{\circ}-39700_{5 / 2}$ & 4394.894 & 100 & 300 & 22747.287 & $8605_{5 / 2}-31353_{3 / 2}^{\circ}$ \\
\hline & & & & $14484_{11 / 2}^{\circ}-37063_{9 / 2}$ & 4393.059 & 401 & 400 & 22756.788 & $26770_{11 / 2}^{\circ}-49527_{13 / 2}$ \\
\hline 4426.050 & 1 & 50 & 22587.166 & $29720_{3 / 2}^{\circ}-52307_{3 / 2}$ & 4392.611 & 1 & 8 & 22759.109 & $22139_{9 / 2}^{\circ}-44898_{7 / 2}$ \\
\hline 4425.983 & 8 & 200 & 22587.508 & $26770_{11 / 2}^{\circ}-49357_{11 / 2}$ & 4391.110 & 600 & 1000 & 22766.889 & $4490_{5 / 2}^{\circ}-27257_{7 / 2}$ \\
\hline 4425.114 & 2 & 25 & 22591.944 & $25414_{11 / 2}^{\circ}-48006_{9 / 2}$ & 4390.580 & 1 & 50 & 22769.637 & $29095_{5 / 2}^{\circ}-51865_{5 / 2}$ \\
\hline 4423.933 & $25 \mathrm{~b}$ & $50 \mathrm{~b}$ & 22597.975 & $15242_{9 / 2}^{\circ}-37840_{9 / 2}$ & 4390.409 & 2 & 15 & 22770.524 & $24132_{3 / 2}^{\circ}-46902_{5 / 2}^{\circ}$ \\
\hline 4423.903 & $20 \mathrm{~b}$ & $40 \mathrm{~b}$ & 22598.128 & $11576_{3 / 2}^{0}-34174_{5 / 2}$ & 4389.438 & 1 & & 22775.561 & $28587_{5 / 2}^{\circ}-51362_{5 / 2}$ \\
\hline 4422.781 & 50 & 100 & 22603.861 & $1859_{3 / 2}-24463_{5 / 2}^{\circ}$ & 4388.999 & 3 & & 22777.839 & $14275_{9 / 2}^{\circ}-37053_{11 / 2}$ \\
\hline 4421.831 & 5 & 100 & 22608.717 & $30310_{11 / 2}^{\circ}-52918_{13 / 2}$ & 4388.402 & 10 & 150 & 22780.938 & $14349_{1 / 2}-37130_{1 / 2}^{\circ}$ \\
\hline 4421.664 & 1 & 5 & 22609.571 & $15236_{3 / 2}-37846_{5 / 2}^{\circ}$ & 4387.502 & 2 & 50 & 22785.611 & $20310_{5 / 2}^{\circ}-43096_{5 / 2}$ \\
\hline 4421.544 & 4001 & 200 & 22610.184 & $1521_{5 / 2}-24132_{3 / 2}^{\circ}$ & 4387.479 & 3 & & 22785.730 & $20158_{5 / 2}-42944_{7 / 2}^{\circ}$ \\
\hline 4420.737 & 5 & 4 & 22614.312 & $11116_{7 / 2}^{\circ}-33730_{5 / 2}$ & 4387.104 & 5 & 100 & 22787.678 & $16564_{11 / 2}^{\circ}-39352_{11 / 2}$ \\
\hline 4419.322 & 2 & 10 & 22621.552 & $23730_{9 / 2}^{\circ}-46352_{7 / 2}$ & 4386.107 & 2 & & 22792.857 & $22513_{5 / 2}^{\circ}-45306_{3 / 2}$ \\
\hline 4419.006 & 5 & 200 & 22623.170 & $17272_{9 / 2}^{\circ}-39895_{9 / 2}$ & 4384.019 & 5 & 5 & 22803.713 & $17771_{11 / 2}^{\circ}-40574_{11 / 2}$ \\
\hline 4418.661 & 40 & 200 & 22624.936 & $16818_{7 / 2}-39443_{9 / 2}^{\circ}$ & 4383.933 & 1 & 3 & 22804.160 & $36812_{1 / 2}-59616_{3 / 2}^{\circ}$ \\
\hline 4416.236 & 100 & 300 & 22637.360 & $10572_{9 / 2}^{\circ}-33209_{7 / 2}$ & 4382.007 & 2 & & 22814.183 & $20989_{9 / 2}^{\circ}-43803_{7 / 2}$ \\
\hline 4415.592 & 3 & $10 \mathrm{~h}$ & 22640.662 & $21131_{3 / 2}^{\circ}-43772_{5 / 2}$ & 4381.860 & 800 & 1000 & 22814.949 & $6700_{9 / 2}^{\circ}-29515_{9 / 2}$ \\
\hline 4414.853 & 1 & 8 & 22644.451 & $17722_{9 / 2}^{\circ}-40367_{9 / 2}$ & 4381.402 & 75 & 200 & 22817.333 & $4146_{7 / 2}-26963_{7 / 2}^{\circ}$ \\
\hline 4414.623 & 15 & 200 & 22645.631 & $16906_{\tau / 2}^{\circ}-39552_{9 / 2}$ & 4380.144 & 2 & & 22823.887 & $19594_{1 / 2}-42418_{3 / 2}^{\circ}$ \\
\hline 4413.373 & $5 b$ & 150 & 22652.045 & $15453_{7 / 2}^{\circ}-38105_{5 / 2}$ & 4379.941 & 2 & 1 & 22824.944 & $23730_{9 / 2}^{\circ}-46555_{11 / 2}$ \\
\hline 4412.891 & 100 & 200 & 22654.519 & $6691_{3 / 2}^{\circ}-29345_{5 / 2}$ & 4379.587 & 3 & 8 & 22826.789 & $17121_{3 / 2}^{\circ}-39948_{1 / 2}$ \\
\hline 4412.797 & 50 & 100 & 22655.001 & $22834_{7 / 2}-45489_{9 / 2}^{\circ}$ & 4378.957 & 3 & 4 & 22830.073 & $15349_{11 / 2}^{\circ}-38179_{9 / 2}$ \\
\hline 4412.739 & 300 & 400 & 22655.299 & $6168_{7 / 2}^{\circ}-28823_{5 / 2}$ & 4378.833 & 4 & 15 & 22830.720 & $16033_{5 / 2}^{\circ}-38863_{5 / 2}$ \\
\hline 4412.533 & $50 \mathrm{~b}$ & $400 \mathrm{~b}$ & 22656.357 & $13468_{9 / 2}^{\circ}-36125_{9 / 2}$ & 4377.313 & 40 & 200 & 22838.647 & $20288_{11 / 2}^{\circ}-43127_{11 / 2}$ \\
\hline 4411.641 & 3 & 50 & 22660.938 & $17983_{5 / 2}^{\circ}-40644_{5 / 2}$ & 4377.261 & 3 & 25 & 22838.919 & $12902_{3 / 2}^{\circ}-35741_{5 / 2}$ \\
\hline 4410.488 & 20 & 200 & 22666.862 & $17272_{9 / 2}^{\circ}-39939_{11 / 2}$ & 4375.588 & 10 & 100 & 22847.651 & $17722_{9 / 2}^{\circ}-40570_{7 / 2}$ \\
\hline 4410.410 & 5 & 200 & 22667.263 & $20288_{11 / 2}^{\circ}-42955_{9 / 2}$ & 4375.022 & 8 & 15 & 22850.607 & $4113_{5 / 2}-26963_{7 / 2}^{\circ}$ \\
\hline 4409.005 & 15 & 50 & 22674.486 & $14790_{7 / 2}^{\circ}-37465_{5 / 2}$ & 4374.785 & 150 & 400 & 22851.845 & $4113_{5 / 2}-26965_{3 / 2}^{\circ}$ \\
\hline 4408.769 & 1 & 8 & 22675.700 & $21131_{3 / 2}^{\circ}-43807_{3 / 2}$ & 4373.901 & 100 & 200 & 22856.463 & $9720_{7 / 2}^{\circ}-32576_{7 / 2}$ \\
\hline 4407.510 & 1 & 4 & 22682.177 & $27787_{9 / 2}^{\circ}-50470_{9 / 2}$ & 4372.814 & 2 & 20 & 22862.145 & $24309_{11 / 2}^{\circ}-47171_{9 / 2}$ \\
\hline 4406.997 & 1 & 8 & 22684.817 & $24463_{5 / 2}^{\circ}-47148_{3 / 2}$ & 4371.767 & 4 & 4 & 22867.620 & $9061_{5 / 2}-31928_{3 / 2}^{\circ}$ \\
\hline 4406.785 & 2 & 20 & 22685.908 & $17837_{1 / 2}^{\circ}-40523_{1 / 2}$ & 4370.773 & 4 & 2 & 22872.820 & $34019_{3 / 2}-56892_{5 / 2}^{\circ}$ \\
\hline 4406.429 & 2 & 50 & 22687.741 & $23697_{7 / 2}^{\circ}-46385_{7 / 2}$ & 4370.011 & 4 & 2 & 22876.809 & $33209_{7 / 2}-56086_{9 / 2}^{\circ}$ \\
\hline 4406.329 & 1 & 10 & 22688.256 & $19248_{5 / 2}^{\circ}-41936_{3 / 2}$ & 4369.317 & $50 \mathrm{~b}$ & $200 \mathrm{~b}$ & 22880.442 & $33354_{5 / 2}^{\circ}-56235_{3 / 2}$ \\
\hline 4405.724 & 1 & 25 & 22691.371 & $25607_{9 / 2}^{\circ}-48298_{7 / 2}$ & & & & & $8378_{7 / 2}^{\circ}-31259_{5 / 2}$ \\
\hline 4404.917 & 2 & 25 & 22695.529 & $16033_{5 / 2}^{\circ}-38728_{5 / 2}$ & 4369.061 & 5 & 20 & 22881.783 & $22513_{5 / 2}^{\circ}-45395_{7 / 2}$ \\
\hline 4402.736 & 4 & 100 & 22706.771 & $15786_{5 / 2}-38493_{5 / 2}^{\circ}$ & 4368.706 & 2 & & 22883.642 & $23697_{7 / 2}^{\circ}-46581_{5 / 2}$ \\
\hline 4401.992 & 2 & 25 & 22710.609 & $14101_{1 / 2}^{\circ}-36812_{1 / 2}$ & 4368.314 & 1 & & 22885.695 & $19050_{3 / 2}^{\circ}-41936_{3 / 2}^{\circ}$ \\
\hline 4401.658 & 10 & 200 & 22712.332 & $15453_{7 / 2}^{\circ}-38165_{7 / 2}$ & 4366.961 & 75 & 50 & 22892.786 & $8460_{3 / 2}-31353_{3 / 2}^{\circ}$ \\
\hline 4401.209 & 3 & 100 & 22714.649 & $18973_{7 / 2}^{\circ}-41688_{7 / 2}$ & 4364.187 & 2 & 25 & 22907.337 & $28923_{5 / 2}^{\circ}-51830_{7 / 2}$ \\
\hline 4401.050 & 1 & 8 & 22715.470 & $22106_{5 / 2}-44821_{5 / 2}^{\circ}$ & 4362.361 & 5 & 25 & 22916.925 & $20310_{5 / 2}^{\circ}-43227_{5 / 2}^{\circ}$ \\
\hline 4400.646 & 1 & 2 & 22717.555 & $34174_{5 / 2}-56892_{5 / 2}^{\circ}$ & 4361.792 & 10 & 100 & 22919.915 & $14545_{5 / 2}^{\circ}-37465_{5 / 2}^{\circ}$ \\
\hline 4400.384 & 20 & 150 & 22718.908 & $7001_{3 / 2}-29720_{3 / 2}^{\circ}$ & 4361.307 & 1001 & 200 & 22922.464 & $15242_{9 / 2}^{\circ}-38165_{7 / 2}$ \\
\hline 4399.093 & 10 & 300 & 22725.575 & $20288_{11 / 2}-43014_{13 / 2}$ & 4359.804 & 1 & & 22930.366 & $37063_{9 / 2}-59993_{9 / 2}^{\circ}$ \\
\hline 4398.837 & 1 & 5 & 22726.897 & $15453_{7 / 2}^{\circ}-38179_{9 / 2}$ & 4359.127 & 5 & 25 & 22933.927 & $20310_{5 / 2}^{\circ}-43244_{3 / 2}$ \\
\hline 4398.020 & 1 & 25 & 22731.119 & $24414_{3 / 2}^{\circ}-47145_{1 / 2}$ & 4358.815 & 5 & 40 & 22935.569 & $18973_{7 / 2}^{\circ}-41909_{9 / 2}$ \\
\hline 4397.914 & 75 & 300 & 22731.667 & $6700_{9 / 2}^{\circ}-29431_{7 / 2}$ & 4358.668 & 4 & & 22936.342 & $15453_{7 / 2}^{\circ}-38389_{7 / 2}$ \\
\hline 4397.752 & 2 & 1 & 22732.504 & $19912_{13 / 2}^{\circ}-42644_{13 / 2}$ & 4358.549 & $50 \mathrm{~s}$ & 100 & 22936.968 & $12219_{3 / 2}-35156_{5 / 2}^{\circ}$ \\
\hline 4397.260 & 1 & 15 & 22735.048 & $29095_{5 / 2}^{\circ}-51830_{7 / 2}$ & 4357.928 & 2 & & 22940.237 & $\begin{array}{l}17983_{5 / 2}^{\circ}-40923_{5 / 2} \\
37053_{11 / 2}-59993_{9 / 2}^{\circ}\end{array}$ \\
\hline
\end{tabular}


TABLE 3. Classified lines of Th II-Continued

\begin{tabular}{|c|c|c|c|c|c|c|c|c|c|}
\hline \multirow{2}{*}{$\begin{array}{c}\text { W avelength } \\
\AA\end{array}$} & \multicolumn{2}{|c|}{ Intensity } & \multirow{2}{*}{$\begin{array}{l}\text { Wavenumber } \\
\mathbf{c m}^{-1}\end{array}$} & \multirow{2}{*}{ Classification } & \multirow{2}{*}{$\begin{array}{c}\text { Wavelength } \\
\AA\end{array}$} & \multicolumn{2}{|c|}{ Intensity } & \multirow{2}{*}{$\begin{array}{c}\text { Wavenumber } \\
\mathbf{c m}^{-1}\end{array}$} & \multirow{2}{*}{ Classification } \\
\hline & Lamp & Spark & & & & Lamp & Spark & & \\
\hline 4357.614 & $100 \mathrm{~b}$ & 150 & 22941.890 & $1521_{5 / 2}-24463_{5 / 2}^{\circ}$ & 4327.228 & 50 & 75 & 23102.986 & $4146_{7 / 2}-27249_{7 / 2}^{\circ}$ \\
\hline 4357.074 & 4 & 8 & 22944.733 & $13248_{9 / 2}-36193_{9 / 2}^{\circ}$ & 4327.091 & 25 & 75 & 23103.717 & $4490_{5 / 2}^{\circ}-27593_{5 / 2}$ \\
\hline 4355.911 & 2 & 8 & 22950.859 & $17460_{5 / 2}^{\circ}-40411_{7 / 2}$ & 4326.576 & 2 & 2 & 23106.467 & $20989_{9 / 2}^{\circ}-44096_{9 / 2}^{\circ}$ \\
\hline 4355.320 & 100 & 300 & 22953.973 & $8018_{3 / 2}-30972_{5 / 2}^{\circ}$ & 4326.341 & 2 & 5 & 23107.722 & $20120_{5 / 2}^{\circ}-43227_{5 / 2}$ \\
\hline 4353.405 & $75 b$ & 200 & 22964.070 & $10673_{5 / 2}^{\circ}-33637_{7 / 2}$ & 4326.179 & 3 & 8 & 23108.588 & $18568_{1 / 2}^{\circ}-41676_{3 / 2}^{\circ}$ \\
\hline 4352.818 & 40 & 75 & 22967.167 & $11576_{3 / 2}^{\circ}-34543_{5 / 2}$ & 4325.920 & 2 & & 23109.971 & $17460_{5 / 2}^{\circ}-40570_{7 / 2}$ \\
\hline 4352.677 & 50 & 200 & 22967.911 & $12488_{9 / 2}^{\circ}-35456_{9 / 2}^{\circ}$ & 4325.663 & 2 & & 23111.344 & $22014_{11 / 2}^{o}-45126_{9 / 2}$ \\
\hline 4352.182 & 5 & 10 & 22970.523 & $36328_{3 / 2}-59299_{5 / 2}^{\circ}$ & 4325.189 & 2 & & 23113.877 & $24757_{9 / 2}^{\circ}-47871_{7 / 2}$ \\
\hline \multirow[t]{2}{*}{4351.073} & 3 & & 22976.378 & $20120_{5 / 2}^{\circ}-43096_{5 / 2}$ & 4324.958 & 4 & & 23115.112 & $22106_{5 / 2}-45221_{5 / 2}^{\circ}$ \\
\hline & & & & & 4324.442 & 4 & 4 & 23117.870 & $20686_{5 / 2}^{\circ}-43803_{7 / 2}^{\circ}$ \\
\hline 4350.572 & 4 & 8 & 22979.024 & $12219_{3 / 2}-35198_{1 / 2}^{\circ}$ & 4323.165 & 1 & & 23124.698 & $20120_{5 / 2}^{\circ}-43244_{3 / 2}$ \\
\hline 4348.943 & $4 \mathrm{~s}$ & 8 & 22987.631 & $16564_{11 / 2}^{\circ}-39552_{9 / 2}$ & 4323.043 & 8 & 25 & 23125.351 & $15710_{3 / 2}^{\circ}-38836_{3 / 2}$ \\
\hline 4348.713 & 3 & & 22988.847 & $16906_{7 / 2}^{\circ}-39895_{9 / 2}^{\circ}$ & 4322.788 & 1 & 1 & 23126.715 & $20120_{5 / 2}^{\circ}-43246_{7 / 2}$ \\
\hline 4348.312 & 20 & 40 & 22990.967 & $13818_{7 / 2}^{\circ}-36809_{\tau / 2}^{\circ}$ & 4322.386 & 2 & 1 & 23128.866 & $20158_{5 / 2}-43287_{3 / 2}^{\circ}$ \\
\hline 4348.236 & 5 & 8 & 22991.368 & $9585_{5 / 2}^{\circ}-32576_{7 / 2}$ & 4322.001 & 3 & 10 & 23130.926 & $23730_{9 / 2}^{\circ}-46861_{11 / 2}^{\circ}$ \\
\hline 4347.430 & 1 & & 22995.631 & $24873_{5 / 2}^{\circ}-47869_{3 / 2}^{\circ}$ & 4321.012 & 8 & 20 & 23136.220 & $4113_{5 / 2}-27249_{7 / 2}^{\circ}$ \\
\hline 4347.239 & $25 \mathrm{~h}$ & 200 & 22996.641 & $26963_{7 / 2}^{\circ}-49960_{7 / 2}$ & 4320.657 & 3 & 20 & 23138.121 & $9711_{7 / 2}-32850_{5 / 2}^{\circ}$ \\
\hline 4347.193 & 20 & 200 & 22996.885 & $14790_{7 / 2}^{\circ}-37787_{7 / 2}$ & 4320.590 & 50 & 300 & 23138.480 & $12902_{3 / 2}^{\circ}-36040_{1 / 2}^{\circ}$ \\
\hline 4347.092 & 1 & & 22997.419 & $24873_{5 / 2}^{\circ}-47871_{7 / 2}$ & 4320.449 & 5 & 200 & 23139.235 & $17272_{9 / 2}^{\circ}-40411_{7 / 2}^{\circ}$ \\
\hline 4346.262 & 5 & 15 & 23001.811 & $14275_{9 / 2}^{\circ}-37277_{7 / 2}^{\circ}$ & 4320.126 & 200 & 500 & 23140.965 & $4490_{5 / 2}^{\circ}-27631_{3 / 2}$ \\
\hline 4345.052 & 10 & 40 & 23008.216 & $17983_{5 / 2}^{\circ}-40991_{3 / 2}$ & 4319.097 & 10 & 300 & 23146.478 & $15242_{9 / 2}^{\circ}-38389_{7 / 2}$ \\
\hline 4344.984 & 3 & 20 & 23008.576 & $23697_{7 / 2}^{\circ}-46706_{7 / 2}$ & 4318.959 & 2 & 50 & 23147.218 & $20080_{7 / 2}^{\circ}-43227_{5 / 2}^{\circ}$ \\
\hline 4344.326 & 400 & 300 & 23012.061 & $0_{3 / 2}-23012_{3 / 2}^{\circ}$ & 4318.293 & 50 & 300 & 23150.787 & $9585_{5 / 2}^{\circ}-32736_{7 / 2}$ \\
\hline 4343.951 & 200 & 300 & 23014.048 & $1859_{3 / 2}-24873_{5 / 2}^{\circ}$ & 4317.756 & 2 & 8 & 23153.667 & $11116_{\tau / 2}^{\circ}-34270_{9 / 2}$ \\
\hline \multirow[t]{2}{*}{4343.602} & 50 & 200 & 23015.897 & $30994_{7 / 2}^{\circ}-54010_{7 / 2}^{\circ}$ & 4317.670 & 2 & 10 & 23154.128 & $14790_{7 / 2}^{\circ}-37945_{5 / 2}$ \\
\hline & & & & $9720_{7 / 2}^{\circ}-32736_{7 / 2}$ & 4317.222 & 3 & 20 & 23156.531 & $17121_{3 / 2}^{\circ}-40278_{3 / 2}$ \\
\hline 4343.238 & 10 & 20 & 23017.826 & $15710_{3 / 2}^{\circ}-38728_{5 / 2}^{\circ}$ & 4315.946 & 25 & 100 & 23163.377 & $12902_{3 / 2}^{\circ}-36065_{5 / 2}^{\circ}$ \\
\hline 4342.255 & 100 & 200 & 23023.036 & $12570_{7 / 2}-35593_{7 / 2}^{\circ}$ & 4315.706 & 4 & 25 & 23164.665 & $15453_{7 / 2}^{\circ}-38617_{9 / 2}^{\circ}$ \\
\hline 4341.992 & 1 & & 23024.431 & $24982_{7 / 2}^{\circ}-48006_{9 / 2}^{\circ}$ & 4315.579 & 4 & 8 & 23165.347 & $8460_{3 / 2}-31625_{1 / 2}^{\circ}$ \\
\hline 4341.030 & 50 & 300 & 23029.533 & $23187_{13 / 2}^{\circ}-46216_{11 / 2}$ & 4314.010 & 5 & 8 & 23173.771 & $6700_{9 / 2}^{\circ}-29873_{7 / 2}^{\circ}$ \\
\hline 4339.650 & $4 \mathrm{~h}$ & 8 & 23036.856 & $12488_{9 / 2}^{\circ}-35525_{11 / 2}$ & 4313.308 & 15 & 50 & 23177.543 & $6168_{7 / 2}^{\circ}-29345_{5 / 2}$ \\
\hline 4338.383 & 4 & 10 & 23043.584 & $16818_{7 / 2}-39861_{5 / 2}^{\circ}$ & 4312.156 & 3 & 5 & 23183.735 & $17460_{5 / 2}^{\circ}-40644_{5 / 2}$ \\
\hline 4337.860 & 5 & 10 & 23046.362 & $15710_{3 / 2}^{\circ}-38757_{3 / 2}$ & 4310.644 & 2 & 40 & 23191.867 & $23187_{13 / 2}^{\circ}-46378_{13 / 2}$ \\
\hline 4337.384 & 100 & 800 & 23048.891 & $15242_{9 / 2}^{\circ}-38291_{7 / 2}^{\circ}$ & 4310.550 & 1 & & 23192.372 & $24132_{3 / 2}^{\circ}-47324_{5 / 2}^{\circ}$ \\
\hline 4336.494 & 3 & & 23053.621 & $30956_{9 / 2}^{\circ}-54010_{7 / 2}$ & 4310.179 & 5 & 25 & 23194.369 & $8605_{5 / 2}-31800_{7 / 2}^{\circ}$ \\
\hline 4335.797 & 50 & 200 & 23057.327 & $12488_{9 / 2}^{\circ}-35545_{9 / 2}$ & 4309.991 & 100 & 800 & 23195.380 & $14484_{11 / 2}^{\circ}-37679_{11 / 2}$ \\
\hline 4335.687 & $25 b$ & $200 \mathrm{~b}$ & 23057.912 & $11116_{7 / 2}^{\circ}-34174_{5 / 2}$ & & & & & $10189_{11 / 2}^{\circ}-33384_{9 / 2}$ \\
\hline 4335.312 & $25 b$ & 200 & 23059.907 & $12485_{7 / 2}^{\circ}-35545_{9 / 2}$ & 4309.215 & 1 & & 23199.557 & $15236_{3 / 2}-38436_{3 / 2}^{\circ}$ \\
\hline 4334.316 & 5 & 5 & 23065.206 & $10572_{9 / 2}^{\circ}-33637_{7 / 2}$ & 4308.255 & 8 & 15 & 23204.727 & $8605_{5 / 2}-31810_{5 / 2}^{\circ}$ \\
\hline 4334.254 & 5 & 5 & 23065.536 & $18816_{13 / 2}^{\circ}-41882_{13 / 2}$ & 4307.956 & 2 & 10 & 23206.337 & $21297_{5 / 2}^{\circ}-44503_{7 / 2}^{\circ}$ \\
\hline 4333.933 & 50 & 150 & 23067.244 & $22028_{15 / 2}^{\circ}-45095_{15 / 2}$ & 4306.365 & $200 \mathrm{~b}$ & $200 \mathrm{~b}$ & 23214.911 & $19912_{13 / 2}^{\circ}-43127_{11 / 2}$ \\
\hline 4331.924 & 751 & 200 & 23077.942 & $14484_{11 / 2}^{\circ}-37562_{11 / 2}$ & 4306.159 & 1 & 5 & 23216.021 & $21682_{7 / 2}^{\circ}-44898_{7 / 2}^{\circ}$ \\
\hline 4330.351 & 2 & & 23086.325 & $20686_{5 / 2}^{\circ}-43772_{5 / 2}$ & 4305.328 & 2 & 100 & 23220.502 & $27249_{7 / 2}^{\circ}-50470_{9 / 2}$ \\
\hline 4329.907 & 3 & 10 & 23088.692 & $28587_{5 / 2}^{\circ}-51676_{3 / 2}$ & 4304.070 & 2 & 5 & 23227.289 & $15144_{3 / 2}^{\circ}-38372_{3 / 2}^{\circ}$ \\
\hline 4329.489 & 40 & 100 & 23090.921 & $14484_{11 / 2}^{\circ}-37575_{13 / 2}$ & 4302.109 & 2 & 40 & 23237.876 & $25607_{9 / 2}^{\circ}-48844_{9 / 2}$ \\
\hline 4329.107 & 10 & 50 & 23092.959 & $22642_{9 / 2}^{\circ}-45735_{11 / 2}$ & 4301.280 & 3 & 10 & 23242.355 & $14545_{5 / 2}^{\circ}-37787_{7 / 2}$ \\
\hline 4328.686 & 40 & 100 & 23095.205 & $17272_{9 / 2}^{\circ}-40367_{9 / 2}^{\circ}$ & 4300.793 & 8 & 150 & 23244.987 & $13818_{\tau / 2}^{\circ}-37063_{9 / 2}^{\circ}$ \\
\hline 4328.286 & 15 & 50 & 23097.339 & $22513_{5 / 2}^{\circ}-45610_{5 / 2}$ & 4300.700 & 2 & 3 & 23245.489 & $9711_{7 / 2}-32957_{7 / 2}^{\circ}$ \\
\hline 4327.546 & 5 & 50 & 23101.288 & $17121_{3 / 2}^{\circ}-40222_{3 / 2}$ & 4300.135 & 2 & 10 & 23248.544 & $18973_{7 / 2}^{\circ}-42222_{7 / 2}^{\circ}$ \\
\hline 4327.448 & 4 & 50 & 23101.811 & $19912_{13 / 2}^{\circ}-43014_{13 / 2}$ & 4299.435 & 3 & 50 & 23252.329 & $14349_{1 / 2}-37601_{3 / 2}^{\circ}$ \\
\hline
\end{tabular}


TABLE 3. Classified lines of Th II-Continued

\begin{tabular}{|c|c|c|c|c|c|c|c|c|c|}
\hline \multirow{2}{*}{$\begin{array}{c}\text { Wavelength } \\
\AA\end{array}$} & \multicolumn{2}{|c|}{ Intensity } & \multirow{2}{*}{$\begin{array}{c}\text { Wavenumber } \\
\text { cm }^{-1}\end{array}$} & \multirow{2}{*}{ Classification } & \multirow{2}{*}{$\begin{array}{c}\text { Wavelength } \\
\AA\end{array}$} & \multicolumn{2}{|c|}{ Intensity } & \multirow{2}{*}{$\begin{array}{l}\text { Wavenumber } \\
\text { cm }^{-1}\end{array}$} & \multirow{2}{*}{ Classification } \\
\hline & Lamp & Spark & & & & Lamp & Spark & & \\
\hline 4298.891 & 25 & 50 & 23255.271 & $21297_{5 / 2}^{\circ}-44552_{5 / 2}$ & 4271.546 & 3 & 20 & 23404.141 & $14275_{9 / 2}^{\circ}-37679_{11 / 2}$ \\
\hline 4298.826 & 20 & $200 \mathrm{~b}$ & 23255.623 & $12485_{7 / 2}^{\circ}-35741_{5 / 2}$ & 4271.240 & 3 & 50 & 23405.818 & $24463_{5 / 2}^{\circ}-47869_{3 / 2}$ \\
\hline 4298.283 & 1 & & 23258.560 & $14349_{1 / 2}-37607_{1 / 2}^{\circ}$ & 4271.103 & $40 \mathrm{~b}$ & 200 & 23406.568 & $1859_{3 / 2}-25266_{1 / 2}^{\circ}$ \\
\hline 4297.365 & $25 b$ & 75 & 23263.529 & $6168_{7 / 2}^{\circ}-29431_{7 / 2}$ & 4271.084 & $20 \mathrm{~b}$ & & 23406.672 & $12472_{5 / 2}^{\circ}-35878_{7 / 2}$ \\
\hline 4296.580 & 3 & 10 & 23267.779 & $15349_{11 / 2}^{\circ}-38617_{9 / 2}$ & 4270.911 & 1 & 10 & 23407.620 & $24463_{5 / 2}^{\circ}-47871_{7 / 2}$ \\
\hline 4295.125 & 8 & 100 & 23275.661 & $15453_{7 / 2}^{\circ}-38728_{5 / 2}$ & 4270.326 & 50 & 200 & 23410.827 & $15453_{7 / 2}^{\circ}-38863_{5 / 2}$ \\
\hline 4295.035 & 20 & & 23276.149 & $17771_{11 / 2}^{\circ}-41047_{9 / 2}$ & 4267.716 & 4 & 15 & 23425.144 & $16033_{5 / 2}^{\circ}-39458_{7 / 2}$ \\
\hline 4293.218 & 1 & & 23286.000 & $19050_{3 / 2}^{\circ}-42336_{5 / 2}$ & 4267.518 & 20 & 50 & 23426.231 & $12902_{3 / 2}^{\circ}-36328_{3 / 2}$ \\
\hline 4293.089 & 1 & 3 & 23286.700 & $14275_{9 / 2}^{\circ}-37562_{11 / 2}$ & 4267.381 & 2 & 5 & 23426.983 & $11116_{7 / 2}^{\circ}-34543_{5 / 2}$ \\
\hline 4292.509 & $10 \mathrm{~b}$ & 8 & 23289.846 & $4113_{5 / 2}-27403_{3 / 2}^{\circ}$ & 4266.823 & 1 & & 23430.047 & $25414_{11 / 2}^{\circ}-48844_{9 / 2}$ \\
\hline 4291.382 & 4 & 40 & 23295.962 & $11725_{\mathrm{i} / 2}^{\circ}-35021_{3 / 2}$ & 4265.661 & 1 & & 23436.429 & $27787_{9 / 2}^{\circ}-51224_{9 / 2}$ \\
\hline 4290.829 & 1 & & 23298.965 & $33384_{9 / 2}-56683_{7 / 2}^{\circ}$ & 4265.488 & 8 & 15 & 23437.380 & $1111_{7 / 2}^{\circ}-34553_{9 / 2}$ \\
\hline 4290.169 & 15 & 50 & 23302.549 & $17272_{9 / 2}^{\circ}-40574_{11 / 2}^{\circ}$ & 4265.034 & 5 & 25 & 23439.874 & $18568_{1 / 2}^{\circ}-42008_{1 / 2}$ \\
\hline 4288.047 & 20 & 200 & 23314.080 & $14790_{7 / 2}^{\circ}-38105_{5 / 2}$ & 4264.943 & 5 & & 23440.375 & $14101_{1 / 2}^{\circ}-37542_{3 / 2}$ \\
\hline 4287.683 & 4 & 75 & 23316.059 & $24982_{7 / 2}^{\circ}-48298_{7 / 2}$ & 4264.374 & 41 & 100 & 23443.502 & $21682_{\tau / 2}^{\circ}-45126_{9 / 2}$ \\
\hline 4286.699 & 5 & 200 & 23321.411 & $22834_{7 / 2}-46155_{5 / 2}^{\circ}$ & 4264.105 & 20 & 50 & 23444.981 & $11576_{3 / 2}^{\circ}-35021_{3 / 2}$ \\
\hline 4286.187 & $15 b$ & $200 \mathrm{~b}$ & 23324.197 & $17722_{9 / 2}^{\circ}-41047_{9 / 2}$ & 4263.355 & 200 & 75 & 23449.105 & $9400_{5 / 2}-32850_{5 / 2}^{\circ}$ \\
\hline 4285.450 & 3 & 8 & 23328.208 & $1859_{3 / 2}-25188_{3 / 2}^{\circ}$ & 4262.739 & 5 & 3 & 23452.494 & $17771_{11 / 2}^{\circ}-41223_{11 / 2}$ \\
\hline 4285.181 & 15 & 200 & 23329.672 & $18816_{13 / 2}^{\circ}-42146_{11 / 2}$ & 4261.273 & 50 & 4 & 23460.562 & $1521_{5 / 2}-24982_{7 / 2}^{\circ}$ \\
\hline 4284.975 & 50 & 200 & 23330.794 & $16564_{11 / 2}^{\circ}-39895_{9 / 2}$ & 4260.936 & 4 & & 23462.418 & $18214_{3 / 2}^{\circ}-41676_{3 / 2}$ \\
\hline 4284.927 & 3 & 5 & 23331.056 & $13250_{5 / 2}-36581_{3 / 2}^{\circ}$ & 4259.857 & 3 & & 23468.360 & $8460_{3 / 2}-31928_{3 / 2}^{\circ}$ \\
\hline 4284.514 & $10 \mathrm{~b}$ & 10 & 23333.305 & $13250_{5 / 2}-36583_{7 / 2}^{\circ}$ & 4258.828 & 2 & & 23474.031 & $33209_{7 / 2}-56683_{7 / 2}^{\circ}$ \\
\hline 4284.431 & 3 & 8 & 23333.756 & $16033_{5 / 2}^{\circ}-39366_{5 / 2}$ & 4258.466 & 3 & & 23476.026 & $6244_{1 / 2}-29720_{3 / 2}^{\circ}$ \\
\hline 4284.215 & 2 & 8 & 23334.933 & $8018_{3 / 2}-31353_{3 / 2}^{\circ}$ & 4256.094 & 50 & 10 & 23489.109 & $9720_{7 / 2}^{\circ}-33209_{7 / 2}$ \\
\hline 4284.181 & 3 & 1 & 23335.118 & $13248_{9 / 2}-36583_{7 / 2}^{\circ}$ & 4254.739 & 1 & & 23496.590 & $20310_{5 / 2}^{\circ}-43807_{3 / 2}$ \\
\hline 4283.519 & 150 & 400 & 23338.724 & $9238_{9 / 2}^{\circ}-32576_{7 / 2}$ & 4254.451 & 75 & 8 & 23498.180 & $9238_{9 / 2}^{\circ}-32736_{7 / 2}$ \\
\hline 4282.370 & 101 & 200 & 23344.986 & $17983_{5 / 2}^{\circ}-41328_{5 / 2}$ & 4254.169 & 1 & & 23499.738 & $30994_{7 / 2}^{\circ}-54493_{5 / 2}$ \\
\hline & & & & $15236_{3 / 2}-38581_{5 / 2}^{\circ}$ & 4254.021 & 5 & & 23500.555 & $17722_{9 / 2}^{\circ}-41223_{11 / 2}$ \\
\hline 4282.042 & $300 \mathrm{~b}$ & $500 \mathrm{~b}$ & 23346.774 & $6168_{7 / 2}^{\circ}-29515_{g / 2}$ & 4253.975 & 2 & & 23500.810 & $14790_{7 / 2}^{\circ}-38291_{7 / 2}$ \\
\hline 4281.758 & 2 & 2 & 23348.323 & $28587_{5 / 2}^{\circ}-51935_{5 / 2}$ & 4253.867 & 15 & 8 & 23501.406 & $10673_{5 / 2}^{\circ}-34174_{5 / 2}^{\circ}$ \\
\hline 4281.416 & 150 & 400 & 23350.188 & $8460_{3 / 2}-31810_{5 / 2}^{\circ}$ & 4253.259 & $3 b$ & & 23504.766 & $17983_{5 / 2}^{\circ}-41488_{7 / 2}$ \\
\hline 4281.068 & 200 & 500 & 23352.086 & $1521_{5 / 2}-24873_{5 / 2}^{\circ}$ & 4253.239 & $2 \mathrm{~b}$ & & 23504.876 & $16906_{7 / 2}^{\circ}-40411_{7 / 2}$ \\
\hline 4280.253 & 3 & 8 & 23356.532 & $14484_{11 / 2}^{\circ}-37840_{9 / 2}$ & 4253.017 & 1 & & 23506.103 & $26963_{7 / 2}^{\circ}-50470_{9 / 2}$ \\
\hline 4280.221 & 5 & 8 & 23356.707 & $10855_{7 / 2}-34212_{5 / 2}^{\circ}$ & 4252.876 & 1 & & 23506.883 & $21682_{7 / 2}^{\circ}-45189_{5 / 2}$ \\
\hline 4278.507 & 5 & 50 & 23366.064 & $16818_{7 / 2}-40184_{7 / 2}^{\circ}$ & 4251.958 & 1 & & 23511.957 & $15324_{1 / 2}^{\circ}-38836_{3 / 2}$ \\
\hline 4278.110 & 1 & & 23368.232 & $18568_{1 / 2}^{\circ}-41936_{3 / 2}^{\circ}$ & 4251.781 & 1 & & 23512.936 & $15349_{11 / 2}^{\circ}-38862_{11 / 2}$ \\
\hline 4277.313 & 600 & 500 & 23372.586 & $0_{3 / 2}-23372_{3 / 2}^{\circ}$ & 4251.592 & 2 & & 23513.982 & $20989_{9 / 2}^{\circ}-44503_{7 / 2}$ \\
\hline 4277.039 & 40 & 100 & 23374.083 & $9585_{5 / 2}^{\circ}-32959_{3 / 2}$ & 4250.341 & $100 \mathrm{~b}$ & & 23520.902 & $4490_{5 / 2}^{\circ}-28011_{3 / 2}$ \\
\hline 4276.966 & 1001 & 300 & 23374.482 & $16564_{11 / 2}^{\circ}-39939_{11 / 2}$ & 4250.014 & 1 & & 23522.712 & $17121_{3 / 2}^{\circ}-40644_{5 / 2}$ \\
\hline & & & & $9202_{7 / 2}^{\circ}-32576_{7 / 2}$ & 4249.969 & 3 & & 23522.961 & $10379_{9 / 2}-33902_{7 / 2}^{\circ}$ \\
\hline 4276.909 & 4 & & 23374.794 & $15242_{9 / 2}^{\circ}-38617_{9 / 2}$ & 4249.678 & 75 & 10 & 23524.572 & $7828_{1 / 2}-31353_{3 / 2}^{\circ}$ \\
\hline 4276.808 & 100 & 400 & 23375.346 & $8378_{7 / 2}^{\circ}-31754_{5 / 2}$ & 4247.989 & 150 & 100 & 23533.925 & $9202_{7 / 2}^{\circ}-32736_{7 / 2}$ \\
\hline 4275.439 & 3 & 3 & 23382.830 & $9238_{9 / 2}^{\circ}-32620_{11 / 2}$ & 4247.598 & 50 & 5 & 23536.091 & $4490_{5 / 2}^{\circ}-28026_{5 / 2}$ \\
\hline 4275.240 & 2 & & 23383.919 & $23012_{3 / 2}^{\circ}-46395_{3 / 2}$ & 4246.363 & 3 & & 23542.936 & $15324_{1 / 2}^{\circ}-38867_{1 / 2}$ \\
\hline 4275.203 & 2 & & 23384.121 & $23518_{7 / 2}^{\circ}-46902_{5 / 2}$ & 4244.824 & 2 & & 23551.472 & $25266_{1 / 2}^{\circ}-48817_{3 / 2}$ \\
\hline 4274.321 & 50 & & 23388.947 & $14790_{\tau / 2}^{\circ}-38179_{9 / 2}$ & 4243.925 & 75 & 15 & 23556.461 & $9400_{5 / 2}-32957_{7 / 2}^{\circ}$ \\
\hline 4274.024 & 100 & 300 & 23390.572 & $12488_{9 / 2}^{\circ}-35878_{\tau / 2}$ & 4243.674 & 1 & & 23557.854 & $35741_{5 / 2}-59299_{5 / 2}^{\circ}$ \\
\hline & & & & $15349_{11 / 2}^{\circ}-38740_{11 / 2}$ & 4243.375 & 2 & & 23559.514 & $14545_{5 / 2}^{\circ}-38105_{5 / 2}$ \\
\hline 4273.357 & 300 & 800 & 23394.223 & $8378_{7 / 2}^{\circ}-31773_{9 / 2}$ & 4242.720 & 15 & 4 & 23563.151 & $7001_{3 / 2}-30564_{1 / 2}^{\circ}$ \\
\hline 4271.913 & 3 & 25 & 23402.130 & $17121_{3 / 2}^{\circ}-40523_{1 / 2}$ & 4242.338 & 5 & 3 & 23565.272 & $14275_{9 / 2}^{\circ}-37840_{9 / 2}$ \\
\hline
\end{tabular}


TABLE 3. Classified lines of Th II-Continued

\begin{tabular}{|c|c|c|c|c|c|c|c|c|c|}
\hline \multirow{2}{*}{$\begin{array}{c}\text { Wavelength } \\
\AA\end{array}$} & \multicolumn{2}{|c|}{ Intensity } & \multirow{2}{*}{$\begin{array}{l}\text { Wavenumber } \\
\mathbf{c m}^{-1}\end{array}$} & \multirow{2}{*}{ Classification } & \multirow{2}{*}{$\begin{array}{c}\text { Wavelength } \\
\AA\end{array}$} & \multicolumn{2}{|c|}{ Intensity } & \multirow{2}{*}{$\begin{array}{c}\text { Wavenumber } \\
\mathbf{c m}^{-1}\end{array}$} & \multirow{2}{*}{ Classification } \\
\hline & Lamp & Spark & & & & Lamp & Spark & & \\
\hline 4241.865 & 2 & & 23567.900 & $22685_{7 / 2}^{\circ}-46253_{9 / 2}$ & 4214.073 & 1 & & 23723.329 & $20080_{7 / 2}^{\circ}-43803_{7 / 2}$ \\
\hline 4240.676 & 4 & 1 & 23574.508 & $22642_{9 / 2}^{\circ}-46216_{11 / 2}^{\circ}$ & 4213.994 & 3 & & 23723.773 & $23187_{13 / 2}^{\circ}-46910_{13 / 2}$ \\
\hline 4240.594 & 40 & 5 & 23574.964 & $6213_{9 / 2}-29788_{9 / 2}^{\circ}$ & 4213.146 & 1 & 2 & 23728.548 & $20080_{7 / 2}^{\circ}-43809_{9 / 2}$ \\
\hline 4239.673 & 5 & 1 & 23580.085 & $12485_{7 / 2}^{\circ}-36065_{5 / 2}$ & 4212.452 & 2 & & 23732.457 & $29788_{9 / 2}^{\circ}-53520_{9 / 2}^{\circ}$ \\
\hline 4239.030 & 2 & & 23583.662 & $20969_{7 / 2}^{\circ}-44552_{5 / 2}$ & 4211.514 & 10 & 10 & 23737.743 & $16906_{7 / 2}^{\circ}-40644_{5 / 2}$ \\
\hline 4238.978 & 5 & 4 & 23583.951 & $15144_{3 / 2}^{\circ}-38728_{5 / 2}$ & 4210.400 & 1 & & 23744.023 & $30101_{7 / 2}^{\circ}-53845_{5 / 2}$ \\
\hline 4238.884 & 1 & & 23584.474 & $13468_{9 / 2}^{\circ}-37053_{11 / 2}$ & 4208.890 & $200 \mathrm{~b}$ & 200 & 23752.542 & $6700_{9 / 2}^{\circ}-30452_{9 / 2}$ \\
\hline 4237.685 & 2 & & 23591.147 & $23012_{3 / 2}^{\circ}-46603_{5 / 2}$ & 4207.922 & 1 & & 23758.006 & $21131_{3 / 2}^{\circ}-44889_{3 / 2}$ \\
\hline 4237.657 & 1 & & 23591.303 & $22106_{5 / 2}-45697_{3 / 2}^{\circ}$ & 4206.656 & 5 & 10 & 23765.156 & $17722_{9 / 2}^{\circ}-41488_{7 / 2}^{\circ}$ \\
\hline 4237.113 & 5 & 5 & 23594.332 & $13468_{9 / 2}^{\circ}-37063_{9 / 2}^{\circ}$ & 4201.847 & 20 & 100 & 23792.354 & $8018_{3 / 2}-31810_{5 / 2}^{\circ}$ \\
\hline 4236.915 & 2 & & 23595.434 & $22139_{9 / 2}^{\circ}-45735_{11 / 2}$ & 4201.602 & 8 & 8 & 23793.742 & $18214_{3 / 2}^{\circ}-42008_{1 / 2}$ \\
\hline 4236.390 & 8 & 3 & 23598.358 & $14790_{7 / 2}^{\circ}-38389_{7 / 2}$ & 4201.005 & 1 & & 23797.123 & $7828_{1 / 2}-31625_{1 / 2}^{\circ}$ \\
\hline 4235.849 & 1 & & 23601.372 & $21297_{5 / 2}^{\circ}-44898_{7 / 2}$ & 4199.015 & 3 & 10 & 23808.401 & $13468_{9 / 2}^{\circ}-37277_{7 / 2}$ \\
\hline 4234.984 & 25 & 5 & 23606.193 & $10673_{5 / 2}^{\circ}-34279_{7 / 2}$ & 4.197 .936 & 2 & 2 & 23814.520 & $9400_{5 / 2}-33215_{3 / 2}^{\circ}$ \\
\hline 4234.752 & 5 & & 23607.486 & $8018_{3 / 2}-31625_{1 / 2}^{\circ}$ & 4195.949 & 3 & 15 & 23825.797 & $15242_{9 / 2}^{\circ}-39068_{7 / 2}$ \\
\hline 4234.375 & 3 & & 23609.588 & $15786_{5 / 2}-39396_{7 / 2}^{\circ}$ & 4195.832 & 4 & 75 & 23826.462 & $14545_{5 / 2}^{\circ}-38372_{3 / 2}$ \\
\hline 4234.302 & 8 & 3 & 23609.995 & $11116_{7 / 2}^{\circ}-34726_{7 / 2}$ & 4195.557 & 4 & 75 & 23828.023 & $18816_{13 / 2}^{\circ}-42644_{13 / 2}^{\circ}$ \\
\hline 4234.083 & 1 & & 23611.216 & $22642_{9 / 2}^{\circ}-46253_{9 / 2}$ & 4194.082 & 2 & 4 & 23836.403 & $16818_{7 / 2}-40654_{5 / 2}^{\circ}$ \\
\hline 4233.511 & 2 & & 23614.406 & $20686_{5 / 2}^{\circ}-44300_{3 / 2}$ & 4191.995 & 1 & & 23848.270 & $19248_{5 / 2}^{\circ}-43096_{5 / 2}$ \\
\hline 4233.287 & 25 & 20 & 23615.656 & $15453_{7 / 2}^{\circ}-39068_{7 / 2}^{\circ}$ & 4191.826 & 5 & 10 & 23849.231 & $11576_{3 / 2}^{\circ}-35425_{1 / 2}^{\circ}$ \\
\hline 4232.525 & $3 b$ & & 23619.907 & $15242_{9 / 2}^{\circ}-38862_{11 / 2}$ & 4190.562 & 1 & & 23856.425 & $12472_{5 / 2}^{\circ}-36328_{3 / 2}$ \\
\hline 4231.983 & 3 & & 23622.932 & $12570_{7 / 2}-36193_{9 / 2}^{\circ}$ & 4188.580 & 2 & 5 & 23867.713 & $17460_{5 / 2}^{\circ}-41328_{5 / 2}$ \\
\hline 4231.794 & 4 & & 23623.987 & $9585_{5 / 2}^{\circ}-33209_{7 / 2}$ & 4188.103 & 1 & 2 & 23870.432 & $10673_{5 / 2}^{\circ}-34543_{5 / 2}$ \\
\hline 4231.174 & 3 & & 23627.449 & $17771_{11 / 2}^{o}-41398_{9 / 2}$ & 4.185 .035 & 1 & 2 & 23887.931 & $6213_{9 / 2}-30101_{7 / 2}^{\circ}$ \\
\hline 4229.454 & 75 & 15 & 23637.057 & $12488_{9 / 2}^{\circ}-36125_{9 / 2}^{\circ}$ & 4184.903 & 3 & 4 & 23888.684 & $20288_{11 / 2}^{o}-44177_{11 / 2}$ \\
\hline 4228.988 & 5 & 4 & 23639.662 & $12485_{7 / 2}^{\circ}-36125_{9 / 2}$ & 4.184 .709 & 4 & 25 & 23889.791 & $14275_{9 / 2}^{\circ}-38165_{7 / 2}$ \\
\hline 4228.701 & $10 \mathrm{~b}$ & & 23641.266 & $4146_{7 / 2}-27787_{9 / 2}^{\circ}$ & 4184.282 & 2 & & 23892.229 & $21297_{5 / 2}^{\circ}-45189_{5 / 2}$ \\
\hline 4227.654 & $4 b$ & 5 & 23647.121 & $13818_{7 / 2}^{\circ}-37465_{5 / 2}^{\circ}$ & 4183.562 & 5 & 15 & 23896.341 & $9061_{5 / 2}-32957_{7 / 2}^{\circ}$ \\
\hline 4226.726 & 3 & & 23652.312 & $20120_{5 / 2}^{\circ}-43772_{5 / 2}$ & 4183.414 & 1 & & 23897.186 & $27631_{3 / 2}-51528_{3 / 2}^{\circ}$ \\
\hline 4225.803 & 5 & & 23657.479 & $21131_{3 / 2}^{\circ}-44789_{1 / 2}$ & 4182.155 & 5 & 50 & 23904.380 & $14275_{9 / 2}^{\circ}-38179_{9 / 2}$ \\
\hline 4224.614 & 15 & 5 & 23664.137 & $9720_{7 / 2}^{\circ}-33384_{9 / 2}$ & 4181.348 & 2 & 4 & 23908.994 & $22355_{1 / 2}^{o}-46264_{3 / 2}$ \\
\hline 4224.238 & 10 & 5 & 23666.243 & $1521_{5 / 2}-25188_{3 / 2}^{\circ}$ & & & & & $20989_{9 / 2}^{\circ}-44898_{7 / 2}^{\circ}$ \\
\hline 4224.142 & 2 & & 23666.781 & $22685_{7 / 2}^{\circ}-46352_{7 / 2}$ & 4181.161 & 2 & 5 & 23910.063 & $12902_{3 / 2}^{\circ}-36812_{1 / 2}^{\circ}$ \\
\hline 4222.749 & 4 & & 23674.588 & $23187_{13 / 2}^{\circ}-46861_{11 / 2}$ & 4181.077 & 2 & 3 & 23910.543 & $8018_{3 / 2}-31928_{3 / 2}^{\circ}$ \\
\hline 4222.579 & 3 & & 23675.541 & $17722_{9 / 2}^{\circ}-41398_{9 / 2}^{\circ}$ & 4181.041 & 2 & 1 & 23910.749 & $27357_{9 / 2}^{\circ}-51268_{7 / 2}$ \\
\hline 4222.185 & 2 & & 23677.750 & $18568_{1 / 2}^{\circ}-42246_{1 / 2}^{\circ}$ & 4179.958 & 75 & 150 & 23916.944 & $9720_{7 / 2}^{\circ}-33637_{7 / 2}$ \\
\hline 4219.678 & 4 & & 23691.818 & $20080_{7 / 2}^{\circ}-43772_{5 / 2}$ & 4179.713 & 100 & 150 & 23918.346 & $1521_{5 / 2}-25440_{5 / 2}^{\circ}$ \\
\hline 4219.414 & 2 & & 23693.300 & $19594_{1 / 2}-43287_{3 / 2}^{\circ}$ & 4178.060 & 150 & 300 & 23927.809 & $7331_{5 / 2}^{\circ}-31259_{5 / 2}$ \\
\hline 4219.380 & 5 & 5 & 23693.491 & $17983_{5 / 2}^{\circ}-41676_{3 / 2}$ & 4176.895 & 2 & & 23934.483 & $27593_{5 / 2}-51528_{3 / 2}^{\circ}$ \\
\hline 4218.998 & 3 & & 23695.636 & $\begin{array}{l}14484_{11 / 2}^{\circ}-38179_{9 / 2} \\
24757_{9 / 2}^{\circ}-48453_{7 / 2}\end{array}$ & 4176.333 & 2 & 5 & 23937.703 & $14790_{7 / 2}^{\circ}-38728_{5 / 2}$ \\
\hline & & & & & 4175.482 & 1 & & 23942.582 & $26965_{3 / 2}^{\circ}-50907_{3 / 2}$ \\
\hline 4218.820 & 1 & & 23696.636 & $31800_{\bar{\tau} / 2}^{\circ}-55496_{9 / 2}$ & 4173.959 & 1 & & 23951.318 & $17272_{9 / 2}^{\circ}-41223_{11 / 2}$ \\
\hline 4218.636 & 2 & & 23697.669 & $24309_{11 / 2}^{\circ}-48006_{9 / 2}$ & 4173.530 & 41 & 4 & 23953.780 & $9400_{5 / 2}-33354_{5 / 2}^{\circ}$ \\
\hline 4218.539 & 10 & 10 & 23698.214 & $10572_{9 / 2}^{\circ}-34270_{9 / 2}$ & 4170.783 & 4 & 25 & 23969.556 & $13818_{7 / 2}^{\circ}-37787_{7 / 2}^{\circ}$ \\
\hline 4218.224 & 3 & & 23699.984 & $22685_{\tau / 2}^{\circ}-46385_{7 / 2}$ & 4170.469 & 25 & 50 & 23971.361 & $9238_{9 / 2}^{\circ}-33209_{7 / 2}^{\circ}$ \\
\hline 4218.187 & 8 & 5 & 23700.192 & $11725_{1 / 2}^{\circ}-35425_{1 / 2}$ & 4168.633 & 10 & 100 & 23981.919 & $10572_{9 / 2}^{\circ}-34553_{9 / 2}$ \\
\hline 4217.759 & 4 & 1 & 23702.597 & $20686_{5 / 2}^{\circ}-44388_{5 / 2}$ & 4167.275 & 2 & 15 & 23989.733 & $15710_{3 / 2}^{\circ}-39700_{5 / 2}$ \\
\hline 4214.629 & 4 & & 23720.199 & $22014_{11 / 2}^{\circ}-45735_{11 / 2}$ & 4166.493 & $2 \mathrm{~h}$ & 2 & 23994.236 & $18118_{3 / 2}-42112_{3 / 2}^{\circ}$ \\
\hline 4214.537 & 5 & 8 & 23720.717 & $17727_{11 / 2}-41447_{11 / 2}^{\circ}$ & 4165.070 & 3 & 10 & 24002.434 & $15349_{11 / 2}^{\circ}-39352_{11 / 2}$ \\
\hline 4214.227 & 1 & & 23722.462 & $15144_{3 / 2}^{\circ}-38867_{1 / 2}$ & 4164.253 & 10 & 50 & 24007.143 & $9202_{7 / 2}^{\circ}-33209_{7 / 2}$ \\
\hline
\end{tabular}


TABLE 3. Classified lines of Th II-Continued

\begin{tabular}{|c|c|c|c|c|c|c|c|c|c|}
\hline \multirow{2}{*}{$\begin{array}{c}\text { Wavelength } \\
\AA\end{array}$} & \multicolumn{2}{|c|}{ Intensity } & \multirow{2}{*}{$\begin{array}{c}\text { Wavenumber } \\
\mathbf{c m}^{-1}\end{array}$} & \multirow{2}{*}{ Classification } & \multirow{2}{*}{$\begin{array}{c}\text { Wavelength } \\
\AA\end{array}$} & \multicolumn{2}{|c|}{ Intensity } & \multirow{2}{*}{$\begin{array}{c}\text { Wavenumber } \\
\mathbf{c m}^{-1}\end{array}$} & \multirow{2}{*}{ Classification } \\
\hline & Lamp & Spark & & & & Lamp & Spark & & \\
\hline 4163.733 & 5 & 75 & 24010.141 & $16564_{11 / 2}^{\circ}-40574_{11 / 2}$ & 4132.979 & 1 & & 24188.800 & $24132_{3 / 2}^{\circ}-48320_{5 / 2}$ \\
\hline 4163.645 & 20 & 200 & 24010.648 & $9720_{7 / 2}^{\circ}-33730_{5 / 2}$ & 4132.754 & 150 & 200 & 24190.116 & $9711_{7 / 2}-33902_{7 / 2}^{\circ}$ \\
\hline 4163.179 & 3 & 15 & 24013.336 & $12570_{7 / 2}-36583_{7 / 2}^{\circ}$ & 4132.287 & 2 & & 24.192 .850 & $20310_{5 / 2}^{\circ}-44503_{7 / 2}$ \\
\hline 4162.678 & 10 & 100 & 24016.226 & $14275_{9 / 2}^{\circ}-38291_{7 / 2}$ & 4132.090 & 2 & & 24194.003 & $19050_{3 / 2}^{\circ}-43244_{3 / 2}$ \\
\hline 4161.587 & 2 & 5 & 24022.522 & $13818_{7 / 2}^{\circ}-37840_{9 / 2}$ & 4131.529 & 5 & 10 & 24197.289 & $18816_{13 / 2}^{\circ}-43014_{13 / 2}^{\circ}$ \\
\hline 4159.657 & 10 & 100 & 24033.668 & $14484_{11 / 2}^{\circ}-38517_{13 / 2}$ & 4131.425 & 50 & 100 & 24197.898 & $8378_{7 / 2}^{\circ}-32576_{7 / 2}$ \\
\hline 4156.987 & 1 & & 24049.104 & $22106_{5 / 2}-46155_{5 / 2}^{\circ}$ & 4130.663 & 5 & 3 & 24202.361 & $15349_{11 / 2}^{\circ}-39552_{9 / 2}$ \\
\hline 4156.512 & $100 \mathrm{~b}$ & 150 & 24051.852 & $9585_{5 / 2}^{\circ}-33637_{7 / 2}$ & 4129.914 & $3 \mathrm{~b}$ & 4 & 24206.751 & $17121_{3 / 2}^{\circ}-41328_{5 / 2}$ \\
\hline 4156.235 & 50 & 100 & 24053.455 & $10673_{5 / 2}^{\circ}-34726_{7 / 2}$ & 4128.912 & 1 & & 24.212 .625 & $20686_{5 / 2}^{\circ}-44898_{7 / 2}$ \\
\hline 4155.474 & 1 & & 24057.860 & $21131_{3 / 2}^{\circ}-45189_{5 / 2}^{\circ}$ & 4128.300 & 3 & 3 & 24216.215 & $17460_{5 / 2}^{\circ}-41676_{3 / 2}$ \\
\hline 4153.722 & 2 & & 24068.007 & $22513_{5 / 2}^{\circ}-46581_{5 / 2}$ & 4127.289 & 3 & & 24222.146 & $15144_{3 / 2}^{\circ}-39366_{5 / 2}$ \\
\hline 4151.447 & 4 & 5 & 24081.196 & $10189_{11 / 2}^{\circ}-34270_{9 / 2}$ & 4126.607 & 1 & & 24226.149 & $24463_{5 / 2}^{\circ}-48689_{3 / 2}$ \\
\hline 4150.482 & 4 & 5 & 24086.795 & $14349_{1 / 2}-38436_{3 / 2}^{\circ}$ & 4124.666 & 5 & 15 & 24237.549 & $15710_{3 / 2}^{\circ}-39948_{1 / 2}^{\circ}$ \\
\hline 4150.369 & 4 & 8 & 24087.451 & $24757_{9 / 2}^{\circ}-48844_{9 / 2}^{\circ}$ & 4124.513 & 5 & 10 & 24238.449 & $22014_{11 / 2}^{o}-46253_{9 / 2}^{\circ}$ \\
\hline 4149.986 & $200 \mathrm{~b}$ & 300 & 24089.674 & $10572_{9 / 2}^{\circ}-34661_{11 / 2}^{\circ}$ & 4123.953 & 2 & & 24241.740 & $20310_{5 / 2}^{\circ}-44552_{5 / 2}$ \\
\hline 4149.711 & 4 & 5 & 24091.270 & $15305_{9 / 2}-39396_{7 / 2}^{\circ}$ & 4123.530 & 40 & 25 & 24244.226 & $8605_{5 / 2}-32850_{5 / 2}^{\circ}$ \\
\hline 4148.773 & 2 & & 24096.717 & $6213_{9 / 2}-30310_{11 / 2}^{\circ}$ & 4123.401 & 2 & & 24244.985 & $16033_{5 / 2}^{\circ}-40278_{3 / 2}^{\circ}$ \\
\hline 4148.684 & 4 & & 24097.234 & $4146_{7 / 2}-28243_{5 / 2}^{\circ}$ & 4123.270 & 2 & & 24245.755 & $22139_{9 / 2}^{\circ}-46385_{7 / 2}^{\circ}$ \\
\hline 4148.425 & 2 & & 24098.738 & $17837_{1 / 2}^{o}-41936_{3 / 2}^{o}$ & 4122.965 & 8 & 15 & 24247.549 & $15453_{7 / 2}^{\circ}-39700_{5 / 2}^{\circ}$ \\
\hline 4148.344 & 20 & 50 & 24099.209 & $15453_{7 / 2}^{\circ}-39552_{9 / 2}^{\circ}$ & 4122.673 & $5 b$ & & 24249.266 & $25246_{9 / 2}^{7 / 2}-49495_{9 / 2}^{\circ}$ \\
\hline 4148.180 & 200 & 150 & 24100.162 & $7828_{1 / 2}-31928_{3 / 2}^{\circ}$ & 4121.857 & 5 & 10 & 24254.067 & $18973_{7 / 2}^{\circ}-43227_{j / 2}$ \\
\hline 4148.026 & 4 & 10 & 24101.056 & $18118_{3 / 2}-42219_{5 / 2}^{\circ}$ & 4120.014 & 3 & 4 & 24264.916 & $19912_{13 / 2}^{\circ}-44177_{11 / 2}^{\circ}$ \\
\hline 4146.664 & 2 & & 24108.972 & $26626_{i / 2}^{o}-50735_{3 / 2}$ & 4119.390 & 2 & 2 & 24268.592 & $20120_{5 / 2}^{\circ}-44388_{5 / 2}^{\circ}$ \\
\hline 4146.256 & 1 & & 24111.345 & $17771_{11 / 2}^{\circ}-41882_{13 / 2}$ & 4118.594 & 5 & 10 & 24273.282 & $13406_{13 / 2}^{\circ}-37679_{11 / 2}$ \\
\hline 4146.069 & 2 & & 24112.432 & $25414_{11 / 2}^{\circ}-49527_{13 / 2}$ & 4117.839 & 4 & 4 & 24277.732 & $35021_{3 / 2}-59299_{5 / 2}^{\circ}$ \\
\hline 4145.836 & $2 \mathbf{b}$ & & 24113.787 & $14275_{9 / 2}^{\circ}-38389_{7 / 2}$ & & & & & $14790_{7 / 2}^{\circ}-39068_{7 / 2}$ \\
\hline 4145.664 & 1 & & 24114.788 & $35878_{7 / 2}-59993_{9 / 2}^{\circ}$ & 4116.714 & $500 \mathrm{~b}$ & 400 & 24284.367 & $6168_{7 / 2}^{\circ}-30452_{9 / 2}$ \\
\hline 4145.197 & 1 & & 24117.504 & $12570_{7 / 2}-36687_{5 / 2}^{\circ}$ & 4116.311 & 4 & 4 & 24286.744 & $13818_{7 / 2}^{\circ}-38105_{5 / 2}^{\circ}$ \\
\hline 4144.453 & 5 & 2 & 24121.834 & $20686_{5 / 2}^{\circ}-44807_{7 / 2}^{\circ}$ & 4115.144 & 81 & & 24293.632 & $9061_{5 / 2}-33354_{5 / 2}^{\circ}$ \\
\hline 4143.603 & 3 & 5 & 24126.782 & $13818_{7 / 2}^{\circ}-37945_{5 / 2}^{\circ}$ & 4114.145 & 2 & & 24299.530 & $18118_{3 / 2}-42418_{3 / 2}^{\circ}$ \\
\hline 4142.970 & 5 & 3 & 24130.468 & $4113_{5 / 2}-28243_{5 / 2}^{\circ}$ & 4113.557 & 5 & & 24303.004 & $24309_{11 / 2}^{\circ}-48612_{13 / 2}$ \\
\hline 4142.700 & 300 & 100 & 24132.041 & $0_{3 / 2}-24132_{3 / 2}^{\circ}$ & 4112.485 & 1 & & 24309.339 & $15242_{9 / 2}^{\circ}-39552_{9 / 2}$ \\
\hline 4142.475 & 50 & 150 & 24133.352 & $14484_{11 / 2}^{o}-38617_{9 / 2}$ & 4112.313 & 5 & 15 & 24310.355 & $18816_{13 / 2}^{\circ}-43127_{11 / 2}^{\circ}$ \\
\hline 4141.947 & 1 & & 24136.428 & $20989_{9 / 2}^{\circ}-45126_{9 / 2}^{\circ}$ & 4111.986 & 3 & & 24312.288 & $23012_{3 / 2}^{\circ}-47324_{5 / 2}^{\circ}$ \\
\hline 4141.631 & 50 & 75 & 24138.269 & $17771_{11 / 2}^{\circ}-41909_{9 / 2}$ & 4111.458 & 3 & & 24315.411 & $11725_{1 / 2}^{\circ}-36040_{1 / 2}^{0 / 2}$ \\
\hline 4141.225 & 2 & & 24140.636 & $16906_{7 / 2}^{\circ}-41047_{9 / 2}$ & 4111.427 & 3 & 2 & 24315.594 & $27631_{3 / 2}-51946_{5 / 2}^{\circ}$ \\
\hline 4140.385 & 50 & 100 & 24145.533 & $9585_{5 / 2}^{\circ}-33730_{5 / 2}^{\circ}$ & 4111.133 & 4 & 5 & 24317.333 & $22106_{5 / 2}-464.23_{3 / 2}^{\circ}$ \\
\hline 4140.235 & 200 & 150 & 24146.408 & $9238_{9 / 2}^{\circ}-33384_{9 / 2}^{\circ}$ & 4110.968 & 4 & 4 & 24318.309 & $14545_{5 / 2}^{\circ}-38863_{5 / 2}^{\circ}$ \\
\hline 4139.934 & 2 & & 24148.164 & $25266_{1 / 2}^{o}-49414_{3 / 2}^{0}$ & 4110.633 & 15 & 15 & 24320.291 & $6244_{1 / 2}-30564_{1 / 2}^{\circ}$ \\
\hline 4139.758 & 1 & & 24149.190 & $26586_{3 / 2}^{\circ}-50735_{3 / 2}^{\circ}$ & 4110.512 & 40 & 25 & 24321.006 & $12488_{9 / 2}^{\circ}-36809_{\tau / 2}$ \\
\hline 4138.841 & 5 & 5 & 24154.541 & $10572_{9 / 2}^{\circ}-34726_{7 / 2}$ & 4110.075 & 5 & 4 & 24323.592 & $12485_{7 / 2}^{\circ}-36809_{7 / 2}$ \\
\hline 4137.886 & 2 & & 24160.115 & $16564_{11 / 2}^{\circ}-40724_{13 / 2}$ & 4108.420 & 500 & 150 & 24333.391 & $4490_{5 / 2}^{\circ}-28823_{5 / 2}^{\circ}$ \\
\hline 4137.612 & 2 & & 24161.715 & $20288_{11 / 2}^{\circ}-44450_{9 / 2}$ & 4107.793 & 5 & 8 & 24337.105 & $12472_{5 / 2}^{\circ}-36809_{7 / 2}^{\circ}$ \\
\hline 4137.068 & 2 & & 24164.892 & $11576_{3 / 2}^{\circ}-35741_{5 / 2}$ & 4107.370 & 20 & 8 & 24339.611 & $11116_{7 / 2}^{\circ}-35456_{9 / 2}^{\circ}$ \\
\hline 4136.393 & $75 b$ & $20 \mathrm{~b}$ & 24168.836 & $13406_{13 / 2}^{\circ}-37575_{13 / 2}$ & 4106.950 & 4 & & 24342.100 & $14275_{9 / 2}^{\circ}-38617_{9 / 2}^{\circ}$ \\
\hline 4136.101 & 2 & & 24170.542 & $12219_{3 / 2}-36390_{3 / 2}^{\circ}$ & 4106.120 & $5 b$ & $5 b$ & 24347.020 & $13818_{7 / 2}^{\circ}-38165_{7 / 2}^{\circ}$ \\
\hline 4134.989 & 2 & & 24177.042 & $19050_{3 / 2}^{\circ}-43227_{5 / 2}$ & 4105.911 & $25 b$ & 50 & 24348.260 & $10673_{5 / 2}^{\circ}-35021_{3 / 2}$ \\
\hline 4134.414 & 1 & & 24180.404 & $20120_{5 / 2}^{\circ}-44300_{3 / 2}^{\circ}$ & 4105.037 & $2 \mathrm{~b}$ & & 24353.444 & $17983_{5 / 2}^{\circ}-42336_{5 / 2}^{\circ}$ \\
\hline 4134.106 & $100 \mathrm{~b}$ & $100 \mathrm{~b}$ & 24182.205 & $9202_{7 / 2}^{\circ}-33384_{9 / 2}^{\circ}$ & & & & & $25607_{9 / 2}^{\circ}-49960_{7 / 2}^{\circ}$ \\
\hline 4133.396 & 2 & & 24186.359 & $17722_{9 / 2}^{\circ}-41909_{9 / 2}^{\circ}$ & 4104.381 & 150 & 75 & 24357.336 & $8378_{7 / 2}^{\circ}-32736_{7 / 2}^{\circ}$ \\
\hline
\end{tabular}


TABLE 3. Classified lines of Th II-Continued

\begin{tabular}{|c|c|c|c|c|c|c|c|c|c|}
\hline \multirow{2}{*}{$\begin{array}{c}\text { Wavelength } \\
\AA\end{array}$} & \multicolumn{2}{|c|}{ Intensity } & \multirow{2}{*}{$\begin{array}{c}\text { Wavenumber } \\
\mathbf{c m}^{-1}\end{array}$} & \multirow{2}{*}{ Classification } & \multirow{2}{*}{$\begin{array}{c}\text { Wavelength } \\
\AA\end{array}$} & \multicolumn{2}{|c|}{ Intensity } & \multirow{2}{*}{$\begin{array}{c}\text { Wavenumber } \\
\mathbf{c m}^{-1}\end{array}$} & \multirow{2}{*}{ Classification } \\
\hline & Lamp & Spark & & & & Lamp & Spark & & \\
\hline \multirow[t]{2}{*}{4103.669} & \multirow[t]{2}{*}{$5 b$} & \multirow{5}{*}{15} & \multirow[t]{2}{*}{24361.562} & $13818_{7 / 2}^{\circ}-38179_{9 / 2}$ & 4075.709 & 5 & \multirow[t]{2}{*}{8} & 24528.682 & \multirow{10}{*}{$\begin{array}{r}9202_{7 / 2}^{\circ}-33730_{5 / 2}^{\circ} \\
30310_{11 / 2}^{\circ}-54845_{9 / 2}^{\circ} \\
35456_{9 / 2}-59993_{9 / 2}^{\circ} \\
16033_{5 / 2}^{\circ}-40570_{7 / 2}^{\circ} \\
22014_{11 / 2}^{\circ}-46555_{11 / 2}^{\circ} \\
15349_{11 / 2}^{\circ}-39895_{9 / 2}^{\circ} \\
9720_{7 / 2}^{\circ}-34270_{9 / 2}^{\circ} \\
30484_{11 / 2}-55038_{9 / 2}^{\circ} \\
17121_{3 / 2}^{\circ}-41676_{3 / 2}^{\circ} \\
9720_{7 / 2}^{\circ}-34279_{7 / 2}^{\circ}\end{array}$} \\
\hline & & & & $12219_{3 / 2}-36581_{3 / 2}^{\circ}$ & 4074.642 & 1 & & 24535.106 & \\
\hline 4103.264 & 5 & & 24363.966 & $22014_{11 / 2}^{\circ}-46378_{13 / 2}$ & 4074.245 & 2 & 4 & 24537.496 & \\
\hline 4103.112 & 4 & & 24364.869 & $10189_{11 / 2}^{\circ}-34553_{9 / 2}$ & & & & & \\
\hline 4102.462 & 2 & & 24368.729 & $17983_{5 / 2}^{\circ}-42352_{5 / 2}$ & 4073.708 & 10 & 40 & 24540.731 & \\
\hline 4101.326 & 4 & \multirow[t]{2}{*}{5} & 24375.479 & $17771_{11 / 2}^{\circ}-42146_{11 / 2}$ & 4072.907 & 10 & 5 & 24545.557 & \\
\hline 4100.847 & $10 \mathrm{~b}$ & & 24378.326 & $16033_{5 / 2}^{\circ}-40411_{7 / 2}$ & 4072.178 & 3 & \multirow[t]{3}{*}{1} & 24549.951 & \\
\hline 4100.820 & $50 \mathrm{~b}$ & 75 & 24378.486 & $14484_{11 / 2}^{0}-38862_{11 / 2}$ & 4071.466 & 5 & & 24554.244 & \\
\hline 4098.932 & 50 & \multirow[t]{2}{*}{20} & 24389.715 & $8460_{3 / 2}-32850_{5 / 2}^{\circ}$ & 4071.302 & 1 & & 24555.233 & \\
\hline 4098.093 & 2 & & 24394.708 & $24982_{7 / 2}^{\circ}-49377_{7 / 2}$ & 4070.671 & 15 & 3 & 24559.040 & \\
\hline 4097.691 & $15 b$ & 10 & 24397.102 & $15786_{5 / 2}-40184_{7 / 2}^{\circ}$ & 4069.757 & 15 & 3 & 24564.555 & $1859_{3 / 2}-26424_{5 / 2}^{\circ}$ \\
\hline 4096.332 & 8 & 2 & 24405.196 & $20989_{9 / 2}^{\circ}-45395_{7 / 2}$ & 4069.299 & 4 & & 24567.320 & $15710_{3 / 2}^{\circ}-40278_{3 / 2}$ \\
\hline 4094.747 & 500 & 200 & 24414.642 & $0_{3 / 2}-24414_{3 / 2}^{\circ}$ & 4069.202 & 300 & 200 & 24567.905 & $6691_{3 / 2}^{\circ}-31259_{5 / 2}$ \\
\hline 4094.490 & 3 & & 24416.174 & $17272_{9 / 2}^{\circ}-41688_{7 / 2}$ & 4068.025 & 5 & 3 & 24575.013 & $12488_{9 / 2}^{\circ}-37063_{9 / 2}$ \\
\hline 4093.556 & 3 & 3 & 24421.745 & $16906_{7 / 2}^{\circ}-41328_{5 / 2}$ & 4067.876 & 10 & & 24575.913 & $14790_{7 / 2}^{\circ}-39366_{5 / 2}$ \\
\hline 4093.392 & 20 & 10 & 24422.724 & $7331_{5 / 2}^{\circ}-31754_{5 / 2}^{\circ}$ & 4067.594 & 15 & 4 & 24577.617 & $12485_{7 / 2}^{\circ}-37063_{9 / 2}$ \\
\hline 4093.327 & 2 & & 24423.111 & $20080_{7 / 2}^{\circ}-44503_{7 / 2}$ & 4066.169 & 1 & & 24586.230 & $30452_{9 / 2}-55038_{9 / 2}^{\circ}$ \\
\hline 4093.255 & 2 & & 24423.541 & $17722_{9 / 2}^{\circ}-42146_{11 / 2}$ & 4066.003 & 8 & 4 & 24587.234 & $14275_{9 / 2}^{\circ}-38862_{11 / 2}$ \\
\hline 4092.338 & $2 \mathrm{~s}$ & & 24429.014 & $1116_{7 / 2}^{\circ}-35545_{9 / 2}$ & 4065.687 & 401 & 8 & 24589.145 & $9585_{5 / 2}^{\circ}-34174_{5 / 2}$ \\
\hline 4092.314 & 1 & & 24429.157 & $17771_{11 / 2}^{\circ}-42200_{9 / 2}$ & 4065.254 & 4 & 4 & 24591.764 & $17727_{11 / 2}-42319_{9 / 2}^{\circ}$ \\
\hline 4091.530 & 5 & 4 & 24433.838 & $9585_{5 / 2}^{\circ}-34019_{3 / 2}$ & 4064.610 & 4 & 2 & 24595.660 & $13250_{5 / 2}-37846_{5 / 2}^{\circ}$ \\
\hline 4091.347 & 50 & 8 & 24434.931 & $9202_{7 / 2}^{\circ}-33637_{7 / 2}$ & 4063.755 & 3 & & 24600.835 & $23697_{7 / 2}^{\circ}-48298_{7 / 2}$ \\
\hline 4090.367 & 3 & & 24440.785 & $4146_{7 / 2}-28587_{5 / 2}^{\circ}$ & 4063.691 & 5 & 4 & 24601.222 & $14484_{11 / 2}^{\circ}-39085_{13 / 2}$ \\
\hline 4090.103 & 21 & & 24442.362 & $15453_{7 / 2}^{\circ}-39895_{9 / 2}$ & 4063.054 & 100 & & 24605.079 & $14545_{5 / 2}^{\circ}-39150_{3 / 2}$ \\
\hline 4088.108 & 1 & & 24454.290 & $9720_{7 / 2}^{\circ}-34174_{5 / 2}$ & 4062.661 & 20 & & 24607.459 & $4113_{5 / 2}-28720_{3 / 2}^{\circ}$ \\
\hline 4086.520 & 500 & 150 & 24463.793 & $0_{3 / 2}-24463_{5 / 2}^{\circ}$ & 4062.305 & 8 & & 24609.616 & $8605_{5 / 2}-33215_{3 / 2}^{\circ}$ \\
\hline 4086.412 & 5 & 8 & 24464.439 & $11576_{3 / 2}^{\circ}-36040_{1 / 2}$ & 4059.888 & 50 & & 24624.267 & $7001_{3 / 2}-31625_{1 / 2}^{\circ}$ \\
\hline 4086.336 & 3 & & 24464.894 & $14275_{9 / 2}^{\circ}-38740_{11 / 2}$ & 4059.815 & 4 & & 24624.709 & $11116_{7 / 2}^{\circ}-35741_{5 / 2}$ \\
\hline 4085.815 & 8 & 8 & 24468.014 & $12219_{3 / 2}-36687_{5 / 2}^{\circ}$ & 4059.766 & 3 & & 24625.007 & $15236_{3 / 2}-39861_{5 / 2}^{\circ}$ \\
\hline 4085.042 & 300 & 200 & 24472.644 & $10189_{i 1 / 2}^{\circ}-34661_{11 / 2}$ & 4057.776 & 25 & 50 & 24637.083 & $17272_{9 / 2}^{\circ}-41909_{9 / 2}$ \\
\hline 4084.953 & 10 & 10 & 24473.177 & $27357_{9 / 2}^{\circ}-51830_{7 / 2}$ & 4057.327 & 50 & & 24639.809 & $12902_{3 / 2}^{\circ}-37542_{3 / 2}$ \\
\hline 4084.815 & $2 \mathbf{b}$ & & 24474.004 & $4113_{5 / 2}-28587_{5 / 2}^{\circ}$ & 4057.034 & 4 & & 24641.589 & $20969_{7 / 2}^{\circ}-45610_{5 / 2}$ \\
\hline 4084.499 & 2 & 2 & 24475.897 & $17460_{5 / 2}^{\circ}-41936_{3 / 2}$ & 4054.765 & 3 & 2 & 24655.377 & $14101_{1 / 2}^{\circ}-38757_{3 / 2}$ \\
\hline 4084.278 & 3 & 3 & 24477.221 & $17722_{9 / 2}^{\circ}-42200_{9 / 2}$ & 4054.185 & 4 & 20 & 24658.905 & $16564_{11 / 2}^{\circ}-41223_{11 / 2}$ \\
\hline 4084.008 & 1 & & 24478.840 & $21131_{3 / 2}^{\circ}-45610_{5 / 2}$ & 4052.443 & 8 & 10 & 24669.504 & $21682_{7 / 2}^{\circ}-46352_{7 / 2}$ \\
\hline 4083.388 & $4 b$ & 8 & 24482.556 & $16564_{11 / 2}^{\circ}-41047_{9 / 2}$ & 4051.277 & 2 & & 24676.604 & $18568_{1 / 2}^{\circ}-43244_{3 / 2}$ \\
\hline 4082.256 & 20 & & 24489.345 & $11576_{3 / 2}^{\circ}-36065_{5 / 2}$ & 4049.825 & 8 & 8 & 24685.452 & $15786_{5 / 2}-40472_{3 / 2}^{\circ}$ \\
\hline 4081.853 & 3 & $20 \mathrm{~h}$ & 24491.763 & $20158_{5 / 2}-44650_{7 / 2}^{\circ}$ & 4049.747 & 10 & 2 & 24685.927 & $24132_{3 / 2}^{\circ}-48817_{3 / 2}^{\circ}$ \\
\hline 4081.829 & 4 & & 24491.907 & $16906_{7 / 2}^{\circ}-41398_{9 / 2}$ & 4048.030 & 15 & 50 & 24696.398 & $13468_{9 / 2}^{\circ}-38165_{7 / 2}$ \\
\hline 4080.975 & 3 & & 24497.032 & $\begin{array}{l}20310_{5 / 2}^{\circ}-44807_{7 / 2} \\
23372_{3 / 2}^{\circ}-47869_{3 / 2}^{\circ}\end{array}$ & 4045.627 & 75 & 50 & 24711.066 & $6168_{7 / 2}^{\circ}-30879_{7 / 2}$ \\
\hline & & & & & 4043.852 & 1 & & 24721.913 & $22139_{9 / 2}^{\circ}-46861_{11 / 2}$ \\
\hline 4080.590 & 2 & & 24499.343 & $17722_{9 / 2}^{\circ}-42222_{7 / 2}$ & 4043.128 & 200 & 40 & 24726.340 & $1859_{3 / 2}-26586_{3 / 2}^{\circ}$ \\
\hline 4080.470 & 2 & & 24500.064 & $9711_{7 / 2}-34212_{5 / 2}^{\circ}$ & 4042.973 & 8 & 75 & 24727.288 & $20080_{7 / 2}^{\circ}-44807_{7 / 2}$ \\
\hline 4080.297 & 5 & 4 & 24501.102 & $9400_{5 / 2}-33902_{7 / 2}^{\circ}$ & 4041.811 & 10 & 15 & 24734.396 & $14101_{1 / 2}^{\circ}-38836_{3 / 2}$ \\
\hline 4079.900 & 4 & 8 & 24503.486 & $20686_{5 / 2}^{\circ}-45189_{5 / 2}$ & 4041.207 & 200 & 400 & 24738.093 & $10855_{7 / 2}-35593_{9 / 2}^{\circ}$ \\
\hline 4079.603 & 4 & 5 & 24505.270 & $15710_{3 / 2}^{\circ}-40216_{5 / 2}$ & 4040.391 & 40 & 20 & 24743.089 & $6213_{9 / 2}-30956_{9 / 2}^{\circ}$ \\
\hline 4079.434 & 1 & & 24506.286 & $13250_{5 / 2}-37756_{7 / 2}^{\circ}$ & 4039.431 & 40 & 15 & 24748.969 & $8605_{5 / 2}-33354_{5 / 2}^{\circ}$ \\
\hline 4079.135 & 4 & 4 & 24508.082 & $13248_{9 / 2}-37756_{7 / 2}^{\circ}$ & 4038.903 & 1 & & 24752.205 & $11576_{3 / 2}^{\circ}-36328_{3 / 2}$ \\
\hline 4077.103 & 1 & & 24520.296 & $25440_{5 / 2}^{\circ}-49960_{z / 2}$ & 4038.427 & 8 & & 24755.122 & $8460_{3 / 2}-33215_{3 / 2}^{\circ}$ \\
\hline 4076.633 & 2 & & 24523.123 & $14545_{5 / 2}^{\circ}-39068_{7 / 2}$ & 4038.351 & 50 & 25 & 24755.588 & $34543_{5 / 2}-59299_{5 / 2}^{\circ}$ \\
\hline
\end{tabular}


TABLE 3. Classified lines of Th II-Continued

\begin{tabular}{|c|c|c|c|c|c|c|c|c|c|}
\hline \multirow{2}{*}{$\begin{array}{c}\text { Wavelength } \\
\AA\end{array}$} & \multicolumn{2}{|c|}{ Intensity } & \multirow{2}{*}{$\begin{array}{c}\text { Wavenumber } \\
\mathbf{c m}^{-1}\end{array}$} & \multirow{2}{*}{ Classification } & \multirow{2}{*}{$\begin{array}{c}\text { Wavelength } \\
\AA\end{array}$} & \multicolumn{2}{|c|}{ Intensity } & \multirow{2}{*}{$\begin{array}{c}\text { Wavenumber } \\
\mathbf{c m}^{-1}\end{array}$} & \multirow{2}{*}{ Classification } \\
\hline & Lamp & Spark & & & & Lamp & Spark & & \\
\hline 4037.434 & 4 & 4 & 24761.210 & $14790_{7 / 2}^{\circ}-39552_{9 / 2}$ & 4007.019 & 200 & 300 & 24949.155 & $11116_{7 / 2}^{\circ}-36065_{5 / 2}$ \\
\hline 4037.259 & 25 & 50 & 24762.284 & $11116_{7 / 2}^{\circ}-35878_{7 / 2}^{\circ}$ & 4006.874 & 1 & & 24950.058 & $17272_{9 / 2}^{\circ}-42222_{7 / 2}^{\circ}$ \\
\hline 4037.132 & 8 & 4 & 24763.063 & $15453_{7 / 2}^{\circ}-40216_{5 / 2}^{\circ}$ & 4006.378 & 200 & 100 & 24953.146 & $10572_{9 / 2}^{\circ}-35525_{11 / 2}^{\circ}$ \\
\hline 4036.565 & $300 \mathrm{~b}$ & 300 & 24766.541 & $1859_{3 / 2}-26626_{1 / 2}^{\circ}$ & 4005.571 & 50 & 100 & 24958.173 & $9585_{5 / 2}^{\circ}-34543_{5 / 2}$ \\
\hline 4035.236 & 3 & & 24774.698 & $27787_{9 / 2}^{\circ}-52562_{7 / 2}$ & 4005.528 & 50 & $500 \mathrm{~b}$ & 24958.441 & $15453_{7 / 2}^{\circ}-40411_{7 / 2}$ \\
\hline 4034.919 & 75 & 25 & 24776.644 & $4146_{7 / 2}-28923_{5 / 2}^{\circ}$ & & & & & $16033_{5 / 2}^{\circ}-40991_{3 / 2}$ \\
\hline 4034.364 & 2 & & 24780.052 & $23518_{7 / 2}^{\circ}-48298_{7 / 2}^{\circ}$ & 4003.307 & 300 & 600 & 24972.288 & $9202_{7 / 2}^{\circ}-34174_{5 / 2}^{\circ}$ \\
\hline 4034.246 & 200 & 200 & 24780.777 & $6213_{9 / 2}-30994_{7 / 2}^{\circ}$ & 4003.104 & 100 & 200 & 24.973 .554 & $10572_{9 / 2}^{\circ}-35545_{9 / 2}^{\circ}$ \\
\hline 4032.893 & 10 & 10 & 24789.091 & $12488_{9 / 2}^{\circ}-37277_{7 / 2}^{\circ}$ & 4002.106 & 3 & 2 & 24979.782 & $12485_{7 / 2}^{\circ}-37465_{5 / 2}$ \\
\hline 4032.238 & 2 & & 24793.117 & $14275_{9 / 2}^{\circ}-39068_{7 / 2}$ & 4001.732 & 100 & 20 & 24982.116 & $4113_{5 / 2}-29095_{5 / 2}^{\circ}$ \\
\hline 4032.035 & 2 & & 24794.366 & $23697_{7 / 2}^{\circ}-48492_{5 / 2}$ & 3999.944 & 20 & 10 & 24993.283 & $12472_{5 / 2}^{\circ}-37465_{5 / 2}$ \\
\hline 4031.781 & 3 & & 24795.928 & $17722_{9 / 2}^{\circ}-42518_{7 / 2}^{\circ}$ & 3997.977 & 15 & 15 & 25005.580 & $8378_{7 / 2}^{\circ}-33384_{9 / 2}$ \\
\hline 4031.225 & 3 & & 24799.347 & $13818_{7 / 2}^{\circ}-38617_{9 / 2}^{\circ}$ & 3997.865 & 200 & 200 & 25006.280 & $9720_{7 / 2}^{\circ}-34726_{7 / 2}$ \\
\hline 4029.518 & 20 & 10 & 24809.853 & $4113_{5 / 2}-28923_{5 / 2}^{\circ}$ & 3997.467 & $40 \mathrm{~b}$ & 100 & 25008.770 & $11116_{7 / 2}^{\circ}-36125_{9 / 2}^{\circ}$ \\
\hline 4029.322 & 25 & 40 & 24811.060 & $9400_{5 / 2}-34212_{5 / 2}^{\circ}$ & 3996.061 & 150 & 300 & 25017.569 & $15349_{11 / 2}^{o}-40367_{9 / 2}$ \\
\hline 4029.025 & 10 & 50 & 24812.889 & $15710_{3 / 2}^{\circ}-40523_{1 / 2}$ & 3995.701 & 2 & & 25019.823 & $34279_{7 / 2}-59299_{5 / 2}^{\circ}$ \\
\hline 4028.629 & 50 & 40 & 24815.328 & $24309_{11 / 2}^{\circ}-49124_{11 / 2}$ & 3995.030 & 2 & & 25024.025 & $25607_{9 / 2}^{\circ}-5063 l_{11 / 2}^{\circ}$ \\
\hline 4027.651 & 15 & 75 & 24821.353 & $14545_{5 / 2}^{\circ}-39366_{5 / 2}$ & 3994.54 .9 & 600 & 200 & 25027.038 & $0_{3 / 2}-25027_{1 / 2}^{\circ}$ \\
\hline 4027.412 & 8 & 25 & 24822.826 & $13468_{9 / 2}^{\circ}-38291_{7 / 2}^{\circ}$ & 3994.34 .1 & 10 & 200 & 25028.341 & $17722_{9 / 2}^{\circ}-42751_{7 / 2}^{\circ}$ \\
\hline 4027.341 & 50 & 75 & 24823.264 & $9720_{7 / 2}^{\circ}-34543_{5 / 2}$ & 3993.722 & 201 & 10 & 25032.221 & $9238_{9 / 2}^{\circ}-34270_{9 / 2}^{\circ}$ \\
\hline 4026.043 & 10 & & 24831.267 & $20969_{7 / 2}^{\circ}-45800_{5 / 2}$ & 3992.273 & $100 \mathrm{~h}$ & 150 & 25041.306 & $9238_{9 / 2}^{\circ}-34279_{7 / 2}$ \\
\hline 4025.944 & 10 & 3 & 24831.877 & $8018_{3 / 2}-32850_{5 / 2}^{\circ}$ & 3992.046 & 8 & 20 & 25042.730 & $12902_{3 / 2}^{\circ}-37945_{5 / 2}^{\circ}$ \\
\hline 4025.654 & $100 \mathrm{~b}$ & $300 \mathrm{~b}$ & 24833.666 & $9720_{7 / 2}^{\circ}-34553_{9 / 2}^{\circ}$ & 3991.601 & $4 b$ & & 25045.521 & $13818_{7 / 2}^{\circ}-38863_{5 / 2}^{\circ}$ \\
\hline 4025.620 & 25 & $8 b$ & 24833.876 & $16564_{i 1 / 2}^{\circ}-41398_{9 / 2}$ & 3991.066 & 10 & 15 & 25048.879 & $14101_{1 / 2}^{\circ}-39150_{3 / 2}$ \\
\hline 4024.467 & 25 & 40 & 24840.990 & $9061_{5 / 2}-33902_{7 / 2}^{\circ}$ & 3990.118 & 2 & & 25054.830 & $21297_{5 / 2}^{\circ}-46352_{7 / 2}$ \\
\hline 4023.765 & 1 & & 24845.324 & $29515_{9 / 2}-54360_{9 / 2}^{\circ}$ & 3989.568 & 2 & & 25058.284 & $17460_{5 / 2}^{\circ}-42518_{7 / 2}$ \\
\hline 4023.542 & $2 \mathbf{b}$ & & 24846.701 & $22014_{11 / 2}^{\circ}-46861_{11 / 2}^{\circ / 2}$ & 3988.845 & 200 & 200 & 25062.826 & $6691_{3 / 2}^{\circ}-31754_{5 / 2}^{\circ}$ \\
\hline 4022.091 & $100 \mathrm{~b}$ & 75 & 24855.665 & $4490_{5 / 2}^{\circ}-29345_{5 / 2}$ & 3988.599 & 100 & 25 & 25064.371 & $1521_{5 / 2}-26586_{3 / 2}^{\circ}$ \\
\hline 4020.915 & 3 & & 24862.934 & $25607_{9 / 2}^{\circ}-50470_{9 / 2}^{\circ}$ & 3988.025 & $200 \mathrm{~b}$ & $500 \mathrm{~b}$ & 25067.979 & $9202_{7 / 2}^{\circ}-34270_{9 / 2}^{\circ}$ \\
\hline 4020.100 & 5 & 10 & 24867.974 & $14484_{11 / 2}^{\circ}-39352_{11 / 2}$ & 3987.706 & 50 & 50 & 25069.984 & $12472_{5 / 2}^{\circ}-37542_{3 / 2}^{\circ}$ \\
\hline 4019.129 & $1000 \mathrm{~b}$ & 800 & 24873.982 & $0_{3 / 2}-24873_{5 / 2}^{\circ}$ & 3987.483 & 10 & 15 & 25071.386 & $15144_{3 / 2}^{\circ}-40216_{5 / 2}$ \\
\hline 4018.768 & 5 & 50 & 24876.216 & $17460_{5 / 2}^{\circ}-42336_{5 / 2}$ & 3987.219 & $200 \mathrm{~b}$ & 75 & 25073.046 & $34543_{5 / 2}-59616_{3 / 2}^{\circ}$ \\
\hline 4017.709 & 2 & & 24882.773 & $22028_{15 / 2}^{\circ}-46910_{13 / 2}$ & 3987.071 & 5 & 3 & 25073.977 & $12488_{9 / 2}^{\circ}-37562_{11 / 2}^{\circ}$ \\
\hline 4017.486 & 75 & 50 & 24884.155 & $10572_{9 / 2}^{\circ}-35456_{9 / 2}^{\circ}$ & 3985.464 & 15 & 40 & 25084.087 & $20310_{5 / 2}^{\circ}-45395_{7 / 2}$ \\
\hline 4016.459 & 1 & & 24890.517 & $16033_{5 / 2}^{\circ}-40923_{5 / 2}$ & 3985.408 & 2 & & 25084.439 & $27593_{5 / 2}-52678_{5 / 2}^{\circ}$ \\
\hline 4016.438 & 1 & & 24890.647 & $24982_{7 / 2}^{\circ}-49873_{5 / 2}$ & 3985.065 & 2 & 8 & 25086.598 & $24873_{5 / 2}^{\circ}-49960$ \\
\hline 4016.299 & 5 & 50 & 24891.509 & $17460_{5 / 2}^{\circ}-42352_{5 / 2}$ & 3984.620 & 10 & & 25089.400 & $22642_{9 / 2}^{\circ}-47731_{9 / 2}^{\circ}$ \\
\hline 4015.826 & 15 & 15 & 24894.441 & $8460_{3 / 2}-33354_{5 / 2}^{\circ}$ & 3984.373 & 50 & 25 & 25090.955 & $6168_{7 / 2}^{\circ}-31259_{5 / 2}^{\circ}$ \\
\hline 4015.589 & 2 & 10 & 24895.910 & $22014_{1 / 2}^{\circ}-46910_{13 / 2}$ & 3982.232 & 75 & 25 & 25104.445 & $14790_{7 / 2}^{\circ}-39895_{9 / 2}$ \\
\hline 4015.142 & 21 & & 24898.681 & $15324_{i / 2}^{\circ}-40222_{3 / 2}$ & 3982.100 & 200 & 40 & 25105.277 & $1859_{3 / 2}-26965_{3 / 2}^{\circ}$ \\
\hline 4014.937 & 1 & & 24899.952 & $26965_{3 / 2}^{\circ}-51865_{5 / 2}$ & 3981.534 & 10 & 20 & 25108.846 & $6244_{1 / 2}-31353_{3 / 2}^{\circ}$ \\
\hline 4014.511 & 75 & 25 & 24902.595 & $1521_{5 / 2}-26424_{5 / 2}^{\circ}$ & 3981.105 & 150 & 200 & 25111.551 & $13406_{13 / 2}^{\circ}-38517_{13 / 2}$ \\
\hline 4013.257 & 15 & 40 & 24910.376 & $12219_{3 / 2}-37130_{1 / 2}^{\circ}$ & 3980.753 & 8 & 100 & 25113.772 & $24381_{7 / 2}-49495_{9 / 2}^{\circ}$ \\
\hline 4011.775 & $50 \mathrm{~b}$ & $100 \mathrm{~b}$ & 24919.578 & $12902_{3 / 2}^{\circ}-37821_{3 / 2}$ & 3980.143 & 5 & 15 & 25117.621 & $15453_{7 / 2}^{\circ}-40570_{7 / 2}$ \\
\hline 4010.984 & 2 & & 24924.492 & $20686_{5 / 2}^{\circ}-45610_{5 / 2}$ & 3979.038 & 200 & 200 & 25124.596 & $34174_{5 / 2}-59299_{5 / 2}^{\circ}$ \\
\hline 4010.531 & 15 & 8 & 24927.307 & $7001_{3 / 2}-31928_{3 / 2}^{\circ}$ & 3977.795 & 3 & 15 & 25132.447 & $21131_{3 / 2}^{\circ}-46264_{3 / 2}$ \\
\hline 4010.427 & 4 & 20 & 24927.954 & $17272_{9 / 2}^{\circ}-42200_{9 / 2}$ & 3976.414 & 100 & 200 & 25141.175 & $9585_{5 / 2}^{\circ}-34726_{7 / 2}$ \\
\hline 4010.326 & 1 & & 24928.582 & $29431_{\tau / 2}-54360_{9 / 2}^{\circ}$ & 3975.222 & 20 & 75 & 25148.714 & $13468_{9 / 2}^{\circ}-38617_{9 / 2}^{\circ}$ \\
\hline 4009.535 & 4 & 10 & 24933.499 & $15710_{3 / 2}^{\circ}-40644_{5 / 2}$ & 3974.873 & 1 & & 25150.922 & $9061_{5 / 2}-34212_{5 / 2}^{\circ}$ \\
\hline 4008.743 & 2 & & 24938.425 & $26424_{5 / 2}^{\circ}-51362_{5 / 2}^{\circ}$ & 3974.391 & 20 & 50 & 25153.972 & $31924_{11 / 2}^{\circ}-57078_{9 / 2}^{o}$ \\
\hline
\end{tabular}


TABLE 3. Classified lines of Th II-Continued

\begin{tabular}{|c|c|c|c|c|c|c|c|c|c|}
\hline \multirow{2}{*}{$\begin{array}{c}\text { Wavelength } \\
\AA\end{array}$} & \multicolumn{2}{|c|}{ Intensity } & \multirow{2}{*}{$\begin{array}{c}\text { Wavenumber } \\
\mathbf{c m}^{-1}\end{array}$} & \multirow{2}{*}{ Classification } & \multirow{2}{*}{$\begin{array}{c}\text { Wavelength } \\
\AA\end{array}$} & \multicolumn{2}{|c|}{ Intensity } & \multirow{2}{*}{$\begin{array}{c}\text { Wavenumber } \\
\mathrm{cm}^{-1}\end{array}$} & \multirow{2}{*}{ Classification } \\
\hline & Lamp & Spark & & & & Lamp & Spark & & \\
\hline 3974.224 & 15 & 100 & 25155.029 & $14545_{5 / 2}^{\circ}-39700_{5 / 2}$ & 3945.818 & 75 & 300 & 25336.116 & $10189_{11 / 2}^{\circ}-35525_{11 / 2}$ \\
\hline 3973.993 & 1 & & 25156.491 & $22014_{11 / 2}^{\circ}-47171_{9 / 2}^{\circ}$ & 3945.506 & 200 & 300 & 25338.120 & $10855_{7 / 2}-36193_{9 / 2}^{\circ}$ \\
\hline 3969.854 & 5 & 50 & 25182.719 & $14275_{9 / 2}^{\circ}-39458_{7 / 2}$ & 3943.688 & 100 & 200 & 25349.800 & $12472_{5 / 2}^{\circ}-37821_{3 / 2}$ \\
\hline 3969.826 & 3 & 10 & 25182.896 & $19912_{13 / 2}^{\circ}-45095_{15 / 2}$ & 3943.395 & 100 & 300 & 25351.684 & $9202_{7 / 2}^{\circ}-34553_{9 / 2}$ \\
\hline 3969.561 & 75 & 50 & 25184.577 & $26762_{3 / 2}-51946_{5 / 2}^{\circ}$ & 3943.258 & 20 & 100 & 25352.564 & $12488_{9 / 2}^{\circ}-37840_{9 / 2}^{\circ}$ \\
\hline 3969.529 & 5 & 50 & 25184.780 & $17771_{11 / 2}^{\circ}-42955_{9 / 2}$ & 3942.855 & 8 & 40 & 25355.155 & $12485_{7 / 2}^{\circ}-37840_{9 / 2}$ \\
\hline 3969.337 & 5 & 50 & 25185.999 & $20120_{5 / 2}^{\circ}-45306_{3 / 2}^{\circ}$ & 3942.700 & $5 b$ & 10 & 25356.152 & $17771_{11 / 2}^{o}-43127_{11 / 2}$ \\
\hline 3969.289 & 10 & 10 & 25186.303 & $12570_{7 / 2}-37756_{7 / 2}^{\circ}$ & 3942.668 & $100 \mathrm{~b}$ & 50 & 25356.358 & $22513_{5 / 2}^{\circ}-47869_{3 / 2}$ \\
\hline 3969.002 & 200 & 150 & 25188.124 & $0_{3 / 2}-25188_{3 / 2}^{\circ}$ & 3942.639 & 50 & 50 & 25356.544 & $10189_{11 / 2}^{\circ}-35545_{9 / 2}^{\circ}$ \\
\hline 3968.484 & 100 & 400 & 25191.412 & $12488_{9 / 2}^{\circ}-37679_{11 / 2}$ & 3942.042 & $5 b$ & 10 & 25360.385 & $18816_{13 / 2}^{\circ}-44177_{11 / 2}^{\circ}$ \\
\hline 3967.212 & 50 & 200 & 25199.489 & $15324_{1 / 2}^{\circ}-40523_{1 / 2}$ & 3941.725 & 25 & 25 & 25362.424 & $20989_{9 / 2}^{\circ}-46352_{7 / 2}$ \\
\hline 3966.707 & 3 & & 25202.697 & $12902_{3 / 2}^{\circ}-38105_{5 / 2}$ & 3941.360 & 3 & 40 & 25364.773 & $22642_{9 / 2}^{\circ}-48006_{9 / 2}$ \\
\hline 3966.231 & 15 & 25 & 25205.722 & $10673_{5 / 2}^{\circ}-35878_{7 / 2}^{\circ}$ & 3940.369 & 2 & 25 & 25371.152 & $23697_{7 / 2}^{\circ}-49068_{5 / 2}^{\circ}$ \\
\hline 3966.054 & 1 & & 25206.847 & $30879_{7 / 2}-56086_{9 / 2}^{\circ}$ & 3939.793 & 15 & 50 & 25374.861 & $15349_{11 / 2}^{\circ}-40724_{13 / 2}$ \\
\hline 3964.865 & 40 & 75 & 25214.406 & $10379_{9 / 2}-35593_{7 / 2}^{\circ}$ & 3938.779 & 100 & 100 & 25381.393 & $6244_{1 / 2}-31625_{1 / 2}^{\circ}$ \\
\hline 3964.738 & 200 & $200 \mathrm{~d}$ & 25215.213 & $17121_{3 / 2}^{\circ}-42336_{5 / 2}$ & 3938.726 & 20 & 100 & 25381.735 & $12219_{3 / 2}-37601_{3 / 2}^{\circ}$ \\
\hline 3963.468 & 40 & 100 & 25223.293 & $10379_{9 / 2}-35602_{11 / 2}^{\circ}$ & 3938.580 & $5 b$ & & 25382.676 & $8460_{3 / 2}-33843_{3 / 2}^{\circ}$ \\
\hline 3963.220 & 40 & 200 & 25224.871 & $15349_{11 / 2}^{\circ}-40574_{11 / 2}$ & 3938.421 & 15 & 15 & 25383.701 & $4490_{5 / 2}^{\circ}-29873_{7 / 2}$ \\
\hline 3962.921 & 3 & 20 & 25226.774 & $20989_{9 / 2}^{\circ}-46216_{11 / 2}$ & 3937.923 & 100 & 200 & 25386.910 & $7828_{1 / 2}-33215_{3 / 2}^{\circ}$ \\
\hline 3962.336 & 50 & $200 \mathrm{~b}$ & 25230.498 & $17121_{3 / 2}^{\circ}-42352_{5 / 2}^{\circ}$ & 3937.040 & $200 \mathrm{~s}$ & 300 & 25392.604 & $10673_{5 / 2}^{\circ}-36065_{5 / 2}^{\circ}$ \\
\hline 3961.966 & 4 & 75 & 25232.855 & $17722_{9 / 2}^{\circ}-42955_{9 / 2}$ & 3936.838 & 1 & & 25393.907 & $23730_{9 / 2}^{\circ}-49124_{11 / 2}$ \\
\hline 3961.503 & 2 & & 25235.804 & $15236_{3 / 2}-40472_{3 / 2}^{\circ}$ & 3936.604 & 75 & 40 & 25395.416 & $30101_{7 / 2}^{\circ}-55496_{9 / 2}$ \\
\hline 3960.955 & $2 b$ & & 25239.295 & $18568_{1 / 2}^{\circ}-43807_{3 / 2}$ & 3936.125 & 1 & & 25398.507 & $29095_{5 / 2}^{\circ}-54493_{5 / 2}$ \\
\hline 3960.360 & $4 b$ & $25 \mathrm{~b}$ & 25243.087 & $17771_{1 / 12}^{\circ}-43014_{13 / 2}$ & 3935.945 & $10 \mathrm{~h}$ & 200 & 25399.668 & $26770_{11 / 2}^{\circ}-52170_{11 / 2}$ \\
\hline 3960.333 & $100 \mathrm{~b}$ & $200 \mathrm{~b}$ & 25243.259 & $13250_{5 / 2}-38493_{5 / 2}^{\circ}$ & 3935.631 & 20 & 200 & 25401.695 & $16818_{7 / 2}-42219_{5 / 2}^{\circ}$ \\
\hline 3960.019 & 8 & 8 & 25245.260 & $7331_{5 / 2}^{\circ}-32576_{7 / 2}$ & 3935.240 & 2 & 20 & 25404.219 & $17722_{9 / 2}^{\circ}-43127_{11 / 2}^{\circ}$ \\
\hline 3959.222 & 8 & 40 & 25250.342 & $13818_{7 / 2}^{\circ}-39068_{7 / 2}^{\circ}$ & 3934.175 & 5 & 50 & 25411.096 & $14484_{11 / 2}^{\circ}-39895_{9 / 2}$ \\
\hline 3959.024 & 1 & 10 & 25251.605 & $26424_{5 / 2}^{\circ}-51676_{3 / 2}$ & 3933.571 & $2 \mathbf{b}$ & & 25414.997 & $18973_{\pi / 2}^{\circ}-44388_{5 / 2}^{\circ}$ \\
\hline 3958.014 & 1 & & 25258.049 & $28587_{5 / 2}^{\circ}-53845_{5 / 2}$ & 3933.353 & 5 & 50 & 25416.406 & $20969_{7 / 2}^{\circ}-46385_{7 / 2}^{\circ}$ \\
\hline 3957.480 & 2 & & 25261.457 & $17983_{5 / 2}^{\circ}-43244_{3 / 2}$ & 3932.226 & 200 & 250 & 25423.690 & $9238_{9 / 2}^{\circ}-34661_{11 / 2}^{\circ}$ \\
\hline 3957.157 & 10 & & 25263.519 & $20989_{9 / 2}^{\circ}-46253_{9 / 2}$ & 3932.002 & 15 & 300 & 25425.138 & $14790_{7 / 2}^{\circ}-40216_{5 / 2}$ \\
\hline 3956.690 & 300 & 300 & 25266.500 & $0_{3 / 2}-25266_{1 / 2}^{o}$ & 3931.257 & 2 & 100 & 25429.957 & $25594_{1 / 2}^{\circ}-51024_{3 / 2}^{\circ}$ \\
\hline 3956.593 & 100 & 500 & 25267.120 & $10189_{i 1 / 2}^{\circ}-35456_{9 / 2}$ & 3929.669 & 800 & 500 & 25440.233 & $0_{3 / 2}-25440_{5 / 2}^{\circ}$ \\
\hline 3955.252 & 25 & & 25275.686 & $12570_{\pi / 2}-37846_{\bar{s} / 2}^{\circ}$ & 3929.192 & 2 & & 25443.321 & $1521_{5 / 2}-26965_{3 / 2}^{\circ}$ \\
\hline 3955.101 & 5 & 15 & 25276.651 & $14275_{9 / 2}^{\circ}-39552_{9 / 2}$ & 3928.940 & $5 b$ & & 25444.953 & $9711_{\pi / 2}-35156_{\pi / 2}^{\circ}$ \\
\hline 3954.591 & 1 & & 25279.911 & $34019_{3 / 2}-59299_{5 / 2}^{\circ}$ & 3928.698 & 4 & 20 & 25446.520 & $20288_{11 / 2}^{0}-45735_{11 / 2}$ \\
\hline 3954.464 & 10 & 25 & 25280.723 & $15710_{3 / 2}^{\circ}-40991_{3 / 2}$ & 3928.239 & 1 & & 25449.493 & $21131_{3 / 2}^{\circ}-46581_{5 / 2}^{\circ}$ \\
\hline 3953.970 & 2 & & 25283.881 & $21297_{5 / 2}^{\circ}-46581_{5 / 2}$ & 3927.421 & 50 & 300 & 25454.794 & $14484_{11 / 2}^{\circ}-39939_{11 / 2}$ \\
\hline 3953.900 & 2 & & 25284.329 & $20969_{\pi / 2}^{\circ}-46253_{9 / 2}$ & 3927.176 & 100 & 300 & 25456.382 & $13406_{13 / 2}^{\circ}-38862_{11 / 2}$ \\
\hline 3952.901 & 2 & & 25290.719 & $17460_{5 / 2}^{\circ}-42751_{7 / 2}$ & 3926.706 & 50 & 200 & 25459.429 & $12485_{7 / 2}^{\circ}-37945_{5 / 2}$ \\
\hline 3952.448 & 3 & 8 & 25293.617 & $16906_{7 / 2}^{\circ}-42200_{9 / 2}$ & 3924.987 & 20 & 40 & 25470.579 & $15453_{7 / 2}^{\circ}-40923_{5 / 2}$ \\
\hline 3951.515 & 300 & 400 & 25299.589 & $12488_{9 / 2}^{\circ}-37787_{\tau / 2}$ & 3924.628 & 3 & 2 & 25472.909 & $12472_{5 / 2}^{\circ}-37945_{5 / 2}^{\circ}$ \\
\hline 3951.107 & 40 & 200 & 25302.202 & $12485_{\tau / 2}^{\circ}-37787_{\tau / 2}$ & 3922.218 & 50 & 50 & 25488.560 & $9238_{9 / 2}^{\circ}-34726_{7 / 2}^{\circ}$ \\
\hline 3950.088 & 1 & & 25308.729 & $23012_{3 / 2}^{\circ}-48320_{5 / 2}$ & 3922.098 & 8 & 40 & 25489.340 & $20310_{5 / 2}^{\circ}-45800_{5 / 2}$ \\
\hline 3949.206 & 4 & 25 & 25314.381 & $20080_{7 / 2}^{\circ}-45395_{7 / 2}$ & 3921.019 & 5 & 10 & 25496.354 & $12219_{3 / 2}-37716_{1 / 2}^{\circ}$ \\
\hline 3948.999 & $8 \mathrm{~b}$ & & 25315.708 & $\begin{array}{l}16906_{7 / 2}^{\circ}-42222_{7 / 2} \\
12472_{/ / 2}^{\circ}-37787_{7 / 2}\end{array}$ & $\begin{array}{l}3920.951 \\
3920.520\end{array}$ & $\begin{array}{l}15 \\
5 b\end{array}$ & 25 & $\begin{array}{l}25496.796 \\
25499.599\end{array}$ & $\begin{array}{l}24463_{5 / 2}^{\circ}-49960_{7 / 2}^{\circ} \\
15144_{3 / 2}^{\circ}-40644_{5 / 2}^{\circ}\end{array}$ \\
\hline 3948.964 & $200 \mathrm{~b}$ & 500 & 25315.932 & $9238_{9 / 2}^{\circ}-34553_{9 / 2}$ & 3920.312 & 50 & 300 & 25500.952 & $16818_{7 / 2}-42319_{9 / 2}^{\circ}$ \\
\hline 3946.596 & 2 & & 25331.122 & $13250_{5 / 2}-38581_{5 / 2}^{\circ}$ & 3920.170 & 1 & & 25501.876 & $19050_{3 / 2}^{\circ}-44552_{5 / 2}$ \\
\hline 3946.144 & 150 & 500 & 25334.023 & $13406_{1: 3 / 2}^{\circ}-38740_{11 / 2}$ & 3918.285 & 10 & 75 & 25514.144 & $13250_{5 / 2}-38764_{7 / 2}^{\circ}$ \\
\hline
\end{tabular}


TABle 3. Classified lines of Th II-Continued

\begin{tabular}{|c|c|c|c|c|c|c|c|c|c|}
\hline \multirow{2}{*}{$\begin{array}{c}\text { Wavelength } \\
\AA\end{array}$} & \multicolumn{2}{|c|}{ Intensity } & \multirow{2}{*}{$\begin{array}{c}\text { Wavenumber } \\
\mathbf{c m}^{-1}\end{array}$} & \multirow{2}{*}{ Classification } & \multirow{2}{*}{$\begin{array}{c}\text { Wavelength } \\
\AA\end{array}$} & \multicolumn{2}{|c|}{ Intensity } & \multirow{2}{*}{$\begin{array}{c}\text { Wavenumber } \\
\mathbf{c m}^{-1}\end{array}$} & \multirow{2}{*}{ Classification } \\
\hline & Lamp & Spark & & & & Lamp & Spark & & \\
\hline 3918.011 & 20 & 100 & 25515.928 & $13248_{9 / 2}-38764_{7 / 2}^{\circ}$ & 3888.460 & $3 \mathrm{~b}$ & 8 & 25709.836 & $20686_{5 / 2}^{\circ}-46395_{3 / 2}$ \\
\hline 3916.796 & 50 & 300 & 25523.843 & $17722_{9 / 2}^{\circ}-43246_{7 / 2}$ & 3888.048 & 2 & & 25712.561 & $24757_{9 / 2}^{\circ}-50470_{9 / 2}$ \\
\hline 3916.724 & 75 & 200 & 25524.312 & $9202_{7 / 2}^{\circ}-34726_{7 / 2}$ & 3887.434 & 3 & 15 & 25716.622 & $22014_{11 / 2}^{\circ}-47731_{9 / 2}$ \\
\hline 3915.770 & 8 & 40 & 25530.531 & $20158_{5 / 2}-45689_{7 / 2}^{\circ}$ & 3885.766 & 75 & 50 & 25727.661 & $1521_{5 / 2}-27249_{7 / 2}^{\circ}$ \\
\hline 3914.470 & 10 & 75 & 25539.009 & $20158_{5 / 2}-45697_{3 / 2}^{\circ}$ & 3885.639 & 8 & 20 & 25728.501 & $10855_{7 / 2}-36583_{7 / 2}^{\circ}$ \\
\hline 3913.823 & 75 & 20 & 25543.231 & $1859_{3 / 2}-27403_{3 / 2}^{\circ}$ & 3885.151 & 3 & 50 & 25731.733 & $22139_{9 / 2}^{\circ}-47871_{7 / 2}^{\circ}$ \\
\hline 3913.195 & 1 & & 25547.330 & $25188_{3 / 2}^{\circ}-50735_{3 / 2}$ & 3884.949 & 2 & & 25733.071 & $27787_{9 / 2}^{\circ}-53520_{9 / 2}^{\circ}$ \\
\hline 3913.005 & 75 & 300 & 25548.571 & $13818_{7 / 2}^{\circ}-39366_{5 / 2}$ & 3884.819 & 500 & 500 & 25733.932 & $19912_{13 / 2}^{\circ}-45646_{15 / 2}$ \\
\hline 3912.282 & 75 & 200 & 25553.292 & $10572_{9 / 2}^{\circ}-36125_{9 / 2}$ & 3884.523 & 50 & 200 & 25735.893 & $9720_{7 / 2}^{\circ}-35456_{9 / 2}$ \\
\hline 3911.299 & 15 & 25 & 25559.714 & $19248_{5 / 2}^{\circ}-44807_{7 / 2}$ & 3884.318 & 2 & 10 & 25737.251 & $20969_{7 / 2}^{\circ}-46706_{7 / 2}$ \\
\hline 3908.488 & 3 & 50 & 25578.096 & $20686_{5 / 2}^{\circ}-46264_{3 / 2}$ & 3882.214 & 1 & & 25751.199 & $18214_{3 / 2}^{\circ}-43965_{1 / 2}$ \\
\hline 3907.905 & 10 & 50 & 25581.912 & $16564_{11 / 2}^{\circ}-42146_{11 / 2}$ & 3882.142 & 100 & 150 & 25751.677 & $8460_{3 / 2}-34212_{5 / 2}^{\circ}$ \\
\hline 3907.336 & 10 & 10 & 25585.637 & $9400_{5 / 2}-34986_{3 / 2}^{\circ}$ & 3881.497 & 50 & & 25755.956 & $9400_{5 / 2}-35156_{5 / 2}^{\circ}$ \\
\hline 3906.828 & 3 & & 25588.964 & $15236_{3 / 2}-40825_{1 / 2}^{\circ}$ & 3879.796 & 15 & 15 & 25767.248 & $17460_{5 / 2}^{\circ}-43227_{5 / 2}$ \\
\hline 3906.033 & 3 & & 25594.172 & $15453_{7 / 2}^{\circ}-41047_{9 / 2}^{\circ}$ & 3878.047 & 20 & 15 & 25778.869 & $15144_{3 / 2}^{\circ}-40923_{5 / 2}$ \\
\hline 3905.924 & 15 & 25 & 25594.886 & $0_{3 / 2}-25594_{1 / 2}^{o}$ & 3877.238 & 5 & 15 & 25784.247 & $17460_{5 / 2}^{\circ}-43244_{3 / 2}$ \\
\hline 3905.356 & 3 & 50 & 25598.609 & $26963_{7 / 2}^{\circ}-52562_{7 / 2}$ & 3876.946 & 1 & & 25786.189 & $17460_{5 / 2}^{\circ}-43246_{7 / 2}$ \\
\hline 3905.186 & 150 & 300 & 25599.723 & $13468_{9 / 2}^{\circ}-39068_{7 / 2}$ & 3876.510 & 2 & & 25789.089 & $17983_{5 / 2}^{\circ}-43772_{5 / 2}$ \\
\hline 3904.200 & 10 & 15 & 25606.188 & $8605_{5 / 2}-34212_{5 / 2}^{\circ}$ & 3875.518 & 4 & 5 & 25795.690 & $8378_{7 / 2}^{\circ}-34174_{5 / 2}$ \\
\hline 3904.082 & 200 & 200 & 25606.962 & $4113_{5 / 2}-29720_{3 / 2}^{\circ}$ & 3873.981 & 1 & 10 & 25805.925 & $23012_{3 / 2}^{\circ}-48817_{3 / 2}^{\circ}$ \\
\hline 3903.764 & 2 & & 25609.048 & $19880_{9 / 2}-45489_{9 / 2}^{\circ}$ & 3873.950 & 5 & 10 & 25806.131 & $12485_{7 / 2}^{\circ}-38291_{7 / 2}$ \\
\hline 3902.731 & 3 & & 25615.826 & $20288_{11 / 2}^{\circ}-45904_{9 / 2}$ & 3872.721 & 200 & 600 & 25814.320 & $10379_{9 / 2}-36193_{9 / 2}^{\circ}$ \\
\hline 3902.474 & 8 & 40 & 25617.513 & $15710_{3 / 2}^{\circ}-41328_{5 / 2}$ & 3872.358 & 25 & 100 & 25816.740 & $11725_{1 / 2}^{\circ}-37542_{3 / 2}$ \\
\hline 3902.120 & 20 & 100 & 25619.837 & $14275_{9 / 2}^{\circ}-39895_{9 / 2}$ & 3871.925 & 3 & 10 & 25819.627 & $12472_{5 / 2}^{\circ}-38291_{7 / 2}$ \\
\hline 3901.152 & 75 & 200 & 25626.194 & $12219_{3 / 2}-37846_{5 / 2}^{\circ}$ & 3871.781 & 1 & & 25820.587 & $17983_{5 / 2}^{\circ}-43803_{7 / 2}$ \\
\hline 3900.878 & 400 & 400 & 25627.994 & $7331_{5 / 2}^{\circ}-32959_{3 / 2}$ & 3871.455 & 8 & 200 & 25822.762 & $19912_{13 / 2}^{\circ}-45735_{11 / 2}$ \\
\hline 3900.131 & $50 \mathrm{~s}$ & 100 & 25632.902 & $12472_{5 / 2}^{\circ}-38105_{5 / 2}$ & 3871.245 & 5 & 25 & 25824.163 & $17983_{5 / 2}^{\circ}-43807_{3 / 2}$ \\
\hline 3900.037 & 1 & $40 \mathrm{~b}$ & 25633.520 & $30452_{9 / 2}-56086_{9 / 2}^{\circ}$ & 3871.142 & 3 & & 25824.850 & $8018_{3 / 2}-33843_{3 / 2}^{\circ}$ \\
\hline 3899.674 & 2 & & 25635.906 & $17460_{5 / 2}^{\circ}-43096_{5 / 2}$ & 3871.073 & 25 & 15 & 25825.310 & $9720_{7 / 2}^{\circ}-35545_{9 / 2}$ \\
\hline 3899.318 & 1 & & 25638.247 & $21297_{5 / 2}^{\circ}-46935_{3 / 2}$ & 3870.923 & 5 & 8 & 25826.311 & $12902_{3 / 2}^{\circ}-38728_{5 / 2}^{\circ}$ \\
\hline 3898.488 & $4 b$ & & 25643.705 & $16033_{5 / 2}^{\circ}-41676_{3 / 2}$ & 3869.971 & 75 & 100 & 25832.664 & $10855_{7 / 2}-36687_{5 / 2}^{\circ}$ \\
\hline 3898.062 & 1 & & 25646.507 & $23730_{9 / 2}^{\circ}-49377_{7 / 2}$ & 3868.161 & 1 & & 25844.751 & $16906_{7 / 2}^{\circ}-42751_{7 / 2}$ \\
\hline 3897.456 & 1 & & 25650.495 & $19248_{5 / 2}^{\circ}-44898_{7 / 2}$ & 3867.883 & 2 & & 25846.609 & $14101_{1 / 2}^{\circ}-39948_{1 / 2}$ \\
\hline 3895.982 & 1 & & 25660.199 & $22014_{11 / 2}^{\circ}-47675_{11 / 2}$ & 3867.849 & 20 & 300 & 25846.836 & $15144_{3 / 2}^{\circ}-40991_{3 / 2}^{\circ}$ \\
\hline 3895.854 & 1 & & 25661.042 & $25607_{9 / 2}^{\circ}-51268_{7 / 2}$ & 3867.696 & 1 & 8 & 25847.858 & $26424_{5 / 2}^{\circ}-52272_{7 / 2}$ \\
\hline 3894.899 & 15 & 40 & 25667.334 & $15324_{1 / 2}^{\circ}-40991_{3 / 2}$ & 3867.577 & 50 & 75 & 25848.654 & $7001_{3 / 2}-32850_{5 / 2}^{\circ}$ \\
\hline 3894.408 & 10 & 40 & 25670.570 & $14545_{5 / 2}^{\circ}-40216_{5 / 2}$ & 3867.197 & 1 & & 25851.193 & $21297_{5 / 2}^{\circ}-47148_{3 / 2}$ \\
\hline 3893.423 & 10 & 75 & 25677.064 & $12488_{9 / 2}^{\circ}-38165_{\pi / 2}$ & 3866.876 & $15 b$ & 50 & 25853.339 & $14790_{7 / 2}^{\circ}-40644_{5 / 2}$ \\
\hline 3893.111 & 20 & 75 & 25679.122 & $13406_{13 / 2}^{\circ}-39085_{13 / 2}$ & 3866.657 & 1 & & 25854.803 & $12902_{3 / 2}^{\circ}-38757_{3 / 2}$ \\
\hline 3893.046 & lb & & 25679.551 & $23697_{7 / 2}^{\circ}-49377_{7 / 2}$ & 3866.632 & 3 & 5 & 25854.971 & $17272_{9 / 2}^{\circ}-43127_{11 / 2}$ \\
\hline 3893.026 & 20 & 75 & 25679.683 & $\begin{array}{l}20158_{5 / 2}-45838_{3 / 2}^{\circ} \\
12485_{7 / 2}^{\circ}-38165_{7 / 2}^{\circ}\end{array}$ & $\begin{array}{l}3866.064 \\
3864.993\end{array}$ & $\begin{array}{l}5 \\
3 b\end{array}$ & 200 & $\begin{array}{l}25858.769 \\
25865.935\end{array}$ & $\begin{array}{ll}23518_{7 / 2}^{\circ} & -49377_{7 / 2} \\
14545_{5 / 2}^{\circ} & -40411_{7 / 2}\end{array}$ \\
\hline 3892.432 & 4 & 15 & 25683.601 & $17272_{9 / 2}^{\circ}-42955_{9 / 2}$ & 3864.370 & $25 b$ & 75 & 25870.105 & $8460_{3 / 2}-34330_{1 / 2}^{\circ}$ \\
\hline 3892.307 & 75 & 100 & 25684.426 & $6244_{1 / 2}-31928_{3 / 2}^{\circ}$ & 3864.118 & 2 & 25 & 25871.792 & $20989_{9 / 2}^{\circ}-46861_{11 / 2}$ \\
\hline 3891.053 & 75 & 100 & 25692.704 & $11116_{7 / 2}^{\circ}-36809_{7 / 2}$ & 3863.593 & 4 & & 25875.307 & $15453_{7 / 2}^{\circ}-41328_{5 / 2}$ \\
\hline 3890.981 & 10 & 50 & 25693.179 & $12472_{5 / 2}^{\circ}-38165_{7 / 2}$ & 3863.405 & 500 & 600 & 25876.566 & $6700_{9 / 2}^{\circ}-32576_{7 / 2}$ \\
\hline 3890.821 & 151 & 25 & 25694.236 & $12485_{\pi / 2}^{\circ}-38179_{9 / 2}$ & 3863.205 & 5 & 5 & 25877.906 & $7331_{5 / 2}^{\circ}-33209_{7 / 2}$ \\
\hline 3890.351 & $4 b$ & 10 & 25697.340 & $15349_{11 / 2}^{\circ}-41047_{9 / 2}$ & 3862.701 & $20 \mathrm{~b}$ & 25 & 25881.282 & $1521_{5 / 2}-27403_{3 / 2}^{\circ}$ \\
\hline 3890.057 & 1 & & 25699.282 & $20686_{5 / 2}^{\circ}-46385_{7 / 2}$ & 3862.654 & $200 \mathrm{~b}$ & 200 & 25881.597 & $9711_{7 / 2}-35593_{7 / 2}^{\circ}$ \\
\hline 3888.673 & 50 & $100 \mathrm{~h}$ & 25708.428 & $25027_{1 / 2}^{o}-50735_{3 / 2}$ & 3862.557 & 5 & & 25882.247 & $13818_{7 / 2}^{\circ}-39700_{5 / 2}$ \\
\hline
\end{tabular}


TABLE 3. Classified lines of Th II-Continued

\begin{tabular}{|c|c|c|c|c|c|c|c|c|c|}
\hline \multirow{2}{*}{$\begin{array}{c}\text { Wavelength } \\
\AA\end{array}$} & \multicolumn{2}{|c|}{ Intensity } & \multirow{2}{*}{$\begin{array}{c}\text { Wavenumber } \\
\mathbf{c m}^{-1}\end{array}$} & \multirow{2}{*}{ Classification } & \multirow{2}{*}{$\begin{array}{c}\text { Wavelength } \\
\AA\end{array}$} & \multicolumn{2}{|c|}{ Intensity } & \multirow{2}{*}{$\begin{array}{c}\text { Wavenumber } \\
\mathbf{c m}^{-1}\end{array}$} & \multirow{2}{*}{ Classification } \\
\hline & Lamp & Spark & & & & Lamp & Spark & & \\
\hline 3862.398 & $100 \mathrm{~b}$ & $150 \mathrm{~b}$ & 25883.312 & $13468_{9 / 2}^{\circ}-39352_{11 / 2}$ & 3826.022 & 10 & 75 & 26129.393 & $12488_{9 / 2}^{\circ}-38617_{9 / 2}$ \\
\hline 3862.052 & 2 & 100 & 25885.631 & $33730_{5 / 2}-59616_{3 / 2}^{\circ}$ & 3825.546 & 2 & & 26132.644 & $14790_{7 / 2}^{\circ}-40923_{5 / 2}$ \\
\hline 3861.540 & $25 b$ & 50 & 25889.063 & $11576_{3 / 2}^{\circ}-37465_{5 / 2}$ & 3825.034 & 150 & 400 & 26136.142 & $10673_{5 / 2}^{\circ}-36809_{7 / 2}$ \\
\hline 3859.931 & 50 & 200 & 25899.855 & $12472_{5 / 2}^{\circ}-38372_{3 / 2}$ & 3824.759 & 5 & 50 & 26138.021 & $26424_{5 / 2}^{\circ}-52562_{7 / 2}$ \\
\hline 3859.840 & 300 & 500 & 25900.466 & $8378_{7 / 2}^{\circ}-34279_{7 / 2}$ & 3824.645 & 5 & 25 & 26138.800 & $19050_{3 / 2}^{\circ}-45189_{5 / 2}$ \\
\hline 3859.747 & 50 & 200 & 25901.090 & $12488_{9 / 2}^{\circ}-38389_{7 / 2}$ & 3823.873 & 1 & & 26144.077 & $20120_{5 / 2}^{\circ}-46264_{3 / 2}$ \\
\hline 3857.507 & 4 & 200 & 25916.130 & $26762_{3 / 2}-52678_{5 / 2}^{\circ}$ & 3823.584 & 75 & 300 & 26146.053 & $13250_{5 / 2}-39396_{7 / 2}^{\circ}$ \\
\hline 3857.347 & 10 & 50 & 25917.205 & $12472_{5 / 2}^{\circ}-38389_{7 / 2}$ & 3823.321 & 15 & 100 & 26147.852 & $13248_{9 / 2}-39396_{7 / 2}^{\circ}$ \\
\hline 3856.188 & 3 & 25 & 25924.994 & $18973_{7 / 2}^{\circ}-44898_{7 / 2}$ & 3822.149 & $300 \mathrm{~b}$ & $200 \mathrm{~b}$ & 26155.869 & $9585_{5 / 2}^{\circ}-35741_{5 / 2}$ \\
\hline 3856.113 & 5 & 10 & 25925.498 & $9061_{5 / 2}-34986_{3 / 2}^{\circ}$ & 3821.755 & 20 & 50 & 26158.566 & $9720_{7 / 2}^{\circ}-35878_{7 / 2}$ \\
\hline 3856.060 & 2 & & 25925.855 & $28243_{5 / 2}^{\circ}-54169_{7 / 2}$ & 3821.431 & 200 & 500 & 26160.784 & $11116_{7 / 2}^{\circ}-37277_{7 / 2}$ \\
\hline 3854.920 & 3 & & 25933.521 & $20969_{7 / 2}^{\circ}-46902_{5 / 2}$ & 3821.032 & 2 & & 26163.515 & $27357_{9 / 2}^{\circ}-53520_{9 / 2}$ \\
\hline 3854.876 & 10 & 150 & 25933.817 & $12902_{3 / 2}^{\circ}-38836_{3 / 2}$ & 3819.941 & $3 b$ & 40 & 26170.988 & $23187_{13 / 2}^{\circ}-49357_{11 / 2}$ \\
\hline 3854.510 & 300 & 600 & 25936.280 & $10189_{11 / 2}^{\circ}-36125_{9 / 2}$ & 3819.339 & 8 & 8 & 26175.113 & $8378_{7 / 2}^{\circ}-34553_{9 / 2}$ \\
\hline 3854.319 & $2 \mathrm{~b}$ & 8 & 25937.565 & $23187_{13 / 2}^{\circ}-49124_{11 / 2}$ & 3819.286 & 3 & 8 & 26175.476 & $23697_{7 / 2}^{\circ}-49873_{5 / 2}$ \\
\hline 3853.437 & 1 & & 25943.502 & $24463_{5 / 2}^{\circ}-50407_{7 / 2}$ & 3819.161 & 2 & & 26176.332 & $14101_{1 / 2}^{\circ}-40278_{3 / 2}$ \\
\hline 3853.083 & 2 & & 25945.885 & $13406_{13 / 2}^{\circ}-39352_{11 / 2}$ & 3818.099 & 5 & 25 & 26183.613 & $15144_{3 / 2}^{\circ}-41328_{5 / 2}$ \\
\hline 3852.958 & 40 & 200 & 25946.727 & $11116_{7 / 2}^{\circ}-37063_{9 / 2}$ & 3817.366 & 8 & 100 & 26188.641 & $21682_{7 / 2}^{\circ}-47871_{7 / 2}$ \\
\hline 3850.768 & 2 & & 25961.483 & $12902_{3 / 2}^{\circ}-38863_{5 / 2}$ & 3817.291 & 5 & 40 & 26189.155 & $16033_{5 / 2}^{\circ}-42222_{7 / 2}$ \\
\hline 3850.281 & 20 & 150 & 25964.767 & $\begin{array}{l}20288_{11 / 2}^{\circ}-46253_{9 / 2} \\
12902_{3 / 2}^{\circ}-38867_{1 / 2}^{\circ}\end{array}$ & 3816.793 & 10 & 40 & 26192.572 & $\begin{array}{r}9400_{5 / 2}-35593_{7 / 2}^{\circ} \\
21131_{3 / 2}^{\circ}-47324_{5 / 2}^{\circ}\end{array}$ \\
\hline 3850.132 & 75 & 200 & 25965.771 & $11576_{3 / 2}^{\circ}-37542_{3 / 2}$ & 3816.607 & 5 & 10 & 26193.849 & $8018_{3 / 2}-34212_{5 / 2}^{\circ}$ \\
\hline 3850.092 & 2 & & 25966.041 & $15710_{3 / 2}^{\circ}-41676_{3 / 2}$ & 3816.561 & 8 & 75 & 26194.164 & $12570_{7 / 2}-38764_{7 / 2}^{\circ}$ \\
\hline 3849.542 & 1 & & 25969.751 & $17837_{1 / 2}^{\circ}-43807_{3 / 2}$ & 3815.026 & 75 & 200 & 26204.704 & $10379_{9 / 2}-36583_{7 / 2}^{\circ}$ \\
\hline 3848.823 & 2 & & 25974.602 & $17272_{9 / 2}^{\circ}-43246_{7 / 2}$ & 3813.666 & 100 & 150 & 26214.048 & $7001_{3 / 2}-33215_{3 / 2}^{\circ}$ \\
\hline 3846.832 & 10 & 8 & 25988.046 & $4113_{5 / 2}-30101_{7 / 2}^{\circ}$ & 3813.400 & 1 & & 26215.877 & $18973_{7 / 2}^{\circ}-45189_{5 / 2}$ \\
\hline 3846.753 & 2 & & 25988.579 & $30956_{9 / 2}^{\circ}-56945_{11 / 2}$ & 3813.324 & 5 & 200 & 26216.399 & $20686_{5 / 2}^{\circ}-46902_{5 / 2}$ \\
\hline 3846.247 & 10 & 200 & 25991.998 & $22014_{11 / 2}^{\circ}-48006_{9 / 2}$ & 3813.068 & 400 & 500 & 26218.159 & $9238_{9 / 2}^{\circ}-35456_{9 / 2}$ \\
\hline 3845.535 & 8 & 300 & 25996.810 & $20158_{5 / 2}-46155_{5 / 2}^{\circ}$ & 3812.300 & 1 & & 26223.441 & $25607_{9 / 2}^{\circ}-51830_{7 / 2}$ \\
\hline 3845.223 & 10 & 15 & 25998.920 & $28923_{5 / 2}^{\circ}-54922_{3 / 2}$ & 3811.975 & 3 & & 26225.677 & $15710_{3 / 2}^{\circ}-41936_{3 / 2}$ \\
\hline 3843.415 & 4 & 10 & 26011.150 & $12570_{\pi / 2}-38581_{5 / 2}^{\circ}$ & 3811.355 & 3 & 20 & 26229.943 & $23730_{9 / 2}^{\circ}-49960_{7 / 2}$ \\
\hline 3842.919 & $25 b$ & & 26014.507 & $7828_{1 / 2}-33843_{3 / 2}^{\circ}$ & 3810.558 & 2 & & 26235.429 & $15453_{7 / 2}^{\circ}-41688_{7 / 2}$ \\
\hline 3841.960 & 300 & 800 & 26021.000 & $9720_{7 / 2}^{\circ}-35741_{5 / 2}$ & 3810.497 & 2 & & 26235.849 & $25440_{5 / 2}^{\circ}-51676_{3 / 2}$ \\
\hline 3841.358 & $4 b$ & 10 & 26025.078 & $14545_{5 / 2}^{\circ}-40570_{\tau / 2}$ & 3810.295 & 15 & 5 & 26237.239 & $10572_{9 / 2}^{\circ}-36809_{7 / 2}$ \\
\hline 3839.744 & $1000 \mathrm{~b}$ & $800 \mathrm{~b}$ & 26036.017 & $6700_{9 / 2}^{\circ}-32736_{\tau / 2}$ & 3809.834 & 75 & 300 & 26240.414 & $14484_{11 / 2}^{\circ}-40724_{13 / 2}$ \\
\hline 3838.964 & 1 & & 26041.307 & $20310_{5 / 2}^{\circ}-46352_{7 / 2}$ & 3809.629 & 1 & & 26241.826 & $24982_{7 / 2}^{\circ}-51224_{9 / 2}$ \\
\hline 3838.850 & 2 & & 26042.080 & $23372_{3 / 2}^{\circ}-49414_{3 / 2}$ & 3809.457 & 1 & & 26243.011 & $12485_{7 / 2}^{\circ}-38728_{5 / 2}$ \\
\hline 3837.794 & 2 & & 26049.246 & $16906_{7 / 2}^{\circ}-42955_{9 / 2}$ & 3809.132 & 3 & 25 & 26245.250 & $15242_{9 / 2}^{\circ}-41488_{7 / 2}$ \\
\hline 3834.080 & 2 & & 26074.479 & $20310_{5 / 2}^{\circ}-46385_{7 / 2}$ & 3809.081 & 1 & & 26245.601 & $11576_{3 / 2}^{\circ}-37821_{3 / 2}$ \\
\hline 3833.693 & 50 & 100 & 26077.111 & $13818_{\tau / 2}^{\circ}-39895_{9 / 2}^{\circ}$ & 3808.688 & 4 & & 26248.310 & $12902_{3 / 2}^{\circ}-39150_{3 / 2}$ \\
\hline 3833.231 & 10 & 50 & 26080.254 & $16564_{11 / 2}^{\circ}-42644_{13 / 2}$ & 3808.512 & 3 & & 26249.523 & $20686_{5 / 2}^{\circ}-46935_{3 / 2}$ \\
\hline 3832.789 & 25 & 200 & 26083.261 & $13468_{9 / 2}^{\circ}-39552_{9 / 2}^{\circ}$ & 3808.128 & 50 & 200 & 26252.169 & $12488_{9 / 2}^{\circ}-38740_{11 / 2}$ \\
\hline 3832.347 & 2 & & 26086.270 & $17722_{9 / 2}^{\circ}-43809_{9 / 2}$ & 3807.875 & 300 & 800 & 26253.914 & $9202_{7 / 2}^{\circ}-35456_{9 / 2}$ \\
\hline 3831.737 & 100 & 500 & 26090.422 & $14484_{11 / 2}^{\circ}-40574_{11 / 2}$ & 3807.671 & 3 & 100 & 26255.320 & $19050_{3 / 2}^{\circ}-45306_{3 / 2}$ \\
\hline 3831.525 & 8 & 25 & 26091.866 & $14275_{9 / 2}^{\circ}-40367_{9 / 2}$ & 3807.500 & 3 & & 26256.499 & $12472_{5 / 2}^{\circ}-38728_{5 / 2}$ \\
\hline 3830.946 & 25 & 50 & 26095.809 & $9061_{5 / 2}-35156_{5 / 2}^{\circ}$ & 3806.266 & 5 & 75 & 26265.011 & $20158_{5 / 2}-46423_{3 / 2}^{\circ}$ \\
\hline 3830.836 & $25 b$ & 100 & 26096.558 & $11725_{1 / 2}^{\circ}-37821_{3 / 2}$ & 3806.230 & 2 & & 26265.260 & $20120_{5 / 2}^{\circ}-46385_{7 / 2}$ \\
\hline 3830.511 & 25 & 100 & 26098.773 & $14545_{5 / 2}^{\circ}-40644_{5 / 2}$ & 3805.969 & 8 & 200 & 26267.061 & $20288_{11 / 2}^{\circ}-46555_{11 / 2}$ \\
\hline 3829.415 & $50 \mathrm{~b}$ & 40 & 26106.242 & $17121_{3 / 2}^{\circ}-43227_{5 / 2}$ & & & & & $25414_{11 / 2}^{\circ}-51681_{9 / 2}$ \\
\hline 3826.949 & 100 & 400 & 26123.064 & $14349_{1 / 2}-40472_{3 / 2}^{\circ}$ & 3805.820 & 200 & 400 & 26268.089 & $6691_{3 / 2}^{\circ}-32959_{3 / 2}$ \\
\hline
\end{tabular}


TABLE 3. Classified lines of Th II-Continued

\begin{tabular}{|c|c|c|c|c|}
\hline \multirow{2}{*}{$\begin{array}{c}\text { Wavelength } \\
\AA\end{array}$} & \multicolumn{2}{|c|}{ Intensity } & \multirow{2}{*}{$\begin{array}{c}\text { Wavenumber } \\
\mathbf{c m}^{-1}\end{array}$} & \multirow{2}{*}{ Classification } \\
\hline & Lamp & Spark & & \\
\hline 3805.341 & $2 b$ & $50 \mathrm{~b}$ & 26271.396 & $27249_{7 / 2}^{\circ}-53520_{9 / 2}$ \\
\hline 3805.314 & 5001 & $400 \mathrm{~b}$ & 26271.582 & $20080_{7 / 2}^{\circ}-46352_{7 / 2}$ \\
\hline 3804.995 & 100 & 200 & 26273.785 & $12219_{3 / 2}-38493_{5 / 2}^{\circ}$ \\
\hline 3804.335 & $8 \mathrm{~h}$ & 20 & 26278.343 & $18816_{13 / 2}^{\circ}-45095_{15 / 2}$ \\
\hline 3803.369 & 2 & & 26285.017 & $12472_{5 / 2}^{\circ}-38757_{3 / 2}$ \\
\hline 3802.320 & 1 & & 26292.268 & $20310_{5 / 2}^{\circ}-46603_{5 / 2}$ \\
\hline 3802.147 & 25 & 75 & 26293.465 & $9585_{5 / 2}^{\circ}-35878_{7 / 2}$ \\
\hline 3801.916 & 10 & 50 & 26295.062 & $14275_{9 / 2}^{\circ}-40570_{\tau / 2}$ \\
\hline 3801.324 & 15 & 150 & 26299.157 & $14275_{9 / 2}^{\circ}-40574_{11 / 2}$ \\
\hline 3800.588 & 10 & 400 & 26304.250 & $19912_{13 / 2}^{\circ}-46216_{11 / 2}$ \\
\hline 3800.371 & 75 & 200 & 26305.752 & $7331_{5 / 2}^{\circ}-33637_{7 / 2}$ \\
\hline 3800.113 & 20 & 20 & 26307.538 & $9238_{9 / 2}^{\circ}-35545_{9 / 2}$ \\
\hline 3799.489 & 3 & & 26311.858 & $17460_{5 / 2}^{\circ}-43772_{5 / 2}$ \\
\hline 3799.422 & 2 & & 26312.322 & $8018_{3 / 2}-34330_{1 / 2}^{\circ}$ \\
\hline 3798.459 & 4 & 15 & 26318.993 & $16033_{5 / 2}^{\circ}-42352_{5 / 2}$ \\
\hline 3798.426 & 200 & 75 & 26319.221 & $23518_{7 / 2}^{\circ}-49837_{9 / 2}$ \\
\hline 3797.718 & 2 & & 26324.128 & $21682_{7 / 2}^{\circ}-48006_{9 / 2}$ \\
\hline 3797.564 & 2 & & 26325.195 & $17771_{11 / 2}^{\circ}-44096_{9 / 2}$ \\
\hline 3797.453 & 15 & 200 & 26325.965 & $15786_{5 / 2}-42112_{3 / 2}^{\circ}$ \\
\hline 3796.185 & 1 & 5 & 26334.758 & $\begin{array}{l}28587_{5 / 2}^{\circ}-54922_{3 / 2} \\
22355_{1 / 2}^{\circ}-48689_{3 / 2}^{\circ}\end{array}$ \\
\hline 3795.678 & 3 & 75 & 26338.276 & $18214_{3 / 2}^{\circ}-44552_{5 / 2}$ \\
\hline 3794.951 & 2 & & 26343.321 & $9202_{7 / 2}^{\circ}-35545_{9 / 2}$ \\
\hline 3794.646 & 4 & 20 & 26345.438 & $9720_{7 / 2}^{\circ}-36065_{5 / 2}$ \\
\hline 3794.428 & 2 & & 26346.952 & $17460_{5 / 2}^{\circ}-43807_{3 / 2}$ \\
\hline 3794.316 & 150 & 100 & 26347.730 & $8378_{7 / 2}^{\circ}-34726_{7 / 2}$ \\
\hline 3794.150 & 100 & 200 & 26348.882 & $11116_{7 / 2}^{\circ}-37465_{5 / 2}$ \\
\hline 3793.309 & 2 & & 26354.724 & $23518_{7 / 2}^{\circ}-49873_{5 / 2}$ \\
\hline 3792.312 & $15 b$ & 200 & 26361.652 & $12219_{3 / 2}-38581_{5 / 2}^{\circ}$ \\
\hline 3791.972 & 15 & 100 & 26364.016 & $12472_{5 / 2}^{\circ}-38836_{3 / 2}$ \\
\hline 3791.296 & 100 & 300 & 26368.717 & $11576_{3 / 2}^{\circ}-37945_{5 / 2}$ \\
\hline 3790.459 & 3 & & 26374.539 & $12488_{9 / 2}^{\circ}-38862_{11 / 2}$ \\
\hline 3789.951 & 25 & 200 & 26378.075 & $14545_{5 / 2}^{\circ}-40923_{5 / 2}$ \\
\hline 3789.610 & 2 & 15 & 26380.448 & $24982_{7 / 2}^{\circ}-51362_{5 / 2}$ \\
\hline 3789.563 & 4 & & 26380.775 & $8605_{5 / 2}-34986_{3 / 2}^{\circ}$ \\
\hline 3789.413 & 2 & & 26381.819 & $27787_{9 / 2}^{\circ}-54169_{7 / 2}$ \\
\hline 3789.122 & $500 \mathrm{~b}$ & 400 & 26383.845 & $1859_{3 / 2}-28243_{5 / 2}^{\circ}$ \\
\hline 3788.358 & 50 & 100 & 26389.166 & $4490_{5 / 2}^{\circ}-30879_{\tau / 2}$ \\
\hline 3788.062 & 1 & & 26391.228 & $16564_{11 / 2}^{\circ}-42955_{9 / 2}$ \\
\hline 3787.995 & 1 & & 26391.695 & $12472_{5 / 2}^{\circ}-38863_{5 / 2}$ \\
\hline 3787.478 & 10 & 100 & 26395.297 & $20310_{5 / 2}^{\circ}-46706_{\pi / 2}$ \\
\hline 3787.116 & 8 & 200 & 26397.820 & $13818_{7 / 2}^{\circ}-40216_{5 / 2}$ \\
\hline 3786.881 & 100 & 200 & 26399.458 & $7331_{5 / 2}^{\circ}-33730_{5 / 2}$ \\
\hline 3786.076 & 2 & & 26405.071 & $9720_{7 / 2}^{\circ}-36125_{9 / 2}$ \\
\hline 3785.913 & 100 & 300 & 26406.208 & $17771_{11 / 2}^{\circ}-44177_{11 / 2}$ \\
\hline 3785.600 & 500 & 500 & 26408.391 & $616_{\pi / 2}^{\circ}-32576_{\tau / 2}$ \\
\hline 3785.428 & 2 & & 26409.591 & $25266_{1 / 2}^{\circ}-51676_{3 / 2}$ \\
\hline 3783.752 & 4 & 10 & 26421.289 & $18973_{\pi / 2}^{\circ}-45395_{\pi / 2}$ \\
\hline 3783.660 & $100 \mathrm{~b}$ & 100 & 26421.932 & $14101_{1 / 2}^{\circ}-40523_{1 / 2}$ \\
\hline 3783.295 & 200 & 300 & 26424.481 & $0_{3 / 2}-26424_{5 / 2}^{\circ}$ \\
\hline
\end{tabular}

\begin{tabular}{|c|c|c|c|c|}
\hline \multirow{2}{*}{$\begin{array}{c}\text { Wavelength } \\
\AA\end{array}$} & \multicolumn{2}{|c|}{ Intensity } & \multirow{2}{*}{$\begin{array}{c}\text { Wavenumber } \\
\mathbf{c m}^{-1}\end{array}$} & \multirow{2}{*}{ Classification } \\
\hline & Lamp & Spark & & \\
\hline 3783.012 & 150 & 400 & 26426.457 & $13468_{9 / 2}^{\circ}-39895_{9 / 2}$ \\
\hline 3782.108 & 5 & & 26432.774 & $15786_{5 / 2}-42219_{5 / 2}^{\circ}$ \\
\hline 3780.282 & 3 & & 26445.541 & $15242_{9 / 2}^{\circ}-41688_{7 / 2}$ \\
\hline 3779.712 & 1 & & 26449.529 & $16564_{11 / 2}^{\circ}-43014_{13 / 2}$ \\
\hline 3778.744 & 10 & 75 & 26456.305 & $15453_{\% / 2}^{\circ}-41909_{9 / 2}$ \\
\hline 3777.286 & 3 & 40 & 26466.516 & $19912_{13 / 2}^{\circ}-46378_{13 / 2}$ \\
\hline 3775.901 & 8 & 200 & 26476.224 & $14349_{1 / 2}-40825_{1 / 2}^{\circ}$ \\
\hline 3775.312 & 10 & 25 & 26480.354 & $9585_{5 / 2}^{\circ}-36065_{5 / 2}$ \\
\hline 3774.924 & 1 & & 26483.076 & $20120_{5 / 2}^{\circ}-46603_{5 / 2}$ \\
\hline 3774.202 & $100 \mathrm{~b}$ & 500 & 26488.142 & $19880_{9 / 2}-46368_{9 / 2}^{\circ}$ \\
\hline 3773.758 & 300 & 400 & 26491.259 & $10572_{9 / 2}^{\circ}-37063_{9 / 2}$ \\
\hline 3772.429 & 1 & & 26500.591 & $20080_{\pi / 2}^{\circ}-46581_{\tilde{s} / 2}$ \\
\hline 3772.236 & 100 & 200 & 26501.947 & $7828_{1 / 2}-34330_{1 / 2}^{\circ}$ \\
\hline 3771.201 & 1 & & 26509.220 & $6700_{9 / 2}^{\circ}-33209_{7 / 2}$ \\
\hline 3768.778 & 3 & 10 & 26526.263 & $8460_{3 / 2}-34986_{3 / 2}^{\circ}$ \\
\hline 3768.435 & 20 & 100 & 26528.677 & $11576_{3 / 2}^{\circ}-38105_{5 / 2}$ \\
\hline 3768.003 & 3 & 5 & 26531.719 & $17272_{9 / 2}^{\circ}-43803_{7 / 2}$ \\
\hline 3767.946 & $8 \mathrm{~b}$ & 50 & 26532.120 & $15144_{3 / 2}^{\circ}-41676_{3 / 2}$ \\
\hline 3767.898 & $300 \mathrm{~s}$ & 500 & 26532.458 & $9061_{5 / 2}-35593_{\tau / 2}^{\circ}$ \\
\hline 3767.868 & 4 & & 26532.669 & $13406_{13 / 2}^{\circ}-39939_{11 / 2}$ \\
\hline 3767.513 & 2 & & 26535.169 & $15710_{3 / 2}^{\circ}-42246_{1 / 2}$ \\
\hline 3767.252 & 2 & & 26537.007 & $17272_{9 / 2}^{\circ}-43809_{9 / 2}$ \\
\hline 3767.201 & 10 & 200 & 26537.367 & $14790_{7 / 2}^{\circ}-41328_{5 / 2}$ \\
\hline 3765.535 & $8 \mathrm{~h}$ & 20 & 26549.108 & $13818_{7 / 2}^{\circ}-40367_{9 / 2}$ \\
\hline 3765.129 & 3 & 10 & 26551.970 & $19248_{5 / 2}^{\circ}-45800_{5 / 2}$ \\
\hline 3764.070 & 2 & 50 & 26559.440 & $15349_{11 / 2}^{\circ}-41909_{9 / 2}$ \\
\hline 3763.581 & 15 & $300 \mathrm{~b}$ & 26562.891 & $14484_{11 / 2}^{\circ}-41047_{9 / 2}$ \\
\hline 3763.554 & 2 & 25 & 26563.082 & $25607_{9 / 2}^{\circ}-52170_{11 / 2}$ \\
\hline 3763.320 & 40 & 300 & 26564.733 & $16818_{\tau / 2}-43382_{\tau / 2}^{\circ}$ \\
\hline 3762.885 & $400 \mathrm{~b}$ & 600 & 26567.804 & $6168_{7 / 2}^{\circ}-32736_{\tau / 2}$ \\
\hline 3762.679 & 1 & & 26569.259 & $17983_{5 / 2}^{\circ}-44552_{5 / 2}$ \\
\hline 3762.417 & 1 & 20 & 26571.109 & $29515_{9 / 2}-56086_{9 / 2}^{\circ}$ \\
\hline 3762.012 & 1 & & 26573.969 & $21297_{5 / 2}^{\circ}-47871_{\pi / 2}$ \\
\hline 3761.887 & 2 & & 26574.852 & $18214_{3 / 2}^{\circ}-44789_{1 / 2}$ \\
\hline 3761.101 & 50 & 200 & 26580.406 & $12488_{9 / 2}^{\circ}-39068_{\tau / 2}$ \\
\hline 3760.733 & 1 & & 26583.007 & $12485_{\pi / 2}^{\circ}-39068_{\tau / 2}$ \\
\hline 3760.295 & $3 \mathrm{~b}$ & & 26586.103 & $20120_{5 / 2}^{\circ}-46706_{7 / 2}$ \\
\hline 3760.271 & $50 \mathrm{~b}$ & $25 b$ & 26586.273 & $0_{3 / 2}-26586_{3 / 2}^{\circ}$ \\
\hline 3758.822 & 4 & 40 & 26596.521 & $12472_{5 / 2}^{\circ}-39068_{7 / 2}$ \\
\hline 3757.276 & 1 & & 26607.465 & $14790_{7 / 2}^{\circ}-41398_{9 / 2}$ \\
\hline 3756.889 & 1 & & 26610.205 & $24414_{3 / 2}^{\circ}-51024_{3 / 2}$ \\
\hline 3756.757 & 8 & 50 & 26611.141 & $13250_{5 / 2}-39861_{5 / 2}^{\circ}$ \\
\hline 3754.843 & 1 & & 26624.705 & $20310_{5 / 2}^{\circ}-46935_{3 / 2}$ \\
\hline 3754.592 & 200 & 200 & 26626.485 & $0_{3 / 2}-26626_{i / 2}^{\circ}$ \\
\hline 3752.568 & 500 & 800 & 26640.846 & $9238_{9 / 2}^{\circ}-35878_{7 / 2}$ \\
\hline 3751.758 & 8 & 40 & 26646.597 & $11725_{1 / 2}^{\circ}-38372_{3 / 2}$ \\
\hline 3750.658 & 2 & 40 & 26654.412 & $29431_{7 / 2}-56086_{9 / 2}^{\circ}$ \\
\hline 3748.975 & 200 & 100 & 26666.378 & $15242_{9 / 2}^{\circ}-41909_{9 / 2}$ \\
\hline 3748.282 & 50 & 200 & 26671.308 & $1116_{7 / 2}^{\circ}-37787_{7 / 2}$ \\
\hline 3747.706 & 2 & 20 & 26675.407 & $18214_{3 / 2}^{\circ}-44889_{3 / 2}$ \\
\hline
\end{tabular}


TABlE 3. Classified lines of Th II-Continued

\begin{tabular}{|c|c|c|c|c|c|c|c|c|c|}
\hline \multirow{2}{*}{$\begin{array}{c}\text { Wavelength } \\
\AA\end{array}$} & \multicolumn{2}{|c|}{ Intensity } & \multirow{2}{*}{$\begin{array}{c}\text { Wavenumber } \\
\mathbf{c m}^{-1}\end{array}$} & \multirow{2}{*}{ Classification } & \multirow{2}{*}{$\begin{array}{c}\text { Wavelength } \\
\AA\end{array}$} & \multicolumn{2}{|c|}{ Intensity } & \multirow{2}{*}{$\begin{array}{c}\text { Wavenumber } \\
\mathbf{c m}^{-1}\end{array}$} & \multirow{2}{*}{ Classification } \\
\hline & Lamp & Spark & & & & Lamp & Spark & & \\
\hline 3747.539 & 300 & 500 & 26676.595 & $9202_{7 / 2}^{\circ}-35878_{7 / 2}$ & 3721.824 & $800 \mathrm{~b}$ & 500 & 26860.906 & $1859_{3 / 2}-28720_{3 / 2}^{\circ}$ \\
\hline 3747.270 & 1 & & 26678.511 & $12472_{5 / 2}^{\circ}-39150_{3 / 2}$ & 3721.344 & 8 & 40 & 26864.371 & $10189_{11 / 2}^{\circ}-37053_{11 / 2}$ \\
\hline 3747.170 & 3 & 10 & 26679.222 & $17771_{1 / 2 / 2}^{\circ}-44450_{9 / 2}$ & 3721.137 & $2 \mathrm{~b}$ & 2 & 26865.865 & $16906_{7 / 2}^{\circ}-43772_{5 / 2}$ \\
\hline 3746.463 & 25 & 50 & 26684.257 & $6700_{9 / 2}^{\circ}-33384_{9 / 2}$ & 3720.700 & 10 & 75 & 26869.020 & $10673_{5 / 2}^{\circ}-37542_{3 / 2}$ \\
\hline 3746.227 & 5 & 75 & 26685.938 & $17121_{3 / 2}^{\circ}-43807_{3 / 2}$ & 3720.307 & $200 s$ & 400 & 26871.859 & $9711_{7 / 2}-36583_{7 / 2}^{\circ}$ \\
\hline 3745.968 & 400 & 500 & 26687.783 & $7331_{5 / 2}^{\circ}-34019_{3 / 2}$ & 3720.214 & 150 & 100 & 26872.530 & $12570_{7 / 2}-39443_{9 / 2}^{\circ}$ \\
\hline 3744.735 & 200 & 200 & 26696.570 & $8460_{3 / 2}-35156_{5 / 2}^{\circ}$ & 3719.976 & $300 \mathrm{~b}$ & 500 & 26874.249 & $10189_{11 / 2}^{\circ}-37063_{9 / 2}$ \\
\hline 3744.652 & 15 & 200 & 26697.162 & $14790_{7 / 2}^{\circ}-41488_{\tau / 2}$ & 3719.693 & 20 & 200 & 26876.294 & $15236_{3 / 2}-42112_{3 / 2}^{\circ}$ \\
\hline 3743.806 & 100 & 200 & 26703.194 & $18118_{3 / 2}-44821_{5 / 2}^{\circ}$ & 3719.152 & 1 & 1 & 26880.204 & $26965_{3 / 2}^{\circ}-53845_{5 / 2}$ \\
\hline 3743.506 & 20 & 200 & 26705.334 & $10572_{9 / 2}^{\circ}-37277_{7 / 2}$ & 3719.057 & 10 & 5 & 26880.890 & $4113_{5 / 2}-30994_{7 / 2}^{\circ}$ \\
\hline 3742.228 & 5 & 200 & 26714.454 & $10855_{7 / 2}-37569_{9 / 2}^{\circ}$ & 3719.012 & 3 & 5 & 26881.215 & $12485_{7 / 2}^{\circ}-39366_{5 / 2}$ \\
\hline 3741.183 & $1000 \mathrm{r}$ & 600 & 26721.916 & $1521_{5 / 2}-28243_{5 / 2}^{\circ}$ & 3718.806 & 1 & 2 & 26882.704 & $24982_{7 / 2}^{\circ}-51865_{5 / 2}$ \\
\hline 3740.854 & 40 o & 300 & 26724.266 & $11116_{7 / 2}^{\circ}-37840_{9 / 2}$ & 3718.656 & 10 & 200 & 26883.789 & $15453_{7 / 2}^{\circ}-42336_{5 / 2}$ \\
\hline 3740.411 & 15 & 20 & 26727.431 & $1859_{3 / 2}-28587_{5 / 2}^{\circ}$ & 3718.168 & 75 & 200 & 26887.317 & $9238_{9 / 2}^{\circ}-36125_{9 / 2}$ \\
\hline 3738.847 & 300 & 300 & 26738.611 & $8460_{3 / 2}-35198_{1 / 2}^{\circ}$ & 3717.830 & 15 & 200 & 26889.762 & $14101_{1 / 2}^{\circ}-40991_{3 / 2}$ \\
\hline 3738.758 & $3 \mathrm{~b}$ & 400 & 26739.248 & $14484_{11 / 2}^{\circ}-41223_{11 / 2}$ & 3716.773 & 4 & 5 & 26897.408 & $16906_{7 / 2}^{\circ}-43803_{7 / 2}$ \\
\hline 3738.103 & $8 b$ & & 26743.933 & $6213_{9 / 2}-32957_{7 / 2}^{\circ}$ & 3716.542 & $2 \mathrm{~b}$ & & 26899.080 & $15453_{7 / 2}^{\circ}-42352_{5 / 2}$ \\
\hline 3737.647 & 3 & & 26747.196 & $15453_{7 / 2}^{\circ}-42200_{9 / 2}$ & 3716.214 & 4 & 2 & 26901.454 & $10855_{7 / 2}-37756_{7 / 2}^{\circ}$ \\
\hline 3737.334 & 1 & & 26749.435 & $19050_{3 / 2}^{\circ}-45800_{5 / 2}$ & 3716.088 & 1 & & 26902.366 & $20969_{7 / 2}^{\circ}-47871_{7 / 2}$ \\
\hline 3736.936 & 15 & 200 & 26752.284 & $13818_{7 / 2}^{\circ}-40570_{7 / 2}$ & 3715.911 & 3 & 2 & 26903.648 & $15242_{9 / 2}^{\circ}-42146_{11 / 2}$ \\
\hline 3735.511 & 3 & 15 & 26762.490 & $20969_{7 / 2}^{\circ}-47731_{9 / 2}$ & 3715.721 & 3 & 25 & 26905.023 & $17272_{9 / 2}^{\circ}-44177_{11 / 2}$ \\
\hline 3734.698 & 1 & 2 & 26768.315 & $30310_{1 / 2}^{\circ}-57078_{9 / 2}$ & 3714.455 & 1 & & 26914.193 & $14484_{11 / 2}^{\circ}-41398_{9 / 2}$ \\
\hline 3734.598 & $100 \mathrm{~b}$ & $200 \mathrm{~b}$ & 26769.032 & $4490_{5 / 2}^{\circ}-31259_{5 / 2}$ & 3714.338 & 2 & 3 & 26915.041 & $24309_{11 / 2}^{\circ}-51224_{9 / 2}$ \\
\hline 3734.566 & $3 b$ & $10 \mathrm{~b}$ & 26769.261 & $15453_{7 / 2}^{\circ}-42222_{7 / 2}$ & 3714.291 & $8 \mathrm{~b}$ & 200 & 26915.382 & $17983_{5 / 2}^{\circ}-44898_{7 / 2}$ \\
\hline 3734.414 & 1 & & 26770.351 & $21682_{\pi / 2}^{\circ}-48453_{\tau / 2}$ & 3712.533 & $40 \mathrm{~b}$ & $25 b$ & 26928.127 & $17460_{5 / 2}^{\circ}-44388_{5 / 2}$ \\
\hline 3734.232 & 1 & & 26771.656 & $14275_{9 / 2}^{\circ}-41047_{9 / 2}$ & 3711.780 & 3 & 2 & 26933.589 & $13250_{5 / 2}-40184_{7 / 2}^{\circ}$ \\
\hline 3734.128 & 1 & & 26772.401 & $23697_{7 / 2}^{\circ}-50470_{9 / 2}$ & 3711.304 & 300 & 500 & 26937.044 & $6700_{9 / 2}^{\circ}-33637_{7 / 2}$ \\
\hline 3732.736 & 2 & & 26782.385 & $20120_{5 / 2}^{\circ}-46902_{5 / 2}$ & 3710.552 & 5 & 50 & 26942.503 & $13468_{9 / 2}^{\circ}-40411_{7 / 2}$ \\
\hline 3732.679 & 3 & 20 & 26782.794 & $14545_{5 / 2}^{\circ}-41328_{5 / 2}$ & 3709.817 & 3 & 8 & 26947.841 & $7331_{5 / 2}^{\circ}-34279_{7 / 2}$ \\
\hline 3732.473 & 1 & & 26784.272 & $33209_{7 / 2}-59993_{9 / 2}^{\circ}$ & 3709.624 & 5 & 200 & 26949.242 & $19912_{13 / 2}^{\circ}-46861_{11 / 2}$ \\
\hline 3731.425 & 8 & 75 & 26791.794 & $15144_{3 / 2}^{\circ}-41936_{3 / 2}$ & 3709.310 & 2 & & 26951.524 & $17837_{1 / 2}^{\circ}-44789_{1 / 2}$ \\
\hline 3731.354 & $4 \mathrm{~b}$ & & 26792.304 & $10673_{5 / 2}^{\circ}-37465_{5 / 2}$ & 3708.754 & 5 & 150 & 26955.564 & $16818_{7 / 2}-43773_{7 / 2}^{\circ}$ \\
\hline 3730.748 & 75 & 500 & 26796.656 & $15349_{11 / 2}^{\circ}-42146_{11 / 2}$ & 3708.671 & $5 b$ & 75 & 26956.167 & $17771_{11 / 2}^{\circ}-44727_{11 / 2}$ \\
\hline 3730.532 & 2 & & 26798.207 & $12902_{3 / 2}^{\circ}-39700_{5 / 2}$ & 3707.428 & 75 & 75 & 26965.205 & $0_{3 / 2}-26965_{3 / 2}^{\circ}$ \\
\hline 3729.678 & 25 & 50 & 26804.343 & $24463_{5 / 2}^{\circ}-51268_{7 / 2}$ & 3706.046 & 8 & 200 & 26975.260 & $18214_{3 / 2}^{\circ}-45189_{5 / 2}$ \\
\hline 3728.990 & 1 & & 26809.289 & $21682_{7 / 2}^{\circ}-48492_{5 / 2}$ & 3705.942 & 5 & 50 & 26976.017 & $9711_{7 / 2}-36687_{5 / 2}^{\circ}$ \\
\hline 3728.965 & 1 & & 26809.468 & $29873_{7 / 2}-56683_{7 / 2}^{\circ}$ & 3704.966 & 25 & 200 & 26983.123 & $15236_{3 / 2}-42219_{5 / 2}^{\circ}$ \\
\hline 3726.946 & 1 & & 26823.991 & $17272_{9 / 2}^{\circ}-44096_{9 / 2}$ & 3704.342 & $20 \mathrm{~h}$ & 100 & 26987.668 & $8605_{5 / 2}-35593_{7 / 2}^{\circ}$ \\
\hline 3726.724 & 200 & 200 & 26825.589 & $4146_{\pi / 2}-30972_{\bar{\sigma} / 2}^{\circ}$ & 3704.085 & 100 & 200 & 26989.541 & $9400_{5 / 2}-36390_{3 / 2}^{\circ}$ \\
\hline 3726.659 & 25 & 50 & 26826.057 & $12570_{7 / 2}-39396_{\pi / 2}^{\circ}$ & 3703.990 & 100 & 300 & 26990.233 & $10572_{9 / 2}^{\circ}-37562_{11 / 2}$ \\
\hline 3726.197 & 3 & 50 & 26829.383 & $18816_{13 / 2 / 2}^{\circ}-45646_{15 / 2}$ & 3703.907 & 75 & 200 & 26990.838 & $10855_{7 / 2}-37846_{5 / 2}^{\circ}$ \\
\hline 3726.099 & 2 & 1 & 26830.089 & $22014_{i 1 / 2}^{\circ}-48844_{9 / 2}$ & 3702.864 & 10 & 300 & 26998.440 & $19912_{13 / 2}^{\circ}-46910_{13 / 2}$ \\
\hline 3725.875 & 3 & 100 & 26831.702 & $19594_{1 / 2}-46426_{1 / 2}^{0}$ & 3702.067 & -3 & 15 & 27004.252 & $17722_{9 / 2}^{\circ}-44727_{11 / 2}$ \\
\hline 3725.822 & 1 & 3 & 26832.084 & $25440_{\bar{s} / 2}^{\circ}-52272_{\pi / 2}$ & 3700.766 & 50 & 500 & 27013.745 & $15305_{9 / 2}-42319_{9 / 2}^{\circ}$ \\
\hline 3724.733 & 40 & 200 & 26839.928 & $17460_{5 / 2}^{\circ}-44300_{3 / 2}$ & 3700.526 & 1 & 2 & 27015.497 & $22106_{5 / 2}-49121_{7 / 2}^{\circ}$ \\
\hline 3724.498 & 75 & 50 & 26841.622 & $7001_{3 / 2}-33843_{3 / 2}^{\circ}$ & 3700.462 & 25 & 75 & 27015.964 & $19248_{5 / 2}^{\circ}-46264_{3 / 2}$ \\
\hline 3724.299 & 3 & 1 & 26843.056 & $7331_{5 / 2}^{\circ}-34174_{5 / 2}$ & 3698.755 & 5 & 200 & 27028.432 & $20120_{5 / 2}^{\circ}-47148_{3 / 2}$ \\
\hline 3723.657 & 100 & 200 & 26847.684 & $4146_{7 / 2}-30994_{7 / 2}^{\circ}$ & 3698.301 & $10 \mathrm{~b}$ & 8 & 27031.750 & $11725_{1 / 2}^{\circ}-38757_{3 / 2}$ \\
\hline 3723.291 & 20 & 200 & 26850.323 & $15349_{11 / 2}^{\circ}-42200_{9 / 2}$ & 3697.234 & 2 & & 27039.551 & $6691_{3 / 2}^{\circ}-33730_{5 / 2}$ \\
\hline 3722.115 & $300 \mathrm{~b}$ & 500 & 26858.806 & $4113_{5 / 2}-30972_{5 / 2}^{\circ}$ & 3697.030 & 100 & 200 & 27041.043 & $6168_{7 / 2}^{\circ}-33209_{7 / 2}$ \\
\hline
\end{tabular}


TABLE 3. Classified lines of Th $\mathrm{II}-$ Continued

\begin{tabular}{|c|c|c|c|c|}
\hline \multirow{2}{*}{$\begin{array}{c}\text { Wavelength } \\
\AA\end{array}$} & \multicolumn{2}{|c|}{ Intensity } & \multirow{2}{*}{$\begin{array}{c}\text { Wavenumber } \\
\mathrm{cm}^{-1}\end{array}$} & \multirow{2}{*}{ Classification } \\
\hline & Lamp & Spark & & \\
\hline 3696.647 & 50 & 200 & 27043.845 & $9400_{5 / 2}-36444_{3 / 2}^{\circ}$ \\
\hline 3696.316 & 1 & 1 & 27046.266 & $26963_{7 / 2}^{\circ}-54010_{7 / 2}$ \\
\hline 3695.974 & 100 & 300 & 27048.769 & $1116_{7 / 2}^{\circ}-38165_{7 / 2}$ \\
\hline 3694.785 & 1 & 2 & 27057.473 & $27787_{9 / 2}^{\circ}-54845_{9 / 2}$ \\
\hline 3693.901 & 50 & 300 & 27063.948 & $12488_{9 / 2}^{\circ}-39552_{9 / 2}$ \\
\hline 3693.693 & 100 & 100 & 27065.472 & $1521_{5 / 2}-28587_{5 / 2}^{\circ}$ \\
\hline 3693.640 & 1 & & 27065.860 & $15453_{7 / 2}^{\circ}-42518_{7 / 2}$ \\
\hline 3693.544 & $4 b$ & & 27066.564 & $12485_{7 / 2}^{\circ}-39552_{9 / 2}$ \\
\hline 3692.075 & 50 & 200 & 27077.333 & $8378_{7 / 2}^{\circ}-35456_{9 / 2}$ \\
\hline 3690.486 & 150 & 300 & 27088.991 & $9720_{7 / 2}^{\circ}-36809_{7 / 2}$ \\
\hline 3690.070 & $5 b$ & 20 & 27092.045 & $17460_{5 / 2}^{\circ}-44552_{5 / 2}$ \\
\hline 3688.812 & 8 & 40 & 27101.284 & $15144_{3 / 2}^{\circ}-42246_{1 / 2}$ \\
\hline 3688.756 & $100 \mathrm{~b}$ & $150 \mathrm{~b}$ & 27101.695 & $13468_{9 / 2}^{\circ}-40570_{7 / 2}$ \\
\hline 3688.266 & 3 & 2 & 27105.296 & $13818_{7 / 2}^{\circ}-40923_{5 / 2}$ \\
\hline 3688.198 & 4 & 10 & 27105.796 & $13468_{9 / 2}^{\circ}-40574_{11 / 2}$ \\
\hline 3687.671 & $10 \mathrm{~h}$ & 75 & 27109.669 & $22014_{11 / 2}^{\circ}-49124_{11 / 2}$ \\
\hline 3687.524 & $100 \mathrm{~b}$ & 100 & 27110.750 & $11725_{1 / 2}^{\circ}-38836_{3 / 2}$ \\
\hline 3686.980 & 20 & 100 & 27114.750 & $10673_{5 / 2}^{\circ}-37787_{7 / 2}$ \\
\hline 3686.494 & 1 & & 27118.325 & $14790_{7 / 2}^{\circ}-41909_{9 / 2}$ \\
\hline 3685.867 & 8 & 100 & 27122.937 & $14275_{9 / 2}^{\circ}-41398_{9 / 2}$ \\
\hline 3683.936 & 5 & 75 & 27137.154 & $19248_{5 / 2}^{\circ}-46385_{7 / 2}$ \\
\hline 3683.723 & 5 & 1 & 27138.723 & $8018_{3 / 2}-35156_{5 / 2}^{\circ}$ \\
\hline 3683.313 & 20 & 200 & 27141.744 & $11725_{1 / 2}^{\circ}-38867_{1 / 2}$ \\
\hline 3682.846 & 2 & 2 & 27145.185 & $23518_{7 / 2}^{\circ}-50663_{5 / 2}$ \\
\hline 3682.350 & 25 & 200 & 27148.842 & $10673_{5 / 2}^{\circ}-37821_{3 / 2}$ \\
\hline 3681.880 & 100 & 200 & 27152.307 & $11576_{3 / 2}^{\circ}-38728_{5 / 2}$ \\
\hline 3681.180 & 10 & 200 & 27157.470 & $15786_{5 / 2}-42944_{7 / 2}^{\circ}$ \\
\hline 3681.103 & 5 & 10 & 27158.038 & $7828_{1 / 2}-34986_{3 / 2}^{\circ}$ \\
\hline 3680.540 & 2 & 3 & 27162.192 & $21682_{7 / 2}^{\circ}-48844_{9 / 2}$ \\
\hline 3679.710 & 200 & 500 & 27168.319 & $\begin{array}{l}29515_{9 / 2}-56683_{7 / 2}^{\circ} \\
13406_{13 / 2}^{\circ}-40574_{11 / 2}^{\circ}\end{array}$ \\
\hline 3678.776 & 4 & 2 & 27175.217 & $1111_{7 / 2}^{\circ}-38291_{7 / 2}$ \\
\hline 3678.273 & 5 & 20 & 27178.933 & $17121_{3 / 2}^{\circ}-44300_{3 / 2}$ \\
\hline 3678.047 & 150 & 150 & 27180.603 & $9400_{5 / 2}-36581_{3 / 2}^{\circ}$ \\
\hline 3678.021 & 150 & 150 & 27180.795 & $\begin{aligned} 8018_{3 / 2} & -35198_{1 / 2}^{\circ} \\
11576_{3 / 2}^{\circ} & -38757_{3 / 2}^{\circ}\end{aligned}$ \\
\hline 3677.915 & 200 & 300 & 27181.578 & $15236_{3 / 2}-42418_{3 / 2}^{\circ}$ \\
\hline 3677.742 & 100 & 200 & 27182.857 & $9400_{5 / 2}-36583_{7 / 2}^{\circ}$ \\
\hline 3677.089 & 2 & 2 & 27187.684 & $22685_{7 / 2}^{\circ}-49873_{5 / 2}$ \\
\hline 3676.823 & 2 & 2 & 27189.651 & $16906_{7 / 2}^{0}-44096_{9 / 2}$ \\
\hline 3676.690 & 20 & 100 & 27190.634 & $10379_{9 / 2}-37569_{9 / 2}^{\circ}$ \\
\hline 3675.567 & 500 & 500 & 27198.942 & $1521_{5 / 2}-28720_{3 / 2}^{\circ}$ \\
\hline 3674.648 & 2 & 2 & 27205.744 & $26963_{7 / 2}^{\circ}-54169_{7 / 2}$ \\
\hline 3673.793 & 150 & 300 & 27212.075 & $7331_{5 / 2}^{\circ}-34543_{5 / 2}$ \\
\hline 3673.615 & 2 & & 27213.394 & $19050_{3 / 2}^{\circ}-46264_{3 / 2}$ \\
\hline 3673.411 & 2 & & 27214.905 & $12485_{7 / 2}^{\circ}-39700_{5 / 2}$ \\
\hline 3673.283 & $400 \mathrm{~b}$ & $300 \mathrm{~b}$ & 27215.853 & $10572_{9 / 2}^{\circ}-37787_{7 / 2}$ \\
\hline 3673.255 & $40 \mathrm{~b}$ & $75 b$ & 27216.061 & $6168_{7 / 2}^{\circ}-33384_{9 / 2}$ \\
\hline 3672.950 & 5 & 20 & 27218.321 & $22139_{9 / 2}^{\circ}-49357_{11 / 2}$ \\
\hline 3672.466 & 1 & 2 & 27221.908 & $13250_{5 / 2}-40472_{3 / 2}^{\circ}$ \\
\hline
\end{tabular}

\begin{tabular}{|c|c|c|c|c|}
\hline \multirow{2}{*}{$\begin{array}{c}\text { Wavelength } \\
\AA\end{array}$} & \multicolumn{2}{|c|}{ Intensity } & \multirow{2}{*}{$\begin{array}{l}\text { Wavenumber } \\
\mathbf{c m}^{-1}\end{array}$} & \multirow{2}{*}{ Classification } \\
\hline & Lamp & Spark & & \\
\hline 3672.205 & 2 & & 27223.842 & $9585_{5 / 2}^{\circ}-36809_{7 / 2}$ \\
\hline 3671.164 & 10 & 50 & 27231.562 & $17272_{9 / 2}^{\circ}-44503_{7 / 2}$ \\
\hline 3670.057 & 300 & 150 & 27239.775 & $4113_{5 / 2}-31353_{3 / 2}^{\circ}$ \\
\hline 3669.403 & 4 & 101 & 27244.630 & $16564_{11 / 2}^{\circ}-43809_{9 / 2}$ \\
\hline 3666.373 & $3 b$ & 2 & 27267.146 & $17121_{3 / 2}^{\circ}-44388_{5 / 2}^{\circ}$ \\
\hline 3666.150 & 2 & 2 & 27268.804 & $10572_{9 / 2}^{\circ}-37840_{9 / 2}$ \\
\hline 3665.723 & 20 & 100 & 27271.980 & $10673_{5 / 2}^{\circ}-37945_{5 / 2}$ \\
\hline 3665.181 & 15 & 100 & 27276.013 & $15242_{9 / 2}^{\circ}-42518_{7 / 2}$ \\
\hline 3663.702 & $300 \mathrm{~b}$ & $500 \mathrm{~b}$ & 27287.024 & $9400_{5 / 2}-36687_{5 / 2}^{\circ}$ \\
\hline 3663.318 & 2 & 3 & 27289.884 & $24982_{7 / 2}^{\circ}-52272_{7 / 2}$ \\
\hline 3662.631 & 2 & 1 & 27295.003 & $15349_{11 / 2}^{\circ}-42644_{13 / 2}$ \\
\hline 3662.562 & 2 & 3 & 27295.517 & $25440_{5 / 2}^{\circ}-52735_{3 / 2}$ \\
\hline 3662.191 & 151 & 25 & 27298.282 & $15453_{7 / 2}^{\circ}-42751_{7 / 2}$ \\
\hline 3660.117 & $4 b$ & 3 & 27313.750 & $12902_{3 / 2}^{\circ}-40216_{5 / 2}$ \\
\hline 3659.508 & 3001 & $500 \mathrm{~s}$ & 27318.296 & $13406_{13 / 2}^{\circ}-40724_{13 / 2}$ \\
\hline 3659.208 & 1 & & 27320.535 & $12902_{3 / 2}^{\circ}-40222_{3 / 2}^{\circ}$ \\
\hline 3658.226 & 100 & 200 & 27327.869 & $6691_{3 / 2}^{\circ}-34019_{3 / 2}$ \\
\hline 3658.063 & $100 \mathrm{~b}$ & $200 \mathrm{~b}$ & 27329.087 & $7001_{3 / 2}-34330_{1 / 2}^{\circ}$ \\
\hline 3656.199 & 50 & 300 & 27343.019 & $9720_{7 / 2}^{\circ}-37063_{9 / 2}$ \\
\hline 3655.624 & 1 & & 27347.320 & $17460_{5 / 2}^{\circ}-44807_{7 / 2}$ \\
\hline 3655.027 & 2 & 2 & 27351.786 & $20969_{7 / 2}^{\circ}-48320_{5 / 2}$ \\
\hline 3654.605 & 2 & 2 & 27354.945 & $19248_{5 / 2}^{\circ}-46603_{5 / 2}$ \\
\hline 3654.572 & 4 & 20 & 27355.192 & $17771_{11 / 2}^{\circ}-45126_{9 / 2}$ \\
\hline 3653.602 & 50 & 50 & 27362.454 & $8378_{7 / 2}^{\circ}-35741_{5 / 2}$ \\
\hline 3652.536 & 100 & 300 & 27370.440 & $7828_{1 / 2}-35198_{1 / 2}^{\circ}$ \\
\hline 3652.224 & 1 & 20 & 27372.778 & $\begin{array}{l}32620_{11 / 2}-59993_{9 / 2}^{\circ} \\
24309_{11 / 2}^{\circ}-51681_{9 / 2}^{\circ}\end{array}$ \\
\hline 3652.167 & 200 & 500 & 27373.205 & $10189_{11 / 2}^{\circ}-37562_{11 / 2}$ \\
\hline 3651.825 & 5 & 50 & 27375.768 & $12902_{3 / 2}^{\circ}-40278_{3 / 2}$ \\
\hline 3651.468 & 4 & 75 & 27378.445 & $18973_{7 / 2}^{\circ}-46352_{7 / 2}$ \\
\hline 3650.765 & 100 & 300 & 27383.717 & $9061_{5 / 2}-36444_{3 / 2}^{\circ}$ \\
\hline 3650.502 & $4 b$ & 3 & 27385.689 & $15710_{3 / 2}^{\circ}-43096_{5 / 2}$ \\
\hline 3649.249 & 200 & 400 & 27395.092 & $7331_{5 / 2}^{\circ}-34726_{7 / 2}$ \\
\hline 3648.634 & 5 & 300 & 27399.710 & $18816_{13 / 2}^{\circ}-46216_{11 / 2}$ \\
\hline 3648.420 & $100 \mathrm{~b}$ & 150 & 27401.317 & $1521_{5 / 2}-28923_{5 / 2}^{\circ}$ \\
\hline 3648.172 & 150 & 200 & 27403.180 & $0_{3 / 2}-27403_{3 / 2}^{\circ}$ \\
\hline 3647.646 & $200 \mathrm{~b}$ & 200 & 27407.131 & $12488_{9 / 2}^{\circ}-39895_{9 / 2}$ \\
\hline 3647.369 & 10 & 100 & 27409.213 & $14790_{7 / 2}^{\circ}-42200_{9 / 2}$ \\
\hline 3647.299 & 15 & 100 & 27409.739 & $12485_{7 / 2}^{\circ}-39895_{9 / 2}$ \\
\hline 3647.046 & 10 & 40 & 27411.640 & $\begin{array}{l}18973_{7 / 2}^{\circ}-46385_{7 / 2} \\
17983_{5 / 2}^{\circ}-45395_{7 / 2}\end{array}$ \\
\hline 3646.883 & 15 & 100 & 27412.865 & $14275_{9 / 2}^{\circ}-41688_{7 / 2}$ \\
\hline 3645.268 & 1 & 2 & 27425.010 & $14484_{11 / 2}^{\circ}-41909_{9 / 2}$ \\
\hline 3645.235 & 2 & & 27425.258 & $11725_{1 / 2}^{\circ}-39150_{3 / 2}$ \\
\hline 3644.714 & 8 & 200 & 27429.178 & $17460_{5 / 2}^{\circ}-44889_{3 / 2}$ \\
\hline 3644.461 & 1 & & 27431.082 & $17121_{3 / 2}^{\circ}-44552_{5 / 2}$ \\
\hline 3644.431 & 15 & 5 & 27431.308 & $14790_{7 / 2}^{\circ}-42222_{7 / 2}$ \\
\hline 3644.348 & 20 & 150 & 27431.933 & $10673_{5 / 2}^{\circ}-38105_{5 / 2}$ \\
\hline 3642.888 & 3 & 8 & 27442.927 & $20288_{11 / 2}^{\circ}-47731_{9 / 2}$ \\
\hline 3641.882 & 1 & 10 & 27450.507 & $24414_{3 / 2}^{\circ}-51865_{5 / 2}$ \\
\hline
\end{tabular}


TABLE 3. Classified lines of Th II-Continued

\begin{tabular}{|c|c|c|c|c|c|c|c|c|c|}
\hline \multirow{2}{*}{$\begin{array}{c}\text { Wavelength } \\
\AA\end{array}$} & \multicolumn{2}{|c|}{ Intensity } & \multirow{2}{*}{$\begin{array}{c}\text { Wavenumber } \\
\mathrm{cm}^{-1}\end{array}$} & \multirow{2}{*}{ Classification } & \multirow{2}{*}{$\begin{array}{c}\text { Wavelength } \\
\AA\end{array}$} & \multicolumn{2}{|c|}{ Intensity } & \multirow{2}{*}{$\begin{array}{c}\text { Wavenumber } \\
\mathbf{c m}^{-1}\end{array}$} & \multirow{2}{*}{ Classification } \\
\hline & Lamp & Spark & & & & Lamp & Spark & & \\
\hline 3641.841 & 10 & 300 & 27450.816 & $12488_{9 / 2}^{\circ}-39939_{11 / 2}$ & 3617.731 & $8 b$ & 2 & 27633.755 & $14275_{9 / 2}^{\circ}-41909_{9 / 2}$ \\
\hline 3641.287 & 3 & 20 & 27454.993 & $17272_{9 / 2}^{\circ}-44727_{11 / 2}$ & 3617.615 & 1 & 2 & 27634.641 & $20686_{5 / 2}^{\circ}-48320_{5 / 2}$ \\
\hline 3641.113 & 15 & 200 & 27456.305 & $13250_{5 / 2}-40706_{7 / 2}^{\circ}$ & 3617.117 & 200 & 500 & 27638.445 & $10855_{7 / 2}-38493_{5 / 2}^{\circ}$ \\
\hline 3640.873 & 3 & 2 & 27458.115 & $13248_{9 / 2}-40706_{7 / 2}^{\circ}$ & 3617.017 & 100 & 500 & 27639.209 & $15305_{9 / 2}-42944_{7 / 2}^{\circ}$ \\
\hline 3640.184 & 1 & 1 & 27463.312 & $20989_{9 / 2}^{\circ}-48453_{7 / 2}$ & 3616.697 & 8 & 200 & 27641.655 & $12219_{3 / 2}-39861_{5 / 2}^{\circ}$ \\
\hline 3639.587 & 2 & 3 & 27467.816 & $28923_{5 / 2}^{\circ}-56391_{5 / 2}$ & 3615.372 & 25 & 150 & 27651.785 & $10189_{11 / 2}^{\circ}-37840_{9 / 2}$ \\
\hline 3639.446 & 300 & 500 & 27468.880 & $6168_{7 / 2}^{\circ}-33637_{7 / 2}$ & 3615.132 & 400 & 500 & 27653.621 & $4146_{7 / 2}-31800_{7 / 2}^{\circ}$ \\
\hline 3637.686 & 5 & 100 & 27482.170 & $16906_{7 / 2}^{\circ}-44388_{5 / 2}$ & 3615.040 & 2 & & 27654.324 & $19248_{5 / 2}^{\circ}-46902_{5 / 2}$ \\
\hline 3637.555 & 75 & 200 & 27483.160 & $6691_{3 / 2}^{\circ}-34174_{5 / 2}$ & 3614.009 & 75 & 500 & 27662.213 & $14484_{11 / 2}^{\circ}-42146_{11 / 2}$ \\
\hline 3636.918 & 1 & 2 & 27487.973 & $27357_{9 / 2}^{\circ}-54845_{9 / 2}$ & 3613.778 & 100 & 150 & 27663.981 & $4146_{7 / 2}-31810_{5 / 2}^{\circ}$ \\
\hline 3636.566 & 20 & 200 & 27490.634 & $10189_{11 / 2}^{\circ}-37679_{11 / 2}$ & 3613.611 & 2 & 3 & 27665.260 & $23697_{7 / 2}^{\circ}-51362_{5 / 2}$ \\
\hline 3636.357 & 4 & 2 & 27492.214 & $10673_{5 / 2}^{\circ}-38165_{7 / 2}$ & 3612.113 & 4 & 25 & 27676.733 & $14545_{5 / 2}^{\circ}-42222_{7 / 2}$ \\
\hline 3636.172 & 5 & 50 & 27493.613 & $23730_{9 / 2}^{\circ}-51224_{9 / 2}$ & 3610.793 & 100 & 200 & 27686.850 & $4113_{5 / 2}-31800_{7 / 2}^{\circ}$ \\
\hline 3635.420 & 15 & 400 & 27499.299 & $22028_{15 / 2}^{\circ}-49527_{13 / 2}$ & 3610.562 & 2 & & 27688.622 & $6213_{9 / 2}-33902_{7 / 2}^{\circ}$ \\
\hline 3635.327 & 8 & 10 & 27500.003 & $8378_{7 / 2}^{\circ}-35878_{7 / 2}$ & 3610.398 & 75 & 200 & 27689.879 & $7331_{5 / 2}^{\circ}-35021_{3 / 2}$ \\
\hline 3635.242 & 20 & 400 & 27500.646 & $15786_{5 / 2}-43287_{3 / 2}^{\circ}$ & 3610.125 & 50 & 200 & 27691.973 & $9585_{5 / 2}^{\circ}-37277_{7 / 2}$ \\
\hline 3634.953 & 1 & & 27502.832 & $15453_{7 / 2}^{\circ}-42955_{9 / 2}^{\circ}$ & 3610.036 & $200 \mathrm{~b}$ & 300 & 27692.656 & $13406_{13 / 2}^{\circ}-41099_{15 / 2}$ \\
\hline 3634.214 & 10 & & 27508.425 & $15242_{9 / 2}^{\circ}-42751_{7 / 2}$ & 3609.444 & 1000 & 600 & 27697.198 & $4113_{5 / 2}-31810_{5 / 2}^{\circ}$ \\
\hline 3634.002 & 1 & & 27510.029 & $13818_{7 / 2}^{\circ}-41328_{5 / 2}^{\circ}$ & 3609.224 & 100 & 300 & 27698.886 & $10673_{5 / 2}^{\circ}-38372_{3 / 2}$ \\
\hline 3633.740 & 1 & 2 & 27512.013 & $27526_{9 / 2}-55038_{9 / 2}^{\circ}$ & 3607.910 & 2 & & 27708.974 & $27787_{9 / 2}^{\circ}-55496_{9 / 2}^{\circ}$ \\
\hline 3633.683 & 1 & 2 & 27512.445 & $22014_{11 / 2}^{\circ}-49527_{13 / 2}$ & 3607.392 & 10 & 150 & 27712.952 & $15242_{9 / 2}^{\circ}-42955_{9 / 2}$ \\
\hline 3633.369 & 2 & 15 & 27514.822 & $24757_{9 / 2}^{\circ}-52272_{7 / 2}$ & 3607.009 & 2 & & 27715.895 & $14484_{11 / 2}^{\circ}-42200_{9 / 2}$ \\
\hline 3633.077 & 3 & 2 & 27517.034 & $15710_{3 / 2}^{\circ}-43227_{5 / 2}^{\circ}$ & 3606.963 & 2 & & 27716.248 & $10673_{5 / 2}^{\circ}-38389_{7 / 2}$ \\
\hline 3632.625 & 75 & 100 & 27520.457 & $9061_{5 / 2}-36581_{3 / 2}^{\circ}$ & 3606.696 & 3 & & 27718.300 & $20288_{11 / 2}^{\circ}-48006_{9 / 2}^{\circ}$ \\
\hline 3631.151 & 4 & 5 & 27531.629 & $16564_{11 / 2}^{\circ}-44096_{9 / 2}$ & 3606.505 & 2 & & 27719.768 & $10572_{9 / 2}^{\circ}-38291_{7 / 2}$ \\
\hline 3630.677 & 2 & & 27535.223 & $23372_{3 / 2}^{\circ}-50907_{3 / 2}$ & 3605.654 & 20 & 200 & 27726.310 & $10855_{7 / 2}-38581_{5 / 2}^{\circ}$ \\
\hline 3630.613 & 15 & 15 & 27535.708 & $17272_{9 / 2}^{\circ}-44807_{7 / 2}$ & 3605.440 & 15 & 20 & 27727.956 & $14790_{7 / 2}^{\circ}-42518_{7 / 2}$ \\
\hline 3629.278 & 4 & 2 & 27545.837 & $14790_{7 / 2}^{\circ}-42336_{5 / 2}$ & 3605.296 & 2 & & 27729.063 & $17460_{5 / 2}^{\circ}-45189_{5 / 2}$ \\
\hline 3627.799 & 5 & 40 & 27557.066 & $9720_{7 / 2}^{\circ}-37277_{7 / 2}$ & 3605.117 & 3 & & 27730.440 & $12485_{7 / 2}^{\circ}-40216_{5 / 2}$ \\
\hline 3627.658 & 2 & 1 & 27558.138 & $21131_{3 / 2}^{\circ}-48689_{3 / 2}$ & 3604.039 & $8 b$ & & 27738.734 & $18816_{13 / 2}^{\circ}-46555_{11 / 2}^{\circ}$ \\
\hline 3627.591 & 4 & 50 & 27558.646 & $20310_{5 / 2}^{\circ}-47869_{3 / 2}$ & 3603.620 & 10 & 100 & 27741.959 & $12902_{3 / 2}^{\circ}-40644_{5 / 2}$ \\
\hline 3627.265 & 10 & 50 & 27561.123 & $14790_{\pi / 2}^{\circ}-42352_{5 / 2}$ & 3603.362 & 50 & 200 & 27743.946 & $12472_{5 / 2}^{\circ}-40216_{5 / 2}$ \\
\hline 3627.150 & 8 & 75 & 27561.997 & $18816_{13 / 2}^{\circ}-46378_{13 / 2}$ & 3603.204 & 300 & 300 & 27745.162 & $9720_{7 / 2}^{\circ}-37465_{5 / 2}^{\circ}$ \\
\hline 3627.073 & 15 & 20 & 27562.582 & $6168_{\tau / 2}^{\circ}-33730_{5 / 2}$ & 3603.031 & 20 & 50 & 27746.494 & $8378_{7 / 2}^{\circ}-36125_{9 / 2}$ \\
\hline 3626.090 & 25 & 50 & 27570.054 & $6700_{9 / 2}^{\circ}-34270_{9 / 2}$ & 3602.936 & 5 & & 27747.226 & $11116_{\pi / 2}^{\circ}-38863_{5 / 2}$ \\
\hline 3625.931 & $75 b$ & $75 b$ & 27571.263 & $9238_{9 / 2}^{\circ}-36809_{7 / 2}^{\circ}$ & 3602.646 & 1 & & 27749.459 & $20120_{5 / 2}^{\circ}-47869_{3 / 2}$ \\
\hline 3625.628 & 500 & 300 & 27573.567 & $1521_{5 / 2}-29095_{5 / 2}^{\circ}$ & 3602.478 & 4 & 5 & 27750.754 & $12472_{5 / 2}^{\circ}-40222_{3 / 2}$ \\
\hline 3625.536 & 5 & 20 & 27574.267 & $11576_{3 / 2}^{\circ}-39150_{3 / 2}$ & 3601.980 & 5 & 50 & 27754.590 & $13468_{9 / 2}^{\circ}-41223_{11 / 2}$ \\
\hline 3624.896 & 300 & 400 & 27579.135 & $6700_{9 / 2}^{\circ}-34279_{7 / 2}^{\circ}$ & 3601.034 & 100 & 500 & 27761.881 & $17727_{11 / 2}-45489_{9 / 2}^{\circ}$ \\
\hline 3624.776 & 2 & 2 & 27580.048 & $24982_{7 / 2}^{\circ}-52562_{7 / 2}$ & 3600.816 & 8 & 100 & 27763.562 & $14349_{1 / 2}-42112_{3 / 2}^{\circ}$ \\
\hline 3624.014 & 3 & 4 & 27585.847 & $18214_{3 / 2}^{\circ}-45800_{5 / 2}$ & 3600.606 & 2 & 3 & 27765.181 & $22642_{9 / 2}^{\circ}-50407_{7 / 2}$ \\
\hline 3623.847 & 1 & 3 & 27587.118 & $31800_{7 / 2}^{\circ}-59387_{7 / 2}^{\circ}$ & 3599.872 & 5 & 75 & 27770.842 & $16033_{5 / 2}^{\circ}-43803_{7 / 2}$ \\
\hline 3623.034 & 8 & 20 & 27593.308 & $10572_{9 / 2}^{\circ}-38165_{7 / 2}$ & 3599.355 & 10 & 100 & 27774.831 & $15453_{7 / 2}^{\circ}-43227_{5 / 2}^{\circ}$ \\
\hline 3621.168 & 3 & 5 & 27607.527 & $18973_{7 / 2}^{\circ}-46581_{5 / 2}$ & 3599.030 & 5 & 50 & 27777.339 & $15349_{11 / 2}^{\circ}-43127_{11 / 2}$ \\
\hline 3621.118 & 200 & 500 & 27607.908 & $10572_{9 / 2}^{\circ}-38179_{9 / 2}$ & 3596.901 & 8 & 20 & 27793.780 & $15453_{7 / 2}^{\circ}-43246_{7 / 2}$ \\
\hline 3620.569 & 4 & 1 & 27612.094 & $11116_{7 / 2}^{\circ}-38728_{5 / 2}$ & 3595.326 & 20 & 100 & 27805.955 & $12472_{5 / 2}^{\circ}-40278_{3 / 2}$ \\
\hline 3620.502 & $25 b$ & 4 & 27612.605 & $16564_{11 / 2}^{\circ}-44177_{11 / 2}$ & 3594.110 & 50 & 75 & 27815.363 & $4113_{5 / 2}-31928_{3 / 2}^{\circ}$ \\
\hline 3620.370 & 100 & 200 & 27613.612 & $12570_{7 / 2}-40184_{7 / 2}^{\circ}$ & 3593.880 & 25 & 300 & 27817.143 & $13406_{13 / 2}^{\circ}-41223_{11 / 2}$ \\
\hline 3619.709 & 20 & 100 & 27618.654 & $10673_{5 / 2}^{\circ}-38291_{7 / 2}$ & 3593.855 & $8 b$ & & 27817.336 & $10572_{9 / 2}^{\circ}-38389_{7 / 2}$ \\
\hline 3619.356 & 4 & 40 & 27621.348 & $12902_{3 / 2}^{\circ}-40523_{1 / 2}$ & 3593.392 & 1 & 2 & 27820.920 & $22139_{9 / 2}^{\circ}-49960_{7 / 2}$ \\
\hline
\end{tabular}


TABLE 3. Classified lines of Th II-Continued

\begin{tabular}{|c|c|c|c|c|c|c|c|c|c|}
\hline \multirow{2}{*}{$\begin{array}{c}\text { Wavelength } \\
\AA\end{array}$} & \multicolumn{2}{|c|}{ Intensity } & \multirow{2}{*}{$\begin{array}{c}\text { Wavenumber } \\
\mathbf{c m}^{-1}\end{array}$} & \multirow{2}{*}{ Classification } & \multirow{2}{*}{$\begin{array}{c}\text { Wavelength } \\
\AA\end{array}$} & \multicolumn{2}{|c|}{ Intensity } & \multirow{2}{*}{$\begin{array}{c}\text { Wavenumber } \\
\mathbf{c m}^{-1}\end{array}$} & \multirow{2}{*}{ Classification } \\
\hline & Lamp & Spark & & & & Lamp & Spark & & \\
\hline 3593.150 & 2 & 3 & 27822.794 & $22014_{11 / 2}^{\circ}-49837_{9 / 2}$ & 3559.964 & $50 \mathrm{~b}$ & $25 b$ & 28082.151 & $8605_{5 / 2}-36687_{5 / 2}^{\circ}$ \\
\hline 3592.826 & $150 \mathrm{~b}$ & 100 & 27825.303 & $9238_{9 / 2}^{\circ}-37063_{9 / 2}^{\circ}$ & 3559.940 & $50 \mathrm{~b}$ & 500 & 28082.340 & $12488_{9 / 2}^{\circ}-40570_{7 / 2}$ \\
\hline 3592.487 & 2 & 50 & 27827.929 & $22642_{9 / 2}^{\circ}-50470_{9 / 2}^{\circ}$ & 3559.730 & 25 & 100 & 28083.997 & $12570_{7 / 2}-40654_{5 / 2}^{\circ}$ \\
\hline 3591.906 & 3 & & 27832.430 & $16818_{7 / 2}-44650_{7 / 2}^{\circ}$ & 3559.614 & 150 & 200 & 28084.912 & $12485_{7 / 2}^{\circ}-40570_{7 / 2}$ \\
\hline 3591.607 & 3 & 3 & 27834.747 & $14101_{i / 2}^{\circ}-41936_{3 / 2}$ & 3559.450 & $300 \mathrm{~b}$ & 500 & 28086.206 & $6244_{1 / 2}-34330_{1 / 2}^{\circ}$ \\
\hline 3589.358 & 150 & 300 & 27852.187 & $6691_{3 / 2}^{\circ}-34543_{5 / 2}^{\circ}$ & 3559.415 & $25 b$ & $50 \mathrm{~b}$ & 28086.482 & $4490_{5 / 2}^{\circ}-32576_{7 / 2}$ \\
\hline 3589.153 & $50 \mathrm{~b}$ & 200 & 27853.777 & $6700_{9 / 2}^{\circ}-34553_{9 / 2}^{\circ}$ & & & & & $12488_{9 / 2}^{\circ}-40574_{11 / 2}^{\circ}$ \\
\hline 3589.127 & $5 b$ & & 27853.979 & $17272_{9 / 2}^{\circ}-45126_{9 / 2}^{\circ}$ & 3558.842 & 3 & 25 & 28091.004 & $13818_{7 / 2}^{\circ}-41909_{9 / 2}$ \\
\hline 3588.974 & 1 & & 27855.167 & $20989_{9 / 2}^{\circ}-48844_{9 / 2}$ & 3558.471 & 2 & 50 & 28093.933 & $18816_{13 / 2}^{\circ}-46910_{13 / 2}$ \\
\hline 3588.640 & 3 & 2 & 27857.759 & $9711_{7 / 2}-37569_{9 / 2}^{\circ}$ & 3558.344 & 2 & 3 & 28094.935 & $19050_{3 / 2}^{\circ}-47145_{1 / 2}$ \\
\hline 3588.300 & 50 & 75 & 27860.399 & $1859_{3 / 2}-29720_{3 / 2}^{\circ}$ & 3558.120 & 1 & & 28096.704 & $15710_{3 / 2}^{\circ}-43807_{3 / 2}$ \\
\hline 3588.216 & 20 & 75 & 27861.051 & $9202_{7 / 2}^{\circ}-37063_{9 / 2}$ & 3557.898 & 1 & & 28098.457 & $12472_{5 / 2}^{\circ}-40570_{7 / 2}$ \\
\hline 3587.051 & 2 & & 27870.099 & $13818_{7 / 2}^{\circ}-41688_{7 / 2}^{\circ}$ & 3557.727 & 2 & & 28099.808 & $20969_{7 / 2}^{\circ}-49068_{5 / 2}$ \\
\hline 3586.941 & 4 & 50 & 27870.954 & $14275_{9 / 2}^{\circ}-42146_{11 / 2}$ & 3557.464 & 100 & 300 & 28101.885 & $6168_{7 / 2}^{\circ}-34270_{9 / 2}$ \\
\hline 3585.884 & 10 & 40 & 27879.169 & $12488_{9 / 2}^{\circ}-40367_{9 / 2}^{\circ}$ & 3556.313 & 15 & 100 & 28110.980 & $6168_{7 / 2}^{\circ}-34279_{7 / 2}^{\circ}$ \\
\hline 3585.769 & 40 & 75 & 27880.063 & $9585_{5 / 2}^{\circ}-37465_{5 / 2}$ & 3555.101 & 75 & 100 & 28120.563 & $9720_{7 / 2}^{\circ}-37840_{9 / 2}$ \\
\hline 3585.549 & 5 & 20 & 27881.774 & $12485_{7 / 2}^{\circ}-40367_{9 / 2}^{\circ}$ & 3554.823 & 5 & $15 b$ & 28122.762 & $17272_{9 / 2}^{\circ}-45395_{7 / 2}$ \\
\hline 3585.050 & 20 & 40 & 27885.655 & $16564_{11 / 2}^{\circ}-44450_{9 / 2}$ & 3554.644 & 4 & 8 & 28124.178 & $11576_{3 / 2}^{\circ}-39700_{5 / 2}^{\circ}$ \\
\hline 3583.036 & 8 & 200 & 27901.328 & $16906_{7 / 2}^{\circ}-44807_{7 / 2}$ & 3553.377 & 50 & 200 & 28134.206 & $9711_{7 / 2}-37846_{5 / 2}^{\circ}$ \\
\hline 3582.391 & 3 & 2 & 27906.352 & $14101_{1 / 2}^{o}-42008_{1 / 2}$ & 3553.110 & 100 & 300 & 28136.320 & $12570_{7 / 2}-40706_{7 / 2}^{\circ}$ \\
\hline 3582.217 & 1 & & 27907.707 & $26586_{3 / 2}^{\circ}-54493_{5 / 2}$ & 3551.869 & 8 & 150 & 28146.151 & $15236_{3 / 2}-43382_{5 / 2}^{\circ}$ \\
\hline 3582.006 & 100 & 300 & 27909.351 & $10855_{7 / 2}-38764_{7 / 2}^{\circ}$ & 3550.292 & 50 & 200 & 28158.653 & $12485_{7 / 2}^{\circ}-40644_{5 / 2}$ \\
\hline 3580.226 & $25 b$ & 200 & 27923.227 & $12488_{9 / 2}^{\circ}-40411_{7 / 2}$ & 3549.736 & 40 & 200 & 28163.063 & $10673_{5 / 2}^{\circ}-38836_{3 / 2}^{\circ}$ \\
\hline 3580.043 & 3 & 10 & 27924.654 & $14275_{9 / 2}^{\circ}-42200_{9 / 2}$ & 3549.179 & 1 & & 28167.483 & $23697_{7 / 2}^{\circ}-51865_{5 / 2}$ \\
\hline 3579.414 & 5 & & 27929.561 & $13468_{9 / 2}^{\circ}-41398_{9 / 2}^{\circ}$ & 3548.589 & 2 & & 28172.166 & $12472_{5 / 2}^{\circ}-40644_{5 / 2}$ \\
\hline 3579.334 & 1001 & 200 & 27930.185 & $8460_{3 / 2}-36390_{3 / 2}^{\circ}$ & 3548.175 & 1 & & 28175.453 & $24132_{3 / 2}^{\circ}-52307_{3 / 2}$ \\
\hline 3578.164 & 20 & 200 & 27939.318 & $12472_{5 / 2}^{\circ}-404.11_{7 / 2}^{\circ}$ & 354.5 .390 & 50 & 200 & 28197.585 & $7001_{3 / 2}-35198_{1 / 2}^{\circ}$ \\
\hline 3577.214 & 10 & 5 & 27946.737 & $14275_{9 / 2}^{\circ}-42222_{7 / 2}$ & & & & & $18973_{7 / 2}^{\circ}-47171_{9 / 2}$ \\
\hline 3575.930 & 2 & 4 & 27956.772 & $9585_{5 / 2}^{\circ}-37542_{3 / 2}$ & 3545.284 & 100 & 75 & 28198.428 & $1521_{5 / 2}-29720_{3 / 2}^{\circ}$ \\
\hline 3575.320 & $300 \mathrm{~b}$ & 400 & 27961.541 & $6700_{9 / 2}^{\circ}-34661_{11 / 2}^{\circ}$ & 3545.180 & 50 & 200 & 28199.255 & $13248_{9 / 2}-41447_{11 / 2}$ \\
\hline 3573.811 & 2 & 2 & 27973.347 & $14545_{5 / 2}^{\circ}-42518_{7 / 2}$ & 3544.993 & $40 \mathrm{~b}$ & 75 & 28200.742 & $9400_{5 / 2}-37601_{3 / 2}^{\circ}$ \\
\hline 3573.508 & 40 & 100 & 27975.719 & $8605_{5 / 2}-36581_{3 / 2}^{\circ}$ & 3544.775 & 10 & 40 & 28202.477 & $9585_{5 / 2}^{\circ}-37787_{7 / 2}$ \\
\hline 3573.220 & 100 & 300 & 27977.974 & $8605_{5 / 2}-36583_{7 / 2}^{\circ}$ & 3544.361 & 10 & 50 & 28205.771 & $14545_{5 / 2}^{\circ}-42751_{7 / 2}$ \\
\hline 3572.392 & 150 & 400 & 27984.459 & $8460_{3 / 2}-36444_{3 / 2}^{\circ}$ & 3542.639 & $40 \mathrm{~b}$ & 50 & 28219.481 & $13468_{9 / 2}^{\circ}-41688_{7 / 2}^{\circ}$ \\
\hline 3572.299 & 40 & 150 & 27985.187 & $7001_{3 / 2}-34986_{3 / 2}^{\circ}$ & 3542.620 & $40 \mathrm{~b}$ & 50 & 28219.632 & $16906_{7 / 2}^{\circ}-45126_{9 / 2}^{\circ}$ \\
\hline 3571.810 & 2 & 2 & 27989.018 & $22642_{9 / 2}^{\circ}-50631_{11 / 2}$ & 3542.301 & 4 & & 28222.173 & $22513_{5 / 2}^{\circ}-50735_{3 / 2}$ \\
\hline 3571.572 & 40 & 200 & 27990.883 & $10189_{11 / 2}^{\circ}-38179_{9 / 2}$ & 3542.201 & 15 & 25 & 28222.970 & $11725_{1 / 2}^{\circ}-39948_{1 / 2}$ \\
\hline 3569.918 & 3 & 4 & 28003.852 & $16818_{7 / 2}-44821_{5 / 2}^{\circ}$ & 3541.969 & 10 & 4 & 28224.818 & $9720_{7 / 2}^{\circ}-37945_{5 / 2}^{\circ}$ \\
\hline 3569.620 & 50 & 150 & 28006.189 & $6168_{\tau / 2}^{\circ}-34174_{5 / 2}$ & 3541.615 & 100 & 200 & 28227.640 & $8460_{3 / 2}-36687_{5 / 2}^{\circ}$ \\
\hline 3567.965 & 10 & 150 & 28019.180 & $13468_{9 / 2}^{\circ}-41488_{7 / 2}$ & 3540.312 & 1 & & 28238.028 & $23697_{7 / 2}^{\circ}-51935_{5 / 2}^{\circ}$ \\
\hline 3567.701 & $50 \mathrm{~b}$ & 75 & 28021.253 & $12902_{3 / 2}^{\circ}-40923_{5 / 2}$ & 3540.047 & 5 & 2 & 28240.142 & $20080_{7 / 2}^{\circ}-48320_{5 / 2}$ \\
\hline 3567.047 & 40 & 150 & 28026.390 & $6700_{9 / 2}^{\circ}-34726_{7 / 2}$ & 3539.587 & 1000 & 500 & 28243.812 & $0_{3 / 2}-28243_{5 / 2}^{\circ}$ \\
\hline 3565.721 & 10 & 5 & 28036.812 & $18118_{3 / 2}-46155_{5 / 2}^{\circ}$ & 3539.322 & 800 & 400 & 28245.927 & $4490_{5 / 2}^{\circ}-32736_{7 / 2}$ \\
\hline 3565.398 & $75 b$ & 200 & 28039.352 & $9238_{9 / 2}^{\circ}-37277_{7 / 2}^{\circ}$ & 3538.501 & 15 & 10 & 28252.480 & $12219_{3 / 2}-40472_{3 / 2}^{\circ}$ \\
\hline 3564.712 & $25 b$ & & 28044.748 & $18816_{13 / 2}^{\circ}-46861_{11 / 2}$ & 3538.211 & 50 & 100 & 28254.796 & $15710_{3 / 2}^{\circ}-43965_{1 / 2}$ \\
\hline 3564.706 & 50 & 200 & 28044.795 & $9711_{7 / 2}-37756_{7 / 2}^{\circ}$ & 3537.158 & 400 & 300 & 28263.207 & $9202_{7 / 2}^{\circ}-37465_{5 / 2}$ \\
\hline 3564.599 & 8 & 40 & 28045.637 & $10572_{9 / 2}^{\circ}-38617_{9 / 2}$ & 3536.632 & $50 \mathrm{~b}$ & 100 & 28267.410 & $16033_{5 / 2}^{\circ}-44300_{3 / 2}$ \\
\hline 3561.571 & 10 & 4 & 28069.480 & $26424_{5 / 2}^{\circ}-54493_{5 / 2}$ & 3536.609 & 3 & & 28267.594 & $22139_{9 / 2}^{\circ}-50407_{7 / 2}^{\circ}$ \\
\hline 3560.857 & 5 & & 28075.109 & $9202_{\tau / 2}^{\circ}-37277_{7 / 2}$ & 3535.863 & 1 & & 28273.558 & $19050_{3 / 2}^{\circ}-47324_{5 / 2}^{\circ}$ \\
\hline 3560.264 & 2 & 2 & 28079.785 & $21297_{5 / 2}^{\circ}-49377_{7 / 2}$ & 3535.329 & 8 & 2 & 28277.828 & $21682_{7 / 2}^{\circ}-49960_{7 / 2}$ \\
\hline
\end{tabular}


TABLE 3. Classified lines of Th $\mathrm{II}-$ Continued

\begin{tabular}{|c|c|c|c|c|c|c|c|c|c|}
\hline \multirow{2}{*}{$\begin{array}{c}\text { Wavelength } \\
\AA\end{array}$} & \multicolumn{2}{|c|}{ Intensity } & \multirow{2}{*}{$\begin{array}{c}\text { Wavenumber } \\
\mathrm{cm}^{-1}\end{array}$} & \multirow{2}{*}{ Classification } & \multirow{2}{*}{$\begin{array}{c}\text { Wavelength } \\
\AA\end{array}$} & \multicolumn{2}{|c|}{ Intensity } & \multirow{2}{*}{$\begin{array}{c}\text { Wavenumber } \\
\mathbf{c m}^{-1}\end{array}$} & \multirow{2}{*}{ Classification } \\
\hline & Lamp & Spark & & & & Lamp & Spark & & \\
\hline 3533.710 & 50 & 25 & 28290.784 & $10572_{9 / 2}^{\circ}-38862_{11 / 2}$ & 3512.351 & 10 & 25 & 28462.818 & $17272_{9 / 2}^{\circ}-45735_{11 / 2}$ \\
\hline 3532.984 & 10 & 100 & 28296.597 & $24381_{7 / 2}-52678_{5 / 2}^{\circ}$ & 3511.562 & 400 & 150 & 28469.213 & $4490_{5 / 2}^{\circ}-32959_{3 / 2}$ \\
\hline 3531.928 & 50 & 300 & 28305.057 & $18118_{3 / 2}-46423_{3 / 2}^{\circ}$ & 3511.387 & 3 & & 28470.632 & $16033_{5 / 2}^{\circ}-44503_{7 / 2}$ \\
\hline 3531.867 & 40 & 4 & 28305.546 & $14790_{7 / 2}^{\circ}-43096_{5 / 2}^{\circ / 2}$ & 3511.277 & 20 & 50 & 28471.524 & $14484_{11 / 2}^{\circ}-42955_{9 / 2}$ \\
\hline 3531.645 & 100 & 150 & 28307.325 & $18118_{3 / 2}-46426_{1 / 2}^{\circ}$ & 3510.755 & $8 b$ & & 28475.757 & $14275_{9 / 2}^{\circ}-42751_{7 / 2}$ \\
\hline 3531.049 & 3 & & 28312.103 & $23518_{7 / 2}^{\circ}-51830_{7 / 2}$ & 3510.535 & 50 & 40 & 28477.542 & $10673_{5 / 2}^{\circ}-39150_{3 / 2}$ \\
\hline 3530.134 & 8 & & 28319.441 & $15453_{\pi / 2}^{\circ}-43772_{5 / 2}$ & 3509.953 & 10 & 25 & 28482.263 & $17771_{11 / 2}^{\circ}-46253_{9 / 2}$ \\
\hline 3529.922 & 2 & & 28321.142 & $24414_{3 / 2}^{\circ}-52735_{3 / 2}$ & 3509.824 & 5 & & 28483.310 & $15324_{1 / 2}^{\circ}-43807_{3 / 2}$ \\
\hline 3528.953 & 300 & 400 & 28328.918 & $10189_{11 / 2}^{\circ}-38517_{13 / 2}^{\circ}$ & 3509.189 & 25 & 40 & 28488.464 & $16906_{7 / 2}^{\circ}-45395_{7 / 2}$ \\
\hline 3528.819 & 150 & 40 & 28329.994 & $6691_{3 / 2}^{\circ}-35021_{3 / 2}$ & 3509.123 & 10 & & 28489.000 & $17121_{3 / 2}^{\circ}-45610_{5 / 2}$ \\
\hline 3528.092 & 8 & 2 & 28335.831 & $26586_{3 / 2}^{\circ}-54922_{3 / 2}$ & 3508.819 & 1 & & 28491.468 & $22139_{9 / 2}^{\circ}-50631_{11 / 2}$ \\
\hline 3527.361 & 10 & & 28341.704 & $11116_{7 / 2}^{\circ}-39458_{7 / 2}$ & 3508.687 & 5 & 25 & 28492.540 & $23372_{3 / 2}^{\circ}-51865_{5 / 2}$ \\
\hline 3526.253 & 25 & 75 & 28350.609 & $18973_{7 / 2}^{\circ}-47324_{5 / 2}$ & 3508.180 & 20 & 40 & 28496.658 & $10572_{9 / 2}^{\circ}-39068_{7 / 2}$ \\
\hline 3525.868 & $5 \mathrm{~h}$ & 75 & 28353.704 & $21131_{3 / 2}^{\circ}-49485_{1 / 2}$ & 3506.903 & 8 & & 28507.034 & $20310_{5 / 2}^{\circ}-48817_{3 / 2}$ \\
\hline 3525.629 & $40 \mathrm{~h}$ & 75 & 28355.626 & $16033_{5 / 2}^{\circ}-44388_{5 / 2}$ & 3506.852 & 50 & 300 & 28507.449 & $15236_{3 / 2}-43744_{1 / 2}^{\circ}$ \\
\hline 3525.605 & $8 b$ & & 28355.819 & $9400_{5 / 2}-37756_{7 / 2}^{\circ}$ & 3505.493 & 100 & 200 & 28518.500 & $13818_{7 / 2}^{\circ}-42336_{5 / 2}$ \\
\hline 3525.551 & $8 \mathrm{~h}$ & $4 \mathrm{~h}$ & 28356.254 & $15453_{7 / 2}^{\circ}-43809_{9 / 2}^{\circ}$ & 3505.384 & 10 & 15 & 28519.387 & $12472_{5 / 2}^{\circ}-40991_{3 / 2}$ \\
\hline 3525.122 & 25 & & 28359.704 & $9585_{5 / 2}^{\circ}-37945_{5 / 2}$ & 3505.350 & 5 & 10 & 28519.663 & $9585_{5 / 2}^{\circ}-38105_{5 / 2}$ \\
\hline 3524.230 & 3 & & 28366.882 & $18214_{3 / 2}^{\circ}-46581_{5 / 2}$ & 3504.100 & 2 & & 28529.837 & $14484_{11 / 2}^{\circ}-43014_{13 / 2}$ \\
\hline 3524.068 & 2 & & 28368.186 & $20989_{9 / 2}^{\circ}-49357_{11 / 2}^{\circ}$ & 3504.037 & 25 & 75 & 28530.350 & $17722_{9 / 2}^{\circ}-46253_{9 / 2}^{\circ}$ \\
\hline 3523.615 & 8 & 8 & 28371.833 & $20120_{5 / 2}^{\circ}-48492_{5 / 2}$ & 3503.615 & 100 & 200 & 28533.786 & $13818_{7 / 2}^{\circ}-42352_{5 / 2}$ \\
\hline 3523.196 & 10 & 8 & 28375.207 & $6168_{7 / 2}^{\circ}-34543_{5 / 2}$ & 3502.778 & $100 \mathrm{~b}$ & 200 & 28540.604 & $9061_{5 / 2}-37601_{3 / 2}^{\circ}$ \\
\hline 3522.569 & 3 & & 28380.258 & $22355_{i / 2}^{\circ}-50735_{3 / 2}$ & 3502.701 & 10 & 10 & 28541.232 & $10855_{7 / 2}-39396_{7 / 2}^{\circ}$ \\
\hline 3522.367 & $5 b$ & & 28381.885 & $13818_{7 / 2}^{\circ}-42200_{9 / 2}^{\circ}$ & 3502.648 & 1 & & 28541.663 & $23730_{9 / 2}^{\circ}-52272_{7 / 2}$ \\
\hline 3521.912 & 800 & 300 & 28385.552 & $10379_{9 / 2}-38764_{7 / 2}^{\circ}$ & 3501.945 & 25 & 25 & 28547.393 & $7331_{5 / 2}^{\circ}-35878_{7 / 2}$ \\
\hline 3521.688 & 4 & & 28387.357 & $20989_{9 / 2}^{\circ}-49377_{7 / 2}^{\circ}$ & 3501.642 & 15 & 25 & 28549.863 & $9238_{9 / 2}^{\circ}-37787_{7 / 2}$ \\
\hline 3521.514 & 4 & & 28388.760 & $18214_{3 / 2}^{\circ}-46603_{5 / 2}$ & 3501.455 & 200 & 200 & 28551.388 & $10189_{11 / 2}^{\circ}-38740_{11 / 2}$ \\
\hline 3520.801 & 1 & & 28394.509 & $22513_{5 / 2}^{\circ}-50907_{3 / 2}$ & 3500.843 & 20 & 100 & 28556.379 & $20288_{i 1 / 2}^{\circ}-48844_{9 / 2}$ \\
\hline 3519.869 & 1 & & 28402.027 & $17983_{5 / 2}^{\circ}-46385_{7 / 2}$ & 3500.531 & 40 & 75 & 28558.924 & $12488_{9 / 2}^{\circ}-41047_{9 / 2}$ \\
\hline 3519.741 & 5 & & 28403.060 & $25607_{9 / 2}^{\circ}-54010_{7 / 2}^{\circ}$ & 3500.267 & 25 & 200 & 28561.078 & $15242_{9 / 2}^{\circ}-43803_{7 / 2}^{\circ}$ \\
\hline 3519.688 & 50 & 200 & 28403.488 & $16818_{7 / 2}-45221_{5 / 2}^{\circ}$ & 3500.206 & 10 & 4 & 28561.575 & $16564_{i 1 / 2}^{\circ}-45126_{9 / 2}$ \\
\hline 3519.482 & 1 & & 28405.150 & $25440_{5 / 2}^{\circ}-53845_{5 / 2}^{\circ}$ & 3499.986 & 200 & 200 & 28563.371 & $8018_{3 / 2}-36581_{3 / 2}^{\circ}$ \\
\hline 3518.902 & $25 b$ & & 28409.832 & $7331_{5 / 2}^{\circ}-35741_{5 / 2}$ & 3498.989 & $75 b$ & $100 \mathrm{~b}$ & 28571.509 & $9720_{7 / 2}^{\circ}-38291_{7 / 2}$ \\
\hline 3518.713 & 4 & & 28411.357 & $20080_{\pi / 2}^{\circ}-48492_{5 / 2}$ & 3498.478 & 1 & & 28575.682 & $21297_{5 / 2}^{\circ}-49873_{5 / 2}$ \\
\hline 3518.558 & $4 \mathrm{~s}$ & & 28412.609 & $17983_{5 / 2}^{\circ}-46395_{3 / 2}$ & 3498.008 & 100 & 75 & 28579.522 & $1521_{5 / 2}-30101_{7 / 2}^{\circ}$ \\
\hline 3516.903 & 15 & & 28425.979 & $12902_{3 / 2}^{\circ}-41328_{5 / 2}$ & 3497.955 & 15 & 50 & 28579.955 & $9585_{5 / 2}^{\circ}-38165_{7 / 2}$ \\
\hline 3516.823 & 200 & 75 & 28426.626 & $8018_{3 / 2}-36444_{3 / 2}^{\circ}$ & 3497.686 & 15 & 200 & 28582.153 & $22642_{9 / 2}^{\circ}-51224_{9 / 2}$ \\
\hline 3516.576 & 50 & 40 & 28428.623 & $10189_{11 / 2}^{\circ}-38617_{9 / 2}$ & 3497.460 & 8 & 40 & 28584.000 & $11116_{7 / 2}^{\circ}-39700_{5 / 2}$ \\
\hline 3516.354 & 200 & 300 & 28430.417 & $8378_{i / 2}^{\circ}-36809_{7 / 2}$ & 3497.262 & 50 & 200 & 28585.618 & $9202_{7 / 2}^{\circ}-37787_{7 / 2}$ \\
\hline 3515.849 & 15 & 25 & 28434.501 & $12219_{3 / 2}-40654_{5 / 2}^{\circ}$ & 3497.010 & 40 & 200 & 28587.678 & $10855_{7 / 2}-39443_{9 / 2}^{\circ}$ \\
\hline 3515.706 & 75 & 100 & 28435.657 & $1116_{7 / 2}^{\circ}-39552_{9 / 2}$ & 3496.762 & 8 & 15 & 28589.705 & $15710_{3 / 2}^{\circ}-44300_{3 / 2}$ \\
\hline 3515.555 & 8 & & 28436.879 & $14790_{7 / 2}^{\circ}-43227_{5 / 2}$ & 3495.158 & 25 & 4 & 28602.825 & $9238_{9 / 2}^{\circ}-37840_{9 / 2}$ \\
\hline 3515.424 & 10 & & 28437.938 & $12485_{7 / 2}^{\circ}-40923_{5 / 2}$ & 3494.815 & 25 & 10 & 28605.632 & $12219_{3 / 2}-40825_{1 / 2}^{\circ}$ \\
\hline 3515.224 & 15 & & 28439.556 & $23730_{9 / 2}^{\circ}-52170_{11 / 2}^{\circ}$ & 3493.518 & 500 & 200 & 28616.252 & $7828_{1 / 2}-36444_{3 / 2}^{\circ}$ \\
\hline 3514.960 & 100 & 100 & 28441.692 & $9238_{9 / 2}^{\circ}-37679_{11 / 2}^{\circ}$ & 3493.084 & 8 & 20 & 28619.807 & $17983_{5 / 2}^{\circ}-46603_{5 / 2}$ \\
\hline 3514.526 & $300 \mathrm{~b}$ & 200 & 28445.204 & $9400_{5 / 2}-37846_{5 / 2}^{\circ}$ & 3492.898 & 5 & 10 & 28621.331 & $19248_{5 / 2}^{\circ}-47869_{3 / 2}$ \\
\hline 3513.755 & 20 & 75 & 28451.445 & $12472_{5 / 2}^{\circ}-40923_{5 / 2}$ & 3492.680 & 8 & 25 & 28623.118 & $19248_{5 / 2}^{\circ}-47871_{7 / 2}$ \\
\hline 3513.299 & 15 & 40 & 28455.138 & $22014_{1 / 2}^{\circ}-50470_{9 / 2}$ & 3492.327 & 4 & 5 & 28626.011 & $22642_{9 / 2}^{\circ}-51268_{7 / 2}$ \\
\hline 3513.213 & 50 & 75 & 28455.835 & $14790_{7 / 2}^{\circ}-43246_{7 / 2}$ & 3492.117 & 4 & 3 & 28627.732 & $15144_{3 / 2}^{\circ}-43772_{5 / 2}^{\circ}$ \\
\hline 3512.742 & $100 \mathrm{~b}$ & 200 & 28459.650 & $9720_{7 / 2}^{\circ}-38179_{9 / 2}$ & 3491.580 & 75 & 100 & 28632.135 & $17272_{9 / 2}^{\circ}-45904_{9 / 2}$ \\
\hline
\end{tabular}


TABLE 3. Classified lines of Th $\mathrm{II}-$ Continued

\begin{tabular}{|c|c|c|c|c|c|c|c|c|c|}
\hline \multirow{2}{*}{$\begin{array}{c}\text { Wavelength } \\
\AA\end{array}$} & \multicolumn{2}{|c|}{ Intensity } & \multirow{2}{*}{$\begin{array}{c}\text { Wavenumber } \\
\mathbf{c m}^{-1}\end{array}$} & \multirow{2}{*}{ Classification } & \multirow{2}{*}{$\begin{array}{c}\text { Wavelength } \\
\AA\end{array}$} & \multicolumn{2}{|c|}{ Intensity } & \multirow{2}{*}{$\begin{array}{l}\text { Wavenumber } \\
\mathbf{c m}^{-1}\end{array}$} & \multirow{2}{*}{ Classification } \\
\hline & Lamp & Spark & & & & Lamp & Spark & & \\
\hline 3490.795 & lb & & 28638.574 & $9202_{7 / 2}^{\circ}-37840_{9 / 2}$ & 3473.092 & 4 & & 28784.545 & $17771_{11 / 2}^{\circ}-46555_{11 / 2}$ \\
\hline 3490.656 & 5 & 4 & 28639.714 & $11576_{i 3 / 2}^{\circ}-40216_{5 / 2}^{\circ}$ & 3473.027 & $150 \mathrm{~b}$ & 100 & 28785.084 & $9061_{5 / 2}-37846_{5 / 2}^{\circ}$ \\
\hline 3490.504 & 8 & 25 & 28640.961 & $17727_{11 / 2}-46368_{9 / 2}^{\circ}$ & 3472.842 & 15 & 10 & 28786.618 & $9585_{5 / 2}^{\circ}-38372_{3 / 2}$ \\
\hline 3490.452 & 50 & 100 & 28641.388 & $15324_{1 / 2}^{\circ}-43965_{1 / 2}$ & 3472.759 & 3 & & 28787.306 & $21682_{7 / 2}^{\circ}-50470_{9 / 2}^{\circ}$ \\
\hline 3490.389 & 2 & & 28641.905 & $23012_{3 / 2}^{\circ}-51653_{1 / 2}$ & 3470.788 & 4 & & 28803.653 & $17460_{5 / 2}^{\circ}-46264_{3 / 2}^{\circ}$ \\
\hline 3490.269 & 20 & 100 & 28642.889 & $14484_{11 / 2}^{\circ}-43127_{11 / 2}$ & 3469.919 & $2000 \mathrm{~h}$ & 800 & 28810.866 & $4146_{7 / 2}-32957_{7 / 2}^{\circ}$ \\
\hline 3490.230 & 8 & 10 & 28643.209 & $15453_{7 / 2}^{\circ}-44096_{9 / 2}$ & 3468.966 & 3 & 8 & 28818.781 & $19050_{3 / 2}^{\circ}-4.7869_{3 / 2}$ \\
\hline 3489.829 & 25 & 20 & 28646.501 & $11576_{3 / 2}^{\circ}-40222_{3 / 2}$ & 3468.714 & 25 & 10 & 28820.875 & $15144_{3 / 2}^{\circ}-43965_{1 / 2}$ \\
\hline 3487.884 & 5 & & 28662.475 & $17722_{9 / 2}^{\circ}-46385_{7 / 2}$ & 3468.219 & 800 & 300 & 28824.988 & $6700_{9 / 2}^{\circ}-35525_{11 / 2}$ \\
\hline 3487.845 & 75 & 100 & 28662.795 & $15144_{3 / 2}^{\circ}-43807_{3 / 2}$ & 3467.301 & 3 & & 28832.619 & $17722_{9 / 2}^{\circ}-46555_{11 / 2}$ \\
\hline 3487.805 & 1 & & 28663.124 & $21297_{5 / 2}^{\circ}-49960_{7 / 2}$ & 3466.896 & 20 & 15 & 28835.987 & $20288_{11 / 2}^{\circ}-49124_{11 / 2}$ \\
\hline 3487.700 & 3 & & 28663.987 & $23012_{3 / 2}^{\circ}-51676_{3 / 2}^{\circ}$ & 3465.924 & 200 & 15 & 28844.074 & $4113_{5 / 2}-32957_{7 / 2}^{\circ}$ \\
\hline 3487.081 & 20 & 75 & 28669.075 & $9720_{7 / 2}^{\circ}-38389_{7 / 2}$ & 3465.764 & 1000 & 200 & 28845.406 & $6700_{9 / 2}^{\circ}-35545_{9 / 2}$ \\
\hline 3486.971 & 40 & 100 & 28669.979 & $8460_{3 / 2}-37130_{1 / 2}^{\circ}$ & 3465.471 & 8 & 15 & 28847.844 & $20989_{9 / 2}^{\circ}-49837_{9 / 2}$ \\
\hline 3486.841 & 8 & & 28671.048 & $16818_{7 / 2}-45489_{9 / 2}^{\circ}$ & 3465.262 & 3 & 10 & 28849.584 & $22513_{5 / 2}^{\circ}-51362_{5 / 2}^{\circ}$ \\
\hline 3486.514 & $500 \mathrm{~b}$ & 200 & 28673.737 & $10189_{11 / 2}^{\circ}-38862_{11 / 2}$ & 3465.017 & $50 \mathrm{~b}$ & 75 & 28851.624 & $14275_{9 / 2}^{\circ}-43127_{11 / 2}^{\circ}$ \\
\hline 3486.050 & 31 & & 28677.554 & $13468_{9 / 2}^{\circ}-42146_{11 / 2}$ & 3464.841 & 8 & $20 \mathrm{~h}$ & 28853.090 & $23012_{3 / 2}^{\circ}-51865_{5 / 2}^{\circ}$ \\
\hline 3485.917 & 4 & & 28678.648 & $17121_{3 / 2}^{\circ}-45800_{5 / 2}$ & 3464.471 & 15 & 8 & 28856.171 & $12472_{5 / 2}^{\circ}-41328_{5 / 2}$ \\
\hline 3485.721 & 5 & 5 & 28680.260 & $14275_{9 / 2}^{\circ}-42955_{9 / 2}^{\circ}$ & 3464.409 & 40 & 50 & 28856.687 & $16033_{5 / 2}^{\circ}-44889_{3 / 2}^{\circ}$ \\
\hline 3485.473 & 15 & 50 & 28682.301 & $14545_{5 / 2}^{\circ}-43227_{5 / 2}^{\circ}$ & 3464.228 & 2 & & 28858.195 & $18816_{13 / 2}^{\circ}-47675_{11 / 2}^{\circ}$ \\
\hline 3485.212 & $100 \mathrm{~b}$ & 150 & 28684.449 & $8378_{7 / 2}^{\circ}-37063_{9 / 2}$ & 3463.719 & 500 & 400 & 28862.436 & $13250_{5 / 2}-42112_{3 / 2}^{\circ}$ \\
\hline 3484.414 & 5 & 25 & 28691.018 & $20686_{5 / 2}^{\circ}-49377_{7 / 2}$ & 3463.592 & 20 & 8 & 28863.494 & $15786_{5 / 2}-44650_{7 / 2}^{\circ}$ \\
\hline 3484.080 & 100 & 100 & 28693.768 & $10673_{5 / 2}^{\circ}-39366_{5 / 2}$ & 3463.424 & 1 & & 28864.894 & $23697_{7 / 2}^{\circ}-52562_{7 / 2}^{\circ}$ \\
\hline 3483.589 & 3 & & 28697.813 & $20120_{5 / 2}^{\circ}-48817_{3 / 2}$ & 3462.850 & 800 & 400 & 28869.678 & $9711_{7 / 2}-38581_{5 / 2}^{\circ}$ \\
\hline 3483.409 & $20 \mathrm{~b}$ & 50 & 28699.295 & $14545_{5 / 2}^{\circ}-43244_{3 / 2}$ & 3460.223 & 3 & & 28891.596 & $17460_{5 / 2}^{\circ}-46352_{7 / 2}$ \\
\hline 3483.254 & 10 & 25 & 28700.572 & $13818_{7 / 2}^{\circ}-42518_{7 / 2}^{\circ}$ & 3459.974 & 5 & 15 & 28893.675 & $16906_{\bar{\tau} / 2}^{0}-45800_{5 / 2}$ \\
\hline 3483.169 & 15 & 100 & 28701.273 & $14545_{5 / 2}^{\circ}-43246_{7 / 2}$ & 3459.641 & $75 \mathrm{~b}$ & 10 & 28896.456 & $10189_{11 / 2}^{\circ}-39085_{13 / 2}$ \\
\hline 3483.113 & 8 & 5 & 28701.734 & $11576_{3 / 2}^{\circ}-40278_{3 / 2}$ & 3459.397 & 5 & 4 & 28898.494 & $8378_{7 / 2}^{\circ}-37277_{7 / 2}$ \\
\hline 3482.834 & 20 & 150 & 28704.033 & $16906_{7 / 2}^{\circ}-45610_{5 / 2}$ & 3458.882 & 5 & 8 & 28902.797 & $9202_{7 / 2}^{\circ}-38105_{5 / 2}^{\circ}$ \\
\hline 3482.761 & 100 & 100 & 28704.635 & $1859_{3 / 2}-30564_{1 / 2}^{\circ}$ & 3458.724 & 3 & & 28904.117 & $20969_{7 / 2}^{\circ}-49873_{5 / 2}$ \\
\hline 3482.547 & 200 & 100 & 28706.399 & $9585_{5 / 2}^{\circ}-38291_{7 / 2}$ & 3457.683 & 15 & 8 & 28912.819 & $12485_{\tau / 2}^{\circ}-41398_{9 / 2}$ \\
\hline 3481.003 & 20 & 200 & 28719.131 & $4490_{5 / 2}^{\circ}-33209_{7 / 2}$ & 3456.928 & 8 & 3 & 28919.133 & $17983_{5 / 2}^{\circ}-46902_{5 / 2}$ \\
\hline 3480.798 & 50 & 4 & 28720.822 & $0_{3 / 2}-28720_{3 / 2}^{\circ}$ & 3456.253 & 1 & & 28924.781 & $17460_{5 / 2}^{\circ}-46385_{7 / 2}$ \\
\hline 3480.750 & 2 & & 28721.219 & $18214_{3 / 2}^{\circ}-46935_{3 / 2}$ & 3455.951 & 20 & 40 & 28927.308 & $9238_{9 / 2}^{\circ}-38165_{\tau / 2}$ \\
\hline 3480.549 & 51 & 5 & 28722.877 & $17983_{5 / 2}^{\circ}-46706_{7 / 2}$ & 3455.469 & 1 & & 28931.343 & $18214_{3 / 2}^{\circ}-47145_{1 / 2}$ \\
\hline 3480.350 & 4 & 4 & 28724.519 & $21682_{7 / 2}^{\circ}-50407_{7 / 2}$ & 3455.273 & 20 & 5 & 28932.984 & $13818_{7 / 2}^{\circ}-42751_{7 / 2}$ \\
\hline 3479.912 & 1 & & 28728.135 & $31259_{5 / 2}-59987_{3 / 2}^{\circ}$ & 3455.131 & $4 b$ & & 28934.173 & $18214_{3 / 2}^{\circ}-47148_{3 / 2}$ \\
\hline 3479.172 & $500 \mathrm{~b}$ & 200 & 28734.245 & $6691_{3 / 2}^{\circ}-35425_{1 / 2}$ & 3455.112 & $4 b$ & 20 & 28934.332 & $15242_{9 / 2}^{\circ}-44177_{11 / 2}^{\circ}$ \\
\hline & & & & $7331_{5 / 2}^{\circ}-36065_{5 / 2}$ & 3455.044 & 3 & & 28934.902 & $23372_{3 / 2}^{\circ}-52307_{3 / 2}$ \\
\hline 3478.463 & 150 & 150 & 28740.101 & $13406_{13 / 2}^{\circ}-42146_{11 / 2}$ & 3454.992 & 8 & $25 b$ & 28935.337 & $17460_{5 / 2}^{\circ}-46395_{3 / 2}^{\circ}$ \\
\hline 3478.131 & 150 & 150 & 28742.845 & $9202_{i / 2}^{\circ}-37945_{\tilde{s} / 2}$ & 3454.946 & 8 & 20 & 28935.723 & $15453_{7 / 2}^{\circ}-44388_{5 / 2}$ \\
\hline 3477.704 & 100 & 150 & 28746.374 & $15349_{11 / 2}^{\circ}-44096_{9 / 2}$ & 3454.715 & 50 & 20 & 28937.657 & $19594_{1 / 2}-48532_{1 / 2}^{\circ}$ \\
\hline 3476.902 & 15 & 8 & 28753.004 & $7828_{1 / 2}-36581_{3 / 2}^{\circ}$ & 3454.646 & 10 & 15 & 28938.235 & $14349_{1 / 2}-43287_{3 / 2}^{\circ}$ \\
\hline 3476.540 & $150 \mathrm{~b}$ & 100 & 28755.998 & $6700_{9 / 2}^{\circ}-35456_{9 / 2}^{\circ}$ & 3454.206 & 200 & 15 & 28941.921 & $9238_{9 / 2}^{\circ}-38179_{9 / 2}^{\circ}$ \\
\hline 3476.332 & 5 & 8 & 28757.719 & $18973_{7 / 2}^{\circ}-47731_{9 / 2}$ & 3453.920 & 15 & 5 & 28944.318 & $17272_{9 / 2}^{\circ}-46216_{11 / 2}^{\circ}$ \\
\hline 3475.537 & 25 & 10 & 28764.297 & $20080_{7 / 2}^{\circ}-48844_{9 / 2}$ & 3453.559 & $50 \mathrm{~b}$ & 50 & 28947.343 & $11576_{3 / 2}^{\circ}-40523_{1 / 2}$ \\
\hline 3474.270 & 10 & $50 \mathrm{~b}$ & 28774.786 & $16033_{5 / 2}^{\circ}-44807_{7 / 2}$ & 3453.404 & 5 & & 28948.643 & $20120_{5 / 2}^{\circ}-49068_{5 / 2}$ \\
\hline 3473.780 & 20 & 8 & 28778.845 & $11116_{7 / 2}^{\circ}-39895_{9 / 2}^{\circ}$ & 3452.970 & 4 & & 28952.281 & $17983_{5 / 2}^{\circ}-46935_{3 / 2}$ \\
\hline 3473.610 & 5 & 3 & 28780.253 & $10572_{9 / 2}^{\circ}-39352_{11 / 2}$ & 3452.682 & 400 & 200 & 28954.696 & $6244_{1 / 2}-35198_{1 / 2}^{\circ}$ \\
\hline 3473.422 & 150 & 150 & 28781.811 & $9711_{\pi / 2}-38493_{5 / 2}^{\circ}$ & 3450.948 & 100 & 15 & 28969.244 & $13250_{5 / 2}-42219_{5 / 2}^{\circ}$ \\
\hline
\end{tabular}


TABLE 3. Classified lines of Th $\mathrm{II}-$ Continued

\begin{tabular}{|c|c|c|c|c|c|c|c|c|c|}
\hline \multirow{2}{*}{$\begin{array}{c}\text { Wavelength } \\
\AA\end{array}$} & \multicolumn{2}{|c|}{ Intensity } & \multirow{2}{*}{$\begin{array}{c}\text { Wavenumber } \\
\mathrm{cm}^{-1}\end{array}$} & \multirow{2}{*}{ Classification } & \multirow{2}{*}{$\begin{array}{c}\text { Wavelength } \\
\AA\end{array}$} & \multicolumn{2}{|c|}{ Intensity } & \multirow{2}{*}{$\begin{array}{c}\text { Wavenumber } \\
\mathrm{cm}^{-1}\end{array}$} & \multirow{2}{*}{ Classification } \\
\hline & Lamp & Spark & & & & Lamp & Spark & & \\
\hline 3450.769 & 1 & & 28970.747 & $20989_{9 / 2}^{\circ}-49960_{7 / 2}$ & 3430.124 & 20 & 8 & 29145.109 & $22685_{7 / 2}^{\circ}-51830_{7 / 2}$ \\
\hline 3450.710 & 15 & 4 & 28971.242 & $14275_{9 / 2}^{\circ}-43246_{7 / 2}$ & 3429.903 & $100 \mathrm{~b}$ & 200 & 29146.987 & $4490_{5 / 2}^{\circ}-33637_{7 / 2}$ \\
\hline 3450.108 & 5 & 10 & 28976.297 & $15324_{i / 2}^{\circ}-44300_{3 / 2}$ & 3429.827 & 5 & & 29147.633 & $8460_{3 / 2}-37607_{1 / 2}^{\circ}$ \\
\hline 3449.949 & 200 & 5 & 28977.633 & $9202_{7 / 2}^{\circ}-38179_{9 / 2}^{\circ}$ & 3429.433 & 4 & & 29150.981 & $8605_{5 / 2}-37756_{7 / 2}^{\circ}$ \\
\hline 3449.566 & $2 \mathrm{~b}$ & & 28980.850 & $21682_{7 / 2}^{\circ}-50663_{5 / 2}$ & 3429.390 & 75 & 100 & 29151.347 & $9238_{9 / 2}^{\circ}-38389_{7 / 2}^{\circ}$ \\
\hline 3449.543 & $3 b$ & & 28981.043 & $17272_{9 / 2}^{\circ}-46253_{9 / 2}$ & 3427.993 & 25 & 50 & 29163.226 & $10189_{11 / 2}^{\circ}-39352_{11 / 2}$ \\
\hline 3449.493 & 8 & 4 & 28981.463 & $14790_{7 / 2}^{\circ}-43772_{5 / 2}$ & 3427.464 & 8 & 40 & 29167.727 & $13250_{5 / 2}-42418_{3 / 2}^{\circ}$ \\
\hline 3449.286 & 50 & 300 & 28983.202 & $23187_{13 / 2}^{\circ}-52170_{11 / 2}$ & 3426.988 & 10 & 25 & 29171.778 & $9585_{5 / 2}^{\circ}-38757_{3 / 2}$ \\
\hline 3448.737 & 5 & & 28987.816 & $27403_{3 / 2}^{\circ}-56391_{5 / 2}$ & 3426.177 & 2 & & 29178.683 & $6700_{9 / 2}^{\circ}-35878_{7 / 2}^{\circ}$ \\
\hline 3448.296 & 1 & & 28991.523 & $20969_{7 / 2}^{\circ}-49960_{7 / 2}$ & 3426.144 & 8 & 75 & 29178.964 & $15710_{3 / 2}^{\circ}-44889_{3 / 2}$ \\
\hline 3447.632 & 8 & 2 & 28997.107 & $7331_{5 / 2}^{\circ}-36328_{3 / 2}$ & 3426.058 & 4 & 10 & 29179.697 & $22685_{7 / 2}^{\circ}-51865_{5 / 2}$ \\
\hline 3446.997 & 8 & 4 & 29002.448 & $12485_{7 / 2}^{\circ}-41488_{7 / 2}$ & 3425.944 & 100 & 100 & 29180.668 & $9400_{5 / 2}-38581_{5 / 2}^{\circ}$ \\
\hline 3446.288 & 8 & 2 & 29008.415 & $9720_{7 / 2}^{\circ}-38728_{5 / 2}$ & 3425.185 & $100 \mathrm{~b}$ & 200 & 29187.134 & $9202_{7 / 2}^{\circ}-38389_{7 / 2}$ \\
\hline 3445.743 & 300 & 200 & 29013.003 & $14790_{7 / 2}^{\circ}-43803_{7 / 2}$ & 3423.356 & 5 & 3 & 29202.727 & $12485_{7 / 2}^{\circ}-41688_{7 / 2}$ \\
\hline 3445.389 & 1501 & 150 & 29015.984 & $12472_{5 / 2}^{\circ}-41488_{7 / 2}$ & 3423.129 & 150 & 150 & 29204.664 & $12472_{5 / 2}^{\circ}-41676_{3 / 2}$ \\
\hline 3445.217 & 200 & 100 & 29017.432 & $10379_{9 / 2}-39396_{7 / 2}^{\circ}$ & 3422.809 & 3 & & 29207.394 & $15242_{9 / 2}^{\circ}-44450_{9 / 2}$ \\
\hline 3445.121 & 8 & 3 & 29018.241 & $14790_{7 / 2}^{\circ}-43809_{9 / 2}^{\circ}$ & 3422.576 & 25 & & 29209.382 & $22014_{11 / 2}^{o}-51224_{9 / 2}$ \\
\hline 3444.028 & 25 & 100 & 29027.450 & $10673_{5 / 2}^{\circ}-39700_{5 / 2}$ & 3422.306 & 2 & & 29211.687 & $24309_{11 / 2}^{\circ}-53520_{9 / 2}$ \\
\hline 3443.139 & $50 \mathrm{~b}$ & $20 \mathrm{~b}$ & 29034.944 & $15786_{5 / 2}-44821_{5 / 2}^{\circ}$ & 3422.245 & 1 & & 29212.207 & $19912_{13 / 2}^{\circ}-49124_{11 / 2}$ \\
\hline 3443.110 & $40 \mathrm{~b}$ & $75 b$ & 29035.189 & $9400_{5 / 2}-38436_{3 / 2}^{\circ}$ & 3421.770 & 3 & & 29216.262 & $12472_{5 / 2}^{\circ}-41688_{7 / 2}^{\circ}$ \\
\hline 3441.365 & 200 & 25 & 29049.911 & $6691_{3 / 2}^{\circ}-35741_{5 / 2}$ & 3419.173 & 500 & 300 & 29238.453 & $13406_{13 / 2}^{\circ}-42644_{13 / 2}$ \\
\hline 3441.036 & 100 & 20 & 29052.688 & $9711_{7 / 2}-38764_{7 / 2}^{\circ}$ & 3418.952 & $150 \mathrm{~b}$ & $40 \mathrm{~b}$ & 29240.342 & $8605_{5 / 2}-37846_{5 / 2}^{\circ}$ \\
\hline 3440.909 & 10 & 20 & 29053.761 & $9238_{9 / 2}^{\circ}-38291_{7 / 2}$ & 3418.914 & $100 \mathrm{~b}$ & $40 \mathrm{~b}$ & 29240.667 & $4490_{5 / 2}^{\circ}-33730_{5 / 2}$ \\
\hline & & & & $25440_{5 / 2}^{\circ}-54493_{5 / 2}$ & 3418.776 & 200 & 300 & 29241.848 & $19880_{9 / 2}-49121_{7 / 2}^{\circ}$ \\
\hline 3439.711 & $500 \mathrm{~b}$ & 400 & 29063.879 & $10379_{9 / 2}-39443_{9 / 2}^{\circ}$ & 3418.523 & 4 & & 29244.012 & $15144_{3 / 2}^{\circ}-44388_{5 / 2}^{\circ}$ \\
\hline 3439.232 & 10 & 5 & 29067.927 & $11576_{3 / 2}^{\circ}-40644_{5 / 2}^{\circ}$ & 3418.333 & 3 & & 29245.637 & $17460_{5 / 2}^{\circ}-46706_{\pi / 2}^{\circ}$ \\
\hline 3439.056 & 10 & 4 & 29069.415 & $20288_{1 / 2}^{\circ}-49357_{11 / 2}$ & 3417.794 & 1 & & 29250.249 & $22685_{7 / 2}^{\circ}-51935_{5 / 2}^{\circ}$ \\
\hline 3438.951 & 500 & 300 & 29070.302 & $13248_{9 / 2}-42319_{9 / 2}^{\circ}$ & 3417.724 & 50 & 75 & 29250.848 & $11116_{7 / 2}^{\circ}-40367_{9 / 2}$ \\
\hline 3438.689 & 4 & 10 & 29072.517 & $19248_{5 / 2}^{\circ}-48320_{5 / 2}$ & 3417.126 & 15 & 15 & 29255.967 & $8460_{3 / 2}-37716_{1 / 2}^{\circ}$ \\
\hline 3437,808 & 8 & 20 & 29079.967 & $17272_{9 / 2}^{\circ}-46352_{7 / 2}^{\circ}$ & 3417.001 & 1 & & 29257.037 & $20120_{5 / 2}^{\circ}-49377_{7 / 2}$ \\
\hline 3437.024 & 100 & $150 \mathrm{~b}$ & 29086.600 & $8378_{7 / 2}^{\circ}-37465_{5 / 2}$ & 3416.551 & 1 & & 29260.891 & $15242_{9 / 2}^{\circ}-44503_{7 / 2}$ \\
\hline 3436.679 & $75 b$ & $100 \mathrm{~b}$ & 29089.520 & $9202_{7 / 2}^{\circ}-38291_{7 / 2}$ & 3416.421 & $40 \mathrm{~b}$ & 50 & 29262.004 & $14545_{5 / 2}^{\circ}-43807_{3 / 2}$ \\
\hline 3436.288 & 3 & 2 & 29092.830 & $9400_{5 / 2}-38493_{5 / 2}^{\circ}$ & 3415.487 & $3 \mathbf{b}$ & & 29270.006 & $19050_{3 / 2}^{\circ}-48320_{5 / 2}^{\circ}$ \\
\hline 3435.975 & $800 \mathrm{~b}$ & 200 & 29095.480 & $0_{3 / 2}-29095_{5 / 2}^{\circ}$ & & & & & $26965_{i / 2}^{\circ}-56235_{3 / 2}$ \\
\hline 3435.695 & 31 & 5 & 29097.851 & $17837_{i / 2}^{\circ}-46935_{3 / 2}$ & 3414.979 & 3 & & 29274.360 & $17121_{i 3 / 2}^{\circ}-46395_{3 / 2}$ \\
\hline 3435.193 & 15 & 10 & 29102.103 & $4113_{5 / 2}-33215_{3 / 2}^{\circ}$ & 3414.503 & 100 & 200 & 29278.441 & $9585_{5 / 2}^{\circ}-38863_{5 / 2}$ \\
\hline 3435.004 & 2 & & 29103.705 & $20310_{5 / 2}^{\circ}-49414_{3 / 2}$ & 3413.634 & $3 \mathbf{b}$ & & 29285.894 & $30101_{7 / 2}^{\circ}-59387_{7 / 2}$ \\
\hline 3434.759 & $100 \mathrm{~b}$ & 40 & 29105.780 & $12902_{3 / 2}^{\circ}-42008_{1 / 2}$ & 3413.408 & 100 & 75 & 29287.833 & $6168_{7 / 2}^{\circ}-35456_{9 / 2}^{\circ}$ \\
\hline 3434.266 & 4 & 8 & 29109.959 & $18214_{3 / 2}^{\circ}-47324_{5 / 2}$ & 3412.632 & 4 & & 29294.492 & $20120_{5 / 2}^{\circ}-49414_{3 / 2}$ \\
\hline 3433.999 & $1000 \mathrm{~h}$ & 300 & 29112.222 & $1859_{3 / 2}-30972_{5 / 2}^{\circ}$ & 3412.521 & 3 & & 29295.445 & $23012_{3 / 2}^{\circ}-52307_{3 / 2}^{\circ}$ \\
\hline 3433.946 & 40 & 15 & 29112.671 & $22834_{7 / 2}-51946_{5 / 2}^{\circ}$ & 3412.399 & 25 & 4 & 29296.493 & $20080_{7 / 2}^{\circ}-49377_{7 / 2}$ \\
\hline 3433.890 & 15 & 100 & 29113.146 & $17272_{9 / 2}^{\circ}-46385_{7 / 2}$ & 3411.785 & $200 \mathrm{~b}$ & 100 & 29301.765 & $7828_{1 / 2}-37130_{1 / 2}^{\circ}$ \\
\hline 3432.085 & 5 & 40 & 29128.457 & $22139_{9 / 2}^{\circ}-51268_{7 / 2}^{\circ}$ & 3411.312 & 2 & 5 & 29305.827 & $25188_{3 / 2}^{\circ}-54493_{5 / 2}^{\circ}$ \\
\hline 3431.811 & 50 & 200 & 29130.782 & $20158_{5 / 2}-49289_{5 / 2}^{\circ}$ & 3411.061 & 3 & & 29307.984 & $17837_{1 / 2}^{\circ}-47145_{1 / 2}$ \\
\hline 3431.019 & 15 & 75 & 29137.506 & $13818_{7 / 2}^{\circ}-42955_{9 / 2}$ & 3409.986 & 3 & & 29317.223 & $22513_{5 / 2}^{\circ}-51830_{7 / 2}$ \\
\hline 3430.761 & 5 & 2 & 29139.698 & $17771_{1 / 22}^{\circ}-46910_{13 / 2}$ & 3409.562 & 3 & & 29320.868 & $22355_{1 / 2}^{\circ}-51676_{3 / 2}$ \\
\hline 3430.567 & 15 & 75 & 29141.345 & $8460_{3 / 2}-37601_{3 / 2}^{\circ}$ & 3409.270 & 400 & 200 & 29323.380 & $10572_{9 / 2}^{\circ}-39895_{9 / 2}^{\circ}$ \\
\hline 3430.419 & 8 & 75 & 29142.603 & $17460_{5 / 2}^{\circ}-46603_{5 / 2}$ & 3409.112 & $3 b$ & & 29324.739 & $18973_{7 / 2}^{\circ}-48298_{7 / 2}$ \\
\hline & & & & $17121_{3 / 2}^{\circ}-46264_{3 / 2}$ & 3408.644 & 200 & 150 & 29328.765 & $10855_{7 / 2}-40184_{7 / 2}^{\circ}$ \\
\hline 3430.339 & $8 b$ & 15 & 29143.282 & $9585_{5 / 2}^{\circ}-38728_{5 / 2}$ & 3407.631 & 40 & 200 & 29337.483 & $16818_{7 / 2}-46155_{5 / 2}^{\circ}$ \\
\hline
\end{tabular}


TABLE 3. Classified lines of Th II-Continued

\begin{tabular}{|c|c|c|c|c|c|c|c|c|c|}
\hline \multirow{2}{*}{$\begin{array}{c}\text { Wavelength } \\
\AA\end{array}$} & \multicolumn{2}{|c|}{ Intensity } & \multirow{2}{*}{$\begin{array}{c}\text { Wavenumber } \\
\mathbf{c m}^{-1}\end{array}$} & \multirow{2}{*}{ Classification } & \multirow{2}{*}{$\begin{array}{c}\text { Wavelength } \\
\AA\end{array}$} & \multicolumn{2}{|c|}{ Intensity } & \multirow{2}{*}{$\begin{array}{l}\text { Wavenumber } \\
\mathbf{c m}^{-1}\end{array}$} & \multirow{2}{*}{ Classification } \\
\hline & Lamp & Spark & & & & Lamp & Spark & & \\
\hline 3407.221 & $3 \mathrm{~s}$ & & 29341.013 & $17983_{5 / 2}^{\circ}-47324_{5 / 2}$ & 3387.532 & 2 & & 29511.544 & $24982_{7 / 2}^{\circ}-54493_{5 / 2}$ \\
\hline 3406.730 & 3 & & 29345.242 & $15305_{9 / 2}-44650_{7 / 2}^{\circ}$ & 3386.763 & 10 & 75 & 29518.244 & $18973_{7 / 2}^{\circ}-48492_{5 / 2}$ \\
\hline 3406.365 & 8 & 8 & 29348.386 & $9720_{7 / 2}^{\circ}-39068_{7 / 2}$ & 3386.500 & 500 & 300 & 29520.537 & $9061_{5 / 2}-38581_{5 / 2}^{\circ}$ \\
\hline 3406.242 & 150 & 100 & 29349.446 & $6691_{3 / 2}^{\circ}-36040_{1 / 2}$ & 3385.825 & 8 & 15 & 29526.422 & $9202_{7 / 2}^{\circ}-38728_{5 / 2}^{\circ}$ \\
\hline 3405.607 & 50 & & 29354.918 & $15453_{7 / 2}^{\circ}-44807_{7 / 2}$ & 3385.596 & 8 & 2 & 29528.419 & $14275_{9 / 2}^{\circ}-43803_{7 / 2}$ \\
\hline 3404.798 & 8 & 15 & 29361.893 & $16033_{5 / 2}^{\circ}-45395_{7 / 2}$ & 3385.531 & 800 & 200 & 29528.986 & $4490_{5 / 2}^{\circ}-34019_{3 / 2}$ \\
\hline \multirow[t]{2}{*}{3404.651} & 200 & 100 & 29363.161 & $10189_{11 / 2}^{\circ}-39552_{9 / 2}$ & 3385.206 & 2 & & 29531.821 & $21131_{3 / 2}^{\circ}-50663_{5 / 2}^{\circ}$ \\
\hline & & & & $23372_{3 / 2}^{\circ}-52735_{3 / 2}$ & 3384.991 & 100 & 100 & 29533.696 & $14275_{9 / 2}^{\circ}-43809_{9 / 2}^{\circ}$ \\
\hline 3404.592 & 40 & 40 & 29363.669 & $9400_{5 / 2}-38764_{7 / 2}^{\circ}$ & 3384.095 & 2 & & 29541.516 & $21682_{7 / 2}^{\circ}-51224_{9 / 2}^{\circ}$ \\
\hline 3404.198 & 4 & & 29367.068 & $10572_{9 / 2}^{\circ}-39939_{11 / 2}$ & 3384.001 & 8 & 40 & 29542.336 & $22139_{9 / 2}^{\circ}-51681_{9 / 2}^{\circ}$ \\
\hline 3403.353 & 100 & 25 & 29374.359 & $6691_{3 / 2}^{\circ}-36065_{5 / 2}$ & 3383.149 & $25 b$ & $25 b$ & 29549.776 & $10673_{5 / 2}^{\circ}-40222_{3 / 2}$ \\
\hline 3403.271 & 200 & $200 \mathrm{~b}$ & 29375.067 & $9061_{5 / 2}-38436_{3 / 2}^{\circ}$ & 3383.107 & $100 \mathrm{~b}$ & 200 & 29550.143 & $16818_{7 / 2}-46368_{9 / 2}^{\circ}$ \\
\hline 3403.006 & 15 & 40 & 29377.354 & $15349_{11 / 2}^{\circ}-44727_{11 / 2}$ & 3381.374 & $50 \mathrm{~b}$ & $40 \mathrm{~b}$ & 29565.287 & $9585_{5 / 2}^{\circ}-39150_{3 / 2}$ \\
\hline 3402.698 & $800 \mathrm{~b}$ & $400 \mathrm{~b}$ & 29380.013 & $6213_{9 / 2}-35593_{7 / 2}^{\circ}$ & 3381.343 & $50 \mathrm{~b}$ & $50 \mathrm{~b}$ & 29565.558 & $15324_{i / 2}^{\circ}-44889_{3 / 2}$ \\
\hline 3402.027 & 200 & 200 & 29385.808 & $8460_{3 / 2}-37846_{5 / 2}^{\circ}$ & 3380.500 & 3 & 2 & 29572.931 & $6168_{7 / 2}^{\circ}-35741_{5 / 2}$ \\
\hline 3401.647 & 150 & 150 & 29389.090 & $7001_{3 / 2}-36390_{3 / 2}^{\circ}$ & 3379.981 & 8 & 15 & 29577.472 & $16033_{5 / 2}^{\circ}-45610_{5 / 2}$ \\
\hline 3400.998 & 10 & 15 & 29394.699 & $14349_{1 / 2}-43744_{1 / 2}^{\circ}$ & 3379.292 & 8 & & 29583.502 & $8018_{3 / 2}-37601_{3 / 2}^{\circ}$ \\
\hline 3400.350 & 8 & & 29400.300 & $17771_{1 / 2}^{\circ}-47171_{9 / 2}$ & 3379.090 & 15 & 50 & 29585.270 & $15236_{3 / 2}-44821_{5 / 2}^{\circ}$ \\
\hline 3399.466 & 5 & 2 & 29407.945 & $15144_{3 / 2}^{\circ}-44552_{5 / 2}$ & 3378.905 & 4 & & 29586.890 & $22685_{7 / 2}^{\circ}-52272_{7 / 2}$ \\
\hline 3399.344 & $25 b$ & $75 b$ & 29409.001 & $8378_{\tau / 2}^{\circ}-37787_{\tau / 2}$ & 3378.803 & 5 & 3 & 29587.783 & $27357_{9 / 2}^{\circ}-56945_{11 / 2}$ \\
\hline 3399.284 & 20 & 40 & 29409.520 & $13818_{7 / 2}^{\circ}-43227_{5 / 2}$ & 3378.573 & 500 & 300 & 29589.797 & $8018_{3 / 2}-37607_{1 / 2}^{\circ}$ \\
\hline 3397.953 & 15 & 25 & 29421.039 & $12488_{9 / 2}^{\circ}-41909_{9 / 2}^{\circ}$ & 3377.943 & $2 b$ & & 29595.316 & $15710_{3 / 2}^{\circ}-45306_{3 / 2}^{\circ}$ \\
\hline 3397.844 & 20 & 150 & 29421.983 & $22106_{5 / 2}-51528_{3 / 2}^{\circ}$ & 3377.661 & $10 \mathrm{~b}$ & 15 & 29597.786 & $14790_{7 / 2}^{\circ}-44388_{5 / 2}^{\circ}$ \\
\hline 3397.255 & 3 & & 29427.084 & $26963_{7 / 2}^{\circ}-56391_{5 / 2}$ & 3377.538 & 3 & & 29598.864 & $29788_{9 / 2}^{\circ}-59387_{7 / 2}^{\circ}$ \\
\hline 3397.092 & $15 b$ & 40 & 29428.496 & $13818_{7 / 2}^{\circ}-43246_{7 / 2}$ & 3376.840 & 75 & 50 & 29604.982 & $10673_{5 / 2}^{\circ}-40278_{3 / 2}$ \\
\hline 3396.874 & 2 & & 29430.384 & $25414_{i 1 / 2}^{\circ}-54845_{9 / 2}$ & 3376.529 & 4 & 5 & 29607.709 & $13406_{13 / 2}^{\circ}-43014_{13 / 2}$ \\
\hline 3396.612 & 100 & 100 & 29432.654 & $9061_{5 / 2}-38493_{5 / 2}^{\circ}$ & 3376.411 & 2 & & 29608.744 & $26626_{1 / 2}^{\circ}-56235_{3 / 2}$ \\
\hline 3396.456 & 50 & 100 & 29434.006 & $17272_{9 / 2}^{\circ}-46706_{7 / 2}$ & 3376.049 & 3 & 3 & 29611.918 & $14484_{11 / 2}^{\circ}-44096_{9 / 2}$ \\
\hline 3396.394 & 50 & 100 & 29434.543 & $15786_{5 / 2}-45221_{5 / 2}^{\circ}$ & 3374.582 & 300 & 300 & 29624.791 & $9238_{9 / 2}^{\circ}-38862_{11 / 2}$ \\
\hline 3395.966 & $8 b$ & & 29438.253 & $20969_{7 / 2}^{\circ}-50407_{7 / 2}$ & 3373.964 & 2 & & 29630.217 & $22642_{9 / 2}^{\circ}-52272_{7 / 2}^{\circ}$ \\
\hline 3395.628 & 8 & 50 & 29441.183 & $19050_{3 / 2}^{\circ}-48492_{5 / 2}$ & 3373.165 & 2 & & 29637.235 & $6691_{3 / 2}^{\circ}-36328_{3 / 2}$ \\
\hline 3395.573 & 3 & & 29441.660 & $19248_{5 / 2}^{\circ}-48689_{3 / 2}$ & 3372.955 & 8 & 20 & 29639.081 & $19050_{3 / 2}^{\circ}-48689_{3 / 2}$ \\
\hline 3395.545 & 2 & & 29441.903 & $17460_{5 / 2}^{\circ}-46902_{5 / 2}$ & 3372.702 & 50 & 100 & 29641.304 & $20989_{9 / 2}^{\circ}-50631_{11 / 2}^{\circ}$ \\
\hline 3395.372 & 100 & 150 & 29443.403 & $7001_{3 / 2}-36444_{3 / 2}^{\circ}$ & 3372.334 & 10 & 15 & 29644.538 & $15144_{3 / 2}^{\circ}-44789_{1 / 2}^{\circ}$ \\
\hline 3395.112 & 20 & 100 & 29445.658 & $19912_{13 / 2}^{\circ}-49357_{11 / 2}$ & 3372.097 & 1 & & 29646.622 & $9720_{7 / 2}^{\circ}-39366_{5 / 2}$ \\
\hline 3394.797 & 150 & 200 & 29448.390 & $17722_{9 / 2}^{\circ}-47171_{9 / 2}$ & 3371.795 & $500 \mathrm{~b}$ & 300 & 29649.277 & $12570_{7 / 2}-42219_{5 / 2}^{\circ}$ \\
\hline 3394.640 & 8 & 10 & 29449.752 & $12902_{3 / 2}^{\circ}-42352_{5 / 2}^{\circ}$ & 3371.492 & 10 & 50 & 29651.941 & $16564_{11 / 2}^{\circ}-46216_{11 / 2}$ \\
\hline 3394.583 & 15 & 3 & 29450.246 & $1521_{5 / 2}-30972_{5 / 2}^{\circ}$ & 3371.123 & 2 & & 29655.187 & $18214_{3 / 2}^{\circ}-47869_{3 / 2}^{\circ}$ \\
\hline 3394.145 & 150 & 200 & 29454.047 & $11116_{7 / 2}^{\circ}-40570_{7 / 2}$ & 3371.043 & 1 & & 29655.891 & $15242_{9 / 2}^{\circ}-44898_{7 / 2}$ \\
\hline 3393.500 & 5 & 3 & 29459.645 & $17121_{3 / 2}^{\circ}-46581_{5 / 2}$ & 3370.656 & 2 & & 29659.296 & $14790_{7 / 2}^{\circ}-44450_{9 / 2}$ \\
\hline 3393.230 & 50 & 100 & 29461.989 & $8378_{7 / 2}^{\circ}-37840_{9 / 2}$ & 3370.397 & 10 & & 29661.575 & $9202_{7 / 2}^{\circ}-38863_{5 / 2}$ \\
\hline 3392.958 & $150 \mathrm{~b}$ & $50 \mathrm{~b}$ & 29464.350 & $12472_{5 / 2}^{\circ}-41936_{3 / 2}$ & 3369.077 & 8 & & 29673.196 & $15453_{7 / 2}^{\circ}-45126_{9 / 2}^{\circ}$ \\
\hline 3392.878 & 3 & & 29465.045 & $15324_{1 / 2}^{\circ}-44789_{1 / 2}$ & 3368.908 & 5 & 10 & 29674.684 & $16906_{7 / 2}^{\circ}-46581_{5 / 2}$ \\
\hline 3392.035 & $1000 \mathrm{~d}$ & 1000 & 29472.368 & $1521_{5 / 2}-30994_{i / 2}^{\circ}$ & 3367.819 & $500 \mathrm{~b}$ & $150 \mathrm{~b}$ & 29684.279 & $4490_{5 / 2}^{\circ}-34174_{5 / 2}$ \\
\hline 3391.296 & 5 & 8 & 29478.790 & $15710_{3 / 2}^{\circ}-45189_{5 / 2}$ & 3367.784 & $100 \mathrm{~b}$ & $50 \mathrm{~b}$ & 29684.588 & $9711_{7 / 2}-39396_{7 / 2}^{\circ}$ \\
\hline 3391.131 & 2 & & 29480.224 & $20989_{9 / 2}^{\circ}-50470_{9 / 2}$ & 3367.399 & 8 & 5 & 29687.982 & $17460_{5 / 2}^{\circ}-47148_{3 / 2}$ \\
\hline 3390.780 & 25 & 50 & 29483.276 & $9585_{5 / 2}^{\circ}-39068_{7 / 2}$ & 3367.318 & 5 & & 29688.696 & $16564_{11 / 2}^{\circ}-46253_{9 / 2}$ \\
\hline 3389.640 & 500 & 300 & 29493.191 & $1859_{3 / 2}-31353_{3 / 2}^{\circ}$ & 3367.073 & $2 \mathbf{h}$ & 12 & 29690.856 & $22139_{9 / 2}^{\circ}-51830_{\tau / 2}$ \\
\hline 3388.578 & 100 & 75 & 29502.434 & $9238_{9 / 2}^{\circ}-38740_{11 / 2}$ & 3366.721 & 40 & 100 & 29693.960 & $13250_{5 / 2}-42944_{7 / 2}^{\circ}$ \\
\hline 3387.834 & 4 & & 29508.913 & $30484_{11 / 2}-59993_{9 / 2}^{\circ}$ & 3366.649 & 8 & 200 & 29694.595 & $20969_{7 / 2}^{\circ}-50663_{5 / 2}^{\circ}$ \\
\hline
\end{tabular}


TABle 3. Classified lines of Th II-Continued

\begin{tabular}{|c|c|c|c|c|c|c|c|c|c|}
\hline \multirow{2}{*}{$\begin{array}{c}\text { Wavelength } \\
\AA\end{array}$} & \multicolumn{2}{|c|}{ Intensity } & \multirow{2}{*}{$\begin{array}{c}\text { Wavenumber } \\
\text { cm }^{-1}\end{array}$} & \multirow{2}{*}{ Classification } & \multirow{2}{*}{$\begin{array}{c}\text { Wavelength } \\
\AA\end{array}$} & \multicolumn{2}{|c|}{ Intensity } & \multirow{2}{*}{$\begin{array}{c}\text { Wavenumber } \\
\mathrm{cm}^{-1}\end{array}$} & \multirow{2}{*}{ Classification } \\
\hline & Lamp & Spark & & & & Lamp & Spark & & \\
\hline 3366.517 & 200 & 200 & 29695.759 & $13248_{9 / 2}-42944_{7 / 2}^{\circ}$ & 3351.150 & $50 \mathrm{~b}$ & $50 \mathrm{~b}$ & 29831.928 & $9720_{7 / 2}^{\circ}-39552_{9 / 2}$ \\
\hline 3366.423 & 10 & 20 & 29696.588 & $16906_{7 / 2}^{\circ}-46603_{\bar{s} / 2}$ & 3350.308 & 5 & 4 & 29839.425 & $10572_{9 / 2}^{\circ}-40411_{7 / 2}$ \\
\hline 3365.633 & 15 & 15 & 29703.559 & $9061_{5 / 2}-38764_{7 / 2}^{\circ}$ & 3350.202 & $5 \mathrm{~h}$ & 4 & 29840.369 & $22106_{5 / 2}-51946_{5 / 2}^{\circ}$ \\
\hline 3365.318 & $25 b$ & & 29706.339 & $10189_{11 / 2}^{\circ}-39895_{9 / 2}$ & 3349.883 & 10 & 8 & 29843.210 & $14545_{5 / 2}^{\circ}-44388_{5 / 2}$ \\
\hline 3364.846 & 15 & 15 & 29710.506 & $6168_{7 / 2}^{\circ}-35878_{7 / 2}$ & 3348.992 & $5 b$ & & 29851.150 & $12485_{7 / 2}^{\circ}-42336_{5 / 2}$ \\
\hline 3364.685 & $75 b$ & $200 \mathrm{~b}$ & 29711.928 & $12488_{9 / 2}^{\circ}-42200_{9 / 2}$ & 3348.953 & 100 & 400 & 29851.497 & $10855_{7 / 2}-40706_{7 / 2}^{\circ}$ \\
\hline 3364.587 & 20 & 75 & 29712.793 & $14790_{7 / 2}^{\circ}-44503_{7 / 2}$ & 3347.678 & 2 & & 29862.866 & $24982_{7 / 2}^{\circ}-54845_{9 / 2}$ \\
\hline 3364.388 & 15 & 25 & 29714.550 & $12485_{7 / 2}^{\circ}-42200_{g / 2}$ & 3347.576 & 15 & 15 & 29863.776 & $17460_{5 / 2}^{\circ}-47324_{5 / 2}$ \\
\hline 3363.733 & 50 & 10 & 29720.336 & $0_{3 / 2}-29720_{3 / 2}^{\circ}$ & 3347.478 & 3 & & 29864.651 & $12472_{5 / 2}^{\circ}-42336_{5 / 2}^{\circ}$ \\
\hline 3363.682 & 200 & 100 & 29720.787 & $13406_{13 / 2}^{\circ}-4.3127_{11 / 2}$ & 3346.746 & 8 & 10 & 29871.182 & $18973_{7 / 2}^{\circ}-48844_{9 / 2}$ \\
\hline 3363.066 & 100 & 100 & 29726.231 & $8378_{7 / 2}^{\circ}-38105_{5 / 2}$ & 3346.555 & 150 & 150 & 29872.887 & $9585_{5 / 2}^{\circ}-39458_{7 / 2}$ \\
\hline 3362.674 & 100 & 50 & 29729.696 & $4113_{5 / 2}-33843_{3 / 2}^{\circ}$ & 3345.384 & 5 & 8 & 29883.343 & $15242_{9 / 2}^{\circ}-45126_{9 / 2}^{\circ}$ \\
\hline 3362.521 & $50 \mathrm{~b}$ & 100 & 29731.049 & $9711_{\pi / 2}-39443_{9 / 2}^{\circ}$ & 3345.060 & $5 \mathrm{~h}$ & 8 & 29886.238 & $17983_{5 / 2}^{\circ}-47869_{3 / 2}$ \\
\hline 3362.427 & $15 b$ & 5 & 29731.880 & $23187_{13 / 2}^{\circ}-52918_{13 / 2}$ & 3344.872 & $50 \mathrm{~b}$ & 50 & 29887.917 & $8605_{5 / 2}-38493_{5 / 2}^{\circ}$ \\
\hline 3362.184 & 75 & 200 & 29734.029 & $12488_{9 / 2}^{\circ}-42222_{7 / 2}$ & 3344.670 & 1 & 3 & 29889.722 & $25607_{9 / 2}^{\circ}-55496_{9 / 2}$ \\
\hline \multirow[t]{2}{*}{3361.890} & 10 & 40 & 29736.628 & $15453_{7 / 2}^{\circ}-45189_{5 / 2}$ & 3343.813 & $75 \mathrm{~s}$ & 20 & 29897.383 & $6168_{7 / 2}^{\circ}-36065_{5 / 2}$ \\
\hline & & & & $12485_{7 / 2}^{\circ}-42222_{7 / 2}$ & 3343.617 & 100 & 150 & 29899.135 & $17272_{9 / 2}^{\circ}-47171_{9 / 2}$ \\
\hline 3361.734 & 150 & 150 & 29738.008 & $9720_{7 / 2}^{\circ}-39458_{7 / 2}$ & 3343.545 & 3 & $5 b$ & 29899.779 & $15710_{3 / 2}^{\circ}-45610_{5 / 2}$ \\
\hline 3361.617 & 100 & 200 & 29739.043 & $17727_{11 / 2}-47466_{11 / 2}^{\circ}$ & 3343.258 & $25 b$ & $50 \mathrm{~b}$ & 29902.346 & $15786_{5 / 2}-45689_{\pi / 2}^{\circ}$ \\
\hline 3360.937 & $3 \mathrm{~b}$ & & 29745.060 & $15144_{3 / 2}^{\circ}-44889_{3 / 2}$ & 3342.069 & 200 & 10 & 29912.984 & $8378_{7 / 2}^{\circ}-38291_{7 / 2}$ \\
\hline 3360.371 & 150 & 100 & 29750.070 & $10189_{11 / 2}^{\circ}-39939_{11 / 2}$ & 3340.097 & 2 & & 29930.644 & $11116_{\tau / 2}^{\circ}-41047_{9 / 2}$ \\
\hline 3360.156 & 100 & 50 & 29751.974 & $11576_{3 / 2}^{\circ}-41328_{5 / 2}$ & 3338.828 & 10 & 40 & 29942.019 & $15453_{\tau / 2}^{\circ}-45395_{7 / 2}$ \\
\hline 3360.043 & 3 & & 29752.974 & $20120_{5 / 2}^{\circ}-49873_{5 / 2}$ & 3338.396 & 150 & 200 & 29945.894 & $7331_{5 / 2}^{\circ}-37277_{7 / 2}$ \\
\hline 3359.755 & 20 & 40 & 29755.524 & $4146_{7 / 2}-33902_{7 / 2}^{\circ}$ & 3337.870 & 800 & 150 & 29950.613 & $1859_{3 / 2}-31810_{5 / 2}^{\circ}$ \\
\hline 3359.059 & 15 & 25 & 29761.690 & $14790_{7 / 2}^{\circ}-44552_{5 / 2}$ & 3337.780 & 10 & 10 & 29951.420 & $11725_{1 / 2}^{\circ}-41676_{3 / 2}$ \\
\hline 3358.602 & 500 & 300 & 29765.739 & $1859_{3 / 2}-31625_{1 / 2}^{\circ}$ & 3337.684 & $2 \mathrm{~b}$ & & 29952.282 & $22355_{1 / 2}^{\circ}-52307_{3 / 2}$ \\
\hline \multirow[t]{2}{*}{3358.446} & 10 & 15 & 29767.122 & $16033_{5 / 2}^{\circ}-45800_{5 / 2}$ & 3337.478 & 5 & 50 & 29954.130 & $13818_{\pi / 2}^{\circ}-43772_{5 / 2}$ \\
\hline & & & & $19050_{3 / 2}^{\circ}-48817_{3 / 2}$ & 3337.159 & 50 & 20 & 29956.994 & $6168_{7 / 2}^{\circ}-36125_{9 / 2}$ \\
\hline 3357.765 & 8 & 10 & 29773.159 & $7828_{1 / 2}-37601_{3 / 2}^{\circ}$ & 3337.019 & 1 & & 29958.250 & $14545_{5 / 2}^{\circ}-44503_{7 / 2}$ \\
\hline 3357.403 & 15 & 40 & 29776.369 & $15349_{11 / 2}^{\circ}-45126_{9 / 2}$ & 3336.775 & 8 & 25 & 29960.441 & $17771_{11 / 2}^{\circ}-47731_{9 / 2}$ \\
\hline 3357.233 & $50 \mathrm{~b}$ & 50 & 29777.877 & $13468_{9 / 2}^{\circ}-43246_{\pi / 2}$ & 3336.161 & 50 & 40 & 29965.955 & $14484_{11 / 2}^{\circ}-44450_{y / 2}$ \\
\hline 3357.056 & 25 & 40 & 29779.447 & $7828_{1 / 2}-37607_{1 / 2}^{\circ}$ & 3335.576 & 2 & 2 & 29971.210 & $10673_{5 / 2}^{\circ}-40644_{5 / 2}$ \\
\hline 3356.888 & 10 & 40 & 29780.937 & $17121_{3 / 2}^{\circ}-46902_{5 / 2}$ & 3335.064 & $100 \mathrm{~b}$ & 100 & 29975.811 & $8460_{3 / 2}-38436_{3 / 2}^{\circ}$ \\
\hline 3356.821 & 75 & 75 & 29781.531 & $9585_{5 / 2}^{\circ}-39366_{5 / 2}$ & & & & & $8605_{5 / 2}-38581_{5 / 2}^{\circ}$ \\
\hline 3356.262 & 8 & & 29786.491 & $8378_{7 / 2}^{\circ}-38165_{7 / 2}^{\circ}$ & 3334.879 & 4 & 20 & 29977.474 & $20686_{5 / 2}^{\circ}-50663_{5 / 2}^{\circ}$ \\
\hline 3356.005 & 4 & & 29788.772 & $4113_{5 / 2}-33902_{7 / 2}^{\circ}$ & 3334.603 & 6001 & 300 & 29979.955 & $6213_{9 / 2}-36193_{9 / 2}^{\circ}$ \\
\hline 3355.970 & 10 & 10 & 29789.083 & $4490_{5 / 2}^{\circ}-34279_{7 / 2}$ & 3334.053 & 10 & 25 & 29984.900 & $15236_{3 / 2}-45221_{5 / 2}^{\circ}$ \\
\hline 3355.567 & 50 & 200 & 29792.661 & $25246_{9 / 2}-55038_{9 / 2}^{\circ}$ & 3333.969 & 8 & 10 & 29985.656 & $13818_{7 / 2}^{\circ}-43803_{7 / 2}$ \\
\hline 3355.256 & 100 & 100 & 29795.422 & $10572_{9 / 2}^{\circ}-40367_{9 / 2}$ & 3333.383 & 15 & 15 & 29990.927 & $13818_{7 / 2}^{\circ}-43809_{9 / 2}$ \\
\hline 3354.836 & 5 & 5 & 29799.152 & $10855_{7 / 2}-40654_{5 / 2}^{\circ}$ & 3332.532 & 5 & $3 b$ & 29998.585 & $10572_{9 / 2}^{\circ}-40570_{7 / 2}^{\circ}$ \\
\hline 3354.780 & 8 & 10 & 29799.649 & $16906_{7 / 2}^{\circ}-46706_{7 / 2}$ & 3332.460 & $3 \mathrm{~b}$ & 8 & 29999.234 & $21682_{7 / 2}^{\circ}-51681_{9 / 2}$ \\
\hline 3354.616 & 150 & 100 & 29801.106 & $8378_{7 / 2}^{\circ}-38179_{9 / 2}^{\circ}$ & 3332.399 & 75 & 50 & 29999.783 & $12219_{3 / 2}-42219_{5 / 2}^{\circ}$ \\
\hline 3354.180 & 500 & 200 & 29804.980 & $10379_{9 / 2}-40184_{i / 2}^{\circ}$ & 3332.074 & 20 & 5 & 30002.709 & $10572_{9 / 2}^{\circ}-40574_{11 / 2}$ \\
\hline 3353.948 & 100 & 100 & 29807.041 & $11116_{\overline{7} / 2}^{\circ}-40923_{5 / 2}$ & 3331.429 & 2 & 1 & 30008.517 & $17722_{9 / 2}^{\circ}-47731_{9 / 2}$ \\
\hline 3353.160 & $4 b$ & & 29814.046 & $17121_{3 / 2}^{\circ}-46935_{3 / 2}$ & 3331.207 & 15 & 15 & 30010.517 & $8378_{7 / 2}^{\circ}-38389_{7 / 2}$ \\
\hline 3353.141 & $25 b$ & $50 \mathrm{~b}$ & 29814.215 & $16564_{11 / 2}^{\circ}-46378_{13 / 2}$ & 3330.379 & 0 & 2 & 30017.978 & $19050_{3 / 2}^{\circ}-4.9068_{5 / 2}$ \\
\hline 3352.415 & 5 & 2 & 29820.671 & $14275_{9 / 2}^{\circ}-44096_{9 / 2}$ & 3329.380 & 3 & 15 & 30026.985 & $17121_{3 / 2}^{\circ}-47148_{3 / 2}$ \\
\hline 3351.594 & $20 \mathrm{~s}$ & 25 & 29827.976 & $8018_{3 / 2}-37846_{5 / 2}^{\circ}$ & 3328.976 & 20 & 20 & 30030.629 & $12488_{9 / 2}^{\circ}-42518_{z / 2}$ \\
\hline 3351.288 & 50 & 25 & 29830.699 & $9238_{9 / 2}^{\circ}-39068_{\tau / 2}$ & 3328.845 & 2 & 3 & 30031.811 & $17837_{1 / 2}^{\circ}-47869_{3 / 2}$ \\
\hline 3351.228 & $800 \mathrm{~b}$ & $200 \mathrm{~b}$ & 29831.233 & $1521_{5 / 2}-31353_{3 / 2}^{\circ}$ & 3328.688 & $1 b$ & & 30033.227 & $12485_{7 / 2}^{\circ}-42518_{7 / 2}$ \\
\hline
\end{tabular}


TABLE 3. Classified lines of Th II-Continued

\begin{tabular}{|c|c|c|c|c|c|c|c|c|c|}
\hline \multirow{2}{*}{$\begin{array}{c}\text { Wavelength } \\
\AA\end{array}$} & \multicolumn{2}{|c|}{ Intensity } & \multirow{2}{*}{$\begin{array}{c}\text { Wavenumber } \\
\mathbf{c m}^{-1}\end{array}$} & \multirow{2}{*}{ Classification } & \multirow{2}{*}{$\begin{array}{c}\text { Wavelength } \\
\AA\end{array}$} & \multicolumn{2}{|c|}{ Intensity } & \multirow{2}{*}{$\begin{array}{c}\text { Wavenumber } \\
\mathrm{cm}^{-1}\end{array}$} & \multirow{2}{*}{ Classification } \\
\hline & Lamp & Spark & & & & Lamp & Spark & & \\
\hline 3328.668 & 5 & 8 & 30033.407 & $8460_{3 / 2}-38493_{5 / 2}^{\circ}$ & 3303.481 & 100 & 100 & 30262.386 & $14545_{5 / 2}^{\circ}-44807_{7 / 2}$ \\
\hline 3328.255 & 100 & 75 & 30037.134 & $13250_{5 / 2}-43287_{3 / 2}^{\circ}$ & 3303.410 & 5 & 5 & 30263.036 & $12488_{9 / 2}^{\circ}-42751_{7 / 2}$ \\
\hline 3327.392 & 4 & 5 & 30044.924 & $15144_{3 / 2}^{\circ}-45189_{5 / 2}$ & 3303.126 & 3 & 4 & 30265.638 & $12485_{7 / 2}^{\circ}-42751_{7 / 2}$ \\
\hline 3327.041 & 2 & & 30048.094 & $24873_{5 / 2}^{\circ}-54922_{3 / 2}$ & 3301.823 & 2 & & 30277.582 & $18214_{3 / 2}^{\circ}-48492_{5 / 2}$ \\
\hline 3326.913 & 5 & 15 & 30049.250 & $22513_{5 / 2}^{\circ}-52562_{7 / 2}$ & 3301.785 & 10 & 50 & 30277.930 & $13818_{7 / 2}^{\circ}-44096_{9 / 2}$ \\
\hline 3326.670 & 1 & 5 & 30051.445 & $15786_{5 / 2}-45838_{3 / 2}^{\circ}$ & 3301.743 & 15 & 20 & 30278.315 & $1521_{5 / 2}-31800_{7 / 2}^{\circ}$ \\
\hline 3326.465 & 150 & 40 & 30053.297 & $4490_{5 / 2}^{\circ}-34543_{5 / 2}$ & & & & & $20989_{9 / 2}^{\circ}-51268_{7 / 2}$ \\
\hline 3325.120 & 1000 & 500 & 30065.453 & $4146_{7 / 2}-34212_{5 / 2}^{\circ}$ & 3301.651 & $1500 \mathbf{r}$ & 150 & 30279.159 & $12472_{5 / 2}^{\circ}-42751_{7 / 2}$ \\
\hline 3324.752 & 800 & 400 & 30068.780 & $1859_{3 / 2}-31928_{3 / 2}^{\circ}$ & 3301.349 & 300 & 300 & 30281.929 & $11116_{7 / 2}^{\circ}-41398_{9 / 2}$ \\
\hline 3323.583 & 2 & 5 & 30079.356 & $24414_{3 / 2}^{\circ}-54493_{5 / 2}^{\circ}$ & 3301.264 & 100 & 100 & 30282.708 & $11725_{1 / 2}^{\circ}-42008_{1 / 2}$ \\
\hline 3322.470 & 10 & 15 & 30089.432 & $15710_{3 / 2}^{\circ}-45800_{5 / 2}$ & 3300.616 & $100 \mathrm{~b}$ & $5 b$ & 30288.653 & $1521_{5 / 2}-31810_{5 / 2}^{\circ}$ \\
\hline 3321.707 & 4 & 5 & 30096.344 & $20310_{5 / 2}^{\circ}-50407_{7 / 2}$ & 3299.707 & 2 & & 30296.997 & $16564_{11 / 2}^{\circ}-46861_{11 / 2}$ \\
\hline 3321.451 & 800 & 800 & 30098.663 & $4113_{5 / 2}-34212_{5 / 2}^{\circ}$ & 3299.670 & 50 & 200 & 30297.337 & $26647_{13 / 2}^{\circ}-56945_{11 / 2}$ \\
\hline 3321.254 & 10 & 15 & 30100.448 & $11576_{3 / 2}^{\circ}-41676_{3 / 2}$ & 3299.478 & 1 & & 30299.100 & $20969_{7 / 2}^{\circ}-51268_{7 / 2}$ \\
\hline 3320.301 & 600 & 500 & 30109.088 & $6700_{9 / 2}^{\circ}-36809_{7 / 2}$ & 3298.542 & 1 & & 30307.697 & $18816_{13 / 2}^{\circ}-49124_{11 / 2}$ \\
\hline 3319.629 & 25 & 100 & 30115.183 & $9585_{5 / 2}^{\circ}-39700_{5 / 2}^{\circ}$ & 3298.019 & 8 & 10 & 30312.503 & $23697_{7 / 2}^{\circ}-54010_{7 / 2}$ \\
\hline 3318.984 & 75 & 200 & 30121.035 & $6691_{3 / 2}^{\circ}-36812_{1 / 2}$ & 3297.833 & 200 & 500 & 30314.213 & $9238_{9 / 2}^{\circ}-39552_{9 / 2}^{\circ}$ \\
\hline 3318.958 & 50 & 50 & 30121.271 & $8460_{3 / 2}-38581_{5 / 2}^{\circ}$ & 3297.735 & $3 \mathbf{h}$ & 3 & 30315.114 & $17983_{5 / 2}^{\circ}-48298_{7 / 2}$ \\
\hline 3318.915 & 4 & $10 \mathrm{~b}$ & 30121.661 & $18568_{1 / 2}^{\circ}-48689_{3 / 2}$ & 3297.374 & 150 & 300 & 30318.432 & $10673_{5 / 2}^{\circ}-40991_{3 / 2}$ \\
\hline 3318.118 & $5 \mathrm{~h}$ & 8 & 30128.896 & $19248_{5 / 2}^{\circ}-49377_{7 / 2}$ & 3297.305 & 5 & 8 & 30319.067 & $16033_{5 / 2}^{\circ}-46352_{7 / 2}$ \\
\hline 3318.018 & 21 & 1 & 30129.804 & $26762_{3 / 2}-56892_{5 / 2}^{\circ}$ & 3296.607 & 150 & 200 & 30325.486 & $12902_{3 / 2}^{\circ}-43227_{5 / 2}$ \\
\hline 3317.746 & 75 & 200 & 30132.274 & $13250_{5 / 2}-43382_{5 / 2}^{\circ}$ & 3296.485 & 5 & 10 & 30326.609 & $20080_{7 / 2}^{\circ}-50407_{7 / 2}$ \\
\hline 3317.560 & 50 & 100 & 30133.963 & $7331_{5 / 2}^{\circ}-37465_{5 / 2}$ & 3296.445 & 4 & 8 & 30326.976 & $23518_{7 / 2}^{\circ}-53845_{5 / 2}$ \\
\hline 3316.215 & 100 & 40 & 30146.185 & $6244_{1 / 2}-36390_{3 / 2}^{\circ}$ & 3295.571 & $10 \mathrm{~b}$ & 20 & 30335.019 & $13468_{9 / 2}^{\circ}-43803_{\tau / 2}^{\circ}$ \\
\hline 3316.042 & 3 & 5 & 30147.757 & $23697_{7 / 2}^{\circ}-53845_{5 / 2}$ & 3295.523 & 751 & 1001 & 30335.461 & $9061_{5 / 2}-39396_{7 / 2}^{\circ}$ \\
\hline 3315.971 & 4 & 10 & 30148.403 & $17722_{9 / 2}^{\circ}-47871_{7 / 2}$ & 3295.325 & $200 \mathrm{~b}$ & 500 & 30337.283 & $6244_{1 / 2}-36581_{3 / 2}^{\circ}$ \\
\hline 3315.832 & 25 & 20 & 30149.667 & $9711_{7 / 2}-39861_{5 / 2}^{\circ}$ & 3294.998 & 100 & 300 & 30340.294 & $13468_{9 / 2}^{\circ}-43809_{9 / 2}$ \\
\hline 3315.560 & 10 & 50 & 30152.140 & $15242_{9 / 2}^{\circ}-45395_{7 / 2}$ & 3294.753 & 40 & 100 & 30342.550 & $20288_{11 / 2}^{\circ}-50631_{11 / 2}$ \\
\hline 3315.210 & 5 & 10 & 30155.323 & $22014_{11 / 2}^{\circ}-52170_{11 / 2}^{\circ}$ & 3294.568 & 75 & 50 & 30344.254 & $14545_{5 / 2}^{\circ}-44889_{3 / 2}$ \\
\hline 3314.827 & $300 \mathrm{~b}$ & 300 & 30158.807 & $8605_{5 / 2}-38764_{7 / 2}^{\circ}$ & 3294.362 & 4 & 8 & 30346.151 & $16564_{11 / 2}^{\circ}-46910_{13 / 2}$ \\
\hline 3314.541 & 3 & & 30161.410 & $15144_{3 / 2}^{\circ}-45306_{3 / 2}$ & 3294.244 & 100 & 200 & 30347.238 & $15453_{7 / 2}^{\circ}-45800_{5 / 2}$ \\
\hline 3314.187 & $2 \mathrm{~h}$ & 1 & $3 Q 164.631$ & $9202_{7 / 2}^{\circ}-39366_{5 / 2}$ & 3293.948 & 500 & 500 & 30349.965 & $9202_{7 / 2}^{\circ}-39552_{9 / 2}$ \\
\hline 3313.996 & $10 \mathrm{~h}$ & 50 & 30166.369 & $19248_{5 / 2}^{\circ}-49414_{3 / 2}$ & 3293.698 & 100 & 200 & 30352.269 & $16033_{5 / 2}^{\circ}-46385_{7 / 2}$ \\
\hline 3313.036 & 8 & $5 b$ & 30175.110 & $9720_{7 / 2}^{\circ}-39895_{9 / 2}$ & 3293.654 & 3 & & 30352.674 & $20310_{5 / 2}^{\circ}-50663_{5 / 2}$ \\
\hline 3312.680 & 20 & 15 & 30178.353 & $10189_{11 / 2}^{\circ}-40367_{9 / 2}$ & 3293.595 & 100 & 300 & 30353.218 & $14545_{5 / 2}^{\circ}-44898_{7 / 2}$ \\
\hline 3312.076 & 20 & 150 & 30183.856 & $15305_{9 / 2}-45489_{9 / 2}^{\circ}$ & 3292.844 & 5 & 4 & 30360.140 & $11576_{3 / 2}^{\circ}-41936_{3 / 2}$ \\
\hline 3310.946 & 5 & 8 & 30194.157 & $12902_{3 / 2}^{\circ}-43096_{5 / 2}$ & 3292.551 & $50 \mathrm{~b}$ & $5 w$ & 30362.842 & $16033_{5 / 2}^{\circ}-46395_{3 / 2}$ \\
\hline 3310.497 & 75 & 100 & 30198.252 & $12219_{3 / 2}-42418_{3 / 2}^{\circ}$ & 3292.520 & $1000 \mathrm{~b}$ & $1000 \mathrm{~b}$ & 30363.128 & $6700_{9 / 2}^{\circ}-37063_{9 / 2}$ \\
\hline 3310.249 & $150 \mathrm{~b}$ & 200 & 30200.515 & $6244_{1 / 2}-36444_{3 / 2}^{\circ}$ & 3291.932 & 15 & 40 & 30368.551 & $15786_{5 / 2}-46155_{5 / 2}^{\circ}$ \\
\hline 3310.001 & 4 & 8 & 30202.777 & $17121_{3 / 2}^{\circ}-47324_{5 / 2}$ & 3291.739 & 1000 & 800 & 30370.331 & $6213_{9 / 2}-36583_{7 / 2}^{\circ}$ \\
\hline 3309.135 & 150 & 200 & 30210.681 & $7331_{5 / 2}^{\circ}-37542_{3 / 2}$ & 3291.606 & 50 & 15 & 30371.559 & $11116_{7 / 2}^{\circ}-41488_{7 / 2}$ \\
\hline 3309.016 & 8 & 15 & 30211.768 & $11116_{7 / 2}^{\circ}-41328_{5 / 2}$ & 3291.343 & 8 & 100 & 30373.986 & $12570_{7 / 2}-42944_{7 / 2}^{\circ}$ \\
\hline 3308.085 & $10 \mathrm{~b}$ & 40 & 30220.270 & $9238_{9 / 2}^{\circ}-39458_{7 / 2}$ & 3290.633 & 2 & & 30380.539 & $22355_{1 / 2}^{\circ}-52735_{3 / 2}$ \\
\hline 3307.215 & $3 \mathrm{~h}$ & 8 & 30228.219 & $14275_{9 / 2}^{\circ}-44503_{7 / 2}^{\circ}$ & 3290.250 & 15 & 15 & 30384.075 & $15305_{9 / 2}-45689_{7 / 2}^{\circ}$ \\
\hline 3306.389 & lb & 40 & 30235.771 & $17771_{11 / 2}^{\circ}-48006_{9 / 2}$ & 3290.126 & 150 & 40 & 30385.220 & $15349_{11 / 2}^{\circ}-45735_{11 / 2}$ \\
\hline 3306.054 & 10 & 20 & 30238.835 & $8378_{\tau / 2}^{\circ}-38617_{9 / 2}$ & 3289.675 & 0 & 2 & 30389.386 & $20080_{7 / 2}^{\circ}-50470_{9 / 2}$ \\
\hline 3305.607 & 8 & 20 & 30242.923 & $14484_{11 / 2}^{\circ}-44727_{11 / 2}$ & 3288.159 & 50 & 400 & 30403.396 & $18973_{7 / 2}^{\circ}-49377_{7 / 2}$ \\
\hline 3304.780 & 3 & 4 & 30250.491 & $10673_{5 / 2}^{\circ}-40923_{5 / 2}$ & 3287.789 & 800 & 1000 & 30406.818 & $1521_{5 / 2}-31928_{3 / 2}^{\circ}$ \\
\hline 3304.512 & 4 & 10 & 30252.945 & $21682_{7 / 2}^{\circ}-51935_{5 / 2}$ & 3287.363 & 4 & & 30410.758 & $17460_{5 / 2}^{\circ}-47871_{7 / 2}$ \\
\hline 3304.176 & $50 \mathrm{~b}$ & 25 & 30256.021 & $9202_{7 / 2}^{\circ}-39458_{7 / 2}^{\circ}$ & 3287.083 & 50 & 50 & 30413.348 & $18118_{3 / 2}-48532_{1 / 2}^{\circ}$ \\
\hline
\end{tabular}


TABLE 3. Classified lines of Th II-Continued

\begin{tabular}{|c|c|c|c|c|c|c|c|c|c|}
\hline \multirow{2}{*}{$\begin{array}{c}\text { Wavelength } \\
\AA\end{array}$} & \multicolumn{2}{|c|}{ Intensity } & \multirow{2}{*}{$\begin{array}{c}\text { Wavenumber } \\
\mathbf{c m}^{-1}\end{array}$} & \multirow{2}{*}{ Classification } & \multirow{2}{*}{$\begin{array}{c}\text { Wavelength } \\
\AA\end{array}$} & \multicolumn{2}{|c|}{ Intensity } & \multirow{2}{*}{$\begin{array}{c}\text { Wavenumber } \\
\mathbf{c m}^{-1}\end{array}$} & \multirow{2}{*}{ Classification } \\
\hline & Lamp & Spark & & & & Lamp & Spark & & \\
\hline 3286.583 & 400 & 500 & 30417.975 & $8018_{3 / 2}-38436_{3 / 2}^{\circ}$ & 3263.626 & 15 & 150 & 30631.934 & $13818_{7 / 2}^{\circ}-44450_{9 / 2}$ \\
\hline 3284.776 & 3 & & 30434.708 & $19050_{3 / 2}^{\circ}-49485_{1 / 2}$ & 3263.111 & 4 & & 30636.768 & $15786_{5 / 2}-46423_{3 / 2}^{\circ}$ \\
\hline 3284.310 & 2 & & 30439.026 & $23730_{9 / 2}^{\circ}-54169_{7 / 2}$ & 3263.032 & 100 & 200 & 30637.510 & $9585_{5 / 2}^{\circ}-40222_{3 / 2}$ \\
\hline 3282.979 & 100 & 500 & 30451.366 & $15453_{7 / 2}^{\circ}-45904_{9 / 2}$ & 3262.952 & 8 & 15 & 30638.261 & $21297_{5 / 2}^{\circ}-51935_{5 / 2}$ \\
\hline 3282.948 & 5 & & 30451.654 & $14275_{9 / 2}^{\circ}-44727_{11 / 2}$ & 3262.882 & 5 & 5 & 30638.918 & $12488_{9 / 2}^{\circ}-43127_{11 / 2}$ \\
\hline 3282.231 & 2 & 50 & 30458.306 & $24463_{5 / 2}^{\circ}-54922_{3 / 2}^{\circ}$ & 3262.669 & 800 & 800 & 30640.918 & $6168_{7 / 2}^{\circ}-36809_{7 / 2}$ \\
\hline 3282.130 & 15 & 50 & 30459.243 & $17272_{9 / 2}^{\circ}-47731_{9 / 2}$ & 3262.564 & 15 & 50 & 30641.905 & $14484_{i 1 / 2}^{\circ}-45126_{9 / 2}$ \\
\hline 3281.976 & 25 & & 30460.672 & $9400_{5 / 2}-39861_{5 / 2}^{\circ}$ & 3262.005 & 2 & 5 & 30647.155 & $9720_{7 / 2}^{\circ}-40367_{9 / 2}$ \\
\hline 3281.604 & 2 & & 30464.125 & $28923_{5 / 2}^{\circ}-59387_{7 / 2}$ & 3261.541 & 100 & 200 & 30651.515 & $10572_{9 / 2}^{\circ}-41223_{11 / 2}^{\circ}$ \\
\hline 3281.414 & $100 \mathrm{~b}$ & & 30465.889 & $15144_{3 / 2}^{\circ}-45610_{5 / 2}$ & 3261.148 & 4 & 20 & 30655.209 & $10673_{5 / 2}^{\circ}-41328_{5 / 2}$ \\
\hline 3281.234 & 5 & & 30467.560 & $12488_{9 / 2}^{\circ}-42955_{9 / 2}$ & 3261.114 & 10 & 100 & 30655.528 & $15144_{3 / 2}^{\circ}-45800_{5 / 2}$ \\
\hline 3281.000 & 3 & & 30469.733 & $17983_{5 / 2}^{\circ}-48453_{7 / 2}$ & 3260.941 & $20 \mathrm{~b}$ & & 30657.155 & $24381_{7 / 2}-55038_{9 / 2}^{\circ}$ \\
\hline 3280.954 & 10 & 25 & 30470.160 & $12485_{7 / 2}^{\circ}-42955_{9 / 2}$ & 3260.915 & $50 \mathrm{~b}$ & $200 \mathrm{~b}$ & 30657.399 & $9238_{9 / 2}^{\circ}-39895_{9 / 2}$ \\
\hline 3280.740 & 25 & 40 & 30472.148 & $9711_{7 / 2}-40184_{7 / 2}^{\circ}$ & 3260.478 & 4 & & 30661.508 & $15242_{9 / 2}^{\circ}-45904_{9 / 2}$ \\
\hline 3280.415 & $50 \mathrm{~b}$ & 75 & 30475.166 & $10572_{9 / 2}^{\circ}-41047_{9 / 2}$ & 3259.616 & 100 & 200 & 30669.616 & $11576_{3 / 2}^{\circ}-42246_{1 / 2}$ \\
\hline 3280.372 & 150 & 500 & 30475.566 & $8018_{3 / 2}-38493_{5 / 2}^{\circ}$ & 3259.245 & $50 \mathrm{~b}$ & 50 & 30673.107 & $16033_{5 / 2}^{\circ}-46706_{7 / 2}$ \\
\hline 3279.357 & 10 & 25 & 30484.998 & $8378_{7 / 2}^{\circ}-38863_{5 / 2}^{\circ}$ & 3257.934 & 100 & 200 & 30685.450 & $13818_{7 / 2}^{\circ}-44503_{7 / 2}^{\circ}$ \\
\hline 3278.767 & 150 & 75 & 30490.484 & $7331_{5 / 2}^{\circ}-37821_{3 / 2}^{\circ}$ & 3257.718 & 4 & 5 & 30687.484 & $14101_{1 / 2}^{\circ}-44789_{1 / 2}$ \\
\hline 3278.630 & 3 & & 30491.758 & $23518_{7 / 2}^{\circ}-54010_{7 / 2}$ & 3257.162 & 300 & 400 & 30692.722 & $9585_{5 / 2}^{\circ}-40278_{3 / 2}$ \\
\hline 3278.193 & 10 & 25 & 30495.822 & $9720_{7 / 2}^{\circ}-40216_{5 / 2}$ & 3256.274 & 800 & 800 & 30701.092 & $9238_{9 / 2}^{\circ}-39939_{11 / 2}$ \\
\hline 3277.925 & 20 & 25 & 30498.316 & $9202_{7 / 2}^{\circ}-39700_{5 / 2}$ & 3255.691 & 3 & & 30706.589 & $17983_{5 / 2}^{\circ}-48689_{3 / 2}$ \\
\hline 3276.941 & 8 & & 30507.473 & $24414_{3 / 2}^{\circ}-54922_{3 / 2}$ & 3255.509 & 200 & 200 & 30708.306 & $13468_{9 / 2}^{\circ}-44177_{11 / 2}$ \\
\hline 3276.814 & $3 \mathrm{~h}$ & & 30508.656 & $17983_{5 / 2}^{\circ}-48492_{5 / 2}$ & 3255.283 & 8 & 400 & 30710.438 & $18816_{13 / 2}^{\circ}-49527_{13 / 2}$ \\
\hline 3275.365 & 1 & & 30522.152 & $21131_{3 / 2}^{\circ}-51653_{1 / 2}$ & 3255.014 & 3 & & 30712.976 & $20969_{7 / 2}^{\circ}-51681_{9 / 2}^{\circ}$ \\
\hline 3275.261 & 25 & 40 & 30523.121 & $13250_{5 / 2}-43773_{7 / 2}^{\circ}$ & 3254.810 & 150 & 200 & 30714.901 & $7001_{3 / 2}-37716_{1 / 2}^{\circ}$ \\
\hline 3275.068 & $300 \mathrm{~b}$ & 800 & 30524.920 & $13248_{9 / 2}-43773_{7 / 2}^{\circ}$ & 3254.398 & 3 & & 30718.789 & $19912_{13 / 2}^{\circ}-50631_{11 / 2}^{\circ}$ \\
\hline 3274.404 & 50 & 25 & 30531.110 & $4490_{5 / 2}^{\circ}-35021_{3 / 2}$ & 3252.750 & 15 & 75 & 30734.352 & $13818_{7 / 2}^{\circ}-44552_{5 / 2}$ \\
\hline 3274.266 & 4 & & 30532.396 & $14275_{9 / 2}^{\circ}-44807_{7 / 2}$ & 3252.721 & 15 & 100 & 30734.626 & $17272_{9 / 2}^{\circ}-48006_{9 / 2}$ \\
\hline 3274.192 & 4 & & 30533.086 & $21297_{5 / 2}^{\circ}-51830_{7 / 2}$ & 3251.308 & 5 & 20 & 30747.983 & $17121_{3 / 2}^{\circ}-47869_{3 / 2}$ \\
\hline 3273.916 & 400 & 800 & 30535.660 & $10189_{11 / 2}^{\circ}-40724_{13 / 2}$ & 3250.494 & 2 & & 30755.683 & $12472_{5 / 2}^{\circ}-43227_{5 / 2}^{\circ}$ \\
\hline 3272.995 & 2 & & 30544.252 & $21131_{3 / 2}^{\circ}-51676_{3 / 2}$ & 3250.191 & 10 & 50 & 30758.550 & $12488_{9 / 2}^{\circ}-43246_{7 / 2}$ \\
\hline 3272.577 & 8 & 100 & 30548.154 & $16033_{5 / 2}^{\circ}-46581_{s / 2}$ & 3249.973 & $10 \mathrm{~b}$ & 40 & 30760.613 & $14545_{5 / 2}^{\circ}-45306_{3 / 2}$ \\
\hline 3271.121 & 20 & 150 & 30561.750 & $29431_{\pi / 2}-59993_{9 / 2}^{\circ}$ & 3249.916 & $75 b$ & 100 & 30761.152 & $12485_{7 / 2}^{\circ}-43246_{7 / 2}$ \\
\hline 3270.940 & 5 & & 30563.441 & $8018_{3 / 2}-38581_{5 / 2}^{\circ}$ & 3248.893 & $100 \mathrm{~b}$ & 200 & 30770.838 & $13406_{13 / 2}^{\circ}-44177_{11 / 2}$ \\
\hline 3270.818 & 150 & 100 & 30564.582 & $0_{3 / 2}-30564_{1 / 2}^{\circ}$ & 3248.602 & 40 & 40 & 30773.594 & $7331_{5 / 2}^{\circ}-38105_{5 / 2}$ \\
\hline 3270.234 & 15 & 100 & 30570.039 & $16033_{5 / 2}^{\circ}-46603_{5 / 2}$ & 3248.490 & 75 & 100 & 30774.655 & $12472_{5 / 2}^{\circ}-43246_{7 / 2}$ \\
\hline 3270.193 & 3 & 8 & 30570.423 & $13818_{7 / 2}^{\circ}-44388_{5 / 2}$ & 3248.376 & 50 & 100 & 30775.735 & $11576_{3 / 2}^{\circ}-42352_{5 / 2}$ \\
\hline 3270.041 & 1 & & 30571.844 & $11116_{7 / 2}^{\circ}-41688_{7 / 2}$ & 3247.594 & 150 & 300 & 30783.146 & $9400_{5 / 2}-40184_{7 / 2}^{\circ}$ \\
\hline 3269.470 & 100 & 100 & 30577.183 & $6700_{9 / 2}^{\circ}-37277_{7 / 2}$ & 3247.081 & 4 & & 30788.009 & $14101_{1 / 2}^{\circ}-44889_{3 / 2}$ \\
\hline 3268.855 & 5 & & 30582.935 & $20080_{7 / 2}^{\circ}-50663_{5 / 2}$ & 3246.795 & $15 b$ & 25 & 30790.720 & $8605_{5 / 2}-39396_{7 / 2}^{\circ}$ \\
\hline 3268.146 & 2 & & 30589.570 & $21682_{7 / 2}^{\circ}-52272_{7 / 2}$ & 3246.581 & 20 & 50 & 30792.750 & $11116_{7 / 2}^{\circ}-41909_{9 / 2}$ \\
\hline 3267.368 & 1 & & 30596.853 & $20310_{5 / 2}^{\circ}-50907_{3 / 2}$ & 3246.558 & lb & & 30792.968 & $28823_{5 / 2}-59616_{3 / 2}^{\circ}$ \\
\hline 3267.003 & 200 & 300 & 30600.272 & $7001_{3 / 2}-37601_{3 / 2}^{\circ}$ & 3246.345 & 5 & & 30794.989 & $25440_{5 / 2}^{\circ}-56235_{3 / 2}$ \\
\hline 3266.601 & 5 & & 30604.037 & $14790_{7 / 2}^{\circ}-45395_{7 / 2}$ & 3245.761 & $200 \mathrm{~b}$ & 1000 & 30800.529 & $9061_{5 / 2}-39861_{5 / 2}^{\circ}$ \\
\hline 3266.330 & $5 b$ & & 30606.576 & $7001_{3 / 2}-37607_{1 / 2}^{\circ}$ & 3244.522 & 15 & & 30812.291 & $12570_{7 / 2}-43382_{5 / 2}^{\circ}$ \\
\hline 3266.313 & $40 \mathrm{~b}$ & 100 & 30606.736 & $16564_{11 / 2}^{\circ}-47171_{9 / 2}$ & 3244.236 & 10 & 25 & 30815.007 & $10673_{5 / 2}^{\circ}-41488_{7 / 2}$ \\
\hline 3265.578 & 1501 & 200 & 30613.624 & $7331_{5 / 2}^{\circ}-37945_{5 / 2}$ & 3243.748 & 5 & 50 & 30819.643 & $14790_{7 / 2}^{\circ}-45610_{5 / 2}$ \\
\hline 3264.434 & 100 & 500 & 30624.352 & $12472_{5 / 2}^{\circ}-43096_{5 / 2}$ & 3243.469 & 1 & & 30822.294 & $19050_{3 / 2}^{\circ}-49873_{5 / 2}$ \\
\hline 3264.121 & 100 & 200 & 30627.289 & $13468_{9 / 2}^{\circ}-44096_{9 / 2}^{\circ}$ & 3243.195 & 2 & & 30824.898 & $16906_{7 / 2}^{\circ}-4773 l_{9 / 2}$ \\
\hline 3263.756 & 40 & 40 & 30630.714 & $9585_{5 / 2}^{\circ}-40216_{5 / 2}$ & 3243.070 & 10 & 25 & 30826.086 & $9585_{5 / 2}^{\circ}-40411_{7 / 2}$ \\
\hline
\end{tabular}


TABle 3. Classified lines of Th II-Continued

\begin{tabular}{|c|c|c|c|c|c|c|c|c|c|}
\hline \multirow{2}{*}{$\begin{array}{c}\text { Wavelength } \\
\AA \\
\end{array}$} & \multicolumn{2}{|c|}{ Intensity } & \multirow{2}{*}{$\begin{array}{c}\text { W avenumber } \\
\mathbf{c m}^{-1}\end{array}$} & \multirow{2}{*}{ Classification } & \multirow{2}{*}{$\begin{array}{c}\text { Wavelength } \\
\AA\end{array}$} & \multicolumn{2}{|c|}{ Intensity } & \multirow{2}{*}{$\begin{array}{c}\text { Wavenumber } \\
\mathbf{c m}^{-1}\end{array}$} & \multirow{2}{*}{ Classification } \\
\hline & Lamp & Spark & & & & Lamp & Spark & & \\
\hline 3243.029 & 50 & 25 & 30826.475 & $10572_{9 / 2}^{\circ}-41398_{9 / 2}$ & 3224.488 & 3 & 3 & 31003.723 & $10673_{5 / 2}^{\circ}-41676_{3 / 2}$ \\
\hline 324.2 .251 & 751 & 800 & 30833.872 & $7331_{5 / 2}^{\circ}-38165_{7 / 2}$ & 3223.911 & 5 & 20 & 31009.272 & $14790_{7 / 2}^{\circ}-45800_{5 / 2}$ \\
\hline 3242.176 & 4 & & 30834.585 & $17983_{5 / 2}^{\circ}-48817_{3 / 2}^{\circ}$ & 3223.800 & 25 & 20 & 31010.340 & $4146_{7 / 2}-35156_{5 / 2}^{\circ}$ \\
\hline 3241.830 & 4 & & 30837.876 & $17460_{5 / 2}^{\circ}-48298_{7 / 2}$ & 3223.435 & 4 & 5 & 31013.851 & $9202_{7 / 2}^{\circ}-40216_{5 / 2}$ \\
\hline 3241.612 & 5 & 25 & 30839.950 & $25246_{9 / 2}-56086_{9 / 2}^{\circ}$ & 3223.285 & 20 & 15 & 31015.294 & $10673_{5 / 2}^{\circ}-41688_{7 / 2}$ \\
\hline 3241.533 & 151 & 200 & 30840.702 & $20989_{9 / 2}^{\circ}-51830_{7 / 2}^{\circ}$ & 3221.864 & 20 & 150 & 31028.973 & $15349_{11 / 2}^{\circ}-46378_{13 / 2}$ \\
\hline 3241.484 & 5 & 3 & 30841.168 & $17771_{11 / 2}^{\circ}-48612_{13 / 2}^{\circ}$ & 3221.291 & $400 \mathrm{~b}$ & 40 & 31034.492 & $10189_{11 / 2}^{\circ}-41223_{11 / 2}$ \\
\hline 3241.108 & 400 & 500 & 30844.746 & $7001_{3 / 2}-37846_{5 / 2}^{\circ}$ & 3220.351 & 200 & 300 & 31043.550 & $4113_{5 / 2}-35156_{5 / 2}^{\circ}$ \\
\hline 3240.521 & 15 & 75 & 30850.333 & $9720_{7 / 2}^{\circ}-40570_{7 / 2}$ & 3218.866 & 75 & 150 & 31057.872 & $7331_{5 / 2}^{\circ}-38389_{7 / 2}$ \\
\hline 3240.475 & 100 & 150 & 30850.771 & $6691_{3 / 2}^{\circ}-37542_{3 / 2}$ & 3218.755 & 100 & 40 & 31058.943 & $9585_{5 / 2}^{\circ}-40644_{5 / 2}$ \\
\hline 3240.330 & 8 & 75 & 30852.151 & $17837_{1 / 2}^{\circ}-48689_{3 / 2}$ & 3218.340 & 4 & & 31062.948 & $15305_{9 / 2}-46368_{9 / 2}^{\circ}$ \\
\hline 3240.090 & 8 & 3 & 30854.436 & $18214_{3 / 2}^{\circ}-49068_{5 / 2}$ & 3218.309 & 10 & 40 & 31063.247 & $12902_{3 / 2}^{\circ}-43965_{1 / 2}$ \\
\hline 3239.700 & 4 & & 30858.150 & $10189_{11 / 2}^{\circ}-41047_{9 / 2}$ & 3218.123 & 5 & 15 & 31065.042 & $14545_{5 / 2}^{\circ}-45610_{5 / 2}$ \\
\hline 3239.288 & 100 & 200 & 30862.075 & $6700_{9 / 2}^{\circ}-37562_{11 / 2}$ & 3217.853 & 40 & 100 & 31067.649 & $12219_{3 / 2}-43287_{3 / 2}^{\circ}$ \\
\hline 3238.802 & 10 & 75 & 30866.706 & $15349_{11 / 2}^{\circ}-46216_{11 / 2}$ & 3217.730 & 75 & 150 & 31068.836 & $10379_{9 / 2}-41447_{11 / 2}^{\circ}$ \\
\hline 3238.445 & 4 & 5 & 30870.109 & $12902_{3 / 2}^{\circ}-43772_{5 / 2}$ & 3217.456 & $100 \mathrm{~b}$ & 300 & 31071.482 & $9400_{5 / 2}-40472_{3 / 2}^{\circ}$ \\
\hline 3238.408 & 5 & 4 & 30870.461 & $15710_{3 / 2}^{\circ}-46581_{5 / 2}^{\circ}$ & 3217.432 & $15 b$ & & 31071.714 & $15324_{1 / 2}^{\circ}-46395_{3 / 2}$ \\
\hline 3238.116 & 1000 & 800 & 30873.245 & $4113_{5 / 2}-34986_{3 / 2}^{\circ}$ & 3216.634 & 20 & & 31079.422 & $8378_{7 / 2}^{\circ}-39458_{7 / 2}^{\circ}$ \\
\hline 3237.534 & $2 \mathrm{~b}$ & & 30878.795 & $22642_{9 / 2}^{\circ}-53520_{9 / 2}^{\circ}$ & 3216.530 & 8 & & 31080.427 & $13818_{7 / 2}^{\circ}-44898_{7 / 2}^{\circ}$ \\
\hline 3236.773 & 8 & 100 & 30886.054 & $6244_{1 / 2}-37130_{1 / 2}^{\circ}$ & 3216.013 & 5 & 10 & 31085.423 & $17983_{5 / 2}^{\circ}-49068_{5 / 2}^{\circ}$ \\
\hline 3236.261 & 8 & & 30890.941 & $22028_{15 / 2}^{\circ}-52918_{13 / 2}$ & 3215.778 & 100 & 100 & 31087.694 & $6700_{9 / 2}^{\circ}-37787_{7 / 2}$ \\
\hline 3236.113 & 5 & 5 & 30892.353 & $15710_{3 / 2}^{\circ}-46603_{5 / 2}$ & 3214.478 & 2 & & 31100.267 & $16906_{7 / 2}^{\circ}-48006_{9 / 2}^{\circ}$ \\
\hline 3235.841 & $800 r$ & 500 & 30894.950 & $6168_{7 / 2}^{\circ}-37063_{9 / 2}$ & 3213.574 & 200 & 300 & 31109.015 & $6168_{7 / 2}^{\circ}-37277_{7 / 2}$ \\
\hline 3235.397 & $5 b$ & 50 & 30899.190 & $15453_{7 / 2}^{\circ}-46352_{7 / 2}$ & 3212.812 & 10 & $15 b$ & 31116.393 & $10572_{9 / 2}^{\circ}-41688_{7 / 2}^{\circ}$ \\
\hline 3235.381 & $2 \mathrm{~b}$ & & 30899.342 & $18973_{7 / 2}^{\circ}-49873_{5 / 2}$ & 3212.494 & 3 & & 31119.473 & $14275_{9 / 2}^{\circ}-45395_{7 / 2}$ \\
\hline 3234.888 & 10 & 100 & 30904.051 & $22014_{11 / 2}^{\circ}-52918_{13 / 2}$ & 3212.241 & 2 & & 31121.924 & $17722_{9 / 2}^{\circ}-48844_{9 / 2}$ \\
\hline 3234.771 & 2 & & 30905.169 & $12902_{3 / 2}^{\circ}-43807_{3 / 2}$ & 3212.131 & 20 & 75 & 31122.990 & $9061_{5 / 2}-40184_{/ / 2}^{\circ}$ \\
\hline 3233.626 & 10 & 25 & 30916.112 & $10572_{9 / 2}^{\circ}-41488_{7 / 2}$ & 3211.467 & 8 & 50 & 31129.424 & $9238_{9 / 2}^{\circ}-40367_{9 / 2}$ \\
\hline 3233.506 & 3 & & 30917.259 & $18568_{1 / 2}^{\circ}-49485_{1 / 2}$ & 3210.309 & 300 & 300 & 31140.653 & $6700_{9 / 2}^{\circ}-37840_{9 / 2}$ \\
\hline 3233.334 & 20 & 50 & 30918.904 & $15236_{3 / 2}-46155_{5 / 2}^{\circ}$ & 3209.332 & 10 & 50 & 31150.132 & $15453_{7 / 2}^{\circ}-46603_{5 / 2}^{\circ}$ \\
\hline 3232.797 & 501 & 100 & 30924.040 & $9720_{7 / 2}^{\circ}-40644_{5 / 2}$ & 3208.418 & 2 & & 31159.006 & $19248_{5 / 2}^{\circ}-50407_{\tau / 2}$ \\
\hline 3231.925 & 5 & & 30932.383 & $15453_{7 / 2}^{\circ}-46385_{7 / 2}$ & 3208.026 & 100 & 150 & 31162.813 & $12219_{3 / 2}-43382_{5 / 2}^{\circ}$ \\
\hline 3231.579 & 2 & & 30935.695 & $20288_{11 / 2}^{\circ}-51224_{9 / 2}$ & 3207.784 & 100 & 200 & 31165.164 & $9202_{7 / 2}^{\circ}-40367_{9 / 2}^{\circ}$ \\
\hline 3230.867 & 500 & 400 & 30942.512 & $9711_{7 / 2}-40654_{5 / 2}^{\circ}$ & 3207.202 & 5 & 15 & 31170.819 & $18118_{3 / 2}-49289_{5 / 2}^{\circ}$ \\
\hline 3229.336 & 4 & & 30957.181 & $20310_{5 / 2}^{\circ}-51268_{7 / 2}$ & 3206.933 & $75 b$ & 100 & 31173.434 & $9238_{9 / 2}^{\circ}-4041 \mathrm{i}_{7 / 2}$ \\
\hline 3229.010 & 1000 & 1000 & 30960.306 & $7331_{5 / 2}^{\circ}-38291_{7 / 2}$ & & & & & $8378_{7 / 2}^{\circ}-39552_{9 / 2}^{\circ}$ \\
\hline 3228.542 & 5 & & 30964.794 & $16906_{7 / 2}^{\circ}-47871_{7 / 2}$ & 3206.218 & 8 & 20 & 31180.386 & $20989_{9 / 2}^{\circ}-52170_{11 / 2}$ \\
\hline 3228.342 & 5 & 15 & 30966.712 & $20969_{7 / 2}^{\circ}-51935_{5 / 2}$ & 3206.170 & 10 & 50 & 31180.852 & $17272_{9 / 2}^{\circ}-48453_{7 / 2}^{\circ}$ \\
\hline 3227.774 & $150 \mathrm{~b}$ & $100 \mathrm{~b}$ & 30972.161 & $0_{3 / 2}-30972_{5 / 2}^{\circ}$ & 3205.480 & 5 & 50 & 31187.564 & $24309_{11 / 2}^{o}-55496_{9 / 2}$ \\
\hline 3227.488 & $2 \mathrm{~b}$ & & 30974.906 & $21297_{5 / 2}^{\circ}-52272_{7 / 2}$ & 3205.290 & 25 & 150 & 31189.412 & $15236_{3 / 2}-46426_{1 / 2}^{\circ}$ \\
\hline 3227.419 & 2 & & 30975.568 & $23518_{7 / 2}^{\circ}-54493_{5 / 2}$ & 3205.056 & 5 & & 31191.690 & $15710_{3 / 2}^{\circ}-46902_{5 / 2}$ \\
\hline 3227.008 & 100 & 200 & 30979.513 & $6700_{9 / 2}^{\circ}-37679_{11 / 2}^{\circ}$ & 3204.180 & 10 & 50 & 31200.217 & $18214_{3 / 2}^{\circ}-49414_{3 / 2}^{\circ}$ \\
\hline 3226.940 & 3 & & 30980.166 & $17837_{i / 2}^{\circ}-48817_{3 / 2}$ & 3203.881 & 50 & 200 & 31203.128 & $12570_{7 / 2}-43773_{7 / 2}^{\circ}$ \\
\hline 3226.817 & 1 & & 30981.347 & $13468_{9 / 2}^{\circ}-44450_{9 / 2}^{\circ}$ & 3203.755 & 1 & & 31204.356 & $14101_{i / 2}^{i}-45306_{3 / 2}$ \\
\hline 3226.413 & 100 & 200 & 30985.226 & $9585_{5 / 2}^{\circ}-40570_{7 / 2}$ & 3203.616 & 10 & 150 & 31205.710 & $15349_{11 / 2}^{\circ}-46555_{11 / 2}$ \\
\hline 3226.119 & 100 & 200 & 30988.050 & $8378_{7 / 2}^{\circ}-39366_{5 / 2}$ & 3203.255 & $8 b$ & $20 \mathrm{~b}$ & 31209.226 & $9202_{7 / 2}^{\circ}-40411_{7 / 2}$ \\
\hline 3225.957 & 3 & & 30989.606 & $13818_{\pi / 2}^{\circ}-44807_{\pi / 2}$ & 3203.233 & $20 \mathrm{~b}$ & $100 \mathrm{~b}$ & 31209.441 & $10189_{11 / 2}^{\circ}-41398_{9 / 2}$ \\
\hline 3225.902 & 50 & 50 & 30990.134 & $1859_{3 / 2}-32850_{5 / 2}^{\circ}$ & 3202.125 & 10 & 15 & 31220.239 & $11116_{7 / 2}^{\circ}-42336_{5 / 2}^{1 / 2}$ \\
\hline 3225.657 & 75 & 100 & 30992.488 & $17460_{5 / 2}^{\circ}-48453_{7 / 2}$ & 3201.655 & $2 \mathrm{~b}$ & & 31224.822 & $15710_{3 / 2}^{\circ}-46935_{3 / 2}^{\circ}$ \\
\hline 3225.412 & 400 & 400 & 30994.842 & $9711_{7 / 2}-40706_{7 / 2}^{\circ}$ & 3201.194 & 8 & 20 & 31229.319 & $17460_{5 / 2}^{\circ}-48689_{3 / 2}^{\circ}$ \\
\hline
\end{tabular}


TABLE 3. Classified lines of Th II-Continued

\begin{tabular}{|c|c|c|c|c|c|c|c|c|c|}
\hline \multirow{2}{*}{$\begin{array}{c}\text { Wavelength } \\
\AA\end{array}$} & \multicolumn{2}{|c|}{ Intensity } & \multirow{2}{*}{$\begin{array}{c}\text { Wavenumber } \\
\mathbf{c m}^{-1}\end{array}$} & \multirow{2}{*}{ Classification } & \multirow{2}{*}{$\begin{array}{c}\text { Wavelength } \\
\AA\end{array}$} & \multicolumn{2}{|c|}{ Intensity } & \multirow{2}{*}{$\begin{array}{c}\text { Wavenumber } \\
\mathbf{c m}^{-1}\end{array}$} & \multirow{2}{*}{ Classification } \\
\hline & Lamp & Spark & & & & Lamp & Spark & & \\
\hline 3200.556 & 10 & 25 & 31235.544 & $11116_{7 / 2}^{\circ}-42352_{5 / 2}$ & 3181.758 & 8 & 50 & 31420.078 & $14484_{11 / 2}^{\circ}-45904_{9 / 2}$ \\
\hline 3199.123 & 2 & & 31249.535 & $20686_{5 / 2}^{\circ}-51935_{5 / 2}$ & 3181.189 & 200 & 300 & 31425.698 & $7331_{5 / 2}^{\circ}-38757_{3 / 2}$ \\
\hline 3198.969 & $75 \mathrm{~s}$ & & 31251.039 & $4490_{5 / 2}^{\circ}-35741_{5 / 2}$ & 3180.773 & 3 & 8 & 31429.808 & $13468_{9 / 2}^{\circ}-44898_{7 / 2}$ \\
\hline 3198.694 & $50 \mathrm{~b}$ & & 31253.726 & $6691_{3 / 2}^{\circ}-37945_{5 / 2}$ & 3180.194 & $1000 \mathbf{r}$ & 1500 & 31435.530 & $1521_{5 / 2}-32957_{7 / 2}^{\circ}$ \\
\hline 3198.596 & 4 & 4 & 31254.683 & $14545_{5 / 2}^{\circ}-45800_{5 / 2}$ & 3179.969 & 40 & 100 & 31437.754 & $15710_{3 / 2}^{\circ}-47148_{3 / 2}$ \\
\hline 3198.482 & 100 & 100 & 31255.797 & $8605_{5 / 2}-39861_{5 / 2}^{\circ}$ & 3179.531 & 2 & 5 & 31442.085 & $9202_{7 / 2}^{\circ}-40644_{5 / 2}$ \\
\hline 3198.229 & 100 & 200 & 31258.270 & $13468_{9 / 2}^{\circ}-44727_{11 / 2}^{\circ}$ & 3179.048 & $500 \mathrm{~s}$ & 500 & 31446.862 & $4146_{7 / 2}-35593_{9 / 2}^{\circ}$ \\
\hline 3197.704 & 8 & 2 & 31263.401 & $10673_{5 / 2}^{\circ}-41936_{3 / 2}$ & 3178.781 & 20 & 200 & 31449.503 & $15453_{7 / 2}^{\circ}-46902_{5 / 2}$ \\
\hline 3197.530 & 3 & & 31265.103 & $21297_{5 / 2}^{\circ}-52562_{7 / 2}$ & 3177.876 & 20 & 100 & 31458.459 & $15144_{3 / 2}^{\circ}-46603_{5 / 2}$ \\
\hline 3195.313 & 25 & & 31286.795 & $12485_{7 / 2}^{\circ}-43772_{5 / 2}$ & 3177.769 & 8 & 75 & 31459.518 & $14275_{9 / 2}^{\circ}-45735_{11 / 2}$ \\
\hline 3194.859 & 4 & & 31291.240 & $16033_{5 / 2}^{\circ}-47324_{5 / 2}$ & 3177.485 & 8 & 100 & 31462.330 & $14790_{7 / 2}^{\circ}-46253_{9 / 2}$ \\
\hline 3194.260 & 5 & 40 & 31297.108 & $6168_{7 / 2}^{\circ}-37465_{5 / 2}$ & 3177.382 & 3 & & 31463.350 & $15242_{9 / 2}^{\circ}-46706_{7 / 2}$ \\
\hline 3193.624 & 1 & & 31303.340 & $20969_{7 / 2}^{\circ}-52272_{7 / 2}^{\circ}$ & 3177.346 & 2 & & 31463.706 & $10855_{7 / 2}-42319_{9 / 2}^{\circ}$ \\
\hline 3193.368 & 50 & 200 & 31305.850 & $9400_{5 / 2}-40706_{7 / 2}^{\circ}$ & 3177.198 & 50 & & 31465.172 & $6700_{9 / 2}^{\circ}-38165_{7 / 2}^{\circ}$ \\
\hline 3193.159 & 75 & 200 & 31307.899 & $13818_{7 / 2}^{\circ}-45126_{9 / 2}^{\circ}$ & 3176.568 & 5 & & 31471.412 & $25607_{9 / 2}^{\circ}-57078_{9 / 2}^{\circ}$ \\
\hline 3192.364 & 8 & 20 & 31315.695 & $12488_{9 / 2}^{\circ}-43803_{7 / 2}$ & 3176.506 & 100 & 150 & 31472.026 & $6244_{1 / 2}-37716_{i / 2}^{\circ}$ \\
\hline 3192.099 & 50 & 150 & 31318.295 & $12485_{7 / 2}^{\circ}-43803_{7 / 2}$ & 3175.726 & 400 & 500 & 31479.756 & $6700_{9 / 2}^{\circ}-38179_{9 / 2}$ \\
\hline 3191.843 & $10 \mathrm{~b}$ & 150 & 31320.807 & $13406_{13 / 2}^{\circ}-44727_{11 / 2}$ & 3175.057 & 5 & 50 & 31486.388 & $12902_{3 / 2}^{\circ}-44388_{5 / 2}^{\circ}$ \\
\hline 3191.749 & 5 & 5 & 31321.729 & $8378_{7 / 2}^{\circ}-39700_{5 / 2}^{\circ}$ & 3174.975 & 2 & 2 & 31487.201 & $19248_{5 / 2}^{\circ}-50735_{3 / 2}$ \\
\hline 3191.560 & 2 & 4 & 31323.584 & $12485_{7 / 2}^{\circ}-43809_{9 / 2}$ & 3174.457 & 100 & 200 & 31492.339 & $7001_{3 / 2}-38493_{5 / 2}^{\circ}$ \\
\hline 3191.221 & 150 & 300 & 31326.911 & $9720_{7 / 2}^{\circ}-41047_{9 / 2}$ & 3174.204 & 300 & 200 & 31494.849 & $1859_{3 / 2}-33354_{5 / 2}^{\circ}$ \\
\hline 3191.093 & 300 & 200 & 31328.168 & $1521_{5 / 2}-32850_{5 / 2}^{\circ}$ & 3174.062 & 2 & & 31496.258 & $18973_{7 / 2}^{\circ}-50470_{9 / 2}$ \\
\hline 3190.360 & 75 & 200 & 31335.365 & $12472_{5 / 2}^{\circ}-43807_{3 / 2}$ & 3173.211 & 15 & 100 & 31504.705 & $7331_{5 / 2}^{\circ}-38836_{3 / 2}$ \\
\hline 3190.220 & 10 & 100 & 31336.740 & $9238_{9 / 2}^{\circ}-40574_{11 / 2}^{\circ}$ & 3173.078 & 4 & & 31506.025 & $22014_{11 / 2}^{\circ}-53520_{9 / 2}^{\circ}$ \\
\hline 3190.164 & 200 & 400 & 31337.290 & $10572_{9 / 2}^{\circ}-41909_{9 / 2}^{\circ}$ & 3172.506 & 10 & 100 & 31511.705 & $15349_{11 / 2}^{\circ}-46861_{11 / 2}$ \\
\hline 3190.070 & 300 & 300 & 31338.213 & $9585_{5 / 2}^{\circ}-40923_{5 / 2}$ & 3172.017 & 5 & 8 & 31516.563 & $8378_{\tau / 2}^{\circ}-39895_{9 / 2}$ \\
\hline 3189.038 & 5 & 25 & 31348.355 & $14349_{1 / 2}-45697_{3 / 2}^{\circ}$ & 3171.727 & $15 b$ & 200 & 31519.445 & $11725_{i / 2}^{\circ}-43244_{3 / 2}$ \\
\hline 3188.552 & 10 & 20 & 31353.132 & $0_{3 / 2}-31353_{3 / 2}^{\circ}$ & 3171.658 & 10 & 100 & 31520.130 & $11576_{3 / 2}^{\circ}-43096_{5 / 2}$ \\
\hline 3188.515 & 5 & & 31353.496 & $17771_{11 / 2}^{\circ}-49124_{11 / 2}$ & 3170.428 & 2001 & 1501 & 31532.358 & $7331_{5 / 2}^{\circ}-38863_{5 / 2}$ \\
\hline 3188.307 & 500 & 800 & 31355.542 & $1859_{3 / 2}-33215_{3 / 2}^{\circ}$ & 3169.328 & 300 & 300 & 31543.302 & $6213_{9 / 2}-37756_{7 / 2}^{\circ}$ \\
\hline 3188.234 & $500 \mathrm{~b}$ & 1000 & 31356.259 & $6213_{9 / 2}-37569_{9 / 2}^{\circ}$ & 3168.705 & 4 & & 31549.504 & $23372_{3 / 2}^{\circ}-54922_{3 / 2}$ \\
\hline 3188.118 & 300 & 300 & 31357.400 & $6244_{1 / 2}-37601_{3 / 2}^{\circ}$ & 3168.234 & 1 & & 31554.194 & $20310_{5 / 2}^{\circ}-51865_{5 / 2}$ \\
\hline 3187.478 & 50 & 50 & 31363.696 & $6244_{1 / 2}-37607_{1 / 2}^{\circ}$ & 3167.560 & $50 \mathrm{~b}$ & $400 \mathrm{~b}$ & 31560.908 & $15349_{11 / 2}^{\circ}-46910_{13 / 2}$ \\
\hline 3187.403 & 100 & 150 & 31364.434 & $10855_{7 / 2}-42219_{5 / 2}^{\circ}$ & 3167.529 & $8 \mathrm{~b}$ & $100 \mathrm{~b}$ & 31561.217 & $14790_{7 / 2}^{\circ}-46352_{7 / 2}$ \\
\hline 3187.332 & 3 & & 31365.133 & $20310_{5 / 2}^{\circ}-51676_{3 / 2}$ & 3166.816 & 5 & 25 & 31568.322 & $17121_{3 / 2}^{\circ}-48689_{3 / 2}^{\circ}$ \\
\hline 3187.033 & $5 b$ & & 31368.076 & $22642_{9 / 2}^{\circ}-54010_{7 / 2}$ & 3166.376 & 5 & 5 & 31572.709 & $17272_{9 / 2}^{\circ}-48844_{9 / 2}$ \\
\hline 3187.003 & 50 & 100 & 31368.371 & $9202_{i / 2}^{\circ}-40570_{\pi / 2}$ & & & & & $20989_{9 / 2}^{\circ}-52562_{7 / 2}$ \\
\hline 3186.704 & 3 & & 31371.314 & $13818_{i / 2}^{\circ}-45189_{5 / 2}$ & 3166.197 & 10 & 50 & 31574.494 & $10572_{9 / 2}^{\circ}-42146_{11 / 2}$ \\
\hline 3184.949 & 400 & 800 & 31388.600 & $4490_{5 / 2}^{\circ}-35878_{7 / 2}$ & 3166.098 & 300 & 300 & 31575.481 & $4490_{5 / 2}^{\circ}-36065_{5 / 2}$ \\
\hline 3184.615 & 10 & 150 & 31391.892 & $16906_{7 / 2}^{\circ}-48298_{7 / 2}$ & 3165.819 & 200 & 500 & 31578.264 & $8605_{5 / 2}-40184_{7 / 2}^{\circ}$ \\
\hline 3183.977 & 15 & 150 & 31398.182 & $12902_{3 / 2}^{\circ}-44300_{3 / 2}$ & 3165.624 & 150 & 200 & 31580.209 & $7001_{3 / 2}-38581_{5 / 2}^{\circ}$ \\
\hline 3183.795 & 200 & 200 & 31399.976 & $13250_{5 / 2}-44650_{7 / 2}^{\circ}$ & 3164.952 & 3 & 25 & 31586.914 & $17771_{11 / 2}^{\circ}-49357_{11 / 2}$ \\
\hline 3183.611 & 3 & 40 & 31401.791 & $13248_{9 / 2}-44650_{7 / 2}^{\circ}$ & 3164.482 & 200 & 200 & 31591.605 & $6700_{9 / 2}^{\circ}-38291_{7 / 2}^{\circ}$ \\
\hline 3183.556 & 75 & 200 & 31402.334 & $11116_{7 / 2}^{\circ}-42518_{7 / 2}$ & 3164.306 & 20 & 50 & 31593.362 & $9061_{5 / 2}-40654_{5 / 2}^{\circ}$ \\
\hline 3183.167 & 4 & 8 & 31406.171 & $9585_{5 / 2}^{\circ}-40991_{3 / 2}$ & 3164.201 & 2 & & 31594.411 & $14790_{7 / 2}^{\circ}-46385_{7 / 2}$ \\
\hline 3182.924 & 1 & & 31408.568 & $24982_{7 / 2}^{\circ}-56391_{5 / 2}$ & 3163.510 & 4 & 8 & 31601.311 & $20080_{7 / 2}^{\circ}-51681_{9 / 2}$ \\
\hline 3182.642 & 100 & 200 & 31411.351 & $9061_{5 / 2}-40472_{3 / 2}^{\circ}$ & 3163.248 & 5 & 20 & 31603.929 & $21131_{3 / 2}^{\circ}-52735_{3 / 2}$ \\
\hline 3182.405 & $200 \mathrm{~b}$ & 200 & 31413.691 & $6691_{3 / 2}^{\circ}-38105_{5 / 2}$ & 3163.096 & 5 & 100 & 31605.447 & $28011_{3 / 2}-59616_{3 / 2}^{\circ}$ \\
\hline 3182.357 & $8 b$ & 40 & 31414.164 & $16906_{\bar{\tau} / 2}^{\circ}-48320_{5 / 2}$ & 3162.839 & $150 w$ & 300 & 31608.015 & $9720_{7 / 2}^{\circ}-41328_{5 / 2}^{\circ}$ \\
\hline 3182.236 & 2 & & 31415.359 & $19248_{5 / 2}^{\circ}-50663_{5 / 2}$ & & & & & $12488_{9 / 2}^{\circ}-44096_{9 / 2}$ \\
\hline
\end{tabular}


TABLE 3. Classified lines of Th II-Continued

\begin{tabular}{|c|c|c|c|c|c|c|c|c|c|}
\hline \multirow{2}{*}{$\begin{array}{c}\text { Wavelength } \\
\AA\end{array}$} & \multicolumn{2}{|c|}{ Intensity } & \multirow{2}{*}{$\begin{array}{c}\text { Wavenumber } \\
\mathbf{c m}^{-1}\end{array}$} & \multirow{2}{*}{ Classification } & \multirow{2}{*}{$\begin{array}{c}\text { Wavelength } \\
\AA\end{array}$} & \multicolumn{2}{|c|}{ Intensity } & \multirow{2}{*}{$\begin{array}{c}\text { Wavenumber } \\
\mathbf{c m}^{-1}\end{array}$} & \multirow{2}{*}{ Classification } \\
\hline & Lamp & Spark & & & & Lamp & Spark & & \\
\hline 3162.583 & 20 & 50 & 31610.574 & $12485_{7 / 2}^{\circ}-44096_{9 / 2}$ & 3140.064 & 15 & 75 & 31837.261 & $8378_{7 / 2}^{\circ}-40216_{5 / 2}$ \\
\hline 3162.362 & 2 & & 31612.783 & $19050_{3 / 2}^{\circ}-50663_{5 / 2}$ & 3139.964 & $2 \mathrm{~s}$ & & 31838.275 & $16033_{5 / 2}^{\circ}-47871_{7 / 2}$ \\
\hline 3162.286 & 2 & & 31613.542 & $15710_{3 / 2}^{\circ}-47324_{5 / 2}$ & 3139.866 & 15 & & 31839.268 & $11116_{7 / 2}^{\circ}-42955_{9 / 2}$ \\
\hline 3161.687 & 75 & 200 & 31619.531 & $6168_{7 / 2}^{\circ}-37787_{7 / 2}^{\circ}$ & 3139.306 & $500 \mathrm{~b}$ & 800 & 31844.948 & $9202_{7 / 2}^{\circ}-41047_{9 / 2}$ \\
\hline 3161.511 & 1 & & 31621.292 & $20686_{5 / 2}^{\circ}-52307_{3 / 2}$ & 3139.225 & $100 \mathrm{~b}$ & 200 & 31845.769 & $10673_{5 / 2}^{\circ}-42518_{7 / 2}$ \\
\hline 3161.072 & 10 & 15 & 31625.683 & $0_{3 / 2}-31625_{1 / 2}^{\circ}$ & 3138.767 & 5 & 10 & 31850.416 & $14545_{5 / 2}^{\circ}-46395_{3 / 2}$ \\
\hline 3160.823 & 15 & 50 & 31628.174 & $10572_{9 / 2}^{\circ}-42200_{9 / 2}$ & 3138.580 & 3 & 8 & 31852.314 & $17272_{9 / 2}^{\circ}-49124_{11 / 2}$ \\
\hline 3160.758 & 2 & 4 & 31628.825 & $14275_{9 / 2}^{\circ}-45904_{9 / 2}$ & 3138.314 & 10 & 100 & 31855.013 & $20080_{7 / 2}^{\circ}-51935_{5 / 2}$ \\
\hline 3160.167 & 15 & 100 & 31634.740 & $11116_{7 / 2}^{\circ}-42751_{7 / 2}$ & 3137.172 & 25 & 100 & 31866.609 & $8605_{5 / 2}-40472_{3 / 2}^{\circ}$ \\
\hline 3159.071 & 50 & 200 & 31645.714 & $9061_{5 / 2}-40706_{7 / 2}^{0}$ & 3136.705 & 40 & 150 & 31871.353 & $15453_{7 / 2}^{\circ}-47324_{5 / 2}^{\circ}$ \\
\hline 3158.871 & 5 & 10 & 31647.718 & $17837_{1 / 2}^{\circ}-49485_{1 / 2}$ & 3135.696 & 1 & & 31881.608 & $20288_{11 / 2}^{\circ}-52170_{11 / 2}$ \\
\hline 3158.617 & $75 b$ & $100 \mathrm{~b}$ & 31650.263 & $10572_{9 / 2}^{\circ}-42222_{7 / 2}^{\circ}$ & 3134.896 & $5 \mathrm{~h}$ & 10 & 31889.744 & $17983_{5 / 2}^{\circ}-49873_{5 / 2}$ \\
\hline 3157.918 & 20 & 100 & 31657.268 & $13468_{9 / 2}^{\circ}-45126_{9 / 2}^{\circ}$ & 3134.425 & 150 & 500 & 31894.536 & $14484_{11 / 2}^{\circ}-46378_{13 / 2}$ \\
\hline 3157.296 & $8 \mathrm{~b}$ & & 31663.505 & $22834_{7 / 2}-54497_{7 / 2}^{\circ}$ & 3133.618 & 150 & 200 & 31902.749 & $9585_{5 / 2}^{\circ}-41488_{7 / 2}$ \\
\hline 3157.277 & $3 \mathrm{~b}$ & & 31663.695 & $10673_{5 / 2}^{\circ}-42336_{5 / 2}^{\circ}$ & 3132.902 & $2 \mathrm{~b}$ & & 31910.040 & $23012_{3 / 2}^{\circ}-54922_{3 / 2}^{\circ}$ \\
\hline 3156.400 & 150 & 200 & 31672.493 & $6168_{7 / 2}^{\circ}-37840_{9 / 2}$ & 3132.389 & 8 & 20 & 31915.266 & $14790_{7 / 2}^{\circ}-46706_{7 / 2}^{\circ}$ \\
\hline 3155.830 & 200 & 500 & 31678.213 & $9720_{7 / 2}^{\circ}-41398_{9 / 2}$ & 3132.259 & 50 & 25 & 31916.590 & $12472_{5 / 2}^{\circ}-44388_{5 / 2}^{\circ}$ \\
\hline 3155.754 & 2 & & 31678.976 & $10673_{5 / 2}^{\circ}-42352_{5 / 2}^{\circ}$ & 3132.169 & $8 b$ & 8 & 31917.507 & $6700_{9 / 2}^{\circ}-38617_{9 / 2}^{\circ}$ \\
\hline 3155.589 & 10 & & 31680.632 & $6691_{3 / 2}^{\circ}-38372_{3 / 2}$ & 3131.093 & $15 b$ & & 31928.475 & $15242_{9 / 2}^{\circ}-47171_{9 / 2}^{\circ}$ \\
\hline 3155.189 & $4 \mathrm{~s}$ & 10 & 31684.649 & $19050_{3 / 2}^{\circ}-50735_{3 / 2}^{\circ}$ & 3131.069 & $300 \mathrm{~b}$ & $200 \mathrm{~b}$ & 31928.720 & $0_{3 / 2}-31928_{3 / 2}^{\circ}$ \\
\hline 3154.774 & $300 \mathrm{~b}$ & 500 & 31688.816 & $13406_{13 / 2}^{\circ}-45095_{15 / 2}$ & 3130.539 & 2 & & 31934.125 & $19594_{1 / 2}-51528_{3 / 2}^{\circ}$ \\
\hline 3154.760 & $200 \mathrm{~b}$ & 300 & 31688.957 & $12488_{9 / 2}^{\circ}-44177_{11 / 2}^{\circ}$ & 3130.284 & 5 & 50 & 31936.727 & $6168_{7 / 2}^{\circ}-38105_{5 / 2}$ \\
\hline 3154.325 & $15 b$ & & 31693.327 & $10189_{11 / 2}^{\circ}-41882_{13 / 2}$ & 3130.124 & 2 & & 31938.359 & $16906_{7 / 2}^{\circ}-48844_{9 / 2}^{\circ}$ \\
\hline 3154.301 & $500 \mathrm{~b}$ & 600 & 31693.568 & $1521_{5 / 2}-33215_{3 / 2}^{\circ}$ & 3129.973 & 150 & 200 & 31939.900 & $10379_{9 / 2}-42319_{9 / 2}^{\circ}$ \\
\hline 3154.024 & 8 & 20 & 31696.352 & $17121_{3 / 2}^{\circ}-48817_{3 / 2}$ & 3129.863 & $2 \mathrm{~h}$ & & 31941.022 & $14275_{9 / 2}^{\circ}-46216_{11 / 2}$ \\
\hline 3153.218 & 25 & 50 & 31704.453 & $24381_{7 / 2}-56086_{9 / 2}^{\circ}$ & 3129.289 & 10 & 100 & 31946.881 & $10572_{9 / 2}^{\circ}-42518_{7 / 2}^{\circ}$ \\
\hline 3152.631 & 1 & & 31710.356 & $20120_{5 / 2}^{\circ}-51830_{7 / 2}$ & 3129.258 & 3 & & 31947.197 & $17121_{3 / 2}^{\circ}-49068_{5 / 2}^{\circ}$ \\
\hline 3151.836 & $5 \mathrm{~h}$ & $25 b$ & 31718.354 & $15453_{7 / 2}^{\circ}-47171_{9 / 2}$ & 3128.588 & 5 & 100 & 31954.039 & $17460_{5 / 2}^{\circ}-49414_{3 / 2}$ \\
\hline 3151.647 & 100 & 200 & 31720.256 & $10189_{11 / 2}^{\circ}-41909_{9 / 2}$ & 3128.251 & $2 \mathrm{~h}$ & & 31957.481 & $10189_{11 / 2}^{\circ}-42146_{11 / 2}$ \\
\hline 3151.538 & $8 b$ & 25 & 31721.353 & $9202_{7 / 2}^{\circ}-40923_{5 / 2}^{\circ}$ & 3127.872 & 8 & 20 & 31961.353 & $20310_{5 / 2}^{\circ}-52272_{7 / 2}$ \\
\hline 3150.455 & 200 & 400 & 31732.257 & $14484_{11 / 2}^{\circ}-46216_{11 / 2}$ & 3127.554 & 15 & 100 & 31964.603 & $12485_{7 / 2}^{\circ}-44450_{9 / 2}$ \\
\hline 3149.963 & 20 & 100 & 31737.213 & $7331_{5 / 2}^{\circ}-39068_{7 / 2}$ & 3127.207 & 150 & 300 & 31968.149 & $9720_{7 / 2}^{\circ}-41688_{7 / 2}^{\circ}$ \\
\hline 3149.395 & 3 & 2 & 31742.937 & $9585_{5 / 2}^{\circ}-41328_{5 / 2}$ & 3126.924 & 5 & 10 & 31971.042 & $13250_{5 / 2}-45221_{5 / 2}^{\circ}$ \\
\hline 3146.926 & 2 & & 31767.841 & $9720_{7 / 2}^{\circ}-41488_{7 / 2}$ & 3126.635 & 3 & 8 & 31973.998 & $19050_{3 / 2}^{\circ}-51024_{3 / 2}^{\circ}$ \\
\hline 3146.880 & $3 \mathrm{~b}$ & & 31768.305 & $17727_{11 / 2}-49495_{9 / 2}^{\circ}$ & 3126.267 & 5 & 15 & 31977.761 & $14275_{9 / 2}^{\circ}-46253_{9 / 2}^{\circ}$ \\
\hline 3146.811 & 10 & 50 & 31769.002 & $14484_{11 / 2}^{\circ}-46253_{9 / 2}$ & 3126.204 & 1 & & 31978.405 & $23518_{7 / 2}^{\circ}-55496_{9 / 2}$ \\
\hline 3146.043 & 500 & 500 & 31776.757 & $6168_{\pi / 2}^{\circ}-37945_{\pi / 2}$ & 3126.052 & 50 & 150 & 31979.960 & $11116_{7 / 2}^{\circ}-43096_{5 / 2}^{\circ}$ \\
\hline 3145.281 & 5 & 50 & 31784.455 & $20080_{7 / 2}^{\circ}-51865_{5 / 2}$ & 3125.982 & 1 & & 31980.677 & $22513_{5 / 2}^{\circ}-54493_{5 / 2}$ \\
\hline 3144.702 & 10 & 25 & 31790.307 & $14790_{7 / 2}^{\circ}-46581_{5 / 2}$ & 3125.858 & 10 & 40 & 31981.945 & $13818_{7 / 2}^{\circ}-45800_{5 / 2}^{\circ}$ \\
\hline 3144.641 & 1 & & 31790.924 & $15144_{3 / 2}^{\circ}-46935_{3 / 2}$ & 3125.745 & 200 & 200 & 31983.101 & $1859_{3 / 2}-33843_{3 / 2}^{\circ}$ \\
\hline 3144.506 & 10 & & 31792.288 & $13818_{7 / 2}^{\circ}-45610_{5 / 2}$ & 3125.507 & 500 & 1000 & 31985.537 & $9238_{9 / 2}^{\circ}-41223_{11 / 2}$ \\
\hline 3143.084 & 8 & 20 & 31806.671 & $14545_{5 / 2}^{\circ}-46352_{7 / 2}$ & 3125.321 & 4 & 5 & 31987.440 & $12902_{3 / 2}^{\circ}-44889_{3 / 2}^{\circ}$ \\
\hline 3142.836 & 400 & 600 & 31809.181 & $9238_{9 / 2}^{\circ}-41047_{9 / 2}$ & 3125.208 & 300 & 600 & 31988.597 & $8378_{7 / 2}^{\circ}-40367_{9 / 2}^{\circ}$ \\
\hline 3142.701 & 50 & 40 & 31810.548 & $0_{3 / 2}-31810_{5 / 2}^{\circ}$ & 3124.387 & 500 & 600 & 31997.002 & $6168_{7 / 2}^{\circ}-38165_{7 / 2}^{\circ}$ \\
\hline 3142.538 & 10 & 100 & 31812.197 & $14790_{7 / 2}^{\circ}-46603_{5 / 2}$ & 3123.994 & 8 & & 32001.027 & $15144_{3 / 2}^{\circ}-47145_{1 / 2}$ \\
\hline 3142.209 & 1 & & 31815.528 & $20120_{5 / 2}^{\circ}-51935_{5 / 2}$ & 3123.004 & $100 \mathrm{~b}$ & 150 & 32011.171 & $10189_{11 / 2}^{0}-42200_{9 / 2}$ \\
\hline 3141.847 & 200 & 300 & 31819.194 & $7331_{5 / 2}^{\circ}-39150_{3 / 2}$ & 3122.963 & $600 \mathrm{~b}$ & $800 \mathrm{~b}$ & 32011.591 & $6168_{7 / 2}^{\circ}-38179_{9 / 2}$ \\
\hline 3141.337 & 10 & 100 & 31824.359 & $15324_{1 / 2}^{\circ}-47148_{3 / 2}$ & 3122.914 & $8 \mathrm{~g}$ & & 32012.094 & $8460_{3 / 2}-40472_{3 / 2}^{\circ}$ \\
\hline 3140.940 & 50 & 150 & 31828.382 & $12472_{5 / 2}^{\circ}-44300_{3 / 2}$ & 3122.582 & 3 & & 32015.497 & $12488_{9 / 2}^{\circ}-44503_{7 / 2}^{\circ}$ \\
\hline 3140.494 & 10 & 8 & 31832.902 & $1521_{5 / 2}-33354_{5 / 2}^{\circ}$ & 3122.327 & 10 & & 32018.112 & $12485_{7 / 2}^{\circ}-44503_{7 / 2}^{\circ}$ \\
\hline
\end{tabular}


TABle 3. Classified lines of Th II-Continued

\begin{tabular}{|c|c|c|c|c|c|c|c|c|c|}
\hline \multirow{2}{*}{$\begin{array}{c}\text { Wavelength } \\
\AA\end{array}$} & \multicolumn{2}{|c|}{ Intensity } & \multirow{2}{*}{$\begin{array}{c}\text { Wavenumber } \\
\mathbf{c m}^{-1}\end{array}$} & \multirow{2}{*}{ Classification } & \multirow{2}{*}{$\begin{array}{c}\text { Wavelength } \\
\AA\end{array}$} & \multicolumn{2}{|c|}{ Intensity } & \multirow{2}{*}{$\begin{array}{c}\text { Wavenumber } \\
\mathbf{c m}^{-1}\end{array}$} & \multirow{2}{*}{ Classification } \\
\hline & Lamp & Spark & & & & Lamp & Spark & & \\
\hline 3122.158 & 2 & & 32019.845 & $19248_{5 / 2}^{\circ}-51268_{7 / 2}$ & 3100.937 & 300 & 500 & 32238.961 & $12488_{9 / 2}^{\circ}-44727_{11 / 2}$ \\
\hline 3121.888 & 1 & & 32022.614 & $27593_{5 / 2}-59616_{3 / 2}^{\circ}$ & 3100.797 & $200 \mathrm{~b}$ & 200 & 32240.417 & $13248_{9 / 2}-45489_{9 / 2}^{\circ}$ \\
\hline 3121.169 & 1 & & 32029.990 & $22139_{9 / 2}^{\circ}-54169_{7 / 2}$ & 3099.863 & 150 & 200 & 32250.131 & $9238_{9 / 2}^{\circ}-41488_{7 / 2}^{\circ}$ \\
\hline 3121.011 & 25 & 100 & 32031.612 & $12472_{5 / 2}^{\circ}-44503_{7 / 2}^{\circ}$ & 3099.740 & 100 & 200 & 32251.410 & $12570_{7 / 2}-44821_{5 / 2}^{\circ}$ \\
\hline 3120.912 & 5 & & 32032.628 & $8378_{7 / 2}^{\circ}-40411_{7 / 2}$ & 3099.118 & 1 & & 32257.883 & $19912_{13 / 2}^{\circ}-52170_{11 / 2}$ \\
\hline 3120.639 & 5 & 10 & 32035.430 & $7331_{5 / 2}^{\circ}-39366_{5 / 2}$ & 3098.387 & 40 & 100 & 32265.493 & $8378_{7 / 2}^{\circ}-40644_{5 / 2}$ \\
\hline 3120.607 & 1 & & 32035.759 & $14545_{5 / 2}^{\circ}-46581_{5 / 2}$ & 3098.325 & 20 & 75 & 32266.139 & $13468_{9 / 2}^{\circ}-45735_{11 / 2}^{\circ}$ \\
\hline 3120.455 & 75 & 8 & 32037.319 & $6691_{3 / 2}^{\circ}-38728_{5 / 2}$ & 3097.267 & 200 & 300 & 32277.160 & $4113_{5 / 2}-36390_{3 / 2}^{\circ}$ \\
\hline 3120.168 & 8 & & 32040.266 & $6700_{9 / 2}^{\circ}-38740_{11 / 2}$ & 3097.142 & 20 & & 32278.463 & $15453_{7 / 2}^{\circ}-47731_{9 / 2}$ \\
\hline 3119.526 & $600 \mathrm{~b}$ & 100 & 32046.859 & $4146_{7 / 2}-36193_{9 / 2}^{\circ}$ & 3096.991 & 5 & $10 \mathrm{~b}$ & 32280.037 & $14275_{9 / 2}^{\circ}-46555_{11 / 2}$ \\
\hline 3119.354 & 150 & 200 & 32048.626 & $8605_{5 / 2}-40654_{5 / 2}^{\circ}$ & 3096.963 & 5 & $10 \mathrm{~b}$ & 32280.328 & $16564_{11 / 2}^{\circ}-48844_{9 / 2}$ \\
\hline 3118.478 & $25 b$ & & 32057.629 & $14545_{5 / 2}^{\circ}-46603_{5 / 2}$ & 3096.430 & 150 & 200 & 32285.885 & $9202_{7 / 2}^{\circ}-41488_{7 / 2}$ \\
\hline 3117.683 & 400 & 300 & 32065.803 & $6691_{3 / 2}^{\circ}-38757_{3 / 2}$ & 3096.295 & $10 \mathrm{~b}$ & $15 b$ & 32287.292 & $12902_{3 / 2}^{\circ}-45189_{5 / 2}$ \\
\hline 3117.150 & 15 & 50 & 32071.286 & $14484_{1 / 2}^{\circ}-46555_{11 / 2}^{\circ / 2}$ & 3096.260 & $4.0 \mathrm{~b}$ & 100 & 32287.657 & $16033_{5 / 2}^{\circ}-48320_{5 / 2}^{\circ}$ \\
\hline 3116.626 & 2 & 3 & 32076.678 & $14275_{9 / 2}^{\circ}-46352_{7 / 2}$ & 3095.744 & 10 & 75 & 32293.039 & $17121_{3 / 2}^{\circ}-49414_{3 / 2}$ \\
\hline & & & & $14349_{1 / 2}-46426_{1 / 2}^{\circ}$ & 3095.634 & $3 \mathrm{~b}$ & & 32294.186 & $14101_{1 / 2}^{\circ}-46395_{3 / 2}$ \\
\hline 3116.478 & 200 & 150 & 32078.201 & $10673_{5 / 2}^{\circ}-42751_{7 / 2}$ & 3095.620 & $10 \mathrm{~b}$ & 75 & 32294.333 & $18973_{7 / 2}^{\circ}-51268_{7 / 2}$ \\
\hline 3116.303 & $150 \mathrm{~b}$ & 400 & 32080.002 & $12570_{7 / 2}-44650_{7 / 2}^{\circ}$ & 3094.706 & $40 \mathrm{~b}$ & 100 & 32303.870 & $16818_{7 / 2}-49121_{7 / 2}^{\circ}$ \\
\hline 3115.745 & 40 & 200 & 32085.747 & $17272_{9 / 2}^{\circ}-49357_{11 / 2}^{\circ}$ & 3093.254 & $8 p$ & 8 & 32319.033 & $4490_{5 / 2}^{\circ}-36809_{7 / 2}$ \\
\hline 3115.715 & 2 & & 32086.056 & $13818_{7 / 2}^{\circ}-45904_{9 / 2}$ & 3093.196 & 5 & 8 & 32319.639 & $12488_{9 / 2}^{\circ}-44807_{7 / 2}^{\circ}$ \\
\hline 3115.415 & 8 & 40 & 32089.146 & $10855_{7 / 2}-42944_{7 / 2}^{\circ}$ & 3093.051 & 150 & 100 & 32321.154 & $1521_{5 / 2}-33843_{3 / 2}^{\circ}$ \\
\hline 3115.191 & 5 & 8 & 32091.453 & $9585_{5 / 2}^{\circ}-41676_{3 / 2}^{\circ}$ & 3092.945 & 40 & 100 & 32322.262 & $12485_{7 / 2}^{\circ}-44807_{7 / 2}$ \\
\hline 3114.744 & $3 \mathrm{~h}$ & & 32096.059 & $24982_{7 / 2}^{\circ}-57078_{9 / 2}$ & 3092.452 & 2 & & 32327.414 & $21682_{7 / 2}^{\circ}-54010_{7 / 2}$ \\
\hline 3114.266 & 100 & 200 & 32100.985 & $8605_{5 / 2}-40706_{7 / 2}^{\circ}$ & 3092.332 & 20 & 100 & 32328.669 & $13406_{13 / 2}^{\circ}-45735_{11 / 2}$ \\
\hline 3114.067 & 100 & 100 & 32103.036 & $9585_{5 / 2}^{\circ}-41688_{7 / 2}$ & 3091.653 & $50 \mathrm{~b}$ & 75 & 32335.769 & $12472_{5 / 2}^{\circ}-44807_{\pi / 2}$ \\
\hline 3113.408 & 8 & & 32109.831 & $14275_{9 / 2}^{\circ}-46385_{7 / 2}$ & 3091.290 & 1 & & 32339.566 & $18568_{i / 2}^{\circ}-50907_{3 / 2}$ \\
\hline 3113.242 & 8 & & 32111.543 & $14790_{7 / 2}^{\circ}-46902_{5 / 2}$ & 3090.184 & 20 & 25 & 32351.140 & $9585_{5 / 2}^{\circ}-41936_{3 / 2}$ \\
\hline 3112.941 & 4 & 10 & 32114.647 & $19248_{5 / 2}^{\circ}-51362_{s / 2}$ & 3090.094 & 300 & 300 & 32352.082 & $1859_{3 / 2}-34212_{5 / 2}^{\circ}$ \\
\hline & & & & $17722_{9 / 2}^{\circ}-49837_{9 / 2}$ & 3089.699 & 1 & & 32356.218 & $27631_{3 / 2}-59987_{3 / 2}^{\circ}$ \\
\hline 3112.088 & 75 & 100 & 32123.449 & $6168_{7 / 2}^{\circ}-38291_{7 / 2}^{\circ}$ & 3089.626 & 75 & 75 & 32356.982 & $14545_{5 / 2}^{\circ}-46902_{5 / 2}^{\circ}$ \\
\hline 3111.832 & 50 & 100 & 32126.092 & $9202_{7 / 2}^{\circ}-41328_{5 / 2}$ & 3088.967 & 4 & & 32363.885 & $17121_{3 / 2}^{\circ}-49485_{1 / 2}$ \\
\hline 3111.762 & 50 & 100 & 32126.815 & $7331_{5 / 2}^{\circ}-39458_{7 / 2}$ & 3088.839 & 100 & 100 & 32365.226 & $8460_{3 / 2}-40825_{1 / 2}^{\circ}$ \\
\hline 3111.429 & 15 & 75 & 32130.253 & $11116_{7 / 2}^{\circ}-43246_{7 / 2}$ & 3088.526 & $25 b$ & & 32368.506 & $6700_{9 / 2}^{\circ}-39068_{7 / 2}^{\circ}$ \\
\hline 3110.020 & 600 & 500 & 32144.809 & $6691_{3 / 2}^{\circ}-38836_{3 / 2}$ & 3088.470 & 500 & 500 & 32369.093 & $7331_{5 / 2}^{\circ}-39700_{5 / 2}$ \\
\hline 3108.671 & 5 & & 32158.758 & $15710_{3 / 2}^{\circ}-47869_{3 / 2}^{\circ}$ & 3087.691 & 15 & 10 & 32377.259 & $14484_{11 / 2}^{\circ}-46861_{11 / 2}$ \\
\hline 3108.454 & $40 \mathrm{~b}$ & 200 & 32161.003 & $15305_{9 / 2}-47466_{11 / 2}^{\circ}$ & 3087.275 & 8 & 15 & 32381.622 & $15349_{11 / 2}^{\circ}-47731_{9 / 2}$ \\
\hline 3108.297 & 1000 & 1000 & 32162.627 & $6700_{9 / 2}^{\circ}-38862_{11 / 2}$ & 3087.067 & 2 & & 32383.803 & $10572_{9 / 2}^{\circ}-42955_{9 / 2}$ \\
\hline 3107.855 & 2 & & 32167.201 & $18568_{1 / 2}^{\circ}-50735_{3 / 2}^{\circ}$ & 3086.466 & 3 & 2 & 32390.109 & $14545_{5 / 2}^{\circ}-46935_{3 / 2}$ \\
\hline 3107.345 & 20 & 25 & 32172.480 & $6691_{3 / 2}^{\circ}-38863_{5 / 2}$ & 3086.361 & 8 & & 32391.211 & $22106_{5 / 2}-54497_{\tau / 2}^{\circ}$ \\
\hline 3107.026 & 500 & 500 & 32175.783 & $6691_{3 / 2}^{\circ}-38867_{1 / 2}$ & 3086.147 & 2 & & 32393.457 & $27593_{5 / 2}-59987_{3 / 2}^{\circ}$ \\
\hline 3106.687 & 50 & $200 \mathrm{~b}$ & 32179.294 & $10572_{9 / 2}^{\circ}-42751_{7 / 2}$ & 3085.165 & 4 & & 32403.767 & $12902_{3 / 2}^{\circ}-45306_{3 / 2}$ \\
\hline 3106.652 & 8 & 100 & 32179.657 & $15144_{3 / 2}^{\circ}-47324_{5 / 2}$ & 3084.689 & 1 & & 32408.767 & $22513_{5 / 2}^{\circ}-54922_{3 / 2}^{\circ}$ \\
\hline 3105.747 & $800 \mathrm{~b}$ & 600 & 32189.033 & $9720_{7 / 2}^{\circ}-41909_{9 / 2}$ & 3084.524 & $2 \mathrm{~b}$ & & 32410.501 & $12488_{9 / 2}^{\circ}-44898_{7 / 2}^{\circ}$ \\
\hline 3105.473 & 100 & 150 & 32191.874 & $6244_{1 / 2}-38436_{3 / 2}^{\circ}$ & 3084.335 & 3 & & 32412.487 & $17460_{5 / 2}^{\circ}-49873_{5 / 2}^{\circ}$ \\
\hline 3105.257 & 8 & 5 & 32194.113 & $8460_{3 / 2}-40654_{5 / 2}^{\circ}$ & 3084.279 & 2 & & 32413.075 & $12485_{7 / 2}^{o}-44898_{7 / 2}$ \\
\hline 3105.050 & 100 & 150 & 32196.259 & $9202_{7 / 2}^{\circ}-41398_{9 / 2}$ & 3083.845 & 20 & 20 & 32417.637 & $12472_{5 / 2}^{\circ}-44889_{3 / 2}$ \\
\hline 3104.381 & 5 & 25 & 32203.197 & $22642_{9 / 2}^{\circ}-54845_{9 / 2}^{\circ}$ & 3083.777 & 1 & & 32418.351 & $15453_{\tau / 2}^{\circ}-47871_{z / 2}^{\circ}$ \\
\hline 3102.664 & 500 & 500 & 32221.017 & $6168_{\tau / 2}^{\circ}-38389_{\tau / 2}$ & 3083.624 & 8 & 15 & 32419.960 & $16033_{5 / 2}^{\circ}-48453_{7 / 2}$ \\
\hline 3101.688 & 100 & 200 & 32231.156 & $11576_{3 / 2}^{\circ}-43807_{3 / 2}$ & 3083.298 & 400 & 200 & 32423.387 & $10673_{5 / 2}^{\circ}-43096_{5 / 2}$ \\
\hline 3101.069 & 2 & & 32237.589 & $17722_{9 / 2}^{\circ}-49960_{7 / 2}$ & 3082.995 & $100 \mathrm{~b}$ & 200 & 32426.574 & $12472_{5 / 2}^{\circ}-44898_{7 / 2}$ \\
\hline
\end{tabular}


TABLE 3. Classified lines of Th II-Continued

\begin{tabular}{|c|c|c|c|c|}
\hline \multirow{2}{*}{$\begin{array}{c}\text { Wavelength } \\
\AA\end{array}$} & \multicolumn{2}{|c|}{ Intensity } & \multirow{2}{*}{$\begin{array}{c}\text { Wavenumber } \\
\mathbf{c m}^{-1}\end{array}$} & \multirow{2}{*}{ Classification } \\
\hline & Lamp & Spark & & \\
\hline 3082.606 & 2 & & 32430.666 & $14275_{9 / 2}^{\circ}-46706_{7 / 2}$ \\
\hline 3082.194 & 75 & 150 & 32435.001 & $13818_{7 / 2}^{\circ}-46253_{9 / 2}$ \\
\hline 3082.153 & 75 & 100 & 32435.432 & $13468_{9 / 2}^{\circ}-45904_{9 / 2}$ \\
\hline 3081.982 & 400 & 200 & 32437.232 & $4146_{7 / 2}-36583_{7 / 2}^{\circ}$ \\
\hline 3081.660 & 150 & 100 & 32440.621 & $13248_{9 / 2}-45689_{7 / 2}^{\circ}$ \\
\hline 3080.834 & 50 & & 32449.318 & $6168_{7 / 2}^{\circ}-38617_{9 / 2}$ \\
\hline 3080.730 & 8 & & 32450.413 & $9238_{9 / 2}^{\circ}-41688_{7 / 2}$ \\
\hline 3080.217 & 800 & 600 & 32455.818 & $10189_{11 / 2}^{\circ}-42644_{13 / 2}$ \\
\hline 3079.888 & 150 & 75 & 32459.285 & $6691_{3 / 2}^{\circ}-39150_{3 / 2}$ \\
\hline 3079.042 & 20 & & 32468.203 & $4113_{5 / 2}-36581_{3 / 2}^{\circ}$ \\
\hline 3078.829 & $100 w$ & 200 & 32470.449 & $4113_{5 / 2}-36583_{7 / 2}^{\circ}$ \\
\hline 3078.734 & 50 & $15 b$ & 32471.451 & $16818_{7 / 2}-49289_{5 / 2}^{\circ}$ \\
\hline 3077.932 & 200 & 150 & 32479.911 & $9720_{7 / 2}^{\circ}-42200_{9 / 2}$ \\
\hline 3077.339 & 300 & 200 & 32486.170 & $9202_{7 / 2}^{\circ}-41688_{7 / 2}^{\circ}$ \\
\hline 3077.271 & 4 & & 32486.888 & $21682_{7 / 2}^{\circ}-54169_{7 / 2}^{\circ}$ \\
\hline 3076.035 & 75 & 100 & 32499.941 & $17460_{5 / 2}^{\circ}-49960_{7 / 2}$ \\
\hline 3075.840 & 75 & & 32502.001 & $9720_{7 / 2}^{\circ}-42222_{7 / 2}$ \\
\hline 3075.293 & 8 & & 32507.782 & $9711_{7 / 2}-42219_{5 / 2}^{\circ}$ \\
\hline 3075.057 & 50 & 150 & 32510.277 & $24381_{7 / 2}-56892_{5 / 2}^{\circ}$ \\
\hline 3074.171 & 1 & & 32519.647 & $20158_{5 / 2}-52678_{5 / 2}^{\circ}$ \\
\hline 3074.041 & 1 & & 32521.022 & $18214_{3 / 2}^{\circ}-50735_{3 / 2}$ \\
\hline 3073.432 & 50 & 15 & 32527.465 & $10855_{7 / 2}-43382_{5 / 2}^{\circ}$ \\
\hline 3073.089 & 2 & & 32531.096 & $20989_{9 / 2}^{\circ}-53520_{9 / 2}$ \\
\hline 3072.825 & 400 & 200 & 32533.890 & $13818_{7 / 2}^{\circ}-46352_{7 / 2}$ \\
\hline 3072.115 & 800 & 300 & 32541.409 & $4146_{7 / 2}-36687_{5 / 2}^{\circ}$ \\
\hline 3071.798 & 5 & 10 & 32544.767 & $8378_{7 / 2}^{\circ}-40923_{5 / 2}$ \\
\hline 3071.496 & 1 & & 32547.967 & $21297_{5 / 2}^{\circ}-53845_{5 / 2}$ \\
\hline 3071.195 & 15 & 5 & 32551.157 & $6213_{9 / 2}-38764_{7 / 2}^{\circ}$ \\
\hline 3071.124 & 1 & & 32551.909 & $20969_{\mathrm{\tau} / 2}^{\circ}-53520_{9 / 2}$ \\
\hline 3070.943 & 150 & 150 & 32553.828 & $15453_{7 / 2}^{\circ}-48006_{9 / 2}^{\circ}$ \\
\hline 3070.817 & 800 & 400 & 32555.164 & $10572_{9 / 2}^{\circ}-43127_{11 / 2}$ \\
\hline 3070.330 & 5 & & 32560.327 & $6168_{7 / 2}^{\circ}-38728_{5 / 2}$ \\
\hline 3069.851 & 5 & 5 & 32565.407 & $17272_{9 / 2}^{\circ}-49837_{9 / 2}$ \\
\hline 3069.694 & 5 & & 32567.073 & $13818_{7 / 2}^{\circ}-46385_{7 / 2}$ \\
\hline 3069.255 & 150 & 150 & 32571.731 & $10673_{5 / 2}^{\circ}-43244_{3 / 2}$ \\
\hline 3069.070 & $50 \mathrm{~s}$ & 15 & 32573.694 & $10673_{5 / 2}^{\circ}-43246_{\pi / 2}$ \\
\hline 3068.981 & $300 \mathrm{~b}$ & 150 & 32574.639 & $4113_{5 / 2}-36687_{5 / 2}^{\circ}$ \\
\hline 3068.935 & $20 \mathrm{~b}$ & & 32575.127 & $11725_{1 / 2}^{\circ}-44300_{3 / 2}$ \\
\hline 3068.263 & $15 b$ & $15 b$ & 32582.261 & $19248_{5 / 2}^{\circ}-51830_{7 / 2}$ \\
\hline 3067.909 & 5 & 4 & 32586.021 & $14275_{9 / 2}^{\circ}-46861_{11 / 2}$ \\
\hline 3067.729 & 800 & 500 & 32587.932 & $13250_{5 / 2}-45838_{3 / 2}^{\circ}$ \\
\hline 3066.412 & 150 & 100 & 32601.928 & $12219_{3 / 2}-4482 i_{5 / 2}^{\circ}$ \\
\hline 3066.307 & 4 & 50 & 32603.044 & $14545_{5 / 2}^{\circ}-47148_{3 / 2}$ \\
\hline 3065.930 & 300 & 150 & 32607.053 & $9711_{7 / 2}-42319_{9 / 2}^{\circ}$ \\
\hline 3065.038 & 50 & 10 & 32616.543 & $9720_{7 / 2}^{\circ}-42336_{5 / 2}$ \\
\hline 3063.916 & 2 & & 32628.486 & $15242_{9 / 2}^{\circ}-47871_{7 / 2}$ \\
\hline 3063.744 & 1 & & 32630.318 & $20288_{11 / 2}^{\circ}-52918_{13 / 2}^{\circ}$ \\
\hline 3063.602 & $8 \mathrm{~b}$ & & 32631.830 & $9720_{7 / 2}^{\circ}-42352_{5 / 2}$ \\
\hline 3063.217 & 5 & & 32635.931 & $24309_{11 / 2}^{\circ}-56945_{11 / 2}$ \\
\hline 3063.185 & 10 & & 32636.272 & $8018_{3 / 2}-40654_{5 / 2}^{\circ}$ \\
\hline
\end{tabular}

\begin{tabular}{|c|c|c|c|c|}
\hline \multirow{2}{*}{$\begin{array}{c}\text { Wavelength } \\
\AA\end{array}$} & \multicolumn{2}{|c|}{ Intensity } & \multirow{2}{*}{$\begin{array}{c}\text { Wavenumber } \\
\mathrm{cm}^{-1}\end{array}$} & \multirow{2}{*}{ Classification } \\
\hline & Lamp & Spark & & \\
\hline 3063.126 & $200 \mathrm{~b}$ & 100 & 32636.901 & $9585_{5 / 2}^{\circ}-42222_{7 / 2}$ \\
\hline 3063.029 & 300 & 150 & 32637.934 & $12488_{9 / 2}^{\circ}-45126_{9 / 2}^{\circ}$ \\
\hline 3062.784 & 5 & 3 & 32640.545 & $12485_{7 / 2}^{\circ}-45126_{9 / 2}$ \\
\hline 3061.700 & 800 & 200 & 32652.101 & $6700_{9 / 2}^{\circ}-39352_{11 / 2}$ \\
\hline 3061.345 & 40 & 25 & 32655.887 & $11116_{7 / 2}^{\circ}-43772_{5 / 2}$ \\
\hline 3061.259 & $10 \mathrm{~b}$ & & 32656.805 & $16033_{5 / 2}^{\circ}-48689_{3 / 2}$ \\
\hline 3061.241 & $50 \mathrm{~b}$ & 401 & 32656.997 & $15349_{11 / 2}^{\circ}-48006_{9 / 2}$ \\
\hline 3060.178 & 4.00 & 200 & 32668.340 & $8378_{7 / 2}^{\circ}-41047_{9 / 2}$ \\
\hline 3059.899 & 3 & & 32671.319 & $9238_{9 / 2}^{\circ}-41909_{9 / 2}^{\circ}$ \\
\hline 3059.505 & 3 & & 32675.526 & $6691_{3 / 2}^{\circ}-39366_{5 / 2}$ \\
\hline 3059.321 & 3 & & 32677.491 & $16818_{7 / 2}-49495_{9 / 2}^{\circ}$ \\
\hline 3059.063 & $8 b$ & & 32680.247 & $17983_{5 / 2}^{\circ}-50663_{5 / 2}$ \\
\hline 3058.685 & 3 & & 32684.285 & $17722_{9 / 2}^{\circ}-50407_{7 / 2}$ \\
\hline 3058.426 & $100 \mathrm{~b}$ & $100 \mathrm{~b}$ & 32687.053 & $14484_{11 / 2}^{\circ}-47171_{9 / 2}$ \\
\hline 3058.395 & $8 b$ & $4 b$ & 32687.385 & $11116_{7 / 2}^{\circ}-43803_{7 / 2}$ \\
\hline 3058.307 & 8 & 5 & 32688.325 & $17272_{9 / 2}^{\circ}-49960_{7 / 2}$ \\
\hline 3058.139 & 50 & 8 & 32690.121 & $1521_{5 / 2}-34212_{5 / 2}^{\circ}$ \\
\hline 3057.901 & 25 & 10 & 32692.665 & $11116_{7 / 2}^{\circ}-43809_{9 / 2}$ \\
\hline 3057.837 & 4 & & 32693.349 & $\begin{array}{l}18214_{i / 2}^{\circ}-50907_{3 / 2}^{\circ} \\
23697_{7 / 2}^{\circ}-56391_{5 / 2}^{\circ}\end{array}$ \\
\hline 3057.637 & 100 & 50 & 32695.488 & $6168_{7 / 2}^{\circ}-38863_{5 / 2}$ \\
\hline 3057.311 & 2 & & 32698.974 & $17771_{11 / 2}^{\circ}-50470_{9 / 2}$ \\
\hline 3056.845 & 15 & 10 & 32703.958 & $12485_{7 / 2}^{\circ}-45189_{5 / 2}$ \\
\hline 3056.555 & 5 & 2 & 32707.061 & $9202_{7 / 2}^{\circ}-41909_{9 / 2}$ \\
\hline 3056.445 & 20 & 10 & 32708.238 & $12902_{3 / 2}^{\circ}-45610_{5 / 2}$ \\
\hline 3056.097 & 50 & 15 & 32711.963 & $9400_{5 / 2}-42112_{3 / 2}^{\circ}$ \\
\hline 3056.024 & 2 & & 32712.744 & $21297_{5 / 2}^{\circ}-54010_{7 / 2}$ \\
\hline 3055.944 & 4 & & 32713.600 & $21131_{3 / 2}^{\circ}-53845_{5 / 2}$ \\
\hline 3055.582 & 5 & & 32717.476 & $12472_{5 / 2}^{\circ}-45189_{s / 2}$ \\
\hline 3054.959 & 10 & 8 & 32724.147 & $11576_{3 / 2}^{\circ}-44300_{3 / 2}$ \\
\hline 3054.892 & 5 & 4 & 32724.865 & $15144_{3 / 2}^{\circ}-47869_{3 / 2}$ \\
\hline 3052.771 & 8 & 5 & 32747.601 & $13468_{9 / 2}^{\circ}-46216_{11 / 2}$ \\
\hline 3052.414 & 10 & 4 & 32751.431 & $9585_{5 / 2}^{\circ}-42336_{5 / 2}$ \\
\hline 3052.353 & lb & & 32752.085 & $17983_{\% / 2}^{\circ}-50735_{3 / 2}$ \\
\hline 3051.792 & 150 & 40 & 32758.106 & $6700_{9 / 2}^{\circ}-39458_{7 / 2}$ \\
\hline 3051.340 & 2 & & 32762.958 & $13818_{7 / 2}^{\circ}-46581_{5 / 2}$ \\
\hline 3050.985 & $150 \mathrm{~b}$ & 100 & 32766.770 & $10189_{11 / 2}^{\circ}-42955_{9 / 2}$ \\
\hline 3049.863 & 100 & 50 & 32778.824 & $14545_{5 / 2}^{\circ}-47324_{5 / 2}$ \\
\hline 3049.643 & 100 & 100 & 32781.188 & $15710_{3 / 2}^{\circ}-48492_{5 / 2}^{\circ}$ \\
\hline 3049.347 & 5 & 3 & 32784.370 & $13468_{9 / 2}^{\circ}-46253_{9 / 2}$ \\
\hline 3049.302 & 10 & 5 & 32784.854 & $13818_{7 / 2}^{\circ}-46603_{5 / 2}$ \\
\hline 3049.092 & $500 \mathrm{~b}$ & 200 & 32787.112 & $4490_{5 / 2}^{\circ}-37277_{7 / 2}$ \\
\hline 3048.023 & 5 & 3 & 32798.611 & $9720_{7 / 2}^{\circ}-42518_{7 / 2}$ \\
\hline 3047.206 & 5 & 3 & 32807.404 & $8018_{3 / 2}-40825_{1 / 2}^{\circ}$ \\
\hline 3046.952 & 300 & 200 & 32810.139 & $13406_{13 / 2}^{\circ}-46216_{11 / 2}$ \\
\hline 3046.745 & 5 & 2 & 32812.368 & $11576_{3 / 2}^{\circ}-44388_{5 / 2}$ \\
\hline 3046.564 & 1 & & 32814.317 & $19050_{3 / 2}^{\circ}-51865_{5 / 2}^{\circ}$ \\
\hline 3046.149 & 15 & 5 & 32818.788 & $9400_{5 / 2}-42219_{5 / 2}^{\circ}$ \\
\hline 3045.565 & 200 & 100 & 32825.080 & $10189_{11 / 2}^{\circ}-43014_{13 / 2}$ \\
\hline 3045.069 & 8 & 15 & 32830.427 & $22014_{11 / 2}^{\circ}-54845_{9 / 2}$ \\
\hline
\end{tabular}


TABLE 3. Classified lines of Th II-Continued

\begin{tabular}{|c|c|c|c|c|c|c|c|c|c|}
\hline \multirow{2}{*}{$\begin{array}{c}\text { Wavelength } \\
\AA\end{array}$} & \multicolumn{2}{|c|}{ Intensity } & \multirow{2}{*}{$\begin{array}{c}\text { Wavenumber } \\
\mathbf{c m}^{-1}\end{array}$} & \multirow{2}{*}{ Classification } & \multirow{2}{*}{$\begin{array}{c}\text { Wavelength } \\
\AA\end{array}$} & \multicolumn{2}{|c|}{ Intensity } & \multirow{2}{*}{$\begin{array}{c}\text { Wavenumber } \\
\mathrm{cm}^{-1}\end{array}$} & \multirow{2}{*}{ Classification } \\
\hline & Lamp & Spark & & & & Lamp & Spark & & \\
\hline 3044.750 & 20 & 8 & 32833.867 & $14101_{i / 2}^{\circ}-46935_{3 / 2}$ & 3025.845 & 10 & 2 & 33038.999 & $15453_{7 / 2}^{\circ}-48492_{5 / 2}$ \\
\hline 3043.671 & 2 & & 32845.506 & $15453_{7 / 2}^{\circ}-48298_{7 / 2}$ & 3025.647 & 4 & 4 & 33041.161 & $20969_{7 / 2}^{\circ}-54010_{7 / 2}^{\circ}$ \\
\hline 3043.248 & 100 & 5 & 32850.071 & $0_{3 / 2}-32850_{5 / 2}^{\circ}$ & 3025.623 & 2 & & 33041.423 & $17983_{5 / 2}^{\circ}-51024_{3 / 2}$ \\
\hline 3043.065 & 200 & 100 & 32852.046 & $6700_{9 / 2}^{\circ}-39552_{9 / 2}$ & 3025.391 & $4.0 \mathrm{~b}$ & 25 & 33043.957 & $14101_{1 / 2}^{\circ}-4.7145_{1 / 2}$ \\
\hline 3042.630 & 4 & 4 & 32856.743 & $18973_{7 / 2}^{\circ}-51830_{7 / 2}$ & 3025.131 & 10 & 5 & 33046.796 & $14101_{1 / 2}^{\circ}-47148_{3 / 2}$ \\
\hline 3042.308 & 10 & 4 & 32860.220 & $7001_{3 / 2}-39861_{5 / 2}^{\circ}$ & 3024.669 & $50 \mathrm{~b}$ & 20 & 33051.844 & $9061_{5 / 2}-42112_{3 / 2}^{\circ}$ \\
\hline 3042.086 & 1 & & 32862.618 & $23372_{3 / 2}^{\circ}-56235_{3 / 2}$ & 3024.475 & 5 & 2 & 33053.964 & $16906_{7 / 2}^{\circ}-49960_{7 / 2}$ \\
\hline 3041.608 & 2 & & 32867.783 & $15453_{7 / 2}^{\circ}-48320_{5 / 2}$ & 3023.570 & 8 & 3 & 33063.857 & $11725_{1 / 2}^{\circ}-44789_{1 / 2}$ \\
\hline 3041.163 & 2 & & 32872.592 & $23518_{7 / 2}^{\circ}-56391_{5 / 2}$ & 3022.095 & 200 & 100 & 33079.994 & $7331_{5 / 2}^{\circ}-40411_{7 / 2}$ \\
\hline 3040.177 & 8 & 4 & 32883.253 & $13468_{9 / 2}^{\circ}-46352_{7 / 2}^{\circ}$ & 3022.062 & 1 & & 33080.355 & $14790_{7 / 2}^{\circ}-47871_{7 / 2}$ \\
\hline 3040.049 & 150 & 50 & 32884.637 & $7331_{5 / 2}^{\circ}-40216_{5 / 2}$ & 3021.709 & 2 & & 33084.219 & $13818_{7 / 2}^{\circ}-46902_{5 / 2}$ \\
\hline \multirow[t]{2}{*}{3039.425} & 8 & 5 & 32891.388 & $7331_{5 / 2}^{\circ}-40222_{3 / 2}$ & 3021.488 & $100 \mathrm{~b}$ & 20 & 33086.639 & $13468_{9 / 2}^{\circ}-46555_{11 / 2}^{\circ}$ \\
\hline & & & & $18973_{7 / 2}^{\circ}-51865_{5 / 2}$ & 3020.329 & 3 & 2 & 33099.335 & $10673_{5 / 2}^{\circ}-43772_{5 / 2}$ \\
\hline 3039.017 & 2 & & 32895.804 & $14275_{9 / 2}^{\circ}-47171_{9 / 2}^{\circ}$ & 3019.421 & 200 & 100 & 33109.289 & $8378_{7 / 2}^{\circ}-41488_{7 / 2}^{\circ}$ \\
\hline 3038.825 & 40 & 20 & 32897.882 & $12902_{3 / 2}^{\circ}-45800_{5 / 2}^{\circ}$ & 3018.549 & 4 & 3 & 33118.853 & $12570_{7 / 2}-45689_{7 / 2}^{\circ}$ \\
\hline 3038.598 & 200 & 75 & 32900.340 & $6168_{7 / 2}^{\circ}-39068_{7 / 2}^{\circ / 2}$ & 3018.490 & 150 & 150 & 33119.500 & $13248_{9 / 2}-46368_{9 / 2}^{\circ}$ \\
\hline 3038.164 & 2 & & 32905.039 & $13250_{5 / 2}-46155_{5 / 2}^{\circ}$ & 3017.457 & 4 & 2 & 33130.838 & $10673_{5 / 2}^{\circ}-43803_{7 / 2}^{\circ}$ \\
\hline 3038.007 & 40 & 25 & 32906.740 & $12488_{9 / 2}^{\circ}-45395_{7 / 2}^{\circ}$ & 3017.132 & 100 & 75 & 33134.406 & $10673_{5 / 2}^{\circ}-43807_{3 / 2}^{\circ}$ \\
\hline 3037.842 & 2 & & 32908.527 & $9238_{9 / 2}^{\circ}-42146_{11 / 2}$ & 3016.763 & 4 & 2 & 33138.459 & $12472_{5 / 2}^{\circ}-45610_{5 / 2}^{\circ}$ \\
\hline 3037.766 & 3 & 3 & 32909.350 & $12485_{\tau / 2}^{\circ}-45395_{7 / 2}$ & 3015.725 & 100 & 40 & 33149.865 & $9202_{7 / 2}^{\circ}-42352_{5 / 2}^{\circ}$ \\
\hline 3037.111 & 8 & 5 & 32916.448 & $13468_{9 / 2}^{\circ}-46385_{7 / 2}$ & 3014.926 & 75 & 10 & 33158.650 & $9061_{5 / 2}-42219_{5 / 2}^{\circ}$ \\
\hline 3036.938 & $8 b$ & $3 b$ & 32918.323 & $10855_{7 / 2}-43773_{7 / 2}^{\circ}$ & 3014.405 & 10 & 5 & 33164.380 & $11725_{1 / 2}^{\circ}-44889_{3 / 2}$ \\
\hline 3036.910 & 25 & $8 b$ & 32918.626 & $12570_{7 / 2}-45489_{9 / 2}^{\circ}$ & 3013.599 & 100 & 50 & 33173.250 & $13250_{5 / 2}-46423_{3 / 2}^{\circ}$ \\
\hline 3035.764 & 20 & 20 & 32931.052 & $16906_{7 / 2}^{\circ}-49837_{9 / 2}$ & 3013.343 & 2 & 2 & 33176.068 & $15144_{3 / 2}^{\circ}-48320_{5 / 2}^{\circ}$ \\
\hline 3035.536 & 100 & 20 & 32933.526 & $9585_{5 / 2}^{\circ}-42518_{7 / 2}$ & 3013.000 & 5 & 4 & 33179.845 & $20989_{9 / 2}^{\circ}-54.169_{1 / 2}^{\circ}$ \\
\hline 3035.110 & 300 & 150 & 32938.148 & $10189_{1 / 2}^{\circ}-43127_{11 / 2}^{\circ}$ & 3012.707 & 100 & 75 & 33183.071 & $6213_{9 / 2}-39396_{7 / 2}^{\circ}$ \\
\hline 3034.893 & 10 & 10 & 32940.503 & $14790_{7 / 2}^{\circ}-47731_{9 / 2}$ & 3012.347 & 2 & & 33187.037 & $17837_{i / 2}^{\circ}-51024_{3 / 2}$ \\
\hline 3034.327 & 40 & 15 & 32946.647 & $7331_{5 / 2}^{\circ}-40278_{3 / 2}$ & 3012.014 & 1 & & 33190.706 & $14484_{11 / 2}^{o}-47675_{11 / 2}$ \\
\hline & & & & $17460_{5 / 2}^{\circ}-50407_{7 / 2}^{\circ}$ & 3011.602 & 200 & 40 & 33195.246 & $6700_{9 / 2}^{\circ}-39895_{9 / 2}$ \\
\hline 3034.065 & 500 & 300 & 32949.492 & $8378_{7 / 2}^{\circ}-41328_{5 / 2}$ & 3011.481 & 2 & & 33196.580 & $21297_{5 / 2}^{\circ}-54493_{5 / 2}$ \\
\hline 3032.895 & 15 & 5 & 32962.203 & $9238_{9 / 2}^{\circ}-42200_{9 / 2}$ & 3011.370 & 1 & & 33197.804 & $17272_{9 / 2}^{\circ}-50470_{9 / 2}$ \\
\hline 3032.834 & 2 & & 32962.865 & $26424_{5 / 2}^{\circ}-59387_{\tau / 2}$ & 3011.302 & 5 & 8 & 33198.553 & $6168_{7 / 2}^{\circ}-39366_{5 / 2}^{\circ}$ \\
\hline 3031.955 & 100 & 75 & 32972.422 & $13406_{13 / 2}^{\circ}-46378_{13 / 2}^{\circ}$ & 3010.899 & $8 b$ & 8 & 33202.997 & $17460_{5 / 2}^{\circ}-50663_{5 / 2}$ \\
\hline 3031.699 & $50 \mathrm{~b}$ & 8 & 32975.205 & $4490_{5 / 2}^{\circ}-37465_{5 / 2}$ & 3010.245 & 2 & & 33210.210 & $15242_{9 / 2}^{\circ}-48453_{7 / 2}^{\circ}$ \\
\hline 3031.600 & 5 & 2 & 32976.282 & $11576_{3 / 2}^{\circ}-44552_{5 / 2}$ & 3010.002 & 20 & 20 & 33212.891 & $11576_{3 / 2}^{\circ}-44789_{1 / 2}$ \\
\hline 3031.339 & 10 & 4 & 32979.122 & $15710_{3 / 2}^{\circ}-48689_{3 / 2}^{\circ}$ & 3009.857 & 5 & & 33214.491 & $23730_{9 / 2}^{\circ}-56945_{11 / 2}^{\circ}$ \\
\hline 3031.287 & 75 & 10 & 32979.687 & $11116_{7 / 2}^{\circ}-44096_{9 / 2}$ & 3009.768 & 200 & 50 & 33215.473 & $0_{3 / 2}-33215_{3 / 2}^{\circ}$ \\
\hline 3030.864 & $50 \mathrm{~b}$ & 15 & 32984.290 & $9238_{9 / 2}^{\circ}-42222_{\tau / 2}$ & 3009.733 & 2 & & 33215.859 & $14790_{7 / 2}^{\circ}-48006_{9 / 2}^{\circ}$ \\
\hline 3029.692 & 5 & 2 & 32997.049 & $7828_{1 / 2}-40825_{1 / 2}^{\circ}$ & 3008.497 & 300 & 150 & 33229.505 & $6213_{9 / 2}-39443_{9 / 2}^{\circ}$ \\
\hline 3029.609 & 10 & & 32997.953 & $9202_{7 / 2}^{\circ}-42200_{9 / 2}$ & 3008.277 & 75 & 75 & 33231.935 & $10572_{9 / 2}^{\circ}-43803_{7 / 2}^{\circ}$ \\
\hline 3029.414 & 8 & 4 & 33000.077 & $15453_{7 / 2}^{\circ}-48453_{7 / 2}$ & 3008.226 & 3 & 3 & 33232.498 & $9711_{7 / 2}-42944_{7 / 2}^{\circ}$ \\
\hline 3029.278 & 2 & & 33001.558 & $12219_{3 / 2}-45221_{5 / 2}^{\circ}$ & 3007.950 & 100 & 50 & 33235.547 & $9720_{7 / 2}^{\circ}-42955_{9 / 2}^{\circ}$ \\
\hline 3028.577 & 200 & 150 & 33009.197 & $6691_{3 / 2}^{\circ}-39700_{5 / 2}^{\circ}$ & 3007.799 & 75 & 100 & 33237.216 & $10572_{9 / 2}^{\circ}-43809_{9 / 2}^{\circ}$ \\
\hline 3027.837 & 2 & & 33017.263 & $9400_{5 / 2}-42418_{3 / 2}^{\circ}$ & 3007.643 & $50 \mathrm{~b}$ & $40 \mathrm{~b}$ & 33238.940 & $6700_{9 / 2}^{\circ}-39939_{11 / 2}^{\circ}$ \\
\hline 3027.617 & 15 & 5 & 33019.663 & $8378_{\pi / 2}^{\circ}-41398_{9 / 2}$ & 3007.625 & $100 \mathrm{~b}$ & $50 \mathrm{~b}$ & 33239.139 & $7331_{5 / 2}^{\circ}-40570_{7 / 2}$ \\
\hline 3027.583 & 8 & 3 & 33020.033 & $9202_{7 / 2}^{\circ}-42222_{7 / 2}$ & 3006.932 & 200 & 150 & 33246.799 & $12488_{9 / 2}^{\circ}-45735_{11 / 2}$ \\
\hline 3027.554 & 1 & & 33020.350 & $20989_{9 / 2}^{\circ}-54010_{7 / 2}$ & 3006.008 & 50 & 15 & 33257.018 & $6691_{3 / 2}^{\circ}-39948_{1 / 2}^{\circ}$ \\
\hline 3027.216 & $4 \mathrm{~b}$ & & 33024.036 & $19248_{5 / 2}^{\circ}-52272_{7 / 2}$ & 3005.527 & 10 & 8 & 33262.340 & $15349_{11 / 2}^{\circ}-48612_{13 / 2}^{\circ}$ \\
\hline 3026.575 & 300 & 200 & 33031.030 & $9720_{7 / 2}^{\circ}-42751_{7 / 2}$ & 3004.637 & 20 & 5 & 33272.192 & $11116_{7 / 2}^{\circ}-44388_{5 / 2}^{\circ}$ \\
\hline 3026.150 & 20 & 20 & 33035.669 & $16033_{5 / 2}^{\circ}-49068_{5 / 2}$ & 3004.562 & 2 & & 33273.023 & $16564_{11 / 2}^{\circ}-49837_{9 / 2}$ \\
\hline
\end{tabular}


TABLE 3. Classified lines of Th II-Continued

\begin{tabular}{|c|c|c|c|c|c|c|c|c|c|}
\hline \multirow{2}{*}{$\begin{array}{c}\text { Wavelength } \\
\AA\end{array}$} & \multicolumn{2}{|c|}{ Intensity } & \multirow{2}{*}{$\begin{array}{c}\text { Wavenumber } \\
\mathrm{cm}^{-1}\end{array}$} & \multirow{2}{*}{ Classification } & \multirow{2}{*}{$\begin{array}{c}\text { Wavelength } \\
\AA\end{array}$} & \multicolumn{2}{|c|}{ Intensity } & \multirow{2}{*}{$\begin{array}{c}\text { Wavenumber } \\
\mathrm{cm}^{-1}\end{array}$} & \multirow{2}{*}{ Classification } \\
\hline & Lamp & Spark & & & & Lamp & Spark & & \\
\hline 3004.398 & 2 & & 33274.839 & $17460_{5 / 2}^{\circ}-50735_{3 / 2}$ & 2983.817 & 300 & 150 & 33504.344 & $13406_{13 / 2}^{\circ}-46910_{13 / 2}$ \\
\hline 3003.851 & 8 & 3 & 33280.898 & $9238_{9 / 2}^{\circ}-42518_{7 / 2}$ & 2983.664 & 50 & 25 & 33506.062 & $13818_{7 / 2}^{\circ}-47324_{5 / 2}$ \\
\hline 3003.036 & 8 & 5 & 33289.930 & $6168_{7 / 2}^{\circ}-39458_{7 / 2}$ & 2983.572 & $150 \mathrm{~b}$ & 75 & 33507.095 & $8605_{5 / 2}-42112_{3 / 2}^{\circ}$ \\
\hline 3002.401 & $200 \mathrm{~b}$ & 100 & 33296.970 & $1859_{3 / 2}-35156_{5 / 2}^{\circ}$ & 2983.531 & $10 \mathrm{~b}$ & 3 & 33507.555 & $9720_{7 / 2}^{\circ}-43227_{5 / 2}$ \\
\hline 3002.259 & 2 & 3 & 33298.545 & $18973_{7 / 2}^{\circ}-52272_{7 / 2}$ & 2983.213 & 15 & 4 & 33511.127 & $9585_{5 / 2}^{\circ}-43096_{5 / 2}$ \\
\hline 3001.264 & 200 & 100 & 33309.584 & $8378_{7 / 2}^{\circ}-41688_{7 / 2}$ & 2983.018 & 100 & 25 & 33513.317 & $9238_{9 / 2}^{\circ}-42751_{7 / 2}^{\circ}$ \\
\hline 3000.969 & 10 & 8 & 33312.858 & $7331_{5 / 2}^{\circ}-40644_{5 / 2}$ & 2982.198 & 10 & 5 & 33522.532 & $14484_{11 / 2}^{\circ}-48006_{9 / 2}$ \\
\hline 3000.920 & 15 & 10 & 33313.402 & $11576_{3 / 2}^{\circ}-44889_{3 / 2}$ & 2982.048 & 50 & 8 & 33524.218 & $10572_{9 / 2}^{\circ}-44096_{9 / 2}^{\circ}$ \\
\hline 3000.845 & 15 & 3 & 33314.235 & $19248_{5 / 2}^{\circ}-52562_{7 / 2}$ & 2982.003 & 40 & 5 & 33524.724 & $6691_{3 / 2}^{\circ}-40216_{5 / 2}$ \\
\hline 3000.814 & 3 & & 33314.579 & $12485_{7 / 2}^{\circ}-45800_{5 / 2}$ & 2981.843 & 100 & 25 & 33526.523 & $9720_{7 / 2}^{\circ}-43246_{7 / 2}$ \\
\hline 3000.627 & 3 & 2 & 33316.655 & $9202_{7 / 2}^{\circ}-42518_{7 / 2}$ & 2981.550 & $15 \mathrm{~g}$ & 4 & 33529.818 & $14790_{7 / 2}^{\circ}-48320_{5 / 2}$ \\
\hline \multirow[t]{2}{*}{2999.962} & 1 & & 33324.040 & $20686_{5 / 2}^{\circ}-54010_{7 / 2}$ & 2981.492 & 200 & 100 & 33530.470 & $8378_{7 / 2}^{\circ}-41909_{9 / 2}$ \\
\hline & & & & $14545_{5 / 2}^{\circ}-47869_{3 / 2}$ & 2981.399 & 100 & 25 & 33531.516 & $6691_{3 / 2}^{\circ}-40222_{3 / 2}$ \\
\hline 2999.801 & 100 & 100 & 33325.828 & $14545_{5 / 2}^{\circ}-47871_{7 / 2}$ & 2981.337 & 100 & 40 & 33532.213 & $6168_{7 / 2}^{\circ}-39700_{5 / 2}$ \\
\hline 2999.272 & 75 & 10 & 33331.706 & $4490_{5 / 2}^{\circ}-37821_{3 / 2}$ & 2981.139 & 3 & & 33534.440 & $20310_{5 / 2}^{\circ}-53845_{5 / 2}$ \\
\hline 2999.094 & 400 & 150 & 33333.684 & $11116_{7 / 2}^{\circ}-44450_{9 / 2}$ & 2980.335 & 150 & 25 & 33543.486 & $9400_{5 / 2}-42944_{7 / 2}^{\circ}$ \\
\hline 2998.611 & 8 & & 33339.053 & $1859_{3 / 2}-35198_{1 / 2}^{\circ}$ & 2980.185 & 15 & & 33545.174 & $15144_{3 / 2}^{\circ}-48689_{3 / 2}$ \\
\hline 2997.871 & 1 & 1 & 33347.282 & $15144_{3 / 2}^{\circ}-48492_{5 / 2}$ & & & & & $17722_{9 / 2}^{\circ}-51268_{7 / 2}$ \\
\hline 2997.819 & 2 & 3 & 33347.861 & $23730_{9 / 2}^{\circ}-57078_{9 / 2}$ & 2979.837 & 8 & 2 & 33549.092 & $9202_{7 / 2}^{\circ}-42751_{7 / 2}$ \\
\hline 2997.354 & 2 & & 33353.034 & $13818_{7 / 2}^{\circ}-47171_{9 / 2}$ & 2978.860 & 2 & & 33560.094 & $23518_{7 / 2}^{\circ}-57078_{9 / 2}^{\circ}$ \\
\hline 2997.327 & 1 & & 33353.334 & $18816_{13 / 2}^{\circ}-52170_{11 / 2}$ & 2978.564 & 2 & & 33563.429 & $16906_{7 / 2}^{\circ}-50470_{9 / 2}$ \\
\hline 2996.986 & $200 \mathrm{~s}$ & 100 & 33357.129 & $9061_{5 / 2}-42418_{3 / 2}^{\circ}$ & 2978.497 & 3 & & 33564.184 & $17460_{5 / 2}^{\circ}-51024_{3 / 2}$ \\
\hline 2996.911 & 5 & 4 & 33357.964 & $15710_{3 / 2}^{\circ}-49068_{5 / 2}$ & 2977.030 & 8 & 2 & 33580.723 & $11725_{1 / 2}^{\circ}-45306_{3 / 2}$ \\
\hline 2996.829 & 1 & & 33358.877 & $17272_{9 / 2}^{\circ}-50631_{11 / 2}$ & 2976.648 & 100 & 10 & 33585.032 & $12570_{7 / 2}-46155_{5 / 2}^{\circ}$ \\
\hline 2996.561 & 1 & & 33361.860 & $12902_{3 / 2}^{\circ}-46264_{3 / 2}$ & 2976.497 & $8 b$ & & 33586.736 & $6691_{3 / 2}^{\circ}-40278_{3 / 2}$ \\
\hline 2996.216 & 8 & & 33365.701 & $15324_{i / 2}^{\circ}-48689_{3 / 2}$ & 2976.320 & 2 & & 33588.733 & $18973_{7 / 2}^{\circ}-52562_{7 / 2}$ \\
\hline 2995.270 & 50 & 15 & 33376.239 & $9720_{7 / 2}^{\circ}-43096_{5 / 2}$ & 2976.019 & 200 & 75 & 33592.130 & $7331_{5 / 2}^{\circ}-40923_{5 / 2}$ \\
\hline 2994.980 & 1 & & 33379.470 & $17983_{5 / 2}^{\circ}-51362_{5 / 2}$ & 2974.860 & 4 & & 33605.217 & $10572_{9 / 2}^{\circ}-44177_{11 / 2}$ \\
\hline 2994.585 & 8 & 2 & 33383.873 & $6168_{7 / 2}^{\circ}-39552_{9 / 2}$ & 2974.418 & 10 & 1 & 33610.211 & $4146_{7 / 2}-37756_{7 / 2}^{\circ}$ \\
\hline 2994.283 & 1 & & 33387.240 & $11116_{7 / 2}^{\circ}-44503_{7 / 2}$ & 2974.091 & 75 & 20 & 33613.906 & $8605_{5 / 2}-42219_{5 / 2}^{\circ}$ \\
\hline 2993.801 & 400 & 200 & 33392.615 & $13468_{9 / 2}^{\circ}-46861_{11 / 2}$ & 2974.011 & 500 & 100 & 33614.810 & $4490_{5 / 2}^{\circ}-38105_{5 / 2}$ \\
\hline 2993.631 & 150 & 100 & 33394.511 & $10379_{9 / 2}-43773_{7 / 2}^{\circ}$ & 2973.926 & 2 & 2 & 33615.771 & $15453_{i / 2}^{\circ}-49068_{i / 2}$ \\
\hline 2991.696 & 100 & 75 & 33416.110 & $12488_{9 / 2}^{\circ}-45904_{9 / 2}$ & 2973.536 & 400 & 100 & 33620.180 & $10189_{11 / 2}^{\circ}-43809_{9 / 2}$ \\
\hline 2991.461 & 1 & & 33418.735 & $12485_{\pi / 2}^{\circ}-45904_{9 / 2}$ & 2972.895 & $8 b$ & 4 & 33627.429 & $10673_{5 / 2}^{\circ}-44300_{3 / 2}$ \\
\hline 2991.062 & $400 \mathrm{~b}$ & 75 & 33423.192 & $4146_{\pi / 2}-37569_{9 / 2}^{\circ}$ & 2972.224 & $400 \mathrm{~b}$ & 100 & 33635.020 & $1521_{5 / 2}-35156_{5 / 2}^{\circ}$ \\
\hline 2989.907 & 50 & 15 & 33436.103 & $11116_{i / 2}^{\circ}-44552_{5 / 2}$ & 2971.481 & 400 & 40 & 33643.430 & $4113_{5 / 2}-37756_{7 / 2}^{\circ}$ \\
\hline 2989.599 & 1 & & 33439.548 & $18214_{3 / 2}^{\circ}-51653_{1 / 2}$ & 2970.632 & 20 & & 33653.045 & $7001_{3 / 2}-40654_{5 / 2}^{\circ}$ \\
\hline 2989.537 & 3 & & 33440.241 & $20080_{7 / 2}^{\circ}-53520_{9 / 2}$ & 2970.066 & 10 & 3 & 33659.457 & $9585_{5 / 2}^{\circ}-43244_{3 / 2}$ \\
\hline 2988.232 & $500 \mathrm{~b}$ & 200 & 33454.845 & $4490_{5 / 2}^{\circ}-37945_{5 / 2}$ & 2970.011 & 10 & 2 & 33660.081 & $7331_{5 / 2}^{\circ}-40991_{3 / 2}$ \\
\hline 2987.350 & 8 & 2 & 33464.721 & $1521_{5 / 2}-34986_{3 / 2}^{\circ}$ & 2969.892 & $50 \mathrm{~b}$ & 8 & 33661.429 & $9585_{5 / 2}^{\circ}-43246_{7 / 2}$ \\
\hline 2986.786 & $100 \mathrm{~b}$ & 40 & 33471.040 & $7001_{3 / 2}-40472_{3 / 2}^{\circ}$ & 2969.378 & 100 & 8 & 33667.256 & $6700_{9 / 2}^{\circ}-40367_{9 / 2}$ \\
\hline 2985.815 & 2 & & 33481.925 & $22014_{11 / 2}^{\circ}-55496_{9 / 2}$ & 2969.064 & 75 & 8 & 33670.816 & $9711_{7 / 2}-43382_{5 / 2}^{\circ}$ \\
\hline 2985.321 & lb & & 33487.465 & $19248_{5 / 2}^{\circ}-52735_{3 / 2}$ & 2968.687 & 800 & 150 & 33675.092 & $4490_{5 / 2}^{\circ}-38165_{7 / 2}$ \\
\hline 2985.243 & 400 & 75 & 33488.340 & $4113_{5 / 2}-37601_{3 / 2}^{\circ}$ & 2968.348 & 101 & 1 & 33678.938 & $12902_{3 / 2}^{\circ}-46581_{5 / 2}^{\circ}$ \\
\hline 2984.774 & $10 \mathrm{~b}$ & & 33493.602 & $12902_{3 / 2}^{\circ}-46395_{3 / 2}$ & 2967.823 & 101 & 4 & 33684.895 & $19050_{3 / 2}^{\circ}-52735_{3 / 2}$ \\
\hline 2984.765 & $8 b$ & 8 & 33493.703 & $15324_{1 / 2}^{\circ}-48817_{3 / 2}$ & 2967.254 & 8 & 2 & 33691.354 & $11116_{7 / 2}^{\circ}-44807_{7 / 2}$ \\
\hline 2984.642 & 8 & 3 & 33495.083 & $15349_{11 / 2}^{\circ}-48844_{9 / 2}$ & 2966.561 & 2 & & 33699.224 & $20310_{5 / 2}^{\circ}-54010_{7 / 2}$ \\
\hline 2984.145 & 3 & 2 & 33500.661 & $16906_{\pi / 2}^{\circ}-50407_{\tau / 2}$ & 2966.530 & 5 & & 33699.576 & $4146_{7 / 2}-37846_{5 / 2}^{\circ}$ \\
\hline 2984.090 & 4 & 3 & 33501.279 & $17722_{9 / 2}^{\circ}-51224_{9 / 2}$ & 2966.423 & 5 & & 33700.792 & $12902_{3 / 2}^{\circ}-46603_{5 / 2}$ \\
\hline 2983.979 & 2 & & 33502.525 & $15786_{5 / 2}-49289_{5 / 2}^{\circ}$ & 2966.405 & 2 & & 33700.996 & $14790_{7 / 2}^{\circ}-48492_{5 / 2}$ \\
\hline
\end{tabular}


TABLE 3. Classified lines of Th II-Continued

\begin{tabular}{|c|c|c|c|c|}
\hline \multirow{2}{*}{$\begin{array}{c}\text { Wavelength } \\
\AA\end{array}$} & \multicolumn{2}{|c|}{ Intensity } & \multirow{2}{*}{$\begin{array}{l}\text { Wavenumber } \\
\mathrm{cm}^{-1}\end{array}$} & \multirow{2}{*}{ Classification } \\
\hline & Lamp & Spark & & \\
\hline 2966.282 & 2 & & 33702.394 & $13468_{9 / 2}^{\circ}-47171_{9 / 2}$ \\
\hline 2966.005 & 4 & & 33705.541 & $22685_{7 / 2}^{\circ}-56391_{5 / 2}$ \\
\hline 2965.500 & 300 & 75 & 33711.281 & $6700_{9 / 2}^{\circ}-40411_{7 / 2}$ \\
\hline 2965.118 & 75 & 10 & 33715.624 & $10673_{5 / 2}^{\circ}-44388_{5 / 2}$ \\
\hline 2964.924 & 200 & 50 & 33717.829 & $9238_{9 / 2}^{\circ}-42955_{9 / 2}$ \\
\hline 2964.624 & 2 & & 33721.242 & $18214_{3 / 2}^{\circ}-51935_{5 / 2}$ \\
\hline 2964.112 & $100 \mathrm{~b}$ & 20 & 33727.066 & $6168_{7 / 2}^{\circ}-39895_{9 / 2}$ \\
\hline 2964.005 & 75 & 15 & 33728.283 & $12488_{9 / 2}^{\circ}-46216_{11 / 2}$ \\
\hline 2963.876 & 50 & 20 & 33729.751 & $11576_{3 / 2}^{\circ}-45306_{3 / 2}$ \\
\hline 2963.608 & 75 & 8 & 33732.801 & $4113_{5 / 2}-37846_{5 / 2}^{\circ}$ \\
\hline 2963.046 & 5 & 2 & 33739.199 & $18568_{1 / 2}^{\circ}-52307_{3 / 2}$ \\
\hline 2961.842 & 1 & & 33752.914 & $14545_{5 / 2}^{\circ}-48298_{7 / 2}$ \\
\hline 2961.783 & $4 b$ & & 33753.586 & $9202_{7 / 2}^{\circ}-42955_{9 / 2}$ \\
\hline 2961.273 & 8 & & 33759.399 & $8460_{3 / 2}-42219_{5 / 2}^{\circ}$ \\
\hline 2960.806 & 5 & & 33764.724 & $20080_{7 / 2}^{\circ}-53845_{5 / 2}$ \\
\hline 2960.779 & 15 & 5 & 33765.032 & $12488_{9 / 2}^{\circ}-46253_{9 / 2}$ \\
\hline 2960.549 & $2 b$ & & 33767.654 & $12485_{7 / 2}^{\circ}-46253_{9 / 2}$ \\
\hline 2960.536 & $5 b$ & & 33767.803 & $14101_{1 / 2}^{\circ}-47869_{3 / 2}$ \\
\hline 2959.934 & 8 & & 33774.670 & $\begin{array}{l}15710_{3 / 2}^{\circ}-49485_{1 / 2} \\
15349_{11 / 2}^{\circ}-49124_{11 / 2}\end{array}$ \\
\hline 2959.883 & 15 & $100 \mathrm{~b}$ & 33775.252 & $14545_{5 / 2}^{\circ}-48320_{5 / 2}$ \\
\hline 2959.449 & 4 & & 33780.205 & $25607_{9 / 2}^{\circ}-59387_{7 / 2}$ \\
\hline 2959.275 & 10 & 75 & 33782.191 & $11116_{7 / 2}^{\circ}-44898_{7 / 2}$ \\
\hline 2958.565 & 8 & 200 & 33790.298 & $21131_{3 / 2}^{\circ}-54922_{3 / 2}$ \\
\hline 2958.409 & 10 & 50 & 33792.080 & $12472_{5 / 2}^{\circ}-46264_{3 / 2}$ \\
\hline 2958.139 & $200 \mathrm{~b}$ & 200 & 33795.164 & $10855_{7 / 2}-44650_{7 / 2}^{\circ}$ \\
\hline 2957.916 & 300 & 400 & 33797.712 & $12570_{7 / 2}-46368_{9 / 2}^{\circ}$ \\
\hline 2957.580 & 500 & 500 & 33801.551 & $4490_{5 / 2}^{\circ}-38291_{7 / 2}$ \\
\hline 2957.059 & 10 & 150 & 33807.506 & $17460_{\overline{3} / 2}^{\circ}-51268_{7 / 2}$ \\
\hline 2956.631 & 40 & 150 & 33812.400 & $8605_{5 / 2}-42418_{3 / 2}^{\circ}$ \\
\hline 2956.484 & 1 & & 33814.081 & $21682_{7 / 2}^{\circ}-55496_{9 / 2}$ \\
\hline 2956.257 & 8 & 2 & 33816.678 & $15305_{9 / 2}-49121_{7 / 2}^{\circ}$ \\
\hline 2955.847 & 100 & 200 & 33821.368 & $8378_{7 / 2}^{\circ}-42200_{9 / 2}$ \\
\hline 2955.601 & $100 \mathrm{~s}$ & 300 & 33824.183 & $7001_{3 / 2}-40825_{1 / 2}^{\circ}$ \\
\hline 2955.255 & 2 & & 33828.143 & $18118_{3 / 2}-51946_{5 / 2}^{\circ}$ \\
\hline 2955.036 & 150 & 300 & 33830.650 & $10673_{5 / 2}^{\circ}-44503_{7 / 2}$ \\
\hline 2954.887 & 100 & 200 & 33832.356 & $6691_{3 / 2}^{\circ}-40523_{1 / 2}$ \\
\hline 2954.369 & $8 \mathbf{h}$ & 2 & 33838.287 & $17837_{1 / 2}^{\circ}-51676_{3 / 2}$ \\
\hline 2954.218 & 3 & & 33840.017 & $16033_{5 / 2}^{\circ}-49873_{5 / 2}$ \\
\hline 2953.917 & 5 & 1 & 33843.465 & $8378_{7 / 2}^{\circ}-42222_{7 / 2}$ \\
\hline 2953.595 & 10 & 100 & 33847.154 & $17983_{5 / 2}^{\circ}-51830_{7 / 2}$ \\
\hline 2952.584 & 4 & 2 & 33858.743 & $20310_{5 / 2}^{\circ}-54169_{7 / 2}$ \\
\hline 2952.129 & 50 & 200 & 33863.962 & $12488_{9 / 2}^{\circ}-46352_{7 / 2}$ \\
\hline 2951.902 & 20 & 50 & 33866.566 & $12485_{7 / 2}^{\circ}-46352_{7 / 2}$ \\
\hline 2951.562 & 25 & 150 & 33870.467 & $6700_{9 / 2}^{\circ}-40570_{7 / 2}$ \\
\hline 2951.203 & 200 & 300 & 33874.587 & $6700_{9 / 2}^{\circ}-40574_{11 / 2}$ \\
\hline 2951.052 & 4 & 751 & 33876.320 & $20969_{7 / 2}^{\circ}-54845_{9 / 2}$ \\
\hline 2950.883 & 4 & & 33878.260 & $10572_{9 / 2}^{\circ}-44450_{9 / 2}$ \\
\hline 2950.769 & 10 & 50 & 33879.569 & $10673_{5 / 2}^{\circ}-44552_{5 / 2}$ \\
\hline 2950.725 & 10 & 50 & 33880.074 & $12472_{5 / 2}^{\circ}-46352_{7 / 2}$ \\
\hline
\end{tabular}

\begin{tabular}{|c|c|c|}
\hline \multirow{2}{*}{$\begin{array}{c}\text { Wavelength } \\
\AA\end{array}$} & \multicolumn{2}{|c|}{ Intensity } \\
\hline & Lamp & Spark \\
\hline 2950.576 & 10 & 50 \\
\hline 2950.437 & 200 & 300 \\
\hline 2950.150 & 1 & \\
\hline 2949.930 & 300 & 300 \\
\hline 2949.487 & 10 & 25 \\
\hline 2949.240 & 50 & 150 \\
\hline 2949.068 & 500 & 300 \\
\hline 2948.793 & 8 & 100 \\
\hline 2948.711 & 1 & \\
\hline 2948.518 & 2 & 2 \\
\hline 2948.362 & 5 & 50 \\
\hline 2948.332 & 4 & 1 \\
\hline 2947.843 & 8 & 150 \\
\hline 2946.922 & 2 & \\
\hline 2946.896 & 4 & 25 \\
\hline 2946.607 & 20 & 200 \\
\hline 2946.231 & 10 & \\
\hline 2945.899 & 5 & \\
\hline 2944.950 & 75 & 150 \\
\hline 2944.471 & 10 & $150 \mathrm{~b}$ \\
\hline 2944.448 & 4 & \\
\hline 2944.391 & 5 & 3 \\
\hline 2943.962 & 25 & 75 \\
\hline 2943.867 & 3 & \\
\hline 2943.210 & 8 & 10 \\
\hline 2942.860 & $800 \mathrm{~h}$ & $500 \mathrm{~h}$ \\
\hline 2942.628 & $200 \mathrm{~b}$ & 200 \\
\hline 2941.890 & 75 & 200 \\
\hline 2941.337 & 100 & 200 \\
\hline 2940.674 & 100 & 300 \\
\hline 2940.587 & 100 & 300 \\
\hline 2940.303 & 10 & 50 \\
\hline 2939.616 & $75 \mathrm{~b}$ & 300 \\
\hline 2939.555 & $100 \mathrm{~b}$ & 300 \\
\hline 2938.336 & 10 & 75 \\
\hline 2938.104 & 100 & 150 \\
\hline 2937.441 & $100 \mathrm{~b}$ & 300 \\
\hline 2937.361 & 20 & 200 \\
\hline 2936.467 & $400 \mathrm{~b}$ & $500 \mathrm{~b}$ \\
\hline 2936.192 & 400 & 300 \\
\hline 2935.812 & 40 & 200 \\
\hline 2935.752 & 20 & 100 \\
\hline 2935.658 & 50 & 200 \\
\hline 2934.992 & 150 & 200 \\
\hline 2934.578 & 5 & 20 \\
\hline 2934.507 & 5 & 50 \\
\hline 2934.135 & 200 & 200 \\
\hline 2934.012 & 10 & 100 \\
\hline 2933.099 & $200 \mathrm{~b}$ & 200 \\
\hline
\end{tabular}

\subsection{6 \\ 33907.571}

33913.196

33923.794

33924.094

33927.421

33931.750

33935.574

33946.509

33952.031

33952.297

33952.954

33957.901

33958.997

33966.577

33970.617

33973.295

33981.817

33988.206

33995.868 33996.874 34000.158

34008.103

34008.809

34022.917

34025.604

34033.283

34034.210

34044.571

34047.760 34052.166 34052.862 34053.953 34061.680 34066.485 34067.309 34071.628 34073.056 34083.662
Classification

$4490_{5 / 2}^{\circ}-38372_{3 / 2}$

$9061_{5 / 2}-42944_{7 / 2}^{\circ}$ $9400_{5 / 2}-43287_{3 / 2}^{\circ}$ $9238_{9 / 2}^{\circ}-43127_{11 / 2}$ $9202_{7 / 2}^{\circ}-43096_{5 / 2}$ $12488_{9 / 2}^{\circ}-46385_{7 / 2}^{\circ}$ $4490_{5 / 2}^{\circ}-38389_{7 / 2}$ $17460_{5 / 2}^{\circ}-51362_{5 / 2}$ $17121_{3 / 2}^{\circ}-51024_{3 / 2}$ $16564_{11 / 2}^{\circ}-50470_{9 / 2}$

$10189_{11 / 2}^{\circ}-44096_{9 / 2}$ $14545_{5 / 2}^{\circ}-48453_{7 / 2}$ $12472_{5 / 2}^{\circ}-46385_{7 / 2}$ $13818_{7 / 2}^{\circ}-47731_{9 / 2}$ $12472_{5 / 2}^{\circ}-46395_{3 / 2}$ $15144_{3 / 2}^{\circ}-49068_{5 / 2}$ $16033_{5 / 2}^{\circ}-49960_{7 / 2}$ $10572_{9 / 2}^{\circ}-44503_{7 / 2}$ $12219_{3 / 2}-46155_{5 / 2}^{\circ}$ $14545_{5 / 2}^{\circ}-48492_{5 / 2}$

$17272_{9 / 2}^{\circ}-51224_{9 / 2}$ $17983_{5 / 2}^{\circ}-51935_{5 / 2}$ $6691_{3 / 2}^{\circ}-40644_{5 / 2}$ $8460_{3 / 2}-42418_{3 / 2}^{\circ}$ $17722_{9 / 2}^{\circ}-51681_{9 / 2}$ $10855_{7 / 2}-44821_{5 / 2}^{\circ}$ $6213_{9 / 2}-40184_{7 / 2}^{\circ}$ $8378_{7 / 2}^{\circ}-42352_{5 / 2}$ $9400_{5 / 2}-43382_{5 / 2}^{\circ}$ $10189_{11 / 2}^{\circ}-44177_{11 / 2}$

$17272_{9 / 2}^{\circ}-51268_{7 / 2}$ $7331_{5 / 2}^{\circ}-41328_{5 / 2}$ $12902_{3 / 2}^{\circ}-46902_{5 / 2}$ $15349_{11 / 2}^{\circ}-49357_{11 / 2}$ $9238_{9 / 2}^{\circ}-43246_{7 / 2}$ $14275_{9 / 2}^{\circ}-48298_{7 / 2}$ $9202_{7 / 2}^{\circ}-43227_{5 / 2}$ $12902_{3 / 2}^{\circ}-46935_{3 / 2}$ $11576_{3 / 2}^{\circ}-45610_{5 / 2}$ $9202_{7 / 2}^{\circ}-43246_{7 / 2}$

$6168_{7 / 2}^{\circ}-40216_{5 / 2}$ $9720_{7 / 2}^{\circ}-43772_{5 / 2}$ $15236_{3 / 2}-49289_{5 / 2}^{\circ}$ $14790_{7 / 2}^{\circ}-48844_{9 / 2}$ $9711_{7 / 2}-43773_{7 / 2}^{\circ}$ $16564_{11 / 2}^{\circ}-50631_{11 / 2}$ $12488_{9 / 2}^{\circ}-46555_{11 / 2}$ $1521_{5 / 2}-35593_{7 / 2}^{\circ}$ $11116_{7 / 2}^{\circ}-45189_{5 / 2}$ $9720_{7 / 2}^{\circ}-43803_{7 / 2}$ 
TABLE 3. Classified lines of Th $\mathrm{II}-$ Continued

\begin{tabular}{|c|c|c|c|c|}
\hline \multirow{2}{*}{$\begin{array}{c}\text { Wavelength } \\
\AA\end{array}$} & \multicolumn{2}{|c|}{ Intensity } & \multirow{2}{*}{$\begin{array}{l}\text { Wavenumber } \\
\mathrm{cm}^{-1}\end{array}$} & \multirow{2}{*}{ Classification } \\
\hline & Lamp & Spark & & \\
\hline 2932.644 & 100 & 400 & 34088.950 & $9720_{7 / 2}^{\circ}-43809_{9 / 2}$ \\
\hline 2932.293 & 3 & & 34093.030 & $18214_{3 / 2}^{\circ}-52307_{3 / 2}$ \\
\hline 2932.147 & 50 & 100 & 34094.727 & $8018_{3 / 2}-42112_{3 / 2}^{\circ}$ \\
\hline 2932.073 & 3 & 3 & 34095.588 & $12485_{7 / 2}^{\circ}-46581_{5 / 2}$ \\
\hline 2931.519 & 4 & & 34102.031 & $18816_{13 / 2}^{\circ}-52918_{13 / 2}$ \\
\hline 2931.045 & $4 \mathrm{~h}$ & 2 & 34107.546 & $17722_{9 / 2}^{\circ}-51830_{7 / 2}$ \\
\hline 2930.911 & 300 & 300 & 34109.105 & $12472_{5 / 2}^{\circ}-46581_{5 / 2}$ \\
\hline 2930.190 & 5 & 10 & 34117.497 & $12485_{7 / 2}^{\circ}-46603_{5 / 2}$ \\
\hline 2929.297 & 100 & 150 & 34.127 .898 & $14484_{11 / 2}^{\circ}-48612_{13 / 2}$ \\
\hline 2929.027 & 1 & & 34131.044 & $12472_{5 / 2}^{\circ}-46603_{5 / 2}$ \\
\hline 2928.254 & 600 & 500 & 34140.053 & $8378_{7 / 2}^{\circ}-42518_{7 / 2}$ \\
\hline 2927.883 & $5 b$ & 25 & 34144.379 & $14545_{5 / 2}^{\circ}-48689_{3 / 2}$ \\
\hline 2926.956 & 15 & 50 & 34155.192 & $10572_{9 / 2}^{\circ}-44727_{11 / 2}$ \\
\hline 2926.831 & 10 & & 34.156 .651 & $7331_{5 / 2}^{\circ}-41488_{7 / 2}$ \\
\hline 2926.435 & 10 & 50 & 34161.273 & $15324_{1 / 2}^{\circ}-49485_{1 / 2}$ \\
\hline 2926.349 & 5 & 3 & 34162.277 & $15710_{3 / 2}^{\circ}-49873_{5 / 2}$ \\
\hline 2925.903 & 3 & & 34167.484 & $18568_{i / 2}^{\circ}-52735_{3 / 2}$ \\
\hline 2925.051 & $600 \mathrm{~b}$ & 600 & 34177.436 & $15349_{11 / 2}^{\circ}-49527_{13 / 2}^{\circ}$ \\
\hline 2924.604 & 1 & & 34.182 .659 & $14349_{1 / 2}-48532_{1 / 2}^{\circ}$ \\
\hline 2924.572 & 8 & $50 \mathrm{~b}$ & 34.183 .033 & $20310_{5 / 2}^{\circ}-54493_{5 / 2}^{\circ}$ \\
\hline 2924.228 & 5 & 8 & 34.187 .054 & $9585_{5 / 2}^{\circ}-43772_{5 / 2}$ \\
\hline 2924.103 & 75 & 100 & 34188.516 & $13818_{7 / 2}^{\circ}-48006_{9 / 2}$ \\
\hline 2923.950 & $5 b$ & & 34190.304 & $15305_{9 / 2}-49495_{9 / 2}^{\circ}$ \\
\hline 2923.199 & 4 & 3 & 34.199 .088 & $6168_{7 / 2}^{\circ}-40367_{9 / 2}$ \\
\hline 2922.990 & 100 & 100 & 34201.533 & $8018_{3 / 2}-42219_{5 / 2}^{\circ}$ \\
\hline 2922.799 & 150 & 150 & 34203.768 & $12219_{3 / 2}-46423_{3 / 2}^{\circ}$ \\
\hline 2922.602 & 300 & 300 & 34206.073 & $\begin{array}{l}12219_{3 / 2}-46426_{1 / 2}^{\circ} \\
13468_{9 / 2}^{\circ}-47675_{11 / 2}^{\circ}\end{array}$ \\
\hline 2922.094 & 10 & 3 & 34212.020 & $0_{3 / 2}-34212_{5 / 2}^{\circ}$ \\
\hline 2921.802 & 3 & & 34.215 .439 & $17460_{5 / 2}^{\circ}-51676_{3 / 2}$ \\
\hline 2921.622 & 200 & 300 & 34217.546 & $13248_{9 / 2}-47466_{11 / 2}^{\circ}$ \\
\hline 2921.534 & $100 \mathrm{~b}$ & 150 & 34218.58 & $9585_{\tilde{5} / 2}^{\circ}-43803_{\tau / 2}$ \\
\hline 2921.365 & 100 & 200 & 34220.56 & $12485_{\tau / 2}^{\circ}-46706_{\tau / 2}$ \\
\hline 2921.083 & 75 & 100 & 34.223 .860 & $11576_{3 / 2}^{\circ}-45800_{5 / 2}$ \\
\hline 2920.932 & 200 & 200 & 34225.629 & $10673_{5 / 2}^{\circ}-44898_{7 / 2}$ \\
\hline 2920.856 & 5 & 3 & 34226.520 & $9061_{5 / 2}-43287_{3 / 2}^{\circ}$ \\
\hline 2920.717 & 8 & 5 & 34228.148 & $6244_{1 / 2}-40472_{3 / 2}^{\circ}$ \\
\hline 2920.369 & $150 \mathrm{~b}$ & 100 & 34232.227 & $6691_{3 / 2}^{\circ}-40923_{5 / 2}$ \\
\hline 2920.212 & 8 & 5 & 34234.067 & $12472_{\% / 2}^{\circ}-46706_{7 / 2}$ \\
\hline 2920.056 & 40 & 40 & 34235.896 & $10572_{9 / 2}^{\circ}-44807_{7 / 2}$ \\
\hline 2919.841 & 400 & 300 & 34238.417 & $4490_{5 / 2}^{\circ}-38728_{5 / 2}$ \\
\hline 2919.597 & 1 & & 34241.279 & $17121_{3 / 2}^{\circ}-51362_{5 / 2}$ \\
\hline 2919.439 & $15 b$ & 8 & 34243.132 & $6168_{\tau / 2}^{\circ}-40411_{\tau / 2}$ \\
\hline 2919.175 & 20 & $100 \mathrm{~b}$ & 34246.228 & $12902_{3 / 2}^{\circ}-47148_{3 / 2}$ \\
\hline 2917.898 & 150 & 200 & 34261.215 & $10189_{11 / 2}^{\circ}-44450_{9 / 2}$ \\
\hline 2917.787 & 150 & $150 \mathrm{~b}$ & 34262.519 & $13468_{9 / 2}^{\circ}-47731_{9 / 2}$ \\
\hline 2917.411 & $800 \mathbf{r}$ & 300 & 34266.934 & $4490_{5 / 2}^{\circ}-38757_{3 / 2}$ \\
\hline 2917.034 & 150 & 200 & 34271.363 & $10379_{9 / 2}-44650_{\pi / 2}^{\circ}$ \\
\hline 2916.945 & 10 & 15 & 34272.408 & $14545_{5 / 2}^{\circ}-48817_{3 / 2}$ \\
\hline 2916.484 & 40 & 50 & 34277.825 & $14790_{7 / 2}^{\circ}-49068_{5 / 2}$ \\
\hline
\end{tabular}

\begin{tabular}{|c|c|c|c|c|}
\hline \multirow{2}{*}{$\begin{array}{c}\text { Wavelength } \\
\AA\end{array}$} & \multicolumn{2}{|c|}{ Intensity } & \multirow{2}{*}{$\begin{array}{l}\text { Wavenumber } \\
\mathbf{c m}^{-1}\end{array}$} & \multirow{2}{*}{ Classification } \\
\hline & Lamp & Spark & & \\
\hline 2916.429 & 10 & & 34278.472 & $11116_{7 / 2}^{\circ}-45395_{7 / 2}$ \\
\hline 2915.926 & 3 & 2 & 34.284 .385 & $7828_{1 / 2}-42112_{3 / 2}^{\circ}$ \\
\hline 2915.536 & 3 & & 34288.970 & $17983_{5 / 2}^{\circ}-52272_{7 / 2}$ \\
\hline 2914.582 & 50 & 20 & 34300.193 & $6691_{3 / 2}^{\circ}-40991_{3 / 2}$ \\
\hline 2914.342 & 300 & 100 & 34303.018 & $22642_{9 / 2}^{\circ}-56945_{11 / 2}$ \\
\hline 2913.097 & 3 & & 34317.678 & $16906_{7 / 2}^{\circ}-51224_{9 / 2}$ \\
\hline 2912.756 & 150 & 200 & 34321.695 & $9061_{5 / 2}-43382_{5 / 2}^{\circ}$ \\
\hline 2912.661 & 100 & 75 & 34322.814 & $4113_{5 / 2}-38436_{3 / 2}^{\circ}$ \\
\hline 2912.328 & 3 & & 34326.739 & $10572_{9 / 2}^{\circ}-44898_{7 / 2}$ \\
\hline 2912.009 & $400 \mathrm{~b}$ & 200 & 34330.499 & $0_{3 / 2}-34330_{1 / 2}^{\circ}$ \\
\hline 2911.321 & 200 & 150 & 34338.611 & $8605_{5 / 2}-42944_{7 / 2}^{\circ}$ \\
\hline 2911.137 & 4 & 3 & 34340.782 & $15144_{3 / 2}^{\circ}-49485_{1 / 2}$ \\
\hline 2910.747 & 20 & 20 & 34345.383 & $7331_{5 / 2}^{\circ}-41676_{3 / 2}$ \\
\hline 2910.596 & 4001 & 300 & 34347.164 & $4146_{7 / 2}-38493_{5 / 2}^{\circ}$ \\
\hline 2909.767 & 25 & 40 & 34356.950 & $7331_{5 / 2}^{\circ}-41688_{7 / 2}$ \\
\hline 2909.456 & 8 & 15 & 34360.622 & $14484_{11 / 2}^{\circ}-48844_{9 / 2}$ \\
\hline 2909.378 & 8 & 5 & 34361.543 & $16906_{\overline{7} / 2}^{\circ}-51268_{7 / 2}$ \\
\hline 2908.984 & 10 & 50 & 34366.197 & $10855_{7 / 2}-45221_{5 / 2}^{\circ}$ \\
\hline 2908.672 & 4 & 10 & 34369.883 & $17460_{5 / 2}^{\circ}-51830_{7 / 2}$ \\
\hline 2908.436 & $2 \mathrm{~g}$ & & 34372.672 & $9400_{5 / 2}-43773_{7 / 2}^{\circ}$ \\
\hline 2908.383 & $10 \mathrm{~b}$ & & $34373: 298$ & $12488_{9 / 2}^{\circ}-46861_{11 / 2}$ \\
\hline 2908.359 & $150 \mathrm{~b}$ & 100 & 34373.582 & $4490_{5 / 2}^{\circ}-38863_{5 / 2}$ \\
\hline 2908.314 & $10 \mathrm{~b}$ & $50 \mathrm{~b}$ & 34374.114 & $16033_{5 / 2}^{\circ}-50407_{7 / 2}$ \\
\hline 2908.156 & 8 & 4 & 34375.981 & $9720_{7 / 2}^{\circ}-44096_{9 / 2}$ \\
\hline 2907.781 & 40 & 10 & 34380.414 & $4113_{5 / 2}-38493_{5 / 2}^{\circ}$ \\
\hline 2906.122 & 50 & 200 & 34400.040 & $8018_{3 / 2}-42418_{3 / 2}^{\circ}$ \\
\hline 2905.931 & 75 & 100 & 34402.301 & $6168_{7 / 2}^{\circ}-40570_{7 / 2}$ \\
\hline 2905.745 & 4 & 8 & 34404.503 & $17460_{5 / 2}^{\circ}-51865_{5 / 2}$ \\
\hline 2905.303 & 4 & 8 & 34409.737 & $17272_{9 / 2}^{\circ}-51681_{9 / 2}$ \\
\hline 2904.999 & 50 & 40 & 34413.338 & $20080_{7 / 2}^{\circ}-54493_{5 / 2}$ \\
\hline 2904.702 & 5 & 15 & 34416.856 & $12485_{\pi / 2}^{\circ}-46902_{5 / 2}$ \\
\hline 2904.429 & 5 & & 34420.091 & $15453_{\pi / 2}^{\circ}-49873_{\tilde{s} / 2}$ \\
\hline 2904.267 & 10 & $50 \mathrm{~b}$ & 34422.011 & $12902_{3 / 2}^{\circ}-47324_{5 / 2}$ \\
\hline 2903.166 & 200 & 150 & 34435.064 & $4146_{7 / 2}-38581_{5 / 2}^{\circ}$ \\
\hline 2903.052 & 8 & 20 & 34436.416 & $22642_{9 / 2}^{\circ}-57078_{9 / 2}$ \\
\hline 2902.144 & 2 & 5 & 34447.190 & $17722_{9 / 2}^{\circ}-52170_{11 / 2}$ \\
\hline 2901.378 & 3 & & 34456.284 & $16906_{7 / 2}^{\circ}-51362_{5 / 2}$ \\
\hline 2900.772 & 4 & 10 & 34463.482 & $12472_{5 / 2}^{\circ}-46935_{3 / 2}$ \\
\hline 2900.367 & 5 & 8 & 34468.294 & $4113_{5 / 2}-38581_{5 / 2}^{\circ}$ \\
\hline 2899.720 & 600 & 400 & 34475.985 & $6168_{\overline{7} / 2}^{\circ}-40644_{5 / 2}$ \\
\hline 2899.369 & 50 & 150 & 34480.158 & $13818_{7 / 2}^{\circ}-48298_{7 / 2}$ \\
\hline 2898.729 & 1 & & 34487.771 & $15349_{11 / 2}^{\circ}-49837_{9 / 2}$ \\
\hline 2898.262 & 20 & 40 & 34493.327 & $6213_{9 / 2}-40706_{7 / 2}^{\circ}$ \\
\hline 2898.202 & 10 & 15 & 34494.042 & $11116_{7 / 2}^{\circ}-45610_{5 / 2}^{\circ}$ \\
\hline 2897.493 & 3 & 5 & 34502.481 & $13818_{7 / 2}^{\circ}-48320_{5 / 2}$ \\
\hline 2897.112 & $40 \mathrm{~b}$ & $20 \mathrm{~b}$ & 34507.019 & $20989_{9 / 2}^{\circ}-55496_{9 / 2}$ \\
\hline 2897.069 & $75 b$ & $100 \mathrm{~b}$ & 34507.531 & $15453_{7 / 2}^{\circ}-49960_{\tau / 2}$ \\
\hline 2896.315 & 4 & 10 & 34516.514 & $10673_{5 / 2}^{\circ}-45189_{5 / 2}$ \\
\hline 2895.913 & 2 & 5 & 34521.305 & $18214_{3 / 2}^{\circ}-52735_{3 / 2}$ \\
\hline 2895.747 & 4 & 10 & 34523.284 & $14545_{5 / 2}^{\circ}-49068_{5 / 2}$ \\
\hline
\end{tabular}


TABLE 3. Classified lines of Th II - Continued

\begin{tabular}{|c|c|c|c|c|}
\hline \multirow{2}{*}{$\begin{array}{c}\text { Wavelength } \\
\AA\end{array}$} & \multicolumn{2}{|c|}{ Intensity } & \multirow{2}{*}{$\begin{array}{l}\text { Wavenumber } \\
\mathbf{c m}^{-1}\end{array}$} & \multirow{2}{*}{ Classification } \\
\hline & Lamp & Spark & & \\
\hline 2895.370 & 1 & & 34527.779 & $20969_{7 / 2}^{\circ}-55496_{9 / 2}$ \\
\hline 2895.136 & 300 & 150 & 34530.570 & $1859_{3 / 2}-36390_{3 / 2}^{\circ}$ \\
\hline 2894.988 & 3 & 75 & 34532.335 & $17121_{3 / 2}^{\circ}-51653_{1 / 2}$ \\
\hline 2894.501 & $25 b$ & $20 \mathrm{~b}$ & 34538.145 & $10189_{11 / 2}^{\circ}-44727_{11 / 2}$ \\
\hline 2894.446 & 10 & 8 & 34538.801 & $11725_{1 / 2}^{\circ}-46264_{3 / 2}$ \\
\hline 2893.747 & 2 & 5 & 34547.143 & $18973_{7 / 2}^{\circ}-53520_{9 / 2}$ \\
\hline 2893.157 & 3 & 5 & 34554.188 & $10572_{9 / 2}^{\circ}-45126_{g / 2}$ \\
\hline 2892.815 & 1 & & 34558.273 & $17272_{9 / 2}^{\circ}-51830_{7 / 2}$ \\
\hline 2892.171 & 50 & 100 & 34565.968 & $9238_{9 / 2}^{\circ}-43803_{7 / 2}$ \\
\hline 2891.815 & 150 & 100 & 34570.223 & $9202_{7 / 2}^{\circ}-43772_{5 / 2}$ \\
\hline 2891.730 & 150 & 150 & 34571.239 & $9238_{9 / 2}^{\circ}-43809_{9 / 2}^{\circ}$ \\
\hline 2891.249 & 300 & 300 & 34576.990 & $\begin{aligned} 22106_{5 / 2} & -56683_{7 / 2}^{\circ} \\
8378_{7 / 2}^{\circ} & -42955_{9 / 2}^{\circ}\end{aligned}$ \\
\hline 2891.128 & 15 & 10 & 34578.438 & $4490_{5 / 2}^{\circ}-39068_{7 / 2}$ \\
\hline 2890.888 & 15 & 20 & 34581.308 & $6244_{1 / 2}-40825_{1 / 2}^{\circ}$ \\
\hline 2890.589 & 3 & & 34584.885 & $1859_{3 / 2}-36444_{3 / 2}^{\circ}$ \\
\hline 2890.480 & $5 b$ & $10 \mathrm{~b}$ & 34586.189 & $14790_{7 / 2}^{\circ}-49377_{7 / 2}$ \\
\hline 2890.316 & 1 & & 34588.151 & $14101_{1 / 2}^{\circ}-48689_{3 / 2}$ \\
\hline 2890.188 & 5 & 4 & 34589.683 & $7828_{1 / 2}-42418_{3 / 2}^{\circ}$ \\
\hline 2889.564 & 1 & & 34597.152 & $19248_{5 / 2}^{\circ}-53845_{5 / 2}$ \\
\hline 2889.181 & $100 \mathrm{r}$ & 25 & 34601.739 & $9202_{7 / 2}^{\circ}-43803_{7 / 2}$ \\
\hline 2888.904 & 5 & $5 b$ & 34605.056 & $7331_{5 / 2}^{\circ}-41936_{3 / 2}$ \\
\hline 2887.818 & 600 & 500 & 34618.069 & $4146_{7 / 2}-38764_{7 / 2}^{\circ}$ \\
\hline 2886.785 & 2 & 2 & 34630.456 & $16033_{5 / 2}^{\circ}-50663_{5 / 2}$ \\
\hline 2886.506 & 100 & 150 & 34633.803 & $10855_{7 / 2}-45489_{9 / 2}^{\circ}$ \\
\hline 2886.242 & 100 & 100 & 34636.971 & $6691_{3 / 2}^{\circ}-41328_{5 / 2}$ \\
\hline 2885.968 & 5 & 10 & 34640.260 & $14484_{11 / 2}^{\circ}-49124_{11 / 2}$ \\
\hline 2885.048 & 800 & 500 & 34651.305 & $4113_{5 / 2}-38764_{7 / 2}^{\circ}$ \\
\hline 2884.289 & 600 & 400 & 34660.423 & $4490_{5 / 2}^{\circ}-39150_{3 / 2}$ \\
\hline 2883.618 & $50 \mathrm{~b}$ & 20 & 34668.488 & $9720_{7 / 2}^{\circ}-44388_{5 / 2}$ \\
\hline 2883.446 & 5 & 4 & 34670.556 & $11725_{1 / 2}^{\circ}-46395_{3 / 2}$ \\
\hline 2883.184 & 2 & & 34673.707 & $13818_{7 / 2}^{\circ}-48492_{5 / 2}$ \\
\hline 2882.959 & 2 & & 34676.412 & $12472_{5 / 2}^{\circ}-47148_{3 / 2}$ \\
\hline 2882.511 & 100 & 50 & 34681.802 & $8605_{5 / 2}-43287_{3 / 2}^{\circ}$ \\
\hline 2882.402 & 1 & & 34683.113 & $12488_{9 / 2}^{\circ}-47171_{9 / 2}$ \\
\hline 2882.354 & 8 & 8 & 34683.691 & $11116_{7 / 2}^{\circ}-45800_{5 / 2}$ \\
\hline 2882.191 & 1 & & 34685.652 & $12485_{7 / 2}^{\circ}-47171_{9 / 2}$ \\
\hline 2882.008 & $300 \mathrm{~s}$ & 300 & 34687.854 & $11576_{3 / 2}^{\circ}-46264_{3 / 2}$ \\
\hline 2881.137 & 100 & 150 & 34698.341 & $6700_{9 / 2}^{\circ}-41398_{9 / 2}$ \\
\hline 2880.803 & 1 & & 34702.363 & $16033_{5 / 2}^{\circ}-50735_{3 / 2}$ \\
\hline 2880.313 & 1 & & 34708.267 & $21682_{7 / 2}^{\circ}-56391_{5 / 2}$ \\
\hline 2879.958 & 2 & & 34712.545 & $9061_{5 / 2}-43773_{7 / 2}^{\circ}$ \\
\hline 2879.531 & 40 & 75 & 34717.692 & $8378_{\tau / 2}^{\circ}-43096_{5 / 2}$ \\
\hline 2879.203 & $50 \mathrm{~b}$ & $20 \mathrm{~b}$ & 34721.647 & $1859_{3 / 2}-36581_{3 / 2}^{\circ}$ \\
\hline 2879.181 & $10 \mathrm{~b}$ & $10 \mathrm{~b}$ & 34721.912 & $10673_{\bar{\pi} / 2}^{\circ}-45395_{\pi / 2}$ \\
\hline 2878.644 & $50 \mathrm{~b}$ & 75 & 34728.389 & $15144_{3 / 2}^{\circ}-49873_{5 / 2}$ \\
\hline 2877.390 & 5 & & 34743.523 & $17121_{3 / 2}^{\circ}-51865_{5 / 2}$ \\
\hline 2876.658 & 3 & & 34752.364 & $17983_{5 / 2}^{\circ}-52735_{3 / 2}$ \\
\hline 2876.417 & $200 \mathrm{~b}$ & 150 & 34755.275 & $6168_{\tau / 2}^{\circ}-40923_{5 / 2}$ \\
\hline 2875.641 & 25 & 200 & 34764.654 & $20080_{\tau / 2}^{\circ}-54845_{9 / 2}$ \\
\hline
\end{tabular}

\begin{tabular}{|c|c|c|c|c|}
\hline \multirow{2}{*}{$\begin{array}{c}\text { Wavelength } \\
\AA\end{array}$} & \multicolumn{2}{|c|}{ Intensity } & \multirow{2}{*}{$\begin{array}{c}\text { Wavenumber } \\
\mathbf{c m}^{-1}\end{array}$} & \multirow{2}{*}{ Classification } \\
\hline & Lamp & Spark & & \\
\hline 2874.753 & 2 & 3 & 34775.392 & $16906_{7 / 2}^{\circ}-51681_{9 / 2}$ \\
\hline 2874.624 & 15 & 40 & 34776.952 & $8605_{5 / 2}-43382_{5 / 2}^{\circ}$ \\
\hline 2873.714 & $25 b$ & 75 & 34787.965 & $6700_{9 / 2}^{\circ}-41488_{7 / 2}$ \\
\hline 2873.168 & 2 & & 34794.575 & $19050_{3 / 2}^{\circ}-53845_{5 / 2}$ \\
\hline 2872.441 & 21 & & 34803.381 & $9585_{5 / 2}^{\circ}-44388_{5 / 2}$ \\
\hline 2872.267 & 2 & & 34805.489 & $22139_{9 / 2}^{\circ}-56945_{11 / 2}$ \\
\hline 2871.754 & 3 & & 34811.706 & $17460_{5 / 2}^{\circ}-52272_{7 / 2}$ \\
\hline 2871.105 & 5 & 10 & 34819.575 & $11576_{3 / 2}^{\circ}-46395_{3 / 2}$ \\
\hline 2870.822 & 40 & 75 & 34823.007 & $10572_{9 / 2}^{\circ}-45395_{7 / 2}$ \\
\hline 2870.406 & $800 \mathrm{r}$ & 300 & 34828.054 & $1859_{3 / 2}-36687_{5 / 2}^{\circ}$ \\
\hline 2870.285 & $8 \mathrm{~h}$ & 8 & 34829.522 & $13468_{9 / 2}^{\circ}-48298_{7 / 2}$ \\
\hline 2870.049 & 15 & 10 & 34832.386 & $9720_{7 / 2}^{\circ}-44552_{5 / 2}$ \\
\hline 2869.916 & 100 & 200 & 34834.000 & $10855_{7 / 2}-45689_{7 / 2}^{\circ}$ \\
\hline 2869.528 & 2 & 3 & 34838.710 & $12485_{7 / 2}^{\circ}-47324_{5 / 2}$ \\
\hline 2868.679 & 40 & 100 & 34849.020 & $8378_{7 / 2}^{\circ}-43227_{5 / 2}^{\circ}$ \\
\hline 2868.416 & 5 & & 34852.215 & $12472_{5 / 2}^{\circ}-47324_{5 / 2}$ \\
\hline 2867.920 & 5 & 10 & 34858.242 & $9238_{9 / 2}^{\circ}-44096_{9 / 2}^{\circ}$ \\
\hline 2867.120 & 5 & & 34867.968 & $8378_{7 / 2}^{\circ}-43246_{7 / 2}$ \\
\hline 2867.067 & 10 & 5 & 34868.613 & $1521_{5 / 2}-36390_{3 / 2}^{\circ}$ \\
\hline 2867.029 & 5 & 5 & 34869.075 & $14545_{5 / 2}^{\circ}-49414_{3 / 2}$ \\
\hline 2866.652 & 15 & 150 & 34873.660 & $14484_{11 / 2}^{\circ}-49357_{11 / 2}$ \\
\hline 2866.407 & 25 & 20 & 34876.641 & $4490_{5 / 2}^{\circ}-39366_{5 / 2}$ \\
\hline 2865.242 & 15 & 20 & 34890.821 & $7331_{5 / 2}^{\circ}-42222_{7 / 2}$ \\
\hline 2864.980 & 2 & & 34894.012 & $9202_{7 / 2}^{\circ}-44096_{9 / 2}^{\circ}$ \\
\hline 2864.657 & 50 & 200 & 34897.946 & $\begin{array}{l}17837_{i / 2}^{\circ}-52735_{3 / 2} \\
17272_{9 / 2}^{\circ}-52170_{11 / 2}^{\circ}\end{array}$ \\
\hline 2863.069 & 1 & & 34917.301 & $24381_{7 / 2}-59299^{\circ} / 2$ \\
\hline 2862.732 & 1 & & 34.921 .412 & $19248_{5 / 2}^{\circ}-54169_{7 / 2}$ \\
\hline 2862.646 & 3 & & 34922.461 & $8460_{3 / 2}-43382_{5 / 2}^{\circ}$ \\
\hline 2862.610 & $300 \mathrm{r}$ & 20 & 34922.900 & $1521_{5 / 2}-36444_{3 / 2}^{\circ}$ \\
\hline 2862.527 & $8 b$ & 75 & 34923.912 & $16906_{\pi / 2}^{\circ}-51830_{7 / 2}$ \\
\hline 2862.007 & 5 & & 34930.257 & $22014_{11 / 2}^{\circ}-56945_{11 / 2}$ \\
\hline 2861.442 & $8 b$ & & 34937.154 & $10189_{11 / 2}^{\circ}-45126_{9 / 2}$ \\
\hline 2861.415 & 300 & 300 & 34937.484 & $10673_{5 / 2}^{\circ}-45610_{5 / 2}$ \\
\hline 2861.331 & $100 \mathrm{~b}$ & $150 \mathrm{~b}$ & 34938.509 & $9711_{7 / 2}-44650_{7 / 2}^{\circ}$ \\
\hline 2861.271 & $15 b$ & $25 b$ & 34939.242 & $9238_{9 / 2}^{\circ}-44177_{11 / 2}$ \\
\hline 2859.691 & 1 & & 34958.545 & $16906_{7 / 2}^{\circ}-51865_{5 / 2}^{0}$ \\
\hline 2858.979 & 5 & 3 & 34967.251 & $\begin{array}{r}12902_{3 / 2}^{\circ}-47869_{3 / 2}^{\circ} \\
9585_{5 / 2}^{\circ}-44552_{5 / 2}^{\circ}\end{array}$ \\
\hline 2858.915 & 15 & 10 & 34968.034 & $4490_{5 / 2}^{\circ}-39458_{7 / 2}^{\circ}$ \\
\hline 2857.599 & 2 & 3 & 34984.137 & $13468_{9 / 2}^{\circ}-48453_{7 / 2}$ \\
\hline 2857.491 & 50 & 75 & 34985.459 & $6691_{3 / 2}^{\circ}-41676_{3 / 2}^{\circ}$ \\
\hline 2857.398 & 8 & 4 & 34986.597 & $0_{3 / 2}-34986_{3 / 2}^{\circ}$ \\
\hline 2857.262 & 2 & & 34988.263 & $6700_{9 / 2}^{\circ}-41688_{7 / 2}^{\circ}$ \\
\hline 2856.300 & 2 & & 35000.046 & $17272_{9 / 2}^{\circ}-52272_{7 / 2}$ \\
\hline 2855.904 & $15 b$ & 100 & 35004.899 & $11576_{3 / 2}^{\circ}-46581_{5 / 2}$ \\
\hline 2855.867 & $10 \mathrm{~b}$ & $50 \mathrm{~b}$ & 35005.352 & $7331_{5 / 2}^{\circ}-42336_{5 / 2}$ \\
\hline 2854.918 & 10 & 100 & 35016.988 & $15453_{7 / 2}^{\circ}-50470_{9 / 2}$ \\
\hline 2854.620 & 5 & 10 & 35020.643 & $7331_{5 / 2}^{\circ}-42352_{5 / 2}$ \\
\hline 2854.297 & 2 & & 35024.606 & $15710_{3 / 2}^{\circ}-50735_{3 / 2}^{\circ}$ \\
\hline
\end{tabular}


TABLE 3. Classified lines of Th II-Continued

\begin{tabular}{|c|c|c|c|c|c|c|c|c|c|}
\hline \multirow{2}{*}{$\begin{array}{c}\text { Wavelength } \\
\AA\end{array}$} & \multicolumn{2}{|c|}{ Intensity } & \multirow{2}{*}{$\begin{array}{c}\text { Wavenumber } \\
\mathrm{cm}^{-1}\end{array}$} & \multirow{2}{*}{ Classification } & \multirow{2}{*}{$\begin{array}{c}\text { Wavelength } \\
\AA\end{array}$} & \multicolumn{2}{|c|}{ Intensity } & \multirow{2}{*}{$\begin{array}{c}\text { Wavenumber } \\
\mathbf{c m}^{-1}\end{array}$} & \multirow{2}{*}{ Classification } \\
\hline & Lamp & Spark & & & & Lamp & Spark & & \\
\hline 2854.134 & 100 & 200 & 35026.606 & $13818_{7 / 2}^{\circ}-48844_{9 / 2}$ & 2832.314 & $1000 \mathbf{r}$ & 400 & 35296.436 & $4146_{7 / 2}-39443_{9 / 2}^{\circ}$ \\
\hline 2853.933 & 2 & & 35029.073 & $16906_{7 / 2}^{\circ}-51935_{5 / 2}^{\circ}$ & 2832.012 & 10 & 25 & 35300.200 & $10855_{7 / 2}-46155_{5 / 2}^{\circ}$ \\
\hline 2853.338 & 1 & & 35036.377 & $18973_{7 / 2}^{\circ}-54010_{7 / 2}$ & 2831.206 & 15 & $100 \mathrm{~b}$ & 35310.249 & $10379_{9 / 2}-45689_{7 / 2}^{\circ}$ \\
\hline 2852.799 & 1 & & 35042.996 & $14484_{11 / 2}^{\circ}-49527_{13 / 2}^{\circ}$ & 2830.997 & 3 & & 35312.855 & $14101_{1 / 2}^{\circ}-4.9414_{3 / 2}$ \\
\hline 2852.501 & 15 & 150 & 35046.657 & $14790_{7 / 2}^{\circ}-49837_{9 / 2}$ & 2830.955 & 3 & & 35313.380 & $9585_{5 / 2}^{\circ}-44898_{7 / 2}$ \\
\hline 2851.443 & 200 & 150 & 35059.660 & $1521_{5 / 2}-36581_{3 / 2}^{\circ}$ & 2830.905 & 1 & & 35314.003 & $15710_{3 / 2}^{\circ}-51024_{3 / 2}$ \\
\hline 2851.260 & $600 \mathrm{r}$ & 300 & 35061.910 & $1521_{5 / 2}-36583_{7 / 2}^{\circ}$ & 2830.683 & 5 & 8 & 35316.772 & $6691_{3 / 2}^{\circ}-42008_{1 / 2}^{\circ}$ \\
\hline 2849.592 & 2 & 2 & 35082.433 & $14275_{9 / 2}^{\circ}-49357_{11 / 2}$ & 2830.441 & 200 & 150 & 35319.792 & $6168_{7 / 2}^{\circ}-41488_{7 / 2}^{\circ}$ \\
\hline 2849.169 & 8 & & 35087.641 & $9720_{7 / 2}^{\circ}-44807_{7 / 2}$ & 2829.932 & 25 & 100 & 35326.144 & $11576_{3 / 2}^{\circ}-46902_{5 / 2}$ \\
\hline 2848.686 & 1 & & 35093.590 & $21297_{5 / 2}^{\circ}-56391_{5 / 2}$ & 2829.645 & 3 & & 35329.727 & $16033_{5 / 2}^{\circ}-51362_{5 / 2}^{\circ}$ \\
\hline 2848.013 & $10 \mathrm{~b}$ & 75 & 35101.882 & $17460_{5 / 2}^{\circ}-52562_{7 / 2}$ & 2829.433 & 20 & 50 & 35332.374 & $10572_{9 / 2}^{\circ}-45904_{9 / 2}$ \\
\hline 2847.890 & 2 & & 35103.398 & $21131_{3 / 2}^{\circ}-56235_{3 / 2}$ & 2827.988 & 100 & 150 & 35350.427 & $9202_{7 / 2}^{\circ}-44552_{5 / 2}$ \\
\hline 2847.354 & 150 & 200 & 35110.006 & $10379_{9 / 2}-45489_{9 / 2}^{\circ}$ & 2827.755 & 20 & 150 & 35353.339 & $14484_{11 / 2}^{\circ}-49837_{9 / 2}$ \\
\hline 2847.231 & $5 b$ & & 35111.523 & $7001_{3 / 2}-42112_{3 / 2}^{\circ}$ & 2827.280 & 10 & 75 & 35359.279 & $11576_{3 / 2}^{\circ}-46935_{3 / 2}$ \\
\hline 2846.757 & 1 & & 35117.369 & $16564_{11 / 2}^{\circ}-51681_{9 / 2}$ & 2826.855 & 500 & 200 & 35364.594 & $8018_{3 / 2}-43382_{5 / 2}^{\circ}$ \\
\hline 2846.531 & 15 & 50 & 35120.157 & $15349_{11 / 2}^{\circ}-50470_{9 / 2}$ & 2825.941 & $15 b$ & 100 & 35376.032 & $13468_{9 / 2}^{\circ}-48844_{9 / 2}^{\circ}$ \\
\hline 2845.966 & 3 & & 35127.129 & $10673_{5 / 2}^{\circ}-45800_{5 / 2}^{\circ}$ & 2825.375 & 25 & 1001 & 35383.119 & $12488_{9 / 2}^{\circ}-47871_{7 / 2}^{\circ}$ \\
\hline 2845.835 & $15 b$ & 75 & 35128.746 & $16818_{7 / 2}-51946_{5 / 2}^{\circ}$ & 2825.172 & 10 & 5 & 35385.661 & $12485_{7 / 2}^{\circ}-47871_{7 / 2}$ \\
\hline 2845.186 & 15 & 50 & 35136.758 & $11116_{7 / 2}^{\circ}-46253_{9 / 2}$ & 2824.536 & 8 & 2 & 35393.628 & $8378_{7 / 2}^{\circ}-43772_{5 / 2}^{\circ}$ \\
\hline 2843.553 & 8 & 2 & 35156.936 & $0_{3 / 2}-35156_{5 / 2}^{\circ}$ & 2824.365 & 1 & & 35395.771 & $21682_{7 / 2}^{\circ}-57078_{9 / 2}^{\circ}$ \\
\hline 2843.304 & $15 b$ & $75 b$ & 35160.015 & $6168_{7 / 2}^{\circ}-41328_{5 / 2}$ & 2824.232 & 8 & 10 & 35397.438 & $12472_{5 / 2}^{\circ}-47869_{3 / 2}$ \\
\hline 2843.056 & 10 & 20 & 35163.081 & $10572_{9 / 2}^{\circ}-45735_{11 / 2}^{\circ}$ & 2824.091 & 4 & & 35399.205 & $12472_{5 / 2}^{\circ}-47871_{7 / 2}^{\circ}$ \\
\hline 2842.812 & 800 & 200 & 35166.099 & $1521_{5 / 2}-36687_{5 / 2}^{\circ}$ & 2823.553 & 50 & 100 & 35405.950 & $9720_{7 / 2}^{\circ}-45126_{9 / 2}^{\circ}$ \\
\hline 2842.674 & 20 & 50 & 35167.806 & $8605_{5 / 2}-43773_{7 / 2}^{\circ}$ & 2823.132 & 25 & 75 & 35411.229 & $15324_{1 / 2}^{\circ}-50735_{3 / 2}$ \\
\hline 2842.530 & 2 & & 35169.588 & $14790_{7 / 2}^{\circ}-49960_{7 / 2}$ & 2822.832 & 15 & 40 & 35414.992 & $14545_{5 / 2}^{\circ}-49960_{7 / 2}$ \\
\hline 2841.811 & 200 & 50 & 35178.485 & $9720_{7 / 2}^{\circ}-44898_{7 / 2}^{\circ}$ & 2822.741 & 2 & & 35416.134 & $20080_{\tau / 2}^{\circ}-55496_{9 / 2}^{\circ}$ \\
\hline 2841.162 & 200 & 200 & 35186.521 & $9202_{7 / 2}^{\circ}-44388_{5 / 2}$ & 2822.688 & 25 & 150 & 35416.799 & $7001_{3 / 2}-42418_{3 / 2}^{\circ}$ \\
\hline 2841.087 & 8 & 3 & 35187.450 & $7331_{5 / 2}^{\circ}-42518_{7 / 2}$ & 2822.559 & 20 & 150 & 35418.418 & $12902_{3 / 2}^{\circ}-48320_{5 / 2}^{\circ}$ \\
\hline 2840.154 & 200 & 150 & 35199.008 & $0_{3 / 2}-35198_{1 / 2}^{\circ}$ & 2822.446 & 15 & 50 & 35419.835 & $7331_{5 / 2}^{\circ}-42751_{7 / 2}$ \\
\hline 2839.605 & 20 & 100 & 35205.813 & $13406_{13 / 2}^{\circ}-48612_{13 / 2}$ & 2822.407 & 10 & $10 \mathrm{~b}$ & 35420.325 & $11725_{1 / 2}^{o}-47145_{1 / 2}$ \\
\hline 2839.335 & 25 & 40 & 35209.161 & $6700_{9 / 2}^{\circ}-41909_{9 / 2}$ & 2822.359 & 20 & 25 & 35420.927 & $9400_{5 / 2}-44821_{5 / 2}^{\circ}$ \\
\hline 2839.240 & 200 & 150 & .35210 .339 & $4490_{5 / 2}^{\circ}-39700_{5 / 2}$ & 2822.275 & 2 & & 35421.982 & $20969_{7 / 2}^{\circ}-56391_{5 / 2}^{\circ}$ \\
\hline 2839.084 & 10 & 10 & 35212.273 & $9238_{9 / 2}^{\circ}-44450_{9 / 2}^{\circ}$ & 2822.181 & $15 b$ & 75 & 35423.161 & $11725_{1 / 2}^{\circ}-47148_{3 / 2}$ \\
\hline 2838.593 & 8 & 5 & 35218.364 & $7001_{3 / 2}-42219_{5 / 2}^{\circ}$ & 2822.025 & 200 & 200 & 35425.119 & $8378_{\tau / 2}^{\circ}-43803_{7 / 2}$ \\
\hline 2838.255 & 10 & 15 & 35222.558 & $9585_{5 / 2}^{\circ}-44807_{7 / 2}$ & 2821.602 & $100 \mathrm{~b}$ & 200 & 35430.430 & $8378_{7 / 2}^{\circ}-43809_{9 / 2}$ \\
\hline 2837.886 & 3 & & 35227.138 & $15242_{9 / 2}^{\circ}-50470_{9 / 2}$ & 2820.589 & 2 & & 35443.154 & $19050_{3 / 2}^{\circ}-54493_{5 / 2}$ \\
\hline 2837.641 & $10 \mathrm{~b}$ & 5 & 35230.179 & $6168_{7 / 2}^{\circ}-41398_{9 / 2}^{\circ / 2}$ & 2820.335 & 200 & 150 & 35446.346 & $6700_{9 / 2}^{\circ}-42146_{11 / 2}^{\circ}$ \\
\hline 2837.295 & $1000 \mathrm{r}$ & 500 & 35234.475 & $6213_{9 / 2}-41447_{11 / 2}^{0}$ & 2819.322 & 200 & 200 & 35459.081 & $7828_{1 / 2}-43287_{3 / 2}^{\circ}$ \\
\hline 2837.201 & $8 b$ & 5 & 35235.642 & $11116_{7 / 2}^{\circ}-46352_{7 / 2}$ & 2818.875 & 3 & 2 & 35464.704 & $11116_{7 / 2}^{\circ}-46581_{5 / 2}$ \\
\hline 2836.593 & 2 & & 35243.194 & $12488_{9 / 2}^{\circ}-47731_{9 / 2}^{\circ}$ & 2818.505 & 5 & & 35469.359 & $9720_{7 / 2}^{\circ}-45189_{5 / 2}$ \\
\hline 2836.435 & $75 b$ & 20 & 35245.157 & $6691_{3 / 2}^{\circ}-41936_{3 / 2}$ & 2817.135 & 20 & 50 & 35486.607 & $11116_{7 / 2}^{\circ}-46603_{5 / 2}$ \\
\hline 2836.205 & 25 & 15 & 35248.015 & $9202_{7 / 2}^{\circ}-44450_{9 / 2}$ & 2816.927 & 20 & 50 & 35489.228 & $9238_{9 / 2}^{\circ}-44727_{11 / 2}^{\circ}$ \\
\hline 2836.047 & 500 & 300 & 35249.979 & $4146_{7 / 2}-39396_{7 / 2}^{\circ}$ & 2816.070 & 100 & 150 & 35500.027 & $6700_{9 / 2}^{\circ}-42200_{9 / 2}^{\circ}$ \\
\hline 2835.305 & 2 & & 35259.203 & $21131_{3 / 2}^{\circ}-56391_{5 / 2}$ & 2815.312 & 5 & 4 & 35509.585 & $9711_{7 / 2}-45221_{5 / 2}^{\circ}$ \\
\hline 2834.776 & 25 & 40 & 35265.783 & $9238_{9 / 2}^{\circ}-44503_{7 / 2}$ & 2815.050 & 10 & 15 & 35512.890 & $10855_{7 / 2}-46368_{9 / 2}^{\circ}$ \\
\hline 2834.530 & 50 & 75 & 35268.843 & $11116_{7 / 2}^{\circ}-46385_{7 / 2}$ & 2814.576 & 50 & 150 & 35518.870 & $15144_{3 / 2}^{\circ}-50663_{5 / 2}^{\circ}$ \\
\hline 2834.482 & 150 & 100 & 35269.441 & $8018_{3 / 2}-43287_{3 / 2}^{\circ}$ & 2814.480 & $25 b$ & 75 & 35520.081 & $6168_{7 / 2}^{\circ}-41688_{7 / 2}^{\circ}$ \\
\hline 2834.025 & 3 & 3 & 35275.128 & $17460_{5 / 2}^{\circ}-52735_{3 / 2}$ & 2814.473 & $2 \mathrm{~b}$ & & 35520.170 & $18973_{7 / 2}^{\circ}-54493_{5 / 2}^{\circ}$ \\
\hline 2833.532 & 15 & 150 & 35281.265 & $15349_{11 / 2}^{\circ}-50631_{11 / 2}$ & 2814.319 & 300 & 200 & 35522.114 & $6700_{9 / 2}^{\circ}-42222_{7 / 2}$ \\
\hline 2833.334 & 200 & 100 & 35283.730 & $8460_{3 / 2}-43744_{1 / 2}^{\circ}$ & 2812.424 & $15 b$ & 100 & 35546.047 & $10189_{11 / 2}^{\circ}-45735_{11 / 2}$ \\
\hline
\end{tabular}


TABLE 3. Classified lines of Th II-Continued

\begin{tabular}{|c|c|c|c|c|c|c|c|c|c|}
\hline \multirow{2}{*}{$\begin{array}{c}\text { Wavelength } \\
\AA\end{array}$} & \multicolumn{2}{|c|}{ Intensity } & \multirow{2}{*}{$\begin{array}{c}\text { Wavenumber } \\
\mathrm{cm}^{-1}\end{array}$} & \multirow{2}{*}{ Classification } & \multirow{2}{*}{$\begin{array}{c}\text { Wavelength } \\
\AA\end{array}$} & \multicolumn{2}{|c|}{ Intensity } & \multirow{2}{*}{$\begin{array}{c}\text { Wavenumber } \\
\mathrm{cm}^{-1}\end{array}$} & \multirow{2}{*}{ Classification } \\
\hline & Lamp & Spark & & & & Lamp & Spark & & \\
\hline 2812.187 & 2 & & 35549.042 & $20686_{5 / 2}^{\circ}-56235_{3 / 2}$ & 2788.687 & 10 & 50 & 35848.596 & $12472_{5 / 2}^{\circ}-48320_{5 / 2}$ \\
\hline 2811.744 & $8 b$ & & 35554.643 & $6691_{3 / 2}^{\circ}-42246_{1 / 2}$ & 2788.077 & 10 & 15 & 35856.439 & $1859_{3 / 2}-37716_{1 / 2}^{\circ}$ \\
\hline 2811.413 & 5 & 10 & 35558.829 & $13818_{7 / 2}^{\circ}-49377_{7 / 2}$ & 2787.666 & 5 & 50 & 35861.725 & $14545_{5 / 2}^{\circ}-50407_{7 / 2}$ \\
\hline 2811.157 & 3 & 5 & 35562.067 & $14275_{9 / 2}^{\circ}-49837_{9 / 2}$ & 2787.131 & 50 & 75 & 35868.608 & $6244_{1 / 2}-42112_{3 / 2}^{\circ}$ \\
\hline 2810.580 & 4 & 20 & 35569.367 & $11576_{3 / 2}^{\circ}-47145_{1 / 2}$ & 2786.915 & 10 & 150 & 35871.388 & $13250_{5 / 2}-49121_{7 / 2}^{\circ}$ \\
\hline 2810.358 & 4 & & 35572.177 & $11576_{3 / 2}^{\circ}-47148_{3 / 2}$ & 2786.684 & 8 & 75 & 35874.362 & $15349_{11 / 2}^{\circ}-51224_{9 / 2}$ \\
\hline 2809.460 & 1 & & 35583.547 & $15324_{1 / 2}^{\circ}-50907_{3 / 2}$ & 2786.239 & 4 & 50 & 35880.091 & $15144_{3 / 2}^{\circ}-51024_{3 / 2}$ \\
\hline 2808.996 & $200 \mathrm{~b}$ & 200 & 35589.424 & $9061_{5 / 2}-44650_{7 / 2}^{\circ}$ & 2785.603 & 4 & $20 \mathrm{~b}$ & 35888.282 & $9238_{9 / 2}^{\circ}-45126_{9 / 2}$ \\
\hline 2808.894 & 8 & & 35590.716 & $15144_{3 / 2}^{\circ}-50735_{3 / 2}$ & 2785.544 & 5 & $15 b$ & 35889.042 & $13468_{9 / 2}^{\circ}-49357_{11 / 2}$ \\
\hline 2808.864 & 25 & 20 & 35591.096 & $10673_{5 / 2}^{\circ}-46264_{3 / 2}$ & 2784.975 & 15 & 75 & 35896.375 & $7331_{5 / 2}^{\circ}-43227_{5 / 2}$ \\
\hline 2807.827 & 200 & 200 & 35604.240 & $9585_{5 / 2}^{\circ}-45189_{5 / 2}$ & 2784.060 & 8 & 75 & 35908.172 & $10673_{5 / 2}^{\circ}-46581_{5 / 2}$ \\
\hline 2807.715 & 100 & 100 & 35605.661 & $9202_{7 / 2}^{\circ}-44807_{7 / 2}$ & 2783.924 & 5 & 10 & 35909.926 & $15453_{7 / 2}^{\circ}-51362_{5 / 2}$ \\
\hline 2807.048 & 10 & 50 & 35614.121 & $17121_{3 / 2}^{\circ}-52735_{3 / 2}$ & 2783.662 & 0 & 15 & 35913.306 & $7331_{5 / 2}^{\circ}-43244_{3 / 2}$ \\
\hline 2806.879 & 10 & 25 & 35616.265 & $14790_{7 / 2}^{\circ}-50407_{7 / 2}$ & 2783.489 & $10 \mathrm{~b}$ & 75 & 35915.537 & $7828_{1 / 2}-43744_{i / 2}^{\circ}$ \\
\hline 2805.721 & 4 & & 35630.964 & $18214_{3 / 2}^{\circ}-53845_{5 / 2}$ & 2783.053 & 50 & 75 & 35921.164 & $4490_{5 / 2}^{\circ}-40411_{7 / 2}$ \\
\hline 2804.652 & 10 & 3 & 35644.544 & $10572_{9 / 2}^{\circ}-46216_{11 / 2}$ & 2782.824 & 0 & 20 & 35924.119 & $9202_{7 / 2}^{\circ}-45126_{9 / 2}$ \\
\hline 2804.580 & 10 & 3 & 35645.459 & $6691_{3 / 2}^{\circ}-42336_{5 / 2}$ & 2782.364 & 4 & 15 & 35930.058 & $10673_{5 / 2}^{\circ}-46603_{5 / 2}$ \\
\hline 2803.760 & 2 & & 35655.883 & $16906_{\tau / 2}^{\circ}-52562_{7 / 2}$ & 2781.353 & 2 & 8 & 35943.118 & $15710_{3 / 2}^{\circ}-51653_{1 / 2}$ \\
\hline 2803.376 & $50 \mathrm{~b}$ & 50 & 35660.767 & $9238_{9 / 2}^{\circ}-44898_{7 / 2}$ & 2780.694 & $15 b$ & 75 & 35951.636 & $13406_{13 / 2}^{\circ}-49357_{11 / 2}$ \\
\hline 2802.278 & 4.0 & 20 & 35674.739 & $9720_{7 / 2}^{\circ}-45395_{7 / 2}$ & 2780.410 & 3 & 75 & 35955.308 & $20989_{9 / 2}^{\circ}-56945_{11 / 2}^{\circ}$ \\
\hline \multirow[t]{2}{*}{2801.938} & 50 & 25 & 35679.068 & $10673_{5 / 2}^{\circ}-46352_{7 / 2}$ & 2779.671 & $2 \mathrm{~b}$ & $10 \mathrm{~b}$ & 35964.867 & $12488_{9 / 2}^{\circ}-48453_{7 / 2}$ \\
\hline & & & & $14790_{7 / 2}^{\circ}-504.70_{9 / 2}$ & 2779.646 & 1 & $10 \mathrm{~b}$ & 35965.190 & $15710_{3 / 2}^{\circ}-51676_{3 / 2}^{\circ}$ \\
\hline 2801.766 & 8 & & 35681.258 & $10572_{9 / 2}^{\circ}-46253_{9 / 2}^{\circ}$ & 2779.476 & 2 & 10 & 35967.389 & $12485_{7 / 2}^{\circ}-48453_{7 / 2}$ \\
\hline 2800.569 & 200 & 100 & 35696.508 & $9202_{7 / 2}^{\circ}-44898_{7 / 2}$ & 2778.704 & 10 & 75 & 35977.382 & $9711_{7 / 2}-45689 \frac{2}{7 / 2}$ \\
\hline 2800.249 & 10 & 15 & 35700.587 & $15324_{1 / 2}^{\circ}-51024_{3 / 2}$ & 2778.426 & 1 & 5 & 35980.981 & $12472_{5 / 2}^{\circ}-48453_{7 / 2}$ \\
\hline 2799.334 & 0 & 8 & 35712.256 & $10673_{5 / 2}^{\circ}-46385_{7 / 2}$ & 2778.401 & 1 & 5 & 35981.305 & $15242_{9 / 2}^{\circ}-51224_{9 / 2}$ \\
\hline 2799.110 & 50 & 75 & 35715.114 & $4146_{7 / 2}-39861_{5 / 2}^{\circ}$ & 2778.055 & $5 b$ & 15 & 35985.786 & $14484_{11 / 2}^{\circ}-50470_{9 / 2}$ \\
\hline 2798.921 & 3 & 10 & 35717.525 & $8378_{\tau / 2}^{\circ}-44096_{9 / 2}$ & 2778.020 & 10 & 25 & 35986.240 & $1859_{3 / 2}-37846_{5 / 2}^{\circ}$ \\
\hline 2798.866 & 2 & 10 & 35718.227 & $13406_{13 / 2}^{\circ}-49124_{11 / 2}$ & 2777.929 & 3 & 50 & 35987.4 .19 & $9202_{7 / 2}^{\circ}-45189_{5 / 2}$ \\
\hline 2798.661 & 50 & 50 & 35720.843 & $9585_{5 / 2}^{\circ}-45306_{3 / 2}$ & 2777.807 & 0 & 4 & 35988.999 & $10379_{9 / 2}-46368_{9 / 2}^{\circ}$ \\
\hline 2798.499 & 5 & 15 & 35722.911 & $10673_{5 / 2}^{\circ}-46395_{3 / 2}$ & 2776.470 & 0 & 4 & 36006.328 & $12485_{7 / 2}^{\circ}-48492_{5 / 2}$ \\
\hline 2798.263 & 20 & 100 & 35725.924 & $8018_{3 / 2}-43744_{1 / 2}^{\circ}$ & 2776.196 & 3 & 5 & 36009.882 & $8378_{7 / 2}^{\circ}-44388_{5 / 2}$ \\
\hline 2797.736 & 200 & 150 & 35732.653 & $4490_{5 / 2}^{\circ}-40222_{3 / 2}$ & 2775.459 & 0 & 75 & 36019.443 & $13818_{7 / 2}^{\circ}-49837_{9 / 2}$ \\
\hline 2797.020 & 50 & 150 & 35741.800 & $1859_{3 / 2}-37601_{3 / 2}^{\circ}$ & 2774.840 & 10 & 75 & 36027.478 & $10189_{11 / 2}^{\circ}-46216_{11 / 2}$ \\
\hline 2796.509 & 20 & 25 & 35748.330 & $4113_{5 / 2}-39861_{5 / 2}^{\circ}$ & 2774.498 & 20 & 8 & 36031.919 & $6168_{7 / 2}^{\circ}-42200_{9 / 2}$ \\
\hline 2795.528 & 5 & 25 & 35760.874 & $9061_{5 / 2}-44821_{5 / 2}^{\circ}$ & 2774.411 & 1 & 5 & 36033.048 & $10673_{5 / 2}^{\circ}-46706_{7 / 2}$ \\
\hline 2795.195 & 10 & 25 & 35765.134 & $7331_{5 / 2}^{\circ}-43096_{5 / 2}$ & 2774.065 & 50 & 50 & 36037.54 & $4146_{7 / 2}-40184_{7 / 2}^{\circ}$ \\
\hline 2794.716 & 0 & 3 & 35771.264 & $15453_{7 / 2}^{\circ}-51224_{9 / 2}$ & 2773.951 & 5 & 75 & 36039.024 & $13250_{5 / 2}-49289_{5 / 2}^{\circ}$ \\
\hline 2794.255 & 25 & 100 & 35777.165 & $9711_{\pi / 2}-45489_{9 / 2}^{\circ}$ & 2773.523 & 3 & 8 & 36044.585 & $8605_{5 / 2}-44650_{7 / 2}^{\circ}$ \\
\hline 2794.012 & 4 & 50 & 35780.277 & $10572_{9 / 2}^{\circ}-46352_{7 / 2}^{\circ}$ & 2773.022 & 10 & 25 & 36051.096 & $6700_{9 / 2}^{\circ}-42751_{7 / 2}$ \\
\hline 2793.567 & 4 & $15 p$ & 35785.976 & $11116_{7 / 2}^{0}-46902_{5 / 2}$ & 2772.808 & 1 & 4 & 36053.879 & $6168_{7 / 2}^{\circ}-42222_{7 / 2}$ \\
\hline 2793.432 & 1 & 8 & 35787.705 & $12902_{3 / 2}^{\circ}-48689_{3 / 2}$ & 2772.741 & 2 & 8 & 36054.750 & $13818_{7 / 2}^{\circ}-49873_{5 / 2}$ \\
\hline 2792.674 & 2 & 8 & 35797.418 & $16033_{5 / 2}^{\circ}-51830_{\tau / 2}$ & & & & & $11116_{7 / 2}^{\circ}-47171_{9 / 2}$ \\
\hline 2791.670 & 3 & 10 & 35810.292 & $12488_{9 / 2}^{\circ}-48298_{7 / 2}$ & 2772.010 & 50 & 25 & 36064.257 & $10189_{11 / 2}^{\circ}-46253_{9 / 2}$ \\
\hline 2791.432 & $8 \mathrm{~b}$ & 75 & 35813.345 & $10572_{9 / 2}^{\circ}-46385_{\tau / 2}$ & 2771.515 & $50 \mathrm{~b}$ & $100 \mathrm{~b}$ & 36070.698 & $4113_{5 / 2}-40184_{7 / 2}^{\circ}$ \\
\hline 2791.007 & 50 & 75 & 35818.799 & $6700_{9 / 2}^{\circ}-42518_{7 / 2}$ & 2771.469 & $20 \mathrm{~b}$ & $100 \mathrm{~b}$ & 36071.297 & $8378_{7 / 2}^{\circ}-44450_{9 / 2}$ \\
\hline 2790.868 & 3 & 10 & 35820.582 & $9400_{5 / 2}-45221_{5 / 2}^{\circ}$ & 2770.815 & 75 & 100 & 36079.810 & $1521_{5 / 2}-37601_{3 / 2}^{\circ}$ \\
\hline 2790.420 & 5 & 50 & 35826.333 & $12472_{5 / 2}^{\circ}-48298_{7 / 2}$ & 2770.134 & 2 & 20 & 36088.680 & $20989_{9 / 2}^{\circ}-57078_{9 / 2}$ \\
\hline 2789.981 & 1 & 4 & 35831.970 & $16033_{5 / 2}^{\circ}-51865_{5 / 2}$ & 2768.841 & 50 & 150 & 36105.531 & $6213_{9 / 2}-42319_{9 / 2}^{\circ}$ \\
\hline 2789.729 & 0 & 5 & 35835.206 & $12485_{\pi / 2}^{\circ}-48320_{5 / 2}$ & 2768.106 & 0 & 3 & 36115.118 & $20120_{5 / 2}^{\circ}-56235_{3 / 2}$ \\
\hline
\end{tabular}


TABLE 3. Classified lines of Th II-Continued

\begin{tabular}{|c|c|c|c|c|c|c|c|c|c|}
\hline \multirow{2}{*}{$\begin{array}{c}\text { Wavelength } \\
\AA\end{array}$} & \multicolumn{2}{|c|}{ Intensity } & \multirow{2}{*}{$\begin{array}{c}\text { Wavenumber } \\
\mathrm{cm}^{-1}\end{array}$} & \multirow{2}{*}{ Classification } & \multirow{2}{*}{$\begin{array}{c}\text { Wavelength } \\
\AA\end{array}$} & \multicolumn{2}{|c|}{ Intensity } & \multirow{2}{*}{$\begin{array}{l}\text { Wavenumber } \\
\mathrm{cm}^{-1}\end{array}$} & \multirow{2}{*}{ Classification } \\
\hline & Lamp & Spark & & & & Lamp & Spark & & \\
\hline 2767.884 & 3 & 15 & 36118.014 & $14545_{5 / 2}^{\circ}-50663_{5 / 2}$ & 2738.816 & 15 & 20 & 36501.327 & $4490_{5 / 2}^{\circ}-40991_{3 / 2}$ \\
\hline 2767.669 & 0 & 41 & 36120.820 & $13406_{13 / 2}^{\circ}-49527_{13 / 2}$ & 2738.324 & 20 & 50 & 36507.885 & $4146_{7 / 2}-40654_{5 / 2}^{\circ}$ \\
\hline 2767.357 & 4 & 25 & 36124.892 & $8378_{7 / 2}^{\circ}-44503_{7 / 2}$ & 2738.224 & 1 & 10 & 36509.218 & $15144_{3 / 2}^{\circ}-51653_{1 / 2}$ \\
\hline 2766.637 & 3 & 5 & 36134.293 & $10572_{9 / 2}^{\circ}-46706_{7 / 2}$ & 2738.119 & 1 & 8 & 36510.618 & $17983_{5 / 2}^{\circ}-54493_{5 / 2}$ \\
\hline 2765.124 & 50 & 150 & 36154.064 & $4490_{5 / 2}^{\circ}-40644_{5 / 2}$ & 2737.422 & 15 & 75 & 36519.914 & $8378_{7 / 2}^{\circ}-44898_{7 / 2}^{\circ}$ \\
\hline 2764.640 & 20 & 150 & 36160.393 & $9061_{5 / 2}-45221_{5 / 2}^{\circ}$ & 2737.185 & 0 & 3 & 36523.076 & $18973_{7 / 2}^{\circ}-55496_{9 / 2}$ \\
\hline 2764.180 & 2 & $5 b$ & 36166.410 & $12902_{3 / 2}^{\circ}-49068_{5 / 2}$ & 2736.710 & 2 & 25 & 36529.415 & $16033_{5 / 2}^{\circ}-52562_{7 / 2}$ \\
\hline 2763.605 & $50 \mathrm{~b}$ & 100 & 36173.934 & $6244_{1 / 2}-42418_{3 / 2}^{\circ}$ & 2736.439 & 8 & 20 & 36533.032 & $9720_{7 / 2}^{\circ}-46253_{9 / 2}$ \\
\hline 2762.835 & 3 & 151 & 36184.016 & $9720_{7 / 2}^{\circ}-45904_{9 / 2}$ & 2736.177 & 4 & 5 & 36536.530 & $6691_{3 / 2}^{\circ}-43227_{5 / 2}$ \\
\hline 2762.402 & 8 & 25 & 36189.687 & $10189_{11 / 2}^{\circ}-46378_{13 / 2}$ & 2735.837 & 20 & 500 & 36541.070 & $4113_{5 / 2}-40654_{5 / 2}^{\circ}$ \\
\hline 2762.041 & 1 & 3 & 36194.417 & $14275_{9 / 2}^{\circ}-50470_{9 / 2}$ & 2735.197 & 1 & 10 & 36549.620 & $17460_{5 / 2}^{\circ}-54010_{7 / 2}$ \\
\hline 2761.021 & 2 & 8 & 36207.787 & $11116_{7 / 2}^{\circ}-47324_{5 / 2}^{\circ}$ & 2735.060 & 4 & 15 & 36551.451 & $12570_{7 / 2}-49121_{7 / 2}^{\circ}$ \\
\hline 2760.391 & 20 & 200 & 36216.051 & $8605_{5 / 2}-44821_{5 / 2}^{\circ}$ & 2734.907 & 3 & 4 & 36553.495 & $6691_{3 / 2}^{\circ}-43244_{3 / 2}$ \\
\hline 2759.720 & 3 & 15 & 36224.856 & $15710_{3 / 2}^{\circ}-51935_{5 / 2}^{\circ}$ & 2734.405 & 50 & 75 & 36560.206 & $4146_{7 / 2}-40706_{7 / 2}^{\circ}$ \\
\hline 2759.405 & 5 & 75 & 36228.991 & $15453_{7 / 2}^{\circ}-51681_{9 / 2}$ & 2733.529 & 3 & 15 & 36571.922 & $14790_{7 / 2}^{\circ}-51362_{5 / 2}$ \\
\hline 2758.958 & 15 & 15 & 36234.860 & $1521_{5 / 2}-37756_{7 / 2}^{\circ}$ & 2733.205 & 15 & 25 & 36576.257 、 & $1859_{3 / 2}-38436_{3 / 2}^{\circ}$ \\
\hline 2757.900 & 0 & 8 & 36248.760 & $17272_{9 / 2}^{\circ}-53520_{9 / 2}^{\circ}$ & 2732.808 & 50 & 50 & 36581.570 & $0_{3 / 2}-36581_{3 / 2}^{\circ}$ \\
\hline 2757.382 & 8 & 15 & 36255.569 & $6700_{9 / 2}^{\circ}-42955_{9 / 2}$ & 2732.702 & 15 & 50 & 36582.989 & $6168_{7 / 2}^{\circ}-42751_{7 / 2}$ \\
\hline 2756.855 & 2 & 8 & 36262.499 & $10673_{5 / 2}^{\circ}-46935_{3 / 2}$ & 2731.918 & 10 & 25 & 36593.487 & $4113_{5 / 2}-40706_{7 / 2}^{\circ}$ \\
\hline 2755.955 & 4 & 50 & 36274.341 & $16033_{5 / 2}^{\circ}-52307_{3 / 2}^{\circ}$ & 2730.308 & $8 \mathrm{~b}$ & $150 \mathrm{~b}$ & 36615.064 & $11116_{7 / 2}^{\circ}-47731_{9 / 2}^{\circ}$ \\
\hline 2755.561 & 0 & 3 & 36279.527 & $18214_{3 / 2}^{\circ}-54493_{5 / 2}$ & 2730.261 & $15 b$ & $150 \mathrm{~b}$ & 36615.694 & $8605_{5 / 2}-45221_{5 / 2}^{\circ}$ \\
\hline 2755.051 & 4 & 15 & 36286.243 & $7001_{3 / 2}-43287_{3 / 2}^{\circ}$ & 2729.327 & 15 & 150 & 36628.224 & $9061_{5 / 2}-45689_{\tau / 2}^{\circ}$ \\
\hline 2754.890 & 2 & 10 & 36288.363 & $9400_{5 / 2}-45689_{7 / 2}^{\circ}$ & 2729.053 & 4 & 5 & 36631.901 & $9720_{7 / 2}^{\circ}-46352_{7 / 2}$ \\
\hline 2754.801 & 1 & 10 & 36289.536 & $10572_{9 / 2}^{\circ}-46861_{11 / 2}$ & 2728.909 & 20 & 25 & 36633.834 & $1859_{3 / 2}-38493_{5 / 2}^{\circ}$ \\
\hline 2754.520 & 2 & $5 b$ & 36293.238 & $11576_{3 / 2}^{\circ}-47869_{3 / 2}$ & 2728.701 & 0 & $10 \mathrm{~b}$ & 36636.626 & $9061_{5 / 2}-45697_{3 / 2}^{\circ}$ \\
\hline 2754.252 & 4 & 15 & 36296.769 & $9400_{5 / 2}-45697_{3 / 2}^{\circ}$ & 2727.578 & 0 & 3 & 36651.709 & $13818_{7 / 2}^{\circ}-50470_{9 / 2}$ \\
\hline 2753.092 & 5 & 50 & 36312.061 & $12219_{3 / 2}-48532_{1 / 2}^{\circ}$ & 2727.231 & 5 & 20 & 36656.373 & $9711_{7 / 2}-46368_{9 / 2}^{\circ}$ \\
\hline 2752.166 & 75 & 100 & 36324.279 & $1521_{5 / 2}-37846_{5 / 2}^{\circ}$ & 2726.482 & 15 & 75 & 36666.442 & $9238_{9 / 2}^{\circ}-45904_{9 / 2}^{\circ}$ \\
\hline 2751.754 & 2 & 50 & 36329.717 & $15324_{1 / 2}^{\circ}-51653_{1 / 2}$ & 2726.027 & 2 & 10 & 36672.562 & $10189_{11 / 2}^{\circ}-46861_{11 / 2}$ \\
\hline 2751.572 & 4 & 3 & 36332.120 & $15349_{11 / 2}^{\circ}-51681_{9 / 2}$ & 2725.559 & 4 & 15 & 36678.858 & $9585_{5 / 2}^{\circ}-46264_{3 / 2}$ \\
\hline 2750.538 & 1 & 4 & 36345.777 & $12472_{5 / 2}^{\circ}-48817_{3 / 2}$ & 2724.881 & 50 & 200 & 36687.984 & $0_{3 / 2}-36687_{5 / 2}^{\circ}$ \\
\hline 2750.174 & 2 & 4 & 36350.587 & $6168_{7 / 2}^{\circ}-42518_{7 / 2}$ & 2723.821 & 0 & $10 \mathrm{~d}$ & 36702.261 & $9202_{\tau / 2}^{\circ}-45904_{9 / 2}^{\circ}$ \\
\hline 2749.713 & 8 & 100 & 36356.681 & $12488_{9 / 2}^{\circ}-48844_{9 / 2}$ & 2723.416 & 0 & 5 & 36707.719 & $18214_{3 / 2}^{\circ}-54922_{3 / 2}$ \\
\hline 2749.531 & 50 & 100 & 36359.088 & $4113_{5 / 2}-40472_{3 / 2}^{\circ}$ & 2723.317 & 4 & 75 & 36709.053 & $17460_{5 / 2}^{\circ}-54169_{7 / 2}$ \\
\hline 2749.355 & 25 & 25 & 36361.415 & $8460_{3 / 2}-44821_{5 / 2}^{\circ}$ & 2722.571 & 0 & 10 & 36719.111 & $12570_{\tau / 2}-49289_{\tilde{\tau} / 2}^{\circ}$ \\
\hline 2748.805 & 5 & $50 \mathrm{~b}$ & 36368.690 & $13468_{9 / 2}^{\circ}-49837_{9 / 2}$ & 2722.468 & 3 & 10 & 36720.500 & $15144_{3 / 2}^{\circ}-51865_{5 / 2}$ \\
\hline 2748.137 & 2 & 10 & 36377.530 & $15453_{\tau / 2}^{\circ}-51830_{7 / 2}$ & 2722.380 & $25 b$ & $50 \mathrm{~b}$ & 36721.687 & $1859_{3 / 2}-38581_{5 / 2}^{\circ}$ \\
\hline 2747.847 & 8 & 50 & 36381.369 & $7001_{3 / 2}-43382_{5 / 2}^{\circ}$ & & & & & $10189_{11 / 2}^{\circ}-46910_{13 / 2}$ \\
\hline 2747.586 & 3 & 50 & 36384.825 & $17460_{5 / 2}^{\circ}-53845_{5 / 2}$ & 2722.313 & 5 & 50 & 36722.591 & $14545_{5 / 2}^{\circ}-51268_{7 / 2}$ \\
\hline 2747.156 & 75 & 100 & 36390.520 & $0_{3 / 2}-36390_{3 / 2}^{\circ}$ & 2722.216 & 0 & $20 \mathrm{~b}$ & 36723.899 & $17121_{3 / 2}^{\circ}-53845_{5 / 2}$ \\
\hline 2745.527 & 25 & 25 & 36412.110 & $15453_{7 / 2}^{\circ}-51865_{5 / 2}$ & 2721.691 & 20 & $200 b$ & 36730.982 & $6213_{9 / 2}-42944_{7 / 2}^{\circ}$ \\
\hline 2744.247 & 3 & 4 & 36429.093 & $8378_{7 / 2}^{\circ}-44807_{7 / 2}$ & 2721.172 & 1 & 5 & 36737.988 & $17272_{9 / 2}^{\circ}-54010_{7 / 2}$ \\
\hline 2743.499 & 1 & 5 & 36439.025 & $15242_{9 / 2}^{\circ}-51681$ & 2721.022 & 0 & 4 & 36740.013 & $14484_{11 / 2}^{\circ}-51224_{9 / 2}$ \\
\hline 2743.155 & $3 \mathrm{~b}$ & 10 & 36443.594 & $9711_{\tau / 2}-46155_{5 / 2}^{\circ}$ & 2720.822 & 0 & 15 & 36742.713 & $7001_{3 / 2}-43744_{1 / 2}^{\circ}$ \\
\hline 2743.063 & 50 & 50 & 36444.816 & $0_{3 / 2}-36444_{3 / 2}^{\circ}$ & 2720.687 & 0 & 4 & 36744.536 & $11576_{3 / 2}^{\circ}-48320_{5 / 2}$ \\
\hline 2740.712 & 1 & 4 & 36476.077 & $7331_{5 / 2}^{\circ}-43807_{3 / 2}$ & 2720.627 & 0 & 10 & 36745.347 & $22642_{9 / 2}^{\circ}-59387_{7 / 2}$ \\
\hline 2740.469 & 3 & 50 & 36479.311 & $14545_{5 / 2}^{\circ}-51024_{3 / 2}$ & 2720.471 & 5 & 15 & 36747.454 & $8378_{7 / 2}^{\circ}-45126_{9 / 2}$ \\
\hline 2740.218 & 1 & 15 & 36482.653 & $15453_{\pi / 2}^{\circ}-51935_{5 / 2}$ & 2719.929 & 15 & 75 & 36754.776 & $11116_{7 / 2}^{\circ}-47871_{7 / 2}$ \\
\hline 2739.545 & 0 & 50 & 36491.614 & $13468_{9 / 2}^{\circ}-49960_{7 / 2}$ & 2719.449 & 4 & 25 & 36761.263 & $8460_{3 / 2}-45221_{5 / 2}^{\circ}$ \\
\hline 2739.133 & 2 & 4 & 36497.103 & $9238_{9 / 2}^{\circ}-45735_{11 / 2}$ & 2717.251 & 3 & 15 & 36790.998 & $15144_{3 / 2}^{\circ}-51935_{5 / 2}$ \\
\hline
\end{tabular}


TABLE 3. Classified lines of Th II-Continued

\begin{tabular}{|c|c|c|c|c|c|c|c|c|c|}
\hline \multirow{2}{*}{$\begin{array}{c}\text { Wavelength } \\
\AA\end{array}$} & \multicolumn{2}{|c|}{ Intensity } & \multirow{2}{*}{$\begin{array}{c}\text { Wavenumber } \\
\mathrm{cm}^{-1}\end{array}$} & \multirow{2}{*}{ Classification } & \multirow{2}{*}{$\begin{array}{c}\text { Wavelength } \\
\AA\end{array}$} & \multicolumn{2}{|c|}{ Intensity } & \multirow{2}{*}{$\begin{array}{c}\text { Wavenumber } \\
\mathrm{cm}^{-1}\end{array}$} & \multirow{2}{*}{ Classification } \\
\hline & Lamp & Spark & & & & Lamp & Spark & & \\
\hline 2716.578 & 5 & 25 & 36800.112 & $9585_{5 / 2}^{\circ}-46385_{7 / 2}$ & 2690.311 & 25 & 20 & 37159.392 & $10572_{9 / 2}^{\circ}-47731_{9 / 2}$ \\
\hline \multirow[t]{2}{*}{2716.328} & 50 & 100 & 36803.499 & $19880_{9 / 2}-56683_{7 / 2}^{\circ}$ & 2690.102 & 1 & $15 b$ & 37162.279 & $13468_{9 / 2}^{\circ}-50631_{11 / 2}$ \\
\hline & & & & $8018_{3 / 2}-44821_{5 / 2}^{\circ}$ & 2690.064 & 3 & $15 b$ & 37162.804 & $15144_{3 / 2}^{\circ}-52307_{3 / 2}^{\circ}$ \\
\hline 2715.156 & 0 & $10 \mathrm{~b}$ & 36819.384 & $15453_{7 / 2}^{\circ}-52272_{7 / 2}^{\alpha}$ & 2689.371 & 2 & 5 & 37172.379 & $7331_{5 / 2}^{\circ}-44503_{7 / 2}^{\circ}$ \\
\hline 2715.082 & 8 & $100 \mathrm{~b}$ & 36820.387 & $15349_{11 / 2}^{\circ}-52170_{11 / 2}$ & 2688.667 & 3 & 15 & 37182.112 & $9720_{7 / 2}^{\circ}-46902_{5 / 2}$ \\
\hline 2713.768 & 0 & $25 b$ & 36838.215 & $4490_{5 / 2}^{\circ}-41328_{5 / 2}$ & 2688.582 & 2 & 3 & 37183.287 & $9202_{7 / 2}^{\circ}-46385_{7 / 2}$ \\
\hline 2713.243 & 4 & 50 & 36845.342 & $13818_{7 / 2}^{\circ}-50663_{5 / 2}$ & 2688.337 & 8 & 15 & 37186.676 & $4490_{5 / 2}^{\circ}-41676_{3 / 2}$ \\
\hline 2712.084 & 3 & 20 & 36861.087 & $9720_{7 / 2}^{\circ}-46581_{5 / 2}^{\circ}$ & 2687.903 & 3 & 20 & 37192.679 & $22106_{5 / 2}-59299_{5 / 2}^{\circ}$ \\
\hline 2711.448 & $15 \mathrm{~b}$ & 75 & 36869.733 & $12488_{9 / 2}^{\circ}-49357_{11 / 2}$ & 2687.533 & $3 b$ & $8 b$ & 37197.800 & $14484_{11 / 2}^{o}-51681_{9 / 2}$ \\
\hline 2711.130 & 0 & $5 s$ & 36874.057 & $22513_{\pi / 2}^{\circ}-59387_{7 / 2}$ & 2687.500 & 5 & $10 \mathrm{~b}$ & 37198.257 & $10673_{5 / 2}^{\circ}-47871_{7 / 2}$ \\
\hline 2710.474 & 5 & 50 & 36882.981 & $9720_{7 / 2}^{\circ}-46603_{5 / 2}$ & 2687.125 & $50 \mathrm{~b}$ & 100 & 37203.447 & $8018_{3 / 2}-45221_{5 / 2}^{\circ}$ \\
\hline 2710.034 & 2 & 25 & 36888.969 & $12488_{9 / 2}^{\circ}-49377_{7 / 2}$ & 2685.586 & 3 & 15 & 37224.766 & $13406_{13 / 2}^{\circ}-50631_{11 / 2}$ \\
\hline 2709.934 & 2 & 8 & 36890.331 & $11116_{7 / 2}^{\circ}-48006_{9 / 2}$ & 2685.024 & 0 & $8 b$ & 37232.557 & $8605_{5 / 2}-45838_{3 / 2}^{\circ}$ \\
\hline 2709.843 & 3 & 25 & 36891.569 & $12485_{7 / 2}^{\circ}-49377_{7 / 2}$ & 2684.669 & 5 & 8 & 37237.480 & $8460_{3 / 2}-45697_{3 / 2}^{\circ}$ \\
\hline 2709.407 & 2 & 251 & 36897.505 & $17272_{9 / 2}^{\circ}-54169_{7 / 2}$ & 2684.288 & 50 & 100 & 37242.765 & $1521_{5 / 2}-38764_{7 / 2}^{\circ}$ \\
\hline 2708.176 & 50 & 100 & 36914.276 & $1521_{5 / 2}-38436_{3 / 2}^{\circ}$ & 2682.018 & 0 & 5 & 37274.285 & $6691_{3 / 2}^{\circ}-43965_{1 / 2}$ \\
\hline 2708.071 & 5 & $25 b$ & 36915.707 & $11576_{3 / 2}^{\circ}-48492_{5 / 2}$ & 2680.210 & 3 & 10 & 37299.428 & $10572_{9 / 2}^{\circ}-47871_{7 / 2}$ \\
\hline 2707.525 & 8 & 10 & 36923.152 & $14101_{1 / 2}^{\circ}-51024_{3 / 2}$ & 2678.934 & 15 & 50 & 37317.193 & $9585_{5 / 2}^{\circ}-46902_{5 / 2}$ \\
\hline 2706.408 & 2 & 25 & 36938.390 & $13468_{9 / 2}^{\circ}-50407_{7 / 2}$ & 2678.752 & 0 & 20 & 37319.728 & $15242_{9 / 2}^{\circ}-52562_{7 / 2}^{\circ}$ \\
\hline 2704.489 & 3 & 50 & 36964.598 & $11725_{1 / 2}^{\circ}-48689_{3 / 2}$ & 2677.544 & 2 & 10 & 37336.564 & $11116_{7 / 2}^{\circ}-48453_{7 / 2}$ \\
\hline 2703.957 & 75 & 200 & 36971.871 & $1521_{5 / 2}-38493_{5 / 2}^{\circ}$ & 2676.623 & 3 & 25 & 37349.411 & $12488_{9 / 2}^{\circ}-49837_{9 / 2}$ \\
\hline 2703.189 & 3 & 15 & 36982.374 & $10189_{11 / 2}^{\circ}-47171_{9 / 2}$ & 2676.559 & 3 & 25 & 37350.304 & $9585_{5 / 2}^{\circ}-46935_{3 / 2}$ \\
\hline 2702.922 & 5 & 25 & 36986.027 & $9720_{7 / 2}^{\circ}-46706_{7 / 2}$ & 2676.440 & 3 & $25 \mathrm{~s}$ & 37351.964 & $12485_{7 / 2}^{\circ}-49837_{9 / 2}$ \\
\hline 2702.852 & 2 & 15 & 36986.985 & $19248_{5 / 2}^{\circ}-56235_{3 / 2}$ & 2675.670 & 5 & 50 & 37362.713 & $9061_{5 / 2}-46423_{3 / 2}^{\circ}$ \\
\hline 2702.202 & 2 & $4 b$ & 36995.882 & $9585_{5 / 2}^{\circ}-46581_{5 / 2}$ & 2674.988 & 0 & $10 \mathrm{~d}$ & 37372.238 & $17121_{3 / 2}^{\circ}-54493_{5 / 2}$ \\
\hline 2702.052 & 4 & 5 & 36997.935 & $4490_{5 / 2}^{\circ}-41488_{7 / 2}$ & 2674.580 & 0 & 5 & 37377.939 & $8460_{3 / 2}-45838_{3 / 2}^{\circ}$ \\
\hline 2701.818 & 8 & 75 & 37001.139 & $13468_{9 / 2}^{\circ}-50470_{9 / 2}$ & 2673.281 & 2 & 4 & 37396.100 & $6700_{9 / 2}^{\circ}-44096_{9 / 2}$ \\
\hline 2700.602 & 4 & 15 & 37017.799 & $9585_{5 / 2}^{0}-46603_{5 / 2}$ & 2672.945 & 50 & 40 & 37400.80 & $9202_{7 / 2}^{\circ}-46603_{5 / 2}$ \\
\hline 2700.080 & 0 & 100 & 37024.955 & $15710_{3 / 2}^{\circ}-52735_{3 / 2}^{\circ}$ & 2672.171 & 4 & 5 & 37411.634 & $15324_{i / 2}^{o}-52735_{3 / 2}$ \\
\hline 2699.012 & 3 & 50 & 37039.605 & $14790_{7 / 2}^{\circ}-51830_{7 / 2}$ & 2671.469 & 4 & 50 & 37421.464 & $8378_{7 / 2}^{\circ}-45800_{5 / 2}^{\circ}$ \\
\hline 2698.730 & $15 b$ & 75 & 37043.475 & $6244_{1 / 2}-43287_{3 / 2}^{\circ}$ & 2669.695 & 3 & 5 & 37446.329 & $4490_{5 / 2}^{\circ}-41936_{3 / 2}$ \\
\hline 2698.169 & 5 & $15 \mathrm{~s}$ & 37051.177 & $9202_{7 / 2}^{\circ}-46253_{9 / 2}$ & 2669.447 & 3 & 25 & 37449.808 & $13818_{7 / 2}^{\circ}-51268_{7 / 2}$ \\
\hline 2697.720 & 3 & 8 & 37057.343 & $7331_{5 / 2}^{\circ}-44388_{5 / 2}$ & 2667.544 & 8 & 15 & 37476.522 & $7331_{5 / 2}^{\circ}-44807_{7 / 2}$ \\
\hline 2697.546 & 25 & 75 & 37059.733 & $1521_{5 / 2}-38581_{5 / 2}^{\circ}$ & 2667.504 & 2 & 4 & 37477.084 & $6700_{9 / 2}^{\circ}-44177_{11 / 2}^{\circ}$ \\
\hline 2696.827 & 8 & 75 & 37069.613 & $12219_{3 / 2}-49289_{5 / 2}^{\circ}$ & 2667.193 & 0 & 5 & 37481.454 & $14790_{7 / 2}^{\circ}-52272_{7 / 2}$ \\
\hline 2696.174 & 10 & 25 & 37078.591 & $6168_{7 / 2}^{\circ}-43246_{7 / 2}$ & 2666.412 & 2 & 5 & 37492.432 & $11576_{3 / 2}^{\circ}-49068_{5 / 2}$ \\
\hline 2695.988 & 2 & 3 & 37081.149 & $6691_{3 / 2}^{\circ}-43772_{5 / 2}$ & 2665.884 & 5 & 50 & 37499.857 & $6244_{1 / 2}-43744_{1 / 2}^{\circ}$ \\
\hline 2695.810 & 25 & 75 & 37083.597 & $8605_{\pi / 2}-45689_{\tau / 2}^{\circ}$ & 2664.051 & 5 & $15 b$ & 37525.657 & $8378_{7 / 2}^{\circ}-45904_{9 / 2}$ \\
\hline 2695.553 & 50 & 150 & 37087.132 & $10379_{9 / 2}-47466_{11 / 2}^{0}$ & 2662.861 & 20 & 75 & 37542.426 & $10189_{11 / 2}^{\circ}-47731_{9 / 2}$ \\
\hline 2695.203 & 50 & 100 & 37091.948 & $8605_{5 / 2}-45697_{3 / 2}^{\circ}$ & 2662.343 & 10 & 75 & 37549.730 & $8605_{5 / 2}-46155_{5 / 2}^{\circ}$ \\
\hline 2695.017 & 8 & 50 & 37094.508 & $9061_{5 / 2}-46155_{5 / 2}^{\circ}$ & 2662.171 & 1 & 8 & 37552.156 & $14101_{1 / 2}^{\circ}-51653_{1 / 2}$ \\
\hline 2694.341 & 5 & 15 & 37103.814 & $6700_{9 / 2}^{\circ}-43803_{7 / 2}$ & 2661.969 & 0 & 5 & 37555.005 & $14275_{9 / 2}^{\circ}-51830_{7 / 2}^{\circ}$ \\
\hline 2693.959 & 30 & 30 & 37109.07 & $6700_{9 / 2}^{\circ}-43809_{9 / 2}^{\circ}$ & 2661.724 & 0 & 4 & 37558.462 & $7331_{5 / 2}^{\circ}-44889_{3 / 2}^{\circ}$ \\
\hline 2693.628 & 2 & $100 \mathrm{~b}$ & 37113.635 & $11576_{3 / 2}^{\circ}-48689_{3 / 2}$ & 2661.386 & 15 & 75 & 37563.232 & $9585_{5 / 2}^{\circ}-47148_{3 / 2}$ \\
\hline 2693.099 & 3 & 8 & 37120.925 & $9585_{5 / 2}^{\circ}-46706_{\tau / 2}$ & 2661.090 & 1 & 25 & 37567.410 & $7331_{5 / 2}^{\circ}-44898_{7 / 2}$ \\
\hline 2692.416 & 75 & 200 & 37130.341 & $0_{3 / 2}-37130_{1 / 2}^{\circ}$ & 2660.965 & 1 & 8 & 37569.174 & $15349_{11 / 2}^{\circ}-52918_{13 / 2}$ \\
\hline 2691.513 & 0 & 81 & 37142.798 & $19248_{5 / 2}^{\circ}-56391_{5 / 2}$ & 2660.680 & 0 & 4 & 37573.198 & $17272_{9 / 2}^{\circ}-54845_{9 / 2}$ \\
\hline 2691.374 & $8 \mathrm{~b}$ & 15 & 37144.716 & $14790_{\pi / 2}^{\circ}-51935_{5 / 2}^{\circ}$ & 2660.596 & 15 & 25 & 37574.384 & $14101_{1 / 2}^{o}-51676_{3 / 2}$ \\
\hline 2691.172 & 10 & 75 & 37147.504 & $9238_{9 / 2}^{\circ}-46385_{7 / 2}$ & 2659.417 & 1 & 5 & 37591.041 & $15144_{3 / 2}^{\circ}-52735_{3 / 2}$ \\
\hline 2690.994 & $8 \mathrm{~b}$ & 20 & 37149.961 & $9202_{7 / 2}^{\circ}-46352_{7 / 2}$ & 2658.664 & 25 & 75 & 37601.687 & $0_{3 / 2}-37601_{3 / 2}^{\circ}$ \\
\hline
\end{tabular}


TABLE 3. Classified lines of Th II-Continued

\begin{tabular}{|c|c|c|c|c|c|c|c|c|c|}
\hline \multirow{2}{*}{$\begin{array}{c}\text { Wavelength } \\
\AA \\
\end{array}$} & \multicolumn{2}{|c|}{ Intensity } & \multirow{2}{*}{$\begin{array}{c}\text { Wavenumber } \\
\mathrm{cm}^{-1}\end{array}$} & \multirow{2}{*}{ Classification } & \multirow{2}{*}{$\begin{array}{c}\text { Wavelength } \\
\AA\end{array}$} & \multicolumn{2}{|c|}{ Intensity } & \multirow{2}{*}{$\begin{array}{c}\text { Wavenumber } \\
\mathrm{cm}^{-1}\end{array}$} & \multirow{2}{*}{ Classification } \\
\hline & Lamp & Spark & & & & Lamp & Spark & & \\
\hline \multirow[t]{2}{*}{2658.490} & 4 & $10 \mathrm{~b}$ & 37604.149 & $9720_{7 / 2}^{\circ}-47324_{5 / 2}$ & 2629.620 & 3 & 50 & 38016.971 & $14545_{5 / 2}^{\circ}-52562_{7 / 2}$ \\
\hline & & & & $6168_{7 / 2}^{\circ}-43772_{5 / 2}$ & 2629.365 & 0 & 3 & 38020.658 & $18214_{3 / 2}^{\circ}-56235_{3 / 2}$ \\
\hline 2658.216 & 8 & 15 & 37608.024 & $0_{3 / 2}-37607_{1 / 2}^{\circ}$ & 2628.918 & 0 & 8 & 38027.122 & $6700_{9 / 2}^{\circ}-44727_{11 / 2}$ \\
\hline 2656.262 & 4 & 8 & 37635.688 & $6168_{7 / 2}^{\circ}-43803_{7 / 2}$ & 2628.811 & 20 & 100 & 38028.670 & $4490_{5 / 2}^{\circ}-42518_{7 / 2}$ \\
\hline 2655.887 & 3 & 20 & 37641.002 & $6168_{7 / 2}^{\circ}-43809_{9 / 2}$ & 2627.557 & 2 & 50 & 38046.818 & $13818_{7 / 2}^{\circ}-51865_{5 / 2}$ \\
\hline 2655.408 & 0 & 10 & 37647.791 & $10673_{5 / 2}^{\circ}-48320_{5 / 2}$ & 2626.394 & 15 & 25 & 38063.665 & $7331_{5 / 2}^{\circ}-45395_{7 / 2}$ \\
\hline 2653.163 & 2 & 25 & 37679.645 & $16818_{7 / 2}-54497_{7 / 2}^{\circ}$ & 2626.098 & 0 & 20 & 38067.955 & $15453_{7 / 2}^{\circ}-53520_{9 / 2}$ \\
\hline 2652.065 & 15 & 50 & 37695.244 & $8460_{3 / 2}-46155_{5 / 2}^{\circ}$ & 2625.737 & 25 & 100 & 38073.188 & $4146_{7 / 2}-42219_{5 / 2}^{\circ}$ \\
\hline 2651.907 & 4 & 3 & 37697.490 & $6691_{3 / 2}^{\circ}-44388_{5 / 2}$ & 2624.582 & 0 & $3 b$ & 38089.942 & $21297_{5 / 2}^{\circ}-59387_{7 / 2}$ \\
\hline 2651.703 & 2 & 20 & 37700.390 & $9202_{7 / 2}^{\circ}-46902_{5 / 2}$ & 2624.033 & 2 & 3 & 38097.911 & $6691_{3 / 2}^{\circ}-44789_{1 / 2}$ \\
\hline 2651.402 & 1 & 4 & 37704.670 & $21682_{7 / 2}^{\circ}-59387_{7 / 2}$ & 2623.448 & 25 & 100 & 38106.406 & $4113_{5 / 2}-42219_{5 / 2}^{\circ}$ \\
\hline 2650.583 & 25 & 200 & 37716.319 & $0_{3 / 2}-37716_{1 / 2}^{\circ}$ & 2622.686 & 10 & 8 & 38117.477 & $13818_{7 / 2}^{\circ}-51935_{5 / 2}$ \\
\hline 2649.866 & 15 & 100 & 37726.524 & $10572_{9 / 2}^{\circ}-48298_{7 / 2}$ & 2622.361 & 4 & 50 & 38122.201 & $9202_{7 / 2}^{\circ}-47324_{5 / 2}$ \\
\hline 2649.736 & 1 & 5 & 37728.375 & $1111_{7 / 2}^{\circ}-48844_{9 / 2}$ & 2621.506 & 0 & 10 & 38134.633 & $15710_{3 / 2}^{\circ}-53845_{5 / 2}$ \\
\hline 2649.477 & 10 & 25 & 37732.063 & $4490_{5 / 2}^{\circ}-42222_{\pi / 2}$ & 2621.376 & 2 & 50 & 38136.524 & $16033_{5 / 2}^{\circ}-54169_{7 / 2}$ \\
\hline 2648.994 & 1 & 8 & 37738.942 & $9585_{5 / 2}^{\circ}-47324_{5 / 2}$ & 2620.942 & 2 & 15 & 38142.839 & $12488_{9 / 2}^{\circ}-50631_{11 / 2}$ \\
\hline 2648.210 & 3 & 20 & 37750.114 & $6700_{9 / 2}^{\circ}-44450_{9 / 2}^{\circ}$ & 2620.375 & 1 & 8 & 38151.092 & $9720_{7 / 2}^{\circ}-47871_{\pi / 2}$ \\
\hline 2647.844 & 0 & 41 & 37755.332 & $13468_{9 / 2}^{\circ}-51224_{9 / 2}^{\circ}$ & 2619.008 & 10 & 75 & 38171.004 & $15349_{11 / 2}^{\circ}-53520_{9 / 2}$ \\
\hline 2647.507 & 2 & $15 b$ & 37760.138 & $11725_{1 / 2}^{\circ}-49485_{1 / 2}^{\circ}$ & 2618.912 & $25 b$ & 100 & 38172.403 & $4146_{7 / 2}-42319_{9 / 2}^{\circ}$ \\
\hline 2647.423 & 4 & 8 & 37761.335 & $12902_{3 / 2}^{\circ}-50663_{5 / 2}$ & 2618.625 & 0 & 3 & 38176.586 & $18214_{3 / 2}^{\circ}-56391_{5 / 2}^{\circ}$ \\
\hline 2647.382 & 2 & 15 & 37761.920 & $14545_{5 / 2}^{\circ}-52307_{3 / 2}$ & 2617.114 & 20 & 15 & 38198.626 & $6700_{9 / 2}^{\circ}-44898_{7 / 2}$ \\
\hline 2646.543 & 1 & 3 & 37773.891 & $17722_{9 / 2}^{\circ}-55496_{9 / 2}$ & 2616.850 & 10 & 15 & 38202.480 & $8378_{7 / 2}^{\circ}-46581_{5 / 2}$ \\
\hline 2644.775 & 2 & 15 & 37799.141 & $13468_{9 / 2}^{\circ}-51268_{7 / 2}$ & 2616.631 & 1 & 10 & 38205.677 & $14101_{1 / 2}^{\circ}-52307_{3 / 2}$ \\
\hline 2644.681 & 2 & 25 & 37800.484 & $17121_{3 / 2}^{\circ}-54922_{3 / 2}^{\circ}$ & 2615.641 & 2 & 5 & 38220.137 & $7001_{3 / 2}-45221_{5 / 2}^{\circ}$ \\
\hline 2644.460 & 2 & 5 & 37803.643 & $6700_{9 / 2}^{\circ}-44503_{7 / 2}^{\circ}$ & 2615.345 & 3 & 50 & 38224.462 & $17272_{9 / 2}^{\circ}-55496_{9 / 2}^{\circ}$ \\
\hline 2643.461 & 3 & 20 & 37817.929 & $8605_{5 / 2}-46423_{3 / 2}^{\circ}$ & & & & & $8378_{7 / 2}^{\circ}-46603_{5 / 2}^{\circ}$ \\
\hline 2643.385 & 0 & 5 & 37819.016 & $10673_{5 / 2}^{\circ}-48492_{5 / 2}$ & 2612.875 & 3 & 25 & 38260.594 & $11116_{7 / 2}^{\circ}-49377_{7 / 2}$ \\
\hline 2643.280 & 4 & 20 & 37820.518 & $7001_{3 / 2}-44821_{5 / 2}^{\circ}$ & 2612.688 & 2 & 10 & 38263.332 & $12472_{5 / 2}^{\circ}-50735_{3 / 2}$ \\
\hline 2642.401 & 3 & 15 & 37833.098 & $12902_{3 / 2}^{\circ}-50735_{3 / 2}$ & 2612.465 & 0 & 10 & 38266.598 & $10855_{7 / 2}-49121_{7 / 2}^{\circ}$ \\
\hline 2642.037 & 0 & 4 & 37838.311 & $11576_{3 / 2}^{\circ}-49414_{3 / 2}$ & 2612.031 & 0 & 4 & 38272.956 & $10572_{9 / 2}^{\circ}-48844_{9 / 2}^{\circ}$ \\
\hline 2641.488 & 75 & 150 & 37846.174 & $0_{3 / 2}-37846_{5 / 2}^{\circ}$ & 2611.688 & 4 & 50 & 38277.982 & $13250_{5 / 2}-51528_{3 / 2}^{\circ}$ \\
\hline 2640.424 & 2 & 5 & 37861.424 & $6691_{3 / 2}^{\circ}-44552_{5 / 2}$ & & & & & $15242_{9 / 2}^{\circ}-53520_{9 / 2}$ \\
\hline 2640.391 & 5 & 10 & 37861.897 & $4490_{5 / 2}^{\circ}-42352_{5 / 2}$ & 2611.420 & 2 & 5 & 38281.910 & $6168_{7 / 2}^{\circ}-44450_{9 / 2}^{\circ}$ \\
\hline 2640.269 & 10 & 25 & 37863.646 & $13818_{\tau / 2}^{\circ}-51681_{9 / 2}^{\circ}$ & 2611.263 & 2 & 8 & 38284.212 & $9585_{5 / 2}^{\circ}-47869_{3 / 2}$ \\
\hline 2639.879 & 4 & 50 & 37869.240 & $7828_{1 / 2}-45697_{3 / 2}^{\circ}$ & 2611.134 & $2 \mathrm{~b}$ & $10 \mathrm{~b}$ & 38286.103 & $9585_{5 / 2}^{\circ}-47871_{7 / 2}$ \\
\hline 2639.503 & 25 & 50 & 37874.634 & $1521_{5 / 2}-39396_{7 / 2}^{\circ}$ & 2610.412 & 1 & 3 & 38296.692 & $11576_{3 / 2}^{\circ}-49873_{5 / 2}^{\circ}$ \\
\hline 2639.053 & 1 & 5 & 37881.092 & $10572_{9 / 2}^{\circ}-48453_{7 / 2}^{\circ}$ & 2609.857 & 25 & 75 & 38304.835 & $4113_{5 / 2}-42418_{3 / 2}^{\circ}$ \\
\hline 2637.098 & 0 & 8 & 37909.173 & $11576_{3 / 2}^{\circ}-49485_{1 / 2}$ & 2608.321 & 4 & 25 & 38327.391 & $8378_{7 / 2}^{\circ}-46706_{7 / 2}^{\circ}$ \\
\hline 2635.414 & 3 & 10 & 37933.395 & $9238_{9 / 2}^{\circ}-47171_{9 / 2}$ & 2607.775 & 3 & 8 & 38335.415 & $6168_{7 / 2}^{\circ}-44503_{7 / 2}$ \\
\hline 2635.290 & 3 & 15 & 37935.180 & $12472_{5 / 2}^{\circ}-50407_{\tau / 2}$ & 2607.481 & 8 & 25 & 38339.738 & $1521_{5 / 2}-39861_{5 / 2}^{\circ}$ \\
\hline 2635.043 & 0 & 5 & 37938.736 & $16906_{7 / 2}^{\circ}-54845_{9 / 2}$ & 2605.994 & 4 & 25 & 38361.613 & $13468_{9 / 2}^{\circ}-51830_{7 / 2}$ \\
\hline 2634.108 & 2 & 20 & 37952.202 & $11116_{\frac{1}{2}}^{\circ}-49068_{5 / 2}$ & 2603.909 & 3 & 25 & 38392.328 & $15453_{7 / 2}^{\circ}-53845_{5 / 2}^{\circ}$ \\
\hline 2633.327 & 10 & 50 & 37963.457 & $8460_{3 / 2}-46423_{3 / 2}^{\circ}$ & 2603.560 & 0 & 15 & 38397.474 & $17837_{1 / 2}^{o}-56235_{3 / 2}$ \\
\hline 2632.925 & 2 & 10 & 37969.253 & $9202_{7 / 2}^{\circ}-47171_{9 / 2}$ & & & & & $20989_{9 / 2}^{\circ}-59387_{7 / 2}$ \\
\hline 2632.641 & 2 & 15 & 37973.349 & $8378_{7 / 2}^{\circ}-46352_{7 / 2}$ & 2603.014 & 3 & $25 b$ & 38405.528 & $8018_{3 / 2}-46423_{3 / 2}^{\circ}$ \\
\hline 2632.050 & 0 & 3 & 37981.875 & $12488_{9 / 2}^{\circ}-50470_{9 / 2}^{\circ}$ & 2602.154 & 0 & 4 & 38418.220 & $20969_{7 / 2}^{\circ}-59387_{7 / 2}^{\circ}$ \\
\hline 2630.817 & 10 & 50 & 37999.675 & $4113_{5 / 2}-42112_{3 / 2}^{\circ}$ & 2601.820 & 3 & 10 & 38423.151 & $10189_{11 / 2}^{\circ}-48612_{13 / 2}$ \\
\hline 2630.671 & 10 & 8 & 38001.784 & $1859_{3 / 2}-39861_{5 / 2}^{\circ}$ & 2601.630 & 2 & 3 & 38425.957 & $6700_{9 / 2}^{\circ}-45126_{9 / 2}^{\circ}$ \\
\hline 2630.107 & 0 & 5 & 38009.932 & $7828_{1 / 2}-45838_{3 / 2}^{\circ}$ & 2601.045 & 2 & 5 & 38434.599 & $14484_{11 / 2}^{\circ}-52918_{13 / 2}$ \\
\hline 2630.015 & 5 & 75 & 38011.262 & $9720_{7 / 2}^{\circ}-47731_{9 / 2}$ & 2600.935 & $8 \mathrm{~b}$ & 10 & 38436.225 & $0_{3 / 2}-38436_{3 / 2}^{\circ}$ \\
\hline
\end{tabular}


TABlE 3. Classified lines of Th II-Continued

\begin{tabular}{|c|c|c|c|c|c|c|c|c|c|}
\hline \multirow{2}{*}{$\begin{array}{c}\text { Wavelength } \\
\AA\end{array}$} & \multicolumn{2}{|c|}{ Intensity } & \multirow{2}{*}{$\begin{array}{c}\text { Wavenumber } \\
\mathbf{c m}^{-1}\end{array}$} & \multirow{2}{*}{ Classification } & \multirow{2}{*}{$\begin{array}{c}\text { Wavelength } \\
\AA\end{array}$} & \multicolumn{2}{|c|}{ Intensity } & \multirow{2}{*}{$\begin{array}{c}\text { Wavenumber } \\
\mathbf{c m}^{-1}\end{array}$} & \multirow{2}{*}{ Classification } \\
\hline & Lamp & Spark & & & & Lamp & Spark & & \\
\hline 2600.880 & $25 b$ & 25 & 38437.037 & $9238_{9 / 2}^{\circ}-47675_{11 / 2}$ & 2552.923 & 0 & 3 & 39159.036 & $11576_{3 / 2}^{\circ}-50735_{3 / 2}$ \\
\hline 2598.741 & 0 & 10 & 38468.673 & $7331_{5 / 2}^{\circ}-45800_{5 / 2}$ & 2552.281 & 0 & 8 & 39168.885 & $10189_{11 / 2}^{\circ}-49357_{11 / 2}$ \\
\hline 2597.046 & $50 \mathrm{~b}$ & $150 \mathrm{~b}$ & 38493.778 & $0_{3 / 2}-38493_{5 / 2}^{\circ}$ & 2551.934 & 3 & 10 & 39174.211 & $4113_{5 / 2}-43287_{3 / 2}^{\circ}$ \\
\hline 2595.036 & 8 & 20 & 38523.592 & $8378_{7 / 2}^{\circ}-46902_{5 / 2}^{\circ}$ & 2551.234 & 5 & 10 & 39184.96 & $1521_{5 / 2}-40706_{7 / 2}^{\circ}$ \\
\hline 2594.662 & 2 & 20 & 38529.145 & $9202_{7 / 2}^{\circ}-47731_{9 / 2}$ & 2550.749 & 0 & 8 & 39192.409 & $15305_{9 / 2}-54497_{7 / 2}^{\circ}$ \\
\hline 2592.786 & 0 & 15 & 38557.020 & $15453_{7 / 2}^{\circ}-54010_{7 / 2}$ & 2550.255 & 0 & 3 & 39200.000 & $10673_{5 / 2}^{\circ}-49873_{5 / 2}$ \\
\hline 2591.370 & 2 & 5 & 38578.088 & $9720_{7 / 2}^{\circ}-48298_{7 / 2}$ & 2549.987 & 5 & 15 & 39204.120 & $6700_{9 / 2}^{\circ}-45904_{9 / 2}$ \\
\hline 2590.229 & 4 & 20 & 38595.080 & $7828_{1 / 2}-46423_{3 / 2}^{\circ}$ & 2548.134 & 81 & 50 & 39232.62 & $9585_{5 / 2}^{\circ}-48817_{3 / 2}$ \\
\hline 2590.070 & 2 & 5 & 38597.449 & $7828_{1 / 2}-46426_{1 / 2}^{\circ}$ & 2547.898 & 15 & 75 & 39236.261 & $4146_{7 / 2}-43382_{5 / 2}^{\circ}$ \\
\hline 2589.485 & 3 & 8 & 38606.169 & $4490_{5 / 2}^{\circ}-43096_{5 / 2}$ & 2547.016 & 0 & 3 & 39249.847 & $7331_{5 / 2}^{\circ}-46581_{5 / 2}$ \\
\hline 2589.059 & 50 & 100 & 38612.520 & $1859_{3 / 2}-40472_{3 / 2}^{\circ}$ & 2546.953 & 0 & 8 & 39250.818 & $9202_{7 / 2}^{\circ}-48453_{7 / 2}$ \\
\hline 2588.916 & 8 & 20 & 38614.653 & $6691_{3 / 2}^{\circ}-45306_{3 / 2}$ & 2545.744 & 8 & 25 & 39269.458 & $4113_{5 / 2}-43382_{5 / 2}^{\circ}$ \\
\hline 2587.615 & 0 & 4 & 38634.067 & $14101_{\mathrm{i} / 2}^{\circ}-52735_{3 / 2}$ & 2545.339 & 8 & 50 & 39275.70 & $6213_{9 / 2}-45489_{9 / 2}^{\circ}$ \\
\hline 2587.252 & 3 & 20 & 38639.486 & $6168_{7 / 2}^{\circ}-44807_{7 / 2}^{\circ}$ & 2544.364 & 0 & 8 & 39290.755 & $11116_{7 / 2}^{\circ}-50407_{7 / 2}$ \\
\hline 2587.246 & 0 & 20 & 38639.576 & $6168_{7 / 2}^{\circ}-44807_{7 / 2}^{\circ}$ & 2543.217 & 0 & 8 & 39308.474 & $12219_{3 / 2}-51528_{3 / 2}^{\circ}$ \\
\hline 2586.158 & 3 & $25 b$ & 38655.831 & $10189_{11 / 2}^{\circ}-48844_{9 / 2}$ & 2542.871 & 0 & 41 & 39313.822 & $4490_{5 / 2}^{\circ}-43803_{7 / 2}$ \\
\hline 2585.726 & 4 & 5 & 38662.289 & $1521_{5 / 2}-40184_{7 / 2}^{\circ}$ & 2542.642 & 4 & 25 & 39317.36 & $4490_{5 / 2}^{\circ}-43807_{3 / 2}$ \\
\hline 2583.456 & 4 & 20 & 38696.258 & $13250_{5 / 2}-51946_{5 / 2}^{\circ}$ & 2540.865 & 0 & 3 & 39344.858 & $12485_{\tau / 2}^{\circ}-51830_{\tau / 2}$ \\
\hline & & & & $7001_{3 / 2}-45697_{3 / 2}^{\circ}$ & 2540.360 & 2 & $10 \mathrm{~b}$ & 39352.679 & $8378_{7 / 2}^{\circ}-47731_{9 / 2}$ \\
\hline 2582.939 & 0 & $4 b$ & 38704.003 & $10673_{5 / 2}^{\circ}-49377_{7 / 2}^{\circ}$ & 2540.309 & 0 & $8 \mathrm{~b}$ & 39353.469 & $11116_{7 / 2}^{\circ}-50470_{9 / 2}^{\circ}$ \\
\hline 2582.099 & 2 & 20 & 38716.593 & $15453_{7 / 2}^{\circ}-54169_{\tau / 2}$ & 2538.931 & 10 & 20 & 39374.827 & $7331_{5 / 2}^{\circ}-46706_{7 / 2}$ \\
\hline 2580.844 & 0 & 5 & 38735.419 & $9585_{5 / 2}^{\circ}-48320_{5 / 2}$ & 2538.832 & 0 & 5 & 39376.362 & $12570_{7 / 2}-51946_{5 / 2}^{\circ}$ \\
\hline 2580.699 & 10 & 50 & 38737.595 & $4490_{5 / 2}^{\circ}-43227_{5 / 2}$ & 2538.680 & 0 & $3 \mathrm{~s}$ & 39378.719 & $14790_{7 / 2}^{\circ}-54169_{7 / 2}$ \\
\hline 2580.354 & 5 & 50 & 38742.774 & $10379_{9 / 2}-49121_{7 / 2}^{\circ}$ & 2538.047 & 0 & 3 & 39388.540 & $10572_{9 / 2}^{\circ}-49960_{7 / 2}$ \\
\hline 2580.264 & 0 & 15 & 38744.125 & $13818_{7 / 2}^{\circ}-52562_{7 / 2}$ & 2537.759 & 0 & 101 & 39393.010 & $12472_{5 / 2}^{\circ}-51865_{5 / 2}$ \\
\hline 2579.768 & 0 & 5 & 38751.574 & $12902_{3 / 2}^{\circ}-51653_{1 / 2}$ & 2535.873 & 4 & 20 & 39422.306 & $7001_{3 / 2}-46423_{3 / 2}^{\circ}$ \\
\hline 2579.437 & 15 & $75 b$ & 38756.55 & $4490_{5 / 2}^{\circ}-43246_{7 / 2}$ & 2534.589 & 4 & 10 & 39442.275 & $6168_{7 / 2}^{\circ}-45610_{5 / 2}$ \\
\hline 2578.958 & 2 & 25 & 38763.744 & $13406_{13 / 2}^{\circ}-52170_{11 / 2}$ & 2534.193 & 0 & 3 & 39448.438 & $11576_{3 / 2}^{\circ}-51024_{3 / 2}$ \\
\hline 2578.619 & 0 & 10 & 38768.840 & $9238_{9 / 2}^{\circ}-48006_{9 / 2}$ & 2534.091 & 0 & 20 & 39450.026 & $12485_{7 / 2}^{\circ}-51935_{5 / 2}$ \\
\hline 2578.238 & 0 & 4 & 38774.569 & $17460_{5 / 2}^{\circ}-56235_{3 / 2}^{\circ}$ & 2533.871 & 0 & 15 & 39453.451 & $6244_{1 / 2}-45697_{3 / 2}^{\circ}$ \\
\hline 2576.910 & 10 & 50 & 38794.550 & $1859_{3 / 2}-40654_{5 / 2}^{\circ}$ & 2533.224 & 0 & 10 & 39463.527 & $12472_{5 / 2}^{\circ}-51935_{5 / 2}$ \\
\hline 2576.688 & 25 & 75 & 38797.892 & $4146_{7 / 2}-42944_{7 / 2}^{\circ}$ & 2533.152 & 0 & 5 & 39464.648 & $14545_{5 / 2}^{\circ}-54010_{7 / 2}$ \\
\hline 2576.322 & 0 & 75 & 38803.404 & $13468_{9 / 2}^{\circ}-52272_{7 / 2}$ & 2532.432 & 5 & 25 & 39475.86 & $6213_{9 / 2}-45689_{7 / 2}^{\circ}$ \\
\hline 2576.209 & 0 & 8 & 38805.105 & $10572_{9 / 2}^{\circ}-49377_{7 / 2}$ & 2531.883 & 0 & 10 & 39484.427 & $16906_{7 / 2}^{0}-56391_{5 / 2}$ \\
\hline 2574.485 & 15 & 5 & 38831.10 & $4113_{5 / 2}-42944_{7 / 2}^{\circ}$ & 2527.877 & 0 & 15 & 39546.995 & $11116_{7 / 2}^{\circ}-50663_{5 / 2}$ \\
\hline 2573.631 & 0 & 10 & 38843.974 & $11116_{7 / 2}^{\circ}-49960_{7 / 2}$ & 2526.343 & 3 & 25 & 39571.007 & $7331_{5 / 22}^{\circ}-46902_{5 / 2}$ \\
\hline 2570.653 & 0 & 50 & 38888.970 & $16033_{5 / 2}^{\circ}-54922_{3 / 2}$ & 2526.223 & $3 b$ & 15 & 39572.88 & $6691_{3 / 2}^{\circ}-46264_{3 / 2}$ \\
\hline 2566.916 & 0 & 25 & 38945.583 & $8378_{7 / 2}^{\circ}-47324_{5 / 2}$ & 2525.925 & 0 & 25 & 39577.554 & $9711_{7 / 2}-49289_{5 / 2}^{\circ}$ \\
\hline 2566.588 & 50 & 100 & 38950.559 & $1521_{5 / 2}-40472_{3 / 2}^{\circ}$ & 2524.229 & 21 & 3 & 39604.144 & $7331_{5 / 2}^{\circ}-46935_{3 / 2}$ \\
\hline 2565.593 & 75 & 100 & 38965.665 & $1859_{3 / 2}-40825_{i / 2}^{0}$ & 2522.775 & 5 & 15 & 39626.969 & $4146_{7 / 2}-43773_{7 / 2}^{\circ}$ \\
\hline 2562.675 & 0 & 8 & 39010.030 & $11725_{i / 2}^{\circ}-50735_{3 / 2}$ & 2522.713 & 0 & 4 & 39627.942 & $8378_{7 / 2}^{\circ}-48006_{9 / 2}$ \\
\hline 2561.938 & 0 & 75 & 39021.251 & $6168_{7 / 2}^{\circ}-45189_{5 / 2}$ & 2521.777 & 0 & 5 & 39642.650 & $9202_{7 / 2}^{\circ}-48844_{9 / 2}$ \\
\hline 2561.149 & 0 & 25 & 39033.272 & $12902_{3 / 2}^{\circ}-51935_{5 / 2}$ & 2520.660 & 8 & 75 & 39660.216 & $4113_{5 / 2}-43773_{7 / 2}^{\circ}$ \\
\hline 2560.934 & 2 & 75 & 39036.549 & $14484_{1 / 2}^{\circ}-53520_{9 / 2}$ & 2519.848 & 0 & 20 & 39672.995 & $17272_{9 / 2}^{\circ}-56945_{11 / 2}$ \\
\hline 2559.793 & 0 & 10 & 39053.947 & $7331_{5 / 2}^{\circ}-46385_{7 / 2}$ & 2519.288 & 0 & 3 & 39681.813 & $12488_{9 / 2}^{\circ}-52170_{11 / 2}$ \\
\hline 2558.328 & 0 & $10 \mathrm{~b}$ & 39076.310 & $20310_{5 / 2}^{\circ}-59387_{7 / 2}$ & 2519.070 & 0 & 15 & 39685.247 & $6700_{9 / 2}^{\circ}-46385_{7 / 2}$ \\
\hline 2557.024 & 0 & 4 & 39096.236 & $9202_{7 / 2}^{\circ}-48298_{7 / 2}$ & 2516.430 & 0 & 50 & 39726.878 & $12219_{3 / 2}-51946_{5 / 2}^{\circ}$ \\
\hline 2556.480 & 0 & 10 & 39104.555 & $9585_{5 / 2}^{\circ}-48689_{3 / 2}$ & 2515.997 & 0 & 3 & 39733.715 & $15305_{9 / 2}-55038_{9 / 2}^{\circ}$ \\
\hline 2554.649 & 5 & 2 & 39132.58 & $1521_{5 / 2}-40654_{5 / 2}^{\circ}$ & 2515.853 & 0 & 5 & 39735.989 & $6168_{7 / 2}^{\circ}-45904_{9 / 2}$ \\
\hline 2553.243 & 4 & 25 & 39154.128 & $7001_{3 / 2}-46155_{5 / 2}^{\circ}$ & 2511.414 & 0 & 8 & 39806.219 & $17272_{9 / 2}^{\circ}-57078_{9 / 2}$ \\
\hline
\end{tabular}


TABLE 3. Classified lines of Th II-Continued

\begin{tabular}{|c|c|c|c|c|c|c|c|c|c|}
\hline \multirow{2}{*}{$\begin{array}{c}\text { Wavelength } \\
\AA\end{array}$} & \multicolumn{2}{|c|}{ Intensity } & \multirow{2}{*}{$\begin{array}{c}\text { Wavenumber } \\
\mathbf{c m}^{-1}\end{array}$} & \multirow{2}{*}{ Classification } & \multirow{2}{*}{$\begin{array}{c}\text { Wavelength } \\
\AA \\
\end{array}$} & \multicolumn{2}{|c|}{ Intensity } & \multirow{2}{*}{$\begin{array}{c}\text { Wavenumber } \\
\mathrm{cm}^{-1}\end{array}$} & \multirow{2}{*}{ Classification } \\
\hline & Lamp & Spark & & & & Lamp & Spark & & \\
\hline 2511.159 & 5 & 10 & 39810.261 & $4490_{5 / 2}^{\circ}-44300_{3 / 2}$ & 2460.168 & 0 & 2 & 40635.335 & $9202_{7 / 2}^{\circ}-49837_{9 / 2}$ \\
\hline 2509.969 & 3 & 20 & 39829.134 & $9585_{5 / 2}^{\circ}-494.14_{3 / 2}$ & 2459.008 & 8 & 25 & 40654.503 & $0_{3 / 2}-40654_{5 / 2}^{\circ}$ \\
\hline 2509.707 & 0 & 8 & 39833.291 & $12902_{3 / 2}^{\circ}-52735_{3 / 2}$ & 2458.015 & 0 & 5 & 40670.925 & $9202_{7 / 2}^{\circ}-49873_{5 / 2}$ \\
\hline 2507.924 & 10 & 251 & 39861.609 & $0_{3 / 2}-39861_{5 / 2}^{\circ}$ & 2457.746 & $2 \mathbf{b}$ & 10 & 40675.376 & $4146_{7 / 2}-44821_{5 / 2}^{\circ}$ \\
\hline 2505.611 & 8 & 25 & 39898.404 & $4490_{5 / 2}^{\circ}-44388_{5 / 2}$ & 2456.865 & 3 & 25 & 40689.961 & $8378_{7 / 2}^{\circ}-49068_{5 / 2}^{\circ}$ \\
\hline 2504.279 & $10 \mathrm{~b}$ & 75 & 39919.62 & $8378_{7 / 2}^{\circ}-48298_{7 / 2}$ & 2456.380 & 5 & 10 & 40697.994 & $1521_{5 / 2}-42219_{5 / 2}^{\circ}$ \\
\hline 2502.339 & 0 & 4 & 39950.570 & $11725_{1 / 2}^{\circ}-51676_{3 / 2}$ & 2456.290 & $4 b$ & 50 & 40699.485 & $4490_{5 / 2}^{\circ}-45189_{5 / 2}$ \\
\hline 2499.696 & 0 & 5 & 39992.808 & $7331_{5 / 2}^{\circ}-47324_{5 / 2}$ & 2455.904 & 0 & 5 & 40705.882 & $14790_{7 / 2}^{\circ}-55496_{9 / 2}$ \\
\hline 2498.873 & 8 & 75 & 40005.979 & $6700_{9 / 2}^{\circ}-46706_{7 / 2}$ & 2454.890 & 5 & 5 & 40722.694 & $9238_{9 / 2}^{\circ}-49960_{7 / 2}$ \\
\hline 2498.405 & 8 & 20 & 40013.472 & $4490_{5 / 2}^{\circ}-44503_{7 / 2}$ & 2454.195 & 0 & 3 & 40734.225 & $6168_{7 / 2}^{\circ}-46902_{5 / 2}$ \\
\hline 2496.522 & 2 & 10 & 40043.650 & $15453_{7 / 2}^{\circ}-55496_{9 / 2}$ & 2449.087 & 5 & 20 & 40819.178 & $11116_{7 / 2}^{\circ}-51935_{5 / 2}$ \\
\hline 2496.014 & 1 & 5 & 40051.800 & $13468_{9 / 2}^{\circ}-53520_{9 / 2}$ & 2448.694 & 0 & 4 & 40825.729 & $0_{3 / 2}-40825_{1 / 2}^{\circ}$ \\
\hline 2495.563 & 0 & 4 & 40059.038 & $10572_{9 / 2}^{\circ}-50631_{11 / 2}$ & 2444.463 & 10 & 25 & 40896.386 & $15786_{5 / 2}-56683_{7 / 2}^{\circ}$ \\
\hline 2495.454 & 0 & 4 & 40060.787 & $9061_{5 / 2}-49121_{7 / 2}^{\circ}$ & & & & & $1521_{5 / 2}-42418_{3 / 2}^{\circ}$ \\
\hline 2495.354 & 8 & 75 & 40062.39 & $4490_{5 / 2}^{\circ}-44552_{5 / 2}$ & 2443.953 & 10 & 15 & 40904.920 & $4490_{5 / 2}^{\circ}-45395_{7 / 2}$ \\
\hline 2494.775 & 1 & 10 & 40071.690 & $8460_{3 / 2}-48532_{1 / 2}^{\circ}$ & 2437.536 & 0 & 75 & 41012.597 & $14484_{11 / 2}^{\circ}-55496_{9 / 2}$ \\
\hline 2494.452 & 0 & 3 & 4.0076 .878 & $12485_{7 / 2}^{\circ}-52562_{7 / 2}^{\circ}$ & 2436.353 & 0 & 5 & 41032.510 & $12488_{9 / 2}^{\circ}-53520_{9 / 2}^{\circ}$ \\
\hline 2494.416 & 0 & 4 & 40077.457 & $11576_{3 / 2}^{\circ}-51653_{1 / 2}$ & 2432.847 & 8 & 50 & 41091.638 & $10855_{7 / 2}-51946_{5 / 2}^{\circ}$ \\
\hline 2493.616 & 2 & 10 & 40090.313 & $12472_{5 / 2}^{\circ}-52562_{7 / 2}^{\circ}$ & 2431.140 & 8 & 8 & 41120.488 & $4490_{5 / 2}^{\circ}-45610_{5 / 2}$ \\
\hline 2492.538 & 0 & 8 & 40107.651 & $11116_{7 / 2}^{\circ}-51224_{9 / 2}$ & 2429.041 & 0 & 8 & 41156.019 & $6168_{7 / 2}^{\circ}-47324_{5 / 2}^{\circ}$ \\
\hline 2490.596 & 2 & 8 & 40138.921 & $19248_{5 / 2}^{\circ}-59387_{7 / 2}$ & 2426.927 & 0 & 10 & 41191.865 & $10673_{5 / 2}^{\circ}-51865_{5 / 2}$ \\
\hline 2490.104 & 0 & 15 & 40146.852 & $15349_{11 / 2}^{\circ}-55496_{9 / 2}$ & 2423.665 & 0 & 75 & 41247.301 & $13250_{5 / 2}-54497_{7 / 2}^{\circ}$ \\
\hline 2489.736 & 4 & 15 & 40152.785 & $9720_{7 / 2}^{\circ}-49873_{5 / 2}$ & 2422.457 & 2 & 3 & 41267.868 & $9202_{7 / 2}^{\circ}-50470_{9 / 2}$ \\
\hline 2489.607 & 10 & 100 & 40154.866 & $6213_{9 / 2}-46368_{9 / 2}^{\circ}$ & 2422.246 & 3 & 8 & 41271.463 & $8018_{3 / 2}-49289_{5 / 2}^{\circ}$ \\
\hline 2488.554 & 0 & 5 & 40171.856 & $16906_{7 / 2}^{\circ}-57078_{9 / 2}$ & 2419.979 & 0 & 5 & 41310.122 & $4490_{5 / 2}^{\circ}-45800_{5 / 2}$ \\
\hline 2488.087 & 2 & 15 & 40179.395 & $6244_{1 / 2}-46423_{3 / 2}^{\circ}$ & 2418.074 & 5 & 10 & 41342.665 & $4146_{7 / 2}-45489_{9 / 2}^{\circ}$ \\
\hline 2487.322 & 0 & 8 & 40191.752 & $13818_{7 / 2}^{\circ}-54010_{7 / 2}$ & 2413.407 & 25 & 50 & 41422.606 & $1521_{5 / 2}-42944_{7 / 2}^{\circ}$ \\
\hline 2486.691 & 0 & 4 & 40201.950 & $16033_{5 / 2}^{\circ}-56235_{3 / 2}$ & 2412.043 & 0 & 4 & 41446.029 & $1111_{7 / 2}^{\circ}-52562_{7 / 2}$ \\
\hline 2486.126 & 3 & 25 & 40211.085 & $6691_{3 / 2}^{\circ}-46902_{5 / 2}$ & 2411.291 & 0 & 25 & 4.1458 .954 & $8378_{7 / 2}^{\circ}-49837_{9 / 2}$ \\
\hline 2485.764 & 0 & 5 & 40216.941 & $6168_{7 / 2}^{\circ}-46385_{7 / 2}$ & 2411.143 & 0 & 4 & 41461.498 & $9202_{7 / 2}^{\circ}-50663_{5 / 2}$ \\
\hline 2485.055 & 2 & 20 & 40228.42 & $9061_{5 / 2}-49289_{5 / 2}^{\circ}$ & 2407.574 & 5 & $8 b$ & 41522.956 & $1859_{3 / 2}-43382_{5 / 2}^{\circ}$ \\
\hline 2484.330 & 0 & 4 & 40240.153 & $9720_{7 / 2}^{\circ}-49960_{7 / 2}$ & 2407.480 & 0 & $4 b$ & 41524.577 & $12485_{7 / 2}^{\circ}-54010_{7 / 2}$ \\
\hline 2484.074 & 0 & 10 & 40244.300 & $6691_{3 / 2}^{\circ}-46935_{3 / 2}$ & 2407.137 & 10 & 10 & 41530.494 & $7001_{3 / 2}-48532_{1 / 2}^{\circ}$ \\
\hline 2483.955 & 2 & 10 & 40246.228 & $11116_{7 / 2}^{\circ}-51362_{5 / 2}^{\circ}$ & 2406.704 & 0 & $10 \mathrm{~b}$ & 41537.965 & $12472_{5 / 2}^{\circ}-54010_{7 / 2}$ \\
\hline 2483.489 & 0 & 4 & 40253.779 & $15242_{9 / 2}^{\circ}-55496_{9 / 2}$ & 2404.497 & 0 & 50 & 41576.088 & $4113_{5 / 2}-45689_{7 / 2}^{\circ}$ \\
\hline 2482.892 & 0 & 5 & 40263.457 & $12472_{5 / 2}^{\circ}-52735_{3 / 2}^{\circ}$ & 2404.171 & 10 & 50 & 41581.72 & $8378_{7 / 2}^{\circ}-49960_{7 / 2}$ \\
\hline 2481.813 & 2 & 5 & 40280.961 & $10189_{11 / 2}^{\circ}-50470_{9 / 2}^{\circ}$ & 2401.635 & 0 & 3 & 41625.630 & $15453_{7 / 2}^{\circ}-57078_{9 / 2}^{\circ}$ \\
\hline 2481.405 & 2 & 25 & 40287.584 & $9585_{5 / 2}^{\circ}-49873_{5 / 2}$ & 2401.413 & 0 & 3 & 41629.478 & $6691_{3 / 2}^{\circ}-48320_{5 / 2}$ \\
\hline 2479.557 & 0 & 8 & 40317.608 & $4490_{5 / 2}^{\circ}-44807_{7 / 2}^{\circ}$ & 2401.139 & 0 & 4 & 41634.228 & $10673_{5 / 2}^{\circ}-52307_{3 / 2}^{\circ}$ \\
\hline 2477.087 & 0 & 15 & 40357.807 & $16033_{5 / 2}^{\circ}-56391_{5 / 2}$ & 2398.341 & 0 & 4 & 41682.796 & $9585_{5 / 2}^{\circ}-51268_{7 / 2}$ \\
\hline 2476.971 & 8 & 25 & 40359.697 & $1859_{3 / 2}-42219_{5 / 2}^{\circ}$ & 2398.267 & 0 & 5 & 41684.083 & $12485_{7 / 2}^{\circ}-54169_{7 / 2}$ \\
\hline 2476.898 & 0 & 25 & 40360.886 & $14484_{11 / 2}^{\circ}-54845_{9 / 2}^{\prime}$ & 2397.491 & 1 & 4 & 41697.573 & $12472_{5 / 2}^{\circ}-54169_{7 / 2}$ \\
\hline 2471.005 & 1 & 5 & 40457.135 & $6691_{3 / 2}^{\circ}-47148_{3 / 2}^{\circ}$ & 2395.200 & 0 & 3 & 41737.454 & $7331_{5 / 2}^{\circ}-49068_{5 / 2}$ \\
\hline 2470.148 & 0 & 15 & 40471.170 & $6700_{9 / 2}^{\circ}-47171_{9 / 2}$ & 2393.574 & 5 & 3 & 41765.805 & $1521_{5 / 2}-43287_{3 / 2}^{\circ}$ \\
\hline 2468.151 & 5 & 100 & 40503.913 & $4146_{\pi / 2}-44650_{\pi / 2}^{\circ}$ & 2393.111 & 50 & 25 & 41773.88 & $4490_{5 / 2}^{\circ}-46264_{3 / 2}$ \\
\hline 2467.416 & 2 & 15 & 40515.978 & $8605_{5 / 2}-49121_{7 / 2}^{\circ}$ & 2388.143 & 10 & 10 & 41860.779 & $1521_{5 / 2}-43382_{5 / 2}^{\circ}$ \\
\hline 2466.904 & 0 & 5 & 40524.386 & $15710_{3 / 2}^{\circ}-56235_{3 / 2}$ & 2388.073 & 8 & 8 & 41862.006 & $4490_{5 / 2}^{\circ}-46352_{7 / 2}$ \\
\hline 2466.128 & $10 \mathrm{r}$ & 50 & 40537.14 & $4113_{5 / 2}-44650_{\tau / 2}^{\circ}$ & 2386.179 & 0 & 3 & 41895.231 & $4490_{5 / 2}^{\circ}-46385_{7 / 2}^{\circ}$ \\
\hline 2466.065 & 5 & 8 & 40538.172 & $7331_{5 / 2}^{\circ}-47869_{3 / 2}$ & 2384.358 & 5 & 20 & 41927.23 & $12570_{7 / 2}-54497_{7 / 2}^{\circ}$ \\
\hline 2462.333 & 0 & 3 & 40599.609 & $9238_{9 / 2}^{\circ}-49837_{9 / 2}^{\circ}$ & 2379.751 & 0 & 4 & 42008.386 & $12485_{7 / 2}^{\circ}-54493_{5 / 2}$ \\
\hline
\end{tabular}


TABLE 3. Classified lines of Th II-Continued

\begin{tabular}{|c|c|c|c|c|}
\hline \multirow{2}{*}{$\begin{array}{c}\text { Wavelength } \\
\AA\end{array}$} & \multicolumn{2}{|c|}{ Intensity } & \multirow{2}{*}{$\begin{array}{c}\text { Wavenumber } \\
\text { cm }^{-1}\end{array}$} & \multirow{2}{*}{ Classification } \\
\hline & Lamp & Spark & & \\
\hline 2377.830 & 25 & 100 & 42042.321 & $4113_{5 / 2}-46155_{5 / 2}^{\circ}$ \\
\hline 2375.076 & 15 & 25 & 42091.067 & $4490_{5 / 2}^{\circ}-46581_{5 / 2}$ \\
\hline 2373.991 & 1 & 4 & 42110.302 & $9720_{7 / 2}^{\circ}-51830_{\tau / 2}$ \\
\hline 2373.840 & 15 & 50 & 42112.981 & $\begin{array}{r}0_{3 / 2}-42112_{3 / 2}^{\circ} \\
4490_{5 / 2}^{\circ}-46603_{5 / 2}^{\circ}\end{array}$ \\
\hline 2373.723 & 0 & 50 & 42115.057 & $17272_{9 / 2}^{\circ}-59387_{7 / 2}$ \\
\hline 2372.043 & 2 & 8 & 42144.882 & $9720_{7 / 2}^{\circ}-51865_{5 / 2}$ \\
\hline 2371.155 & 0 & 5 & 42160.664 & $9202_{7 / 2}^{\circ}-51362_{5 / 2}$ \\
\hline 2368.047 & 15 & 25 & 42215.994 & $4490_{5 / 2}^{\circ}-46706_{7 / 2}$ \\
\hline 2367.842 & 50 & 100 & 42219.649 & $0_{3 / 2}-42219_{5 / 2}^{\circ}$ \\
\hline 2367.725 & 0 & 3 & 42221.735 & $4146_{7 / 2}-46368_{9 / 2}^{\circ}$ \\
\hline 2366.991 & 10 & 100 & 42234.83 & $9711_{7 / 2}-51946_{5 / 2}^{\circ}$ \\
\hline 2366.040 & 10 & 20 & 42251.801 & $1521_{5 / 2}-43773_{7 / 2}^{\circ}$ \\
\hline 2364.194 & 3 & 10 & 42284.789 & $\begin{array}{l}6168_{7 / 2}^{\circ}-48453_{7 / 2} \\
8378_{7 / 2}^{\circ}-50663_{5 / 2}^{\circ}\end{array}$ \\
\hline 2364.025 & 0 & 25 & 42287.812 & $6244_{1 / 2}-48532_{1 / 2}^{\circ}$ \\
\hline 2362.759 & 3 & 5 & 42310.469 & $4113_{5 / 2}-46423_{3 / 2}^{\circ}$ \\
\hline 2362.021 & 0 & 4 & 42323.687 & $6168_{7 / 2}^{\circ}-48492_{5 / 2}$ \\
\hline 2360.533 & 0 & 3 & 42350.365 & $9585_{5 / 2}^{\circ}-51935_{5 / 2}$ \\
\hline 2356.758 & 10 & 10 & 42418.20 & $0_{3 / 2}-42418_{3 / 2}^{\circ}$ \\
\hline 2355.242 & 15 & 20 & 42445.496 & $4490_{5 / 2}^{\circ}-46935_{3 / 2}$ \\
\hline 2355.003 & 0 & 4 & 42449.803 & $12472_{5 / 2}^{\circ}-54922_{3 / 2}$ \\
\hline 2354.030 & 8 & 25 & 42467.348 & $9061_{5 / 2}-51528_{3 / 2}^{\circ}$ \\
\hline 2349.686 & 0 & 4 & 42545.853 & $9400_{5 / 2}-51946_{5 / 2}^{\circ}$ \\
\hline 2349.344 & 0 & 3 & 42552.046 & $9720_{7 / 2}^{\circ}-52272_{7 / 2}$ \\
\hline 2343.494 & 0 & 10 & 42658.259 & $4490_{5 / 2}^{\circ}-47148_{3 / 2}$ \\
\hline 2343.242 & 1 & 5 & 42662.846 & $9202_{7 / 2}^{\circ}-51865_{5 / 2}$ \\
\hline 2342.873 & 0 & 5 & 42669.565 & $14275_{9 / 2}^{\circ}-56945_{11 / 2}$ \\
\hline 2339.566 & 0 & 4 & 42729.873 & $10189_{11 / 2}^{\circ}-52918_{13 / 2}$ \\
\hline 2336.051 & 0 & 3 & 42794.163 & $6691_{3 / 2}^{\circ}-49485_{1 / 2}$ \\
\hline 2333.684 & 0 & 3 & 42837.564 & $13248_{9 / 2}-56086_{9 / 2}^{\circ}$ \\
\hline 2333.258 & 3 & 10 & 42845.385 & $8378_{7 / 2}^{\circ}-51224_{9 / 2}$ \\
\hline 2330.868 & 4 & 25 & 42889.313 & $8378_{7 / 2}^{\circ}-51268_{7 / 2}$ \\
\hline 2329.057 & 0 & 10 & 42922.660 & $8605_{5 / 2}-51528_{3 / 2}^{\circ}$ \\
\hline 2326.920 & 50 & 50 & 42962.075 & $1859_{3 / 2}-44821_{5 / 2}^{\circ}$ \\
\hline 2325.729 & 0 & 8 & 42984.074 & $8378_{7 / 2}^{\circ}-51362_{5 / 2}$ \\
\hline 2317.451 & 0 & 3 & 43137.602 & $6700_{9 / 2}^{\circ}-49837_{9 / 2}^{\circ}$ \\
\hline 2308.754 & 0 & 3 & 43300.085 & $1521_{5 / 2}-44821_{5 / 2}^{\circ}$ \\
\hline 2308.590 & 4 & 15 & 43303.161 & $8378_{7 / 2}^{\circ}-51681_{9 / 2}$ \\
\hline 2306.573 & 1 & 8 & 43341.025 & $8605_{5 / 2}-51946_{5 / 2}^{\circ}$ \\
\hline 2305.545 & 0 & 8 & 43360.348 & $9202_{7 / 2}^{\circ}-52562_{\tau / 2}$ \\
\hline 2305.474 & 25 & 25 & 43361.683 & $1859_{3 / 2}-45221_{3 / 2}^{\circ}$ \\
\hline 2304.534 & 2 & 4 & 43379.368 & $4490_{5 / 2}^{\circ}-47869_{3 / 2}$ \\
\hline 2300.695 & 0 & 10 & 43451.746 & $8378_{7 / 2}^{\circ}-51830_{7 / 2}$ \\
\hline 2298.855 & 3 & 15 & 43486.521 & $8460_{3 / 2}-51946_{5 / 2}^{\circ}$ \\
\hline 2298.732 & 0 & 4 & 43488.848 & $12902_{3 / 2}^{\circ}-56391_{5 / 2}$ \\
\hline 2297.596 & 0 & 5 & 43510.348 & $8018_{3 / 2}-51528_{3 / 2}^{\circ}$ \\
\hline 2297.491 & 0 & 75 & 43512.337 & $15786_{5 / 2}-59299_{5 / 2}^{\circ}$ \\
\hline 2295.141 & 0 & 3 & 43556.885 & $8378_{7 / 2}^{\circ}-51935_{5 / 2}$ \\
\hline 2291.974 & 0 & 4 & 43617.066 & $9061_{5 / 2}-52678_{5 / 2}^{\circ}$ \\
\hline
\end{tabular}

\begin{tabular}{|c|c|c|c|c|}
\hline \multirow{2}{*}{$\begin{array}{c}\text { Wavelength } \\
\AA\end{array}$} & \multicolumn{2}{|c|}{ Intensity } & \multirow{2}{*}{$\begin{array}{c}\text { Wavenumber } \\
\mathbf{c m}^{-1}\end{array}$} & \multirow{2}{*}{ Classification } \\
\hline & Lamp & Spark & & \\
\hline 2290.644 & 0 & 20 & 43642.388 & $10855_{7 / 2}-54497_{7 / 2}^{\circ}$ \\
\hline 2287.248 & 0 & 4 & 43707.181 & $6700_{9 / 2}^{\circ}-50407_{7 / 2}$ \\
\hline 2285.315 & 5 & 5 & 43744.147 & $0_{3 / 2}-43744_{i / 2}^{\circ}$ \\
\hline 2282.362 & 0 & 5 & 43800.739 & $9720_{7 / 2}^{\circ}-53520_{9 / 2}$ \\
\hline 2281.965 & 4 & 20 & 43808.359 & $4490_{5 / 2}^{\circ}-48298_{7 / 2}$ \\
\hline 2280.425 & 20 & 50 & 43837.941 & $1859_{3 / 2}-45697_{3 / 2}^{\circ}$ \\
\hline 2277.538 & 0 & 3 & 43893.504 & $8378_{7 / 2}^{\circ}-52272_{7 / 2}$ \\
\hline 2276.922 & 0 & 5 & 43905.378 & $12485_{7 / 2}^{\circ}-56391_{5 / 2}$ \\
\hline 2275.594 & 0 & 3 & 43930.998 & $6700_{9 / 2}^{\circ}-50631_{11 / 2}$ \\
\hline 2271.931 & 5 & 8 & 44001.821 & $4490_{5 / 2}^{\circ}-48492_{5 / 2}$ \\
\hline 2270.403 & 0 & 3 & 44031.431 & $7331_{5 / 2}^{\circ}-51362_{5 / 2}$ \\
\hline 2265.919 & 3 & $20 \mathrm{~b}$ & 44118.557 & $10379_{9 / 2}-54497_{7 / 2}^{\circ}$ \\
\hline 2263.408 & 5 & 15 & 44167.497 & $1521_{5 / 2}-45689_{7 / 2}^{\circ}$ \\
\hline 2261.759 & 0 & 4 & 44199.695 & $4490_{5 / 2}^{\circ}-48689_{3 / 2}$ \\
\hline 2255.536 & 0 & 3 & 44321.630 & $12570_{7 / 2}-56892_{5 / 2}^{\circ}$ \\
\hline 2245.131 & 0 & 3 & 44527.018 & $7001_{3 / 2}-51528_{3 / 2}^{\circ}$ \\
\hline 2243.275 & 8 & 20 & 44563.855 & $1859_{3 / 2}-46423_{3 / 2}^{\circ}$ \\
\hline 2238.629 & 0 & 4 & 44656.333 & $10189_{11 / 2}^{\circ}-54845_{9 / 2}$ \\
\hline 2230.360 & 10 & 20 & 44821.879 & $0_{3 / 2}-44821_{5 / 2}^{\circ}$ \\
\hline 2225.268 & 0 & 5 & 44924.433 & $4490_{5 / 2}^{\circ}-49414_{3 / 2}$ \\
\hline 2222.430 & 0 & 3 & 44981.795 & $6700_{9 / 2}^{\circ}-51681_{9 / 2}$ \\
\hline 2222.287 & 0 & 3 & 44984.689 & $6691_{3 / 2}^{\circ}-51676_{3 / 2}^{\circ}$ \\
\hline 2216.767 & 0 & 5 & 45096.695 & $9400_{5 / 2}-54497_{7 / 2}^{\circ}$ \\
\hline 2215.115 & 0 & 3 & 45130.324 & $6700_{9 / 2}^{\circ}-51830_{7 / 2}$ \\
\hline 2214.539 & 1 & 15 & 45142.061 & $8378_{7 / 2}^{\circ}-53520_{9 / 2}$ \\
\hline 2212.980 & 0 & 3 & 45173.859 & $6691_{3 / 2}^{\circ}-51865_{5 / 2}$ \\
\hline 2210.648 & 5 & 20 & 45221.508 & $0_{3 / 2}-45221_{5 / 2}^{\circ}$ \\
\hline 2207.592 & 0 & 5 & 45284.102 & $6244_{1 / 2}-51528_{3 / 2}^{\circ}$ \\
\hline 2206.445 & 0 & 5 & 45307.641 & $10189_{11 / 2}^{\circ}-55496_{9 / 2}$ \\
\hline 2200.186 & 2 & 10 & 45436.517 & $9061_{5 / 2}-54497_{7 / 2}^{\circ}$ \\
\hline 2198.736 & 1 & $10 \mathrm{q}$ & 45466.478 & $8378_{\overline{7} / 2}^{\circ}-53845_{5 / 2}$ \\
\hline 2178.354 & 0 & 8 & 45891.845 & $8605_{5 / 2}-54497_{7 / 2}^{\circ}$ \\
\hline 2177.158 & 2 & 15 & 45917.053 & $4490_{5 / 2}^{\circ}-50407_{7 / 2}^{\circ}$ \\
\hline 2167.808 & 0 & 10 & 46115.076 & $8378_{7 / 2}^{\circ}-54493_{5 / 2}$ \\
\hline 2159.403 & 0 & 3 & 46294.549 & $9202_{7 / 2}^{\circ}-55496_{9 / 2}$ \\
\hline 2153.397 & 2 & 10 & 46423.655 & $0_{3 / 2}-46423_{3 / 2}^{\circ}$ \\
\hline 2151.412 & 0 & 10 & 46466.483 & $8378_{7 / 2}^{\circ}-54845_{9 / 2}$ \\
\hline 2148.266 & 0 & 5 & 46534.522 & $4490_{5 / 2}^{\circ}-51024_{3 / 2}$ \\
\hline 2141.930 & 0 & 4 & 46672.160 & $1859_{3 / 2}-48532_{1 / 2}^{\circ}$ \\
\hline 2132.770 & 2 & 15 & 46872.588 & $4490_{5 / 2}^{\circ}-51362_{5 / 2}$ \\
\hline 2118.875 & 0 & 3 & 47179.930 & $9711_{7 / 2}-56892_{5 / 2}^{\circ}$ \\
\hline 2118.604 & 0 & 3 & 47185.964 & $4490_{5 / 2}^{\circ}-51676_{3 / 2}$ \\
\hline 2111.708 & 0 & 10 & 47340.036 & $4490_{5 / 2}^{\circ}-51830_{7 / 2}$ \\
\hline 2110.148 & 0 & 4 & 47375.030 & $4490_{5 / 2}^{\circ}-51865_{5 / 2}^{\circ}$ \\
\hline 2092.794 & 0 & 3 & 47767.828 & $1521_{5 / 2}-49289_{5 / 2}^{\circ}$ \\
\hline 2091.383 & 0 & 4 & 47800.052 & $4146_{7 / 2}-51946_{5 / 2}^{\circ}$ \\
\hline 2090.632 & 0 & 15 & 47817.221 & $4490_{5 / 2}^{\circ}-52307_{3 / 2}$ \\
\hline 2089.920 & 0 & 15 & 47833.509 & $4113_{5 / 2}-51946_{5 / 2}^{\circ}$ \\
\hline 2088.053 & 0 & 2 & 47876.273 & $9202_{7 / 2}^{\circ}-57078_{9 / 2}^{\circ}$ \\
\hline 2079.541 & 0 & 5 & 48072.217 & $4490_{5 / 2}^{\circ}-52562_{7 / 2}$ \\
\hline
\end{tabular}


TABLE 3. Classified lines of Th II-Continued

\begin{tabular}{|c|c|c|c|c|c|c|c|c|c|}
\hline \multirow{2}{*}{$\begin{array}{c}\text { Wavelength } \\
\AA\end{array}$} & \multicolumn{2}{|c|}{ Intensity } & \multirow{2}{*}{$\begin{array}{c}\text { W avenumber } \\
\mathbf{c m}^{-1}\end{array}$} & \multirow{2}{*}{ Classification } & \multirow{2}{*}{$\begin{array}{c}\text { Wavelength } \\
\AA\end{array}$} & \multicolumn{2}{|c|}{ Intensity } & \multirow{2}{*}{$\begin{array}{c}\text { Wavenumber } \\
\text { cm }^{-1}\end{array}$} & \multirow{2}{*}{ Classification } \\
\hline & Lamp & Spark & & & & Lamp & Spark & & \\
\hline 2072.081 & 0 & 3 & 48245.266 & $4490_{5 / 2}^{\circ}-52735_{3 / 2}$ & & & & & \\
\hline 2059.848 & 0 & 8 & 48531.747 & $4146_{7 / 2}-52678_{5 / 2}^{\circ}$ & & & & & \\
\hline 2028.185 & 0 & 3 & 49289.296 & $0_{3 / 2}-4 \dot{9} 289_{5 / 2}^{\circ}$ & & & & & \\
\hline 1999.098 & 0 & 3 & 50006.353 & $1521_{5 / 2}-51528_{3 / 2}^{\circ}$ & & & & & \\
\hline 1995.888 & 0 & 10 & 50086.766 & $1859_{3 / 2}-51946_{5 / 2}^{\circ}$ & & & & & \\
\hline
\end{tabular}

